\title{
FEDERAL ENVIRONMENTAL STANDARDS OF POTENTIAL IMPORTANCE TO OPERATIONS AND ACTIVITIES AT U.S. DEPARTMENT OF ENERGY SITES
}

DRAFT

By:

K.M. Fowler

G.R. Bilyard

S.A. Davidson

R.J. Jonas

J. Joseph

JUNE 1993

\begin{abstract}
Prepared for:
U.S. Department of Energy

Assistant Secretary for

Environment, Safety and Health

Office of Environmental Guidance

Air, Water and Radiation Division

Washington, DC 20585

Prepared by:

Pacific Northwest Laboratory

Richland, Washington 99352

Under Contract No. AC06-76RL01830
\end{abstract}




\section{DISCLAIMER}

This report was prepared as an account of work sponsored by an agency of the United States Government. Neither the United States Government nor any agency thereof, nor any of their employees, make any warranty, express or implied, or assumes any legal liability or responsibility for the accuracy, completeness, or usefulness of any information, apparatus, product, or process disclosed, or represents that its use would not infringe privately owned rights. Reference herein to any specific commercial product, process, or service by trade name, trademark, manufacturer, or otherwise does not necessarily constitute or imply its endorsement, recommendation, or favoring by the United States Government or any agency thereof. The views and opinions of authors expressed herein do not necessarily state or reflect those of the United States Government or any agency thereof. 


\section{DISCLAIMER}

Portions of this document may be illegible in electronic image products. Images are produced from the best available original document. 


\section{Acknowledgements}

This document was prepared jointly by staff of the U.S. Department of Energy (DOE) Office of Environmental Guidance and Pacific Northwest Laboratory (PNL). Individuals who provided technical direction, reviewed, and wrote sections of this report include H. Beckert and A. Wa1lo with DOE, and K. M. Fowler, G.R. Bilyard, S. A. Davidson, R. J. Jonas, and J. Joseph with PNL. 


\section{CONTENTS}

ABBREVIATIONS ............................. vi

EXECUTIVE SUMMARY ..................

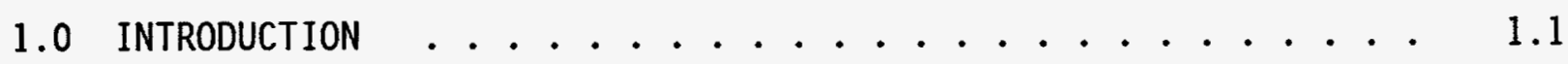

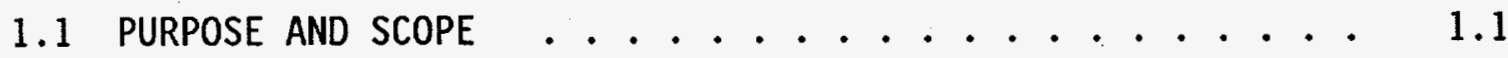

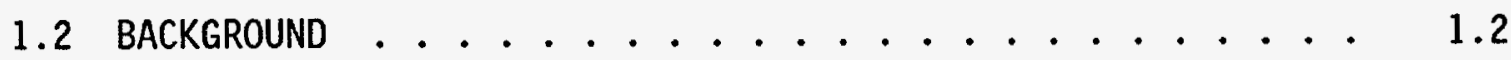

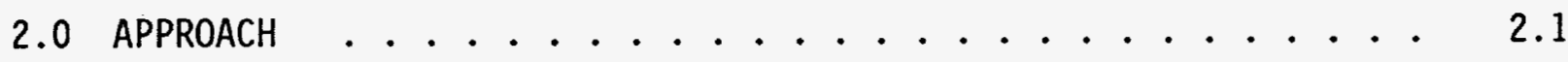

2.1 DETERMINATION OF INFORMATION SOURCES ....... 2.2

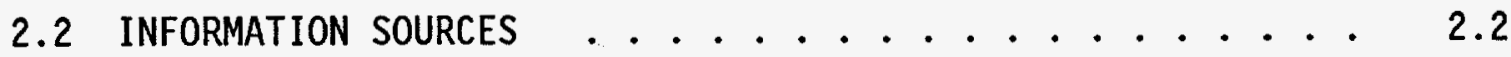

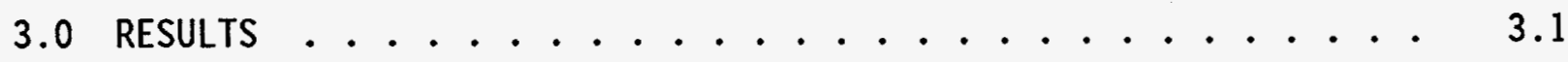

3.1 STANDARDS UNDER THE CLEAN AIR ACT . . . . . . . . 3.1

3.1.1 40 CFR Part 50 - National Primary and Secondary Ambient Air Quality Standards Pursuant to the Clean Air Act . . . . . . . . . 3.4

3.1.2 40 CFR Part 60 - Regulations on Standards of Performance for New Stationary Sources . . . . 3.5

3.1.3 40 CFR Part 61 - Regulations on National Emission Standards for Hazardous Air Pollutants Pursuant to the Clean Air Act ........... 3.10

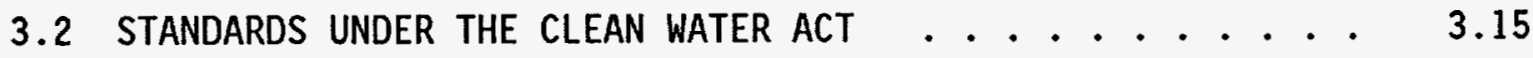

3.2.1 Categorical Standards ........... 3.16

3.2.2 Water Quality Criteria .......... 3.24

3.3 STANDARDS UNDER THE COMPREHENSIVE ENVIRONMENTAL $\ldots \ldots \ldots$
RESPONSE, COMPENSATION, AND LIABILITY ACT . . . . . 3.32

3.4 STANDARDS UNDER THE FEDERAL INSECTICIDE, FUNGICIDE, AND
RODENTICIDE ACT $\ldots \ldots \ldots . \ldots . \ldots . \ldots$

3.4.1 40 CFR 165 - Regulations for the Acceptance of Certain Pesticides and Recommended Procedures for the Disposal and Storage of Pesticides and Pesticides Containers 
3.4.2 40 CFR 170 - Worker Protection Standards for Agricultural Pesticides ..........

3.5 REGULATIONS FOR RADIATION PROTECTION . . . . . . 3.35

3.5.1 10 CFR 60 - Disposal of High-Level Radioactive Wastes in Geologic Repositories Pursuant to the Nuclear Waste Policy Act

3.5.2 10 CFR 72 - Licensing Requirements for the Independent Storage of Spent Nuclear Fuel and High-Level Radioactive Waste Pursuant to the Atomic Energy Act .............

3.5.3 40 CFR 191 - Radiation Protection Standards for Managing and Disposing of Spent Nuclear Fuel, High-Leve 7 and Transuranic Radioactive Waste Pursuant to the Atomic Energy Act ........

3.5.4 40 CFR 192 - Standards for Protection Against Uranium Mill Tailings Pursuant to the Atomic Energy Act

3.6 STANDARDS UNDER THE RESOURCE CONSERVATION AND RECOVERY ACT

3.6.1 40 CFR 261 - Regulations for Identifying Hazardous Waste ...........

3.6.2 40 CFR 264 Subpart F - Releases from Solid Waste Management Units

3.6.3 40 CFR 264/265 Subpart M - Land Treatment ..... 3.47

3.6.4 40 CFR - Land Disposal Restrictions ...... 3.49

3.7 STANDARDS UNDER THE SAFE DRINKING WATER ACT $\ldots \ldots . . . .3 .50$

3.7.1 40 CFR 141 - National Primary Drinking Water Regulations Pursuant to Section 1412 of the Safe Drinking Water Act

3.7.2 40 CFR 143 - National Secondary Drinking Water Regulations Pursuant to Section 1412 of the Safe Drinking Water Act

3.8 STANDARDS UNDER THE TOXIC SUBSTANCES CONTROL ACT $\ldots \ldots . . .109$

3.8.1 Polychlorinated Biphenyls (PCBs) Manufacturing, Processing, Distribution in Commerce, and Use Prohibitions (40 CFR 761) 
3.9 STANDARDS UNDER THE OCCUPATIONAL SAFETY AND HEALTH ACT . . 3.112

3.9.1 29 CFR 1910.120-Hazardous Waste Operations and Emergency Response Sections 1910.120(e) and (p) . . 3.115

3.9.2 29 CFR 1910.1000 - Air Contaminants -- Permissable Exposure Limits ........... 3.119

3.10 STANDARDS IN DOE ORDERS . . . . . . . . . 3.123

3.10 .1 Summary of DOE Orders ......... 3.123

3.10.2 DOE Order 5400.1 - General Environmental 3.125

3.10.3 DOE Order 5400.5 - Radiation Protection of
the Public and the Environment . . . . . 3 3.126

3.10.4 DOE Order 5480.1B - Environment, Safety, and Health Program for Department of Energy Operations ............... 3.136

3.10.5 DOE Order 5480.4 - Environment Protection,
Safety, and Health Protection Standards ... 3.136

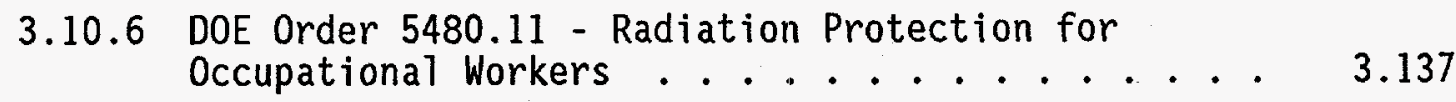

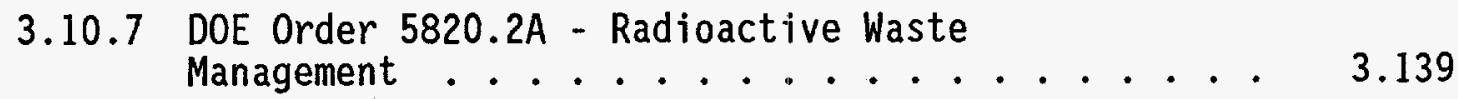

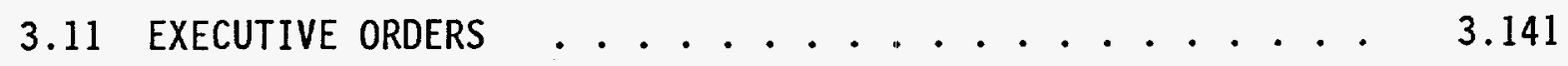

3.11 .1 Presidential Guidance ............ 3.141

4.0 REFERENCES ............................ 4.1

APPENDIX A - AIR CONTAININANTS--PERMISSABLE EXPLOSURE LIMITS . . . A A.1

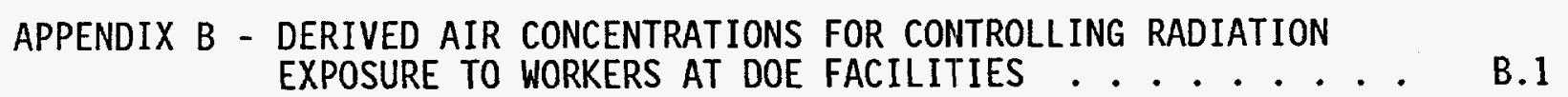

APPENDIX C - SURFACE RADIOACTIVITY GUIDES . . . . . . . . C. C.

APPENDIX D - DERIVED CONCENTRATION GUIDES FOR AIR AND WATER $\cdots \quad 0.1$ 


\section{$\underline{\text { TABLES }}$}

3.1 Summary of Ambient Air Quality Standards . . . . . . . . 3.3

3.2 Summary of National Emission Standards . . . . . . . 3.11

3.3 Summary of Water Quality Criteria . . . . . . . . 3.25

3.4 Reentry Time . . . . . . . . . . . . 3.35

3.5 Release Limits for Containment Requirements . . . . . . . 3.39

3.6 Maximum Concentration of Contaminants for the Toxicity

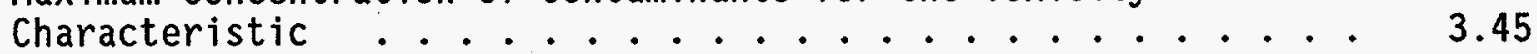

3.7 Limits for Hazardous Constituents in Facility Permits . . . . 3.48

3.8 Annual Cadmium Application Rates ... . . . . . . . 4.48

3.9 Maximum Cumulative Cadmium Application . . . . . . . . 3.49

3.10 Treatment Standards for RCRA Wastes Under Land Disposal
Restrictions . . . . . . . . . . . . . . . . .

3.11 Land Disposal Restrictions for California List Wastes . . . . 3.97

3.12 Maximum Contaminant Levels for Inorganic Chemicals . . . . 3.101

3.13 Maximum Contaminant Levels for Organic Chemicals . . . . 3.102

3.14 Maximum Contaminant Leve]s for Turbidity . . . . . . . . 3.103

3.15 Average Annual Concentrations ............. 3.104

3.16 Maximum Contaminant Levels and Goals . . . . . . . 3.106

3.17 MCLGs for Microbiological Contaminants . . . . . . 3.108

3.18 MCLs for Microbiological Contaminants . . . . . . . 3.108

3.19 Secondary Drinking Water Standards . . . . . . . . 3.108

3.20 Requirements for Decontaminating PCB Spills in Outdoor
Electrical Substations .......................... 
3.21 Requirements for Decontaminating PCB Spills in Other

Restricted Access Areas Other Than Outdoor Electrical

Substations

3.22 Requirements for Decontaminating PCB Spills in Nonrestricted Access Areas

3.23 OSH 1910.120 Hazardous Waste Operations Training Requirements . 3.118

3.24 Quality Factors by Radiation Type . . . . . . . . . . 3.125

3.25 Weighting Factors for Tissues and Organs . . . . . . 3.126

3.26 Summary of Regulations in DOE Orders . . . . . . . . 3.127

3.27 Surface Contamination Guidelines . . . . . . . . . 3.134 


\section{ABBREVIATIONS}

ACL

alternate concentration limit

AEA

Atomic Energy Act

AEC

Atomic Energy Commission

ALARA

as low as reasonably achievable

ARAR

applicable or relevant and appropriate

BAT

best available technology (DOE Orders)

$\mathrm{BCT}$

best conventional pollutant control technology

BDAT

best demonstrated available technology

$\mathrm{BOD}_{5}$

five-day biochemical oxygen demand

BPT

best practicable control technology currently available

CAA

Clean Air Act

CERCLA

Comprehensive Environmental Response, Compensation, and Liability Act

CFR

Code of Federal Regulations

$\mathrm{COD}$

chemical oxygen demand

CWA

Clean Water Act

DAC

derived air concentration

DCG

derived concentration guide

DOE

U.S. Department of Energy

dose e.

dose equivalent

DOT

U.S. Department of Transportation

$\mathrm{dpm}$

disintegrations per minute

EDE

effective dose equivalent

EPA

U.S. Environmental Protection Agency

EP Toxicity

extraction procedure toxicity 
Environment Reporter

ERS

Environmental Restoration Sites

EH

Environmental Safety and Health

ERDA

U.S. Energy Research and Development Administration

FIFRA

Federal Insecticide, Fungicide, and Rodenticide Act

FR

Federal Register

HCS

Hazard Communication Standard

$\mathrm{Hg}$

mercury

HHS

Department of Human and Health Services

$\mathrm{HOC}$

halogenated organic compound

HSWA

Hazardous and Solid Waste Amendments

ICRP

International Commission on Radiological Protection

IOCs

inorganic chemicals

ISFSI

Independent Spent Fuel Storage Installation

keV

kiloelectron-volt

LDR

land disposal restriction

LOEL

lowest observed effect level

MACT

maximum achievable control technology

MCL

maximum contaminant level

MCLG

maximum contaminant level goal

MeV

megaelectron-volt

$\mu \mathrm{Ci} / \mathrm{mL}$

microcurie per milliliter

$\mu \mathrm{g} / \mathrm{L}$

microgram per 7 iter

$\mu \mathrm{g} / \mathrm{m}^{3}$

microgram per cubic meter

$\mu \mathrm{R} / \mathrm{L}$

microrems per hour

$\mathrm{mg} / \mathrm{L}$

milligram per liter 


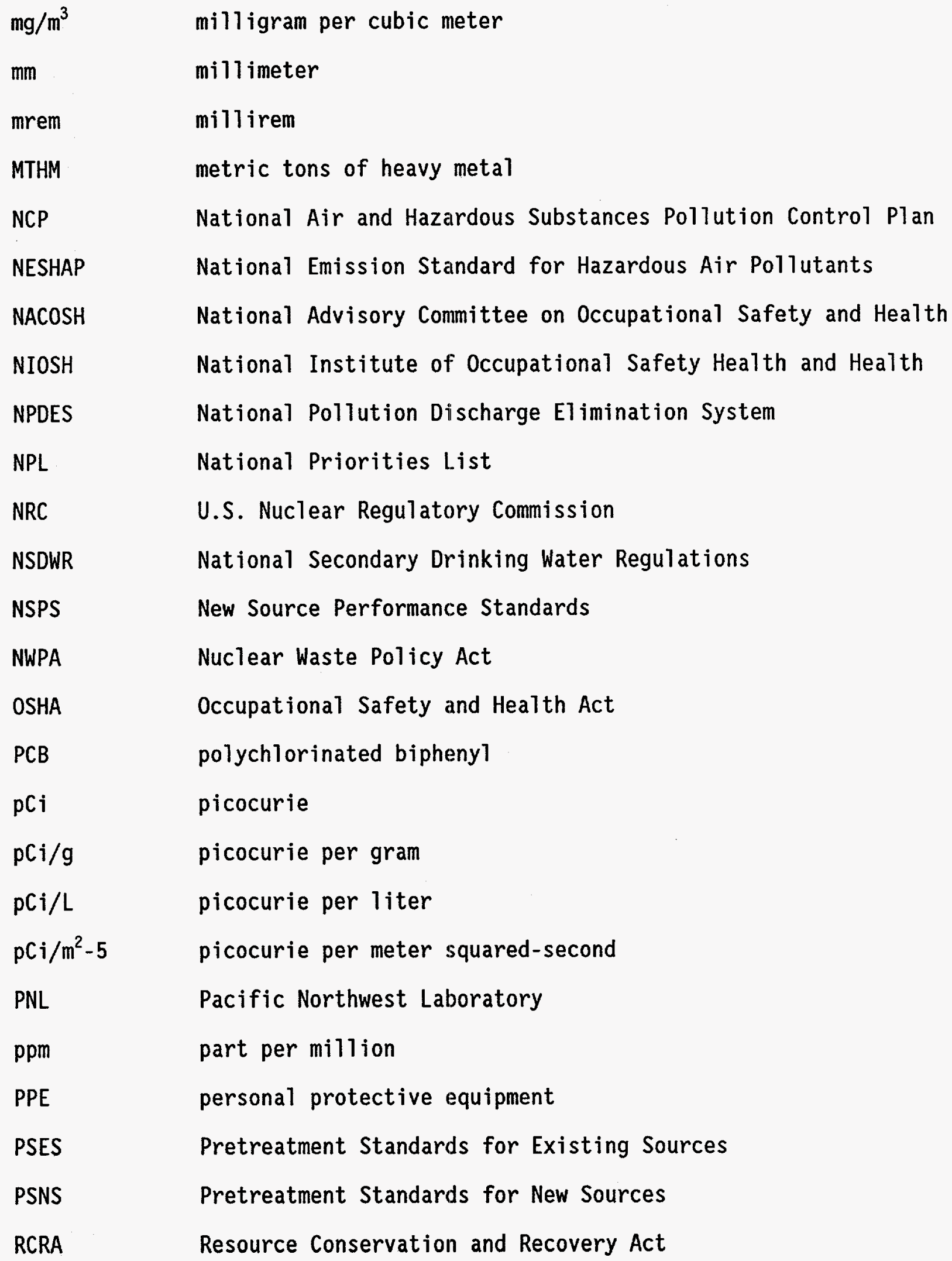




$\begin{array}{ll}\text { RMERC } & \begin{array}{l}\text { retorting or roasting in a thermal processimgt capable of } \\ \text { volatilizing mercury and subsequently condensing the } \\ \text { volatilized mercury for recovery }\end{array} \\ \text { SCBA } & \text { self-contained breathing apparatus } \\ \text { SMCL } & \text { secondary maximum contaminant level } \\ \text { SDWA } & \text { Safe Drinking Water Act } \\ \text { SOCs } & \text { synthetic organic chemicals } \\ \text { SV } & \text { sievert } \\ \text { TC } & \text { toxicity characteristic } \\ \text { TRU } & \text { transuranic } \\ \text { TSCA } & \text { Toxic Substances Control Act } \\ \text { TSD } & \text { treatment, storage, and disposal } \\ \text { TSS } & \text { total suspended solids } \\ \text { UST } & \text { underground storage tank } \\ \text { VHAP } & \text { volatile hazardous air pollutant } \\ \text { VOC } & \text { volatile organic compound } \\ \text { Wb } & \text { welfare-based as defined by the Safe Drinking Water Act, } \\ \text { secondary standards based on aesthetic qualities of drinking } \\ \text { water } \\ \text { working level }\end{array}$




\section{EXECUTIVE SUMMARY}

When conducting environmental restoration, waste management, and decontamination and decommissioning activities, the U.S. Department of Energy (DOE) must consider potentially applicable federal standards. Potentially applicable standards are found in the following statutes and regulations promulgated pursuant to those statutes: the Clean Air Act; the Clean Water Act; the Comprehensive Environmental Response, Compensation, and Liability Act; the Federal Insecticide, Fungicide, and Rodenticide Act; the Atomic Energy Act and Nuclear Waste Policy Act; the Resource Conservation and Recovery Act; the Safe Drinking Water Act; Toxic Substances Control Act; and the Occupational Safety and Health Act. Some DOE Orders and federal Executive Orders also contain potentially applicable standards.

A variety of qualitative and quantitative standards exist in the regulations. In general, quantitative standards are risk-based, technology-based, welfare-based, or a combination thereof. Risk-based standards are typically defined in terms of human health or safety. Risk is often measured as the maximum acceptable probability of unacceptable health effects. Technologybased standards can be defined in a variety of ways. However, all technologybased standards set pollutant discharge limits for categories of discharges based on available pollution control technologies. The environmental and health effects of the discharged pollutants are not considered. The type of technology-based standard may differ with the type of substance being regulated.

Welfare-based standards are defined differently among the various regulations. The Clean Air Act defines public welfare to include factors such as effects on soils, water, crops, vegetation, manmade materials, animals, wildlife, weather, visibility, climate, damage to and deterioration of property, hazards to transportation, as well as effects on economic values, personal comfort, and we17-being. Under the Safe Drinking Water Act, welfare is defined very generalty as factors that may affect the aesthetic qualities of drinking water. 
Some standards are based on a combination of these basic types of standards. A typical example is a technology-based standard founded on a risk-based goal. The acceptable level of risk is determined based on purely health factors. An ample margin of safety for that risk value is based on a combination of health, economic, technological, and other relevant factors. In all standards that are cited in this report and that have a combination of bases, risk is the underlying factor. After risk has been considered, other factors are included in the evaluation.

In some cases the bases for standards are not specified in the regulations. Such standards may be questioned by the public. As it continues its cleanup and remediation activities, DOE must consider a11 of these standards, regardless of their technical bases. 


\subsection{INTRODUCTION}

The U.S. Department of Energy (DOE) is now engaged in a program of environmental restoration nationwide across its 45 sites. It is also bringing its facilities into compliance with environmental regulations, decontaminating and decommissioning unwanted facilities, and constructing new waste management facilities.

One of the most difficult questions that DOE must face in successfully remediating its inactive waste sites, decontaminating and decommissioning its inactive facilities, and operating its waste management facilities is: "What criteria and standards should be met?" Before a specific cleanup action begins, and as a continuing activity during the operation of its waste management facilities, DOE must determine acceptable levels of "cleanliness." This determination must be based on federal, state, and local laws and regulations. However, within the framework of those laws and regulations, considerable latitude exists for setting quantitative cleanup standards (OTA 1989). Hence, in cooperation with the responsible federal, state, and local regulatory agencies, DOE will need acceptable quantitative standards and criteria that apply to its environmental restoration, waste management, and decontamination and decommissioning (D\&D) activities or acceptable procedures for determining such standards and criteria at each of its sites. Acceptable standards or procedures for determining standards will assist DOE in its conduct of ongoing waste management and pending cleanup activities by helping to ensure that those activities are conducted in compliance with applicable laws and regulations and are accepted by the regulatory community and the public.

\subsection{PURPOSE AND SCOPE}

This document reports on the second of three baseline activities that are being conducted as prerequisites to either the development of quantitative standards that could be used by DOE, or consistent procedures for developing such standards. The first and third baseline activities are also briefly discussed in conjuction with the second of the three activities. 
The first baseline activity for determining quantitative standards that are applicable to DOE's environmental restoration, waste management, and D\&D activities is to identify substances that are important to DOE and for which standards and criteria are needed. The second baseline activity for determining applicable standards is addressed in this report: to identify existing quantitative standards that are potentially applicable to DOE's activities. The standards identified in this document were compiled from relevant federal regulations and DOE Orders that are presently in effect. Draft regulations were not considered. State and local regulations are also not considered, al though they may be at a later date.

Although the primary purpose of this report is to compile quantitative standards that are or could be applicable to DOE, qualitative standards are noted where appropriate. The potentially applicable quantitative standards compiled herein are typically risk-based or technology-based. However, other bases (e.g., welfare-based) for standards were encountered and are defined as appropriate below. This report was prepared by the Pacific Northwest Laboratory $(P N L)^{(a)}$ for DOE's Office of Environmental Guidance.

In the third baseline activity, the potentially applicable quantitative standards identified in this report will be matched with the substances identified in the first baseline activity to identify quantitative standards that are applicable to DOE's waste management, decontamination and decommissioning, and environmental restoration activities. When the applicable standards and criteria have been identified, areas in which quantitative standards and criteria need to be developed and technical and procedural issues that are related to the development of needed standards and criteria will be apparent.

\subsection{BACKGROUND}

DOE owns 45 sites in 26 different states where it operates facilities that support national security interests, energy research, environmental restoration, etc. The first site was established in 1942 to support World War

(a) Pacific Northwest Laboratory is operated for the U.S. Department of Energy by Battelle Memorial Institute under Contract DE-AC06-76RLO 1830. 
II efforts and has operated since 1944. Additional sites were added as the nation's security needs increased. These facilities generated, treated, stored, and disposed of hazardous, radioactive, and mixed wastes.

During four decades of operations at its facilities, DOE facilities released chemicals and radioactive substances into the environment via trenches, landfills, pipes and stacks. As a result, cubic miles of soils and groundwater at these sites became contaminated with hazardous, radioactive, and mixed wastes. These contaminated media will require remediation under the Comprehensive Environmental Response, Compensation, and Liability Act (CERCLA) of 1980, or the corrective action requirements of the Resource Conservation and Recovery Act (RCRA) of 1984 (as amended). DOE's sites also contain numerous old production reactors and other facilities that are no longer operable. These facilities must be decontaminated and decommissioned in accordance with RCRA and other statutes.

All operating facilities must also have, and be in compliance with, RCRA operating permits from the U.S. Environmental Protection Agency (EPA) or from the state regulatory agency to which RCRA permitting responsibility has been delegated. Most of DOE's facilities were not constructed to be in compliance with today's environmental requirements. DOE is presently modernizing its facilities by bringing them into compliance with environmental requirements and by decontaminating and decommissioning unwanted facilities.

As stated publicly on numerous occasions, DOE views the minimization, management, and cleanup of waste materials generated from its operations to be one of its most challenging problems. Before DOE can effectively act to minimize, manage, and clean up waste materials, it must understand the present status of its sites, the standards that now apply to those sites, and the operations that are conducted on them. This report compiles the existing risk-based and technology-based standards that may be applicable to DOE's activities and goals. By comparing the inventory of waste materials that are important to DOE operations and activities and this compilation of standards, it will be possible to assess which standards are relevant to DOE's environmental restoration, waste management, and decontamination and decommissioning activities; where multiple standards exist; and where no standards exist. The 
regulatory agency can then $\mathrm{plan}$ and execute actions to ensure that standards needed for its environmental restoration, D\&D, and waste management activities are developed. The regulatory agency can also ensure that unique problems (e.g., radioactive mixed waste) are considered and appropriate actions are taken to develop standards that address these specific problems. 


\subsection{APPROACH}

Standards are generally published in regulations promulgated pursuant to statutes. Hence, the results section, Chapter 3.0 , of this report is divided into subsections that correspond to the applicable statutes. The intent of each statute is discussed briefly, followed by a compilation of potentially applicable standards listed in the numerical order of the Code of Federal Regulations (CFR) sections in which they are found. Potentially applicable standards in DOE Orders are also listed according to the numerical order of the relevant DOE orders.

Most potentially applicable standards and criteria are risk-based or technology-based. However, welfare-based standards and standards having combinations of different bases also exist. The bases for all standards are defined, as appropriate, in this document. These definitions are critical to understanding the bases of the standards because different CFR sections may define the same term (e.g., welfare-based standard) differently. In most cases, the bases for the standards and criteria are explained in the preambles of the Federal Register (FR) notices at the time the regulations were proposed and finalized, or in the preambles to amended regulations. Hence, in most cases, the FR notices must be consulted if more detailed information on the bases for the standards and criteria is desired.

Understanding and documenting the bases for the existing standards is important for determining the appropriate actions that DOE should take in its environmental restoration, decontamination and decommissioning, and waste management activities. Compliance with technology-based standards reduces emissions of, and exposures to, hazardous and radioactive substances. The 1990 Clean Air Act Amendments are an example of technology-based standards. However, the degree to which public health and the environment are protected is often not estimated. For this reason, the EPA moved toward the development of risk-based standards. Similarly, the National Academy of Sciences (NAS, 1989) has recommended that DOE adopt risk-based standards in its environmental restoration, decontamination and decommissioning, and waste management activities to ensure that public health and the environment are protected 
adequately. Hence, for some substances, DOE may find that existing technology-based standards are not adequate and that risk-based standards need to be developed.

Quantitative and qualitative standards were encountered during the preparation of this report. All potentially applicable quantitative standards and all potentially applicable qualitative standards upon which quantitative standards could be based are compiled herein. Vaguely defined qualitative standards (i.e., "shall not degrade...") are not compiled herein because they are too broadly defined to be used alone as a basis for setting quantitative standards. Operational and procedural requirements also were compiled only if they specified target levels for cleanup or remediation activities. All standards compiled below were updated through July 24, 1992.

\subsection{DETERMINATION OF INFORMATION SOURCES}

DOE Orders were the primary means by which relevant regulations and requirements specific to DOE facilities were identified. Order DOE 5480.4 1ists all parts of the CFR that apply to DOE under federal 1aw. All CFR sections 1isted in Order DOE 5480.4 were examined to determine whether they contain standards or criteria that could be applicable to environmental restoration, waste management, and decontamination and decommissioning activities. Because the agency specifies additional requirements that a 11 DOE facilities must meet, relevant DOE Orders were also a major source of information on potentially applicable standards. DOE Orders were an especially important source of standards on exposure to radiation.

\subsection{INFORMATION SOURCES}

The primary sources of information used to compile potentially applicable standards and criteria were the CFR and relevant DOE Orders (as identified in Section 4.8 of this report). Secondary sources of information included the relevant statutes, the FR, the Environment Reporter, Executive Orders, and Publication No. 26 of the International Commission on Radiological Protection (ICRP). 
The CFR is a compilation of Executive Orders and the rules and requirements published by agencies of the federal government. It includes 50 titles, most of which are promulgated to implement federal statutes. Title 10 contains the regulations that affect energy, and Title 40 contains the regulations that affect the environment. Titles are divided into chapters, which are further subdivided into parts dedicated to specjfic regulatory areas.

DOE Orders are internal department documents that set policy and specify procedures for implementing that policy. They may apply to specific sites and facilities or to all areas of DOE operations. In lieu of creating new policies and regulations, DOE Orders may mandate compliance with existing federal, state, and local regulations. Directives contained in DOE Orders may be very specific. During the preparation of this report, DOE Orders were a major source of information on radiation standards for the protection of human health.

The FR is the federal government's newspaper through which draft and final regulations are published, meetings are announced, and legal notices are posted. Regulations published in the FR are typically accompanied by background information and explanatory material that does not become incorporated into the CFR. Information in the FR often explains the intent of the regulation (or specific parts thereof) and the technical basis of the regulations. The FR was a key source of information on the technical bases of various numerical standards.

Executive Orders are the means by which the executive branch assigns responsibilities for implementing policies, statutes, and regulations. The Presidential Guidance entitled "Radiation Protection Guidance to Federal Agencies for Occupational Exposure" (52 FR 2822) contains standards that are potentially applicable to DOE activities.

Publication No. 26 of the ICRP explains the types of risk and sets risk levels for occupational exposure to radioactive contamination. It also defines the process used to set exposure limits and weighing factors for exposure of specific organs and tissues. This process has been adopted in Executive Orders, DOE Orders, and federal regulations for occupational exposure. 


\subsection{RESULTS}

This section covers the standards and regulations mandated by major Federal environmental statutes and orders. The statutes and orders included in this investigation were the Clean Air Act (CAA); the Clean Water Act (CWA); the Comprehensive Environmental Restoration, Compensation, and Liability Act (CERCLA); the Federal Insecticide, Fungicide, and Rodenticide Act (FIFRA); the Atomic Energy Act (AEA); the Nuclear Waste Policy Act (NWPA); the Resource Conservation and Recovery Act (RCRA); the Safe Drinking Water Act (SDWA); the Toxic Substances Control Act (TSCA); the Occupational Safety and Health Act (OSHA); Orders DOE; and Executive Orders. The statutes are presented below in alphabetical order, followed by Orders DOE and Executive Orders.

Within each statute, more than one part of the CFR typically pertains to DOE. For easy reference, CFR parts are arranged in this chapter numerically by statute. To maintain some consistency within the sections, a very general format was set up, beginning with definitions. In cases where it was possible to do so, standards and criteria were summarized in tables, followed by more detailed descriptions of the standards, their bases, and the conditions for their applicability. In general, quantitative standards are listed first, followed by qualitative standards and industry-specific standards. The bases of the standards are included in the discussions.

Because of the variety of formats within the regulations, the format within the descriptions varies substantially in some cases. The summary attempts to clarify the critical details for each standard or criterion. For industry-specific standards that do not apply to DOE at this time, the industry and the appropriate CFR part are listed for future reference. In the event that DOE constructs new facilities, these standards may be pertinent.

\subsection{STANDARDS UNDER THE CLEAN AIR ACT}

The Clean Air Act was enacted in 1970 and was extended and amended in 1977. The Act was also amended in 1990 and those amendments will be a major focus in 1992. Its intent is to "protect and enhance the quality of the nation's air resources in order to promote the public health and welfare and 
the productive capacity of its population." Standards under the Clean Air Act control air quality by two different mechanisms. National ambient air quality standards establish the concentrations of pollutants that are allowed in the surrounding air. Emission standards limit the quantities of specific air pollutants at the source regardless of the ambient air concentrations of those pollutants.

The Clean Air Act is based on the premise that State and local governments bear primary responsibility for preventing and controlling air pollution. Each state is to develop an implementation plan that establishes the regulatory framework and control strategy to attain or maintain the national ambient air quality standards established by EPA. Once approved by EPA, the State implementation $\mathrm{p} 7$ an has the effect and force of Federal 1 aw and may be enforced by EPA in Federal courts. If a state fails to submit a $p l a n$, or submits one that is not acceptable to EPA, EPA must promulgate a $\mathrm{plan}$ for the State. The approved State implementation plan must have provisions not only for ambient air quality, but also for emission limitations for new sources and for EPA-specified hazardous air pollutants. State or local governments may enforce emission standards or limitations that are more stringent than those in effect under Federal new source performance standards or hazardous air pollutant standards.

of the new rules and proposals under the 1990 Clean Air Act Amendments, industrial emission permits are the most critical. Other major rule-makings include air toxics emissions trading, acid rain controls on emissions of sulfur dioxide and nitrogen oxides, and controlling emissions from motor vehicle fueling. The Act lists 189 hazardous chemicals commonly emitted from industrial sources that must be regulated. The act requires EPA to regulate emissions by "source" rather than chemical-by-chemical. For each source category, the agency must develop "maximum achievable control technology" (MACT) standards. The first 40 are to be finalized by November 15, 1992 and the remainder are to be issued within 10 years on a phased schedule. In summary, EPA establishes the levels of air quality standards to be adopted nationwide, but the State dictates the methods for achieving those standards 
through State implementation plans. About 30 states already require various levels of air permits (ER 1992, 22 (39): 2192).

Ambient air quality is regulated by primary and secondary standards (Table 3.1) for the purpose of protecting public health and welfare as defined in 40 CFR part 50. Standards of performance for new stationary sources are industry-specific and are listed under 40 CFR part 60. National emission standards for hazardous air pollutants (NESHAPs) are specific for pollutants and are 1 isted under 40 CFR part 61.

\section{TABLE 3.1. Summary of Ambient Air Quality Standards}

\begin{tabular}{|c|c|c|c|}
\hline \multirow[b]{2}{*}{ Constituent } & \multicolumn{2}{|c|}{ Primary Standard (risk-based) } & \multirow{2}{*}{$\begin{array}{l}\text { Secondary Standard } \\
\text { (welfare-based) }\end{array}$} \\
\hline & Annual Mean ${ }^{(a)}$ & Hourly Maximum & \\
\hline Sulfur 0xides & $\begin{array}{l}80 \mu \mathrm{g} / \mathrm{m}^{3} \\
(0.03 \mathrm{ppm})\end{array}$ & $\begin{array}{l}365 \mu \mathrm{g} / \mathrm{m}^{3(\mathrm{~b})} \\
(0.14 \mathrm{ppm})\end{array}$ & $\begin{array}{l}1300 \mu \mathrm{g} / \mathrm{m}^{3(\mathrm{c})} \\
(0.5 \mathrm{ppm})\end{array}$ \\
\hline Particulates & $50 \mu \mathrm{g} / \mathrm{m}^{3}$ & $150 \mu \mathrm{g} / \mathrm{m}^{3(\mathrm{~d})}$ & same as primary \\
\hline Carbon Monoxide & & $\begin{array}{l}10 \mathrm{mg} / \mathrm{m}^{3}\left(^{(9 \mathrm{ppm})^{(\mathrm{e})}}\right. \\
40 \mathrm{mg} / \mathrm{m}^{3}{(35 \mathrm{ppm})^{(\mathrm{f})}}^{(35}\end{array}$ & \\
\hline Ozone & & $235 \mu \mathrm{g} / \mathrm{m}^{3}(0.12 \mathrm{ppm})^{(\mathrm{g})}$ & same as primary \\
\hline Nitrogen Dioxide & $\begin{array}{l}100 \mu \mathrm{g} / \mathrm{m}^{3} \\
(0.053 \mathrm{ppm})\end{array}$ & & same as primary \\
\hline Lead & $1.5 \mu \mathrm{g} / \mathrm{m}^{3(\mathrm{~h})}$ & & same as primary \\
\hline
\end{tabular}

(a) Annual arithmetic mean concentration.

(b) Maximum 24-hour concentration not to be exceeded more than once per year.

(c) Maximum 3-hour concentration not to be exceeded more than once per year.

(d) 24-hour average concentration not expected to exceed limit more than once per year.

(e) 8-hour average concentration not to be exceeded more than once per year.

(f) 1-hour average concentration not to be exceeded more then once per year.

(g) Maximum hourly average concentrations not expected to exceed limit more than one day per calendar year.

(h) Maximum arithmetic mean averaged over a calendar quarter. 


\subsubsection{CFR part 50 - National Primary and Secondary Ambient Air Quality}

Standards Pursuant to the Clean Air Act

\section{Reference Conditions}

$$
\begin{array}{ll}
\text { Temperature } & =25^{\circ} \mathrm{C} \\
\text { Pressure } & =760 \mathrm{~mm} \mathrm{Hg}
\end{array}
$$

\section{Definitions}

Ambient Air. That portion of the atmosphere, external to buildings, to which the general public has access.

Primary Standards. The levels of air quality that are necessary to protect the public health. These are risk-based standards.

Secondary Standards. The levels of air quality that are necessary to protect the public welfare. These welfare-based standards include factors such as soils, water, crops, vegetation, manmade materials, animals, wildlife, weather, visibility, climate, damage to and deterioration of property, hazards to transportation, and effects on economic values and on personal comfort and well-being [40 CFR part $302(h)$ ].

40 CFR part 50.4 - National Primary Ambient Air Quality Standards for Sulfur Oxides

- $80 \mu \mathrm{g} / \mathrm{m}^{3}$ (0.03 ppm) annual arithmetic mean

- $365 \mu \mathrm{g} / \mathrm{m}^{3}(0.14 \mathrm{ppm})$ maximum 24 -hour concentration not to be exceeded more than once per year

40 CFR part 50.5 - National Secondary Ambient Air Quality Standards for Sulfur 0xides

- $1300 \mu \mathrm{g} / \mathrm{m}^{3}(0.5 \mathrm{ppm})$ maximum 3-hour concentration not to be exceeded more than once per year

40 CFR part 50.6 - National Primary and Secondary Ambient Air Quality Standards for Particulate Matter

- $50 \mu \mathrm{g} / \mathrm{m}^{3}$ annual arithmetic mean concentration

- $150 \mu \mathrm{g} / \mathrm{m}^{3}$ 24-hour average concentration not to be exceeded more than once per calendar year 
40 CFR part 50.8 - National Primary Ambient Air Quality Standards for Carbon Monoxide

- $10 \mathrm{mg} / \mathrm{m}^{3}(9 \mathrm{ppm})$ for an 8-hour average concentration not to be exceeded more than once per year

- $40 \mathrm{mg} / \mathrm{m}^{3}$ (35 ppm) for a 1-hour average concentration not to be exceeded more than once per year

40 CFR part 50.9 - National Primary and Secondary Ambient Air Quality Standards for Ozone

- $235 \mu \mathrm{g} / \mathrm{m}^{3}(0.12 \mathrm{ppm})$ maximum hourly average concentration not to be exceeded more than once a year

40 CFR part 50.11 - National Primary and Secondary Ambient Air Quality Standards for Nitrogen Dioxide

- primary standard $=100 \mu \mathrm{g} / \mathrm{m}^{3}(0.053 \mathrm{ppm})$ annual arithmetic mean concentration

- secondary standard $=100 \mu \mathrm{g} / \mathrm{m}^{3}(0.053 \mathrm{ppm})$ annual arithmetic mean concentration

40 CFR part 50.12 - National Primary and Secondary Ambient Air Quality Standards for Lead

- $1.5 \mu \mathrm{g} / \mathrm{m}^{3}$ maximum arithmetic mean averaged over a calendar quarter

3.1.2 40 CFR part 60 - Regulations on Standards of Performance for New Stationary Sources

The regulations listed below in Subparts A through VWV set performance standards for emissions from new stationary sources. Performance standards vary based on the type of source and the design of the facility. Typically, the substances regulated by these performance standards are particulate matter, sulfur dioxide, nitrogen oxides, carbon monoxide, and volatile organic compounds (VOCs). Because these regulations would only apply to new DOE facilities, the standards promulgated therein are not listed in this document. The titles of the subparts are listed, however, to facilitate retrieval of standards that could be applicable to a new DOE facility. 
Subpart A

Subpart B

Subpart C

Subpart Ca

Subpart Cb

Subpart D

Subpart Da

Subpart Db

Subpart DC

Subpart E

Subpart Ea

Subpart F

Subpart G

Subpart H

Subpart I

Subpart J

Subpart K

Subpart Ka
General Provisions

Adoption and Submittal of State Plans for Designated Facilities

Emission Guidelines and Compliance Times

Emission Guidelines and Compliance Times for Municipal Waste Combustors

Emission Guidelines and Compliance Times for Sulfuric Acid Production Units

Standards of Performance for Fossil-Fuel Fired Steam Generators for Which Construction is Commenced After August 17, 1971

Standards of Performance for Electric Utility Steam Generating Units for Which Construction is Commenced After September 18, 1978

Standards of Performance for Industrial-CommercialInstitutional Steam Generating Units

Standards of Performarce for Small Industrial-CommercialInstitutional Steam Generating Units

Standards of Performance for Incinerators

Standards of Performance for Municipal Waste Combustors

Standards of Performance for Portland Cement Plants

Standards of Performance for Nitric Acid Plants

Standards of Performance for Sulfuric Acid Plants

Standards of Performance for Asphalt Concrete Plants

Standards of Performance for Petroleum Refineries

Standards of Performance for Storage Vessels for Petroleum Liquids for Which Construction, Reconstruction, or Modification Commenced After June 11, 1973, and Prior to May 19, 1978

Standards of Performance for Storage Vessels for Petroleum Liquids for Which Construction, Reconstruction, or Modification Commenced After May 18, 1978, and Prior to JuTy 23, 1984 
Standards of Performance for Volatile Organic Liquid Storage Vessels (including Petroleum Liquid Storage Vessels) for Which Construction, Reconstruction, or Modification Commenced After July 23, 1984

Subpart L Standards of Performance for Secondary Lead Smelters

Subpart M Standards of Performance for Secondary Brass and Bronze Production Plants

Subpart $N$

Standards of Performance for Primary Emissions from Basic Oxygen Process Furnaces for Which Construction is Commenced After June 11, 1973

Subpart $\mathrm{Na}$

Standards of Performance for Secondary Emissions from Basic 0xygen Process Steelmaking Facilities for Which Construction is Commenced After January 20, 1983

Subpart $0 \quad$ Standards of Performance for Sewage Treatment Plants

Subpart P Standards of Performance for Primary Copper Smelters

Subpart Q Standards of Performance for Primary Zinc Smelters

Subpart $\mathrm{R} \quad$ Standards of Performance for Primary Lead Sme1ters

Subpart $S \quad$ Standards of Performance for Primary Aluminum Reduction Plants

Subpart $T$ Standards of Performance for the Phosphate Fertilizer Industry: Wet-Process Phosphoric Acid Plants

Subpart $U$ Standards of Performance for the Phosphate Fertilizer Industry: Superphosphoric Acid Plants

Subpart $V \quad$ Standards of Performance for the Phosphate Fertilizer Industry: Diammonium Phosphate Plants

Subpart $W \quad$ Standards of Performance for the Phosphate Fertilizer Industry: Triple Superphosphate Plants

Subpart $X \quad$ Standards of Performance for the Phosphate Fertilizer Industry: Granular Triple Superphosphate Storage Facilities

Subpart $Y \quad$ Standards of Performance for Coal Preparation Plants

Subpart $Z$ Standards of Performance for Ferroalloy Production Facilities 
Subpart AA Standards of Performance for Steel Plants: Electric Arc Furnaces

Subpart AAa Standards of Performance for Steel Plants: Electric Arc Furnaces and Argon-0xygen Decarburization Vessels

Constructed After August 7, 1983

Subpart BB Standards of Performance for Kraft Pulp Mills

Subpart CC Standards of Performance for Glass Manufacturing Plants

Subpart DD Standards of Performance for Grain Elevators

Subpart EE Standards of Performance for Surface Coating of Metal Furniture

Subpart FF Reserved

Subpart GG Standards of Performance for Stationary Gas Turbines

Subpart HH Standards of Performance for Lime Manufacturing Plants

Subpart KK Standards of Performance for Lead-Acid Battery Manufacturing Plants

Subpart LL Standards of Performance for Metallic Mineral Processing Plants

Subpart MM Standards of Performance for Automobile and Light-Duty Truck Surface Coating Operations

Subpart NN

Standards of Performance for Phosphate Rock Plants

Subpart PP

Standards of Performance for Ammonium Sulfate Manufacture

Subpart QQ

Standards of Performance for the Graphic Arts Industry:

Publication Rotogravure Printing

Subpart RR Standards of Performance for Pressure Sensitive Tape and Label Surface Coating Operations

Subpart SS Standards of Performance for Industrial Surface Coating: Large Appliances

Subpart TT

Standards of Performance for Metal Coil Surface Coating

Subpart UU

Standards of Performance for Asphalt Processing and Asphalt Roofing Manufacture

Subpart VV Standards of Performance for Equipment Leaks of VOC in the Synthetic Organic Chemicals Manufacturing Industry 
Subpart WW Standards of Performance for the Beverage Can Surface Coating Industry

Subpart XX Standards of Performance for Bulk Gasoline Terminals

Subpart AAA Standards of Performance for New Residential Wood Heaters

Subpart BBB Standards of Performance for the Rubber Tire Manufacturing Industry

Subpart CCC Reserved

Subpart DDD Standards of Performance for Volatile Organic Compound (VOC) Emissions from the Polymer Manufacturing Industry

Subpart EEE Reserved

Subpart FFF Standards of Performance for Flexible Vinyl and Urethane Coating and Printing

Subpart GGG Standards of Performance for Equipment Leaks of VOC in Petroleum Refineries

Subpart HHH Standards of Performance for Synthetic Fiber Production Facilities

Subpart III Standards of Performance for Volatile Organic Compound Emissions from the Synthetic Organic Chemical

Manufacturing Industry (SOCMI) Air Oxidation Unit Processes

Subpart JJJ Standards of Performance for Petroleum Dry Cleaners

Subpart KKK Standards of Performance for Equipment Leaks of VOC from Onshore Natural Gas Processing Plants

Subpart LLL Standards of Performance for Onshore Natural Gas Processing: $\mathrm{SO}_{2}$ Emissions

Subpart MMM Reserved

Subpart NNN Standards of Performance for Volatile Organic Compound Emissions from Synthetic Organic Chemical Manufacturing Industry Distillation Operations

Subpart 000 Standards of Performance for Nonmetallic Mineral Processing Plants

Subpart PPP Standards of Performance for Wool Fiberglass Insulation Manufacturing Plants 
Subpart QQQ Standards of Performance for VOC Emissions from Petroleum Refinery Wastewater Systems

Subpart RRR Reserved

Subpart SSS Standards of Performance for Magnetic Tape Coating Facilities

Subpart TTT Standards of Performance for Industrial Surface Coating: Surface Coating of Plastic Parts for Business Machines

Subpart UUU Reserved

Subpart VVV Standards of Performance for Polymetric Coating of Supporting Substrates Facilities

\subsubsection{CFR part 61 - Regulations on National Emission Standards for Hazardous Air Pollutants Pursuant to the Clean Air Act}

National emission standards (Table 3.2) regulate emissions of air pollutants classified as hazardous by EPA. These 1 imits are regulated independentiy of ambient air concentrations of the hazardous substances. For some hazardous substances, emission limits are established based on the type of generating facility as well as the substance itself. 40 CFR part 61 should be consulted for standards applicable to newly constructed facilities of the following types: underground uranium mines, beryllium rocket motors, firing test sites, elemental phosphorus plants, glass manufacturing plants, primary copper smelters, arsenic production facilities, phosphogypsum plants, mill tailings, and facilities that emit vinyl chloride.

\section{Specific Standards within 40 CFR part 61}

The subparts listed below give further details regarding the NESHAPs. All subparts are listed, though some are not applicable to facilities presently operated by DOE.

Subpart A General Provisions

Subpart B National Emission Standards for Radon Emissions from Underground Uranium Mines These are risk-based, construction-specific standards. 
TABLE 3.2. Summary of National Emission Standards

\begin{tabular}{|c|c|c|c|c|}
\hline Contaminant & Facility Type & $\begin{array}{l}\text { Sampling Time } \\
\text { Period } \\
\end{array}$ & $\begin{array}{l}\text { Maximum } \\
\text { Emission }\end{array}$ & $\begin{array}{l}\text { Standard } \\
\text { Type } \\
\end{array}$ \\
\hline Bery11ium & $\begin{array}{l}\text { Stationary source } \\
\text { Stationary source }\end{array}$ & $\begin{array}{l}\text { 24-hr period } \\
\text { 30-day period }\end{array}$ & $\begin{array}{l}10 \mathrm{~g} \\
0.01 \mu \mathrm{g} / \mathrm{m}^{3}\end{array}$ & $n^{(a)}$ \\
\hline \multirow[t]{2}{*}{ Mercury } & $\begin{array}{l}\text { Ore processing facility, } \\
\text { Hg cell chlor-alkali } \\
\text { plants }\end{array}$ & 24 hours & $2300 \mathrm{~g}$ & ns \\
\hline & $\begin{array}{l}\text { Sludge incineration } \\
\text { plants, drying } \\
\text { plants, etc. }\end{array}$ & 24 hours & $3200 \mathrm{~g}$ & ns \\
\hline Radionuclides & DOE facilities & 1 year & $10 \mathrm{mrem} / \mathrm{yr}$ & $r t^{(b)}$ \\
\hline Radon-222 & DOE facilities & & $20 \mathrm{pCi} / \mathrm{m}^{2} \mathrm{~s}$ & rt \\
\hline Benzene & Operational standards & & & $r t$ \\
\hline Benzene & $\begin{array}{l}\text { Coke by-product } \\
\text { Recovery plants }\end{array}$ & & 0 & $r t$ \\
\hline Benzene & Benzene storage vessels & & 0 & rt \\
\hline Equip. Leaks & Operational standards & & & ns \\
\hline Asbestos & Operational standards & & & ns \\
\hline
\end{tabular}

(a) $n s=$ not specified.

(b) $r t=$ "acceptable risk" based on health factors, "ample margin of safety" for that risk level includes evaluation of health, economic, technological, and other relevant factors. This is called the multifactor approach. 54 Federal Register 51656 states that the multifactor approach has already been adopted for the determination of the benzene NESHAP and will become the policy for future NESHAP decisions. The approach will not apply to other EPA programs or to other sections of the CAA.

Subpart C National Emission Standard for Beryllium Emissions from stationary sources to the atmosphere sha77 not exceed $10 \mathrm{~g}$ of beryllium over any 24-hour period, except as provided here. Upon approval from the administrator of the EPA, the ambient concentration limit of beryllium in the vicinity of the stationary source shall be $0.01 \mu \mathrm{g} / \mathrm{m}^{3}$, averaged over a 30-day period. The basis for this standard is not specified.

Subpart D National Emission Standard for Beryllium Rocket Motor Firing Not presently applicable to DOE. 
Subpart E

Subpart $F$

Subpart G

Subpart $H$

Subpart I

Subpart $J$

Subpart K

Subpart L
National Emission Standard for Mercury

Emissions from mercury ore processing facilities and mercury cell chlor-alkali plants shall not exceed $2300 \mathrm{~g}$ of $\mathrm{Hg}$ per 24-hour period. Emissions from sludge incineration plants, sludge drying plants, or plants that process wastewater treatment plant sludges shall not exceed $3200 \mathrm{~g}$ of $\mathrm{Hg}$ per 24-hour period. The basis for this standard is not specified.

National Emission Standard for Vinyl Chloride These standards vary based on the type of vinyl chloride produced and the type of generating plant. Risk/ technology-based standards exist under this subpart for ethylene dichloride plants, vinyl chloride plants, and polyvinyl chloride plants.

\section{Reserved}

National Emission Standards for Emissions of Radionuclides other than Radon from Department of Energy Facilities Emissions to ambient air are not to exceed those amounts that would cause any member of the public to receive in any year an EDE of $10 \mathrm{mrem}$ per year. Doses due to radon220 or radon-222 or decay products are excluded. This is a risk/technology-based standard.

National Emission Standards for Radionuclide Emissions from Facilities Licensed by the Nuclear Regulatory Commission and Federal Facilities Not Covered By Subpart H Not presently applicable to DOE.

National Emission Standard for Equipment Leaks (Fugitive Emission Sources) of Benzene

These are risk/technology-based operational standards based on the type of equipment. The standards are listed under Subpart $V$.

National Emission Standards for Radionuclide Emissions from Elemental Phosphorus Plants

Not presently applicable to DOE.

National Emission Standard for Benzene Emissions from Coke By-Product Recovery Plants

No detectable emissions of benzene are allowed from process vessels, storage tanks, tar-intercepting sumps, light-oil sumps, naphthalene processing, final coolers, and final-cooler cooling towers. By definition, a leak is detected if an instrument reads an organic chemical concentration of more than $500 \mathrm{ppm}$ above a background concentration, or $10,000 \mathrm{ppm}$ or greater total concentration. These are risk/technology-based standards. 
Subpart M

National Emission Standard for Asbestos

These operation-specific standards vary with respect to type of generator or source. Standards exist for: asbestos mills

roadways

manufacturing

demolition and renovation

spraying

fabricating

insulating materials

waste disposal for asbestos mills

waste disposal for manufacturing, demolition, renovation, spraying, and fabricating

inactive waste disposal for asbestos mills and manufacturing and fabricating

air cleaning

reporting

active waste disposal sites

operations that convert asbestos-containing waste

material into non-asbestos (asbestos-free) material

cross-reference to other asbestos regulations

Subpart $N$ National Emission Standard for Inorganic Arsenic Emissions from Glass Manufacturing Plants

Not presently applicable to DOE.

Subpart $0 \quad$ National Emission Standard for Inorganic Arsenic Emissions from Primary Copper Smelters

Not presently applicable to DOE.

Subpart $P \quad$ National Emission Standard for Inorganic Arsenic Emissions from Arsenic Trioxide and Metallic Arsenic Production Facilities

Not presently applicable to DOE.

Subpart Q National Emission Standards for Radon Emissions from Department of Energy Facilities

No source shall emit more than $20 \mathrm{pCi} / \mathrm{m}^{2} s$ of radon-222 as an average for the entire source into the air. This requirement will be part of any Federal Facilities Agreement reached between Environmental Protection Agency and Department of Energy. This is a risk/ technology-based standard.

Subpart R National Emission Standards for Radon Emissions from Phosphogypsum Stacks

Not presently applicable to DOE.

Subpart $\mathbf{R} \quad$ Reserved 
Subpart $T$ National Emission Standards for Radon Emissions from the Disposal of Uranium Mill Tailings

Radon-222 emissions to the ambient air from uranium mil7 tailings pile that are no longer operational shall not exceed $20 \mathrm{pCi} / \mathrm{m}^{2}$ s of Radon-222. Compliance must be achieved within 2 years of operations cessation. This is a risk/technology-based standard.

Subpart U Reserved

Subpart V National Emission Standards for Equipment Leaks (Fugitive Emission Sources)

These are operational standards based on very specific mechanical sources. Monitoring and equipment standards exist for:

pumps

compressors

pressure relief devices in gas/vapor service sampling connection systems

open-ended valves or lines

valves

pressure relief devices in liquid service and

flanges and other connectors

product accumulator vessels

delay of repair

closed-vent systems and control device

and include alternative standards for:

valves in VHAP service - allowable percentage of valves leaking

valves in VHAP service - skip period leak detection and repair

Subpart $W$ National Emission Standards for Radon Emissions from Operating Mill Tailings

Not presently applicable to DOE.

Subpart $X \quad$ Reserved

Subpart $Y$ National Emission Standard for Benzene Emissions from Benzene Storage Vessels

No emissions are allowed from storage vessels with a design capacity greater than or equal to 38 cubic meters $(10,000$ gallons). An emission can be detected visually and is inferred if an instrument reads $500 \mathrm{ppm}$ or more above background levels. This is a risk/technology-based standard.

Subparts Z-AA Reserved 
Subpart BB National Emission Standard for Benzene Emissions from Benzene Transfer Operations

These operating standards apply to facilities which load

liquid containing more than $70 \%$-weight benzene. Monitoring and equipment standards exist for:

loading racks

vent streams

flares

vapor-tight vehicles

marine vessels

collections systems

Subparts CC-EE Reserved

Subpart FF National Emission Standard for Benzene Waste Operations These operational standards apply to owners and operators of chemical manufacturing plants, coke by-product recovery plants, and petroleum refineries and to those facilities which treat, store, or dispose of such waste. Monitoring and equipment standards exist for:

tanks

surface impoundments

containers

individual drain systems

oil-water separators

treatment processes

closed-vent systems and control devices

delay of repair

and include alternative standards for:

tanks

oil-water separators

\subsection{STANDARDS UNDER THE CLEAN WATER ACT}

The legislation commonly referred to as the Clean Water Act was originally enacted in 1972 as the Federal Water Pollution Control Act. It was amended with major revisions in 1977 (i.e., the Clean Water Act), 1981, and 1987. The 1987 amendments are entitled the Water Quality Act of 1987. This legislation was enacted to protect surface waters from point and non-point sources of pollutants. Under this act, all discharges of pollutants must be in compliance with a National Pollutant Discharge Elimination System (NPDES) permit that is issued by the EPA or the State, if the EPA has delegated the authority to issue NPDES permits to the State. Under the Federal Water Pollution Control Act and its amendments, states are required to enforce water 
quality standards that are at least as stringent as those required by the EPA. To date, implementation of the act has emphasized the control or elimination of point source discharges of pollutants. In recent years, the EPA has become increasingly active in attempting to control pollutants from non-point source discharges. The most recent amendment was the Great Lakes Critical Programs Act of 1990 which amended the Federal Water Pollution Control Act with respect to water quality in the Great Lakes. This amendment extends the authorization of the EPA's Great Lakes National Program Office through 1997. This amendment also requires all states to develop remedial action plans for "areas of concern" as specified in the amended Great Lakes Agreement of 1978.

Clean Water Act regulations cited for completion in 1992 include a rule that would establish Federal water quality standards for toxic pollutants. The rule, which was proposed in November 1991, would cover pollutants listed under section 307(a) of the act. Congress will set a high priority on the reauthorization of the CWA in 1992 and on the controversial debate of defining wetlands. Some revisions have been made in the wetlands manual, but the manual may not be completed before the end of 1992 (ER 1991, 22 (39): 2194 2195).

Two general sets of standards have been promulgated under the CWA that may be applicable to DOE activities: categorical standards and water quality criteria. Categorical standards are applicable to various categories of industrial discharges. Standards for the various categories of industrial discharges are each promulgated in a different part of the CFR. Water quality criteria are not promulgated in the CFR, but are published as a separate document that is updated periodically. The most recent water quality criteria were published in 1986 as "Quality Criteria for Water" (EPA 1986).

\subsubsection{Categorical Standards}

Categorical standards are technology-based standards that are applicable to various categories of industrial discharges. Categorical standards are promulgated in 40 CFR parts 405-471. Each CFR part contains a set of standards that is specific to a particular category of industries (e.g., Dairy Products Processing; Battery Manufacturing). Industries regulated under a specific set of categorical standards may be subject to compliance with one of 
up to six categories of standards, depending on the types of pollutants discharged, the age of the facility, the type of manufacturing process, or other characteristics. These six categories are Best Available Technology Economically Achievable (BAT), Best Conventional Pollutant Control Technology (BCT), Best Practicable Control Technology Currently Available (BPT), New Source Performance Standards (NSPS), Pretreatment Standards for Existing Sources (PSES), and Pretreatment Standards for New Sources (PSINS).

The types of pollutants regulated vary greatly among the industries and may include conventional (e.g., dissolved oxygen, suspended solids) and nonconventional pollutants (e.g., toxic substances). The units of measurement upon which compliance is determined also vary greatly. Many standards measure performance as a function of product produced (e.g., kg of pollutant discharged per $1000 \mathrm{~kg}$ of product produced), and hence, are very industryspecific. Other standards measure performance as the concentration of a pollutant in the waste stream (e.g., mg of pollutant per $L$ of effluent) of the specific industry being considered. The categorical standards for each industry may be further subdivided into one or more of six categories of dischargers within the type of industry.

Most of the categorical standards are highly industry-specific and cannot be applied directly to present or future DOE activities. Hence, compilation of those standards is not warranted in this document. Four sets of categorical standards are specifically referenced in Orders DOE, however, and can be applied to present and future DOE activities. The four sets of applicable categorical standards and the pollutants that are regulated therein are identified below.

\section{Applicable Categorical Standards}

Categorical standards that are cited in Orders DOE include standards for the Electroplating Point Source Category, the Steam Electric Power Generating Point Source Category, the Explosives Manufacturing Point Source Category, and the Photographic Point Source Category. The categories of standards, units of measurement for the standards, and pollutants for which standards are promulgated are listed below for each of these four industrial categories. Within each CFR part, multiple standards exist for individual pollutants. The 
applicability of the standards is determined largely by the type of facility and its discharge characteristics. Because multiple standards exist, and because the characteristics of the facility under consideration must be known before the appropriate standards can be determined, numerical standards are not compiled herein. The appropriate CFR part should be consulted to determine which standards are appropriate for each specific facility.

\section{CFR part 413 - Effluent Guidelines and Standards for Electroplating}

Types of Standards: PSES

Units of Standards: $\mathrm{mg} / \mathrm{L}$

Pollutants Regulated:

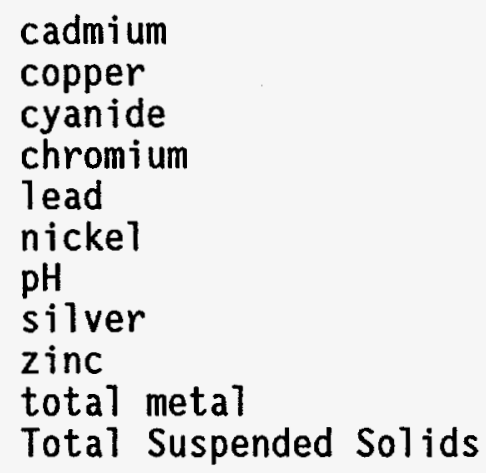

Total Toxic Organics:

acenaphthene

acrolein

acrylonitrile

benzene

benzidine

carbon tetrachloride (tetrachloromethane)

chlorobenzene

1,2,4-trichlorobenzene

hexachlorobenzene

1,2-dichloroethane

1,1,1-trichloroethane

hexachloroethane

1,1,-dichloroethane

1,1,2-trichloroethane

$1,1,2,2$-tetrachloroethane

chloroethane 
Total Toxic Organics (contd):

bis (2-chloroethyl) ether

2-chloroethyl vinyl ether (mixed)

2-chloronaphthalene

2,4,6-trichlorophenol

parachlorometa cresol

chloroform (trichloromethane)

2-chlorophenol

1,2-dichl orobenzene

1,3-dichlorobenzene

1,4-dichlorobenzene

3,3-dichlorobenzidine

1,1-dichloroethylene

1,2-trans-dichloroethylene

2,4-dichlorophenol

1,2-dichloropropane

1,3-dichloropropylene (1,3-dichloropropene)

2,4-dimethylphenol

2,4-dinitrotoluene

2,6-dinitrotoluene

1,2-diphenylhydrazine

ethylbenzene

fluoranthene

4-chlorophenyl phenyl ether

4-bromophenyl phenyl ether

bis (2-chloroisopropyl) ether

bis (2-chloroethoxy) methane

methylene chloride (dichloromethane)

methyl chloride (chloromethane)

methyl bromide (bromomethane)

bromoform (tribromomethane)

dichlorobromomethane

chlorodibromomethane

hexachlorobutadiene

hexach lorocyclopentadiene

isophorone

naphthalene

nitrobenzene

2-nitrophenol

4-nitrophenol

2,4-dinitrophenol

4,6-dinitro-o-cresol

n-nitrosodimethylamine

$n$-nitrosod iphenyl amine

$n$-nitrosodi-n-propylamine

pentachlorophenol

phenol

bis (2-ethylhexy1) phthalate

butyl benzyl phthalate

di-n-butyl phthalate 
Total Toxic Organics (contd):

di-n-octyl phthalate

diethyl phthalate

dimethyl phthalate

1,2-benzanthracene (benzo(a)anthracene)

benzo(a)pyrene (3,4-benzopyrene)

3,4-benzofluoranthene (benzo(b)fluoranthene)

11,12-benzofluoranthene (benzo(k)fluoranthene)

chrysene

acenaphthylene

anthracene

1,12-benzoperylene (benzo(ghi)perylene)

fluorene

phenanthrene

$1,2,5,6$-dibenzanthracene (dibenzo $(a, h)$ anthracene)

indeno $(1,2,3-c d)$ pyrene (2,3-0-phenylene pyrene)

pyrene

tetrachloroethylene

toluene

trichloroethylene

vinyl chloride (chloroethylene)

aldrin

dieldrin

chlordane (technical mixture and metabolites)

4,4-DDT

4,4-DDE ( $p, p-D D X)$

4,4-DDD $(p, p-T D E)$

a)pha-endosulfan

beta-endosulfan

endosulfan sulfate

endrin

endrin aldehyde

heptachlor

heptachlor epoxide

BHC-hexachlorocyclohexane

alpha-BHC

beta-BHC

gamma-BHC

delta-BHC

PCBs-polychlorinated biphenyls

PCB-1242 (aroclor 1242)

PCB-1254 (aroclor 1254)

PCB-1221 (aroclor 1221)

PCB-1232 (aroclor 1232)

PCB-1248 (aroclor 1248)

PCB-1260 (aroclor 1260 )

PCB-1016 (aroclor 1016)

toxaphene

$2,3,7,8$-tetrachlorodibenzo-p-dioxin (TCDD) 
40 CFR part 423 - Effluent Guidelines and Standards for Steam Electric

Power Generating

Types of Standards: BAT; BCT; BPT; NSPS; PSES; PSNS

Units of Standards: $\mathrm{mg} / \mathrm{L}$

Pollutants Regulated:

$0 i 1$ and grease

Total Suspended Solids

chlorine (free available)

chlorine (total residual)

copper (total)

iron (total)

chromium (total)

zinc (total)

Priority Pollutants (by number):

001 acenaphthene

002 acrolein

003 acrylonitrile

004 benzene

005 benzidine

006 carbon tetrachloride (tetrachloromethane)

007 chlorobenzene

008 1,2,4-trichlorobenzene

009 hexachlorobenzene

010 1,2-dichloroethane

011 1,1,1-trichloroethane

012 hexachloroethane

013 1,1,-dichloroethane

014 1,1,2-trichloroethane

015 1,1,2,2-tetrachloroethane

016 chloroethane

018 bis (2-chloroethyl) ether

019 2-chloroethyl vinyl ether (mixed)

020 2-chloronaphthalene

021 2,4,6-trichlorophenol

022 parachlorometa cresol

$023 \mathrm{ch} 7$ oroform (trichloromethane)

024 2-chlorophenol

025 1,2-dichlorobenzene

026 1,3-dichlorobenzene

027 1,4-dichlorobenzene

028 3,3-dichlorobenzidine

029 1,1-dichloroethylene

030 1,2-trans-dichloroethylene

031 2,4-dichloropheno1

032 1,2-dichloropropane 
Priority Pollutants (by number) (contd):

033 1,3-dichloropropylene (1,3-dichloropropene)

034 2,4-dimethylphenol

035 2,4-dinitrotoluene

036 2,6-dinitrotoluene

037 1,2-diphenylhydrazine

038 ethylbenzene

039 fluoranthene

040 4-chlorophenyl phenyl ether

041 4-bromophenyl phenyl ether

042 bis (2-chloroisopropy1) ether

043 bis (2-chloroethoxy) methane

044 methylene chloride (dichloromethane)

045 methyl chloride (chloromethane)

046 methyl bromide (bromomethane)

047 bromoform (tribromomethane)

048 dichlorobromomethane

051 chlorodibromomethane

052 hexachlorobutadiene

053 hexachlorocyclopentadiene

054 isophorone

055 naphthalene

056 nitrobenzene

057 2-nitrophenol

058 4-nitrophenol

059 2,4-dinitrophenol

060 4,6-dinitro-o-cresol

061 n-nitrosodimethylamine

$062 n$-nitrosodiphenylamine

$063 n$-nitrosodi-n-propyl amine

064 pentachlorophenol

065 phenol

066 bis (2-ethylhexy1) phthalate

067 butyl benzyl phthalate

068 di-n-butyl phthalate

069 di-n-octyl phthalate

070 diethyl phthalate

071 dimethyl phthalate

072 1,2-benzanthracene (benzo(a)anthracene)

073 benzo(a)pyrene (3,4-benzopyrene)

074 3,4-benzofluoranthene (benzo(b)fluoranthene)

075 11,12-benzofluoranthene (benzo(b)fluoranthene)

076 chrysene

077 acenaphthylene

078 anthracene

079 1,12-benzoperylene (benzo(ghi)perylene)

080 fluorene

081 phenanthrene

$0821,2,5,6$-dibenzanthracene (dibenzo $(a, h)$ anthracene)

083 indeno $(1,2,3-\mathrm{cd})$ pyrene (2,3-0-phenylene pyrene) 
Priority Pollutants (by number) (contd):

084 pyrene

085 tetrachloroethylene

086 toluene

087 trichloroethylene

088 vinyl chloride (chloroethylene)

089 aldrin

090 dieldrin

091 chlordane (technical mixture and metabolites)

092 4, 4-DDT

093 4, 4-DDE ( $p, p-D D X)$

094 4, 4-DDD ( $p, p-T D E)$

095 alpha-endosulfan

096 beta-endosulfan

097 endosulfan sulfate

098 endrin

099 endrin aldehyde

100 heptach1or

101 heptachlor epoxide (BHC-hexachlorocyclohexane)

102 alpha-BHC

103 beta-BHC

104 gamma-BHC (1 indane)

105 delta-BHC (PCB-polychlorinated biphenyls)

106 PCB-1242 (aroclor 1242)

107 PCB-1254 (aroclor 1254)

108 PCB-1221 (aroclor 1221)

109 PCB-1232 (aroclor 1232)

110 PCB-1248 (aroclor 1248)

111 PCB-1260 (aroclor 1260)

112 PCB-1016 (aroclor 1016)

113 toxaphene

114 antimony

115 arsenic

116 asbestos

117 beryllium

118 cadmium

119 chromium

120 copper

121 cyanide, total

122 lead

123 mercury

124 nicke 1

125 selenium

126 silver

127 thallium

128 zinc

$1292,3,7,8$-tetrachloro-dibenzo-p-dioxin (TCDD) 
40 CFR part 457 - Effluent Guidel ines and Standards for Explosives Manufactuning

Types of Standards: BPT

Units of Standards: $\mathrm{kg} / 1000 \mathrm{~kg}$ of product; $1 \mathrm{~b} / 1000 \mathrm{lb}$ of product;

$\mathrm{pH}$ units

Pollutants Regulated:

$\mathrm{pH}$

chemical oxygen demand (COD)

five day biochemical oxygen demand $\left(\mathrm{BOD}_{5}\right)$

Total Suspended Solids

$0 i 1$ and grease

40 CFR part 459 - Effluent Guidel ines and Standards for Photographic

Processing

Types of Standards: BPT

Units of Standards: $\mathrm{kg} / 1000 \mathrm{~m}^{2}$ of product; $1 \mathrm{~b} / 1000 \mathrm{ft}^{2}$ of product; $\mathrm{pH}$ units

Pollutants Regulated:

$\mathrm{pH}$

silver

cyanide

\subsubsection{Water Quality Criteria}

Water quality criteria (Table 3.3) are risk-based standards that are intended to protect aquatic life and human health. They are applicable to all permitted discharges of effluent to surface waters. The present set of standards was published by the EPA (1986) to be used by its regional offices and by states for the purpose of setting effluent limitations in NPDES permits. The Water Quality Act of 1987 elevated the water quality criteria from guideline to criterion status, thereby making their use mandatory for setting permit limitations. States may develop and implement water quality standards that are stricter than those listed below, but may not allow compliance with standards that are less strict. 
TABLE 3.3. Summary of Water Quality Criteria

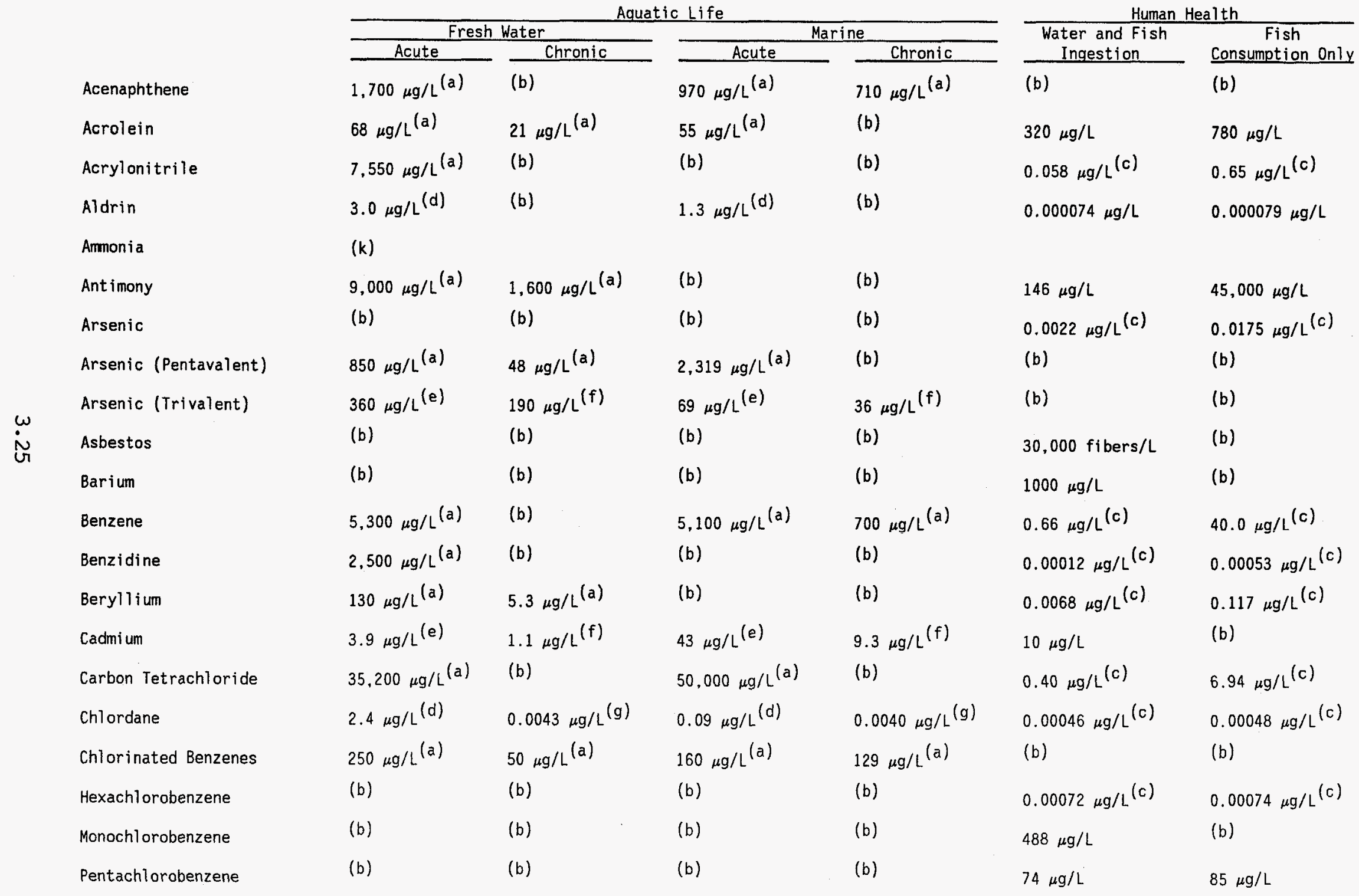


TABLE 3.3. (contd)

Tetrachiorobenzene $1,2,4,5$

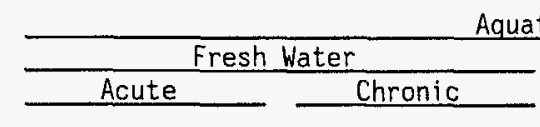

Chlorinated Ethanes

Dichloroethane 1,2

Hexachloroethane

Pentachloroethane

Tetrachlorinated Ethanes

Tetrachloroethane 1,1,2,2

Trichlorinated Ethanes

Trichloroethane 1,1,1

Trichloroethane 1,1,2

Chlorinated Ethylenes

Dichloroethylenes

Tetrachloroethylene

Trichloroethylene

Chlorinated Naphthalenes

Chlorinated Phenols

Chloro-4 Methyl-3 Phenol

Chlorophenol 2

Chlorophenol 4

Dichlorophenol 2,4

Pentachiorophenol (penta)

$$
118,000 \mu \mathrm{g} / \mathrm{L}^{(a)} \quad 20,000 \mu \mathrm{g} / \mathrm{L}^{(a)}
$$$$
980 \mu \mathrm{g} / \mathrm{L}^{(\mathrm{a})} \quad 540 \mu \mathrm{g} / \mathrm{L}^{(\mathrm{a})}
$$$$
7,240 \mu \mathrm{g} / \mathrm{L}^{(a)}
$$$$
1.100 \mu \mathrm{g} / \mathrm{L}^{(a)}
$$$$
9.320 \mu \mathrm{g} / \mathrm{L}^{(a)}
$$

$18.000 \mu \mathrm{g} / \mathrm{L}^{(\mathrm{a})}$

(b)

(b)

(b)

$2,400 \mu \mathrm{g} / \mathrm{L}^{(a)}$

(b)

(b)

$9,400 \mu g / L^{(a)}$

$11.600 \mu \mathrm{g} / \mathrm{L}^{(\mathrm{a})}$

\section{(b)}

$5,280 \mu \mathrm{g} / \mathrm{L}^{(a)}$

$45,000 \mu \mathrm{g} / \mathrm{L}^{(a)}$

$1,600 \mu \mathrm{g} / \mathrm{L}^{(a)}$

$840 \mu \mathrm{g} / \mathrm{L}^{(\mathrm{a})}$

$21,900 \mu \mathrm{g} / \mathrm{L}^{(\mathrm{a})}$

(b)

$4,380 \mu \mathrm{g} / \mathrm{L}(\mathrm{a})$

(b)

$2,020 \mu \mathrm{g} / \mathrm{L}^{(a)}$

$20 \mu \mathrm{g} / \mathrm{L}(\mathrm{e}, \mathrm{h})$

(b)

$2,000 \mu \mathrm{g} / \mathrm{L}^{(a)}$

(b)

$365 \mu \mathrm{g} / \mathrm{L}^{(\mathrm{a})}$

$13 \mu \mathrm{g} / \mathrm{L}(\mathrm{f}, \mathrm{h})$
$30 \mu \mathrm{g} / \mathrm{L}^{(\mathrm{a})}$ (b)

\section{(b)}

(b)

Acute

Marine

Chronic

(b)

$113,000 \mu \mathrm{g} / \mathrm{L}^{(a)} \quad$ (b)

$940 \mu \mathrm{g} / \mathrm{L}^{(\mathrm{a})}$

$390 \mu g / L^{(a)}$

(b)

$9,020 \mu \mathrm{g} / \mathrm{L}^{(a)}$

(b)

$31,200 \mu \mathrm{g} / \mathrm{L}^{(\mathrm{a})}$

(b)

$224,000 \mu \mathrm{g} / \mathrm{L}^{(\mathrm{a})}$

$10,200 \mu \mathrm{g} / \mathrm{L}(\mathrm{a})$

$2,000 \mu \mathrm{g} / \mathrm{L}^{(\mathrm{a})}$

$7.5 \mu \mathrm{g} / \mathrm{L}^{(\mathrm{a})}$ (b)

(b)

$29.700 \mu \mathrm{g} / \mathrm{L}^{(\mathrm{a})}$

(b)

$13 \mu g / L^{(h)}$ (b)

$281 \mu \mathrm{g} / \mathrm{L}^{(\mathrm{a})}$

(b)

(b)

(b)

(b)

(b)

(b)

$450 \mu \mathrm{g} / \mathrm{L}^{(\mathrm{a})}$

(b)

(b)

(b)

(b)

(b)

(b)

$7.9 \mu \mathrm{g} / \mathrm{L}^{(\mathrm{h})}$

$\begin{array}{cc}\begin{array}{c}\text { Huter and Fish } \\ \text { Ingestion }\end{array} & \text { Fish } \\ 38 \mu \mathrm{g} / \mathrm{L} & \end{array}$

$0.94 \mu \mathrm{g} / \mathrm{L}^{(\mathrm{c})}$

$243 \mu \mathrm{g} / \mathrm{L}^{(\mathrm{c})}$

$1.9 \mu \mathrm{g} / \mathrm{L}(\mathrm{c})$

$8.74 \mu \mathrm{g} / \mathrm{L}^{(\mathrm{c})}$

(b)

(b)

(b)

(b)

$0.17 \mu \mathrm{g} / \mathrm{L}^{(\mathrm{c})}$

(b)

$10.7 \mu \mathrm{g} / \mathrm{L}^{(\mathrm{c})}$

(b)

$18,400 \mu \mathrm{g} / \mathrm{L} \quad 1.03 \mu \mathrm{g} / \mathrm{L}$

$0.6 \mu \mathrm{g} / \mathrm{L}^{(\mathrm{c})}$

$41.8 \mu \mathrm{g} / \mathrm{L}(\mathrm{c})$

$0.033 \mu \mathrm{g} / \mathrm{L}(\mathrm{c})$

$1.85 \mu \mathrm{g} / \mathrm{L}^{(\mathrm{c})}$

$0.8 \mu \mathrm{g} / \mathrm{L}(\mathrm{c})$

$8.85 \mu \mathrm{g} / \mathrm{L}^{(\mathrm{c})}$

$2.7 \mu \mathrm{g} / \mathrm{L}(\mathrm{c})$

$80.7 \mu \mathrm{g} / \mathrm{L}^{(\mathrm{c})}$

(b)

(b)

(b)

(b)

(b)

(b)

(b)

$3.090 \mu \mathrm{g} / \mathrm{L}$

(b) 
IABLE 3.3. (contd)

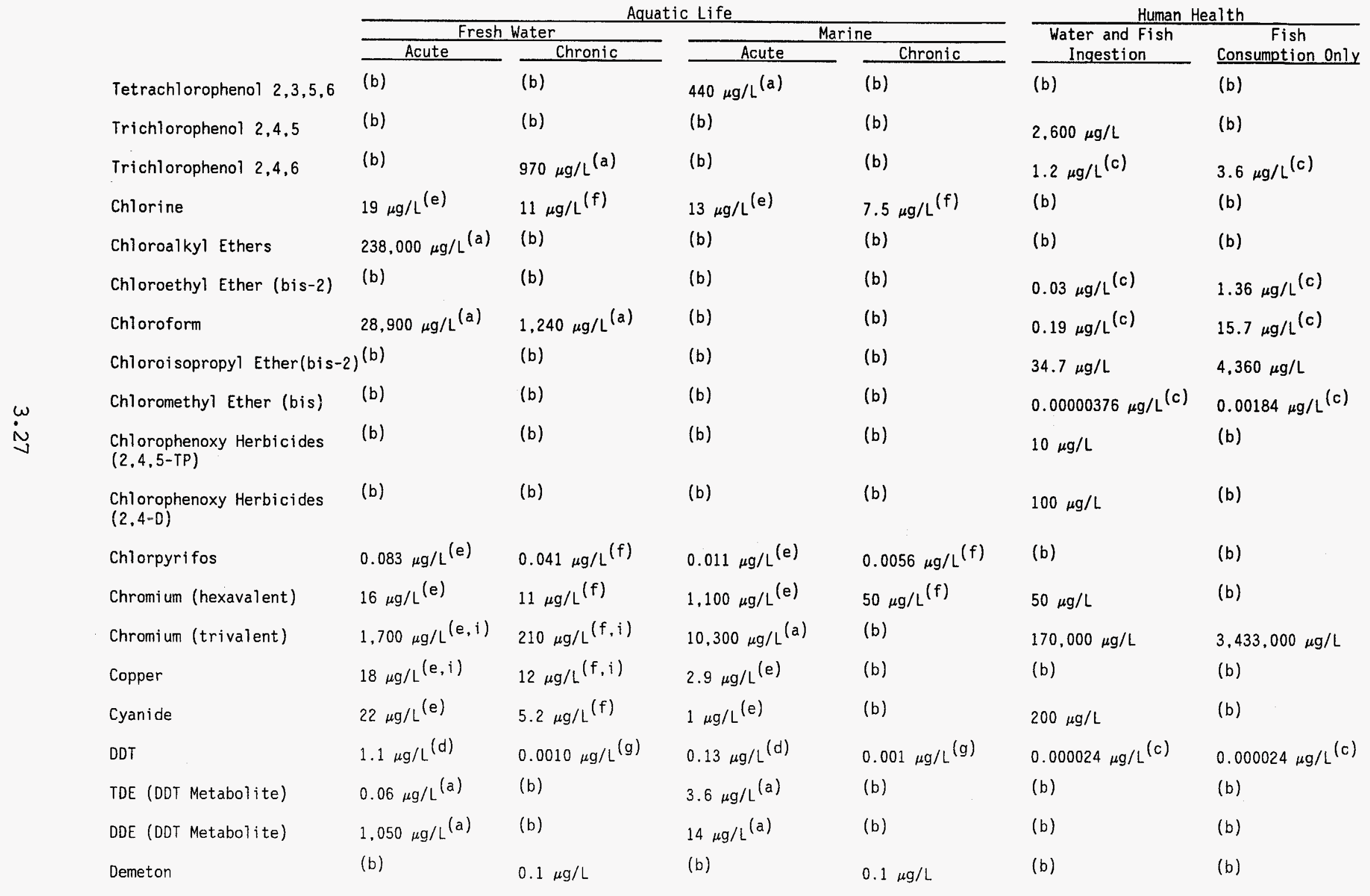


TABLE 3.3. (contd)

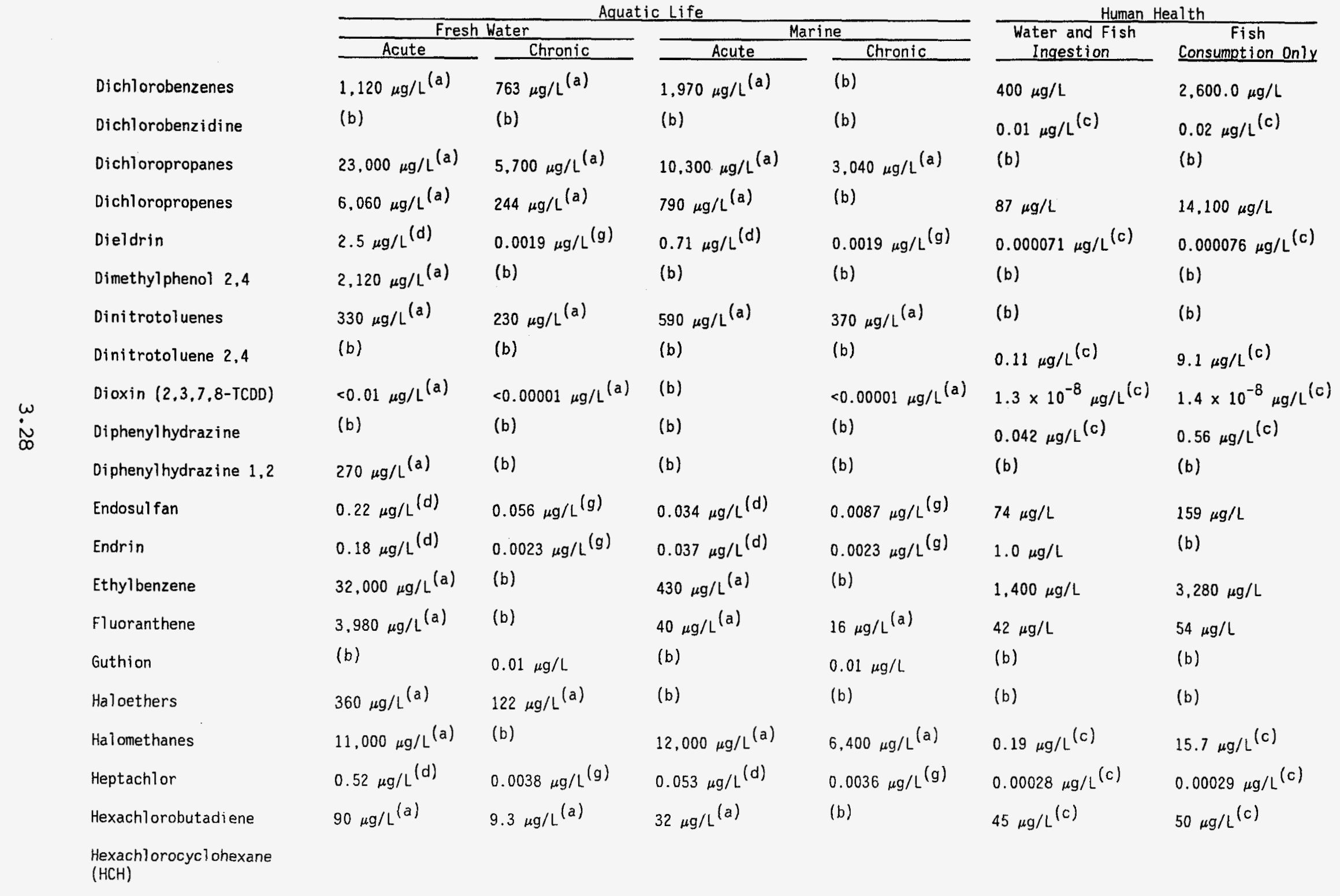


TABLE 3.3. (contd)

\begin{tabular}{|c|c|c|c|c|c|c|}
\hline & \multicolumn{4}{|c|}{ Aquatic Life } & \multicolumn{2}{|c|}{ Human Health } \\
\hline & \multicolumn{2}{|c|}{ Fresh Water } & \multirow{2}{*}{\multicolumn{2}{|c|}{ Chronic }} & \multirow{2}{*}{$\begin{array}{l}\text { Water and Fish } \\
\text { Ingestion }\end{array}$} & \multirow{3}{*}{$\begin{array}{c}\text { Fish } \\
\text { Consumption Only } \\
0.031 \mu \mathrm{g} / \mathrm{L}^{(\mathrm{c})}\end{array}$} \\
\hline & Acute & Chronic & & & & \\
\hline HCH-Alpha & (b) & (b) & (b) & (b) & $0.0092 \mu \mathrm{g} / \mathrm{L}^{(c)}$ & \\
\hline $\mathrm{HCH}-$ Beta & (b) & (b) & (b) & (b) & $0.0163 \mu \mathrm{g} / \mathrm{L}^{(\mathrm{c})}$ & $0.0547 \mu \mathrm{g} / \mathrm{L}^{(\mathrm{c})}$ \\
\hline Lindane (HCH-gamma) & $2.0 \mu \mathrm{g} / \mathrm{L}^{(\mathrm{d})}$ & $0.08 \mu \mathrm{g} / \mathrm{L}^{(g)}$ & $0.16 \mu \mathrm{g} / \mathrm{L}^{(\mathrm{d})}$ & (b) & $0.0186 \mu \mathrm{g} / \mathrm{L}^{(\mathrm{c})}$ & $0.0625 \mu \mathrm{g} / \mathrm{L}^{(\mathrm{c})}$ \\
\hline $\mathrm{HCH}$ (mixture of isomers) & $100 \mu \mathrm{g} / \mathrm{L}^{(a)}$ & (b) & $0.34 \mu \mathrm{g} / \mathrm{L}^{(\mathrm{a})}$ & (b) & $0.0123 \mu \mathrm{g} / \mathrm{L}^{(\mathrm{c})}$ & $0.0414 \mu \mathrm{g} / \mathrm{L}^{(c)}$ \\
\hline Hexachlorocyclopentadi ene & $7.0 \mu \mathrm{g} / \mathrm{L}^{(\mathrm{a})}$ & $5.2 \mu \mathrm{g} / \mathrm{L}^{(\mathrm{a})}$ & $7.0 \mu \mathrm{g} / \mathrm{L}^{(a)}$ & (b) & $206 \mu \mathrm{g} / \mathrm{L}$ & (b) \\
\hline Iron & (b) & $1,000 \mu \mathrm{g} / \mathrm{L}^{(\mathrm{a})}$ & (b) & (b) & $300 \mu g / L^{(j)}$ & (b) \\
\hline Isophorone & $117,000 \mu \mathrm{g} / \mathrm{L}^{(\mathrm{a})}$ & (b) & $12,900 \mu \mathrm{g} / \mathrm{L}^{(\mathrm{a})}$ & (b) & $5,200 \mu \mathrm{g} / \mathrm{L}$ & $520,000 \mu \mathrm{g} / \mathrm{L}$ \\
\hline Lead & $82 \mu \mathrm{g} / \mathrm{L}^{(\mathrm{e})}$ & $3.2 \mu \mathrm{g} / \mathrm{L}^{(f)}$ & $140 \mu \mathrm{g} / \mathrm{L}^{(e)}$ & $5.6 \mu g / L^{(f)}$ & $50 \mu \mathrm{g} / \mathrm{L}$ & (b) \\
\hline Malathion & (b) & $0.1 \mu \mathrm{g} / \mathrm{L}$ & (b) & $0.1 \mu \mathrm{g} / \mathrm{L}$ & (b) & (b) \\
\hline Manganese & (b) & (b) & (b) & (b) & $50 \mu \mathrm{g} / \mathrm{L}^{(j)}$ & $100 \mu \mathrm{g} / \mathrm{L}$ \\
\hline Mercury & $2.4 \mu \mathrm{g} / \mathrm{L}^{(\mathrm{e})}$ & $0.012 \mu \mathrm{g} / \mathrm{L}^{(f)}$ & $2.1 \mu \mathrm{g} / \mathrm{L}^{(e)}$ & $0.025 \mu \mathrm{g} / \mathrm{L}^{(f)}$ & $0.144 \mu \mathrm{g} / \mathrm{L}$ & $0.146 \mu \mathrm{g} / \mathrm{L}$ \\
\hline Methoxychlor & (b) & $0.03 \mu \mathrm{g} / \mathrm{L}$ & (b) & $0.03 \mu \mathrm{g} / \mathrm{L}$ & $100 \mu \mathrm{g} / \mathrm{L}$ & (b) \\
\hline Mirex & (b) & $0.001 \mu \mathrm{g} / \mathrm{L}$ & (b) & $0.001 \mu \mathrm{g} / \mathrm{L}$ & (b) & (b) \\
\hline Naphthalene & $2,300 \mu \mathrm{g} / \mathrm{L}^{(a)}$ & $620 \mu \mathrm{g} / \mathrm{L}^{(a)}$ & $2,350 \mu \mathrm{g} / \mathrm{L}^{(\mathrm{a})}$ & (b) & (b) & (b) \\
\hline Nickel & $1,400 \mu \mathrm{g} / \mathrm{L}^{(\mathrm{d}, \mathrm{i})}$ & $160 \mu \mathrm{g} / \mathrm{L}^{(\mathrm{g}, \mathrm{i})}$ & $75 \mu \mathrm{g} / \mathrm{L}^{(\mathrm{d})}$ & $8.3 \mu \mathrm{g} / \mathrm{L}^{(\mathrm{g})}$ & $13.4 \mu \mathrm{g} / \mathrm{L}$ & $100 \mu \mathrm{g} / \mathrm{L}$ \\
\hline Nitrates & (b) & (b) & (b) & (b) & $10.000 \mu \mathrm{g} / \mathrm{L}$ & (b) \\
\hline Nitrobenzene & $27,000 \mu \mathrm{g} / \mathrm{L}^{(a)}$ & (b) & $6,680 \mu \mathrm{g} / \mathrm{L}^{(a)}$ & (b) & $19.800 \mu \mathrm{g} / \mathrm{L}$ & (b) \\
\hline Nitrophenols & $230 \mu \mathrm{g} / \mathrm{L}^{(a)}$ & $150 \mu \mathrm{g} / \mathrm{L}^{(a)}$ & $4,850 \mu \mathrm{g} / \mathrm{L}^{(\mathrm{a})}$ & (b) & (b) & (b) \\
\hline Dinitrophenols & (b) & (b) & (b) & (b) & $70 \mu \mathrm{g} / \mathrm{L}$ & $14,300 \mu \mathrm{g} / \mathrm{L}$ \\
\hline Dinitro-o-cresol 2,4 & (b) & (b) & (b) & (b) & $13.4 \mu \mathrm{g} / \mathrm{L}$ & $765 \mu \mathrm{g} / \mathrm{L}$ \\
\hline $\begin{array}{l}\text { Nitrosamines } \\
\text { Nitrosodibutylamine }\end{array}$ & $5 B)^{50 \mu g / L}(a)$ & $\begin{array}{l}\text { (b) } \\
\text { (b) }\end{array}$ & $\beta_{B} 300,000 \mu \mathrm{g} / \mathrm{L}^{(a)}$ & $\begin{array}{l}\text { (b) } \\
\text { (b) }\end{array}$ & $\begin{array}{l}\text { (b) } \\
0.0064 \mu \mathrm{g} / \mathrm{L}(\mathrm{c})\end{array}$ & $\begin{array}{l}\text { (b) } \\
0.587 \mu \mathrm{g} / \mathrm{L}^{(\mathrm{c})}\end{array}$ \\
\hline
\end{tabular}


TABLE 3.3. (contd)

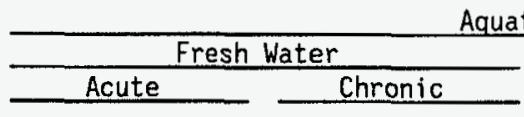

(b)

Nitrosodiethylamine

Ni trosodimethylamine

Nitrosodiphenylamine

Nitrosopyrrolidine

Parathion

Phenol

Phosphorus (elemental)

Phthalate esters

$\omega$

\section{Dibutyl phthalate}

Diethyl phthalate

(b)

(b)

(b)

(b)

$0.065 \mu \mathrm{g} / \mathrm{L}(\mathrm{e})$ $10,200 \mu \mathrm{g} / \mathrm{L}^{(a)}$

(b)

$940 \mu \mathrm{g} / \mathrm{L}^{(\mathrm{a})}$

(b)

(b)

(b)

(b)

(b)

$0.013 \mu \mathrm{g} / \mathrm{L}^{(f)}$

$2,560 \mu \mathrm{g} / \mathrm{L}^{(a)}$

(b)

$3 \mu \mathrm{g} / \mathrm{L}(\mathrm{a})$

(b)

(b)
Dimethyl phthalate

Di-2-ethylhexyl phthalate

Polychlorinated biphenyls

Polynuclear aromatic

hydrocarbons

Selenium inorganic selenite $260 \mu \mathrm{g} / \mathrm{L}$ (d)

inorganic selenate

$760 \mu \mathrm{g} / \mathrm{L}$

Silver

$4.1 \mu \mathrm{g} / \mathrm{L}(d, i)$

Solids (dissolved) and

(b)

(b)

(b)

$2 \mu g / L(a)$

(b)

salinity

Sulfide (hydrogen sulfide, H2S)

(b)

(b)

(b)

(b)

(b)

(b)

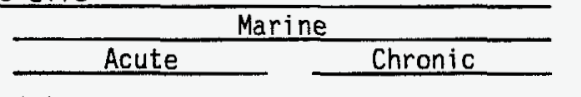

(b)

$0.014 \mu \mathrm{g} / \mathrm{L}^{(\mathrm{g})}$

$35 \mu \mathrm{g} / \mathrm{L}^{(\mathrm{g})}$

$0.12 \mu \mathrm{g} / \mathrm{L}$

$2 \mu g / L^{(g)}$

(b)

(b)

(b)

(b)

(b)

(b)

$5,800 \mu \mathrm{g} / \mathrm{L}^{(a)}$

(b)

$2.944 \mu \mathrm{g} / \mathrm{L}^{(\mathrm{a})}$

(b)

(b)

(b)

(b)

$10 \mu \mathrm{g} / \mathrm{L}^{(\mathrm{a})}$

$300 \mu \mathrm{g} / \mathrm{L}^{(\mathrm{a})}$

$410 \mu g / L^{(d)}$

(b)

$2.3 \mu \mathrm{g} / \mathrm{L}^{(\mathrm{d})}$

(b) (b) (b)

(b)

(b)

$0.03 \mu \mathrm{g} / \mathrm{L}^{(\mathrm{g})}$

(b)

(b)

(b)
Human Health
$\begin{gathered}\text { Water and Fish } \\ \text { Ingestion }\end{gathered}$ Consumption Only

$0.0008 \mu \mathrm{g} / \mathrm{L}(\mathrm{c}) \quad 1.24 \mu \mathrm{g} / \mathrm{L}(\mathrm{c})$

$0.0014 \mu \mathrm{g} / \mathrm{L}^{(\mathrm{c})} \quad 16 \mu \mathrm{g} / \mathrm{L}^{(\mathrm{c})}$

$4.9 \mu \mathrm{g} / \mathrm{L}^{(\mathrm{c})} \quad 16.1 \mu \mathrm{g} / \mathrm{L}^{(c)}$

$0.016 \mu \mathrm{g} / \mathrm{L}^{(\mathrm{c})} \quad 91.9 \mu \mathrm{g} / \mathrm{L}^{(c)}$

(b) (b)

$3,500 \mu \mathrm{g} / \mathrm{L} \quad$ (b)

(b)

(b)

$\begin{array}{lll}0.1 \mu \mathrm{g} / \mathrm{L} & \text { (b) } & \text { (b) } \\ \text { (b) } & \text { (b) } & \text { (b) }\end{array}$

$34,000 \mu \mathrm{g} / \mathrm{L}$

$350,000 \mu \mathrm{g} / \mathrm{L} \quad 1,800,000 \mu \mathrm{g} / \mathrm{L}$

$313.000 \mu \mathrm{g} / \mathrm{L}$

$2,900,000 \mu \mathrm{g} / \mathrm{L}$

$15,000 \mu \mathrm{g} / \mathrm{L}$

$50,000 \mu \mathrm{g} / \mathrm{L}$

$0.000079 \mu \mathrm{g} / \mathrm{L}(\mathrm{c})$

$0.000079 \mu \mathrm{g} / \mathrm{L}^{(\mathrm{c})}$

$0.0028 \mu \mathrm{g} / \mathrm{L}^{(\mathrm{c})}$

$0.0311 \mu \mathrm{g} / \mathrm{L}^{(\mathrm{c})}$

$54 \mu \mathrm{g} / \mathrm{L}(\mathrm{g}) \quad 10 \mu \mathrm{g} / \mathrm{L}$

(b)

(b)

(b)

$50 \mu \mathrm{g} / \mathrm{L}$

(b)

$250,000 \mu \mathrm{g} / \mathrm{L}^{(j)}$

(b)

$2 \mu \mathrm{g} / \mathrm{L}(\mathrm{g}) \quad$ (b)

(b) 
IABLE 3.3. (contd)

Thalli um

Toluene

Toxaphene

Vinyl chloride

Zinc

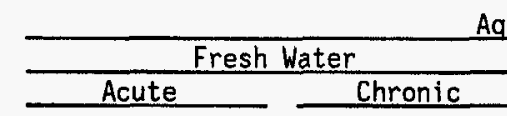

$1.400 \mu \mathrm{g} / \mathrm{L}^{(\mathrm{a})}$

$40 \mu \mathrm{g} / \mathrm{L}^{(\mathrm{a})}$

$17.500 \mu \mathrm{g} / \mathrm{L}^{(a)}$

(b)

$0.73 \mu \mathrm{g} / \mathrm{L}^{(\mathrm{e})}$

(b)

$120 \mu \mathrm{g} / \mathrm{L}^{(e, i)}$

\begin{abstract}
$0.0002 \mu \mathrm{g} / \mathrm{L}^{(f)}$
\end{abstract}
(b)
$110 \mu g / L(f, i)$
Aquatic Life

Marine

$2,130 \mu \mathrm{g} / \mathrm{L}^{(a)}$

(b)

$6,300 \mu \mathrm{g} / \mathrm{L}^{(a)}$

$5,000 \mu \mathrm{g} / \mathrm{L}^{(a)}$

$0.21 \mu \mathrm{g} / \mathrm{L}^{(\mathrm{e})}$

(b)

$95 \mu \mathrm{g} / \mathrm{L}(\mathrm{e})$
$0.0002 \mu \mathrm{g} / \mathrm{L}^{(f)}$

(b)

$86 \mu g / L^{(f)}$

\begin{tabular}{c} 
Human Health \\
\hline $\begin{array}{c}\text { Water and Fish } \\
\text { Ingestion }\end{array}$ Consumption Only
\end{tabular}

$13 \mu \mathrm{g} / \mathrm{L}$

$48 \mu \mathrm{g} / \mathrm{L}$

$14,300 \mu \mathrm{g} / \mathrm{L}$

$0.00071 \mu \mathrm{g} / \mathrm{L}$

$2 \mu \mathrm{g} / \mathrm{L}(\mathrm{c})$

(b)
$424,000 \mu \mathrm{g} / \mathrm{L}$

$0.00073 \mu \mathrm{g} / \mathrm{L}$ $525 \mu \mathrm{g} / \mathrm{L}^{(\mathrm{c})}$

(b)

(a) Data insufficient to derive criteria. Value stated is the lowest observed effect level (LOEL). These concentrations represent apparent threshold levels for acute and/or chronic toxic effects, and are intended to convey information about the degree of toxicity of a pollutant in the absence of established criteria.

(b) Criterion has not been established.

(c) Human health criteria for carcinogens at the $10^{-6}$ risk level, assuming a lifetime consumption of $6.5 \mathrm{~g} /$ day average of contaminated fish and/or shellfish for a $70-\mathrm{kg}$ male.

$\omega$ (d) Not to be exceeded at any time.

(e) Maximum 1-hour average. Not to be exceeded more than an average of once every three years.

(f) Maximum 96-hour (four-day) average. Not to be exceeded more than once every three years.

(g) Maximum 24-hour average. Not to be exceeded more than once every three years.

(h) At $7.8 \mathrm{pH}$

(i) At $100 \mathrm{mg} / \mathrm{L} \mathrm{Ca} \mathrm{CO}$.

(j) Welfare-based. 
Aquatic life is protected by setting standards below which acute or chronic effects of the subject pollutant do not occur in test species. Acute and chronic criteria are determined separately for freshwater and marine biota. Criteria for the protection of human health are set for the ingestion of water and fish tissue and for the ingestion of fish tissue only. For noncarcinogens, the objective of the standards is to prevent adverse health effects in humans. For carcinogens, the objective of the standards is to represent incremental levels of cancer risk that range from $10^{-7}$ to $10^{-5}$. water quality criteria are for individual pollutants; the effects of mixtures of pollutants are not considered. A detailed explanation of the technical bases for water quality criteria is found in 45 FR 79318-79357.

\subsection{STANDARDS UNDER THE COMPREHENSIVE ENVIRONMENTAL RESPONSE, COMPENSATION, AND LIABILITY ACT (CERCLA)}

CERCLA and its related regulations do not promulgate specific standards for environmental restoration. Instead, they mandate that during the feasibility study, the lead agency and support agency identify requirements that are "applicable or relevant and appropriate requirements" (ARARs) to each site (55 FR 8666-8865). Hence, the agencies are given the authority to determine which Federal and State standards should be used at each site during environmental restoration activities.

However, in addition to ARARs, the lead and supporting agencies must al so consider generic standards for toxicants and carcinogens (55 FR 8848):

- "For systematic toxicants, acceptable exposure levels shall represent concentration levels to which the human population, including sensitive subgroups, may be exposed without adverse effect during a lifetime or part of a lifetime, incorporating an adequate margin of safety"

- "For known or suspected carcinogens, acceptable exposure levels are generally concentration levels that represent an excess upper-bound ifetime cancer risk to an individual of between $10^{-4}$ and $10^{-6}$ using information on the relationship between dose and response. The $10^{-6}$ risk level shall be used as the point of departure for determining remediation goals for alternatives when ARARs are not available or are not sufficiently protective because of the presence of multiple contaminants at a site or multiple pathways of exposure" 
Hence, although the regulations pursuant to CERCLA do not set specific numerical standards for environmental restoration, they do set minimum generic standards for systemic toxic substances and carcinogenic substances.

In October of 1991, the EPA announced its goals for expediting Superfund cleanups, with a target of 650 completed cleanups by the year 2000 (ER 1992, $22(39): 2178)$. By the end of 1992 , construction would be complete at 130 sites under the accelerated schedule and at 200 sites by the end of 1993 . The EPA has now completed construction at 65 sites. Of those sites, 25 are 1 isted in the construction completion category, and 40 sites have been deleted from the National Priorities List (NPL). The 1990 revisions to the Superfund National Contingency Plan allow EPA to re-categorize certain "completed" sites into a construction completion category. This category includes sites awaiting deletion from the NPL and sites undergoing long-term remedial actions. In the construction phase of the actions, the National Contingency Plan also permits another category, sites awaiting a five-year review after completion of the remedial action, but EPA removed the five-year requirement in December 1991 (ER $199222(39): 2178)$. The sites listed in this category are now considered awaiting deletion. As a part of the regulatory agenda (ORNL/M-1929/R1, ORNL 1992) EPA proposed to add 30 sites including 6 Federal facility sites, to the NPL. No DOE sites were proposed, but a final ruling will be made in December of 1992. CERCLA will be the topic of interest in a series of congressional hearings in 1992, which will bring about debate over the reauthorization of the statute. CERCLA's current authorization expires at the end of 1994.

\subsection{STANDARDS UNDER THE FEDERAL INSECTICIDE, FUNGICIDE, AND RODENTICIDE ACT}

The Federal Insecticide, Fungicide, and Rodenticide Act has undergone several amendments and name changes, although it is still commonty referred to as FIFRA. FIFRA was amended most recently in 1972 and 1978.

The original act of 1947 marked the beginning of major Federal regulation of the production, storage, distribution, sale, use, and disposal of pesticides and was primarily a pesticide labeling statute. The 1972 amendments added a requirement for the registration of all pesticides, which was 
essentially a means of instituting a premarket clearance system for the sale of pesticides. Registration of pesticides continues to be the primary part of FIFRA, since EPA must determine that the pesticide will not adversely affect people or the environment before approving its registration. The registration of a pesticide can be cancelled by EPA at any time that the pesticide is determined to present a hazard to human health or the environment.

A state can be given primary responsibility for the enforcement of regulations under FIFRA if a cooperative agreement is reached with EPA. EPA restricts the extent to which a state can regulate pesticides, and retains the authority to rescind State responsibility if the State does not comply with statutory requirements or does not adequately enforce the regulations (Wolf 1988).

Standards under FIFRA potentially affect DOE facilities in two ways: through storage and disposal regulations for pesticides, and through worker protection standards.

\subsubsection{CFR part 165 - Regulations for the Acceptance of Certain Pesticides and Recommended Procedures for the Disposal and Storage of Pesticides and Pesticides Containers}

The EPA has the authority to establish regulations and procedures for the disposal and storage of pesticides, pesticide containers, and pesticiderelated wastes to adequately protect public health and the environment. EPA is required by FIFRA to accept for safe disposal any pesticide or related wastes for which registration under FIFRA has been canceled, if the owner of the pesticide so requests.

\subsubsection{CFR part 170 - Worker Protection Standards for Agricultural}

\section{Pesticides}

Unless they are wearing protective clothing, workers may not enter a treated field until sprays have dried or dusts have settled, unless the owner has been specifically exempted from this requirement, or a specific reentry time (see Table 3.4) has been assigned to the pesticide. The owner of the field is responsible for seeing that the restriction is enforced. 
TABLE 3.4. Reentry Time

\begin{tabular}{|c|c|}
\hline ctive Ingredient & Hours \\
\hline $\begin{array}{l}\text { Ethyl parathion } \\
\text { Methyl parathion } \\
\text { Guthion } \\
\text { Demeton } \\
\text { Azodrin } \\
\text { Phosalone } \\
\text { Carbophenothion } \\
\text { Metasystox-R } \\
\text { EPN } \\
\text { Bidrin } \\
\text { Endrin } \\
\text { Ethion }\end{array}$ & $\begin{array}{l}48 \\
48 \\
24 \\
48 \\
48 \\
24 \\
48 \\
48 \\
24 \\
48 \\
48 \\
24\end{array}$ \\
\hline
\end{tabular}

\subsection{REGULATIONS FOR RADIATION PROTECTION}

Standards for radiation protection stem from two laws: the Nuclear Waste Policy Act (NWPA) and the Atomic Energy Act (AEA). The AEA was first promulgated in 1946 to ensure that nuclear energy was developed in a manner consistent with the security of the United States. The AEA mandated the creation of the Atomic Energy Commission (AEC), which was given control of regulations for the production and use of fissionable material. The AEA subsequently underwent several major revisions to reflect changes in nuclear energy research and development in other countries. The first major revision in 1954 opened the development and use of nuclear energy for peaceful purposes to private enterprise. Amendments in 1959 provided for some AEA regulatory responsibilities to be transferred to State governments under federallyapproved programs. In 1964, amendments allowed AEC 1icensees private ownership of special nuclear material. The Uranium Mi11 Tailings Radiation Control Act of 1978 amended AEA by expanding the definition of by-product material to include tailings and waste produced from uranium or thorium ore. The 1983 amendments implemented procedures to improve quality assurance and quality control and mandated quarterly reports to the Secretary of Energy. The AEA has continued to be revised and supplemented by additional statutes.

The NWPA, enacted in 1982, was developed to "provide for the development of repositories for the disposal of high-level radioactive waste and spent 
nuclear fuel, to establish a program of research, development, and demonstration regarding the disposal of high-level radioactive waste and spent nuclear fuel" (42 USC 10101 et. seq.). This Act was the first to establish Federal responsibility for the development of repositories. Under the Act, DOE must evaluate potential sites for a permanent high-level waste repository. Once the NRC approves the proposed site, DOE may begin construction of a monitored retrievable storage facility to provide interim storage for high-level radioactive waste.

The Energy Reorganization Act of 1974 separated licensing and related functions from energy development functions by establishing the Energy Research and Development Administration (ERDA) and the Nuclear Regulatory Commission (NRC). The Act also abolished the AEC and gave the NRC some regulatory authority over ERDA (which later became the U.S. Department of Energy). Unlike the AEA and the NWPA, the Energy Reorganization Act of 1974 did not promulgate regulations containing standards that might be applicable to DOE.

\subsubsection{CFR part 60 - Disposal of High-Level Radioactive Wastes in Geologic Repositories Pursuant to the Nuclear Waste Policy Act}

These performance objectives are based on human health considerations. The specific health factors and the methodology used are not defined in the regulations.

\section{Performance Objectives Before Permanent Closure}

- Geologic repository operations areas must be designed so that any releases or exposures before permanent closure are within the limits specified in any applicable environmental standards (especially 10 CFR part 20).

- Geologic repository operations areas must be designed so that any of the waste could be retrieved at any time, with retrieval operations beginning up to 50 years after waste emplacement began.

\section{Performance Objectives After Permanent Closure}

- Engineered barrier systems must be designed so that containment of high-level waste will be assured during the period when radiation and thermal conditions are dominated by fission product decay. 
This period, determined by the NRC, must be at least 300 years and less than 1,000 years after permanent closure.

- Any release of radionuclides must be a gradual process which results in small fractional releases over long periods of time. The release rate must be less than one part per 100,000 per year of the quantity of that radionuclide calculated to be present 1,000 years after permanent closure.

\subsubsection{CFR part 72 - Licensing Requirements for the Independent Storage of Spent Nuclear Fuel and High-Level Radioactive Waste Pursuant to the Atomic Energy Act}

The design of an Independent Spent Fuel Storage Installation (ISFSI) should be based on public health and safety criteria, but specific health factors and the methodology for determining the standards are not defined in this CFR part.

\section{Annual Dose Equivalent Outside a Controlled Area}

The annual dose equivalent to any individual outside a controlled area must not exceed:
25 mrem to whole body
75 mrem to the thyroid
25 mrem to any other organ

as a result of planned discharges of radioactive materials (except radon and its daughters), direct radiation from ISFSI operations, or any other radiation from uranium fuel cycle operations. Radioactive materials in effluents and direct radiation levels associated with ISFSI operations must have as low as reasonably achievable (ALARA) operational restrictions established.

\section{Doses Outside a Controlled Area Due to Design Accidents}

Any individual at or outside the nearest boundary of the controlled area of an ISFSI site must not receive a dose greater than 5 rem to the whole body or any organ as a result of any design-basis accident. The minimum distance of the nearest boundary of a controlled area to the spent fuel handling and storage facilities must be at least 100 meters. 


\subsubsection{CFR part 191 - Radiation Protection Standards for Managing and Disposing of Spent Nuclear Fuel, High-Level and Transuranic Radioactive Waste Pursuant to the Atomic Energy Act}

The objective of long-term containment follows a non-degradation ideal. Containment should limit the projected releases of radionuclides to the environment so as to "insure that risks to future generations from disposal of these wastes will be no greater than the risks which would have existed if the uranium ore used to create the wastes had not been mined to begin with." (50 FR 38066).

The actual standards set forth in this rule were developed by balancing several considerations. The first was the expected capabilities of waste management and disposal technologies to reduce risks to the public health and environment. Long-term and short-term risks should be factors. A second consideration was consistency with any applicable or related environmental standards for radiation exposure. Third, to assess the acceptability of residual risks that might be allowed by the rule, various benchmarks were evaluated. Finally, a major consideration was public concerns expressed during various phases of the rule-making process, particularly concerns about the uncertainties inherent in the unprecedented time periods involved. The final rule is a combination of all of these factors. EPA states that no one factor predominated in creating the rule. These standards are reasonably achievable and limit risks to levels that are acceptably small (50 FR 38069-38070).

\section{Management and Storage}

- The combined annual dose equivalent to any member of the public from 1) discharges of radioactive material and direct radiation resulting from management and storage of the materials and 2) all operations covered by Part 190, at facilities regulated by the NRC, or Agreement States, must not exceed

25 mrem to the whole body

75 mrem to the thyroid

25 mrem to any other critical organ.

- Any discharges from facilities operated by the DOE must not exceed

25 mrem to the whole body

75 mrem to any critical organ. 
(These limits were equivalent to the standards promulgated under the Clean Air Act in 40 CFR part 61 until these limits were reduced.)

\section{Disposal - Containment}

Disposal systems for spent nuclear fuel or high-level or transuranic radioactive wastes are to be designed so as to provide reasonable assurance that for 10,000 years after disposal the system will

- have a likelihood of less than one chance in 10 of exceeding the quantities calculated according to Table 3.5 .

- have a likelihood of less than one chance in 1,000 of exceeding ten times the quantities calculated according to Table 3.5.

TABLE 3.5. Release Limits for Containment Requirements (cumulative releases to the accessible environment for 10,000 years after disposal)

Radionuclide

Americium-241 or -243

Carbon-14

Cesium-135 or -137

Iodine-129

Neptunium-237

Plutonium-238, $-239,-240$, or -242

Radium-226

Strontium-90

Technetium-99

Thorium -230 or -232

Tin-126

Uranium-233, $-234,-235,-236$, or -238

Any other alpha-emitting radionuclide

with a half-life greater than 20 years

Any other radionuclide with a half-life greater than 20 years that does not emit alpha particles
Release Limit per 1,000

MTHM $^{(a)}$ or Other Unit of Waste (Curies)

(a) metric tons of heavy metal 


\section{Disposal - Individual Protection}

The system must be designed so that the annual dose equivalent to any member of the public for 1,000 years after disposal does not exceed

25 mrem to the whole body

75 mrem to any critical organ.

(These doses are calculated by considering all potential pathways and assuming that individuals consume 2 L/day of drinking water from any significant source of groundwater outside of the controlled area.)

\section{Disposal - Groundwater Protection}

For 1,000 years after disposal, the radionuclide concentrations averaged over any portion of a special source of groundwater must not exceed

- $5 \mathrm{pCi} / \mathrm{L}$ of radium-226 and radium-228

- $15 \mathrm{pCi} / \mathrm{L}$ of alpha-emitting radionuclides (including radium-226 and radium-228 but excluding radon)

- a combined concentration of radionuclides that emit either beta or gamma radiation producing an annual dose equivalent to the total body or any internal organ greater than 4 mrems per year if an individual consumed $2 \mathrm{~L} /$ day of drinking water.

\subsubsection{CFR part 192 - Standards for Protection Against Uranium Mi11}

\section{Tailings Pursuant to the Atomic Energy Act}

The basis for these standards was changed from non-degradation to optimized cost-benefit. The small increase in benefit for health protection under the non-degradation basis was judged to be insufficient to justify the associated large increase in cost. An optimized cost-benefit approach gives longer-term health protection at somewhat higher costs without a reliance on institutional controls. Because health risks cannot be quantified totally, risk models are used. The extent to which health impacts might be avoided under any realistic alternative standards are estimated, and the alternatives that offer the most cost-effective reduction of these impacts are selected (48 FR 596). 


\section{Residual Radioactive Material}

Residual radioactive material must be contained for up to 1,000 years to the extent that is reasonably achievable, or for at least 200 years. The containment structures must provide reasonable assurance that releases of radon-222 from residual radioactive material will not

- exceed an average release rate (averaged over the entire surface of the disposal site over at least a one-year period) of $20 \mathrm{pCi} / \mathrm{m}^{2} / \mathrm{s}$ or

- increase the annual average concentration of radon-222 in air outside the disposal site by more than one-half $\mathrm{pCi} / \mathrm{L}$.

For 1 and and buildings contaminated with post-cleanup residual radioactive materials, the concentration of radium-226 in land averaged over any area of $100 \mathrm{~m}^{2}$ must not exceed the background leve 1 by more than

- $5 \mathrm{pCi} / \mathrm{g}$, averaged over the upper $15 \mathrm{~cm}$ of soil

- $15 \mathrm{pCi} / \mathrm{g}$, averaged over $15 \mathrm{~cm}$-thick layers of soil more than $15 \mathrm{~cm}$ below the surface.

In any occupied or habitable building, the objective of remedial action is to achieve an annual average radon decay product concentration that does not exceed $0.002 \mathrm{WL}$ (WL = working level = any combination of short-1ived radon decay products in one liter of air that will result in the ultimate emission of alpha particles with a total energy of 130 billion electron volts). In any case, the concentration must not exceed $0.03 \mathrm{WL}$, and the level of gamma radiation must not exceed the background level by more than 20 microroentgens per hour.

\section{Management of Uranium By-Products}

Molybdenum and uranium are listed as hazardous under 40 CFR part 26493. They are limited to quantities for emissions not exceeding the following:

- combined radium-226 and radium-228

$5 \mathrm{pCi}$

- gross alpha-particle activity (excluding $15 \mathrm{pCi}$. radon and uranium)

Disposal areas must be designed to provide reasonable assurance that radiological hazards will be controlled for 1,000 years to the extent 
reasonably achievable, and for at least 200 years; and that releases of radon-222 do not exceed an average release rate of $20 \mathrm{pCi} / \mathrm{m}^{2} / \mathrm{s}$.

These requirements do not apply to a site that contains a concentration of radium-226 in land, averaged over areas of $100 \mathrm{~m}^{2}$, that does not exceed the background level by more than

- $5 \mathrm{pCi} / \mathrm{g}$, averaged over the upper $15 \mathrm{~cm}$ below the surface

- $15 \mathrm{pCi} / \mathrm{g}$, averaged over $15 \mathrm{~cm}$-thick layers of soil more than $15 \mathrm{~cm}$ below the surface.

\section{Management of Thorium By-Products}

The above standards for management of uranium by-products apply also to thorium by-products. In addition, provisions applicable to radon-222 also apply to radon-220, and provisions applicable to radium-226 also apply to radium-228.

Operations must be conducted in such a way as to provide reasonable assurance that the annual dose equivalent as a result of exposures to the planned discharge of radioactive materials, radon-220 and its daughters excepted, does not exceed

$$
\begin{aligned}
& 25 \text { mrem to the whole body } \\
& 75 \text { mrem to the thyroid } \\
& 25 \text { mrem to any other organ. }
\end{aligned}
$$

\subsection{STANDARDS UNDER THE RESOURCE CONSERVATION AND RECOVERY ACT}

The Resource Conservation and Recovery Act (RCRA) of 1976 governs the management of solid waste. The purposes of RCRA are 1) to ensure the safe and environmentally sound management of hazardous and nonhazardous solid waste, and 2) to promote resource recovery techniques to provide fuel and minimize waste volume. Substantive requirements that are applicable to the management of solid waste are found in Subtitle $C$, "Hazardous Waste Management"; Subtitle D, "State and Regional Solid Waste Plans;" and Subtitle I, "Regulation of Underground Storage Tanks." In 1984, RCRA's scope and level of detail were expanded by the Hazardous and Solid Waste Amendments (HSWA). Under RCRA, states may develop and enforce their own hazardous waste programs 
in lieu of the Federal program administered by the EPA, provided that the State program is at least as stringent as the Federal program.

The chemical-specific regulatory standards that have been promulgated pursuant to RCRA and that are of interest to DOE are found in 40 CFR part 261 (Regulations for Identifying Hazardous Waste); 40 CFR part 264/265 (Standards for Owners and Operators of Hazardous Waste Treatment, Storage, and Disposal Facilities); and 40 CFR part 268 (Land Disposal Restrictions). The standards in 40 CFR part 261 identify wastes that are subject to regulatory control under the hazardous waste management program of RCRA Subtitle $C$.

RCRA provides quantitative, chemical specific standards for many hazardous constituents in groundwater, and other media. Chemical-specific standards for the monitoring and protection of groundwater are found in $40 \mathrm{CFR}$ part 257 (Appendix I), 40 CFR part 258 Subpart D, 49 CFR part 264 Subpart F. Air emission standards for waste management unit process vents and equipment leaks are found at 40 CFR part 264 subparts AA and BB, and 40 CFR part 265 Subparts $A A$ and $B B$. Air emission and related technical standards for industrial boilers and furnaces that burn hazardous waste are found in 40 CFR part 266 Appendix I through VIII. Chemical specific and technology-based treatment standards for hazardous waste subject to land disposal restrictions are found in 40 CFR part 268.

In addition to RCRA's currently promulgated standards, changes to add new standards and modify existing standards have also been proposed by EPA and may be of concern to DOE. The proposed changes include:

- 40 CFR parts 260 through 270 - EPA is going to propose exemption from regulation for any currently 1 isted wastes that are not hazardous because the concentrations of hazardous constituents are lower than limits established by the proposed by rule.

- 40 CFR part 261 - Seven additional waste streams generated during the production, recovery, and refining of coke are proposed for listing as hazardous waste.

- 40 CFR part 264 Subpart S - "Action" levels, for clean up of soils. water and air, were proposed for the RCRA Subpart $S$ corrective action program. 
- 40 CFR parts 264 and 265 - Air emission standards are proposed for emissions of volatile organic constituents from tanks, impoundments, containers, and miscellaneous units.

- 40 CFR part 268 - Treatment standards are proposed for contaminated debris and other newly listed wastes.

- 40 CFR part 268 - EPA will propose standards that mush be met before contaminated soil or toxicity characteristic waste may be disposed of on 1 and.

Additional information about technical standards, definition, and other provisions in RCRA 40 CFR parts 261, 264, 265, and 268 are discussed in the following sections.

\subsubsection{CFR part 261 - Regulations for Identifying Hazardous Waste}

\section{Definitions}

Solid Waste. A solid waste is any garbage, refuse, sludge from a waste treatment plant or air pollution control facility, and other discarded material that results from industrial, commercial or mining and agricultural operations, or from community activities. Solid waste does not include the following: solid or dissolved material in domestic sewage; solid or dissolved materials in irrigation return flows; industrial discharges that are point sources subject to permits under the Clean Water Act (CWA); or source material, special nuclear materials, or by-products as defined by the Atomic Energy Act (AEA) [RCRA Section 1004(27)].

Hazardous Waste. Hazardous waste is defined as solid waste that causes or contributes to an increase in mortality or an increase in serious illness, or that poses a substantial present or potential hazard to human health or the environment when improperly treated, stored, transported, or disposed of or otherwise managed [RCRA Section $1004(5)$ ]. A solid waste is considered hazardous if it is not excluded by regulation and if it 1) is a listed hazardous waste, 2) exhibits a characteristic of a hazardous waste, or 3) contains a mixture of a solid waste and a listed hazardous waste (40 CFR part 261.3).

Characteristics of Ignitability, Corrosivity, and Reactivity. If a solid waste exhibits the characteristics of either ignitability, corrosivity, 
or reactivity as defined in 40 CFR part 261 , it is considered to be a hazardous waste and is subject to the provisions of RCRA Subtitle $C$.

Toxicity Characteristic. A solid waste exhibits toxicity characteristics if the extract from a representative sample of the waste contains any of the contaminants listed in Table 3.6 at a concentration equal to or greater than the respective value in the table. If the waste contains less than $0.5 \%$ filterable solids, the waste after filtering is considered to be the extract for the purposes of this section. A solid waste that exhibits the toxicity characteristics is considered to be a hazardous waste and is subject to the provisions of RCRA Subtitle C.

TABLE 3.6. Maximum Concentration of Contaminants for the Toxicity Characteristic ( $r b, r b / t b, r b / a b)^{(a)}$

EPA Hazardous Waste Number(a)

D004

D005

D018

D006

D019

D020

D021

D022

D007

D023

D024

D025

D026

D016

D027

D028

D029

D030

D012

D031

D032

D033

D034

D008

D013

D009

D014

D035
Contaminant

Arsenic

Barium

Benzene

Cadmium

Carbon tetrachloride

Chlordane

Chlorobenzene

Chloroform

Chromium

o-Cresol

m-Cresol

p-Cresol

Cresol

2,4-D

1,4-Dichl orobenzene

1,2-Dichloroethane

1,1-Dichloroethylene

2,4-Dinitrotoluene

Endrin

Heptachlor (and its

epoxide)

Hexach Torobenzene

Hexachlorobutadiene

Hexachloroethane

Lead

Lindane

Mercury

Methoxychlor

Methyl ethyl ketone
Level (mg/L)

5.0

100.0

0.5

1.0

0.5

0.03

100.0

6.0

5.0

200.0

200.0

200.0

200.0

10.0

7.5

0.5

0.7

0.13 (b)

0.02

0.008

0.13 (b)

0.5

3.0

5.0

0.4

0.2

10.0

200.0 
IABLE 3.6. (contd)

EPA Hazardous

Waste Number (a)

D036

D037

D038

D010

D011

D039

D015

D040

D041

D042

D017

D043
Contaminant

Nitrobenzene

Pentachlorophenol

Pyridine

Selenium

Silver

Tetrachloroethylene

Toxaphene

Trichloroethylene

2,4,5-Trichlorophenol

2,4,6-Trichlorophenol

2,4,5-TP (Silvex)

Vinyl chloride
Level (mg/L)

2.0

100.0

5.0 (b)

1.0

5.0

0.7

0.5

0.5

400.0

2.0

1.0

0.2

(a) levels for hazardous wastes D018 to D043 are effective September 25, for hazardous wastes D004 to D017 are technology-based standards founded on a risk-based goal ( $\mathrm{rb} / \mathrm{tb})$; standards for hazardous wastes D018 to D043 are risk-based (rb), with the exception of D030, D032, D038 which are default analytical-based standards founded on a riskbased goal ( $r b / a b)$.

(b) The quantitation limit for this waste type is greater than the calculated regulatory limit. The quantitation limit therefore becomes the default regulatory limit (55 FR 11798, March 19, 1990). regulatory limits are, in practice, analytically-based standards founded on a risk-based goal.

Maximum Concentration of Contaminants. The maximum concentrations of contaminants that were determined to exhibit toxicity characteristics for hazardous waste categories D004 to D017 were derived from maximum contaminant levels (MCLs) under the Safe Drinking Water Act are equal to 100 times the SDWA maximum contaminant levels and are, therefore, technology-based standards founded on risk-based goáls.

The regulatory levels for D018 to D043 wastes are based on health-based concentration thresholds and a dilution/attenuation factor that was developed using a subsurface fate and transport model.

\subsubsection{CFR part 264 Subpart F - Releases from Solid Waste Management Units} Hazardous Constituent 
Constituents are those substances 1 isted in 40 CFR part 261 Appendix VIII. In addition, a Groundwater Monitoring List of Hazardous Constituents is in 40 CFR part 264 Appendix IX. The original basis for listing a solid waste as hazardous was the presence of hazardous constituents within that solid waste.

Concentration Limit. Limits are established for hazardous constituents in a RCRA treatment, storage, or TSD facility permit for the purpose of protecting groundwater. EPA Regional Administrator can exclude an Appendix VIII constituent from a facility permit if he/she finds that the constituent is not capable of posing a present or potential hazard to human health or the environment. Concentration limits that are established in a facility permit must not exceed the background levels of that constituent in groundwater, or must not exceed the respective value given in Table 3.7 if the background level is less than the value in the table. Limits are equal to maximum contaminant levels under the SDWA, and are therefore a combination of risk-based and technology-based standards.

Alternate Concentration Limit $(A C L)$. ACL is an alternate limit established in a facility permit in lieu of hazardous-constituent background concentration levels or the levels in Table 3.7 establish an ACL, the EPA Regional Administrator must consider potential adverse effects on the quality of groundwater and of hydraulically connected surface are based on a sitespecific environmental and human health risk assessment and are therefore risk-based standards.

\subsubsection{CFR parts $264 / 265$ Subpart M - Land Treatment}

\section{Definitions}

Land Treatment. Land treatment is the operation of applying hazardous waste onto, or incorporating the waste into the soil so that it is degraded, transformed, or immobilized.

Cadmium Application Rate. Cadmium application rate refers to the amount of cadmium (in waste) that can be applied to land. The regulatory levels in Tables 3.8 and 3.9 are based on human health risk assessment. 
TABLE 3.7. Limits for Hazardous Constituents in Facility Permits ( $r b / t b)^{(a)}$

EPA Hazardous

Waste Number
D004
D005
D006
D007
D008
D009
D010
D011
D012
D013
D014
D015
D016
D017

\begin{tabular}{lll} 
Contaminant & & $\begin{array}{c}\text { Concentration } \\
\text { Limit (mg/L) }\end{array}$ \\
\cline { 1 - 1 } Arsenic & & 0.05 \\
Barium & & 1.0 \\
Cadmium & & 0.01 \\
Chromium & & 0.05 \\
Lead & 0.05 \\
Mercury & & 0.002 \\
Selenium & & 0.01 \\
Silver & & 0.05 \\
Endrin & & 0.0002 \\
Lindane & & 0.004 \\
Methoxychlor & 0.1 \\
Toxaphene & 0.005 \\
2,4-D & & 0.1 \\
2,4,5-TP Silvex & 0.01
\end{tabular}

(a) Standards for hazardous wastes D004 to D017 are technology-based standards founded on a risk-based goal (rb/tb).

TABLE 3.8. Annual Cadmium Application Rates ( $r b$ )

Annual Cadmium Application Rate

Time Period

\begin{tabular}{ll}
\multicolumn{2}{c}{ (kg/hectare) } \\
\cline { 1 - 1 } (a) & (b) \\
0.5 & 2.0 \\
0.5 & 1.25 \\
0.5 & 0.5
\end{tabular}

Before $6 / 30 / 84$

$7 / 1 / 84$ to $12 / 31 / 86$

$1 / 1 / 87$ to present.

0.5

0.5

(a) Applies to 1 and used for production of tobacco, leafy vegetables, or root crops grown for human consumption.

(b) Applies to other food-chain crops. 
TABLE 3.9. Maximum Cumulative Cadmium Application (kg/hectare)

$\begin{array}{ccc}\begin{array}{c}\text { Soil Cation Exchange } \\ \text { Capacity } \\ \text { (milliequivalent/100g) }\end{array} & \begin{array}{c}\text { Background Soil } \\ \mathrm{pH}<6.5\end{array} & \begin{array}{c}\text { Background Soil } \\ \mathrm{pH}>6.5\end{array} \\ {5} } & 5 & 5 \\ 5 \text { to 15 } & 5 & 10 \\ \text { Greater than } 5 & 5 & 20\end{array}$

\subsubsection{CFR part 268 - Land Disposal Restrictions}

\section{Definitions}

California List Hazardous Waste. California list hazardous wastes are subject to 7 and disposal restrictions that specify technologies as treatment standards. The list was derived from California State regulations.

Land Disposal. Land disposal is defined as placement in or on the 1 and. It includes placement in a landfill, surface impoundment, waste pile, injection well, land treatment facility, salt dome/bed formation, underground mine or cave, or placement in a concrete vault for disposal purposes.

Wastewater. Wastewater is defined by EPA to contain less than $1 \%$ total organic carbon and less than $1 \%$ total filterable solids. All other wastes (including soil and debris) are considered not to be wastewater for the purposes of this CFR part, unless specified otherwise.

Soft-Hammer Provisions. Soft-hammer provisions were effective until EPA set treatment standards or until May 8, 1990, when hard-hammer provisions became effective. Under soft-hammer provisions, wastes were allowed to be land disposed only if 1) the disposal unit met RCRA minimum technology requirements and 2) the generator investigated treatment options and used the best treatment that was available and practical.

Hard-Hammer Provisions. Hard-hammer provisions prohibit 1 and disposal of restricted wastes if EPA fails to promulgate treatment standards by the statutory deadlines for solvent-containing, dioxin-containing, and Californialist wastes and by May 8, 1990 for all the scheduled wastes, as defined in 40 CFR part 268. 
Halogenated Organic Compounds (HOCs). HOCs are listed in 40 CFR part 268 Appendix III.

Treatment Standards. Treatment standards are concentration levels or specific technologies that must be achieved prior to disposal of hazardous wastes. Alternatively, EPA may assign to a hazardous waste a "no land disposal" designation. This designation is set when the waste is no longer generated, is totally recycled, is not currently being land disposed, or treatable such that no residual hazardous wastes are produced from the treatment. Treatment standards are based on the best demonstrated available technology (BDAT) for a specific waste. In addition to treatment standards, LDRs contain three other types of restrictions (national capacity extension, soft-hammer restrictions, and hard-hammer restrictions).

Tables 3.10 and 3.11 specify regulatory standards that are applicable to the hazardous wastes listed in 40 CFR part 268.

\section{Burning of Hazardous Waste in Boilers and Industrial Furnaces}

The EPA regulates air emissions from the burning of hazardous waste in boilers and industrial furnaces under 40 CFR parts 260, 261, 264, 265, 266, 270, and 271. These rules control emissions of toxic organic compounds, toxic metals, hydrogen chloride, chlorine gas, and particulate matter from boilers and industrial furnaces burning hazardous waste. The rules also subject owners and operators to the general facility standards applicable to hazardous waste treatment, storage, and disposal facilities (56 FR 7134).

\subsection{STANDARDS UNDER THE SAFE DRINKING WATER ACT}

The SDWA was enacted in 1974 and amended in 1986. To protect public drinking water sources, EPA is required to develop mechanisms to control bacterial and chemical contaminants and to regulate the underground injection of contaminants into groundwater.

The SDWA mandates the creation of primary and secondary standards to regulate the quality of water that is available to the public through community and non-community water systems. Primary standards protect public 
TABLE 3.10. Treatment Standards for RCRA Wastes Under Land Disposal Restrictions (tb)

\begin{tabular}{|c|c|c|c|c|c|}
\hline \multirow[b]{3}{*}{$\begin{array}{l}\text { EPA Hazardous Waste } \\
\text { Number (Name) }\end{array}$} & \multirow[b]{3}{*}{ Constituent ${ }^{(b)}$} & \multicolumn{4}{|c|}{ Regulatory Standard } \\
\hline & & \multicolumn{2}{|c|}{$\begin{array}{l}\text { Concentration in Waste } \\
\text { Extract (40 CFR 268.41) }\end{array}$} & \multicolumn{2}{|c|}{$\begin{array}{c}\text { Concentration in Waste } \\
(40 \text { CFR 268.43) }\end{array}$} \\
\hline & & $\begin{array}{l}\text { Nonwastewater } \\
(\mathrm{mg} / \mathrm{L})\end{array}$ & $\begin{array}{l}\text { Wastewater } \\
\text { (mg/L) }\end{array}$ & $\begin{array}{l}\text { Nonwastewater } \\
(\mathrm{mg} / \mathrm{kg})\end{array}$ & $\begin{array}{l}\text { Wastewater } \\
(\mathrm{mg} / \mathrm{L})\end{array}$ \\
\hline $\begin{array}{l}\text { D001 (Ignitability } \\
\text { Characteristic) }\end{array}$ & $\begin{array}{l}\text { Ignitable liquids based on } 261.21(a)(1)- \\
\text { wastewaters } \\
\text { Ignitable liquids based on } 261.21(a)(1)-\text { Low } \\
\text { TOC Ignitable Liquids Subcategory-less than } 10 \% \\
\text { total organic carbon } \\
\text { Ignitable liquids based on } 261.21(a)(1)-H i g h \\
\text { ToC Ignitable Liquids Subcategory-greater than } \\
\text { or equal to } 10 \% \text { total organic carbon } \\
\text { Ignitable compressed gases based on } 261.21(a)(3) \\
\text { Ignitable reactives } 261.21(\mathrm{a})(2) \\
\text { Oxidizers based on } 261.21(\mathrm{a})(4)\end{array}$ & & & $\begin{array}{l}\text { spec. tech. } \\
\text { spec. tech. } \\
\text { spec. tech. (c) } \\
\text { spec. tech. } \\
\text { spec. tech. }\end{array}$ & spec. tech. \\
\hline $\begin{array}{l}\text { D002 (Corrosivity } \\
\text { Characteristic) }\end{array}$ & $\begin{array}{l}\text { Acid subcategory based on } 261.22(a)(1) \\
\text { Alkaline subcategory based } 261.22(a)(1) \\
\text { Other corrosives based on } 261.22(a)(2) \\
\text { Radioactive High-Level Wastes Generated During the } \\
\text { Reprocessing of Fuel Rods Subcategory }\end{array}$ & & & $\begin{array}{l}\text { spec. tech. } \\
\text { spec. tech. } \\
\text { spec. tech. } \\
\text { spec. tech. }\end{array}$ & $\begin{array}{l}\text { spec. tech. } \\
\text { spec. tech. } \\
\text { spec. tech. }\end{array}$ \\
\hline $\begin{array}{l}\text { D003 (Reactivity } \\
\text { Characteristic) }\end{array}$ & $\begin{array}{l}\text { Cyanides (Total) } \\
\text { Cyanides (Amenable) } \\
\text { Reactive sulfides based on } 261.23(a)(5) \text { : } \\
\text { Explosives based on } 261.23(a)(6) .(7) \text { and (8) } \\
\text { Water reactives based on } 261.23(a)(2),(3), \&(4) \\
\text { Other reactives based on } 261.23(a)(1)\end{array}$ & & & $\begin{array}{l}590 \\
30 \\
\text { spec. tech. } \\
\text { spec. tech. } \\
\text { spec. tech. }\end{array}$ & $\begin{array}{l}\text { Reserved } \\
0.86 \\
\text { spec. tech. } \\
\text { spec. tech. }\end{array}$ \\
\hline $\begin{array}{l}0004 \text { (EP Toxicity } \\
\text { Characteristic) }\end{array}$ & $\begin{array}{l}\text { Arsenic } \\
\text { Radioactive High-Level Wastes Generated During the } \\
\text { Reprocessing of Fuel Rods Subcategory }\end{array}$ & 5.0 & & spec. tech. & 5.0 \\
\hline $\begin{array}{l}\text { D005 (EP Toxicity } \\
\text { Characteristic) }\end{array}$ & $\begin{array}{l}\text { Barium } \\
\text { Radioactive High-Level Wastes Generated During the } \\
\text { Reprocessing of Fuel Rods Subcategory }\end{array}$ & 100 & & spec. tech. & 100 \\
\hline
\end{tabular}




\section{TABLE 3.10. (contd)}

\begin{tabular}{|c|c|}
\hline $\begin{array}{l}\text { EPA Hazardous Waste } \\
\text { Number (Name) }\end{array}$ & Constituent ${ }^{(b)}$ \\
\hline $\begin{array}{l}\text { D006 (EP Toxicity } \\
\text { Characteristic) }\end{array}$ & $\begin{array}{l}\text { Cadmium } \\
\text { Cadmi um containing batteries } \\
\text { Radioactive High-Level Wastes Generated During the } \\
\text { Reprocessing of Fuel Rods Subcategory }\end{array}$ \\
\hline $\begin{array}{l}\text { D007 (EP Toxicity } \\
\text { Characteristic) }\end{array}$ & $\begin{array}{l}\text { Ihromi um (Total) } \\
\text { Radioactive High-Level Wastes Generated During the } \\
\text { Reprocessing of Fuel Rods Subcategory }\end{array}$ \\
\hline $\begin{array}{l}\text { D008 (EP Toxicity } \\
\text { Characteristic) }\end{array}$ & $\begin{array}{l}\text { Lead } \\
\text { Lead acid batteries }(f) \\
\text { Radioactive High-Level Wastes Generated During the } \\
\quad \text { Reprocessing of Fuel Rods Subcategory }\end{array}$ \\
\hline $\begin{array}{l}0009 \text { (EP Toxicity } \\
\text { Characteristic) }\end{array}$ & $\begin{array}{l}\text { Mercury }(g) \\
\text { Mercury }(\mathrm{h}) \\
\text { Mercury } \\
\text { Radioactive High-Level Wastes Generated During the } \\
\text { Reprocessing of Fuel Rods Subcategory } \\
\text { Elemental mercury contaminated with radioactive } \\
\text { materials } \\
\text { Hydraulic oil contaminated with mercury Radioactive } \\
\text { Materials subcategory }\end{array}$ \\
\hline $\begin{array}{l}\text { D010 (EP Toxicity } \\
\text { Characteristic) }\end{array}$ & $\begin{array}{l}\text { Selenium } \\
\text { Radioactive High-Level Wastes Generated During the } \\
\text { Reprocessing of Fuel Rods Subcategory }\end{array}$ \\
\hline $\begin{array}{l}\text { D011 (EP Toxicity } \\
\text { Characteristic) }\end{array}$ & $\begin{array}{l}\text { Silver } \\
\text { Radioactive High-Level Wastes Generated During the } \\
\text { Reprocessing of Fuel Rods Subcategory }\end{array}$ \\
\hline D012 (EP Toxicity Characteristic) & Endrin \\
\hline D013 (EP Toxicity Characteristic) & Lindane \\
\hline
\end{tabular}

\begin{tabular}{|c|c|c|c|}
\hline \multicolumn{2}{|c|}{$\begin{array}{l}\text { Concentration in Waste } \\
\text { Extract ( } 40 \text { CFR 268.41) }\end{array}$} & \multicolumn{2}{|c|}{$\begin{array}{c}\text { Concentration in Waste } \\
(40 \text { CFR 268.43) }\end{array}$} \\
\hline $\begin{array}{c}\begin{array}{c}\text { Nonwastewater } \\
(\mathrm{mg} / \mathrm{L})\end{array} \\
\end{array}$ & $\begin{array}{l}\text { Wastewater } \\
(\mathrm{mg} / \mathrm{L})\end{array}$ & $\begin{array}{l}\text { Nonwastewater } \\
(\mathrm{mg} / \mathrm{kg}) \\
\end{array}$ & $\begin{array}{l}\text { Wastewater } \\
(\mathrm{mg} / \mathrm{L})\end{array}$ \\
\hline 1.0 & & $\begin{array}{l}\text { spec. tech. } \\
\text { spec. tech. }\end{array}$ & 1.0 \\
\hline 5.0 & & spec. tech. & 5.0 \\
\hline 5.0 & & $\begin{array}{l}\text { spec. tech. } \\
\text { spec. tech. }\end{array}$ & 5.0 \\
\hline 0.20 & & $\begin{array}{l}\text { spec. tech. } \\
\text { spec. tech. } \\
\text { spec. tech. } \\
\text { spec. tech. } \\
\text { spec. tech. } \\
\text { spec. tech. }\end{array}$ & 0.20 \\
\hline 5.7 & & spec. tech. & 1.0 \\
\hline 5.0 & & spec. tech. & 5.0 \\
\hline & & 0.13 & spec. tech. \\
\hline & & 0.066 & spec. tech. \\
\hline
\end{tabular}




\section{TABLE 3.10. (contd)}

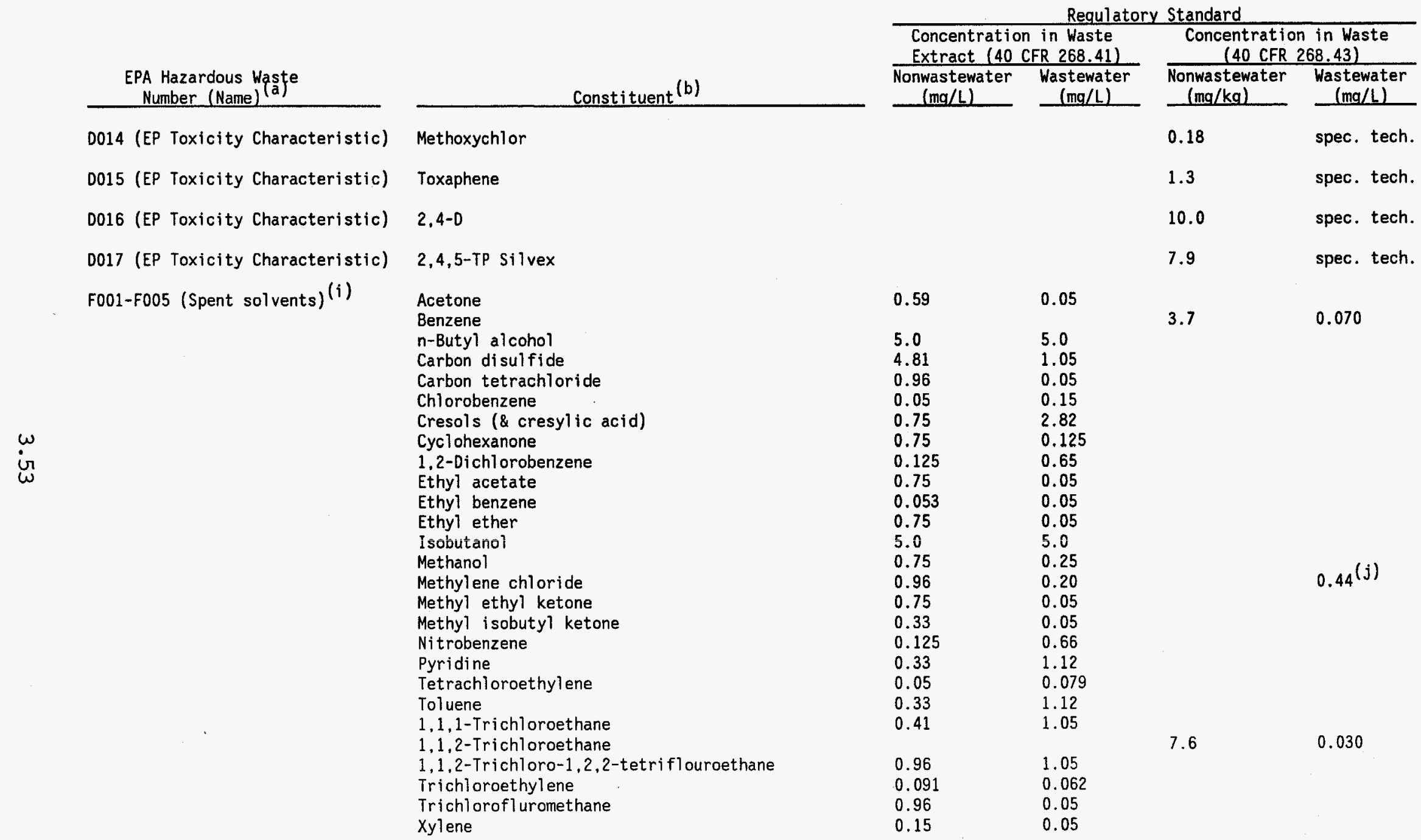


TABLE 3.10. (contd)

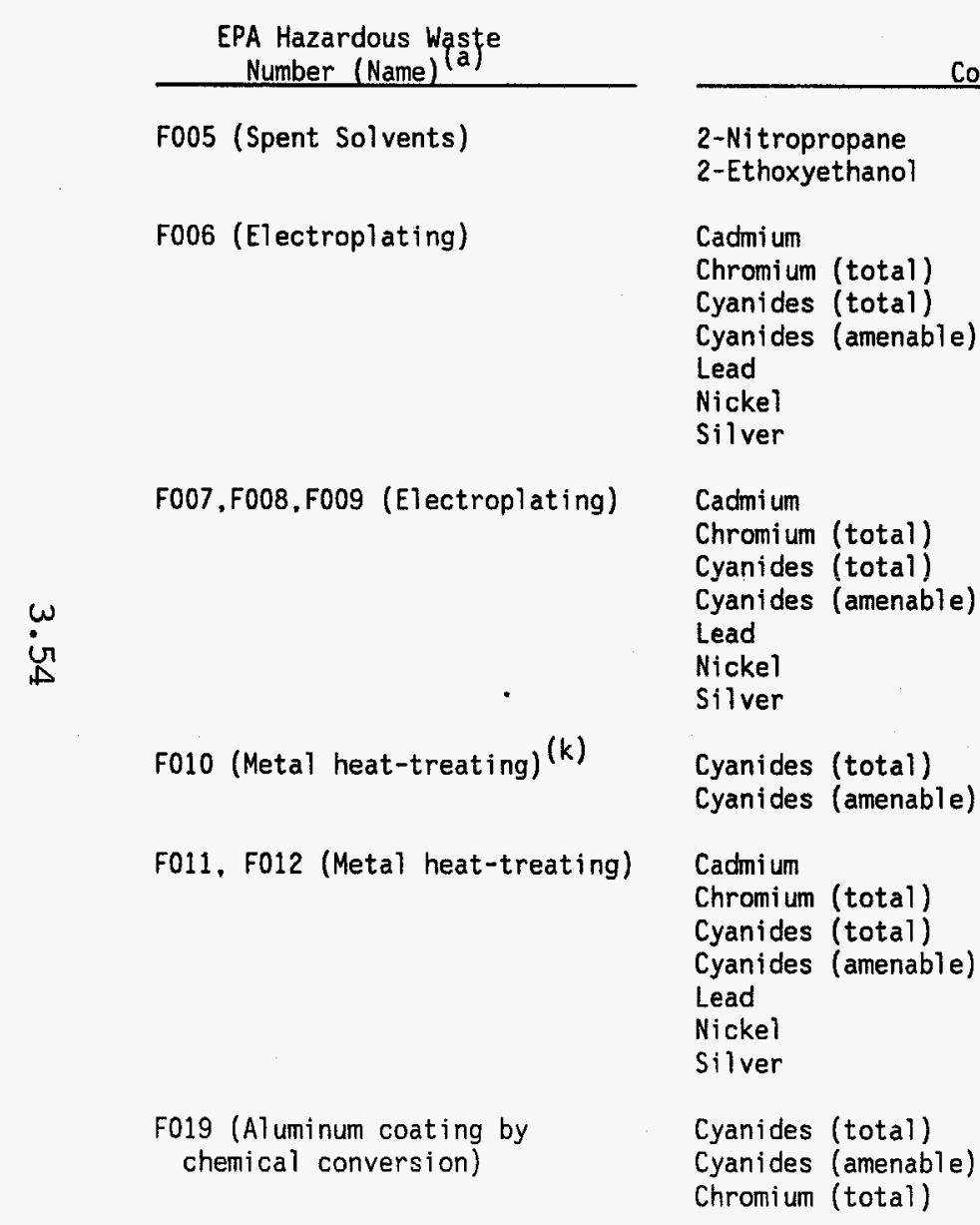

Constituent ${ }^{(b)}$

(2)


IABLE 3.10. (contd) EPA Hazardous Waste
Number (Name)

F020-F023, F026-F028 (Dioxin-

F024 (Chlorinated aliphatic

hydrocarbon prodn.)

$\omega$

un

Regulatory Standard

\begin{tabular}{|c|c|c|c|}
\hline $\begin{array}{l}\text { Concentrati } \\
\text { Extract }(40\end{array}$ & $\bar{e}$ & $\begin{array}{r}\text { Concentrati } \\
\text { (40 CFR }\end{array}$ & $\begin{array}{l}\text { in Waste } \\
68.43)\end{array}$ \\
\hline $\begin{array}{l}\text { nwastewater } \\
\text { (mg/L) }\end{array}$ & $\begin{array}{l}\text { Wastewate } \\
(\mathrm{mg} / \mathrm{L})\end{array}$ & $\begin{array}{l}\text { Nonwastewater } \\
(\mathrm{mg} / \mathrm{kg})\end{array}$ & $\begin{array}{l}\text { Wast } \\
\text { In }\end{array}$ \\
\hline
\end{tabular}

Hexachlorodibenzo-p-dioxins

Hexachlorodibenzofurans

$<1 \mathrm{ppb}$

Pentachlorodibenzo-p-dioxins

$<1 \mathrm{ppb}$

$<1 \mathrm{ppb}$

Pentachlorodibenzofurans

Tetrachiorodibenzo-p-dioxins

Tetrachlorodibenzofurans

2,4,5-Trichlorophenol

2,3,4,6-Tetrachlorophenol

Pentachlorophenol

$\begin{array}{ll}<1 \mathrm{ppb} & <1 \mathrm{ppb} \\ <1 \mathrm{ppb} & <1 \mathrm{ppb}\end{array}$

$<1 \mathrm{ppb} \quad<1 \mathrm{ppb}$

$<1 \mathrm{ppb}<1 \mathrm{ppb}$

$<0.05 \mathrm{ppm} \quad<0.05 \mathrm{ppm}$

$<0.05 \mathrm{ppm} \quad<0.05 \mathrm{ppm}$

$<0.05 \mathrm{ppm} \quad<0.05 \mathrm{ppm}$

$<0.01 \mathrm{ppm} \quad<0.01 \mathrm{ppm}$

Organic standards

2-Chloro-1,3-butadiene

3-Chloropropene

1.1-Dichloroethane

1,2-Dichloroethane

1.2-Dichloropropane

cis-1,3-Dichloropropene

trans-1,3-Dichloropropene

Bis(2-ethyl hexyl)phthalate

Hexachloroethane

Hexachl orodibenzo-furans

Hexachlorodibenzo-p-dioxins

Pentachlorodibenzo-furans

Pentachlorodibenzo-p-dioxins

Tetrachlorodibenzo-furans

Chromium (tota1)

Nickel

co. pipm

spec. tech. spec. tech.

$0.28 \quad 0.28$

$0.28 \quad 0.28$

$0.014 \quad 0.014$

$0.014 \quad 0.014$

$0.014 \quad 0.014$

$0.014 \quad 0.014$

$0.014 \quad 0.014$

$1.8 \quad 0.036$

$1.8 \quad 0.036$

$0.001 \quad 0.001$

$0.001 \quad 0.001$

$0.001 \quad 0.001$

$0.001 \quad 0.001$

$0.001 \quad 0.001$

0.073

0.088

0.35

0.47

F025

Chloroform

1,2-Dichloroethane

1,1-Dichloroethylene

Methylene chloride

Carbon tetrachloride

1,1,2-Trichloroethane

$\begin{array}{ll}6.2 & 0.046 \\ 6.2 & 0.21 \\ 6.2 & 0.025 \\ 31 & 0.089 \\ 6.2 & 0.057 \\ 6.2 & 0.054\end{array}$


TABLE 3.10. (contd) EPA Hazardous Waste
Number (Name)

in

Regulatory Standard

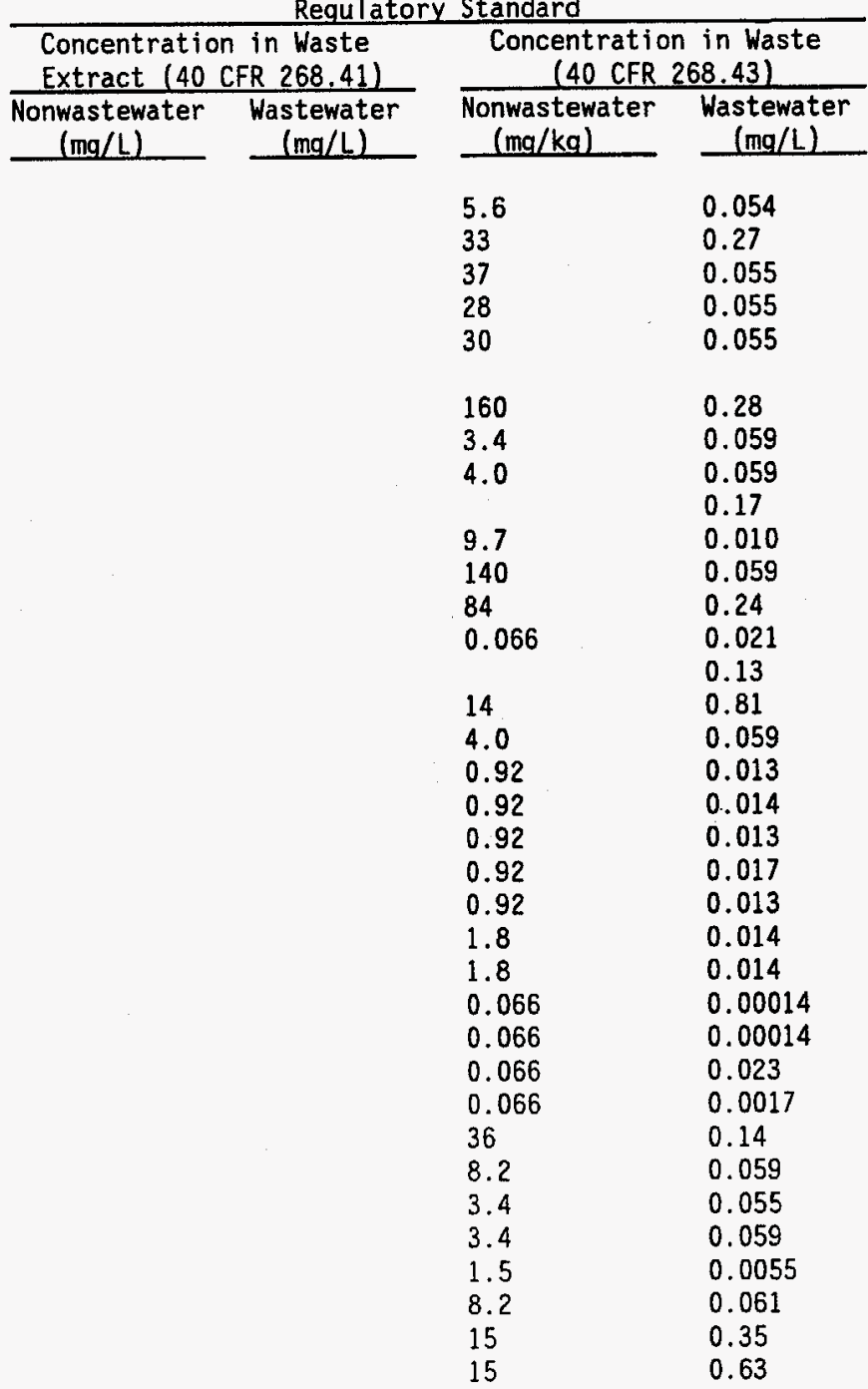

F039 (wastes classified by more than one waste code or from

a mixture of wastes)
Constituent $^{(b)}$

Trichloroethylene

Vinyl chloride

Hexachl orobenzene

Hexachl orobutadiene

Hexachl oroethane

\section{Acetone}

Acenaphtalene

Acenaphthene

Acetonitrile

Acetophenone

-Acetylaminofluorene

Acrylonitrile

Aldrin

4-Aminobipheny]

Aniline

Anthracene.

Aroclor 1016

Aroclor 1221

Aroclor 1232

Aroclor 1242

Aroclor 1248

Aroclor 1254

Aroclor 1260

alpha-BHC

beta-BHC

del ta-BHC

gamma-BHC

Benzene

Benzo(a) anthracene

Benzo(b) fluoranthene

Benzo(k)fluoranthene

Benzo(g,h,i)perylene

Benzo(a)pyrene

Bromodichl oromethane

Bromoform 
TABLE 3.10. (contd)

EPA Hazardous Waste Number (Namela)

Requlatory Standard

\begin{tabular}{|c|c|c|c|}
\hline $\begin{array}{l}\text { Concentratio } \\
\text { Extract }(40\end{array}$ & $\begin{array}{c}\text { in Waste } \\
F R \text { 268.41) }\end{array}$ & $\begin{array}{r}\text { Concentratio } \\
\text { (40 CFR }\end{array}$ & $\begin{array}{l}\text { in Waste } \\
68.43 \text { ) }\end{array}$ \\
\hline $\begin{array}{l}\text { Nonwastewater } \\
(\mathrm{mg} / \mathrm{L})\end{array}$ & $\begin{array}{l}\text { Wastewater } \\
(\mathrm{mg} / \mathrm{L})\end{array}$ & $\begin{array}{l}\text { Nonwastewater } \\
(\mathrm{mg} / \mathrm{kg})\end{array}$ & $\begin{array}{l}\text { Wastewate } \\
(\mathrm{mg} / \mathrm{L})\end{array}$ \\
\hline
\end{tabular}

Bromomethane (methyl bromide)

4-Bromophenyl phenyl ether

n-Butyl alcohol

Butyl benzyl phthalate

2-sec-Butyl-4,6-dini trophenol

Carbon tetrachloride

Carbon disulfide

Chlordane

p-Chloroaniline

Chlorobenzene

Chlorobenzilate

Chlorodibromomethane

Chloroethane

bis(2-Chloroethoxy) methane

bis(2-Chloroethyl) ether

2-Chloroethyl vinyl ether

Chloroform

bis (2-Chloroi sopropyl) ether

p-Chloro-m-cresol

Chloromethane (Methyl chioride)

2-Choronaphthalene

2-Chlorophenol

3-Chloropropene

Chrysene

0 -Cresol

Cresol ( $m$ - and $p$-isomers)

Cyclohexanone

1,2-Dibromo-3-chloropropane

1.2-Dibromoethane (Ethylene dobromide)

Dibromomethane

2,4-Dichlorophenoxyacetic acid (2,4-D)

$0, p^{\prime}-D D D$

$p, p^{\prime}-D D D$

$o, p^{\prime}-D D E$

$p \cdot p^{\prime}-D D E$

$0, p^{\prime}-D D T$ (b)

15

15

2.6

2.5
5.6

0.13

16
5.7

16

.0

7.2

7.2

5.6

7.2

0.044

0.036

0.059

0.11

0.77

0.36

0.11

0.028

0.11

0.72

0.023

0.023

0.031

0.031

0.0039 
TABLE 3.10. (contd)

EPA Hazardous Waste Number (Name) (a)

$\underset{\infty}{\omega}$

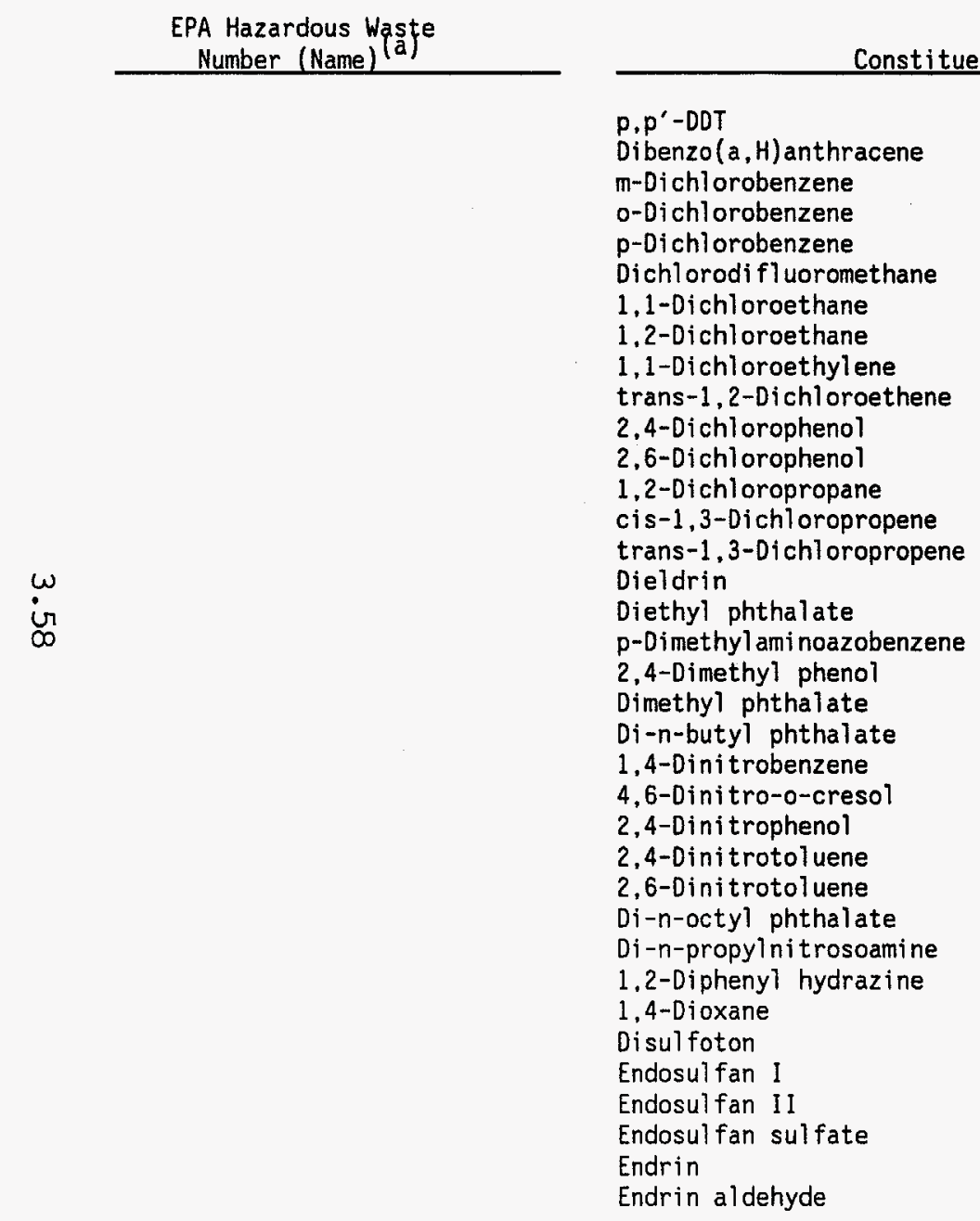

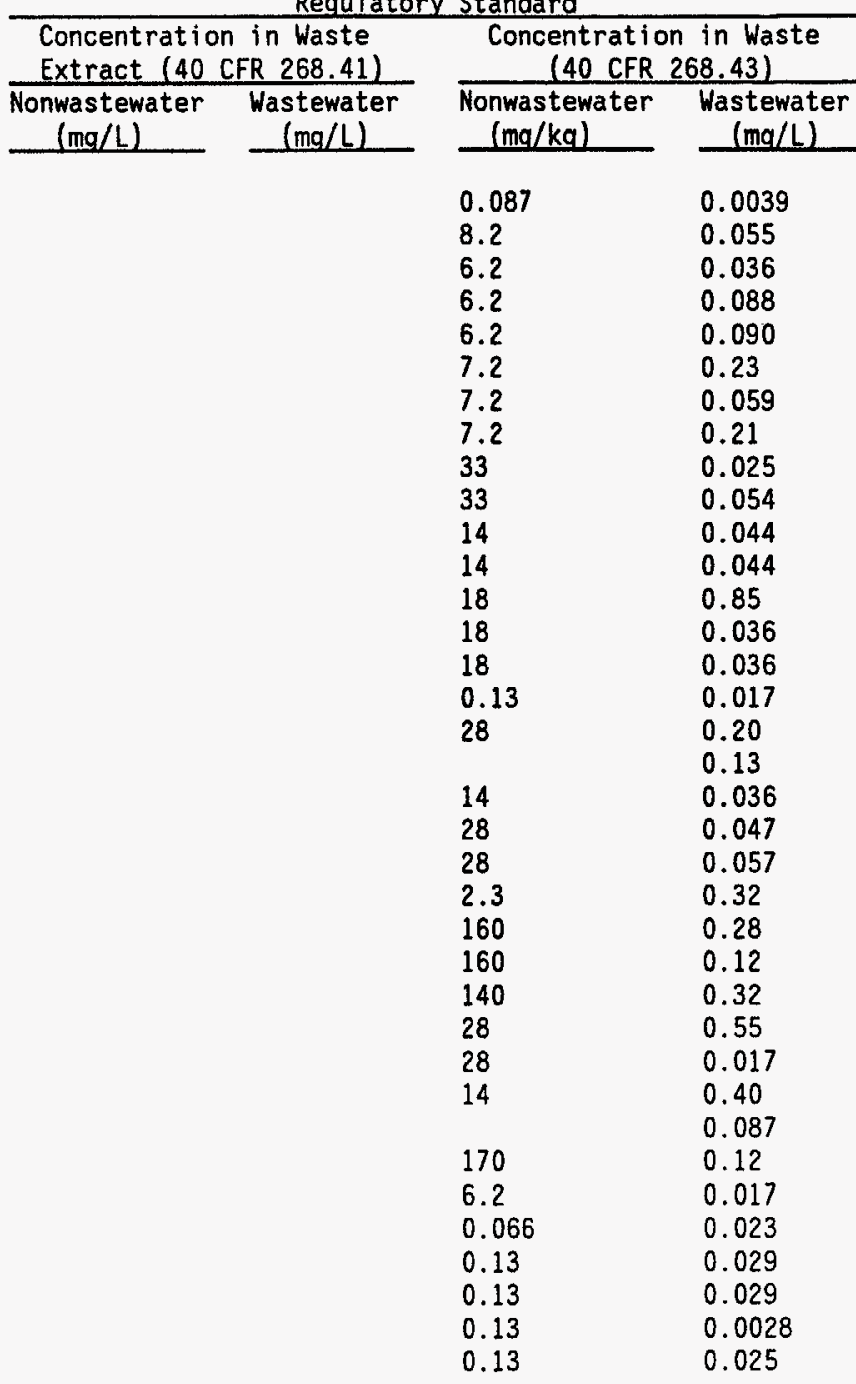


TABLE 3.10. (contd)

EPA Hazardous Waste Number (Name) (a)

Requlatory Standard

Concentration in Waste
Extract (40 CFR 268.41) $\frac{\text { Concentration in Waste }}{\text { (40 CFR 268.43) }}$

Ethyl acetate

Ethyl cyanide

Ethyl benzene

Ethyl ether

bis (2-Ethyl hexyl)phthal ate

Ethyl methacrylate

Ethylene oxide

Famphur

Fluoranthene

Fluorene

Fluorotrichloromethan

Heptachlor

Heptachlor epoxide

Hexachl orobenzene

exachl orobutadiene

Hexachl oroyclopentadiene

Hexachl orodi benzo-furans

Hexachlorodibenzo-p-dioxins

Hexachl oroethane

Hexachloropropene

Indeno $(1,2,3,-c, d)$ pyrene

Iodomethane

Isobutanol

I sodrin

Isosafrole

Kepone

Methacrylonitrile

Methapyrilene

Methoxychlor

3-Methylchol anthrene

4,4-Methylene-bis-(2-chloroaniline)

Methylene chloride

Methyl ethyl ketone

Methyl isobutyl ketone

Methylmethacrylate

Methyl methansulfonate

33

6.0

160

28

160

15

8.2
4.0

33

0.066

0.066

37

28

3.6

0.001

0.001

28

28

8.2

65
170

0.066

2.6

0.13

84

1.5

0.18

15

35

33

36

33
160

$(\mathrm{mg} / \mathrm{L})$

0.34

0.057

0.12

0.28

0.14

0.12

0.017

0.068

0.059

0.020

0.0012

0.0012
0.016

0.016
0.055

0.055

0.057

0.000063

0.000063

0.055

0.035

0.0055

0.019

5.6

0.021

0.081

0.0011

0.24

0.081

0.25

0.0055

0.50

0.089

0.28

0.14

0.14

0.018 
TABLE 3.10. (contd)

EPA Hazardous Waste Number (Name) (a)
Constituent (b)

Methyl parathion

Naphthalene

2-Naphtylamine

p-Nitroaniline

Nitrobenzene

5-Nitro-o-toluidine

4-Nitrophenol

$\mathrm{N}$-Nitrosodi ethylamine

$\mathrm{N}$-Nitrosodimethylamine

$\mathrm{N}$-Nitroso-di-n-butylamine

$\mathrm{N}$-Ni trosomethyl ethyl amine

$\mathrm{N}$-Nitrosomorpholine

N-Nitrosopiperidine

$\mathrm{N}$-Nitrosopyrrolidine

Parathion

Pentachlorobenzene

Pentachlorodi benzo-furans

Pentachlorodibenzo-p-dioxins

Pentachloroni trobenzene

Pentachlorophenol

Phenacet in

Phenanthrene

Phenol

Phorate

Propanenitrile (ethyl cyanide)

Pronamide

Pyrene

Pyridine

Safrole

Silvex $(2,4,5-T P)$

$2,4,5-\mathrm{T}$

1,2,4,5-Tetrachlorobenzene

Tet rachl orodi benzo-furans

Tet rachi orodi benzo-p-dioxins

$2,3,7,8$-Tetrachlorodibenzo-p-dioxin

$1,1,1,2$-Tetrachloroethane
Requlatory Standard

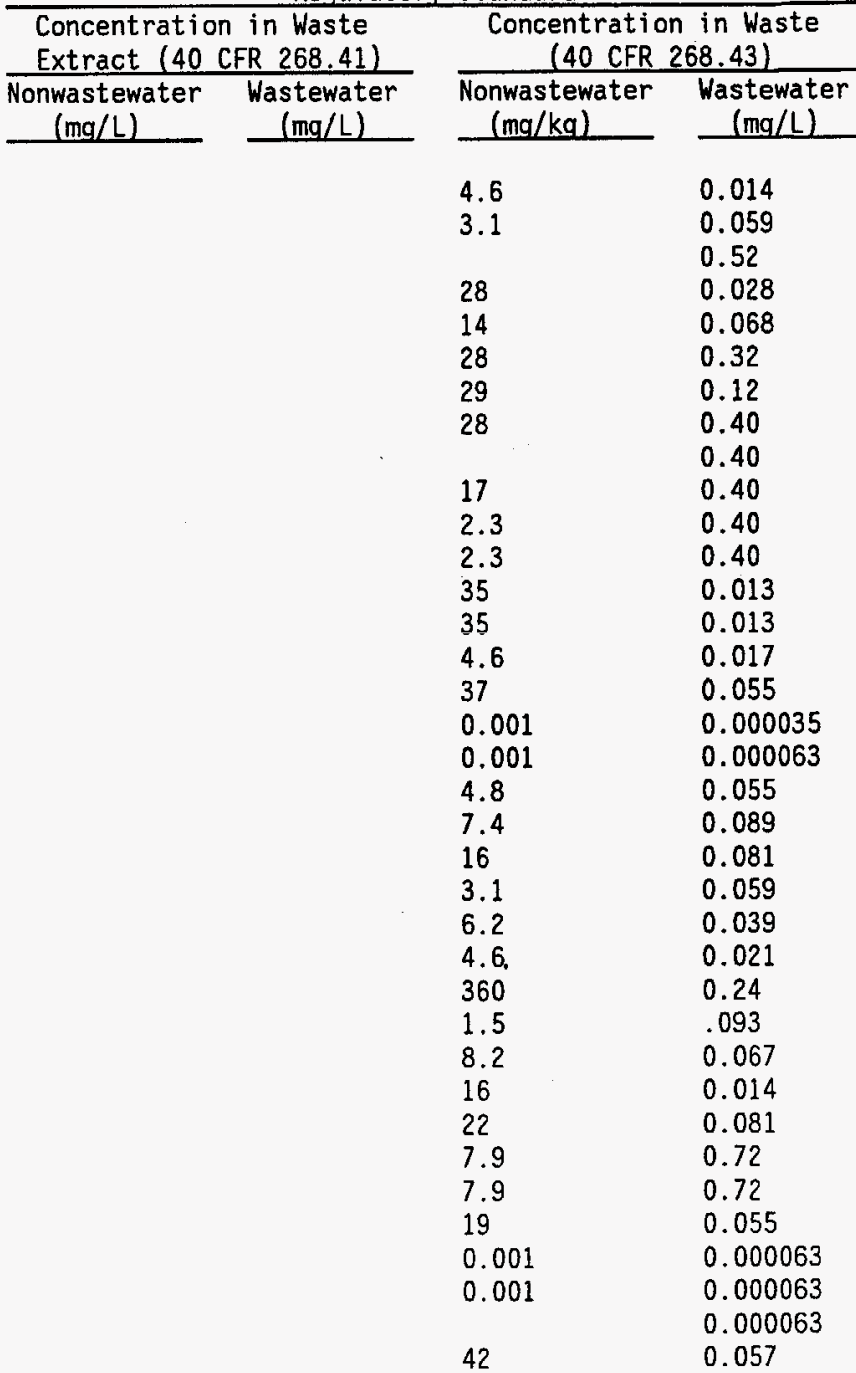




\section{TABLE 3.10. (contd)}

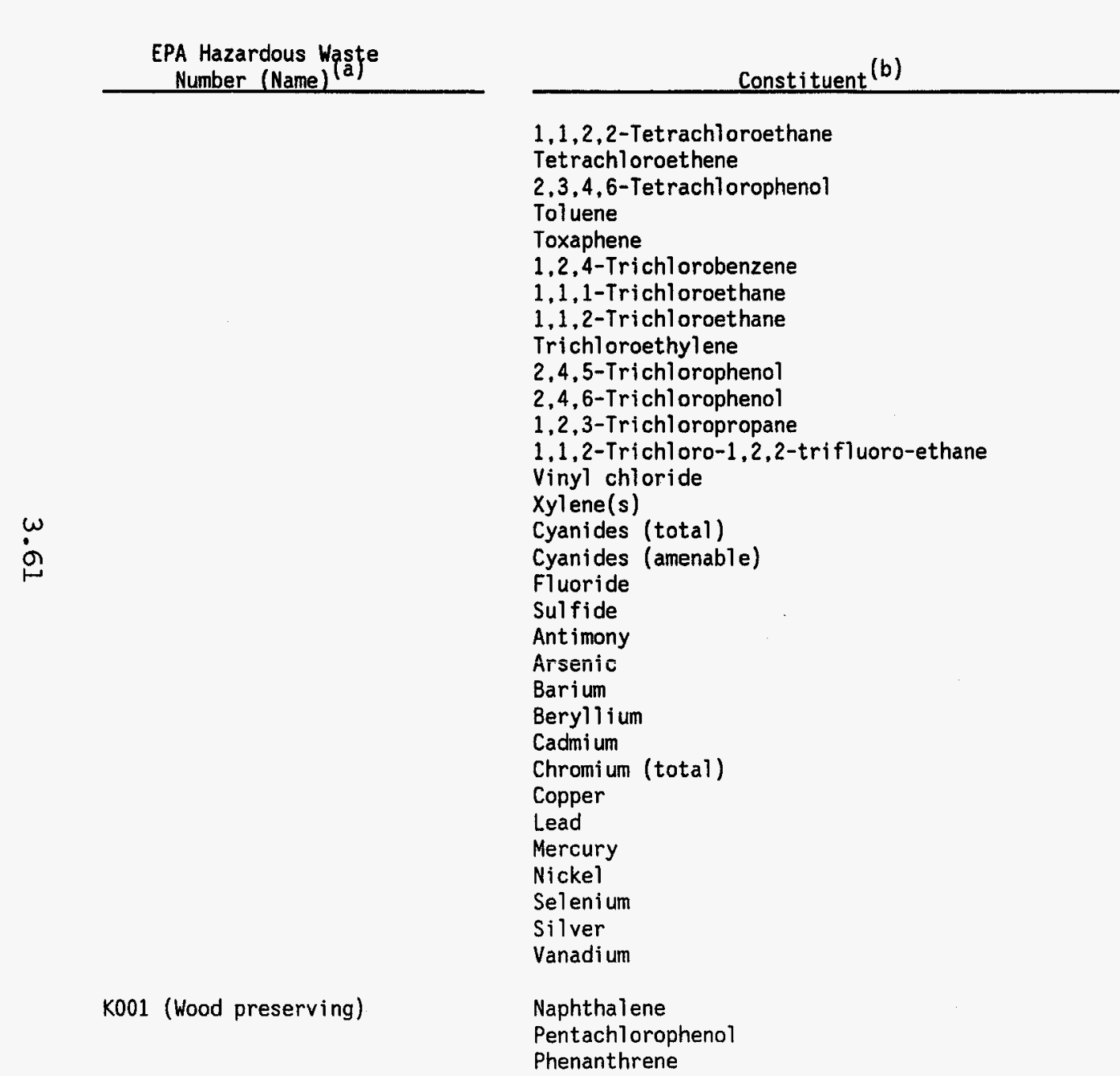

\begin{tabular}{|c|c|c|c|}
\hline \multicolumn{2}{|c|}{$\begin{array}{l}\text { Concentration in Waste } \\
\text { Extract (40 CFR 268.41) }\end{array}$} & \multicolumn{2}{|c|}{$\begin{array}{c}\text { Concentration in Waste } \\
(40 \text { CFR 268.43) }\end{array}$} \\
\hline $\begin{array}{l}\text { Nonwastewater } \\
(\mathrm{mg} / \mathrm{L})\end{array}$ & $\begin{array}{l}\text { Wastewater } \\
(\mathrm{mg} / \mathrm{L})\end{array}$ & $\begin{array}{l}\text { Nonwastewater } \\
(\mathrm{mg} / \mathrm{kg})\end{array}$ & $\begin{array}{l}\text { Wastewater } \\
(\mathrm{mg} / \mathrm{L})\end{array}$ \\
\hline $\begin{array}{l}0.23 \\
5.0 \\
52 \\
\\
0.066 \\
5.2 \\
\\
0.51 \\
0.025 \\
0.32 \\
5.7 \\
0.072\end{array}$ & & $\begin{array}{l}42 \\
5.6 \\
37 \\
28 \\
1.3 \\
19 \\
5.6 \\
5.6 \\
5.6 \\
37 \\
37 \\
28 \\
28 \\
33 \\
28 \\
1.8\end{array}$ & $\begin{array}{l}0.057 \\
0.056 \\
0.030 \\
0.080 \\
0.0095 \\
0.055 \\
0.054 \\
0.054 \\
0.054 \\
0.18 \\
0.035 \\
0.85 \\
0.057 \\
0.27 \\
0.32 \\
1.2 \\
0.86 \\
35 \\
14 \\
1.9 \\
5.0 \\
1.2 \\
0.82 \\
0.20 \\
0.37 \\
1.3 \\
0.28 \\
0.15 \\
0.55 \\
0.82 \\
0.29 \\
0.042\end{array}$ \\
\hline & & $\begin{array}{l}1.5 \\
1.5 \\
1.5\end{array}$ & $\begin{array}{l}0.031 \\
0.031 \\
0.031\end{array}$ \\
\hline
\end{tabular}


TABLE 3.10. (contd)

\begin{tabular}{|c|c|}
\hline $\begin{array}{l}\text { EPA Hazardous Waste } \\
\text { Number (Name) (a) }\end{array}$ & Constituent (b) \\
\hline & $\begin{array}{l}\text { Pyrene } \\
\text { Toluene } \\
\text { Xylenes (total) } \\
\text { Lead }\end{array}$ \\
\hline K002,K003,K004 (pigment prodn.) & $\begin{array}{l}\text { Chromium (total) } \\
\text { Lead }\end{array}$ \\
\hline $\begin{array}{l}\text { K005 (Chrome green pigment } \\
\text { prodn.) }\end{array}$ & $\begin{array}{l}\text { Chromium (total) } \\
\text { Lead } \\
\text { Cyanides (total) }\end{array}$ \\
\hline $\begin{array}{l}\text { K006 (Chrome oxide green } \\
\text { pigment prodn.) }\end{array}$ & $\begin{array}{l}\text { Chromium (total) } \\
\text { Lead }\end{array}$ \\
\hline KO06 (anhydrous) & $\begin{array}{l}\text { Chromium (total) } \\
\text { Lead }\end{array}$ \\
\hline K006 (hydrated) & Chromium (total) \\
\hline KOO7 (Iron blue pigment prodn.) & $\begin{array}{l}\text { Chromium (total) } \\
\text { Lead } \\
\text { Cyanides (total) }\end{array}$ \\
\hline $\begin{array}{l}\text { KOOB (Chrome green oxide } \\
\text { pigment prod.) }\end{array}$ & $\begin{array}{l}\text { Chromium (total) } \\
\text { Lead }\end{array}$ \\
\hline $\begin{array}{l}\text { K009, K010 (Acetaldehyde } \\
\text { prodn.) }\end{array}$ & Chloroform \\
\hline $\begin{array}{l}\text { K011,K013,Ko14 (Acrylonitrile } \\
\text { prodn.) }\end{array}$ & $\begin{array}{l}\text { Acetonitrile } \\
\text { Acrylonitrile } \\
\text { Acrylamide } \\
\text { Benzene } \\
\text { Cyanides (total) }\end{array}$ \\
\hline
\end{tabular}

\begin{tabular}{|c|c|c|c|}
\hline \multicolumn{2}{|c|}{$\begin{array}{l}\text { Concentration in Waste } \\
\text { Extract (40 CFR 268.41) }\end{array}$} & \multicolumn{2}{|c|}{$\begin{array}{c}\text { Concentration in Waste } \\
(40 \text { CFR } 268.43 \text { ) }\end{array}$} \\
\hline $\begin{array}{c}\text { Nonwastewater } \\
(\mathrm{mg} / \mathrm{L})\end{array}$ & $\begin{array}{l}\text { Wastewater } \\
(\mathrm{mg} / \mathrm{L})\end{array}$ & $\begin{array}{l}\text { Nonwastewater } \\
(\mathrm{mg} / \mathrm{kg})\end{array}$ & $\begin{array}{l}\text { Wastewater } \\
(\mathrm{mg} / \mathrm{L})\end{array}$ \\
\hline 0.51 & & $\begin{array}{l}1.5 \\
28 \\
33\end{array}$ & $\begin{array}{l}0.028 \\
0.028 \\
0.032 \\
0.037\end{array}$ \\
\hline $\begin{array}{l}0.094 \\
0.37\end{array}$ & & & $\begin{array}{l}2.9 \\
3.4\end{array}$ \\
\hline $\begin{array}{l}0.094 \\
0.37\end{array}$ & & & $\begin{array}{l}2.9 \\
3.4 \\
0.74\end{array}$ \\
\hline & & & $\begin{array}{l}2.9 \\
3.4\end{array}$ \\
\hline
\end{tabular}

0.094

0.37

5.2

0.094

0.37

0.094

0.37

2.9

0.74

2.9

3.4

0.10

Benzene

Cyanides (total)

$\begin{array}{ll} & 2.9 \\ & 3.4 \\ & 0.74 \\ & \\ & 2.9 \\ & 3.4 \\ & \\ 6.0 & 0.10 \\ & \\ 1.8 & 38 \\ 1.4 & 0.06 \\ 23 & 19 \\ 0.03 & 0.02 \\ 57 & 21\end{array}$


TABLE 3.10. (contd)

EPA Hazardous Waste
Number (Name)

K015 (Benzyl chloride

distillation)

K016 (Carbon tetrachloride prodn.)

$\omega$
on

K017 (Epichlorohydrin prodn.)

K018 (Ethyl chloride prodn.)

019 (Ethylene dichloride prodn.)
Constituent (b)

Anthracene

Benzal chloride

Benzo ( $b$ and/or $k$ ) fluoranthene

Phenanthren

Toluene

Chromi um (total)

Nickel

Lead

Hexachlorobenzene

Hexachlorobutadiene

Hexachl orocycl opentadiene

Hexachloroethane

Tetrachloroethene

1,2-Dichloropropane

1,2,3-Trichloropropane

Bis (2-chloroethyl)ether

Chloroethane

1.1-Dichloroethane

1,2-Dichloroethane

Hexachlorobutadiene

Hexachlorethane

Pentachloroethane

1,1,1-Trichloroethane

1.2-Dichloroethane

Bis (2-chloroethyl) ether

Chlorobenzene

Chloroform

Fluorene

Hexachloroethane

Naphthalene

Phenanthrene

$p$-Dichlorobenzene

Requlatory Standard

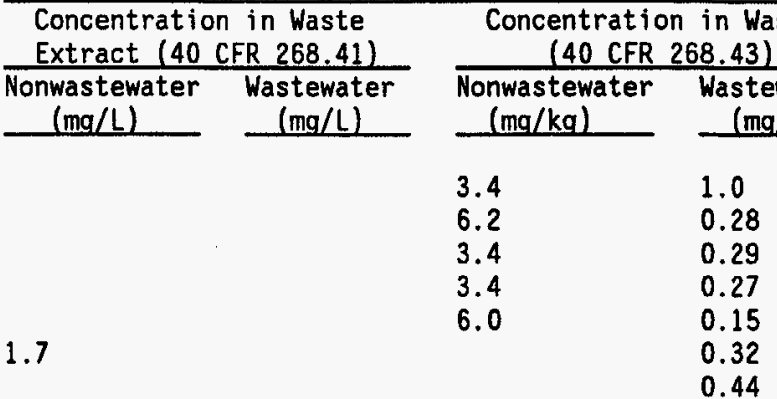

0.2

$\begin{array}{ll}28 & 0.033 \\ 5.6 & 0.007 \\ 5.6 & 0.007 \\ 28 & 0.033 \\ 6.0 & 0.007 \\ & \\ 18 & 0.85 \\ 28 & 0.85 \\ 7.2 & 0.033 \\ & \\ 6.0 & 0.007 \\ 6.0 & 0.007 \\ 6.0 & 0.007 \\ 5.6 & 0.033 \\ 28 & 0.007 \\ 5.6 & 0.007 \\ 6.0 & 0.007 \\ & \\ 6.0 & 0.007 \\ 5.6 & 0.007 \\ 6.0 & 0.006 \\ 6.0 & 0.007 \\ & 0.007 \\ 28 & 0.033 \\ 5.6 & 0.007 \\ 5.6 & 0.007 \\ & 0.008\end{array}$


TABLE 3.10. (contd)

EPA Hazardous Waste Number (Name)

K020 (Vinyl chloride prodn.)

K023,K093,K094 (Phtha) ic

anhydride prodn.) $(\mathrm{k})$

K024 (Phthalic anhydride prodn.)

K025 (Nitrobenzene prodn.)

K026 (Methyl ethyl pyridine prodn.)

K027 (Toluene di i socyanate prodn.)
Tetrachloroethene

1,1,1-Trichloroethane

1,2,4-Trichlorobenzene

1,2,4,5-Tetrachlorobenzene

\section{1,2-Dichloroethane}

$1,1,2,2$-Tetrachloroethane

Tetrachloroethene

Chloroform

Carbon tetrachioride

Antimony

0.23

\begin{tabular}{l} 
Requlatory \\
\hline Concentration in Waste \\
Extract $(40$ CFR 268.41) \\
$\begin{array}{c}\text { Nonwastewater Wastewater } \\
(\mathrm{mg} / \mathrm{L})\end{array} \quad$ (mg/L)
\end{tabular}

$(\mathrm{mg} / \mathrm{L})$

\section{Ácetophenone \\ Toluene}

Diphenylamine

Diphenylnitrosamine

Diphenylamine + Diphenylnitrosamine

Phenol

Chromi um (total)

Nickel

5.2

Phthal ic anhydride

Phthalic anhydride

0.32

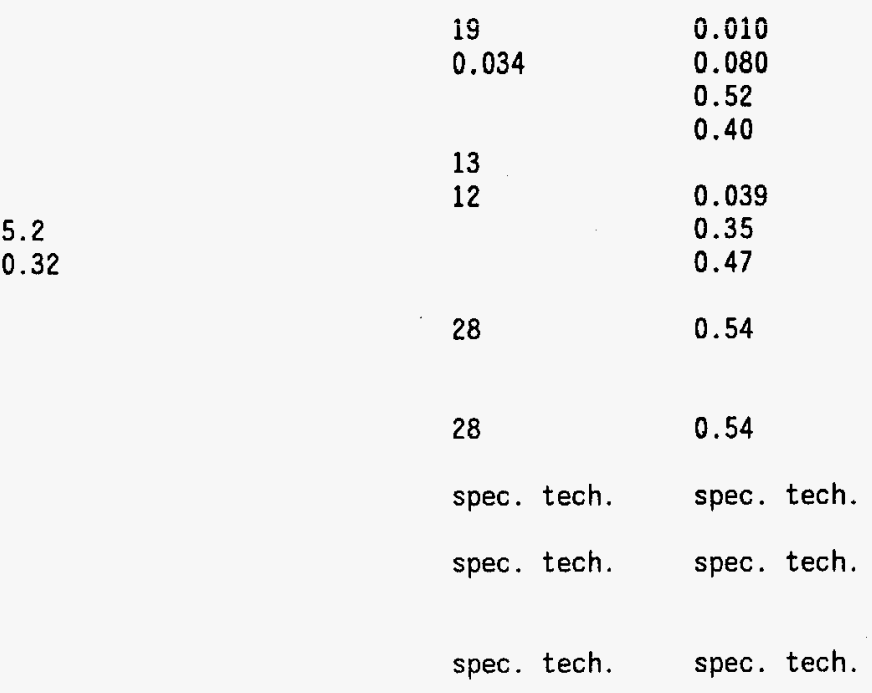

(40 CFR 268.43)

(40 CFR 268.43)

Nonwastewater Wastewater

$(\mathrm{mg} / \mathrm{kg}) \quad(\mathrm{mg} / \mathrm{L})$

$19 \quad 0.023$

0.007

0.007

0.007

$6.0 \quad 0.007$

6.2

$6.2 \quad 0.057$

0.057
0.60 $\begin{array}{ll}6.0 & 0.007 \\ 19 & 0.007\end{array}$ 
IABLE 3.10. (contd)

EPA Hazardous Waste

K028 $\left(1,1,1-{ }^{\text {trichloroethane }}\right.$
prodn. $)$

$\omega \quad$ K029 $(1,1,1-$ trichloroethane

K030 (tri\&tetrachloroethylene prodn.)

K031 (MSMA \& cacodylic acid prodn.)
Constituent (b)

1,1-Dichloroethane

trans-1,2-Dichloroethane

Hexachlorobutadiene

Hexachloroethane

Pentachloroethane

1,1,1,2-Tetrachloroethane

1,1,2,2-Tetrachloroethane

1,1,1-Trichloroethane

1.1,2-Trichloroethane

Tetrachloroethylene

Cadmi um

Chromium (total)

Lead

Nickel

Chloroform

1,2-Dichloroethane

1,1-Dichloroethylene

1,1,1-Trichioroethane

Vinyl chloride

Hexachlorobutadiene

Hexachloroethane

Hexachloropropene

Pentachlorobenzene

Pentachloroethane

1, 2,4,5-Tetrachlorobenzene

Tetrachloroethane

1,2,4-Trichlorobenzene

o-Dichlorobenzene

p-Dichlorobenzene
Requiatory Standard

Concentration in Waste
Extract (40 CFR 268.41)
$\begin{gathered}\text { Nonwastewater Wastewater } \\
(\mathrm{mg} / \mathrm{L})\end{gathered} \quad$\begin{tabular}{l}
$(\mathrm{mg} / \mathrm{L})$ \\
\hline
\end{tabular}

Concentration in Waste
(40 CFR 268.43)
$\begin{gathered}\text { Nonwastewater Wastewater } \\
(\mathrm{mg} / \mathrm{kg})\end{gathered} \quad$\begin{tabular}{l}
$(\mathrm{mq} / \mathrm{L})$ \\
\hline
\end{tabular}

$\begin{array}{ll}6.0 & 0.007\end{array}$

6.0

0.033

$5.6 \quad 0.007$

$28 \quad 0.033$

6.4

0.35

0.037

0.47

0.021

0.088

$6.0 \quad 0.46$

0

0.21

0.025

$\begin{array}{ll}6.0 & 0.054 \\ 6.0 & 0.27\end{array}$

$\begin{array}{ll}5.6 & 0.007\end{array}$

5.6

$28 \quad 0.033$

19

28 
TABLE 3.10. (contd)

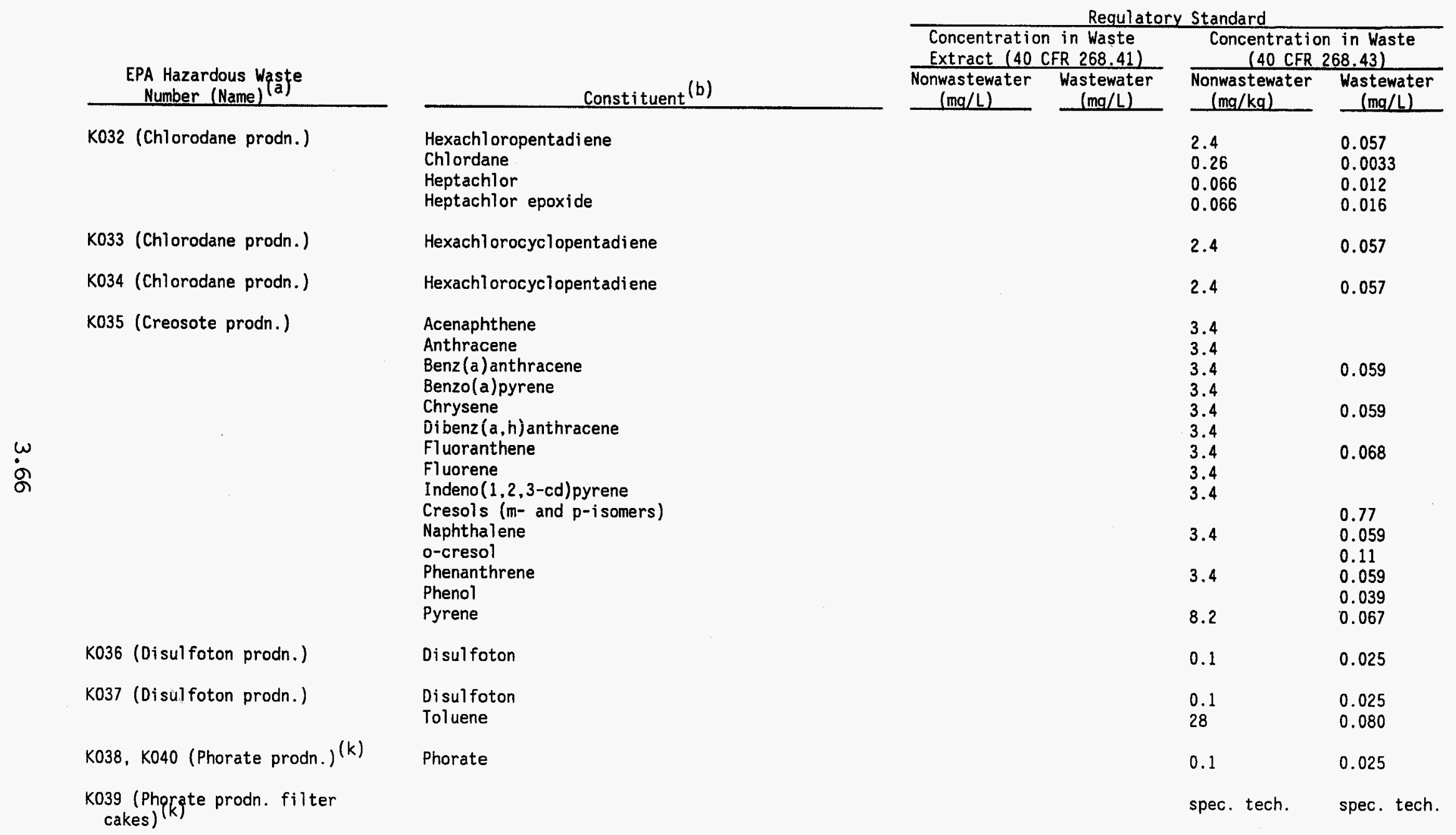


TABLE 3.10. (contd)

\begin{tabular}{|c|c|}
\hline $\begin{array}{l}\text { EPA Hazardous Waste } \\
\text { Number (Name) (a) }\end{array}$ & Constituen \\
\hline K041 (Toxaphene prodn.) & Toxaphene \\
\hline K042 (2,4,5-T prodn.) & $\begin{array}{l}\text { 1,2,4,5-Tetrachlorobenzene } \\
\text { o-Dichlorobenzene } \\
\text { p-Dichlorobenzene } \\
\text { Pentachlorobenzene } \\
\text { 1,2,4-Trichlorobenzene }\end{array}$ \\
\hline K043 $(2,4-0 \text { prodn. })^{(k)}$ & $\begin{array}{l}\text { 2,4-Dichlorophenol } \\
\text { 2,6-0ichlorophenol } \\
\text { 2,4,5-Trichlorophenol } \\
\text { 2,4,6-Trichlorophenol } \\
\text { Tetrachlorophenols (total) } \\
\text { Pentachlorophenol } \\
\text { Tetrachloroethene } \\
\text { Hexachlorodibenzo-p-dioxins } \\
\text { Hexachlorodibenzo-furans } \\
\text { Pentachlorodibenzo-p-dioxins } \\
\text { Pentachlorodibenzo-furans } \\
\text { Tetrachlorodibenzo-p-dioxins } \\
\text { Tetrachlorodibenzo-furans }\end{array}$ \\
\hline
\end{tabular}

KO44 (Explosives prodn.)

K045 (Spent carbon from

explosives treatment)

K046 (Lead-based initiating 7 cmpds.)

K047 (TNT operations)

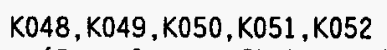
(Petroleum refining industry)
(m) $\begin{aligned} & \text { Chromium (total) } \\ & \text { Nickel }\end{aligned}$
Requlatory Standard

Concentration in Waste
Extract (40 CFR 268.41)
Nonwastewater Wastewater
(mq/L)

Concentration in Waste (40 CFR 268.43)

onwastewater Wastewater

Nonwastewater Wastewater

$(\mathrm{mg} / \mathrm{kg}) \quad(\mathrm{mg} / \mathrm{L})$

$2.6 \quad 0.0095$

$4.4 \quad 0.055$

$\begin{array}{ll}4.4 & 0.055\end{array}$

$4.4 \quad 0.090$

$4.4 \quad 0.055$

$4.4 \quad 0.055$

$0.38 \quad 0.049$

$0.34 \quad 0.013$

$8.2 \quad 0.016$

$\begin{array}{ll}7.6 & 0.039\end{array}$

$0.68 \quad 0.018$

$1.9 \quad 0.22$

$\begin{array}{ll}1.7 & 0.006\end{array}$

$0.001 \quad 0.001$

$0.001 \quad 0.001$

$0.001 \quad 0.001$

$0.001 \quad 0.001$

$0.001 \quad 0.001$

$0.001 \quad 0.001$

spec. tech. spec. tech.

spec. tech. spec. tech.

0.037

spec. tech. spec. tech.

1.7

0.20 
TABLE 3.10. (contd)

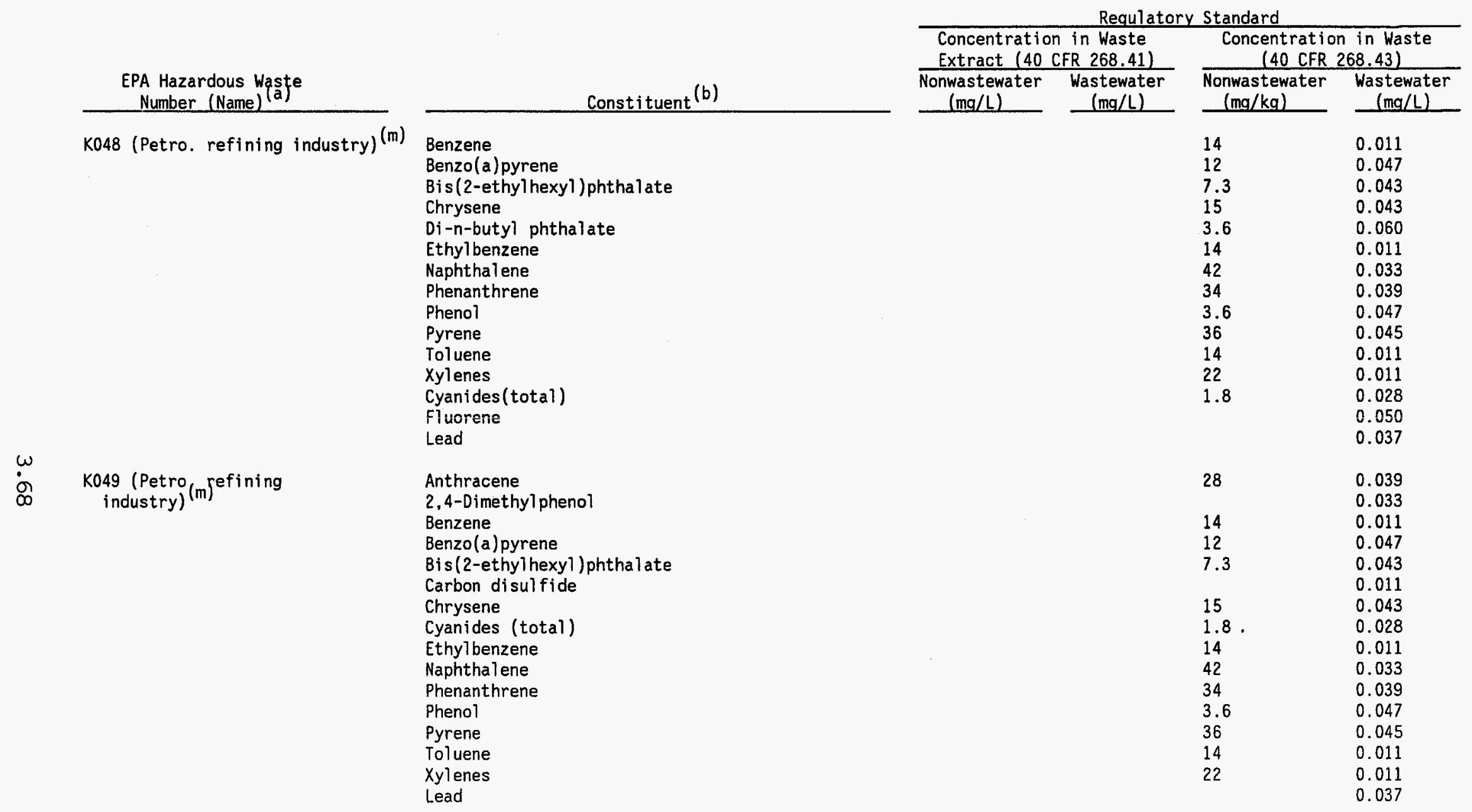




\section{TABLE 3.10. (contd)}

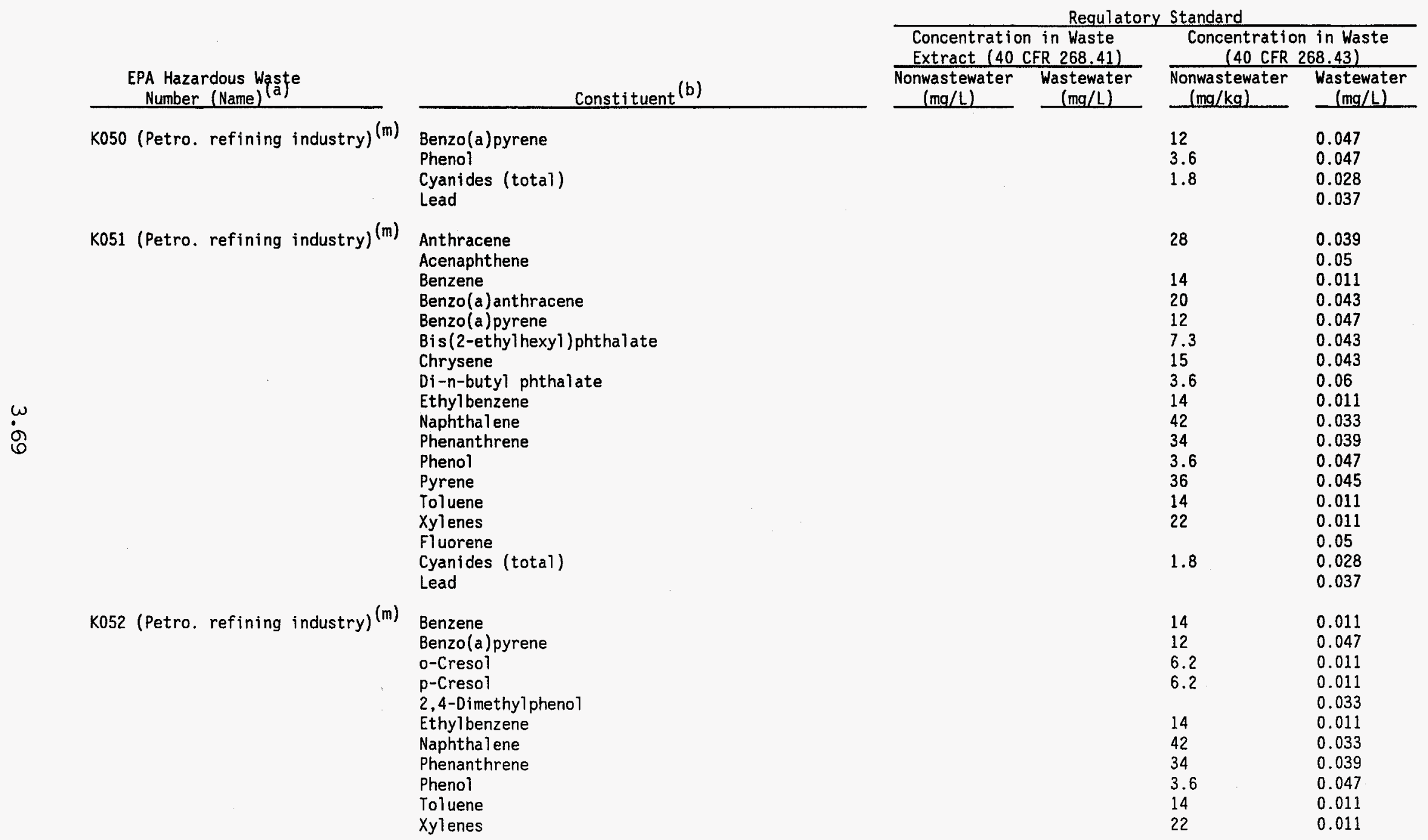


TABLE 3.10. (contd) EPA Hazardous Waste
Number (Name)

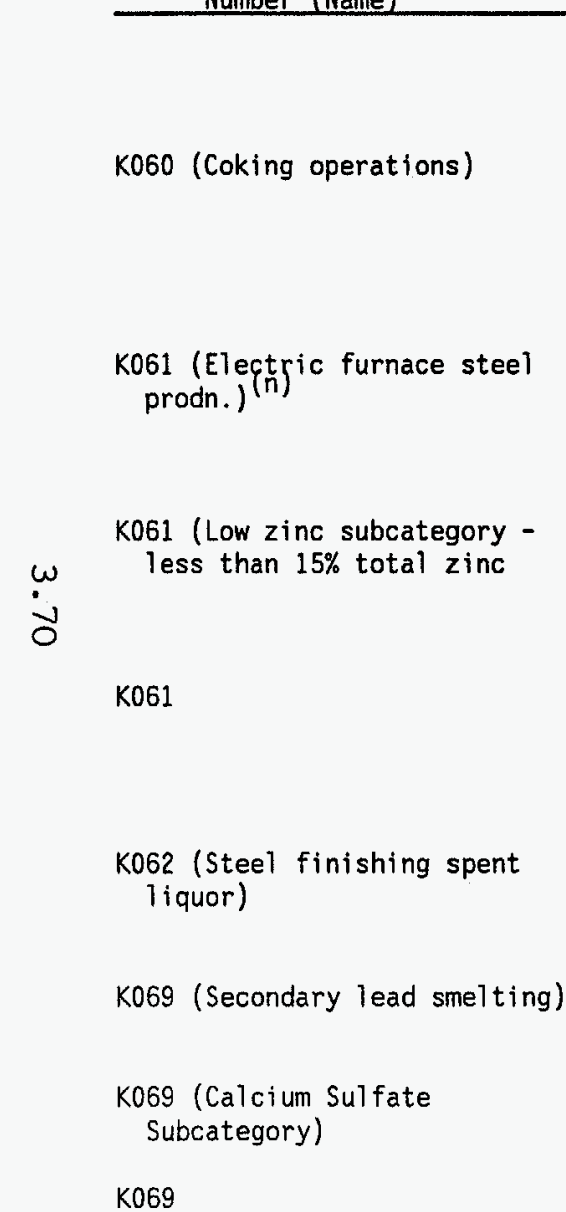

Constituent ${ }^{(b)}$

Cyanides (total)

Lead

$$
\begin{aligned}
& \text { Benzene } \\
& \text { Benzo(a) pyrene } \\
& \text { Naphthal ene } \\
& \text { Phenol } \\
& \text { Cyanides (total) }
\end{aligned}
$$

Emission control dust/sludge from primary production of steel in electric furnaces (high zinc subcategory - greater than or equal to $15 \%$ total zinc)

\section{Cadmi um}

Chromi um (total)

Lead

Nickel

0.14

5.2

0.24

0.32

Cadmi um

Chromium (total)

Lead

Nickel

Chromium (total)

Lead

Nickel

Emission control dust/sludge from secondary lead smelting: Non-Calcium Sulfate subcategory

Cadmium

Lead

Cadmium

Lead

0.094

0.37

0.14

0.24
Regulatory Standard

\begin{tabular}{lc}
\hline Concentration in Waste & Concentration in Waste \\
Extract (40 CFR 268.41) & (40 CFR 268.43) \\
Nonwastewater Wastewater & Nonwastewater Wastewater
\end{tabular}

Nonwastewater Wastewater

Wastewater Water

$(\mathrm{mg} / \mathrm{L})$

$(\mathrm{mg} / \mathrm{L})$

1.8

0.028

0.037

$\begin{array}{ll}0.071 & 0.17 \\ 3.6 & 0.035 \\ 3.4 & 0.028 \\ 3.4 & 0.042 \\ 1.2 & 1.9\end{array}$

spec. tech.

.042

1.9

1.61

0.32

0.51

0.44

0.32

0.04

0.44

spec. tech. 


\section{TABLE 3.10. (contd)}

\begin{tabular}{|c|c|}
\hline $\begin{array}{l}\text { EPA Hazardous Waste } \\
\text { Number (Name) }\end{array}$ & Constituent ${ }^{(b)}$ \\
\hline K071 (Chlorine prodn.) & Mercury \\
\hline $\begin{array}{l}\text { K071 (Low mercury subcategory - } \\
\text { less than } 16 \mathrm{mg} / \mathrm{kg} \text { mercury) }\end{array}$ & Mercury \\
\hline K073 (Chlorine prodn.) & $\begin{array}{l}\text { Carbon tetrachloride } \\
\text { Chloroform } \\
\text { Hexachloroethane } \\
\text { Tetrachloroethene } \\
1,1,1-\text { Trichloroethane }\end{array}$ \\
\hline K083 (Aniline prodn.) & $\begin{array}{l}\text { Benzene } \\
\text { Aniline } \\
\text { Diphenylamine } \\
\text { Diphenylnitrosamine } \\
\text { Diphenylamine + Diphenylnitrosamine } \\
\text { Nitrobenzene } \\
\text { Phenol } \\
\text { Cyclohexanone } \\
\text { Nickel }\end{array}$ \\
\hline $\begin{array}{l}\text { K084 (Veterjnary pharmaceutical } \\
\text { prodn.) }\end{array}$ & Arsenic \\
\hline KO85 (Chlorobenzene prodn.) & $\begin{array}{l}\text { Benzene } \\
\text { Chlorobenzene } \\
\text { o-Dichlorobenzene } \\
\text { m-Dichlorobenzene } \\
\text { p-Dichlorobenzene } \\
1,2,4-\text { Trichlorobenzene } \\
1,2,4,5-\text { Tetrachlorobenzene } \\
\text { Pentachlorobenzene } \\
\text { Hexachlorobenzene } \\
\text { Aroclor } 1016 \\
\text { Aroclor } 1221 \\
\text { Aroclor } 1232\end{array}$ \\
\hline
\end{tabular}

Requlatory Standard

\begin{tabular}{|c|c|c|c|}
\hline \multicolumn{2}{|c|}{$\begin{array}{l}\text { Concentration in Waste } \\
\text { Extract ( } 40 \text { CFR } 268.41)\end{array}$} & \multicolumn{2}{|c|}{$\begin{array}{c}\text { Concentration in Waste } \\
(40 \text { CFR 268.43) }\end{array}$} \\
\hline $\begin{array}{l}\text { Nonwastewater } \\
(\mathrm{mg} / \mathrm{L})\end{array}$ & $\begin{array}{l}\text { Wastewater } \\
(\mathrm{mg} / \mathrm{L})\end{array}$ & $\begin{array}{l}\text { Nonwastewater } \\
(\mathrm{mg} / \mathrm{kg})\end{array}$ & $\begin{array}{l}\text { Wastewater } \\
(\mathrm{mg} / \mathrm{L})\end{array}$ \\
\hline
\end{tabular}

0.030

0.025

\begin{tabular}{|c|c|c|}
\hline & $\begin{array}{l}6.2 \\
6.2 \\
30 \\
6.2 \\
6.2\end{array}$ & $\begin{array}{l}0.057 \\
0.046 \\
0.055 \\
0.056 \\
0.054\end{array}$ \\
\hline & $\begin{array}{l}6.6 \\
14\end{array}$ & $\begin{array}{l}0.14 \\
0.81 \\
0.52 \\
0.40\end{array}$ \\
\hline .088 & $\begin{array}{l}14 \\
14 \\
5.6 \\
30\end{array}$ & $\begin{array}{l}0.068 \\
0.039 \\
0.36 \\
0.47\end{array}$ \\
\hline 5.6 & & 0.79 \\
\hline & $\begin{array}{l}4.4 \\
4.4 \\
4.4 \\
4.4 \\
4.4 \\
4.4 \\
4.4 \\
4.4 \\
4.4 \\
0.92 \\
0.92 \\
0.92\end{array}$ & $\begin{array}{l}0.14 \\
0.057 \\
0.088 \\
0.036 \\
0.090 \\
0.055 \\
0.055 \\
0.055 \\
0.055 \\
0.013 \\
0.014 \\
0.013\end{array}$ \\
\hline
\end{tabular}


TABLE 3.10. (contd) EPA Hazardous Waste
Number (Name)

K086 (Ink prodn.) ${ }^{(0)}$

$\dot{\omega}$
Constituent ${ }^{(b)}$

Aroclor 1242

Aroclor 1248

Aroclor 1254

Aroclor 1260

Acetone

Acetophenone

bis(2-ethylhexyl)phthal ate

n-Butyl alcohol

Butyl benzylphthal ate

Cyclohexanone

1,2-Dichlorobenzene

Diethyl phthalate

Dimethyl phthalate

Di-n-butyl phthalate

Di-n-octyl phthalate

Ethyl acetate

Ethyl benzene

Methanol

Methylene chloride

Methyl ethyl ketone

Methyl isobutyl ketone

Naphthalene

Ni trobenzene

Toluene

1,1,1-Trichloroethane

Trichloroethylene

Xylenes

Cyanides (total)

Chromium (total)

Lead

\subsection{4}

0.37

Acenaphthalene

Benzene

Chrysene

Fluoranthene

Requlatory Standard

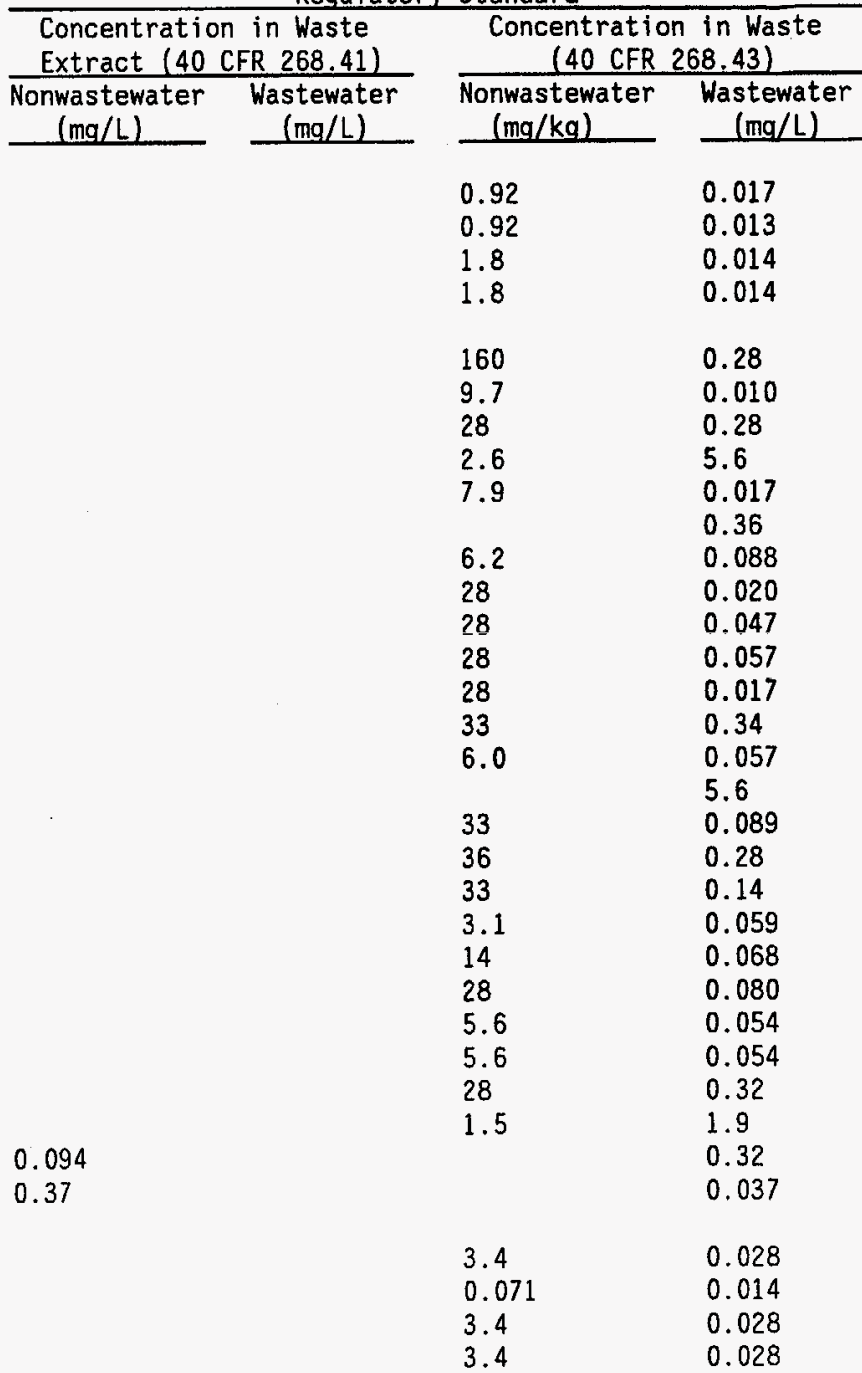


TABLE 3.10. (contd) EPA Hazardous Waste
Number (Name)

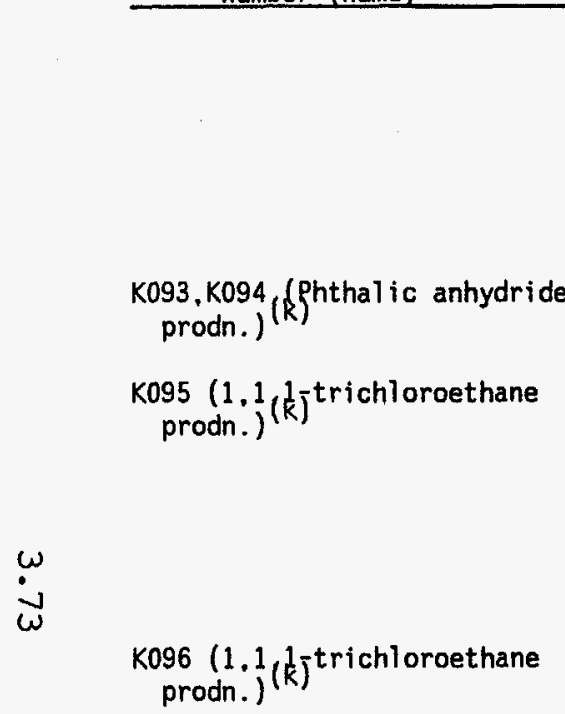

K097 (Chlorodane prodn.)

K098 (Toxaphene prodn.)
Constituent (b)

Ideno (1,2,3-cd) pyrene

Naphthalene

Phenanthrene

Toluene

$X y l e n e s$

Lead

0.51

Phthal ic anhydride

1,1,1,2-Tetrachloroethane

1,1,2,2-Tetrachloroethane

Tetrachloroethene

1,1,2-Trichloroethane

Trichloroethylene

Hexachloroethane

Pentachloroethane

1,3-0ichlorobenzene

Pentachloroethane

1,1,1,2-Tetrachloroethane

1,1,2,2-Tetrachloroethane

Tetrachloroethene

1,2,4-Trichlorobenzene

Trichloroethene

1,1,2-Trichloroethane

Hexachl orocycl opentadi ene

Chlordane

Heptachlor

Heptachlor epoxide

Toxaphene
Requlatory Standard

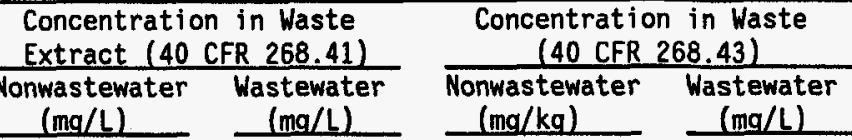

(mg/L)

3.4

3.4

3.4
3.4

0.65

0.070

0.028

0.028

0.028

0.028
0.008

0.014

0.037

28

0.54

$\begin{array}{ll}5.6 & 0.057 \\ 5.6 & 0.057 \\ 6.0 & 0.056 \\ 6.0 & 0.054 \\ 5.6 & 0.054 \\ 28 & 0.055 \\ 5.6 & 0.055 \\ 5.6 & 0.036 \\ 5.6 & 0.055 \\ 5.6 & 0.057 \\ 5.6 & 0.057 \\ 6.0 & 0.056 \\ 19 & 0.055 \\ 5.6 & 0.054 \\ 6.0 & 0.054 \\ 2.4 & 0.057 \\ 0.26 & 0.0033 \\ 0.066 & 0.0012 \\ 0.066 & 0.016 \\ 2.6 & \\ 2.6 & 0.0095\end{array}$


TABLE 3.10. (contd)

\begin{tabular}{|c|c|c|c|c|c|}
\hline \multirow[b]{2}{*}{$\begin{array}{l}\text { EPA Hazardous Waste } \\
\text { Number (Name) }\end{array}$} & \multirow[b]{2}{*}{ Constituent $(\mathrm{b})$} & \multicolumn{2}{|c|}{$\begin{array}{l}\text { Concentration in Waste } \\
\text { Extract (40 CFR 268.41) }\end{array}$} & \multicolumn{2}{|c|}{$\begin{array}{c}\text { Concentration in Waste } \\
(40 \text { CFR 268.43) }\end{array}$} \\
\hline & & $\begin{array}{l}\text { Nonwastewater } \\
(\mathrm{mg} / \mathrm{L})\end{array}$ & $\begin{array}{l}\text { Wastewater } \\
(\mathrm{mg} / \mathrm{L})\end{array}$ & $\begin{array}{l}\text { Nonwastewater } \\
(\mathrm{mg} / \mathrm{kg}) \\
\end{array}$ & $\begin{array}{l}\text { Wastewater } \\
(\mathrm{mg} / \mathrm{L})\end{array}$ \\
\hline KO9g $(2,4-0$ prodn. $)$ & $\begin{array}{l}\text { 2,4-Dichlorophenoxyacetic acid } \\
\text { Hexachlorodibenzo-p-dioxins } \\
\text { Hexachlorodibenzofurans } \\
\text { Pentachlorodibenzo-p-dioxins } \\
\text { Pentachlorodi benzofurans } \\
\text { Tetrachlorodibenzo-p-dioxins } \\
\text { Tetrachlorodi benzofurans }\end{array}$ & & & $\begin{array}{l}1.0 \\
0.001 \\
0.001 \\
0.001 \\
0.001 \\
0.001 \\
0.001\end{array}$ & $\begin{array}{l}1.0 \\
0.001 \\
0.001 \\
0.001 \\
0.001 \\
0.001 \\
0.001\end{array}$ \\
\hline K100 (Secondary lead smelting) & $\begin{array}{l}\text { Cadmi um } \\
\text { Chromi um (total) } \\
\text { Lead }\end{array}$ & $\begin{array}{l}0.066 \\
5.2 \\
0.51\end{array}$ & & & $\begin{array}{l}1.6 \\
0.32 \\
0.51\end{array}$ \\
\hline $\begin{array}{l}\text { K101 (Vet, } \\
\text { prodn.) }(e)^{\text {pharmaceutical }}\end{array}$ & o-Nitroaniline & & & 14 & 0.27 \\
\hline $\begin{array}{l}\text { Ki01, K102 (Vet, pharmaceutical } \\
\text { prodn.) }\end{array}$ & $\begin{array}{l}\text { Arsenic } \\
\text { Cadmium } \\
\text { Chromi um (total) } \\
\text { Lead } \\
\text { Nickel } \\
\text { Mercury }\end{array}$ & $\begin{array}{l}5.6 \\
0.066 \\
5.2 \\
0.51 \\
0.32\end{array}$ & & & $\begin{array}{l}0.79 \\
0.24 \\
0.17 \\
0.082\end{array}$ \\
\hline $\begin{array}{l}\text { K102 (Vet } \\
\text { prodn.) } \\
\text { pharmaceutical }\end{array}$ & Ortho-Nitrophenol & & & 13 & 0.028 \\
\hline K103 (Aniline prodn.) & $\begin{array}{l}\text { Aniline } \\
\text { Benzene } \\
2 \text { 2.4-Dinitrophenol } \\
\text { Nitrobenzene } \\
\text { Phenol }\end{array}$ & & & $\begin{array}{l}5.6 \\
6.0 \\
5.6 \\
5.6 \\
5.6\end{array}$ & $\begin{array}{l}4.5 \\
0.15 \\
0.61 \\
0.073 \\
1.4\end{array}$ \\
\hline K104 (Nitrobenzene/Aniline prodn.) & $\begin{array}{l}\text { Aniline } \\
\text { Benzene } \\
\text { 2,4-Dinitrophenol }\end{array}$ & & & $\begin{array}{l}5.6 \\
6.0 \\
5.6\end{array}$ & $\begin{array}{l}4.5 \\
0.15 \\
0.61\end{array}$ \\
\hline
\end{tabular}




\section{TABLE 3.10. (contd)} EPA Hazardous Waste
Number (Name)

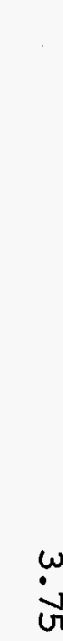

K106 (Low Mercury Subcategoryless than $260 \mathrm{mg} / \mathrm{kg}$ (Merçury-
residues from RMERC)

K106 (Low Mercury Subcategoryless than $260 \mathrm{mg} / \mathrm{kg}$ Megrcurythat are not residues from RMERC)

K113 (Toluenediamine prodn.) ${ }^{(k)}$

K114 (Toluenediamine prodn.) ${ }^{(k)}$

K115 (Toluenediame prodn.) ${ }^{(k)}$

K116 (Toluenediamine prodn.) ${ }^{(k)}$

Constituent $(b)$

Ni trobenzene

Phenol

Cyanides (total)

Benzene

Chlorobenzene

o-Dichlorobenzene

p-Dichlorobenzene

2,4,5-Trichlorophenol

2,4,6-Trichlorophenol

2-Chlorophenol

Phenol

Mercury

Mercury

Wastewater treatment sludge from the mercury cell process in chlorine production: (High Mercury

Subcategory - > = $260 \mathrm{mg} / \mathrm{kg}$ total mercury)

\section{Nickel}

Heavy ends from the purification of toluenediame

in production of toluenediame via hydrogenation

of dinitrotoluene
Requlatory Standard

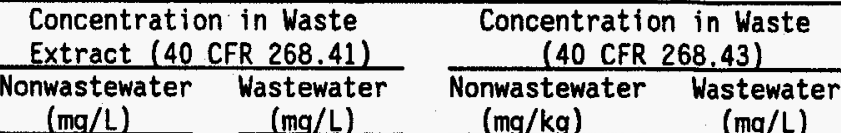

$5.6 \quad 0.073$

$5.6 \quad 0.073$

$\begin{array}{ll}1.8 & 2.7\end{array}$

$4.4 \quad 0.14$

$4.4 \quad 0.057$

$\begin{array}{ll}4.4 & 0.057 \\ 4.4 & 0.088\end{array}$

$\begin{array}{ll}4.4 & 0.088 \\ 4.4 & 0.090\end{array}$

$4.4 \quad 0.18$

$4.4 \quad 0.035$

$4.4 \quad 0.044$

$\begin{array}{ll}4.4 & 0.039\end{array}$

0.20

0.030

0.030

spec. tech. spec. tech

spec. tech. spec. tech.

0.47

spec. tech. spec. tech.

spec. tech.

spec. tech 
TABLE 3.10. (contd)

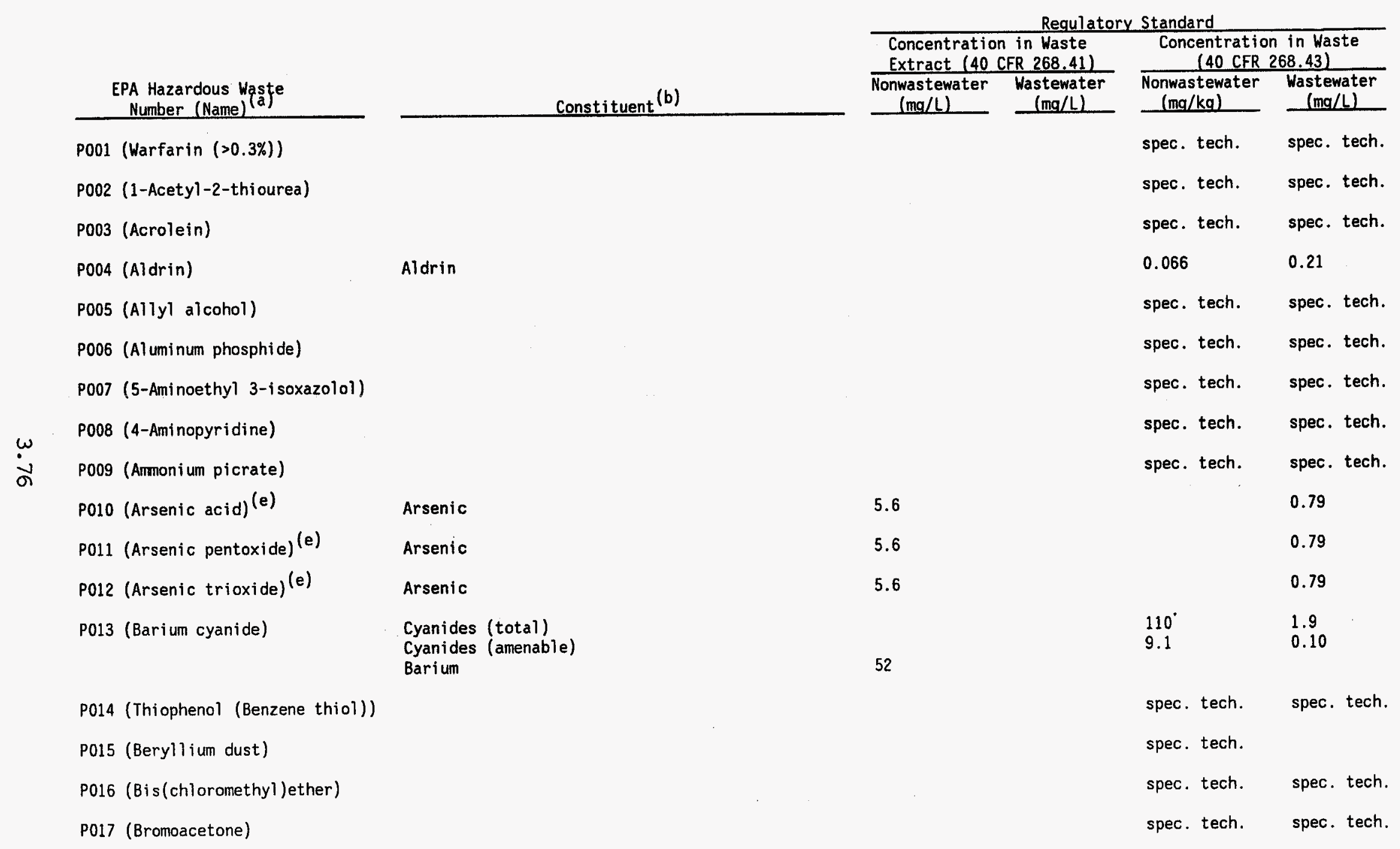




\section{TABLE 3.10. (contd)}

\begin{tabular}{|c|c|}
\hline $\begin{array}{l}\text { EPA Hazardous Waste } \\
\text { Number (Name) }\end{array}$ & Constituent \\
\hline \multicolumn{2}{|l|}{ P018 (Brucine) } \\
\hline $\begin{array}{l}\text { P020 (2-sec-Butyl-4,6- } \\
\text { dinitrophenol) }\end{array}$ & 2-sec-Buty1-4,6-dinitrophenol \\
\hline P021 (Calcium cyanide) & $\begin{array}{l}\text { Cyanides (total) } \\
\text { Cyanides (amenable) }\end{array}$ \\
\hline P022 (Carbon disulfide) & Carbon disulfide \\
\hline \multicolumn{2}{|l|}{ P023 (Chloroacetaldehyde) } \\
\hline PO24 ( $p$-Chloroaniline) & $\mathrm{p}$-Chloroaniline \\
\hline \multicolumn{2}{|l|}{$\begin{array}{l}\text { PO26 (1-(o-Chlorophenyi) } \\
\text { thiourea) }\end{array}$} \\
\hline \multicolumn{2}{|l|}{ P027 (3-Chloropropionitrile) } \\
\hline \multicolumn{2}{|l|}{ P028 (Bensyl chloride) } \\
\hline pozs (Coppét cyañi de) & $\begin{array}{l}\text { Cyanides (total) } \\
\text { Cyanides (amenable) }\end{array}$ \\
\hline $\begin{array}{l}\text { PO3O (Soluble cyanide salts } \\
(\text { n.o.s.)) }\end{array}$ & $\begin{array}{l}\text { Cyanides (total) } \\
\text { Cyanides (amenable) }\end{array}$ \\
\hline \multicolumn{2}{|l|}{ P031 (Cyanogen) } \\
\hline \multicolumn{2}{|l|}{ P033 (Cyanogen chloride) } \\
\hline \multicolumn{2}{|l|}{$\begin{array}{l}\text { P034 (2-Cyclohexyl-4,6- } \\
\text { dinitrophenol) }\end{array}$} \\
\hline P036 (Dichlorophenylarsine) $(e)$ & Arsenic \\
\hline
\end{tabular}

\begin{tabular}{|c|c|c|c|}
\hline \multicolumn{4}{|c|}{ Requlatory Standard } \\
\hline $\begin{array}{l}\text { Concentratio } \\
\text { Extract } 140\end{array}$ & $\begin{array}{l}\text { in Waste } \\
\text { FR 268.411 }\end{array}$ & $\begin{array}{r}\text { Concentrati } \\
\text { (40 CFR }\end{array}$ & $\begin{array}{l}\text { in Waste } \\
68.43)\end{array}$ \\
\hline $\begin{array}{c}\begin{array}{c}\text { Nonwastewater } \\
(\mathrm{mg} / \mathrm{L})\end{array} \\
\end{array}$ & $\begin{array}{l}\text { Wastewater } \\
(\mathrm{mg} / \mathrm{L})\end{array}$ & $\begin{array}{l}\text { Nonwastewater } \\
(\mathrm{mg} / \mathrm{kg})\end{array}$ & $\begin{array}{l}\text { Wastewater } \\
(\mathrm{mg} / \mathrm{L})\end{array}$ \\
\hline
\end{tabular}

spec. tech. spec. tech.

2.5

0.066

$110 \quad 1.9$

$9.1 \quad 0.10$

spec. tech. $\quad 0.014$

spec. tech. spec. tech.

16

0.46

spec. tech. spec. tech.

spec. tech. spec. tech.

spec. tech. spec. tech.

110

$9.1 \quad 0.10$

$110 \quad 1.9$

$9.1 \quad 0.10$

spec. tech. spec. tech.

spec. tech. spec. tech.

spec. tech. spec. tech. 
TABLE 3.10. (contd)

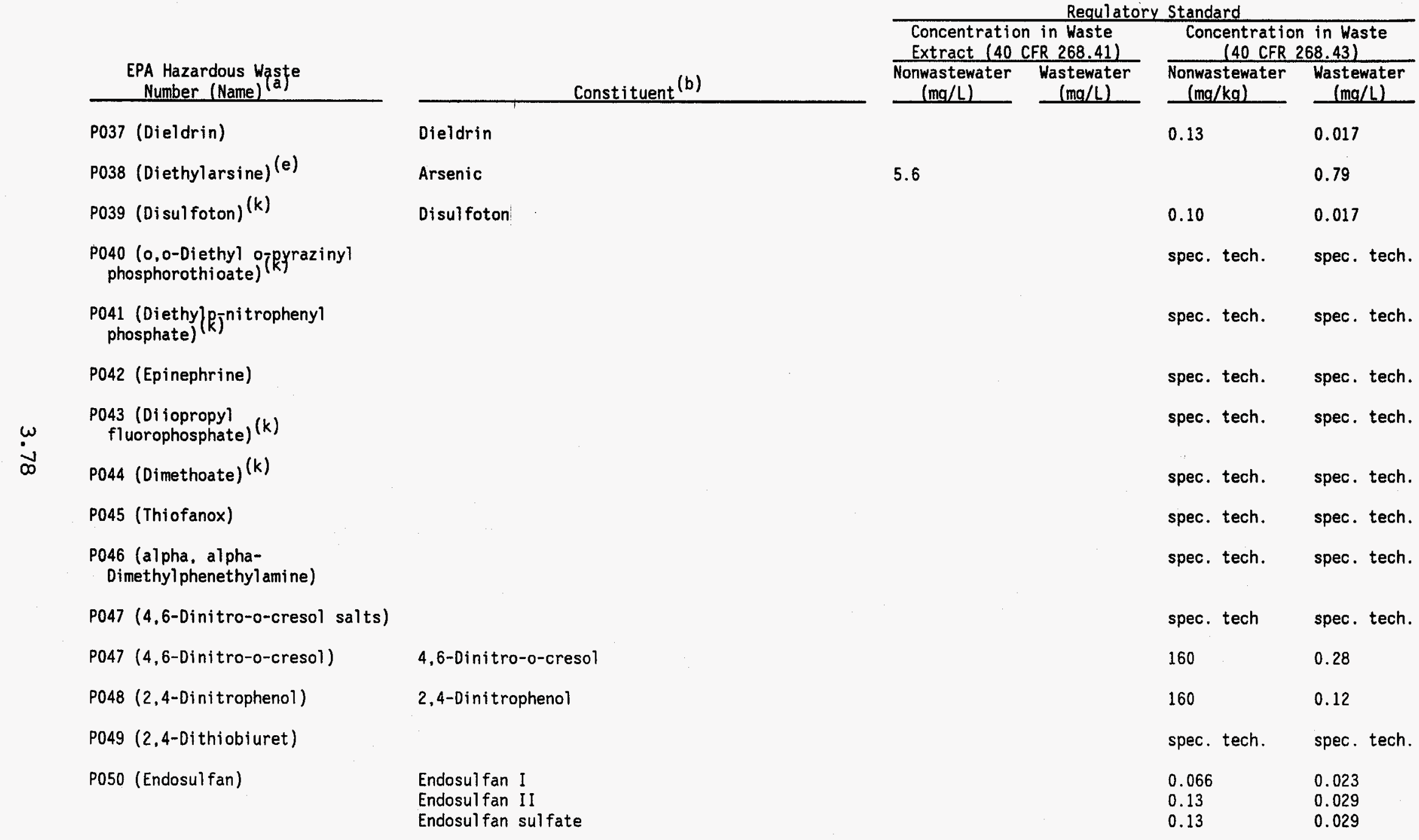


TABLE 3.10. (contd)

\begin{tabular}{|c|c|}
\hline $\begin{array}{l}\text { EPA Hazardous Waste } \\
\text { Number (Name) } \\
\end{array}$ & Constituent $(b)$ \\
\hline P051 (Endrin) & $\begin{array}{l}\text { Endrin } \\
\text { Endrin aldehyde }\end{array}$ \\
\hline \multicolumn{2}{|l|}{ P054 (Aziridine) } \\
\hline P056 (Fluoride) & Fluoride \\
\hline \multicolumn{2}{|l|}{ P057 (Fluoroacetamide) } \\
\hline \multicolumn{2}{|l|}{$\begin{array}{l}\text { P058 (Fluoroacetic acid, } \\
\text { sodium salt) }\end{array}$} \\
\hline P059 (Heptachlor) & $\begin{array}{l}\text { Heptachlor } \\
\text { Heptachlor epoxide }\end{array}$ \\
\hline P060 (Isodrin) & Isodrin \\
\hline \multicolumn{2}{|l|}{$\begin{array}{l}\text { P062 (Hexaethy]tetra- } \\
\text { phosphate) }\end{array}$} \\
\hline P063 (Hydrogen cyanide) & $\begin{array}{l}\text { Cyanides (total) } \\
\text { Cyanides (amenable) }\end{array}$ \\
\hline \multicolumn{2}{|c|}{ P064 (I socyanic acid, ethyl ester) } \\
\hline P065 (Mercury fulminate) ${ }^{(r)}$ & $\begin{array}{l}\text { Mercury } \\
\text { Mercury }(q) \\
\text { Mercury }_{(s)} \\
\text { Mercury fulminate }(t) \\
\text { Mercury fulminate }\end{array}$ \\
\hline \multicolumn{2}{|l|}{ P066 (Methomy1) } \\
\hline P067 (2-Methylaziridine) & \\
\hline P068 (Methyl hydrazine) & \\
\hline
\end{tabular}

\begin{tabular}{|c|c|c|c|}
\hline \multicolumn{2}{|c|}{$\begin{array}{l}\text { Concentration in Waste } \\
\text { Extract (40 CFR 268.41) } \\
\end{array}$} & \multicolumn{2}{|c|}{$\begin{array}{c}\text { Concentration in Waste } \\
(40 \text { CFR } 268.43)\end{array}$} \\
\hline $\begin{array}{l}\text { Nonwastewater } \\
(\mathrm{mg} / \mathrm{L})\end{array}$ & $\begin{array}{l}\text { Wastewater } \\
(\mathrm{mg} / \mathrm{L})\end{array}$ & $\begin{array}{l}\text { Nonwastewater } \\
\text { (mg/kg) }\end{array}$ & $\begin{array}{l}\text { Wastewater } \\
(\mathrm{mg} / \mathrm{L})\end{array}$ \\
\hline & & $\begin{array}{l}0.13 \\
0.13\end{array}$ & $\begin{array}{l}0.0028 \\
0.025\end{array}$ \\
\hline & & spec. tech. & spec. tech. \\
\hline & & spec. tech. & 35 \\
\hline & & spec. tech. & spec. tech. \\
\hline & & spec. tech. & spec. tech. \\
\hline & & $\begin{array}{l}0.066 \\
0.066\end{array}$ & $\begin{array}{l}0.0012 \\
0.016\end{array}$ \\
\hline & & 0.066 & 0.021 \\
\hline & & spec. tech. & spec. tech. \\
\hline & & $\begin{array}{l}110 \\
9.1 \\
\text { spec. tech. }\end{array}$ & $\begin{array}{l}1.9 \\
0.10 \\
\text { spec. tech. }\end{array}$ \\
\hline & & & 0.030 \\
\hline & $\begin{array}{l}0.20 \\
0.025\end{array}$ & & \\
\hline & & $\begin{array}{l}\text { spec. tech. } \\
\text { spec. tech. }\end{array}$ & \\
\hline & & spec. tech. & spec. tech. \\
\hline & & spec. tech. & spec. tech. \\
\hline & & ec. tech & ec. tech. \\
\hline
\end{tabular}




\section{TABLE 3.10. (contd)} EPA Hazardous Waste
Number (Name)

P069 (Methyllactonitrile)

P070 (Aldicarb)

P071 (Methyl parathion) ${ }^{(k)}$

P072 (1-Naphthyl-2-thiourea)

P073 (Nickel carbonyl)

P074 (Nickel cyanide)

P075 (Nicotine and salts)

क् P076 (Nitric oxide)

P077 ( $p$-Nitroaniline)

P078 (Nitrogen dioxide)

P081 (Nitroglycerin)

P082 (N-Nitrosodimethyl amine)

N-Nitrosodimethyl amine

P084 (N-Nitrosomethylvinylamine)

P085 (Octamethyl pyrophosphora-

P087 (Osmium tetroxide) (v)

P088 (Endothal1)

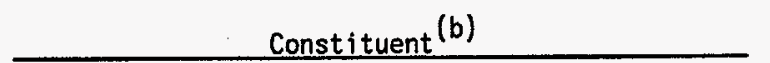

Methyl parathion

Nickel

Nickel

Cyanides (total)

Cyanides (amenable)

p-Nitroaniline
0.32

Concentration in Waste

Extract (40 CFR 268.41)

Nonwastewater Wastewater

(mg/L) $(\mathrm{mg} / \mathrm{L})$

0.32

Concentration in Waste

(40 CFR 268.43)

Nonwastewater Wastewater $(\mathrm{mg} / \mathrm{kg})$ $(m g / L)$

spec. tech. spec. tech. spec. tech. spec. tech.

0.10 0.025

spec. tech. spec. tech.

0.44

110

9.1

1.9

spec. tech.

spec. tech.

spec, tech. spec, tech.

28

0.028

spec. tech.

spec. tech.

spec. tech.

spec. tech.

spec. tech.

0.40

spec. tech.

spec, tech.

spec. tech.

spec. tech.

spec. tech.

spec. tech.

spec, tech. 
TABLE 3.10. (contd)

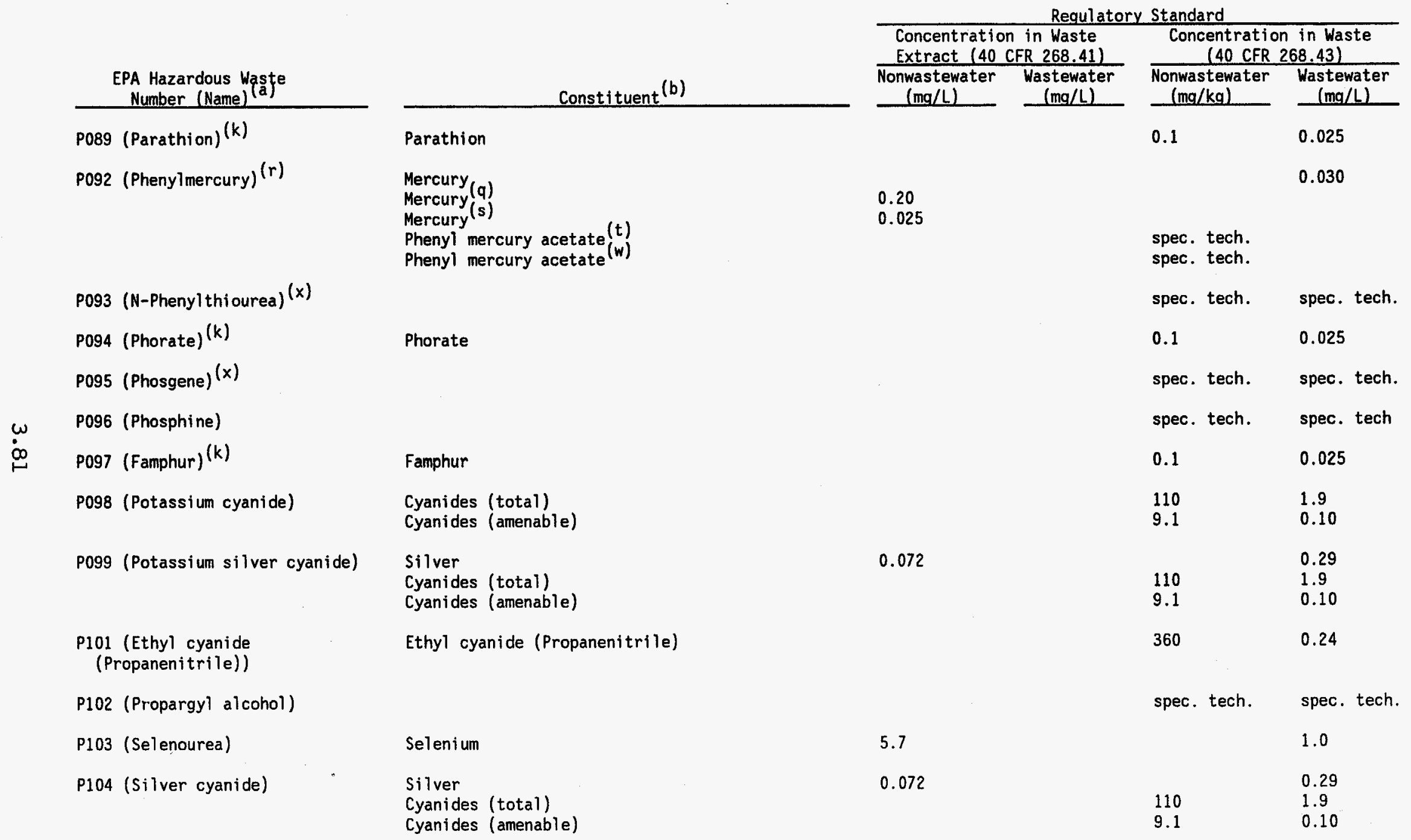


TABLE 3.10. (contd)

\begin{tabular}{|c|c|c|c|c|c|}
\hline \multirow[b]{3}{*}{$\begin{array}{l}\text { EPA Hazardous Waste } \\
\text { Number (Name)) (aj) }\end{array}$} & \multirow[b]{3}{*}{ Constituent $(\mathrm{b})$} & \multicolumn{4}{|c|}{ Requlatory Standard } \\
\hline & & \multicolumn{2}{|c|}{$\begin{array}{l}\text { Concentration in Waste } \\
\text { Extract }(40 \text { CFR 268.41) }\end{array}$} & \multicolumn{2}{|c|}{$\begin{array}{c}\text { Concentration in Waste } \\
\text { (40 CFR 268.43) }\end{array}$} \\
\hline & & $\begin{array}{l}\text { Nonwastewater } \\
\text { (mg/L) } \\
\end{array}$ & $\begin{array}{l}\text { Wastewater } \\
(\mathrm{mg} / \mathrm{L})\end{array}$ & $\begin{array}{l}\text { Nonwastewater } \\
(\mathrm{mg} / \mathrm{kg}) \\
\end{array}$ & $\begin{array}{l}\text { Wastewater } \\
(\mathrm{mg} / L) \\
\end{array}$ \\
\hline P105 (Sodium azide) & & & & spec. tech. & spec. tech. \\
\hline P106 (Sodium cyanide) & $\begin{array}{l}\text { Cyanides (total) } \\
\text { Cyanides (amenable) }\end{array}$ & & & $\begin{array}{l}110 \\
9.1\end{array}$ & $\begin{array}{l}1.9 \\
0.10\end{array}$ \\
\hline P108 (Strychnine and salts) ${ }^{(x)}$ & & & & spec. tech. & spec. tech. \\
\hline $\begin{array}{l}\text { P109 (Tetraethyldithiophyro- } \\
\text { phosphate) }\end{array}$ & & & & spec. tech. & spec. tech. \\
\hline P110 (Tetraethyl lead) & Lead & 0.51 & & & 0.040 \\
\hline P112 (Tetrani tromethane) & & & & spec. tech. & spec. tech. \\
\hline P113 (Thallic oxide) & Thallium & & & spec. tech. & 0.14 \\
\hline P114 (Thallium selenite) & Selenium & 5.7 & & & 1.0 \\
\hline P115 (Thallium(I)sulfate) & Thall i um & & & spec. tech. & 0.14 \\
\hline P116 (Thiosemicarbazide) $(x)$ & & & & spec. tech. & spec. tech. \\
\hline P118 (Trichloromethanethiol) ${ }^{(x)}$ & & & & spec. tech. & spec. tech. \\
\hline P119 (Ammonium vanadate) & Vanadium & & & spec. tech. & 28 \\
\hline P120 (Vanadium pentoxide) & Vanadium & & & spec. tech. & 28 \\
\hline P121 (Zinc cyanide) & $\begin{array}{l}\text { Cyanides (total) } \\
\text { Cyanides (amenable) }\end{array}$ & & & $\begin{array}{l}110 \\
9.1\end{array}$ & $\begin{array}{l}1.9 \\
0.10\end{array}$ \\
\hline P122 (Zinc phosphide $(<10 \%)$ ) & & & & spec. tech. & spec. tech. \\
\hline P123 (Toxaphene) & Toxaphene & & & 1.3 & 0.0095 \\
\hline
\end{tabular}


TABLE 3.10. (contd)

\begin{tabular}{|c|c|}
\hline $\begin{array}{l}\text { EPA Hazardous Waste } \\
\text { Number (Name) }\end{array}$ & Cons \\
\hline \multicolumn{2}{|l|}{ U001 (Acetaldehyde) } \\
\hline U002 (Acetone) & Acetone \\
\hline U003 (Acetonitrile) $(x)$ & Acetonitrile \\
\hline U004 (Acetophenone) & Acetophenone \\
\hline U005 (2-Acetylaminofluorene) & 2-Acetylaminofluorene \\
\hline \multicolumn{2}{|l|}{ U006 (Acetyl chloride) $(x)$} \\
\hline \multicolumn{2}{|l|}{ U007 (Acryl ami de) $(x)$} \\
\hline \multicolumn{2}{|l|}{ U008 (Acrylic acid) } \\
\hline U009 (Acrylonitrile) & Acrylonitrile \\
\hline \multicolumn{2}{|l|}{ U010 (Mitomycin C) ${ }^{(x)}$} \\
\hline \multicolumn{2}{|l|}{ U011 (Amitrole) $(x)$} \\
\hline U012 (Aniline) & Aniline \\
\hline \multicolumn{2}{|l|}{ U014 (Auramine) $(x)$} \\
\hline \multicolumn{2}{|l|}{ U015 (Azaserine) ${ }^{(x)}$} \\
\hline \multicolumn{2}{|l|}{ U016 (Benz(c)acridine) } \\
\hline \multicolumn{2}{|l|}{ U017 (Benzal chloride) ${ }^{(x)}$} \\
\hline U018 (Benz (a) anthracene) & Benz(a)anthracene \\
\hline U019 (Benzene) & Benzene \\
\hline
\end{tabular}


TABLE 3.10. (contd)

\begin{tabular}{|c|c|c|c|c|c|}
\hline \multirow{2}{*}{$\begin{array}{l}\text { EPA Hazardous Waste } \\
\text { Number (Name) } \\
\end{array}$} & \multirow{2}{*}{ Constituent ${ }^{(b)}$} & \multicolumn{4}{|c|}{ Requlatory Standard } \\
\hline & & \multicolumn{2}{|c|}{$\begin{array}{l}\text { Concentration in Waste } \\
\text { Extract ( } 40 \text { CFR 268.41) } \\
\end{array}$} & \multicolumn{2}{|c|}{$\begin{array}{c}\text { Concentration in Waste } \\
(40 \text { CFR 268.43) }\end{array}$} \\
\hline U021 (Benzidine) $(x)$ & & & & spec. tech. & spec. tech. \\
\hline U022 (Benzo(a)pyrene) & Benzo(a)pyrene & & & 8.2 & 0.061 \\
\hline $\begin{array}{l}\text { U024 (Bis (2-Chloroethoxy) } \\
\text { methane) }\end{array}$ & Bis(2-chloroethoxy)methane & & & 7.2 & 0.036 \\
\hline U025 (Bis(2-chloroethyl)ether) & Bis(2-chloroethyl)ether & & & 7.2 & 0.033 \\
\hline U026 (Chlornaphazin) ${ }^{(x)}$ & & & & spec. tech. & spec. tech. \\
\hline U027 (Bis(2-chloroi sopropyl)ether) & Bis(2-chloroi sopropyl)ether & & & 7.2 & 0.055 \\
\hline U030 (4-Bromophenyl phenyl ether) & 4-Bromophenyl phenyl ether & & & 15 & 0.055 \\
\hline U031 (n-Butyl alcohol) & n-Butyl alcohol & & & 2.6 & 5.6 \\
\hline U032 (Calcium chromate) & Chromium (total) & 0.094 & & & 0.32 \\
\hline U033 (Carbonyl fluoride) $(x)$ & & & & spec. tech. & spec. tech. \\
\hline $\begin{array}{l}\text { U034 (Trichlqroacetaldehyde } \\
\text { (chloral)) }\end{array}$ & & & & spec. tech. & spec. tech. \\
\hline U035 (Chlorambucil) $(x)$ & & & & spec. tech. & spec. tech. \\
\hline
\end{tabular}


TABLE 3.10. (contd)

\begin{tabular}{|c|c|c|c|c|c|}
\hline $\begin{array}{l}\text { EPA Hazardous Waste } \\
\text { Number (Name) (ą) }\end{array}$ & Constituent $^{(b)}$ & \multicolumn{4}{|c|}{ Requlatory Standard } \\
\hline U037 (Chlorobenzene) & Chlorobenzene & & & 5.7 & 0.057 \\
\hline U038 (Chlorobenzilate) $(x)$ & Chlorobenzilate & & & spec. tech. & 0.10 \\
\hline $\begin{array}{l}\text { U041 (1-Chloro-2,3- } \\
\text { epoxypropane } \\
\text { (epichlorohydrin)) }\end{array}$ & & & & spec. tech. & spec. tech. \\
\hline v042 (2-Chloroethyl vinyl $(x)$ & 2-Chloroethyl vinyl & & & spec. tech. & 0.057 \\
\hline U043 (Vinyl chloride) & Vinyl chloride & & & 33 & 0.27 \\
\hline $\begin{array}{l}\text { U046 (Ch } \mid(x) \text { omethyl methyl } \\
\text { ether) }\end{array}$ & & & & spec. tech. & spec. tech. \\
\hline U047 (2-Chloronaphthalene) & 2-Chloronaphthalene & & & 5.6 & 0.055 \\
\hline U048 (2-Chlorophenol) & 2-Chlorophenol & & & 5.7 & 0.044 \\
\hline $\begin{array}{l}\text { U049 (4-Chloro-0-toluidine } \\
\text { hydrochloride) }\end{array}$ & & & & spec. tech. & spec. tech. \\
\hline U050 (Chrysene) & Chrysene & & & 8.2 & 0.059 \\
\hline
\end{tabular}


IABLE 3.10. (contd)

\begin{tabular}{|c|c|}
\hline $\begin{array}{l}\text { EPA Hazardous Waste } \\
\text { Number (Name) }\end{array}$ & Constituent (b) \\
\hline U051 (Creosote) & $\begin{array}{l}\text { Naphthalene } \\
\text { Pentachlorophenol } \\
\text { Phenanthrene } \\
\text { Pyrene } \\
\text { Toluene } \\
\text { Xylenes (total) } \\
\text { Lead }\end{array}$ \\
\hline U052 (Cresols (Cresylic acid)) & $\begin{array}{l}\text { o-Cresol } \\
\text { Cresols (m- and p- isomers) }\end{array}$ \\
\hline \multicolumn{2}{|l|}{ U053 (Crotonaldehyde) } \\
\hline \multicolumn{2}{|l|}{ U055 (Cumene) } \\
\hline U056 (Cyclohexane) & Cyclohexane \\
\hline U057 (Cyclohexanone) & Cyclohexanone \\
\hline \multicolumn{2}{|l|}{ U058 (Cyclophosphamide) ${ }^{(y)}$} \\
\hline \multicolumn{2}{|l|}{ U059 (Daunomycin) $(x)$} \\
\hline U060 $(D D D)(x)$ & $\begin{array}{l}0, p^{\prime}-D D D \\
p, p^{\prime}-D D D\end{array}$ \\
\hline U061 $(D D T)^{(x)}$ & $\begin{array}{l}o, p^{\prime}-D D T \\
p, p^{\prime}-D D T \\
0, p^{\prime}-D D D \\
p, p^{\prime}-D D D \\
0, p^{\prime}-D D E \\
p, p^{\prime}-D D E\end{array}$ \\
\hline U062 (Diallate) $(x)$ & \\
\hline
\end{tabular}

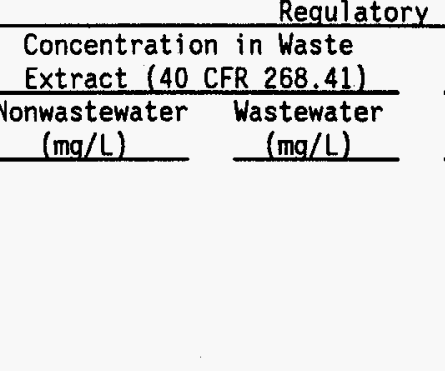

Concentration in Waste
(40 CFR 268.43)
Nonwastewater Wastewater

0.51

$\begin{array}{ll}1.5 & 0.031 \\ 7.4 & 0.18 \\ 1.5 & 0.031 \\ 28 & 0.028 \\ 33 & 0.028 \\ & 0.032 \\ & 0.037 \\ 5.6 & 0.11 \\ 3.2 & 0.77\end{array}$

spec. tech. spec. tech.

spec tech. spec, tech.

spec. tech. spec, tech.

spec. tech. 0.36

spec. tech. spec. tech.

spec. tech. spec. tech.

$0.087 \quad 0.023$

$0.087 \quad 0.023$

$\begin{array}{ll}0.087 & 0.0039\end{array}$

$0.087 \quad 0.0039$

$0.087 \quad 0.023$

$0.087 \quad 0.023$

$0.087 \quad 0.031$

$0.087 \quad 0.031$

spec. tech. spec. tech. 
TABLE 3.10. (contd)

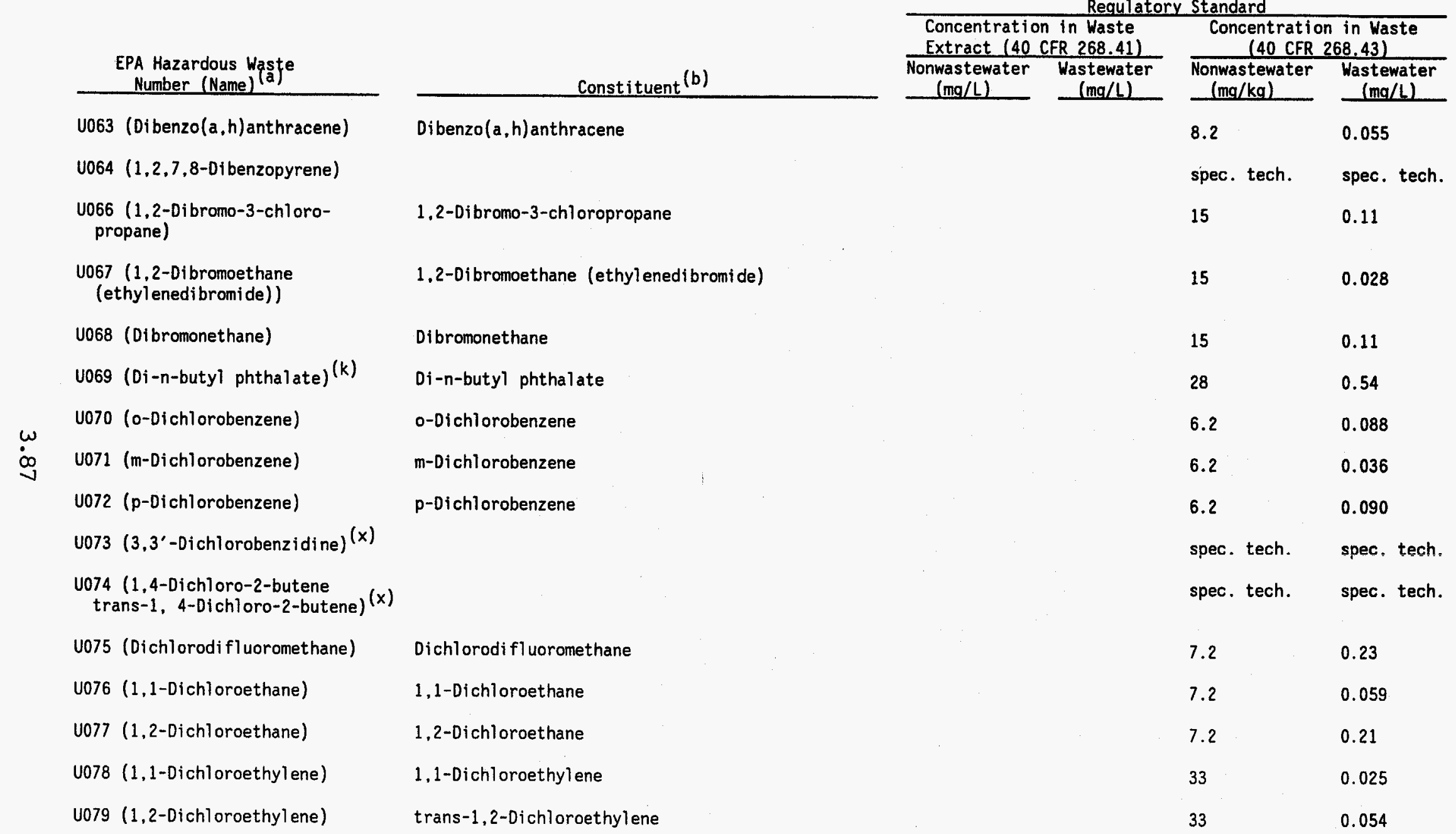




\section{TABLE $2.10 . \quad$ (contd)}

\author{
EPA Hazardous Waste
Number (Name) \\ U080 (Methylene chloride) \\ U081 (2,4-Dichlorophenol) \\ U082 (2,6-Dichlorophenol) \\ U083 (1,2-Dichloropropane) \\ U084 (1,3-Dichloropropene) \\ U085 (1,2,3,4-Diepoxybutane) \\ U086 (N, N-Diethyl hydrazine) \\ $\dot{w}$

$$
\begin{aligned}
& \text { U087 (0.0-diethy1- }\{- \text { methy1- } \\
& \text { di thi ophosphate })(x)
\end{aligned}
$$

$$
\text { U088 (Diethyl phthalate) }{ }^{(k)}
$$ \\ U089 (Diethyl stilbestrol) \\ vogo (Dihydrosafrole) \\ U091 (3,3'-Dimethoxybenzidine) $(x)$ \\ U092 (Dimethylamine) ${ }^{(x)}$

$$
\begin{aligned}
& \text { U093 ( } p-D j \text { methylanimoazoben- } \\
& \text { zene) }
\end{aligned}
$$ \\ U094 (7,12-Dimethyl benz(a)

\begin{tabular}{|c|c|c|c|}
\hline \multicolumn{2}{|c|}{$\begin{array}{l}\text { Concentration in Waste } \\
\text { Extract ( } 40 \text { CFR 268.41) }\end{array}$} & \multicolumn{2}{|c|}{$\begin{array}{c}\text { Concentration in Waste } \\
\text { (40 CFR 268.43) }\end{array}$} \\
\hline $\begin{array}{l}\text { Nonwastewater } \\
(\mathrm{mg} / \mathrm{L})\end{array}$ & $\begin{array}{l}\text { Wastewater } \\
(\mathrm{mg} / \mathrm{L})\end{array}$ & $\begin{array}{l}\text { Nonwastewater } \\
(\mathrm{mg} / \mathrm{kg})\end{array}$ & $\begin{array}{l}\text { Wastewater } \\
(\mathrm{mg} / \mathrm{L})\end{array}$ \\
\hline
\end{tabular} \\ anthracene) \\ V095 $\left(3,3^{\prime}\right.$-Dimethyl benzidine) ${ }^{(x)}$
}

Requlatory Standard

Constituent (b)

$(\mathrm{mg} / \mathrm{L})$

Methylene chloride

33

0.089

2.4-Dichlorophenol

14

0.044

2,6-Dichlorophenol

1,2-Dichloropropane

cis-1,3-Dichloropropylene

trans-1,3--Dichloropropylene

Diethyl phthalate

p-Dimethylanimoazobenzene

14

0.044

18

0.85

18

0.036

18

0.036

spec. tech. spec. tech.

spec. tech. spec. tech.

spec. tech. spec. tech.

28

0.54

spec. tech.

spec. tech.

spec. tech. spec. tech.

spec. tech. spec. tech.

spec. tech. spec. tech.

spec. tech. 0.13

spec. tech. spec. tech.

spec. tech. spec. tech. 
TABLE 3.10. (contd)

\begin{tabular}{|c|c|}
\hline $\begin{array}{l}\text { EPA Hazardous Waste } \\
\text { Number (Name) (a) }\end{array}$ & Constituent (b) \\
\hline \multicolumn{2}{|l|}{$\begin{array}{l}\text { U096 (a,a-Dimethyl benzy } \\
\text { hydroperoxide) }\end{array}$} \\
\hline \multicolumn{2}{|l|}{$\begin{array}{l}\text { U097 (Dimethy)carbomyl } \\
\text { chloride) }\end{array}$} \\
\hline \multicolumn{2}{|l|}{ U098 (1,1-Dimethylhydrazine) } \\
\hline \multicolumn{2}{|l|}{ U099 (1,2-Dimethylhydrazine) } \\
\hline U101 (2,4-Dimethylphenol) & 2,4-Dimethylphenol \\
\hline U102 (Dimethyl phthalate) ${ }^{(k)}$ & Dimethyl phthalate \\
\hline \multicolumn{2}{|l|}{ U103 (Dimethyl sulfate) } \\
\hline U105 (2,4-Dinitrotoluene) & 2,4-Dinitrotoluene \\
\hline U106 (2,6-Dinitrotoluene) & 2,6-Dinitrotoluene \\
\hline U107 (Di-n-octyl phthalate) ${ }^{(k)}$ & Di-n-octyl phthalate \\
\hline U108 (1,4-Di oxane) & 1.4-Dioxane \\
\hline \multicolumn{2}{|l|}{ U109 (1,2-Diphenylhydrazine) } \\
\hline \multicolumn{2}{|l|}{ U110 (Dipropylamine) $)^{(x)}$} \\
\hline U111 (Di-n-propylnitrosoamine) & Di-n-propylnitrosoamine \\
\hline U112 (Ethyl acetate) & Ethyl acetate \\
\hline U113 (Ethyl acrylate) & \\
\hline
\end{tabular}

\begin{tabular}{|c|c|}
\hline $\begin{array}{l}\text { Concentratio } \\
\text { Extract }(40\end{array}$ & $\begin{array}{r}\text { in } \mathrm{Wa} \\
\text { FR } 268 \\
\end{array}$ \\
\hline $\begin{array}{l}\text { Vonwastewater } \\
(\mathrm{mg} / \mathrm{L})\end{array}$ & $\begin{array}{l}\text { Waster } \\
\quad(\mathrm{mg})\end{array}$ \\
\hline
\end{tabular}

$(\mathrm{mg} / \mathrm{L})$ Wastewater 
TABLE 3.10. (contd)

\begin{tabular}{|c|c|c|c|c|c|}
\hline \multirow[b]{3}{*}{$\begin{array}{l}\text { EPA Hazardous Waste } \\
\text { Number (Name) }\end{array}$} & \multirow[b]{3}{*}{ Constituent (b) } & \multicolumn{4}{|c|}{ Regulatory Standard } \\
\hline & & \multicolumn{2}{|c|}{$\begin{array}{l}\text { Concentration in Waste } \\
\text { Extract }(40 \text { CFR 268.41) }\end{array}$} & \multicolumn{2}{|c|}{$\begin{array}{c}\text { Concentration in Waste } \\
\text { (40 CFR 268.43) }\end{array}$} \\
\hline & & $\begin{array}{c}\text { Nonwastewater } \\
(\mathrm{mg} / \mathrm{L})\end{array}$ & $\begin{array}{l}\text { Wastewater } \\
\text { (mg/L) }\end{array}$ & $\begin{array}{l}\text { Nonwastewater } \\
(\mathrm{mg} / \mathrm{kg})\end{array}$ & $\begin{array}{c}\text { Wastewater } \\
(\mathrm{mg} / \mathrm{L})\end{array}$ \\
\hline $\begin{array}{l}\text { U114 (Ethylene bis-dithiocarbamic } \\
\text { acid) }\end{array}$ & & & & spec. tech. & spec. tech. \\
\hline U115 (Ethylene oxide) & & & & spec. tech. & spec. tech. \\
\hline U116 (Ethylene thiourea) ${ }^{(x)}$ & & & & spec. tech. & spec. tech. \\
\hline U117 (Ethy1 ether) & Ethyl ether & & & 160 & 0.12 \\
\hline U118 (Ethyl methacrylate) & Ethyl methacrylate & & & 160 & 0.14 \\
\hline $\begin{array}{l}\text { U119 (Ethyl methane } \\
\text { sulfonate) }\end{array}$ & & & & spec. tech. & spec. tech. \\
\hline U120 (Fluoranthene) & Fluoranthene & & & 8.2 & 0.068 \\
\hline U121 (Trichloromonofluoromethane) & Trichloromonofl uoromethane & & & 33 & 0.020 \\
\hline U122 (Formalaldehyde) & & & & spec. tech. & spec. tech. \\
\hline U123 (Formic acid) & & & & spec. tech. & spec. tech. \\
\hline U124 (Furan) & & & & spec. tech. & spec. tech. \\
\hline U125 (Furfural) & & & & spec. tech. & spec. tech. \\
\hline U126 (Glycidaldehyde) & & & & spec. tech. & spec. tech. \\
\hline U127 (Hexachlorobenzene) & Hexachlorobenzene & & & 37 & 0.055 \\
\hline U128 (Hexachlorobutadiene) & Hexachl orobutadi ene & & & 28 & 0.055 \\
\hline U129 (Lindane) & $\begin{array}{l}\text { alpha-BHC } \\
\text { beta-BHC } \\
\text { del ta-BHC } \\
\text { gamma-BHC (Lindane) }\end{array}$ & & & $\begin{array}{l}0.066 \\
0.066 \\
0.066 \\
0.066\end{array}$ & $\begin{array}{l}0.00014 \\
0.00014 \\
0.023 \\
0.0017\end{array}$ \\
\hline
\end{tabular}




\section{TABLE 3.10. (contd)}

\begin{tabular}{|c|c|}
\hline $\begin{array}{l}\text { EPA Hazardous Waste } \\
\text { Number (Name) }\end{array}$ & Cons \\
\hline $\begin{array}{l}\text { U130 (Hexachlorocyclopenta- } \\
\text { diene) }\end{array}$ & Hexachlorocyclopentadien \\
\hline U131 (Hexachloroethane) & Hexachloroethane \\
\hline U132 (Hexachlorophenene) $(x)$ & \\
\hline U133 (Hydrazine) & \\
\hline U134 (Hydrogen fluoride) & Fluoride \\
\hline U135 (Hydrogen sulfide) & \\
\hline U136 (Cacodylic acid) $(x)$ & Arsenic \\
\hline U137 (Indeno( $1,2,3-c, d)$ pyrene) & Indeno $(1,2,3-c, d)$ pyrene \\
\hline U138 (Iodomethane) & Iodomethane \\
\hline U140 (Isobutyl alcohol) & Isobutyl alcohol \\
\hline U141 (Isosafrole) & Isosafrole \\
\hline U142 (Kepone) & Kepone \\
\hline U143 (Lasiocarpine) ${ }^{(x)}$ & \\
\hline U144 (Lead acetate) & Lead \\
\hline U145 (Lead phosphate) & Lead \\
\hline U146 (Lead subacetate) & Lead \\
\hline
\end{tabular}

Requlatory Standard

Concentration in Waste

Extract (40 CFR 268.41)

Nonwastewater Wastewater

$(\mathrm{mg} / \mathrm{L}) \quad(\mathrm{mg} / \mathrm{L})$

5.6

Concentration in Waste (40 CFR 268.43)

Nonwastewater Wastewater

(mg/kg)

3.6

0.057

28

0.055

spec. tech.

spec. tech.

spec. tech. spec. tech.

spec. tech. 35

spec. tech. spec. tech.

0.79

$8.2 \quad 0.0055$

$65 \quad 0.19$

$170 \quad 5.6$

$2.6 \quad 0.081$

$0.13 \quad 0.0011$

spec. tech. spec. tech.

0.040

0.040

0.040

spec. tech. 
TABLE 3.10. (contd)

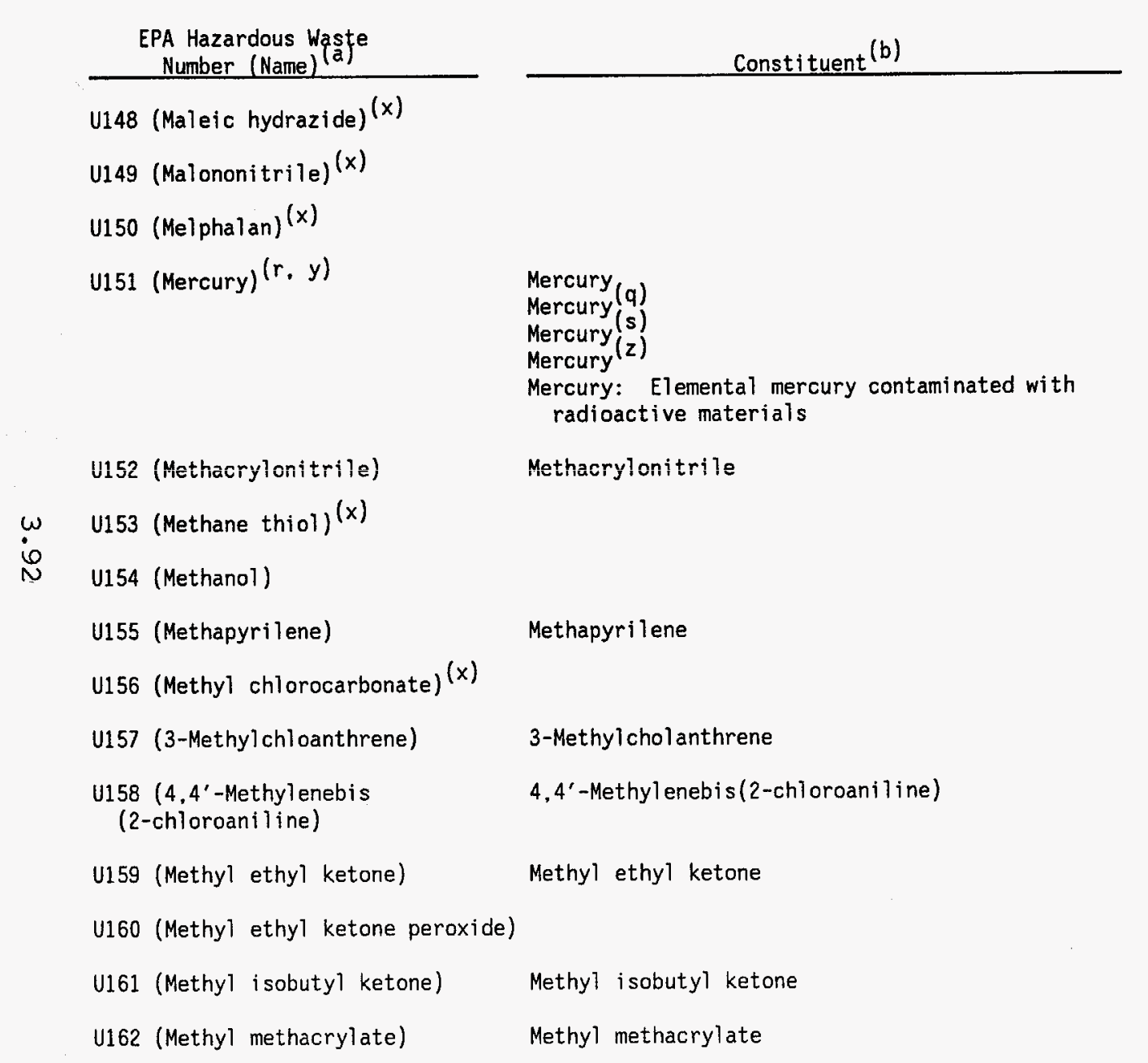

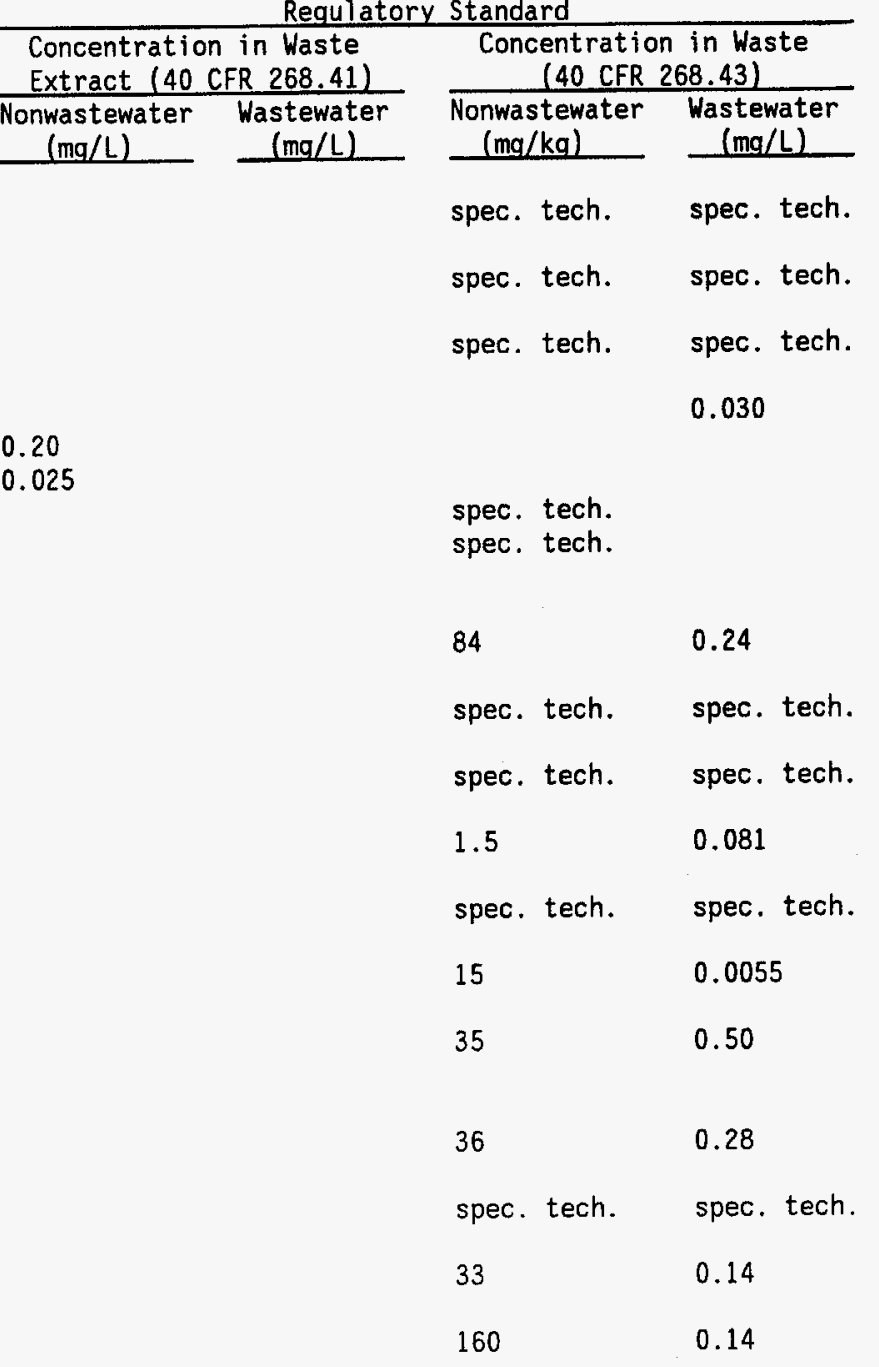


TABLE 3.10. (contd)

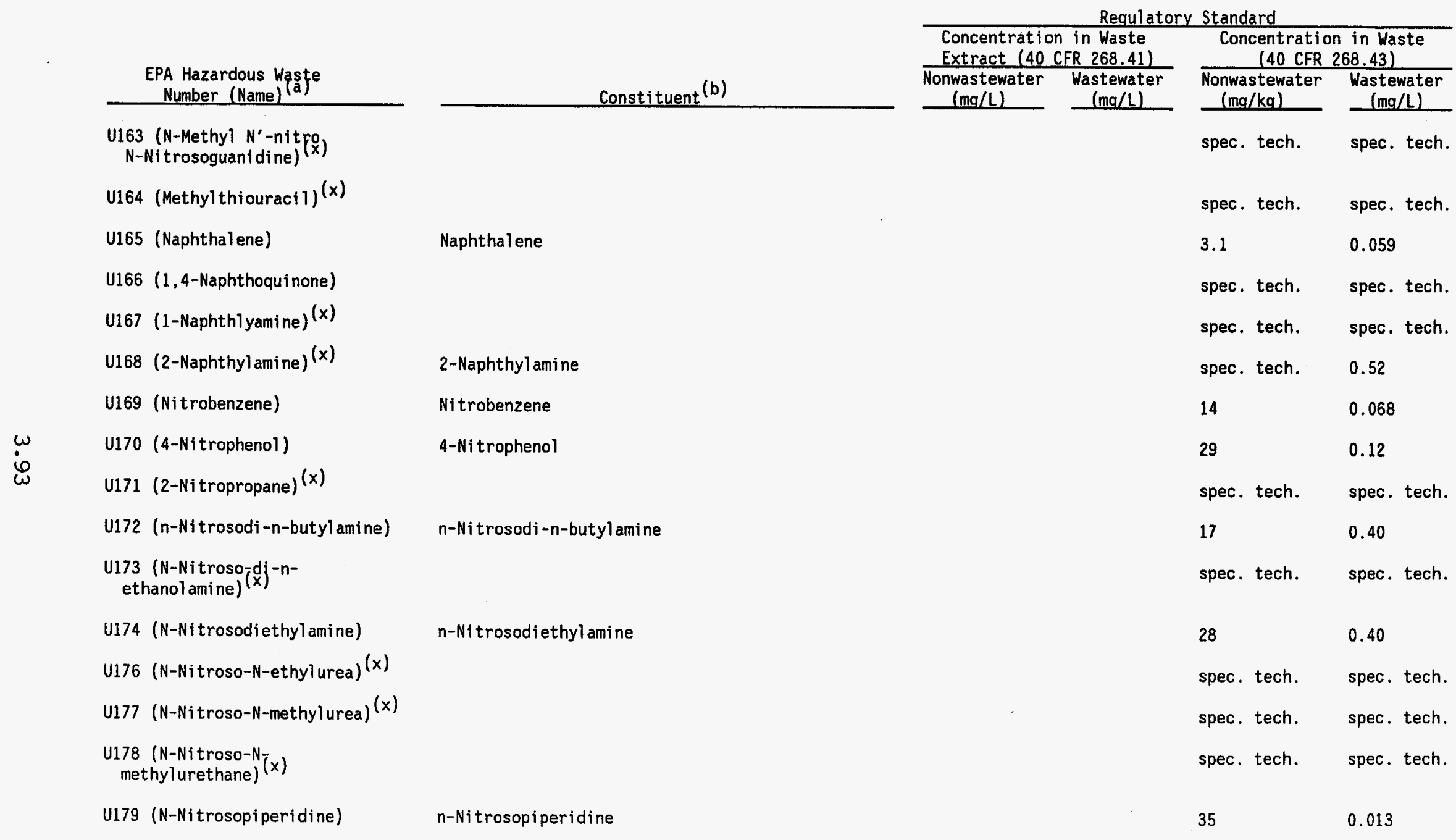




\section{TABLE 3.10. (contd)}

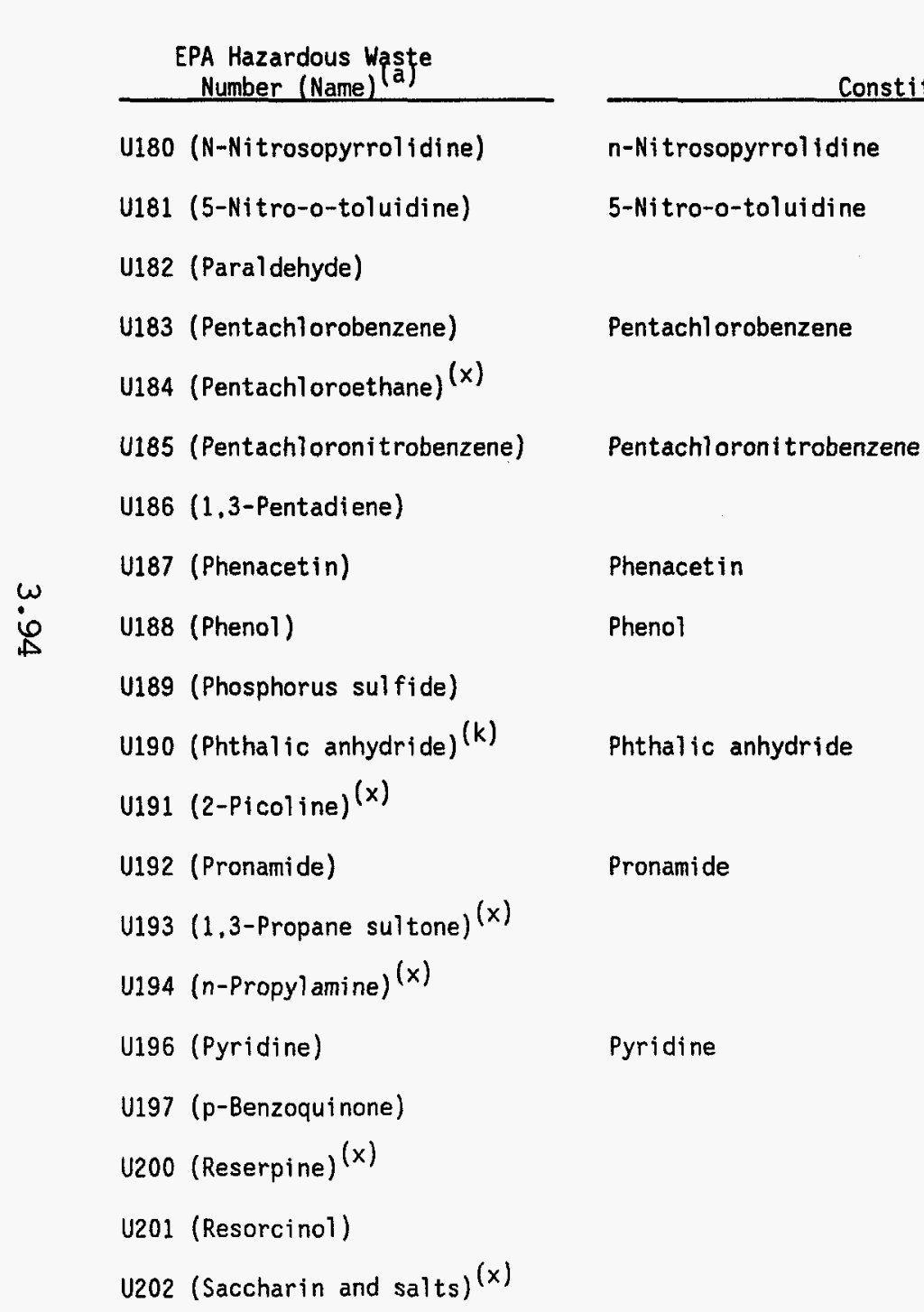

Requlatory Standard

\begin{tabular}{|c|c|}
\hline $\begin{array}{l}\text { Concentration in Waste } \\
\text { Extract }(40 \text { CFR } 268.41)\end{array}$ & $\begin{array}{c}\text { Concentration in Waste } \\
\text { (40 CFR 268.43) }\end{array}$ \\
\hline $\begin{array}{l}\text { nwastewater } \begin{array}{l}\text { Wastewater } \\
(\mathrm{mg} / \mathrm{L})\end{array} \quad \begin{array}{l}(\mathrm{mg} / \mathrm{L}) \\
\end{array}\end{array}$ & $\begin{array}{c}\text { Nonwastewater Wastewat } \\
(\mathrm{mg} / \mathrm{kg})\end{array}$ \\
\hline
\end{tabular}

onwastewater Wastewater

U180 (N-Nitrosopyrrolidine)

\section{(b)}

35

0.013

28

0.32

spec. tech. spec, tech.

37

0.055

spec. tech. spec. tech.

4.8

0.055

spec. tech.

spec, tech.

16

0.081

6.2

0.039

spec. tech.

spec. tech.

28

0.54

spec. tech. spec, tech.

1.5

0.093

spec. tech. spec. tech.

spec. tech. spec. tech.

16

0.014

spec. tech. spec. tech.

spec, tech. spec. tech.

spec. tech. spec. tech.

spec. tech. spec. tech. 
TABLE 3.10. (contd)

\begin{tabular}{|c|c|}
\hline $\begin{array}{l}\text { EPA Hazardous Waste } \\
\text { Number (Name) }\end{array}$ & Constituent $^{(b)}$ \\
\hline U203 (Safrole) & Safrole \\
\hline U204 (Selenium dioxide) & Selenium \\
\hline U205 (Selenium sulfide) & Selenium \\
\hline \multicolumn{2}{|l|}{ U206 (Streptozatocin) ${ }^{(x)}$} \\
\hline U207 (1,2,4,5-Tetrachlorobenzene) & 1,2,4,5-Tetrachlorobenzene \\
\hline U208 (1,1,1,2-Tetrachloroethane) & 1,1,1,2-Tetrachloroethane \\
\hline U209 $(1,1,2,2$-Tetrachloroethane) & 1,1,2,2-Tetrachloroethane \\
\hline U210 (Tetrachloroethylene) & Tetrachioroethylene \\
\hline U211 (Carbon tetrachloride) & Carbon tetrachloride \\
\hline \multicolumn{2}{|l|}{ U213 (Tetrahydrofuran) } \\
\hline U214 (Tha1 i i um(I) acetate) & Thaitium \\
\hline U215 (Tha17 i um(I)carbonate) & Thallium \\
\hline U216 (Thallium(I)chloride) & Thallium \\
\hline U217 (Thal lium(I)nitrate) & Thall ium \\
\hline \multicolumn{2}{|l|}{ U218 (Thi oacetamide) $(x)$} \\
\hline \multicolumn{2}{|l|}{ U219 (Thiourea) $(x)$} \\
\hline U220 (Toluene) & Toluene \\
\hline \multicolumn{2}{|l|}{ U221 (Toluenediamine) ${ }^{(k)}$} \\
\hline $\begin{array}{l}\text { U222 (o-Toluiding } \\
\text { hydrochloride) }\end{array}$ & \\
\hline
\end{tabular}

Regulatory Standard

\begin{tabular}{|c|c|c|c|}
\hline \multicolumn{2}{|c|}{$\begin{array}{l}\text { Concentration in Waste } \\
\text { Extract (40 CFR 268.41) }\end{array}$} & \multicolumn{2}{|c|}{$\begin{array}{c}\text { Concentration in Waste } \\
(40 \text { CFR } 268.43)\end{array}$} \\
\hline $\begin{array}{c}\text { onwastewater } \\
\text { (mg/L) }\end{array}$ & $\begin{array}{l}\text { Wastewater } \\
(\mathrm{mg} / \mathrm{L})\end{array}$ & $\begin{array}{l}\text { Ionwastewater } \\
(\mathrm{mg} / \mathrm{kg})\end{array}$ & $\begin{array}{r}\text { Waste } \\
\text { (mg }\end{array}$ \\
\hline
\end{tabular}

$22 \quad \begin{aligned} & 0.081 \\ & 1.0 \\ & 1.0\end{aligned}$

spec. tech. spec. tech.

$19 \quad 0.055$

$42 \quad 0.057$

$42 \quad 0.057$

$5.6 \quad 0.056$

$5.6 \quad 0.057$

spec. tech. spec. tech.

spec. tech. 0.14

spec. tech. 0.14

spec. tech. 0.14

spec. tech. 0.14

spec. tech. spec. tech.

spec. tech. spec. tech.

$28 \quad 0.080$

spec. tech. spec. tech.

spec. tech. spec, tech. 
IABLE 3.10. (contd)

\begin{tabular}{|c|c|c|c|c|c|}
\hline \multirow[b]{3}{*}{$\begin{array}{l}\text { EPA Hazardous Waste } \\
\text { Number (Name) }\end{array}$} & \multirow[b]{3}{*}{ Constituent $^{(b)}$} & \multicolumn{4}{|c|}{ Regulatory Standard } \\
\hline & & \multicolumn{2}{|c|}{$\begin{array}{l}\text { Concentration in Waste } \\
\text { Extract }(40 \text { CFR 268.41) } \\
\end{array}$} & \multicolumn{2}{|c|}{$\begin{array}{c}\text { Concentration in Waste } \\
\text { (40 CFR 268.43) } \\
\end{array}$} \\
\hline & & $\begin{array}{l}\text { Nonwastewater } \\
(\mathrm{mg} / L) \\
\end{array}$ & $\begin{array}{l}\text { Wastewater } \\
(\mathrm{mg} / \mathrm{L})\end{array}$ & $\begin{array}{l}\text { Nonwastewater } \\
(\mathrm{mg} / \mathrm{kg})\end{array}$ & $\begin{array}{l}\text { Wastewater } \\
(\mathrm{mg} / \mathrm{L}) \\
\end{array}$ \\
\hline U223 (ToTuene di isocyanate) ${ }^{(k)}$ & & & & spec. tech. & spec. tech. \\
\hline U225 (Tribromomethane) & Tribromomethane (Bromoform) & & & 15 & 0.63 \\
\hline U226 (1.1.1-Trichloroethane) & 1,1,1-Trichloroethane & & & 5.6 & 0.054 \\
\hline U227 (1,1,2-Trichloroethane) & 1,1,2-Trichloroethane & & & 5.6 & 0.054 \\
\hline U228 (Trichloroethylene) & Trichloroethylene & & & 5.6 & 0.054 \\
\hline U234 (sym-Trinitrobenzene) $(x)$ & & & & spec. tech. & spec. tech. \\
\hline $\begin{array}{l}\text { U235 (tris- }\left(2 Z^{3-d i b r o m o p r o p y l)}\right. \\
\text { phosphate) }\end{array}$ & tris-(2,3-Dibromopropyl)phosphate & & & 0.1 & 0.025 \\
\hline U236 (Trypan Blue) $(x)$ & & & & spec. tech. & spec. tech. \\
\hline U237 (Uracil mustard) $(x)$ & & & & spec. tech. & spec. tech. \\
\hline U238 (Ethyl carbamate) $(x)$ & & & & spec. tech. & spec. tech. \\
\hline U239 (Xylenes) & Xylenes & & & 28 & 0.32 \\
\hline $\begin{array}{l}\text { U240 }\left(2,4{ }^{4} \text { Dichlorophenoxyacetic }\right. \\
(x)^{-1}\end{array}$ & 2,4-Dichlorophenoxyacetic acid & & & 10 & 0.72 \\
\hline U243 (Hexachloropropene) & Hexachloropropene & & & 28 & 0.035 \\
\hline U244 (Thi ram) $(x)$ & & & & spec. tech. & spec. tech. \\
\hline U246 (Cyanogen bromide) & & & & spec. tech. & spec. tech. \\
\hline U247 (Methoxychlor) & Methoxychior & & & 0.18 & 0.25 \\
\hline U248 (Warfarin $(\geq=3 \%))$ & & & & spec. tech. & spec. tech. \\
\hline U249 (Zinc Phosphide $(<10 \%)$ ) & & & & spec. tech. & spec. tech. \\
\hline $\begin{array}{l}\text { Mixed radipactive/hazardous } \\
\text { wastes }\end{array}$ & & & & $\begin{array}{l}\text { no land } \\
\text { disp. }\end{array}$ & $\begin{array}{l}\text { no land } \\
\text { disp. }\end{array}$ \\
\hline
\end{tabular}


TABLE 3.10. (contd)

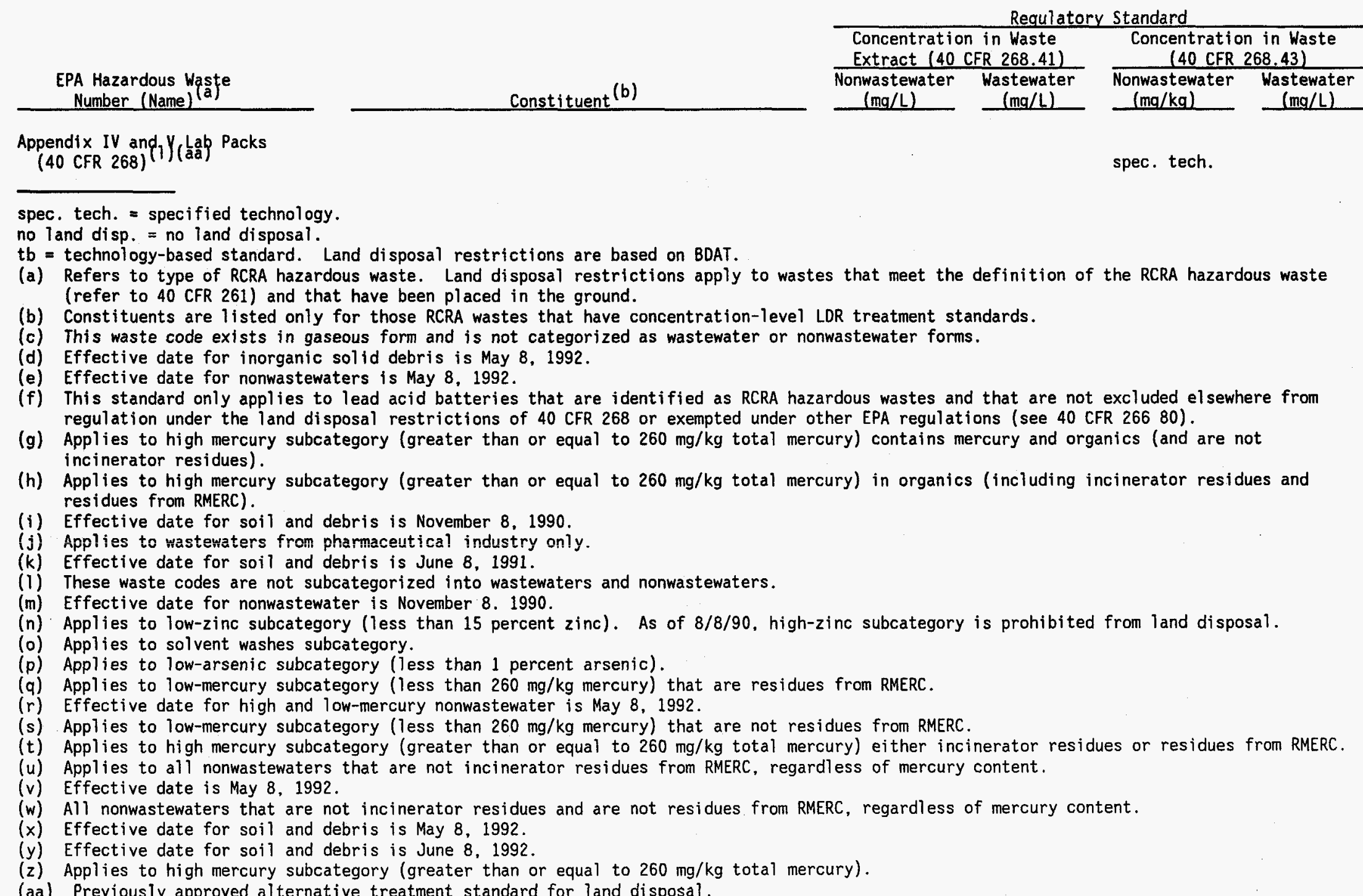


TABLE 3.11. Land Disposal Restrictions for RCRA California List Wastes

Liquid RCRA hazardous waste that contains free cyanides

Liquid RCRA hazardous waste that contains metals

Liquid RCRA hazardous waste that is a corrosive waste

$\omega \quad$ Liquid RCRA hazardous waste

i that contains PCBs in concentration $\geq 50 \mathrm{ppm}$

RCRA hazardous waste that contains halogenated organic compound (HOCs) in the

following subcategories:

\section{Dilute wastewaters}

$(<10,000 \mathrm{mg} / \mathrm{kg})$

Non-dilute wastewaters and non-liquids

RCRA/CERCLA soil and debris

Non-RCRA/CERCLA soil

and debris

\begin{tabular}{|c|c|c|c|}
\hline Constituent & $\begin{array}{c}\text { Prohibition } \\
\text { Level }\end{array}$ & $\begin{array}{l}\text { Type of } \\
\text { Restriction }\end{array}$ & $\begin{array}{l}\text { Effective Date } \\
\text { of Restriction }\end{array}$ \\
\hline Cyanides & $1000 \mathrm{mg} / \mathrm{L}$ & $\begin{array}{l}\text { hard-hammer } \\
\text { restrictions }\end{array}$ & $7 / 8 / 87$ (ns) \\
\hline $\begin{array}{l}\text { Arsenic } \\
\text { Cadmium } \\
\text { Chromium (VI) } \\
\text { Lead } \\
\text { Mercury } \\
\text { Nickel } \\
\text { Selenium } \\
\text { Thallium }\end{array}$ & $\begin{array}{l}500 \mathrm{mg} / \mathrm{L} \\
100 \mathrm{mg} / \mathrm{L} \\
500 \mathrm{mg} / \mathrm{L} \\
500 \mathrm{mg} / \mathrm{L} \\
20 \mathrm{mg} / \mathrm{L} \\
134 \mathrm{mg} / \mathrm{L} \\
100 \mathrm{mg} / \mathrm{L} \\
130 \mathrm{mg} / \mathrm{L}\end{array}$ & $\begin{array}{l}\text { hard-hammer } \\
\text { restrictions }\end{array}$ & $7 / 8 / 87$ (ns) \\
\hline $\mathrm{pH}$ & $\mathrm{pH} \leq 2.0$ & prohibition level & $7 / 8 / 87(t b)$ \\
\hline PCB & $50 \mathrm{ppm}$ & $\begin{array}{l}99.9999 \% \text { destruction } \\
\text { by incineration }\end{array}$ & $7 / 8 / 87(t b)$ \\
\hline \multirow[t]{4}{*}{$\mathrm{HOC}$} & $1000 \mathrm{mg} / \mathrm{kg}$ & $\begin{array}{l}\text { no land disposal } \\
\text { (codified prohibition } \\
\text { level) }\end{array}$ & $7 / 8 / 87(t b)$ \\
\hline & $1000 \mathrm{mg} / \mathrm{kg}$ & $\begin{array}{l}99.99 \% \text { destruction } \\
\text { by incineration }\end{array}$ & $11 / 8 / 88(\mathrm{tb})$ \\
\hline & $1000 \mathrm{mg} / \mathrm{kg}$ & $\begin{array}{l}99.99 \% \text { destruction } \\
\text { by incineration }\end{array}$ & $11 / 8 / 90(\mathrm{tb})$ \\
\hline & $1000 \mathrm{mg} / \mathrm{kg}$ & $\begin{array}{l}99.99 \% \text { destruction } \\
\text { by incineration }\end{array}$ & $11 / 8 / 90(t b)$ \\
\hline
\end{tabular}


health, and secondary standards regulate public welfare. The 1986 amendments mandate the creation of regulatory programs for filtering and disinfecting public water supplies.

On January 30, 1991 the Phase II regulations for 26 synthetic organic chemicals and 7 inorganic chemicals were printed in the Federal Register (56 FR 3526). On June 7, 1991 the national primary drinking water regulations for lead and copper were also printed int he Federal Register (56 FR 26480). Each of these printed texts contained errors which will be corrected by the EPA.

Changes have already been made in 40 CFR parts $141.86,141.91$ and 142.96 and are now effective. Changes in 40 CFR parts 141.80 to 141.85 will become effective on December 2, 1992 (57 FR 28785). On July 17, 1992, the EPA published its final standards for 23 organic and inorganic chemicals. These new standards will establish MCLGs (maximum contaminant level goals) for 18 SOCs (synthetic organic chemicals) and 5 IOCs (inorganic chemicals). The National Primary Drinking Water Regulations (NPDWRs) include monitoring, reporting, public notification requirements, and MCLs for these chemicals (57 FR 31776).

The SDWA is due for reauthorization, but there will be little action in 1992 on comprehensive legislation. EPA's drinking water programs can continue to operate without a reauthorization as long as Congress appropriates funding (ER 1992, 22 (39): 2195).

States may apply to EPA to be granted the primary enforcement responsibility for implementing EPA's regulations that protect drinking water sources. State programs must satisfy five statutory criteria to obtain this responsibility. These criteria include regulations that are no less stringent than EPA's national primary drinking water regulations, adequate provisions for the enforcement of the regulations, recording and reporting procedures that are needed to satisfy EPA regulations, variances and exemptions that are no less stringent than those allowed by SDWA, and plans for providing safe drinking water in emergency conditions. EPA retains the authority to oversee State programs and to take over enforcement if the States fail to do so (Wolf 1988). 
3.7.1 40 CFR part 141 - National Primary Drinking Water Requlations Pursuant to Section 1412 of the Safe Drinking Water Act

Primary drinking water regulations consist of MCLGs and MCLs based on those goals, as defined below.

\section{Definitions}

Maximum Contaminant Level Goal. A MCLG is a level at which "no known or anticipated adverse effect on the health of persons occur and which allows an adequate margin of safety" [SDWA section 1412(b)(1)(B)]. MCLGs are risk-based standards that have only been determined for the contaminants specified in 40 CFR part 141 Subpart F (Table 3.17). MCLGs are nonenforceable health goals.

Maximum Contaminant Level. A MCL is a level set as close to a MCLG as is "feasible with the use of the best technologies, treatment techniques, and other means, which the Administrator finds are generally available (taking costs into consideration)" (SDWA section 1412). Factors include 1) the availability and performance of water treatment technologies; 2) other factors relative to technical feasibility, such as levels of reliable analytical detection; and 3 ) an assessment of the costs of the application of treatment technologies to achieve various concentrations (that would be as close to the MCLG as feasible). The value of the MCL is essentially established by the best generally available technology but is founded on a health-based goal. Thus, it is a combination of a risk-based and technology-based standard.

40 CFR part 141.11 - Maximum Contaminant Levels for Inorganic Chemicals $\langle\mathrm{rb} / \mathrm{tb})$

MCLs for inorganic chemicals are given in Table 3.12.

40 CFR part 141.12 - Maximum Contaminant Levels for Organic Chemicals $(\mathrm{rb} / \mathrm{tb})$

MCLs for organic chemicals are given in Table 3.13.

40 CFR part 141.13 - Maximum Contaminant Levels Turbidity (rb/tb)

MCLs for turbidity are given in Table 3.14 . 
TABLE 3.12. Maximum Contaminant Levels for Inorganic Chemicals

\begin{tabular}{l} 
Contaminant \\
\hline Arsenic \\
Antimony \\
Asbestos \\
Barium \\
Beryllium \\
Cadmium \\
Chromium \\
Cyanide \\
Fluoride \\
Lead \\
Mercury \\
Nickel \\
Nitrate (as N) \\
Nitrite \\
Selenium \\
Silver \\
Sulfate \\
Thallium
\end{tabular}

\begin{tabular}{l} 
Level $(\mathrm{mg} / \mathrm{L})^{(\mathrm{a})}$ \\
\hline 0.05 \\
0.006 \\
7 million ${ }^{(b)}$ \\
fibers/1iter $(\mathrm{b})$ \\
0.004 \\
$0.005(\mathrm{~b})$ \\
$0.1(\mathrm{~b})$ \\
0.2 \\
4.0 \\
0.05 \\
0.002 \\
0.1 \\
10 \\
$1(\mathrm{~b})$ \\
$0.5(\mathrm{~b})$ \\
0.05 \\
Deferred \\
0.002 \\
\end{tabular}

(a) These levels, except those for nitrate, apply only to community water systems. The level for nitrate applies to both community and non-community water systems unless the State allows an exception for a non-community system based on several public health restrictions. With the permission of the State, the nitrate levels must not exceed $20 \mathrm{mg} / \mathrm{L}$.

(b) MCLs are not effective untif July 30, 1992 (56 FR 3526).

\section{CFR part 141.14 - Maximum Microbiological Levels (rb/tb)}

The MCLs for coliform bacteria apply to community and non-community water sources. They are based on the sampling and analytical method used.

\section{Membrane Filter Technique}

If the membrane filter technique is used, the coliform bacteria population shall not exceed any of the following limits:

- an arithmetic mean (of all samples per compliance period) of one per $100 \mathrm{ml}$, unless the State determines that for a variety of reasons one positive sample per month may be excluded from the 
TABLE 3.13. Maximum Contaminant Levels for Organic Chemicals

Contaminant

Chlorinated hydrocarbons:

Endrin

Lindane

Methoxychlor

Toxaphene
Level $(\mathrm{mg} / \mathrm{L})^{(\mathrm{a})}$

0.0002

0.004

0.1

0.005

0.1

0.01

2,4-D

Total Trihalomethanes

(bromodichloromethane,

dibromochloromethane,

tribromomethane, and

trichloromethane)

0.10

Volatile Organic Compounds:

Dichloromethane

0.005

1,2,4-Trichlorobenzene

0.07

1,1,2-Trichloroethane

0.005

Pesticides:

Aldicarb

Aldicarb Sulfoxide

0.003

Aldicarb Sulfone

0.004

Dalapon

0.003

Dinoseb

0.2

Diquat

0.007

Endothall

0.02

Endrin

0.1

Glyphosate

0.002

Oxamyl (Vydate)

0.7

Picloram

0.2

Simazine

0.5

0.004

Other Organic Contaminants:

Benzo(a)pyrene

Di (2-ethylhexy) adipate

0.0002

Di (2-ethyl hexy)phthalate

0.4

Hexachlorobenzene

0.006

0.001

Hexachlorocyclopentadiene

0.001

2,3,7,8-TCDD (Dioxin)

$3 \times 10^{-8}$

(a) The MCLs in this section apply to all community water systems. The MCL for total trihalomethanes is applicable only to community water systems that service 10,000 individuals or more and that add a disinfectant (oxidant) during any part of the drinking water treatment process. 
TABLE 3.14. Maximum Contaminant Levels for Turbidity

Limit $^{(a)}$

5 turbidity units

1 turbidity unit
Measurement type

averaged for 2 consecutive days monthly average ${ }^{(b)}$

(a) Both community and non-community water systems that use surface water sources, in whole or in part, are regulated by these limits.

(b) Five or fewer units may be allowed by the State under certain circumstances.

calculation (this exception is at the primary agency's discretion and applies only to systems required to take 10 or fewer samples per month)

- 4 per $100 \mathrm{ml}$ in more than one sample when less than 20 samples are taken

- 4 per $100 \mathrm{ml}$ in more than $5 \%$ of the samples when 20 or more samples are taken per month.

\section{Fermentation Tube Method}

If the fermentation tube method is used, two different standards exist based on the volume of the sample. When 10-ml sample portions are used, coliform bacteria must not be found in:

- more than $10 \%$ of the portions in any one month, unless the State determines that following a series of checks, one positive sample may be excluded from the calculation (this exception is at the discretion of the State and only applies to those systems required to take 10 or fewer samples per month)

- 3 or more portions in more than one sample when less than 20 samples are collected per month

- 3 or more portions in more than $5 \%$ of the samples when the required number of samples is at least 20 per month.

100-ml Sample Portions

When 100-ml sample portions are used, coliform bacteria must not be found in:

- more than $60 \%$ of the portions in any month, unless the State determines that for a variety of reasons one positive sample may be excluded from the calculation (This exception is at the discretion 
of the State and is only applicable to systems requiring 10 or fewer samples per month)

- 5 portions in more than one sample when less than 5 samples per month are required

- 5 portions in more than $20 \%$ of the samples when at least 5 samples are required per month.

For systems required to take less than 4 samples per month, compliance should be based on a three-month sampling period, except at the discretion of the State, which may maintain the one-month period.

40 CFR part 141.15 - Maximum Contaminant Levels for Radium-226, Radium228, and Gross Alpha Particle Radioactivity in Community Water Systems $(\mathrm{rb} / \mathrm{tb})$

- The MCL for combined radium-226 and radium-228 is $5 \mathrm{pCi} / \mathrm{L}$.

- Gross alpha particle activity excluding radon and uranium but including radium 226 must not exceed $15 \mathrm{pCi} / \mathrm{L}$.

40 CFR part 141.16 - Maximum Contaminant Levels for Beta Particle and Photon Radioactivity from Man-Made Radionuclides in Community Water Systems (rb/tb)

The annual average concentration in drinking water of beta particles and photon radioactivity from man-made radionuclides must not produce an annual dose equivalent greater than $4 \mathrm{mrem} /$ year to the total body or any internal organ. For all radionuclides except those 1 isted in Table 3.15, the concentration causing a 4-mrem dose equivalent is calculated based on a principle of a drinking water intake of 2 L/day and is calculated for the total body or any

TABLE 3.15. Average Annual Concentrations Assumed to Produce a Total Body or Organ Dose of $4 \mathrm{mrem} / \mathrm{yr}$

$\begin{array}{llr}\text { Radionuclide } & \text { Critical Organ } & \frac{\mathrm{pCi} / \mathrm{L}}{20,000} \\ \text { Tritium } & \text { Total Body } & 8 \\ \text { Strontium-90 } & \text { Bone Marrow } & \end{array}$


internal organ. If more than one radionuclide is present, the sum of the annual dose equivalents of all those present must not exceed $4 \mathrm{mrem} /$ year.

40 CFR part 141 Subpart F - Recommended Maximum Contaminant Levels (RMCL) (rb) and 40 CFR part 141 Subpart G - National Revised Primary Drinking Water Regulations: Maximum Contaminant Levels (rb/tb)

MCLs and their respective MCLGs for organic and inorganic compounds are given in Table 3.16 .

40 CFR part 141.52 - MCLGs for Microbiological Contaminants

MCLGs for microbiological contaminants are given in Table 3.17 .

40 CFR part 141.63 - MCLs for Microbiological Contaminants

The MCLs for microbiological and biological contaminants are based on the presence or absence of total coliform bacteria in a sample rather than on the density of total coliform bacteria in a sample (Table 3.18).

In addition, the MCL for total coliform bacteria is violated when any fecal coliform or $E$. coli repeat sample or any total coliform repeat sample following a routine sample is positive.

\section{CFR part 141 Subpart $H$ - Filtration and Disinfection}

Effective 12-31-90, this subpart established treatment techniques in lieu of maximum contaminant levels for

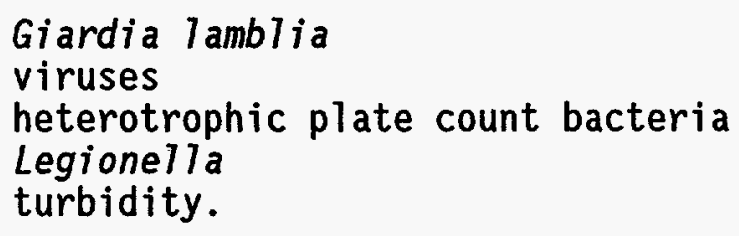

These technologies are required to protect against the potential adverse health effects of exposure to pathogenic organisms.

\subsubsection{CFR part 143 - National Secondary Drinking Water Regulations Pursuant to Section 1412 of the Safe Drinking Water Act}

Secondary drinking water standards given in Table 3.19 are intended to protect public welfare by regulating the aesthetic qualities of water in 
TABLE 3.16. Maximum Contaminant Levels and Goals

\begin{tabular}{|c|c|c|}
\hline Contaminant & $\begin{array}{c}M C L(\mathrm{mg} / \mathrm{L}) \\
(\mathrm{rb} / \mathrm{tb})\end{array}$ & $\begin{array}{l}\text { RMCL or MCL Goal } \\
(\mathrm{mg} / \mathrm{L})(\mathrm{rb}) \\
\end{array}$ \\
\hline $\begin{array}{l}\text { Organic Compounds: } \\
\text { Benzene } \\
\text { Vinyl Chloride } \\
\text { Carbon tetrachloride } \\
\text { 1,2-dichloroethane } \\
\text { Trichloroethylene } \\
\text { 1,1-Dichloroethylene } \\
\text { 1,1,1-Trichloroethane } \\
\text { para-Dichlorobenzene }\end{array}$ & $\begin{array}{l}0.005 \\
0.002 \\
0.005 \\
0.005 \\
0.005 \\
0.007 \\
0.20 \\
0.075\end{array}$ & $\begin{array}{l}0 \\
0 \\
0 \\
0 \\
0 \\
0.007 \\
0.20 \\
0.075\end{array}$ \\
\hline $\begin{array}{l}\text { Volatile Organic Compounds: } \\
\text { Dichloromethane } \\
\text { 1,2,4-Trichlorobenzene } \\
\text { 1,1,2-Trichloroethane } \\
\text { 0-Dichlorobenzene } \\
\text { cis-1,2-Dichloroethylene } \\
\text { trans-1,2-Dichloroethylene } \\
\text { 1,2-Dichloropropane } \\
\text { Ethylbenzene } \\
\text { Monochlorobenzene } \\
\text { Styrene } \\
\text { Tetrachloroethylene } \\
\text { Toluene } \\
\text { Xylenes (total) }\end{array}$ & $\begin{array}{l}0.005 \\
0.07 \\
0.005 \\
0.6 \\
0.07 \\
0.1 \\
0.005 \\
0.7 \\
0.1 \\
0.1 \\
0.005 \\
1 \\
10\end{array}$ & $\begin{array}{l}0^{(a)} \\
0.07^{(a)} \\
0.003^{(a)} \\
0.6 \\
0.07 \\
0.1 \\
0 \\
0.7 \\
0.1 \\
0.1 \\
0 \\
1 \\
10\end{array}$ \\
\hline $\begin{array}{l}\text { Pesticides: } \\
\text { Aldicarb } \\
\text { Aldicarb Sulfoxide } \\
\text { Aldicarb Sulfone } \\
\text { Alachlor } \\
\text { Atrazine } \\
\text { Carbofuran } \\
\text { Chlordane } \\
\text { 1,2-Dibromo-3-chloropropane } \\
\text { (DBCP) }\end{array}$ & $\begin{array}{l}0.003 \\
0.004 \\
0.003 \\
0.002 \\
0.003 \\
0.04 \\
0.002 \\
0.0002\end{array}$ & $\begin{array}{l}0.001^{(b)} \\
0.001^{(b)} \\
0.001^{(b)} \\
0 \\
0.003 \\
0.04 \\
0 \\
0\end{array}$ \\
\hline $\begin{array}{l}2,4-D \\
\text { Dalapon } \\
\text { Dinoseb } \\
\text { Diquat } \\
\text { Endothall } \\
\text { Endrin } \\
\text { Ethylene dibromide (EDB) } \\
\text { Glyphosate } \\
\text { Heptachlor } \\
\text { Heptachlor epoxide }\end{array}$ & $\begin{array}{l}0.071 \\
0.2 \\
0.007 \\
0.02 \\
0.1 \\
0.002 \\
0.00005 \\
0.7 \\
0.0004 \\
0.0002\end{array}$ & $\begin{array}{l}0.07^{a)} \\
0.2^{a)} \\
0.007^{(a)} \\
0.02^{(a)} \\
0.1^{(a)} \\
0.002^{(a)} \\
0 \\
0.7^{(a)} \\
0 \\
0\end{array}$ \\
\hline
\end{tabular}


IABLE 3.16. (contd)

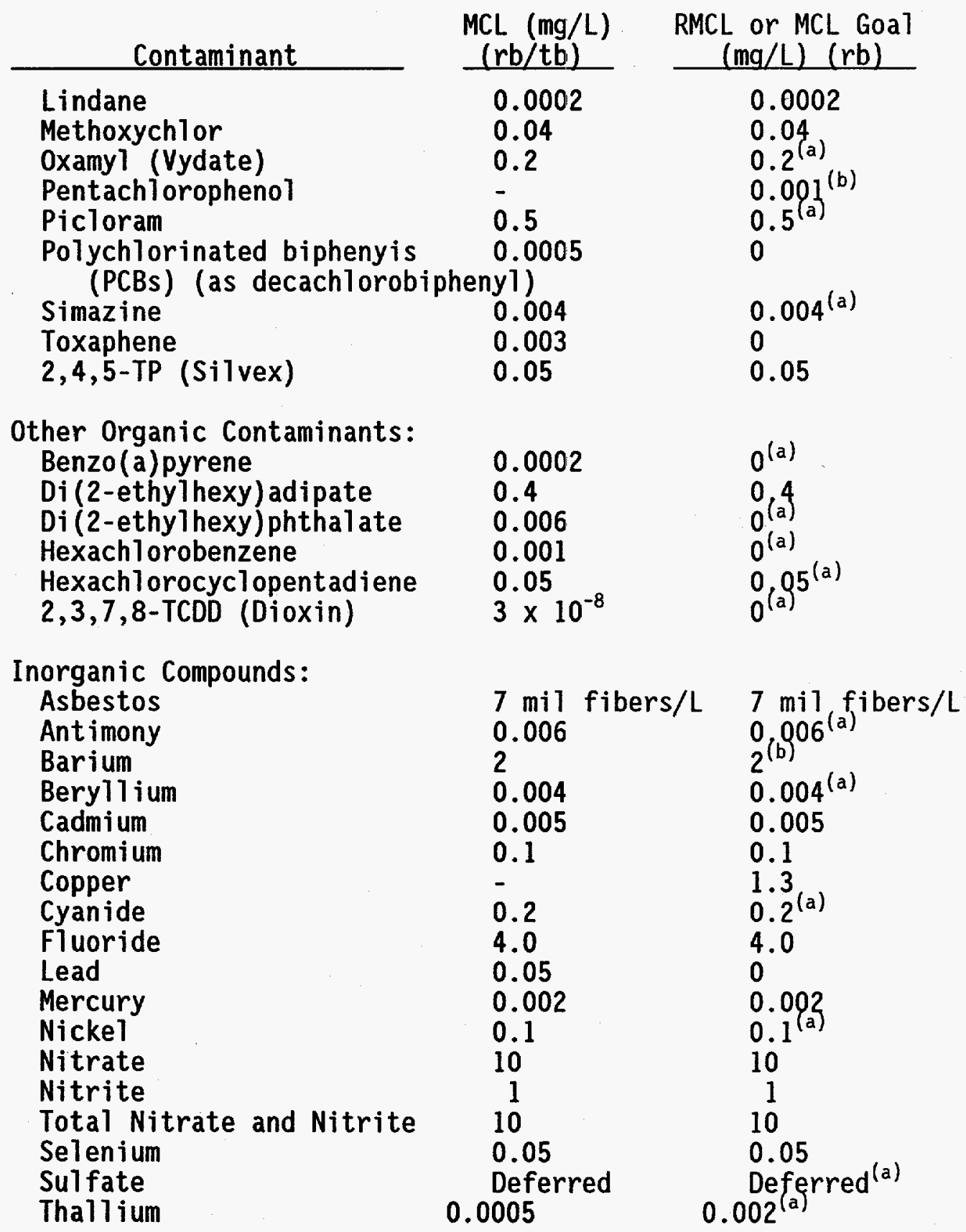

(a) MCLGs not effective until January 17, 1994 (57 FR 31776).

(b) MCLGs not effective until January 1, 1993 (56 FR 30266).

(c) MCLs and MCLGs are not effective unti1 July 30, 1992 (56 FR 3536). 
TABLE 3.17. MCLGs for Microbiological Contaminants ${ }^{(a)}$

\begin{tabular}{lc}
\multicolumn{1}{c}{ Contaminant } & MCLG \\
\hline Giardia lamblia & 0 \\
Viruses & 0 \\
Legionella & 0 \\
Total coliforms (including & \\
fecal coliforms and & \\
Escherichia coli & 0
\end{tabular}

(a) Effective 12-31-90.

TABLE 3.18. MCLs for Microbiological Contaminants ${ }^{(a)}$

Number of Samples

Collected Per Month

$\geq 40$ samples

$<40$ samples
Maximum Positive Results for Compliance

$5.0 \%$ of samples

1 positive sample

(a) Effective 12-31-90.

TABLE 3.19. Secondary Drinking Water Standards

Contaminant

Level (welfare-based)

Aluminum
Chloride
Color
Copper
Corrosivity
Fluoride
Foaming agents
Iron
Manganese
Odor
pH
Silver
Sulfate
Total Dissolved
Solids (TDS)
Zinc

0.05 to $0.2 \mathrm{mg} / \mathrm{L}^{(\mathrm{a})}$

$250 \mathrm{mg} / \mathrm{L}$

15 color units

$1 \mathrm{mg} / \mathrm{L}$

noncorrosive

$2.0 \mathrm{mg} / \mathrm{L}$

$0.5 \mathrm{mg} / \mathrm{L}$

$0.3 \mathrm{mg} / \mathrm{L}$

$0.05 \mathrm{mg} / \mathrm{L}$

3 threshold odor numbers

$6.5-8.5$

$0.1 \mathrm{mg} / \mathrm{L}^{(\mathrm{a})}$

$250 \mathrm{mg} / \mathrm{L}$

$500 \mathrm{mg} / \mathrm{L}$

$5 \mathrm{mg} / \mathrm{L}$

(a) Secondary drinking water standards are not effective until July 30, 1992) (56 FR 3526). 
community and non-community public systems. They are defined in terms of secondary maximum contaminant levels (SMCLs), which are the levels necessary to protect public welfare (welfare-based).

\subsection{STANDARDS UNDER THE TOXIC SUBSTANCES CONTROL ACT}

The Toxic Substances Control Act (TSCA) is intended to regulate chemical substances that present a hazard to human health or the environment. First enacted in 1976, TSCA greatly expanded Federal control of chemicals. The purpose of the Act is to control chemical hazardous at the source before they are released into society or the environment. The broad definition of a chemical substance means that the provisions for testing, premarket notification, and record keeping apply to pure chemical substances, impurities of materials, incidental reaction products, contaminants, co-products, and trace materials. Chemical mixtures are not included in the definition and are thus not subject to TSCA requirements.

TSCA preempts State laws in two specific cases. If EPA requires a chemical to be tested, states may not impose a similar testing requirement. Also, State laws are preempted when EPA has rules that involve the control of hazardous substances, except for disposal, or that affect manufacturers of hazardous substances. This preemption does not apply, however, when the State law is identical to EPA's, is promulgated under another Federal 7 aw, or prohibits the use of the substance in question in the State. EPA may exempt a state from Federal preemption if the State requirement provides a substantially higher degree of protection than the Federal requirement; does not place undue burden on interstate commerce; and does not cause the manufacturer, processor, distributor, or user of the chemical to violate Federal law (Wolf 1988).

On July 12, 1989, EPA issued a final rule under section 6 of TSCA which prohibited, at certain intervals, the future manufacture, importation, processing, and distribution in commerce of all asbestos-containing products, and required labeling of such products (15 USC 2605). On October 18, 1991, the 
United States Court of Appeals for the Fifth Circuit in Corrosion Proof Fittings vs. EPA, CA 5, No. 89-4596, 10/18/91, vacated and remanded most of the rule (57 FR 11364).

Because TSCA achieves its objectives through testing, reporting, and record keeping requirements, the only regulation under TSCA that applies to DOE facilities deals specifically with PCBs.

\subsubsection{Polychlorinated Biphenyls (PCBs) Manufacturing, Processing, Distribution in Commerce, and Use Prohibitions (40 CFR part 761) \\ Definitions}

High-Concentration PCBs. PCBs that contain $500 \mathrm{ppm}$ or greater PCBs, or those substances assumed to contain $500 \mathrm{ppm}$ or greater PCBs in the absence of testing.

High-Contact Surface. A surface that is repeatedly touched, often for relatively long periods of time.

Impervious Solid Surfaces. Solid surfaces that are nonporous and thus are unlikely to absorb spilled PCBs within the short period of time required for cleanup of spills in this policy.

Low-Concentration PCBs. PCBs that are tested and found to contain less than $500 \mathrm{ppm}$ PCBs, or those $\mathrm{PCB}$-containing materials that EPA requires to be assumed to be at concentrations below $500 \mathrm{ppm}$ (i.e., untested mineral oil dielectric fluid).

Nonimpervious Solid Surfaces. Solid surfaces that are porous and are more likely to absorb spilled PCBs prior to completion of the cleanup requirements prescribed in this policy.

$\underline{P C B}$. Any chemical substance that is limited to the biphenyl molecule that has been chlorinated to varying degrees, or any combination that contains such a substance.

Basis for Requirements

The manufacturing, processing, and distribution in commerce of PCBs at concentrations of $50 \mathrm{ppm}$ or greater present an unreasonable risk of injury to 
health. This finding is based on the well-documented human health and environmental hazard of PCB exposure, the high probability of human and environmental exposure to PCBs, the potential hazard of PCB exposure posed by the transportation of PCBs, and the evidence that contamination of the environment by PCBs has spread beyond the areas where they were used.

The cancer potency for PCBs has been estimated by EPA's Cancer Assessment Group and the Office of Toxic Substances. The estimate is based on dermal and inhalation exposure and on possible routes of exposure. "EPA has generally taken an environmentally conservative approach by establishing cleanup requirements based on risk and exposure considerations, and by excluding certain potentially higher-risk spill scenarios from the scope of the policy" (52 FR 10688).

\section{Containment}

With specific listed exceptions, no one may use any PCB in any concentration in other than a totally enclosed manner within the United States unless authorized by the EPA. The exceptions include items that are not included in the definitions of PCB products.

\section{Concentrations Higher Than $50 \mathrm{ppm}$}

PCBs at concentrations of $50 \mathrm{ppm}$ or greater may be processed and distributed in commerce when in compliance with the requirements for disposal in 40 CFR part 761. PCBs at concentrations of $50 \mathrm{ppm}$ or greater that were sold before July 1, 1979 (for purposes other than resale) may be distributed in commerce only in a totally enclosed manner after that date. Any materials contaminated with PCBs because of proximity to or spills from PCB items of a greater than $50 \mathrm{ppm}$ concentration must be decontaminated in accordance with EPA spill cleanup policies before being used or distributed in commerce.

\section{Concentrations Less Than $50 \mathrm{ppm}$}

PCBs at concentrations of less than $50 \mathrm{ppm}$ may be processed and distributed for purposes of disposal without restriction. They may also be exported for disposal. 


\section{Waste $0 \mathrm{il}$}

The use of waste oil that contains any detectable concentration of PCB as a sealant, coating, or dust control agent is prohibited.

\section{Procedural Requirements}

Procedural requirements are defined for storage, monitoring, labeling, and disposal. Operational and design specifications are listed for incinerators used for disposal of PCBS. Approval for the use of an incinerator is granted by the EPA.

Procedures are established for both liquid and non-liquid PCB wastes. Mass air emissions from an incinerator for disposal of non-liquid PCB wastes can be no greater than $0.001 \mathrm{~g} \mathrm{PCB} / \mathrm{kg}$ of the $\mathrm{PCB}$ introduced into the incinerator.

EPA will determine whether or not there is a need for a trial burn before the actual disposal burn.

$\underline{\text { Spills }}$

Requirements for the cleanup of PCB spills vary, based on the size and type of spill. To clean up low-concentration spills involving less than $1 \mathrm{lb}$ of PCBs by weight (less than 270 gal of untested mineral oil), solid surfaces must be double washed/rinsed. All indoor, residential surfaces other than vault areas must be cleaned to $10 \mu \mathrm{g} / 100 \mathrm{~cm}^{2}$ by standard commercial wipe tests. The cleanup of high-concentration spills and low-concentration spills that involve $1 \mathrm{lb}$ or more PCBs by weight (270 gal or more of untested mineral 0i1), depends also on the location of the spill. Requirements for spills in outdoor electrical substations are given in Table 3.20. Table 3.21 gives cleanup standards for spills in restricted areas other than outdoor electrical substations. Spills in nonrestricted access areas are regulated by the limits given in Table 3.22 .

\subsection{STANDARDS UNDER THE OCCUPATIONAL SAFETY AND HEALTH ACT}

The Occupational Safety and Health Act (OSHA) was passed by Congress in 1970. OSHA's specific goal is to maintain a safe and healthy environment 
TABLE 3.20. Requirements for Decontaminating PCB Spi11s in Outdoor Electrical Substations

\begin{tabular}{lll}
\multicolumn{1}{c}{ Medium } & \multicolumn{1}{c}{ Surface } & Maximum Concentration \\
\cline { 3 - 4 } Solid surfaces & Impervious and non-impervious & $100 \mu \mathrm{g} / 100 \mathrm{~cm}^{2}$ \\
Soil & $25 \mathrm{ppm}$ PCBs by weight \\
& $\begin{array}{l}50 \mathrm{ppm} \text { PCB by weight } \\
\text { with label or notice }\end{array}$
\end{tabular}

TABLE 3.21. Requirements for Decontaminating PCB Spills in Other Restricted Access Areas Other Than Outdoor Electrical Substations

\begin{tabular}{|c|c|c|}
\hline$\frac{\text { Medium }}{\text { Solid surfaces }}$ & Surface & Maximum Concentration \\
\hline \multirow[t]{4}{*}{ Solid surfaces } & High-contact & $10 \mu \mathrm{g} / 100 \mathrm{~cm}^{2}$ \\
\hline & $\begin{array}{l}\text { Low-contact, indoor, } \\
\text { impervious }\end{array}$ & $10 \mu \mathrm{g} / 100 \mathrm{~cm}^{2}$ \\
\hline & $\begin{array}{l}\text { Low-contact, indoor, } \\
\text { non-impervious }\end{array}$ & $\begin{array}{l}10 \mu \mathrm{g} / 100 \mathrm{~cm}^{2} \\
- \text { or }- \\
100 \mu \mathrm{g} / 100 \mathrm{~cm}^{2} \\
\text { and encapsulated }\end{array}$ \\
\hline & $\begin{array}{l}\text { Low-contact, outdoor, } \\
\text { impervious and } \\
\text { non-impervious }\end{array}$ & $100 \mu \mathrm{g} / 100 \mathrm{~cm}^{2}$ \\
\hline Soil & & $25 \mathrm{ppm}$ PCBs by weight \\
\hline
\end{tabular}

in the American workplace. Under the Act, the Occupational Safety and Health Administration was created within the Department of Labor. The National Institute of Occupational Safety and Health (NIOSH) was established by the Act as an agency of the Department of Health and Human Services (HHS). The purpose of NIOSH is to give technical assistance and to make recommendations on OSHA standards.

Two standing advisory committees were also formed to address special areas of concern to OSHA: 
TABLE 3.22. Requirements for Decontaminating PCB Spills in Nonrestricted Access Areas

\begin{tabular}{|c|c|c|}
\hline Medium & Surface & Maximum Concentration \\
\hline \multirow[t]{3}{*}{ Solid surfaces } & $\begin{array}{l}\text { Indoor \& high-contact } \\
\text { outdoor }\end{array}$ & $10 \mu \mathrm{g} / 100 \mathrm{~cm}^{2}$ \\
\hline & $\begin{array}{l}\text { Indoor vault areas \& } \\
\text { low-contact, outdoor, } \\
\text { impervious }\end{array}$ & $10 \mu \mathrm{g} / 100 \mathrm{~cm}^{2}$ \\
\hline & $\begin{array}{l}\text { Low-contact, outdoor, } \\
\text { nonimpervious }\end{array}$ & $\begin{array}{l}10 \mu \mathrm{g} / 100 \mathrm{~cm}^{2} \\
- \text { or }- \\
100 \mu \mathrm{g} / 100 \mathrm{~cm}^{2} \\
\text { and encapsulated }\end{array}$ \\
\hline Soil & $\begin{array}{l}\text { Excavated to minimum } \\
\text { depth of } 10 \mathrm{in.}\end{array}$ & $\begin{array}{l}10 \mathrm{ppm} \text { PCBs by weight } \\
\text { (clean soil replacement } \\
\text { to contain >l ppm PCBs) }\end{array}$ \\
\hline
\end{tabular}

- National Advisory Committee on Occupational Safety and Health (NACOSH) - makes recommendations and consults with the Secretary of HHS, and the Secretary of Labor on matters relating to the administration of the Act.

- Advisory Committee on Construction Safety and Health - advises

the Secretary of Labor on formulation of construction safety and health standards and other regulations.

OSHA's regulations can be found in Title 29 of the CFR, parts 1900-1999. When other Federal agencies adopt conflicting occupational safety and health regulations for the workplace, OSHA does not apply. Sections 3(5) and 4(b)(1) of the Act exclude Federal employees from the specific standards and protection of the Act. Although omitted from the general provisions of the Act, Section 19(a) of the OSHA places responsibility on the head of each Federal agency to "establish and maintain an effective and comprehensive occupational safety and health program which is consistent with standards promulgated under Section 6 of the Act..." This responsibility was further emphasized by Executive Order 12196, Section 1-2, February 26, 1980. It requires agencies to implement workplace safety and health standards similar to those required by OSHA. 
The Comprehensive Occupational Safety and Health Reform Act (H.R.3160 and S.1622) was submitted on August 1, 1991, to both the House of Representatives and the Senate. One key point of this legislation is to provide for comprehensive OSHA coverage to all Federal, State, and local employees, and employees working in Federal nuclear facilities under the jurisdiction of the DOE. The Senate Labor Committee referred the bill to the Department of Labor for comment on August 15, 1991; no response had been issued as of Apri1 1, 1992.

Orders DOE 5480.4 and 5483.1A require DOE employees and DOE contractors at government-owned, contractor-operated (GOCO) facilities to comply with OSHA regulations. OSHA's Hazardous Waste Operations and Emergency Response Standard (29 CFR part 1910.120) addresses many specific training requirements for workers. On March 6, 1989, the Department of Labor through its Occupational Safety and Health Administration, published its Hazardous Waste Operations and Emergency Response final rule. The scope of the rule is very broad, covering four major areas/specific worker populations. These areas include (1) cleanup operations at uncontrolled hazardous waste disposal sites that have been identified for cleanup by an environmental health or governmental agency, (2) routine operations at hazardous waste treatment, storage and disposal facilities or those portions of any facility regulated by 40 CFR parts 264 and 265, (3) emergency response operations at sites where hazardous substances have been or may by released, and (4) corrective actions at RCRA sites. The OSHA regulations at 29 CFR part 1910.120 set forth standards that employers must meet for the protection of employees involved in hazardous waste operations.

\subsubsection{CFR part 1910.120 -Hazardous Waste Operations and Emergency Response} Sections $1910.120(e)$ and (p)

These sections establish training requirements for all employees who may be exposed to health or safety hazards while working at environmental restoration sites and RCRA treatment, storage, and disposal (TSD) facilities. DOE also has an obligation to ensure that a11 non-DOE employees/contractors who must enter a DOE environmental restoration site or RCRA TSD facility do so safely. OSHA regulations require that all new employees (those hired after 
March 6, 1989, the effective date of the rule) receive the 24-hour initial training. However, section $1910.120(p)(7)(i i)$ states that employers who can demonstrate that existing employees have had the initial 24-hour training need not require the training again for those employees.

Section $1910.120(c)$

This section requires that environmental restoration sites be characterized to identify potential hazards so that the appropriate training requirements can be determined. An occupational safety and health site evaluation should be performed at all environmental restoration sites, even if actual cleanup is not to begin for several years. Information from this evaluation should be used to determine appropriate site controls, personal protective equipment (PPE), and training requirements for site entry until cleanup work begins.

PPE should be selected based on a number of factors, such as types of site hazards, performance of PPE materials in providing a barrier to these hazards, work activities, task-specific site conditions, and route of potential exposure to hazards (inhalation, skin absorption, ingestion, and eye or skin contact). Appendix B of Section 1910.120 divides PPE into four categories based on the degree of protection:

Level Protection Required

A Respiratory protection, positive pressure self-contained breathing apparatus (SCBA), disposable protective suit

B Respiratory protection, positive pressure SCBA respiratory protection, air purifying respirators

C Respiratory protection, minimal protection (coveral1, boots, safety glasses, etc.)

\section{Site Work Zones}

When cleanup operations begin, site work zones will be established based on information from the site evaluation. Site work zones usually include an exclusion zone, a contamination reduction zone, and a clean zone. 


\section{Exclusion Zone}

An exclusion zone is defined as the contaminated or potentially contaminated area of the environmental restoration site into which entry and exit are regulated at an established check point and within which appropriate levels of protective gear are required. There is a potential for exposure to health and safety hazards in this zone. Exclusion zones may require PPE levels $A, B, C$, or $D$, depending on the types of hazards present.

\section{Contamination Reduction Zone}

The decontamination or contamination reduction zone is the area used to decontaminate the clothing and equipment used by employees and serves as a buffer to prevent contamination from entering the clean zone.

\section{Clean Zone}

The clean zone is an area used as the command center to direct operations at the site. This should also be used to show that "the employer can demonstrate that the operation does not involve employee exposure or the reasonable possibility for employee exposure to safety or health hazards, " in accordance with Section 1910.120(a)(1).

An important part of training workers is to develop a written, sitespecific safety and health plan. Refer to Table 3.23 for training requirements. The plan must be discussed in the training program and must include the following:

- task-specific analyses of safety and health risks

- training assignments for employees

- procedures for using PPE

- medical surveillance requirements

- procedures for air sampling and environmental monitoring

- control measures for eliminating or limiting employees' exposure

- decontamination procedures

- plan for responding to emergencies 
TABLE 3.23. OSHA 1910.120 Hazardous Waste Operations Training Requirements (Source: DOE/EH-0227P, DOE 1991)

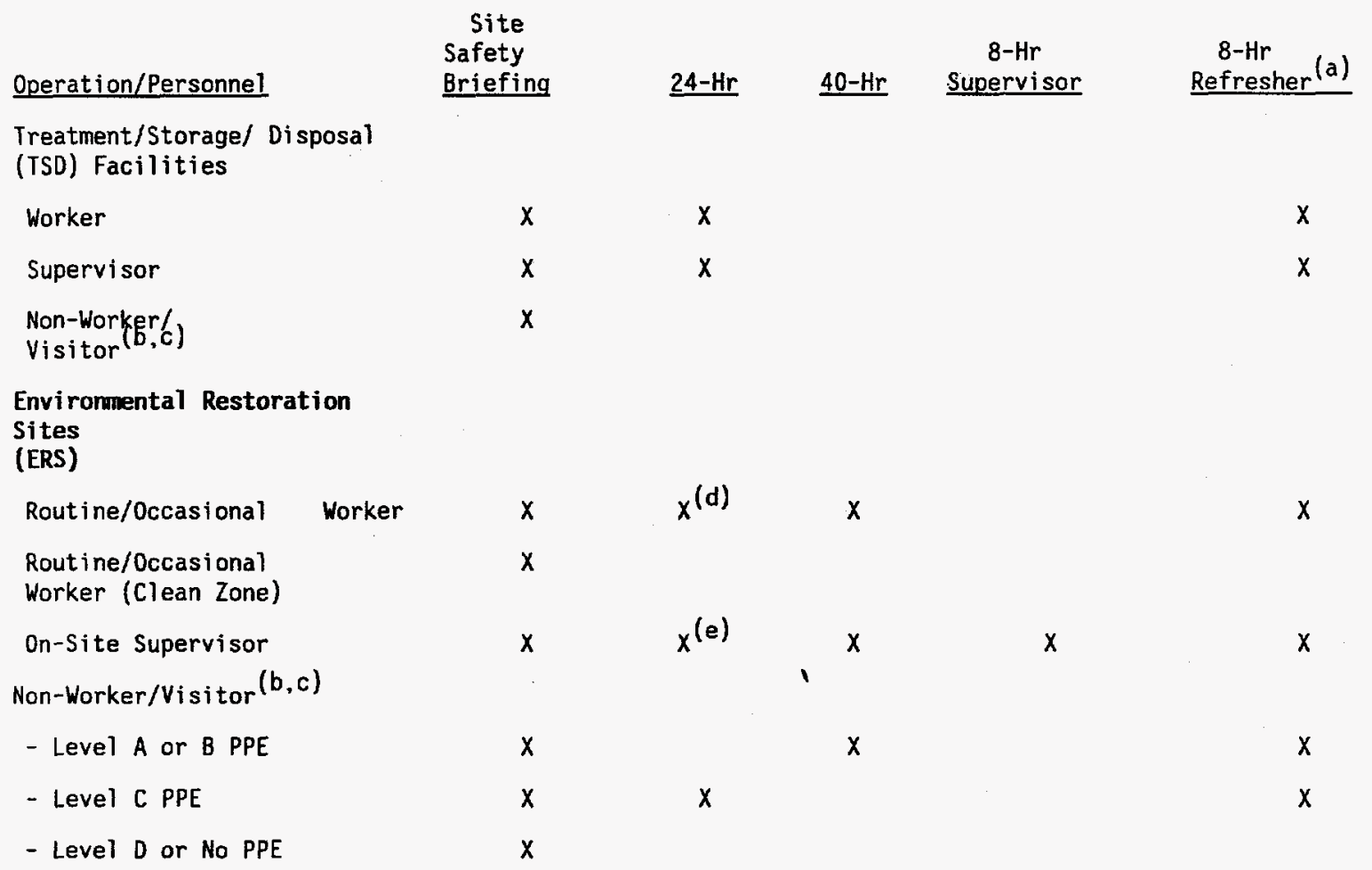

Emergency Response (f)

(a) Annual requirement; however, personnel not receiving refresher training within 3 years of initial training or last refresher course (at a minimum) should repeat the initial course.

(b) All visitors should be issued and instructed on the use of required personal protective equipment (PPE), receive a site-specific safety briefing. and be escorted by trained personnel.

(c) Non-workers are DOE employees and DOE contractors not directly involved with hazardous waste or other TSD/ERS operations (e.g., management, audit, and oversight personnel). Visitors include those covered and not covered by OSHA. Visitors not covered by OSHA who are gained an EH-1 waiver from training requirements should comply with footnote 2 requirements above.

(d) 24-hour training is adequate for these workers only for entry into areas where Level D PPE is sufficient. For routine workers, area must also have been monitored and fully characterized.

(e) Supervisors of ERS on-site workers who require only the 24-hour course need only take the 24-hour initial and 8-hour initial supervisor courses.

(f) Training requirements for emergency response programs are described in OSHA 29 CFR part 1910.120(p)(8) and $(q)$.

- procedures for entering confined spaces

- spil1-containment program

In addition to the components of the safety and health $\mathrm{plan}$, training programs must thoroughly cover the following elements:

- names of personnel responsible for safety and health at the work site 
- work practices that minimize risks form hazards

- safe use of engineering controls and equipment

Employers and their health and safety personnel should become thoroughly familiar with the specific requirements of this regulation. Workers have had the "right to know" about hazardous chemicals in their workplaces since 1983. Called the Hazard Communication Standard (HCS), it has proven to be a powerful standard for workers as long as employers comply. Initially, the standard applied only to manufacturing industries, but OSHA extended HCS to nonmanufacturing industries in August of 1987 . The HCS requires all chemical manufacturers to develop Material Safety Data Sheets (MSDs) for all the hazardous chemicals they produce.

\subsubsection{CFR part 1910.1000 - Air Contaminants -- Permissable Exposure Limits}

These Permissable Exposure Limits protect workers against a variety of health effects which could cause serious material health impairment. The Permissable Exposure Limits were instituted by OSHA using the Recommended Exposure Limits developed by NIOSH and the Threshold Limit Values published by the American Conference of Governmental Industrial Hygienists. Refer to Appendix A for 29 CFR part 1910.1000 Tables Z-1 parts A \& B, Z-2, and Z-3.

\section{CFR part 1910 - Occupational Safety And Health Standards}

Subpart A General

Subpart B Adoption and Extension of Established Federal Standards

Subpart C General Safety and Health Provisions

Subpart D Walking-Working Surfaces

Subpart $E$ Means of Egress

Subpart F Powered Platforms, Manlifts, and Vehicle-Mounted Work Platforms

Subpart G Occupational Health and Environmental Control

Subpart H Hazardous Materials

Subpart I Personal Protective Equipment 
Subpart J General Environmental Controls

Subpart $K \quad$ Medical and First Aid

Subpart $\mathrm{L} \quad$ Fire Protection

Subpart $M \quad$ Compressed Gas and Compressed Air Equipment

Subpart $\mathbf{N} \quad$ Materials Handling and Storage

Subpart $0 \quad$ Machinery and Machine Guarding

Subpart $P \quad$ Hand and Portable Powered Tools and Other Hand-Held Equipment

Subpart $Q \quad$ Welding, Cutting and Brazing

Subpart $R \quad$ Special Industries

Subpart $S \quad$ Electrical

Subpart T Commercial Diving Operations

Subparts U-Y Reserved

29 CFR part 1915 - Occupational Safety And Health Standards For Shipyard Employment

Subpart A General Provisions

Subpart B Explosive and Other Dangerous Atmospheres

Subpart C Surface Preparation and Preservation

Subpart $D \quad$ Welding, Cutting and Heating

Subpart E Scaffolds, Ladders and Other Working Surfaces

Subpart F General Working Conditions

Subpart $G \quad$ Gear and Equipment for Rigging and Materials Handling

Subpart $H \quad$ Tools and Related Equipment

Subpart I Personal Protective Equipment

Subpart J Ship's Machinery and Piping Systems

Subpart K Portable, Unfired Pressure Vessels, Drums and Containers, 
Other Than Ship's Equipment

Subpart L Electrical Machinery

29 CFR part 1918 - Safety And Health Regulations for Longshoring

Subpart A General Provisions

Subpart B Gangways and Gear Certification

Subpart $C \quad$ Means of Access

Subpart D Working Surfaces

Subpart E Opening and Closing Hatches

Subpart F Ship's Cargo Handling Gear

Subpart G Cargo Handling Gear and Equipment Other Than Ship's Gear

Subpart $H \quad H a n d l i n g ~ C a r g o$

Subpart I General Working Conditions

Subpart J Personal Protective Equipment

29 CFR part 1926 - Safety And Health Regulations For Construction

Subpart A General

Subpart B General Interpretations

Subpart C General Safety and Health Provisions

Subpart D Occupational Health and Environmental Controls

Subpart E Personal Protective and Life Saving Equipment

Subpart $F \quad$ Fire Protection and Prevention

Subpart G Signs, Signa7s, and Barricades

Subpart H Materials Handling, Storage, Use, and Disposal

Subpart I Tools - Hand and Power

Subpart $J \quad$ Welding and Cutting

Subpart $K \quad$ Electrical

Subpart L Scaffolding 
Subpart $M$

Floor and Wall Openings

Subpart $N$

Cranes, Derricks, Hoists, Elevators, and Conveyors

Subpart 0

Motor Vehicles, Mechanized Equipment, and Marine Operations

Subpart P

Excavations

Subpart Q

Concrete and Masonry Construction

Subpart R

Steel Erection

Subpart S

Underground Construction, Caissons, Cofferdams and Compressed Air

Subpart $T$ Demolition

Subpart $U \quad B$ Blasting and Use of Explosives

Subpart V Power Transmission and Distribution

Subpart W Rollover Protective Structures; Overhead Protection

Subpart $X \quad$ Stairways and Ladders

29 CFR part 1928 - Occupational Safety And Health Standards For Agriculture

Subpart A General

Subpart B Applicability of Standards

Subpart C Rol1-0ver Protective Standards

Subpart D Safety for Agricultural Equipment

Subparts $\mathrm{E}-\mathrm{H}$ Reserved

Subpart I General Environmental Controls

29 CFR part 1960 - Basic Program Elements For Federal Employee Occupational Safety and Health Programs and Related Matters

Subpart A General

Subpart B Administration

Subpart C Standards

Subpart D Inspection and Abatement 
Subpart E General Services Administration and Other Federal Agencies

Subpart F Occupational Safety and Health Committees

Subpart $G \quad$ Allegations of Reprisal

Subpart $\mathrm{H} \quad$ Training

Subpart I Record keeping and Reporting Requirements

Subpart $J \quad$ Evaluation of Federal Occupational Safety and Health Programs

Subpart K Field Federal Safety and Health Councils

\subsection{STANDARDS IN ORDERS DOE}

DOE establishes its own standards and regulations to supplement those mandated by Federal statutes. In some cases, Orders DOE mandate that the agency should comply with Federal regulations that would otherwise not apply. In other cases, the orders clarify DOE's interpretation of how Federal regulations apply to DOE operations. Each Order DOE deals with a particular aspect of the Department's activities and operations.

This section discusses Orders DOE that apply to hazardous waste remediation, decontamination, and decommissioning activities. Radiation standards for exposed persons are summarized by type of radiation source, and each standard is cross-referenced with the applicable Order DOE. More detailed descriptions of the Orders DOE are then provided.

\subsubsection{Summary of Orders DOE}

Because the terminology used in the Orders DOE can be very specific, the following list of definitions is provided.

\section{Definitions}

Absorbed Dose. The energy imparted to matter by ionizing radiation per unit mass of irradiated material at a specific place in that material.

ALARA (as low as reasonably achievable). As defined for use in order DOE 5400.5, ALARA is the process objective of attaining dose levels as far 
below applicable limits as social, technical, economic, practical, and public policy considerations permit.

Annual Limit on Intake. The quantities of a single radionuclide which, if inhaled or ingested during one year, would irradiate a person to the limiting dose value for control of the workplace.

BAT (Best Available Technology). Selection from among alternative treatment technologies based on evaluation process including factors related to technology, economics, and public policy considerations. At a minimum, the evaluation should include age of equipment and facilities, process employed, engineering aspects of the application of various types of control techniques, process changes, cost of achieving effluent reduction, non-water quality environmental impact, safety considerations, and public policy considerations.

Derived Air Concentration (DAC). Quantity obtained by dividing the annual limit on intake for any given radionuclide by the volume of air breathed by an average worker during a working year.

Derived Concentration Guide (DCG). Concentration of a radionuclide in air or water that, under conditions of continuous exposure for one year by one exposure path, would result in an EDE of $100 \mathrm{mrem}$. DCGs are guides, not release limits; they are screening values for discharges under the BAT process and for making dose estimates.

Dose Equivalent (dose e.). The product of absorbed dose in a tissue times a quality factor. A dose absorbed in a tissue times a quality factor. Dose equivalent allows for the possibility of non-stochastic effects, those effects for which the severity of the effect varies with the dose and for which a threshold may exist.

Effective Dose Equivalent (EDE). The summation of the products of the dose equivalents received by specified organs or tissues of the body and a organ or tissue-specific weighting factor. EDE is a risk-equivalent value that can be used to estimate the health-effects risk of an exposed individual. This measure is based on the possibility of stochastic effects (a malignant or hereditary disease for which the probability of an effect occurring, ragther than its severity, is the critical measure). 
Quality Factor. Quality factors (Table 3.24) are used to calculate dose equivalents from absorbed doses.

Weighting Factor. For a particular tissue or organ, a weighting factor represents the fraction of the total health risk that results from uniform whole-body irradiation. Weighting factors are listed in Table 3.25.

Radiation exposure standards are found in Orders DOE 5480.11, 5400.5, and 5820.2A. A summary of these standards is provided in Table 3.26.

\subsubsection{Order DOE 5400.1 - General Environmental Protection Program (November 9,1988 )}

\section{Purpose}

To establish environmental protection program requirements, authorities, and responsibilities for DOE operations to assure compliance with applicable

TABLE 3.24. Quality Factors by Radiation Type (Order DOE 5480.11)

\begin{tabular}{lc}
\multicolumn{1}{c}{ Radiation Type } & $\begin{array}{c}\text { Quality } \\
\text { Factor }\end{array}$ \\
\hline $\begin{array}{l}\text { X-rays, gamma rays, positrons, } \\
\text { and electrons (including } \\
\text { tritium beta particles) }\end{array}$ & 1 \\
Neutrons, $\leq 10 \mathrm{keV}$ & 3 \\
Neutrons, >10 keV & 10 \\
$\begin{array}{l}\text { Protons and singly charged particles } \\
\text { of unknown energy with rest mass }\end{array}$ & 10 \\
$>$ one atomic mass unit & \\
$\begin{array}{l}\text { Alpha particles and Multiple- } \\
\text { charged particles (and particles } \\
\text { of unknown charge) of unknown } \\
\text { energy }\end{array}$ & 20
\end{tabular}

Note: For neutrons of known energies, more detailed quality factors are given in Order DOE 5480.11. 
TABLE 3.25. Weighting Factors for Tissues and Organs

(Order DOE 5480.11)

$\begin{array}{ll}\text { Gonads } & 0.25 \\ \text { Breasts } & 0.15 \\ \text { Red bone marrow } & 0.12 \\ \text { Lungs } & 0.12 \\ \text { Thyroid } & 0.03 \\ \text { Bone surfaces } & 0.03 \\ \text { Remainder } & 0.30^{(a)}\end{array}$

(a) Includes the other five organs with the highest dose (e.g., liver, kidney, spleen, thymus, adrenal, pancreas, stomach, small intestine, or upper and lower large intestine, but excluding skin, lens of the eye, and extremities). The weighting factor for each of these other five organs is 0.06 .

Federal, State and local environmental protection 1 aws and regulations, Executive Orders, and internal DOE policies.

General Policy

Order DOE 5400.1 states the following regarding environmental protection: "It is DOE policy to conduct its operations in an environmentaliy safe and sound manner. Protection of the environment and the public are responsibilities of paramount importance and concern to DOE. A11 DOE activities should recognize and reflect this concern and public trust. To that end, DOE is firmly committed to ensuring incorporation of national environmental protection goals in the formulation and implementation of DOE programs. It has an equal commitment to advance the goals of restoring and enhancing environmental quality, and ensuring public health."

\subsubsection{Order DOE 5400.5 - Radiation Protection of the Public and the Environment (to Change 1, June 5, 1990)}

\section{Purpose}

To establish standards and requirements for operations of DOE and DOE contractors with respect to protection of members of the public and the environment against undue risk from radiation. 
IABLE 3.26. Summary of Regulations in Orders DOE

Subject

\section{Occupational Workers}

Adult
Adult (lens of the eye)
Adult (any other organ
tissue or extremity)
Planned Special Exposure
Unborn Child
Minors/Students
Public Entering
Controlled Area
Facility Design Limits:
Continuously
Occupied
Non-Continuously
Occupied

Facility Control Limits:

$$
\begin{aligned}
& 0.1 \text { rem } \\
& 5 \text { rem }
\end{aligned}
$$

$0.5 \mathrm{rem}$

$0.1 \mathrm{rem}$

Limit

5 rem

EDE

rb

dose e.

dose e.

EDE

tot. dose .e.

EDE

EDE

dose e.

rb

na

rb

na

rb

5480.11

0.5 mrem

objective

5480.11

1 rem

3 rem

5 rem

50 rem

Emergency Limits:

Exposures

10 rem

25 rem

(Appendix B)

(Appendix C)

Materials and Equipment

Public Dose Limit

Any Member
100 mrem objective

objective

objtv (EDE)

objtv(dose

EDE

dose e.

$\begin{array}{lll}r b & 5480.11 \\ n a & 5480.11 \\ r b & 5480.11 \\ n a & 5480.11\end{array}$

5480.11

5480.11

5480.11

5480.11
DACS

5480.11

5480.11

Basis order DOE

5480.11

5480.11

5480.11

5480.11

5480.11

Volunteers

(Appendix $B)$

na

5480.11

Any Member

(Appendix C)

na

5480.11

EDE

rb $\quad 5400.5$ 
TABLE 3.26. (contd)

Subject

Limit

Type

Basis order DOE

Airborne Emissions

Any Member

$10 \mathrm{mrem}$

EDE

$\mathrm{rb} \quad 5400.5$

Disposal Facilities for Spent Nuclear Fuel, High-level, and Transuranic Wastes

Any

Member

25 mrem

$75 \mathrm{mrem}$

dose e.

na $\quad 5400.5$

dose e.

na $\quad 5400.5$

Drinking Water

Any Member

4 mrem

EDE

rb $\quad 5400.5$

Ra-226+

$\mathrm{Ra}-288$

$5 E-9 \mu \mathrm{Ci} / \mathrm{m} 1$

EDE

rb $\quad 5400.5$

Gross $\alpha$

$1.5 \mathrm{E}-8 \mu \mathrm{Ci} / \mathrm{m}]$

EDE

rb $\quad 5400.5$

Downstream Systems

40 CFR part 141

MCLS

$\mathrm{rb} / \mathrm{tb} \quad 5400.5$

Low-Level Waste Disposal

Any Member

25 mrem

EDE

rb

$5820.2 A$

40 CFR part 61

$5820.2 A$

Inadvertent Intruder

100 mrem

500 mrem

EDE

$r b$

$5820.2 \mathrm{~A}$

EDE

rb $5820.2 A$

Discharges of Liquid Waste

Surface $\mathrm{H}_{2} \mathrm{O}$ w/annual

avg conc rad > DCG

Waterways

$5 \mathrm{pCi} / \mathrm{g}$

above background

$50 \mathrm{pCi} / \mathrm{g}$

above background

BAT

tb $\quad 5400.5$

Native Aquatic

Animal

Organisms

$1 \mathrm{rad}$

absorbed dose

na

5400.5

Sanitary Sewerage

Monthly average > 5XDCG

(Appendix D)

BAT

tb $\quad 5400.5$ 
IABLE 3.26. (contd)

Subject

Limit

Type

Basis Order DOE

Release of Property

Equipment, Materials, and Personal Property

(See Table 3.27)

5400.5

Remediated Properties

40 CFR part 192

5400.5

Residual Radioactivity

Ra-226, Th-230, and Th-232

$5 \mathrm{pCi} / \mathrm{g}$

$15 \mathrm{pCi} / \mathrm{g}$

basic dose

basic dose

na

5400.5

na

5400.5

Airborne Radon:

Annual Average Rn Decay

$0.02 \mathrm{WL}$

$0.03 \mathrm{WL}$

objective

absolute

na

5400.5

na $\quad 5400.5$

Gamma Sources Inside Structures

$20 \mu \mathrm{R} / \mathrm{h}$

above background

basic dose

na $\quad 5400.5$

Air/water

100 mrem

EDE

rb $\quad 5400.5$

Interim Storage Controls $\mathrm{Rn}-222$

$100 \mathrm{pCi} / \mathrm{L}$

conc.

na

5400.5

$30 \mathrm{pCi} / \mathrm{L}$

annual average na

concentration

5400.5

$3 \mathrm{pCi} / \mathrm{L}$

annual average

na

concentration

5400.5

Flux Rate

$20 \mathrm{pCi} / \mathrm{m}^{2}-\mathrm{sec}$

na

5400.5

Long-Term Management

$\mathrm{Rn}-222$

$20 \mathrm{pCi} / \mathrm{m}^{2} / \mathrm{s}$

annual average na release rate

5400.5

Increases

$0.5 \mathrm{pCi} / \mathrm{L}$

annual average na

concentration

5400.5 


\section{DOE Public Dose Limit}

The EDE for members of the public who are exposed to radiation sources as a result of routine DOE activities may not exceed $100 \mathrm{mrem}(1 \mathrm{mSv}$ ) in a year.

DOE operators must report DOE-related EDE contributions of 10 mrem or more in a year.

DOE operators are required to make a reasonable effort to be aware of sources of radiation other than DOE man-made sources (excluding also medical sources, consumer products, residual fallout from past nuclear accidents and weapons test, and naturally occurring radiation sources) which, when combined with the DOE sources, have the potential to exceed contributions of $10 \mathrm{mrem}$ (0.1 mSv) EDE in a year.

Operators must make a reasonable effort to limit the public's EDE from multiple radiation sources to 100 mrem or less in a year.

If avoidance of higher exposures is impracticable, the Operations office of a facility may request specific authorization from EH-1 for a temporary public dose limit higher than $100 \mathrm{mrem}(1 \mathrm{mSv})$, but the 1 imit may not exceed $500 \mathrm{mrem}(5 \mathrm{mSv})$ for the year. The International Commission on Radiological Protection (ICRP) recommends a principal stochastic dose 1 imit of $100 \mathrm{mrem}$ EDE in a year for exposures to the public, and a subsidiary dose limit of $500 \mathrm{mrem}$ $E D E$ in a year, for some years, if the dose averaged over a lifetime does not exceed the principal limit of 100 mrem EDE per year. Specific sources excepted above are also excepted here).

\section{Airborne Emissions}

The EDE for members of the public who are exposed to radioactive materials (including radon-220, radon-222, and their respective decay products) released to the atmosphere as a result of routine DOE activities may not exceed $10 \mathrm{mrem}$ in a year.

The public dose limits above are established by EPA regulation 40 CFR part 61, Subpart $H$, under the Clear Air Act (CAA). Subpart $Q$ and $T$ of 
40 CFR part 61 provide radon flux limits for DOE radium storage and disposal facilities and for DOE inactive uranium mill tailings sites regulated under 40 CFR part 192.

Sources from Management and Storage of Spent Nuclear Fuel, HighLevel, and Transuranic Wastes at Disposal Facilities

Members of the public shall not be exposed to direct radiation or radioactive material released from DOE management and storage activities at a disposal facility for spent nuclear material or for high-level or TRU wastes that are not regulated by the NRC, which would cause them to receive an annual dose equivalent that exceeds 25 mrem to the whole body or a committed dose equivalent that exceeds 75 mrem to any organ.

DOE facilities are in some cases subject to the regulatory requirements of the NRC and the EPA: 10 CFR parts 60 and 72, 40 CFR parts 61,191 , and 192.

\section{Drinking Water Pathway}

Consistent with 40 CFR part 141, drinking water systems supplied by DOE shall not cause persons to receive an EDE greater than $4 \mathrm{mrem} / \mathrm{yr}$. Combined radium-226 and radium-228 shall not exceed $5 \times 10^{-9} \mu \mathrm{Ci} / \mathrm{ml}$ and gross alpha activity (including radium-226, but excluding radon and uranium) shall not exceed $1.5 \times 10^{-8} \mu \mathrm{C} i / \mathrm{ml}$. This dose limit is the EDE for individuals whose exclusive source of drinking water contains radionuclides at a monthly average level of $4 \%$ of the DCG value.

DOE activities shall not cause other drinking water systems downstream of its effluent discharges to exceed the drinking water radiological limits in 40 CFR part 141.

\section{The ALARA Process}

A program for implementing the ALARA process must be developed. Contractors will be required to implement the ALARA process for all DOE activities and facilities that cause public doses.

DOE's primary public dose limit is based on consideration of potential risk or radiation-induced fatal cancers and serious genetic defects. 
The ALARA process includes all factors related to societal, technological, economic, and other public policy considerations at a minimum; factors considered shall include:

- maximum dose to members of the public

- collective dose to the population

- alternative processes

- doses for each process alternative

- costs for each of the technological alternatives

- examination of the changes in cost among alternatives

- changes in societal impact associated with process alternatives.

Qualitative analyses are acceptable because quantifying of the parameters in a cost-benefit analysis is difficult. The bases for such judgments should be documented.

\section{Management and Control of Radioactive Materials}

The management and control radioactive materials differs with the type of material. Gasseous, liquid, and solid wastes are regulated under this part.

Discharges of Liquid Waste to Surface Waters. BAT is the prescribed level of treatment for discharges to surface waters that would otherwise contain at the point of discharge and prior to dilution, radioactive material at annual average concentrations greater than DCG value.

BAT is not required for discharges to surface waters that exhibit radionuclide concentrations below DCG levels. The cost consideration component of the BAT analysis precludes additional treatment in such cases. ALARA provisions are applicable.

Liquid waste that contains radioactive material in the form of settleable solids may be released to natural waterways if the concentration in the waste does not exceed $5 \mathrm{pCi} / \mathrm{g}$ above background level for $\alpha$-emitting radionuclides or $50 \mathrm{pCi} / \mathrm{g}$ above background level of settleable solids for $B$ gamma-emitting radionuclides. 
The absorbed dose to native aquatic animal organisms must not exceed $1 \mathrm{rad} /$ day from exposure to radioactive material discharged in liquid wastes to natural waterways.

Discharges of Liquid Waste to Aquifers and Phaseout of Soil Columns. The BAT selection process must be applied to those liquid waste streams that will continue to be discharged to soil columns. The use of soil columns to retain suspended or dissolved radionuclides from liquid waste streams must be discontinued at the earliest practicable time in favor of an acceptable alternative disposal means. New or increased discharges to active and virgin soil columns are prohibited.

To prevent further spread of previously deposited radionuclides, the discharge of liquids, even when uncontaminated, is prohibited.

Discharges of Liquid Waste to Sanitary Sewerage. If the averaged monthly concentration of radionuclides in liquid waste discharged into sanitary sewerage systems would otherwise be greater than five times the DCG values at the point of discharge, the BAT selection process must be implemented. If the sewerage system is owned by the Federal government the concentrations may be greater than those specified above, except for systems with drain fields. In such cases, ALARA process considerations are required, as is liquid waste treatment prior to discharge to surface water from such a sewerage system.

Release of Property. Real property is subject to the requirements of Section $120(\mathrm{~h})$ of CERCLA and to any other applicable Federal, State, and local requirements. 40 CFR part 192 is applicable to properties remediated by DOE under Title I of the Uranium Mill Tailings Radiation Control Act.

Personal property may be released for unrestricted use if the contamination levels are within the limits in Table 3.27. The concentration limits in Table 3.27 also apply to the release of materials and equipment.

"No guidance is currently available for release of material that has been contaminated in depth such as activated material or smelted contaminated metals" (Order DOE 5400.5). 
TABLE 3.27. Surface Contamination Guidelines (from Order DOE 5400.5)

Radionuclides (b)

Transuranics, I-125, I-129, Ra-226,

Ac-227, $\mathrm{Ra}-228$, Th-228, Th-230, $\mathrm{Pa}-231$.

Th-Natural, Sr-90, I-126, I-131, I-133, Ra-223, Ra-224, U-232, Th-232.

U-Natural, U-235, U-238, and associated decay product, alpha emitters.

Beta-gamma emitters (radionuclides with decay modes other than alpha emission or spontaneous fission) except $\mathrm{Sr}-90$ and others noted above. $(g)$
Allowable Total Residual Surface Contamination $\left(\mathrm{dom} / 100 \mathrm{~cm}^{2}\right)(a)$

Average $^{(c, d)} \quad$ Maximum (d,e) $\quad$ Removable ${ }^{(d, f)}$

Reserved

Reserved

Reserved

1,000

3.000

5,000

15,000

1,000

5,000

15,000

1,000

(a) As used in this table, disintegrations per minute (dpm) means the rate of emission by radioactive material as determined by correcting the counts per minute measured by an appropriated detector for background, efficiency, and geometric factors associated with the instrumentation.

(b) Where surface contamination by both alpha- and beta-gamma-emitting radionuclides exists, the limits established for alpha- and beta-gamma-emitting radionuclides should apply independently.

(c) Measurements of average contamination should not be averaged over an area of more than $1 \mathrm{~m}^{2}$. For objects of less surface area, the average should be derived for each such object.

(d) The average and maximum dose rates associated with surface contamination resulting from beta-gamma emitters should not exceed $0.2 \mathrm{mrad} / \mathrm{h}$ and $1.0 \mathrm{mrad} / \mathrm{h}$, respectively, at $1 \mathrm{~cm}$.

(e) The MCL applies to an area of not more than $100 \mathrm{~cm}^{2}$.

(f) The amount of removable material per $100 \mathrm{~cm}^{2}$ of surface area should be determined by wiping an area of that size with dry filter of soft absorbent paper, applying moderate pressure, and measuring the amount of radioactive material on the wiping with an appropriate instrument of known efficiency. When removable contamination on objects of surface area less than $100 \mathrm{~cm}^{2}$ is determined, the activity per unit area should be based on the actual area and the entire surface should be wiped. It is not necessary to use wiping techniques to measure removable contamination levels if direct scan surveys indicate that the total residual surface contamination levels are within the limits for removable contamination.

(g) This category of radionuclides includes mixed fission products, including the $\mathrm{Sr}-90$ which is present in them. It does not apply to $\mathrm{Sr}-90$ which has been separated from the other fission products or mixtures where the $\mathrm{Sr}-90$ has been enriched.

\section{Guidelines for Residual Radioactive Material. Residual concentrations} of $\mathrm{Ra}-226$, Th-230, and Th-232 should be no more than $5 \mathrm{pCi} / \mathrm{g}$ averaged over the first $15 \mathrm{~cm}$ of soil below the surface and $15 \mathrm{pCi} / \mathrm{g}$ averaged over $15-\mathrm{cm}$-thick layers of soil more than $15 \mathrm{~cm}$ below the surface.

The objective of remedial action for airborne radon decay products shall be an annual average radon decay product concentration that does not exceed 0.02 working level (WL). (A WL is any combination of short-lived radon decay products in $1 \mathrm{~L}$ of air that will result in the emission of $1.3 \times 10^{5} \mathrm{MeV}$ of potential adpha energy.) The concentration shall not exceed $0.03 \mathrm{WL}$ in any case. 
The average level of gamma radiation inside a structure to be released without restrictions shall not exceed the background level by more than $20 \mu \mathrm{R} / \mathrm{h}$. External gamma radiation on open land must comply with the basic limit and the ALARA process.

Residual radionuclides in air and water shall be controlled to the same levels as the public dose limits and as required by applicable Federal and/or State laws.

Interim Storage. Control and stabilization features for interim storage facilities must be designed for an effective life of 50 years with a minimum life of at least 25 years. Controls must be designed so that $\mathrm{Rn}-222$ concentrations will not exceed $100 \mathrm{pCi} / \mathrm{L}$ at any given point, an annual average concentration of $30 \mathrm{pCi} / \mathrm{L}$ over the facility site, or an annual average concentration of $3 \mathrm{pCi} / \mathrm{L}$ at or above any location outside the facility site. Flux rates from the storage of radon-producing wastes must not exceed $20 \mathrm{pCi} / \mathrm{m}^{2}-\mathrm{s}$ (as required by 40 CFR part 61 ).

Concentrations of radionuclides in groundwater and quantities of residual radioactive material must not exceed applicable Federal or State standards.

Access controls must be designed to provide, to the extent reasonable, an effective life of at least 25 years as described in 40 CFR part 192 .

Long Term Management. Control features for long-term management sha11 be designed to provide an effective life of 1000 years, with a minimum life of at least 200 years. Control features shall also be designed to limit Rn-222 emissions from wastes to less than an annual average release rate of 20 $\mathrm{pC} i / \mathrm{m}^{2} / \mathrm{s}$ and to prevent increases in annual average concentration outside the contaminated area by more than $0.5 \mathrm{pCi} / \mathrm{L}$.

Groundwater shall be protected with applicable Federal and State standards.

Access controls should be designed such as those in 40 CFR part 192 and should be effective to the extent reasonable for at least 200 years. 
3.10.4 Order DOE 5480.1B - Environment, Safety, and Health Program for Department of Energy Operations (to change 4, March 27, 1990)

Order DOE 5480.1B establishes the ES\&H program for DOE operations, cancels Order DOE 5480.1A of 8-13-81, and redesignates the chapters of Order DOE $5480.1 A$ to new order numbers.

Individual chapters of Order DOE $5480.1 \mathrm{~A}$ remain in effect until new orders are finalized, even though the order itself has been cancelled.

3.10.5 Order DOE 5480.4 - Environmental Protection, Safety, and Health Protection Standards (to Change 1, Mav 16, 1990)

\section{Purpose}

This order specifies requirements for mandatory environmental protection, safety, and health (ES\&H) standards that are applicable to all DOE and DOE contractor operations.

\section{Employee Safety and Health}

Order DOE 5480.4 and Order DOE 5483.1A are applicable to DOE contractor employee safety and health programs that are not subject to jurisdiction of ihe U.S. Department of Labor, Occupational Safety and Health Administration. (A list of contractors is maintained by the Office of Operational Safety, PE24.) These orders require compliance with OSHA standards in the following regulations: 29 CFR part 1910, 29 CFR part 1915, 29 CFR part 1918, 29 CFR part 1926, 29 CFR part 1928. Those contractors not included on the listing of contractors maintained by the office of Operational Safety are required to comply with 29 CFR part 1905. The Occupational Safety and Health Program for Federal employees is required to comply with 29 CFR part 1960.

Order DOE 5480.1A, Chapter III is applicable to programs that assure the safe packaging of fissile and other radioactive materials. This order requires compliance with transportation and packaging standards, including DOT hazardous materials regulations, 49 CFR parts 100-199. 


\section{Requlations}

Order DOE 5480.4 contains lists of standards that are mandatory for DOE, either as a result of non-DOE Federal or State ES\&H statutes, or as a matter of DOE policy.

\subsubsection{Order DOE 5480.11 - Radiation Protection for Occupational Workers (December 21, 1988)}

\section{General Policy}

Order DOE 5480.11 gives occupational radiation protection standards for DOE and DOE contractors for internal and external exposure of operational workers, unborn children, minors and students; for planned special exposures; and for exposure of members of the public who enter a DOE-controlled area.

\section{Adults}

DOE policy is to maintain radiation exposures within limits of the Order and as far below these limits as is reasonably achievable.

The annual EDE from both internal and external sources received in any year must not exceed 5 rem to the whole body. The annual dose equivalent is not to exceed 15 rem to the lens of the eye, or 50 rem to any other organ, tissue (including skin), or extremity.

\section{Unborn Children}

The total dose equivalent to an unborn child must not exceed 0.5 rem.

\section{Planned Special Exposure}

A planned special exposure is not to exceed 2 times the annual EDE (5 rem) when added to annual occupational dose anticipated for the year. A planned special exposure requires approval.

\section{Minors (including students)}

Individuals under the age of 18 are not allowed in controlled areas where they would be exposed to more than $0.1 \mathrm{rem} / \mathrm{yr}$ from the sum of the committed EDEs from internal irradiation plus the annual EDEs from external irradiation. 


\section{Public Entering Controlled Area}

The EDE from exposure during direct onsite access must not exceed 0.1 rem/yr from internal and external irradiation; the dose equivalent to any tissue (including skin and lens of eye) must not exceed 5 rem per year.

Derived Air Concentrations (DACs) - Appendix B to this report gives the limits for radiation exposures due to inhalation.

Concentrations of radionuclides in drinking water in controlled areas are regulated by standards in 40 CFR part 141 .

\section{Release of Materials and Equipment}

Materials and equipment shall not be released if measurements or prior use suggest that contamination levels exceed the guides specified in Appen$\operatorname{dix} C$ of this report.

Requirements in this order do not apply to induced radioactivity or D\&D release limits.

\section{Design Objectives}

External Radiation. Exposure in continuously occupied areas must be ALARA and must not exceed $0.5 \mathrm{mrem} / \mathrm{hr}$ on average.

Exposure in non-continuously occupied areas must be ALARA and must not exceed $20 \%$ of the EDE of $5 \mathrm{rem} / \mathrm{yr}$ for the whole body or the dose equivalent of $15 \mathrm{rem} / \mathrm{yr}$ to individual organs and tissues.

Internal Radiation. Exposure due to inhalation is to be avoided under normal operating conditions to the extent reasonably achievable.

\section{Emergency Conditions}

The $\mathrm{planned}$ exposure objective is not to exceed $10 \mathrm{rem} / \mathrm{yr}$ for individuals in operations that are essential to reduce potential hazards, to protect health, or to prevent substantial loss of property. The official in charge may allow emergency exposures not to exceed $25 \mathrm{rem} / \mathrm{yr}$ for volunteers. 


\subsubsection{Order DOE 5820.2A - Radioactive Waste Management (September 26, 1988)}

\section{Purpose}

Establishes policies, guidelines, and minimum requirements by which DOE manages its radioactive and mixed waste and contaminated facilities.

\section{High-Level Waste}

Designs for new storage and treatment facilities must meet the requirements of Order DOE 6430.1, applicable EH Orders, and 40 CFR part 264 (Standards for Owners and Operators of Hazardous Waste Treatment, Storage, and Disposal Facilities).

Existing facilities must be reviewed based on the requirements of order DOE 5481.1B

Design-specific standards exist for storage, transfer, and monitoring operations.

Singly-contained pipelines may be used routinely for liquid wastes that have a total radioactivity concentration of less than $0.05 \mathrm{Ci} / \mathrm{gal}$; under certain conditions, these pipelines may be used on a temporary basis for higher activity waste.

Groundwater or vadose zone monitoring wells meeting RCRA requirements in accordance with 40 CFR part 264 shall be installed around clusters of liquid waste storage tanks.

Quality assurance activities shall be consistent with Order DOE 5700.6B. High-level waste operations shall comply with the applicable requirements of the American National Standards Institute/American Society of Mechanical Engineers Nuclear Quality Assurance-1 and other appropriate national consensus standards.

\section{Transuranic Waste (TRU)}

Without regard to source or form, TRU waste is waste that is contaminated with alpha-emitting radionuclides having half-lives exceeding 20 years and in concentrations exceeding $100 \mathrm{nCi} / \mathrm{g}$ at the time of assay. Heads of field elements can determine that other alpha-contaminated wastes, peculiar to a specific site, mush be managed as TRU waste. TRU waste must be stored 
such that radiation exposures are maintained as low as reasonably achievable. While in storage, wastes must be monitored periodically to ensure that they are not releasing their radioactive and/or hazardous constituents.

\section{Low-Leve] Waste}

Disposal must "assure that external exposure to the waste and concentrations of radioactive material which may be released into surface water, ground water, soil, plants and animals results in an EDE that does not exceed $25 \mathrm{mrem} / \mathrm{yr}$ to any member of the public. Releases to the atmosphere shall meet the requirements of 40 CFR part 61 . Reasonable effort should be made to maintain releases of radioactivity in effluents to the general environment as low as is reasonably achievable."

Disposal must "assure that the committed EDEs received by individuals who inadvertently may intrude into the facility after the loss of active institutional control (100 years) will not exceed $100 \mathrm{mrem} / \mathrm{yr}$ for continuous exposure or 500 mrem for a single acute exposure."

Disposal must protect groundwater resources, consistent with Federai, State, and local requirements.

Liquid wastes must be converted to contain as little freestanding and noncorrosive liquid as is reasonably achievable. At no time can the liquid exceed $1 \%$ of the volume when the waste is in a disposal container or $0.5 \%$ of the volume of waste if processed to a stable form.

Waste in a gaseous form must be packaged at a pressure that will not exceed 1.5 atmospheres at $20^{\circ} \mathrm{C}$. The environmental monitoring program must conform with Order DOE 5484.1.

\section{By-Product Material and NORM}

Disposal of wastes that contain by-product material and naturally occurring and accelerator-produced radioactive material in large volumes must be managed according to the requirements of 40 CFR part 192; small volumes may be managed as low-level waste. 


\subsection{EXECUTIVE ORDERS}

52 FR 2822 - Radiation Protection Guidance to Federal Agencies for Occupational Exposure.

\subsubsection{Presidential Guidance}

This Presidential Guidance for workers is based on the ICRP risk-based system.

\section{Occupational Exposure}

Radiation doses received as a result of occupational exposure are not to exceed an EDE of 5 rems (0.05 Sv).

\section{Internal Exposure}

Special care should be given to controlling internal exposure. The anticipated committed EDE from internal exposure plus any annual EDE from external exposure should not exceed 5 rems $(0.05 \mathrm{~Sv})$ in adult workers. The anticipated committed dose equivalent to any organ or tissue should not exceed 50 rems $(0.5 \mathrm{~Sv})$. Committed EDE from internal sources is $H_{E, 50}=\Sigma W_{T} H_{T, 50}$. $W_{T}$ is the weighting factor previously defined. The committed dose equivalent, $H_{T, 50}$, is the sum of all dose equivalents to an organ or tissue $T$ that may accumulate over an individual's anticipated remaining lifetime (assumed 50 years) from radionuclides taken into the body during one year.

\section{Minors}

Occupational dose equivalents to individuals under the age of 18 should not exceed one-tenth of the values defined here for adult workers.

\section{Unborn Children}

Doses to unborn children should not exceed 0.5 rem $(0.005 \mathrm{~Sv})$ during the entire gestation period.

\section{ALARA}

In all cases, occupational exposures should be maintained as low as reasonably achievable. Occupational exposure of workers should not be allowed unless an overall benefit is expected from the activity causing the exposure. 


\section{Basis}

These limits utilize the concept of risk-based weighting of doses to different body parts. Thus, the standards are inherently risk-based. 


\subsection{REFERENCES}

Atomic Energy Act. 1946. P.L. 79-585, as amended. 42 USC 2011 et seq. Clean Air Act. 1963. P.L. 88-206, as amended 1990. 42 USC 7401 et seq. Clean Water Act. 1977. P.L. 92-500, as amended 1990. 33 USC 1251 et seq. Department of Energy. 1991. OSHA Training Requirements for Hazardous Waste Operations. U.S. Department of Energy Office of Environment, Safety and Health. Washington, D.C. pp. 12.

Energy Reorganization Act. 1974. P.L. 93-438, as amended. 42 USC 5811 et seq.

Oak Ridge National Laboratory. 1992. Environmental Regulatory Update Table March/April 1992. Environmental Sciences Division, Oak Ridge, Tennessee.

Federal Insecticide, Fungicide, and Rodenticide Act. 1982. P.L. 92-516, as amended 1990. 7 USC 136 et seq.

National Academy of Sciences. 1989. The Nuclear Weapons Complex - Management for Health, Safety, and the Environment. National Academy Press, Wash-

ington, D.C. pp. 146.

Nuclear Waste Policy Act. 1982. P.L. 97-425, as amended. 42 USC 10101 et seq.

Occupational Safety and Health Act. 1970. P.L. 93-237, as amended. 29 USC 651 et seq.

Resource Conservation and Recovery Act. 1976. P.L. 94-580, as amended. 42 USC 6901 , et seq.

Safe Drinking Water Act. 1974. P.L. 93-523, as amended. 42 USC 300f, et seq.

Toxic Substances Control Act. 1976. P.L. 94-469, as amended. 15 USC 2601, et seq.

U.S. Congress, Office of Technology Assessment. 1989. Coming Clean: Superfund's Problems Can be Solved. OTA-ITE-443, U.S. Government Printing Office, Washington, D.C.

U.S. Environmental Protection Agency (EPA). 1986. Quality Criteria for Water - 1986. EPA 440/5-86-0001, Office of Water Regulations and Standards, Washington, D.C. U.S. Environmental Protection Agency.

Wolf, S. M. 1988. Pollution Law Handbook - A Guide to Federal Environmental Laws. Quorum Books, New York, New York. 


\section{Code of Federal Regulations}

10 CFR 60. 1992. U.S. Nuclear Regulatory Commission. "Disposal of Highlevel Radioactive Wastes in Geologic Repositories." U.S. Code of Federal Regulations.

10 CFR 72. 1992. U.S. Nuclear Regulatory Commission. "Licensing Requirements for the Independent Storage of Spent Nuclear Fuel and High Level Radioactive Waste." U.S. Code of Federal Regulations.

29 CFR 1910. 1991. Occupational Safety and Health Administration, Labor. "Occupational Safety and Health Standards." U.S. Code of Federal Regulations.

29 CFR 1915. 1991. Occupational Safety and Health Administration, Labor. "Occupational Safety and Health Standards for Shipyard Employment." U.S. Code of Federal Regulations.

29 CFR 1918. 1991. Occupational Safety and Health Administration, Labor. "Safety and Health Regulations for Longshoring." U.S. Code of Federal Regulations.

29 CFR 1926. 1991. Occupational Safety and Health Administration, Labor. "Safety and Health Regulations for Construction." U.S. Code of Federal Regulations.

29 CFR 1928. 1991. Occupational Safety and Health Administration, Labor. "Occupational Safety and Health Standards for Agriculture. U.S. Code of rederal Regulations.

40 CFR 50. 1991. U.S. Environmental Protection Agency. "National Primary and Secondary Ambient Air Quality Standards." U.S. Code of Federal Regulations.

40 CFR 60. 1991. U.S. Environmental Protection Agency. "Standards of Performance for New Stationary Sources." U.S. Code of Federal Regulations.

40 CFR 61. 1991. U.S. Environmental Protection Agency. "National Emission Standards for Hazardous Air Pollutants." U.S. Code of Federal Regulations.

40 CFR 141. 1991. U.S. Environmental Protection Agency. "National Primary Drinking Water Regulations." U.S. Code of Federal Regulations.

40 CFR 143. 1991. U.S. Environmental Protection Agency. "National Secondary Drinking Water Regulations." U.S. Code of Federal Regulations.

40 CFR 165. 1991. U.S. Environmental Protection Agency. "Regulations for the Acceptance of Certain Pesticides and Recommended Procedures for the Disposal and Storage of Pesticides and Pesticides Containers." U.S. Code of Federal Regulations. 
40 CFR 170. 1991. U.S. Environmental Protection Agency. "Worker Protection Standards for Agricultural Pesticides." U.S. Code of Federal Regulations.

40 CFR 191. 1991. U.S. Environmental Protection Agency. "Protection Standards for Managing and Disposing of Spent Nuclear Fuel, High-level and Transuranic Radioactive Wastes." U.S. Code of Federal Regulations.

40 CFR 192. 1991. U.S. Environmental Protection Agency. "Standards for Protection Against Uranium Mill Tailings." U.S. Code of Federal Regulations.

40 CFR 261. 1991. U.S. Environmental Protection Agency. "Regulations for Identifying Hazardous Waste." U.S. Code of Federal Regulations.

40 CFR 264. 1991. U.S. Environmental Protection Agency. "Regulations for Owners and Operators of Permitted Hazardous Waste Facilities." U.S. Code of Federal Regulations.

40 CFR 265. 1991. U.S. Environmental Protection Agency. "Interim Status Standards for Owners and Operators of Hazardous Waste Facilities." U.S. Code of Federal Regulations.

40 CFR 268. 1991. U.S. Environmental Protection Agency. "Regulations on Land Disposal Restrictions." U.S. Code of Federal Regulations.

40 CFR 302. 1991. U.S. Environmental Protection Agency. "Designation, Reportable Quantities, and Notification." U.S. Code of Federal Regulations.

40 CFR 761. 1991. U.S. Environmental Protection Agency. "Polychlorinated Biphenyls (PCBs) Manufacturing, Processing, Distribution in Commerce, and Use Prohibitions." U.S. Code of Federal Regulations.

\section{DOE Orders}

DOE 5400.1. 1988. "General Environmental Protection Program." U.S. Department of Energy.

DOE 5400.5. 1989. "Radiation Protection of the Public and the Environment." U.S. Department of Energy.

DOE 5480.1B. 1988. "Environment, Safety, and Health Program for Department of Energy Operations." U.S. Department of Energy. 
DOE 5480.4. 1988. "Environmental Protection, Safety, and Health Protection Standards." U.S. Department of Energy.

DOE 5480.11. 1988. "Radiation Protection for Occupational Workers." U.S. Department of Energy.

DOE 5483.1A. 1983. "Occupational Safety and Health Program for DOE Contractor Employees at Government-Owned Contractor-Operated Facilities." U.S. Department of Energy.

DOE 5820.2A. 1988. "Radioactive Waste Management." U.S. Department of Energy.

\section{Federal Register}

45 FR 74693. November 12, 1980. U.S. Environmental Protection Agency. "Licensing Requirements for the Storage of Spent Fuel in an Independent Spent Fuel Storage Installation." Federal Register.

45 FR 79318. November 28, 1980. U.S. Environmental Protection Agency. "Water Quality Criteria Documents; Availability." Federal Register.

46 FR 7836. January 23, 1981. U.S. Environmental Protection Agency. "Federal Radiation Protection Guidance for Occupational Exposure." Federal Register.

48 FR 590. January 5, 1983. U.S. Environmental Protection Agency. "Standards for Remedial Actions at Inactive Uranium Processing Sites." Federal Register.

50 FR 38066. September 19, 1985. U.S. Environmental Protection Agency. "Environmental Standards for the Management and Disposal of Spent Nuclear Fuel, High-level and Transuranic Radioactive Waste." Federal Register.

50 FR 47156. November 14, 1985. U.S. Environmental Protection Agency "National Primary Drinking Water Regulations; Fluoride." Federal Register.

52 FR 2822. January 27, 1987. U.S. Environmental Protection Agency. "Radiation Protection Guidance to Federal Agencies for Occupational Exposure." Federal Register.

52 FR 5992. February 27, 1987. U.S. Environmental Protection Agency. Definition of "High-level Radioactive Waste." Federal Register.

52 FR 10688. Apri1 2, 1987. U.S. Environmental Protection Agency. "Polychlorinated Biphenyls Spill Cleanup Policy." Federal Register.

52 FR 24634. July 1, 1987. U.S. Environmental Protection Agency. "Revisions to the National Ambient Air Quality Standards for Particulate Matter."

Federal Register. 
54 FR 38044. September 14, 1989. U.S. Environmental Protection Agency. "National Emission Standards for Hazardous Air Pollutants; Benzene Emissions From Maleic Anhydride Plants, Ethylbenzene/ Styrene Plants, Benzene Storage Vessels, Benzene Equipment Leaks, and Coke By-Product Recovery Plants." Federal Register.

54 FR 51654. December 15, 1989. U.S. Environmental Protection Agency. "National Emission Standards for Hazardous Air Pollutants; Radionuclides." Federal Register.

55 FR 8666. March 8, 1990. U.S. Environmental Protection Agency. "National $0 i 1$ and Hazardous Substances Pollution Contingency Plan." Federal Register.

55 FR 8848. March 8, 1990. U.S. Environmental Protection Agency. "National 0 il and Hazardous Substances Pollution Contingency Plan." Federal Register.

55 FR 11798. March 29, 1990. U.S. Environmental Protection Agency. "Hazardous Waste Management System; Identification and Listing of Hazardous Waste; Toxicity Characteristics Revisions; Final Rule." Federal Register.

56 FR 3526. January 30, 1991. U.S. Environmental Protection Agency. "National Primary Drinking Water Regulations - Synthetic Organic Chemicals and Inorganic Chemicals; Monitoring for Unregulated Contaminants; National Primary Drinking Water Regulations Implementation; National Secondary Drinking Water Regulations." Federal Register.

56 FR 7134. February 21, 1991. U.S. Environmental Protection Agency. "Burning of Hazardous Waste in Boilers and Idustrial Furnaces." Federal Register.

56 FR 26460. June 7, 1991. U.S. Environmental Protection Agency. " Maximum Contaminant Level Goals and National Primary Drinking Water Regulations for Lead and Copper." Federal Register.

56 FR 26480. June 7, 1991. U.S. Environmental Protection Agency. "Drinking Water Regulations: Maximum Contaminant Level Goals and National Primary Drinking Water Regulations for Lead and Copper." Federal Register.

56 FR 30266. JuTy 1, 1991. "Drinking Water; National Primary Drinking Water Regulations; Monitoring for Volatile Organic Chemicals; MCLGs and MCLs for Aldicarb, Aldicarb Sulfoxide, Aldicarb Sulfane, Pentachlorophenol, and Barium." Federal Register.

57 FR 11363. April 2, 1991. "Asbestos; Manufacture, Importation, Processing and Distribution Prohibitions; Effect of Court Decision." Federal Register.

57 FR 28785. June 29, 1992. "Drinking Water Regulations: Maximum Contaminant Level Goals and National Primary Drinking Water Regulations for Lead and Copper." Federal Register. 
57 FR 31776. July 17, 1992. "Drinking Water; National Primary Water Regulations - Synthetic Organic Chemicals and Inorganic Chemicals, National Primary Drinking Water Regulations Implementation." Federal Register. 


\section{APPENDIX A}

AIR CONTAMINANTS -- PERMISSIBLE EXPOSURE LIMITS

Title 29 Code of Federal Regulations Part 1910.1000

(as amended July 1, 1992) 
APPENDIX A

\subsection{AIR CONTAMINANTS}

(as amended July 1, 1992)

An employee's exposure to any substance listed in Tables Z- 1-A, Z-2 or Z-3 of this section shall be limited in accordance with the requirements of the following paragraphs of this section.

(a) Table Z-1-A -- (1) Substances in Transitional Limits Columns with limits preceded by "C" -- Ceiling Values. An employee's exposure to any substance in Table Z-1-A under the Transitional Limits columns, the exposure limit of which is preceded by a "C", shall at no time exceed the exposure limit given for that substance in Table Z-1-A under the Transitional Limits columns.

(2) Other Substances in Transitional Limits Columns -- 8- hour Time Weighted Average. An employee's exposure to any substance in Table Z-1-A under the Transitional Limits columns, the exposure limit of which is not preceded by a "C", shall not exceed the 8-hour Time Weighted Average given for that substance in Table Z-1-A under the Transitional Limits columns in any 8-hour work shift of a 40 -hour work week.

(3) Final Rule Limits Columns. An employee's exposure to any substance listed in Table Z-1-A shall not exceed the Time Weighted Average (TWA), Short Term Exposure Limit (STEL) and Ceiling Limit specified for that substance in Table Z-1-A under the Revised Limits columns.

(4) Skin Designation. To prevent or reduce skin absorption, an employee's skin exposure to substances listed in Table Z-1-A with an "X" in one or both of the Skin Designation columns following the substance name sha11 be prevented or reduced to the extent necessary in the circumstances through the use of gloves, coveralls, goggles, or other appropriate personal protective equipment, engineering controls or work practices.

(5) Définitions. The following definitions are applicable to the Final Rule Limits columns of Table Z-1-A: 
(i) Time weighted average (TWA) is the employee's average airborne exposure in any 8-hour work shift of a 40 -hour work week which shall not be exceeded.

(ii) Short term exposure limit (STEL) is the employee's 15-minute time weighted average exposure which shall not be exceeded at any time during a work day unless another time limit is specified in a parenthetical notation below the 1imit. If another time period is specified, the time weighted average exposure over that time period shall not be exceeded at any time during the working day.

(iii) Ceiling is the employee's exposure which shall not be exceeded during any part of the work day. If instantaneous monitoring is not feasible, then the ceiling shall be assessed as a 15 -minute time weighted average exposure which shall not be exceeded at any time over a working day.

(6) Additional Definition. The terms "substance", "air contaminant," and "material" are equivalent in meaning for 29 CFR 1910.1000.

(b) Table Z-2. Table Z-2 is applicable for the transitional period and to the extent set forth in paragraph ( $f$ ) of this section.

(1) 8-hour time weighted averages. An employee's exposure to any material listed in table Z-2, in any 8-hour work shift of a 40-hour work week, shall not exceed the 8-hour time weighted average 1 imit given for that material in Table Z-2.

(2) Acceptable ceiling concentrations. An employee's exposure to a material listed in Table Z-2 shall not exceed at any time during an 8-hour shift the acceptable ceiling concentration limit given for the material in the table, except for a time period, and up to a concentration not exceeding the maximum duration and concentration allowed in the column under "acceptable maximum peak above the ceiling concentration for an 8-hour shift".

(3) Example. During an 8-hour work shift, an employee may be exposed to a concentration of Substance $A$ (with a $10 \mathrm{ppm}$ TWA, $25 \mathrm{ppm}$ ceiling and $50 \mathrm{ppm}$ peak above $25 \mathrm{ppm}$ (but never above $50 \mathrm{ppm}$ ) only for a maximum of 10 minutes. 
Such exposure must be compensated for by exposures to concentrations less than $10 \mathrm{ppm}$ so that the cumulative exposure for the entire 8-hour work shift does not exceed a weighted average of $10 \mathrm{ppm}$.

(c) Table Z-3. Table Z-3 is applicable for the transitional period and to the extent set forth in paragraph ( $f$ ) of this section. An employee's exposure to any substance listed in Table Z-3 in any 8-hour work shift of a 40-hour work week shall not exceed the 8-hour time weighted average limit given for that material in the table.

(d) Computation formulae. The computation formula which shall apply to employee exposure to more than one substance for which 8-hour time weighted averages are 1isted in subpart $Z$ of 29 CFR Part 1910 in order to determine whether an employee is exposed over the regulatory limit is as follows:

(1)(i) The cumulative exposure for an 8-hour work shift shall be computed as follows:

$$
E=(C(a) T(a)+C(b) T(b)+\ldots C(n) T(n)) \div 8
$$

Where: $E$ is the equivalent exposure for the working shift.

$C$ is the concentration during any period of time $T$ where the concentration remains constant.

$T$ is the duration in hours of the exposure at the concentration $C$.

The value of $E$ shall not exceed the 8-hour time weighted average specified in Subpart $Z$ or 29 CFR Part 1910 for the material involved.

(ii) To illustrate the formula prescribed in paragraph (d)(1)(i) of this section, assume that Substance $A$ has an 8 - hour time weighted average limit of $100 \mathrm{ppm}$ noted in Table Z- 1-A. Assume that an employee is subject to the following exposure:

Two hours exposure at $150 \mathrm{p} / \mathrm{m}$

Two hours exposure at $75 \mathrm{p} / \mathrm{m}$

Four hours exposure at $50 \mathrm{p} / \mathrm{m}$ 
Substituting this information in the formula, we have

$$
(2 \times 150+2 \times 75+4 \times 50) \div 8=81.25 \mathrm{p} / \mathrm{m}
$$

Since $81.25 \mathrm{ppm}$ is 1ess than $100 \mathrm{p.p.m}$, the 8 -hour time weighted average limit, the exposure is acceptable.

(2)(i) In case of a mixture of air contaminants an employer shall compute the equivalent exposure as follows:

$$
E m=(C(1) \div L(1)+C(2) \div L(2))+\ldots(C(n) / L(n))
$$

Where: $E(m)$ is the equivalent exposure for the mixture.

$C$ is the concentration of a particular contaminant.

$L$ is the exposure limit for that substance specified in Subpart Z of 29 CFR Part 1910.

The value of $E(m)$ shall not exceed unity (1).

(ii) To illustrate the formula prescribed in paragraph (d)(2)(i) of this

\begin{tabular}{|c|c|c|}
\hline Material & $\begin{array}{l}\text { Actual Concentration } \\
\text { 8-hour exposure } \\
\text { (ppm) }\end{array}$ & $\begin{array}{l}\text { 8-hour TWA } \\
\text { PEL (ppm) }\end{array}$ \\
\hline 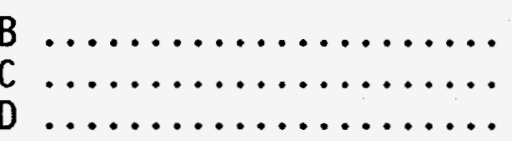 & $\begin{array}{r}500 \\
45 \\
40\end{array}$ & $\begin{array}{r}1000 \\
200 \\
200\end{array}$ \\
\hline
\end{tabular}
section, consider the following exposures:

Substituting in the formula, we have:

$$
\begin{aligned}
& E m=500 \div 1,000+45 \div 200+40 \div 200 \\
& E m=0.500+0.225+0.200 \\
& E m=0.925
\end{aligned}
$$

Since $\mathrm{Em}$ is less than unity (1), the exposure combination is within acceptable limits. 
(e) To achieve compliance with paragraphs (a) through (d) of this section, administrative or engineering controls must first be determined and implemented whenever feasible. When such controls are not feasible to achieve full compliance, protective equipment or any other protective measures shall be used to keep the exposure of employees to air contaminants within the limits prescribed in this section. Any equipment and/or technical measures used for this purpose must be approved for each particular use by a competent industrial hygienist or other technically qualified person. Whenever respirators are used, their use shall comply with ^U 1910.134.

(f) Effective dates, start-up dates and transitional provisions -. (1) Effective date. The effective date for the permissible exposure limits specified in the Final Rule Limits columns of Table Z-1-A is March 1, 1989.

(2) Start-up dates. (i) The permissible exposure limits specified in the Final Rule Limits columns of Table Z-1-A shall be achieved by any reasonable combination of engineering controls, work practices and personal protective equipment effective September 1, 1989, through December 30, 1992. (See Note at end of Table Z-1-A.).

(ii)(A) The permissible exposure limits specified in the final Rule Limits columns of Table Z-1-A shall be achieved by the method of compliance specified in paragraph (e) of this section effective December 31,1992 , if by December 31, 1991 a final rule has been published in the Federal Register amending or determining not to amend paragraph (e) of this section.

(B) If no final rule has been published in the Federal Register by December 31, 1991, amending or determining not to amend paragraph (e) of this section, then the permissible limits specified in the Final Rule Limits columns of Table Z- 1-A shall be achieved by the methods of compliance specified by paragraph (e) of this section effective December 31, 1993, and paragraph $(f)(2)(i)$ of this section shall remain in effect through December $30,1993$.

(iii) The skin designations in the Final Rule Limits columns become effective September 1, 1989. The skin designations in the Transitional Limits columns are in effect from March 1, 1989, through August 31, 1989. 
(3) Transitional provisions. (i) The permissible exposure limits specified in the Transitional Limits columns of Table Z-1-A, Table Z-2 and Table Z-3 shall continue to be achieved by the methods of compliance specified in paragraph (e) of this section through December 30, 1992. If paragraph $(f)(2)(i i)(B)$ of this section takes effect, this provision is extended through December 30, 1993. The permissible exposure Timits specified in Tables Z-2 and Z-3 for substances mentioned in the Final Rule Limits column of Table Z-1-A only by cross reference to Table Z-2 or Z-3 shall remain in effect indefinitely and continue to be achieved by the methods of compliance specified in paragraph (e) of this section.

(ii) The permissible exposure limits specified in the Transitional Limits columns of Table Z-1-A, Z-2 and Z-3 shall be applicable to the extent cross referenced 29 CFR Parts 1915, 1917 and 1918.

(iii) If any new or amended provisions or new or revised limits for any substance or substances are either administratively stayed or judicially stayed or vacated, then the existing provisions or limits for those substances specified in the Transitional Limits columns of Table Z-1-A, Table Z-2 or Table Z-3 shall remain in effect until such stay is lifted, or indefinitely, if the limit is vacated.

(4) Enforcement of the limits are indefinitely stayed for: aluminum alkyls; ethylidene norbornene; hexafluoracetone; mercury (alkyl compounds); oxygen difluoride; phenylphosphine; and sulfur pentafluoride; until OSHA publishes in the Federal Register a notice that a sampling and analytical technique is available. 
TABLE Z-1-A, part 1. Limits For Air Contaminants

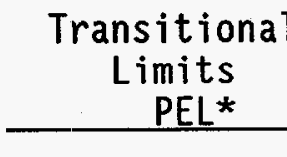

Substance

CAS No. [f]

75-07-0

$64-19-7$

108-24-7

$67-64-1$

$75-05-8$

$53-96-3$

2-Acetylaminofluorine;

see 1910.1014

Acetylene dichloride;

see 1,2-Dichloroethylene

Acetylene tetrabromide

Acetylsalicylic acid

(Aspirin)

Acrolein

79-27-6

1

14

50-78-2

107-02-8

ppm[a] $\mathrm{mg} / \mathrm{m}[3]$

Skin

Acrylamide

Acrylic acid

79-06-1

79-10-7

Acrylonitrile;

see 1910.1045

Aldrin

Allyl alcohol

Allyl chloride

Allyl glycidyl ether (AGE)

Allyl propyl disulfide

alpha-Alumina

Total dust

Respirable fraction

Aluminum (as Al)

Metal

Total dust

Respirable fraction

Pyro powders

Welding fumes***

Soluble salts

Alkyls

4-Aminodiphenyl;

see 1910.1011

107-13-1

309-00-2

107-18-6

107-05-1

106-92-3

2179-59-1

1344-28-1

$$
200
$$

10

1000

40

$\mathrm{mg} / \mathrm{m}[3]$

Designation

-Aminoethanol;

see Ethanolamine

7429-90-5

(C) 10

$\begin{array}{rrr}- & 0.25 & x \\ 2 & 5 & x \\ 1 & 3 & -\end{array}$

$\begin{array}{rrr}- & 0.25 & X \\ 2 & 5 & X \\ 1 & 3 & -\end{array}$

0.25

0.1

$\begin{array}{lll}- & 0.3 & X \\ - & -\end{array}$

360
25

20

2400

70

$\begin{array}{lll}- & 0.3 & X \\ - & -\end{array}$

$\begin{array}{lll}- & 0.3 & X \\ - & -\end{array}$

(C) 45

12

$\begin{array}{lr}- & 15 \\ - & 5\end{array}$

$92-67-1$

$\begin{array}{lr}- & 15 \\ - & 5 \\ - & - \\ - & - \\ - & - \\ - & -\end{array}$

A. 7 
TABLE Z-1-A, part 1. (contd)

\section{Substance}

2-Aminopyridine

Amitrole

Ammonia

Ammonium chloride fume

Ammonium sulfamate

Total dust

Respirable fraction

n-Amyl acetate

sec-Amyl acetate

Aniline and homologs

Anisidine (0-, $p$-isomers)

Antimony and compounds

$$
\text { (as Sb) }
$$

ANTU (alpha Naphthyl-

thiourea)

Arsenic, organic compounds (as As)

Arsenic, inorganic compounds (as As); see 1910.1018

Arsine

Asbestos; see $1910.1001 \& 1910.1101$

Atrazine

Azinphos-methyl

Barium, soluble compounds

$$
\text { (as Ba) }
$$

Barium sulfate

Total dust

Respirable fraction

Benomyl

Total dust

Respirable fraction

Benzene;

applica-

see 1910.1028

ex-
Transitional

Limits

PEL*

CAS No.[f]

504-29-0

$61-82-5$

7664-41-7

12125-02-9

$7773-06-0$

628-63-7

626-38-0

$62-53-3$

29191-52-4

7440-36-0

86-88-4

$7440-38-2$

$7440-38-2$

7784-42-1

Varies

1912-24-9

$86-50-0$

$7440-39-3$

$7727-43-7$

$17804-35-2$

0.05

0.2

$\mathrm{ppm}[\mathrm{a}] \quad \mathrm{mq} / \mathrm{m}[3]$

0.5

50

$\begin{array}{rrr}- & 15 & - \\ - & 5 & - \\ 100 & 525 & - \\ 125 & 650 & - \\ 5 & 19 & X \\ - & 0.5 & X\end{array}$

0.5

0.3

0.5

71-43-2 See Table Z-2 for the limits

ble in the operations or sectors

cluded in $1910.1028[\mathrm{~d}]$
35

0.2

$x$

Skin

Designation

$x$

0.5

15

15

5

A. 8 
TABLE Z-1-A, part 1. (contd)

\section{Substance}

Benzidine;
see 1910.1010
p-Benzoquinone;
see Quinone
Benzo(a)pyrene;
see Coal tar pitch
volatiles
Benzoyl peroxide
Benzyl chloride
Beryllium and beryllium
compounds (as Be)
Biphenyl; see Diphenyl
Bismuth telluride, Undoped
Total dust
Respirable fraction
Bismuth telluride, Se-doped
Borates, tetra,
sodium salts
Anhydrous
Decahydrate
Pentahydrate
Boron oxide
Total dust
Respirable fraction
Boron tribromide
Boron trifluoride
Bromacil
Bromine
Bromine pentafluoride
Bromoform
Butadiene (1,3-
Butadiene)
Butane
Butanethiol; see Butyl
mercaptan
2-Butanone (Methyl
ethyl ketone)
2-Butoxyethanol
n-Butyl-acetate
But

CAS No. [f] 92-87-5

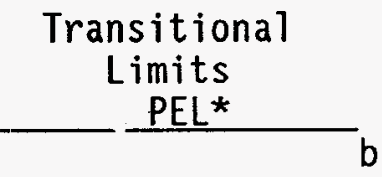

$\mathrm{ppm}[\mathrm{a}] \mathrm{mg} / \mathrm{m}[3]$
Skin

Designation

\author{
94-36-0 \\ $100-44-7$ \\ $7440-41-7$ \\ $1304-82-1$ \\ $1330-43-4$
$1303-96-4$
$12179-04-3$
$1303-86-2$
}

10294-33-4

7637-07-2

314-40-9

$7726-95-6$

$7789-30-2$

$75-25-2$

106-99-0

106-97-8

78-93-3

$111-76-2$

$123-86-4$
1

5

See Table Z-2

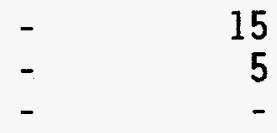

15
5
-

(C) 1

(C) 3

0.1

0.7

0.5

$$
5
$$

1000

2200

15

-

(


TABLE Z-1-A, part 1. (contd)

Substance

sec-Butyl acetate

tert-Butyl acetate

Butyl acrylate

n-Butyl alcohol

sec-Butyl alcohol

tert-Butyl alcohol

Butylamine

tert-Butyl chromate

(as CrO(3))

n-Butyl glycidyl ether (BGE)

n-Buty1 lactate

Buty 1 mercaptan

o-sec-Butylphenol

p-tert-Butyltoluene

Cadmium fume (as $C d$ )

Cadmium dust (as Cd)

Calcium carbonate

Total dust

Respirable fraction

Calcium cyanamide

Calcium hydroxide[i]

Calcium oxide[ $j]$

Calcium silicate

Total dust

Respirable fraction

Calcium sulfate

Total dust

Respirable fraction

Camphor, synthetic

Caprolactam

Dust

Vapor

Captafol (Difolatan[R])

Captan

Carbaryl (Sevin[R])

Carbofuran (Furadan[R])

Carbon black

Carbon dioxide
CAS No.[f]

$105-46-4$

540-88-5

$141-32-2$

$71-36-3$

$78-92-2$

$75-65-0$

109-73-9

1189-85-1

2426-08-6

$138-22-7$

109-79-5

$89-72-5$

98-51-1

7440-43-9

$7440-43-9$

$1317-65-3$

156-62-7

$1305-62-0$

1305-78-8

1344-95-2

$7778-18-9$

$76-22-2$

$105-60-2$

2425-06-1

133-06-2

$63-25-2$

$1563-66-2$

1333-86-4

124-38-9
Transitional

Limits

PEL*

$\mathrm{ppm}[\mathrm{a}] \mathrm{mg} / \mathrm{m}[3]$

950

200

200

950

100

150

100

(C) 5

300

450

300

(C) 15

(C) 0.1

270

50

10

35

10

60

See Table Z-2

See Table Z-2

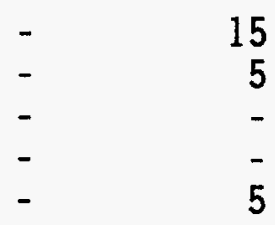

15

5

$-$

5

15

5

15

5

2

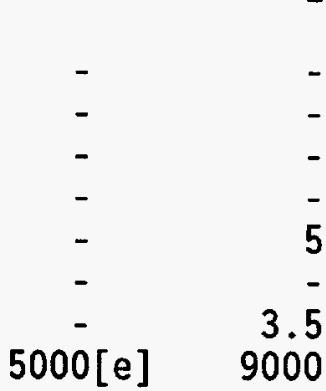

Skin

Designation

$$
\begin{aligned}
& - \\
& - \\
& - \\
& - \\
& \hline
\end{aligned}
$$

X

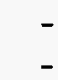


TABLE Z-1-A, part 1. (contd)

Substance

Carbon disulfide

Carbon monoxide

Carbon tetrabromide

Carbon tetrachloride

Carbonyl fluoride

Catechol

(Pyrocatechol)

Cellulose

Total dust

Respirable fraction

Cesium hydroxide

Chlordane

Chlorinated camphene

Chlorinated diphenyl oxide

Chlorine

Chlorine dioxide

Chlorine trifluoride

Chloroacetaldehyde

a-Chloroacetophenone

(Phenacyl chloride)

Chloroacetyl chloride

Chlorobenzene

o-Chlorobenzylidene malononitrile

Chlorobromomethane

2-Chloro-1,3-butadiene; see b-Chloroprene

Chlorodifluoromethane

Chlorodiphenyl (42\% Chlorine) (PCB)

Chlorodiphenyl (54\% Chlorine) (PCB)

1-Chloro, 2,3-epoxypropane; see Epichlorohydrin

2-Chloroethanol; see Ethylene chlorohydrin

Chloroethylene;

see Vinyl chioride

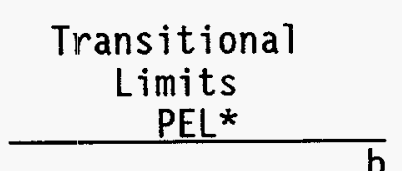

CAS No.[f]

75-15-0

630-08-0

558-13-4

56-23-5

$353-50-4$

120-80-9

9004-34-6

21351-79-1

57-74-9

8001-35-2

55720-99-5

$7782-50-5$

10049-04-4

7790-91-2

107-20-0

532-27-4

79-04-9

108-90-7

2698-41-1

74-97-5

$75-45-6$

53469-21-9

11097-69-1

ppm[a] $\mathrm{mg} / \mathrm{m}[3]^{\mathrm{b}}$

Skin

Designation

$\begin{array}{cc}50 & \text { See Table Z-2 } \\ - & 55 \\ - & - \\ - & \text { See Table Z-2 }\end{array}$

15

0.5

0.5

0.5

(C) 1

(C) 3

0.1

(C) 0.1

(C) 1

(C) 0.4

(C) 3

0.05

0.3

350

0.05

0.4

1050

200

$x$

$0.5 \quad X$ 
TABLE Z-1-A, part 1. (contd)

Substance

Chloroform

(Trichloromethane)

bis(Chloromethyl) ether; see 1910.1008

Chloromethyl methyl ether; see 1910.1006

1-Ch7oro-1-nitropropane

Chloropentafluoroethane

Chloropicrin

beta-Ch 1 oroprene

o-Chlorostyrene

o-Chlorotoluene

2-Chloro-6-trichloromethyl pyridine

Total dust

Respirable fraction

Chlorpyrifos

Chromic acid and chromates (as CrO(3))

Chromium (II) compounds (as $\mathrm{Cr}$ )

Chromium (III) compounds (as $\mathrm{Cr}$ )

$67-66-3$

542-88-1

107-30-2

$600-25-9$

76-15-3

76-06-2

126-99-8

2039-87-4

95-49-8

1929-82-4

2921-88-2

Varies with

compound

$7440-47-3$

$7440-47-3$

$7440-47-3$

Chrysene; see Coal tar pitch volatiles

Clopidol Total dust Respirable fraction

Coal dust (less than $5 \%$ SiO(2)), Respirable quartz fraction

2971-90-6
CAS No.[f]

Transitional

Limits

PEL*

ppm[a] $\mathrm{mg} / \mathrm{m}[3]^{b}$

Skin

Designation

(C) 50

(C) 240

20

100

$0 . \overline{1}$

25

0.7

90

-

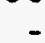

$\begin{array}{lr}- & 15 \\ - & 5\end{array}$

See Table Z-2

0.5

0.5

1

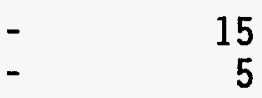

See Table Z-3 
TABLE Z-1-A, part 1. (contd)

Substance

CAS No.[f] ppm[a] $\mathrm{mg} / \mathrm{m}[3]$

Skin

Transitiona 7

Limits

PEL*

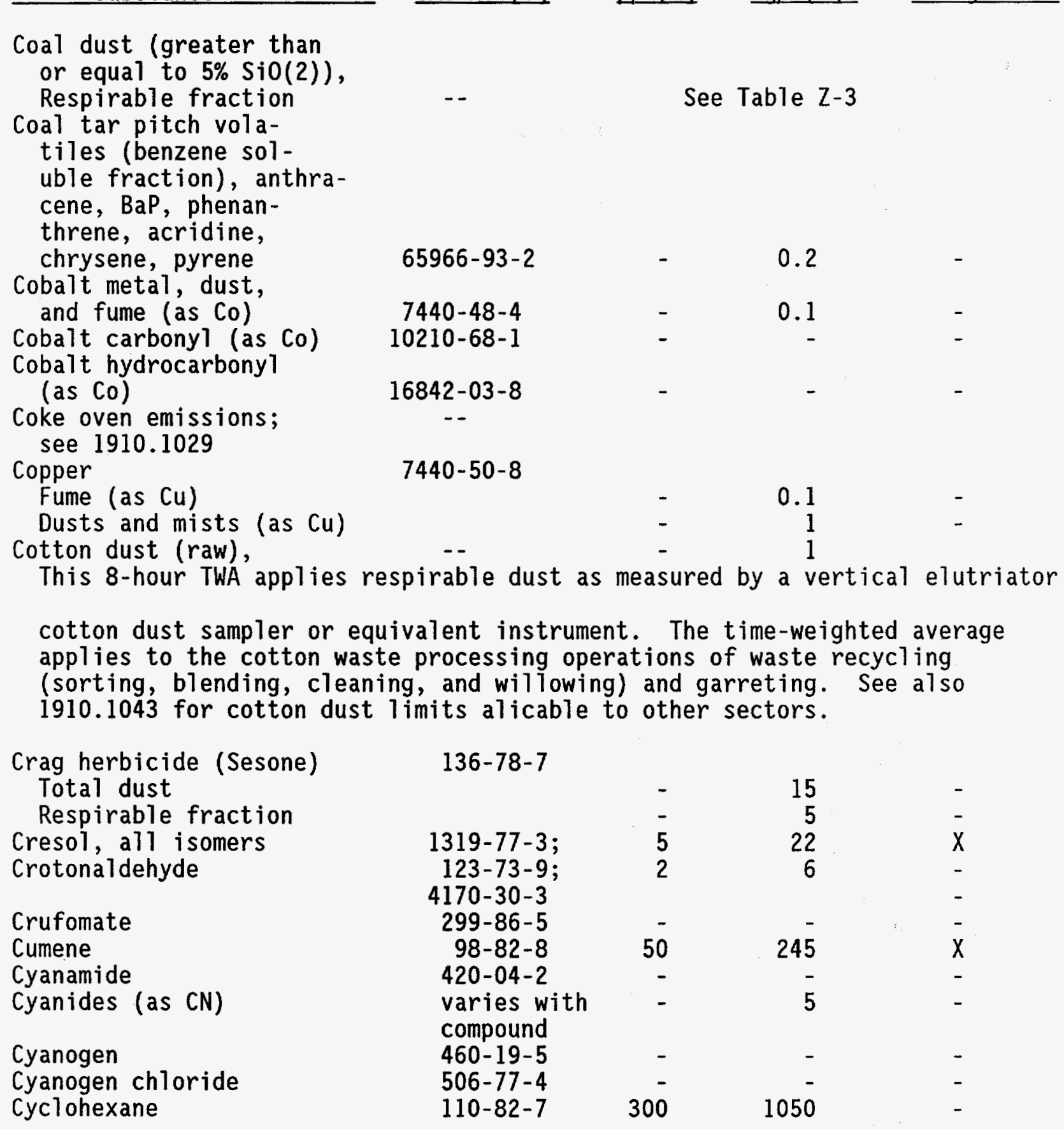


TABLE Z-1-A, part 1. (contd)

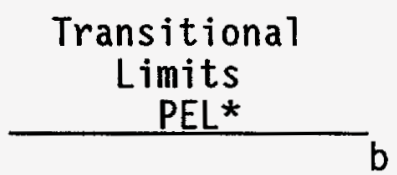

ppm[a] $\mathrm{mg} / \mathrm{m}[3]$

$108-94-1$

$110-83-8$

108-91-8

121-82-4

542-92-7

$287-92-3$

$13121-70-5$

Cyhexatin

2,4-D (Dichlorylphenoxyacetic acid)

Decaborane

Demeton (Systox(R))

Dichlorodiphenyltrichloroethane (DDT)

Dichlorvos (DDVP)

Diacetone alcohol

(4-Hydroxy-4-methy12-pentanone)

1,2-Diaminoethane; see Ethylenediamine

Diazinon

Diazomethane

Diborane

1,2-Dibromo3-chloropropane; see 1910.1044

2-N-Dibutylaminoethanol

Dibutyl phosphate

Dibutyl phthalate

Dichloroacetylene

o-Dichlorobenzene

p-Dichlorobenzene

3, 3'-Dichlorobenzidine; see 1910.1007

Dichlorodifluoromethane
94-75-7

8065-48-3

50-29-3

$62-73-7$

$123-42-2$

333-41-5

$334-88-3$

$19287-45-7$

96-12-8

102-81-8

$107-66-4$

84-74-2

7572-29-4

95-50-1

106-46-7

91-94-1

$75-71-8$
$17702-41-9$

(C) 50

75

(C) 300

450

1000

4950
50

50

300

Skin

Designation

200
200
1015
-
-
200
-
-

10

0.3

0.1

$x$

$X$

$X$
$X$

50

240

0.2

0.1

0.4

0.1

\section{1}


TABLE Z-1-A, part 1. (contd)

Substance

1,3-Dichloro-5,5dimethyl hydantoin

1,1-Dichloroethane

1,2-Dichloroethylene

Dichloroethyl ether

Dichloromethane; see Methylene chloride

Dichloromonofluoromethane

1,1-Dichloro-1-nitroethane

1,2-Dichloropropane; see Propylene dichloride

1,3-Dichloropropene

2,2-Dichloropropionic acid

Dichlorotetrafluoroethane

Dicrotophos

Dicyclopentadiene

Dicyclopentadienyl iron Total dust Respirable fraction

Dieldrin

Diethanolamine

Diethyl amine

2-Diethyl aminoethanol

Diethylene triamine

Diethyl ether; see Ethyl ether

Diethyl ketone

Diethyl phthalate

Difluorodibromomethane

Diglycidyl ether (DGE)

Dihydroxybenzene; see Hydroquinone

Diisobutyl ketone
Transitional

Limits PEL*

CAS No.[f] ppm[a] $\mathrm{mg} / \mathrm{m}[3]$

Skin

Designation
$118-52-5$

$75-34-3$

$540-59-0$

111-44-4

75-43-4

594-72-9

542-75-6

75-99-0

76-14-2

141-66-2

$77-73-6$

102-54-5

60-57-1

111-42-2

109-89-7

$100-37-8$

111-40-0

96-22-0

$84-66-2$

75-61-6

2238-07-5

108-83-8
1000

7000

0.2

400

790

(C) 90

4200

(C) 60

(C) 10

(C) 0.5

(C) 2.8

50

290 


\section{TABLE Z-1-A, part 1. (contd)}

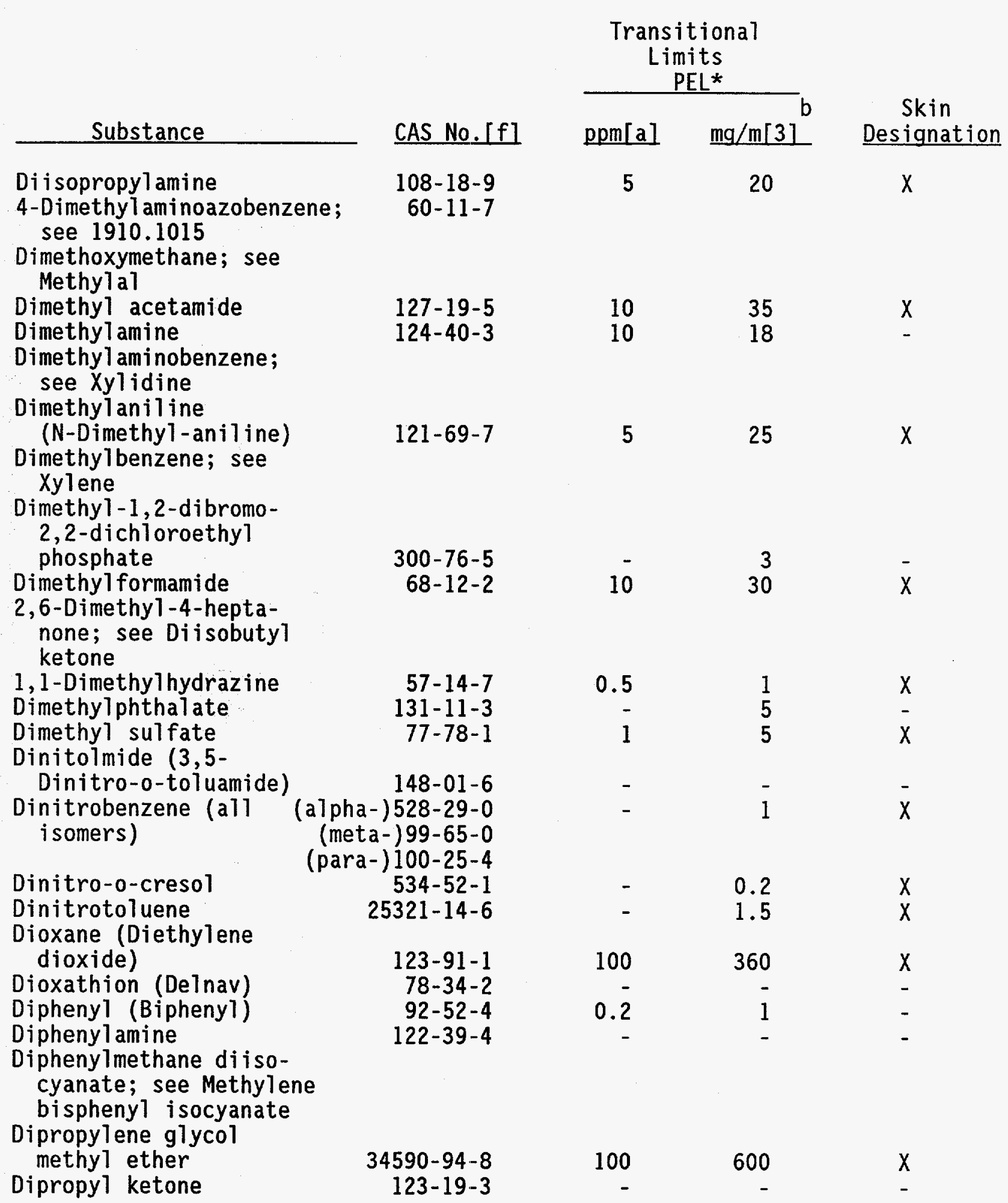


TABLE Z-1-A, part 1. (contd)

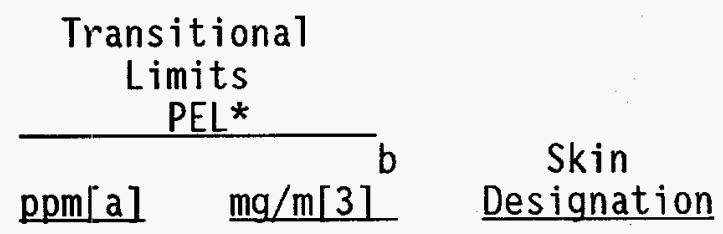

\section{Substance}

Diquat

Di-sec octyl phthalate (Di-2-ethylhexylphthalate)

Disulfiram

Disulfoton

2,6-Di-tert-butyl-pcresol

Diuron

Divinyl benzene

Emery

Total dust

Respirable fraction

Endosulfan

Endrin

Epichlorohydrin

EPN

1,2-Epoxypropane; see

Propylene oxide

2,3-Epoxy-1-propano1; see Glycidol

Ethanethiol; see Ethyl mercaptan

Ethanol amine

Ethion

2-Ethoxyethanol

2-Ethoxyethyl acetate (Cellosolve acetate)

Ethyl acetate

Ethyl acrylate

Ethyl alcohol (Ethanol)

Ethyl amine

Ethyl amyl ketone

(5-Methy 1-3-heptanone)

Ethyl benzene

Ethyl bromide

Ethyl butyl ketone

(3-Heptanone)

Ethyl chloride
CAS No.[f]

85-00-7

117-81-7

97-77-8

298-04-4

128-37-0

$330-54-1$

1321-74-0

112-62-9

115-29-7

72-20-8

106-89-8

2104-64-5

141-43-5

$563-12-2$

$110-80-5$

$111-15-9$

$141-78-6$

$140-88-5$

$64-17-5$

75-04-7

541-85-5

100-41-4

74-96-4

541-86-5

75-00-3
0.1
19
0.5

0.1
19
0.5

0.1
19
0.5
Designation

$$
\begin{aligned}
& x \\
& x \\
& x
\end{aligned}
$$

$\begin{array}{rrr}3 & 6 & - \\ - & - & -\end{array}$

$\begin{array}{rrr}100 & 540 & X \\ 400 & 1400 & - \\ 25 & 100 & X \\ 1000 & 1900 & - \\ 10 & 18 & -\end{array}$

$\begin{array}{rr}25 & 130 \\ 100 & 435 \\ 200 & 890\end{array}$

50

230

1000 
TABLE Z-1-A, part 1. (contd)

Substance

Ethyl ether

Ethyl formate

Ethyl mercaptan

Ethyl silicate

Ethylene chlorohydrin

Ethylenediamine

Ethylene dibromide

Ethylene dichloride

Ethylene glycol

Ethylene glycol

dinitrate[k]

Ethylene glycol methy]

acetate; see Methy1

cellosolve acetate

Ethyleneimine;

see 1910.1012

Ethylene oxide;

see 1910.1047

Ethylidene chloride; see 1,1-Dichloroethane

Ethylidene norbornene

$\mathrm{N}$-Ethylmorpholine

Fenamiphos

Fensulfothion

(Dasanit)

Fenthion

Ferbam

Total dust

Ferrovanadium dust

Fluorides (as F)

Fluorine

Fluorotrichloromethane (Trichloro-

f]uoromethane)

Fonofos

Formaldahyde;

see 1910.1048;

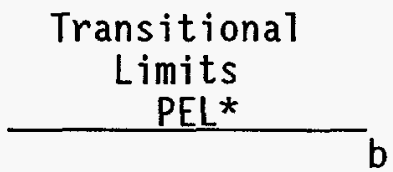

CAS No. [f]

60-29-7

109-94-4

75-08-1

78-10-4

107-07-3

107-15-3

106-93-4

107-06-2

107-21-1

$628-96-6$

151-56-4

$75-21-8$

16219-75-3

$100-74-3$

22224-92-6

115-90-2

55-38-9

14484-64-1

12604-58-9

Varies with

compound

$\mathrm{ppm}[\mathrm{a}] \mathrm{mg} / \mathrm{m}[3]$

Skin

Designation

$400 \quad 1200$

$100 \quad 300$

(C) $10 \quad$ (C) 25
100

100

5

10

850

16

25

See Table Z-2

See Table Z-2

(C) 0.2

(C) 1

$X$
7782-41-4

75-69-4

944--22-9

50-00-0 


\section{TABLE Z-1-A, part 1. (contd)}

\section{Substance}

Formamide

Formic acid

Furfural

Furfuryl alcohol

Gasoline

Germanium tetrahydride

Glutaraldehyde

Glycerin (mist)

Total dust

Respirable fraction

G]ycidol

Glycol monoethyl ether; see 2-Ethoxyethanol

Grain dust (oat, wheat, barley)

Graphite, natural respirable dust

Graphite, synthetic Total dust

Respirable fraction

Guthion[R]; see Azinphos methy 1

Gypsum

Total dust

Respirable fraction

Hafnium

Heptachlor

Heptane (n-Heptane)

Hexachlorobutadiene

Hexachlorocyclo-

pentadiene

Hexachloroethane

Hexachloronaphthalene

Hexafluoroacetone

$n$-Hexane

Hexane isomers
Transitional

Limits

$P E L$ *

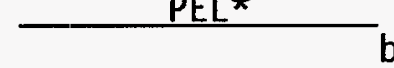

CAS No. [f]

75-12-7

64-18-6

98-01-1

98-00-0

8006-61-9

$7782-65-2$

$111-30-8$

$56-81-5$

$556-52-5$

$\mathrm{ppm}[\mathrm{a}] \mathrm{mg} / \mathrm{m}[3]$

Skin

Designation

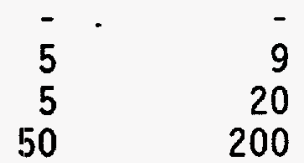

15

50

150

$7782-42-5$

See Table Z-3

15

13397-24-5

$7440-58-6$

$76-44-8$

142-82-5

87-68-3

77-47-4

$67-72-1$

1335-87-1

$684-16-2$

110-54-3

Varies with

compound 
TABLE Z-1-A, part 1. (contd)
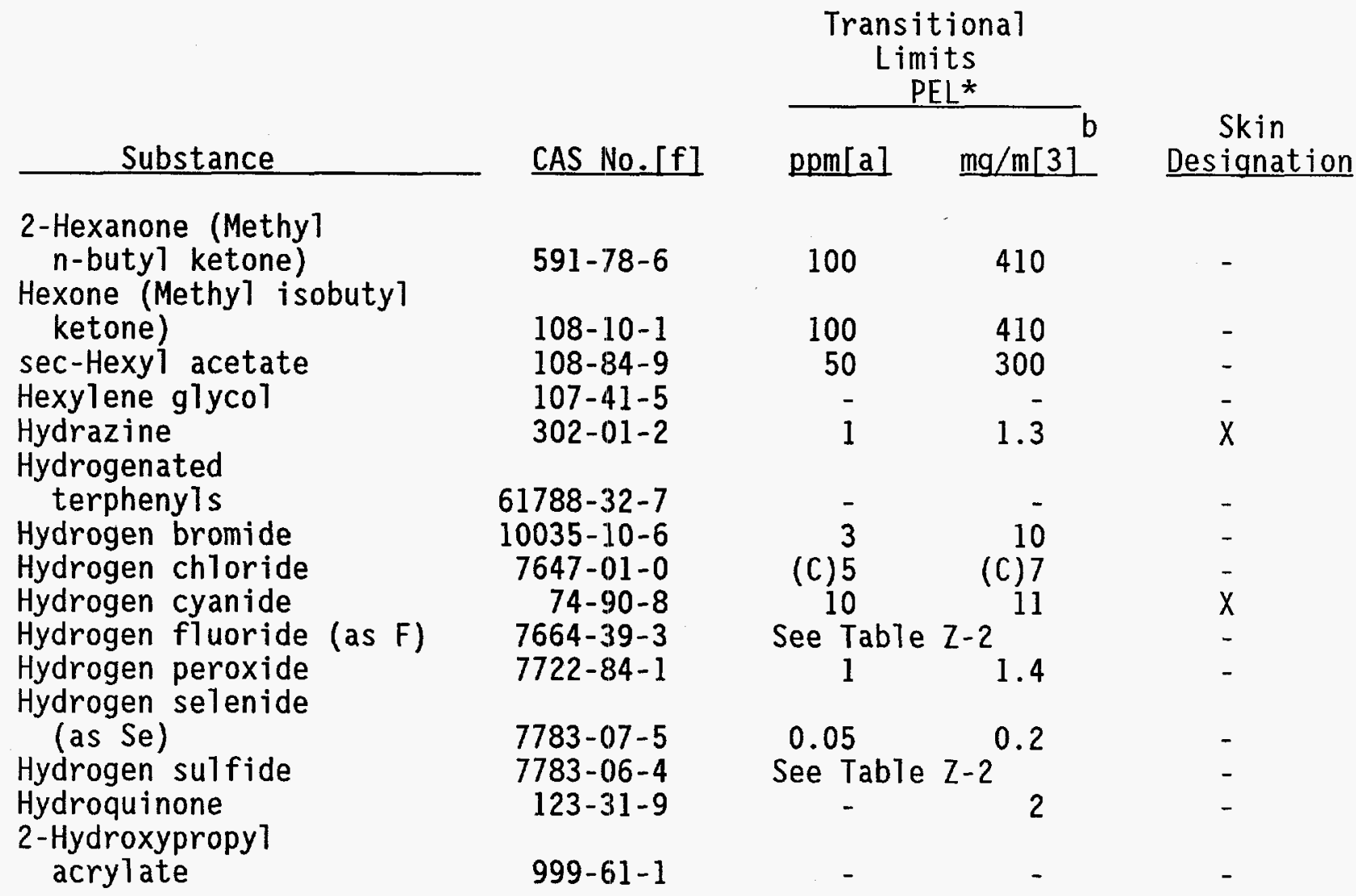

7664-39-3

7722-84-1

7783-07-5

$7783-06-4$

123-31-9

999-61-1

95-13-6

Indene

Indium and compounds

$$
\text { (as In) }
$$

Iodine

Iodoform

Iron oxide fume Total particulate

Iron pentacarbonyl (as

Iron salts (soluble) (as $\mathrm{Fe}$ )

Isoamy 7 acetate Isoamyl alcohol (primary and secondary) Isobuty] acetate Isobuty 7 alcohol Isooctyl alcohol Isophorone

$7440-74-6$ $7553-56-2$

$75-4.7-8$ 1309-37-1

Fe) 13463-40-6

Varies with compound

123-92-2

123-51-3

$110-19-0$

78-83-1

26952-21-6

78-59-1

(C) 0.

(C) 1

100

410

50

300

1.3

Designation

(C) 5

10

(C) 7

See Table Z-2

$\begin{array}{ll}1 & 1.4\end{array}$

0.05

0.2

See Table Z-2

10

100

525

100

360

150

700

100

300

25

140 
TABLE Z-1-A, part 1. (contd)

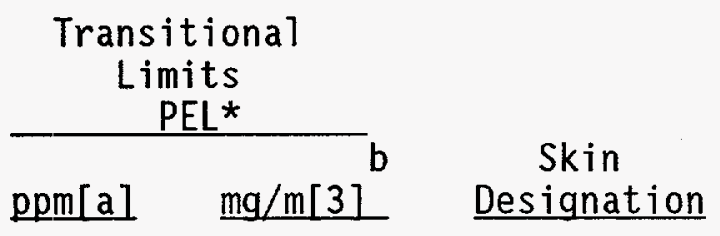

Isophorone diiso-

\section{cyanate}

2-Isopropoxyethanol

Isopropy 1 acetate

Isopropyl alcohol

Isopropyl amine

$\mathrm{N}$-I sopropylan $\mathrm{i} 1 \mathrm{ine}$

Isopropyl ether

Isopropyl glycidyl ether (IGE)

\section{Kaol in \\ Total dust \\ Respirable fraction \\ Ketene}

Lead inorganic (as $\mathrm{Pb}$ ); see 1910.1025

Limestone

Total particulate

Respirable fraction

Lindane

Lithium hydride

L.P.G. (Liquefied petroleum gas)

Magnesite

Total dust

Respirable fraction

Magnesium oxide fume

Total Particulate

Malathion

Total dust

Maleic anhydride

Manganese compounds

(as Mn)

Manganese fume

(as $M n$ )
CAS No. [f]

4098-71-9

109-59-1

108-21-4

$67-63-0$

$75-31-0$

$768-52-5$

108-20-3

4016-14-2

$463-51-4$

7439-92-1

$1317-65-3$

58-89-9

7580-67-8

68476-85-7

546-93-0

$1309-48-4$

$121-75-5$

$108-31-6$

0.25

7439-96-5

7439-96-5

400

50

$0 . \overline{5}$

1000

\section{signation}

950

980

12

2100

240

15

$0.5 \quad 0.9$

15
-
0.5
.025
1800

15

15

15

X

(C) 5

(C) 5 
IABLE Z-1-A, part 1. (contd)

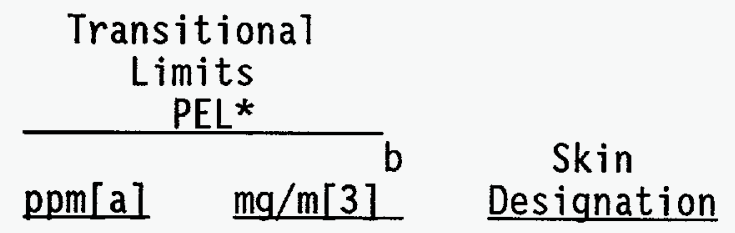

Substance

Manganese cyclopentadienyl tricarbonyl (as $M n$ )

Manganese tetroxide (as Mn)

Marble

Total dust

Respirable fraction

Mercury (aryl and inorganic) (as $\mathrm{Hg}$ )

Mercury (organo)

alkyl compounds (as $\mathrm{Hg}$ )

Mercury (vapor) (as $\mathrm{Hg}$ )

Mesityl oxide

Methacrylic acid

Methanethiol; see Methyl mercaptan

Methomy1 (Lannate)

Methoxychlor Total dust

2-Methoxyethanol; see Methyl cellosolve

4-Methoxyphenol

Methy 7 acetate

Methyl acetylene (Propyne)

Methyl acetylenepropadiene mixture (MAPP)

Methyl acrylate

Methylacrylonitrile

Methylal (Dimethoxymethane)

Methyl alcohol

Methyl amine

Methyl amyl alcohol; see Methyl isobutyl carbinol

Methyl n-amyl ketone

Methyl bromide
CAS No. [f]

12079-65-1

1317-35-7

1317-65-3

\section{ppm}

7439-97-6

7439-97-6

7439-97-6

$141-79-7$

79-4l-4

$16752-77-5$

$72-43-5$
100

15

150-76-5

79-20-9

74-99-7

200

610

1000

1650

96-3.3-3

126-98-7

109-87-5

67-56-1

74-89-5

1000

10

1800

35

1000

200

3100

260

10

12

110-43-0

74-83-9
100

(C) 20
465

(C) 80
15
5

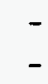$$
\text { - }
$$

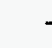


TABLE Z-1-A, part 1. (contd)

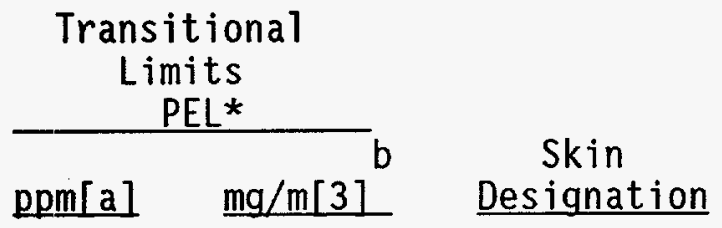

Methyl butyl ketone;

see 2-Hexanone

Methyl cellosolve

(2-Methoxyethanol)

$109-86-4$

25

80

$\mathrm{X}$

Methyl cellosolve acetate (2-Methoxyethy 1 acetate)

Methyl chloride

110-49-6

74-87-3

Methyl chioroform (1,1,1-Trichloroethane)

Methyl 2-cyanoacrylate

Methylcyclohexane

Methylcyclohexanol

o-Methylcyclohexanone

Methyl cyclopentadienyl manganese tricarbony 1 (as Mn)

Methyl demeton

$4,4^{\prime}$-Methylene bis (2-chloroaniline) (MBOCA)

Methylene bis(4-cyclohexyl i socyanate)

Methylene chloride

Methyl ethyl ketone

(MEK); see 2-Butanone

Methyl ethyl ketone peroxide (MEKP)

Methyl formate

Methy 7 hydrazine (Monomethyl hydrazine)

Methyl iodide

Methyl isoamyl ketone

Methyl isobutyl carbinol

Methyl isobutyl ketone; see Hexone

Methyl isocyanate

Methyl isopropyl ketone

$\begin{array}{rrrr}71-55-6 & 350 & 1900 & - \\ 137-05-3 & - & - & - \\ 108-87-2 & 500 & 2000 & - \\ 25639-42-3 & 100 & 470 & - \\ 583-60-8 & 100 & 460 & X\end{array}$

12108-13-3

$8022-00-2$

101-14-4

5124-30-1 75-09-2

1338-23-4

$107-31-3$

60-34-4

74-88-4

$110-12-3$

108-11-2

624-83-9

$563-80-4$
$25 \quad 120 \quad X$

See Table Z-2

See Table Z-2

100

250

(C) 0.2

(C) 0.35

28

100

X

25

0.02

0.05

$x$ 
TABLE Z-1-A, part 1. (contd)

Substance

Methy] mercaptan

Methyl methacrylate

Methyl parathion

Methyl propyl ketone;

see 2-Pentanone

Methyl silicate

alpha-Methyl styrene

Methylene bisphenyl isocyanate (MDI)

Metribuzin

Mica; see Silicates

Molybdenum (as Mo)

Soluble compounds

Insoluble compounds

Total dust

Monocrotophos

(Azodrin[R])

Monomethyl aniline

Morpholine

Naphtha (Coal tar)

Naphthalene

alpha-Naphthyl amine;

see 1910.1004

beta-Naphthyl amine;

see 1910.1009

Nickel carbonyl

(as $\mathrm{Ni}$ )

Nickel, metal and

insoluble

compounds

(as $\mathrm{Ni}$ )

Nickel, soluble

compounds

(as $\mathrm{Ni}$ )

Nicotine

Nitric acid

Nitric oxide

p-Nitroaniline

Nitrobenzene
CAS No. [f]

$$
\begin{array}{r}
74-93-1 \\
80-62-6 \\
298-00-0
\end{array}
$$

$681-84-5$

98-83-9

(C) 100

(C) 480

101-68-8

21087-64-9

7439-98-7

$6923-22-4$

100-61-8

110-91-8

$8030-30-6$

91-20-3

134-32-7

91-59-8

13463-39-3

(C) 0.02

(C) 0.2

Limits

PEL*

$\mathrm{ppm}[\mathrm{a}] \mathrm{mg} / \mathrm{m}[3]$

(C) 20

410

100

Skin

Designation

$\begin{array}{rr}\text { (C) } 0.02 & \text { (C) } 0.2 \\ - & -\end{array}$

5

15

9

70

400

100

10

50

7440-02-0

7440-02-0

54-11-5

7697-37-2

10102-43-9

$100-01-6$

98-95-3

0.001

0.007

$X$
$X$

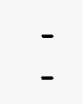

1
0.5

5

30

25

6
5

\begin{tabular}{l}
- \\
$X$ \\
- \\
\hline$X$ \\
$X$
\end{tabular} 
TABLE Z-1-A, part 1. (contd)

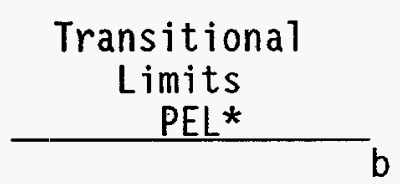

$\mathrm{ppm}[\mathrm{a}] \mathrm{mg} / \mathrm{m}[3]$

CAS No.[f]

100-00-5

92-93-3

4-Nitrodiphenyl; see 1910.1003

Nitroethane

Nitrogen dioxide

Nitrogen trifluoride

Nitroglycerin [1]

Nitromethane

1-Nitropropane

2-Nitropropane

$\mathrm{N}$-Nitrosodimethyl amine; see 1910.1016

Nitrotoluene

0 -isomer

$\mathrm{m}$-isomer

p-isomer

Nitrotrichloromethane; see Chloropicrin

Nonane

79-24-3

10102-44-0

$7783-54-2$

$55-63-0$

$75-52-5$

108-03-2

$79-46-9$

62-79-9

88-72-2;

99-08-1;

99-99-0

111-84-2

Octachloro-

naphthalene

Octane

0il mist, mineral

Osmium tetroxide

$$
\text { (as 0s) }
$$

Oxalic acid

Oxygen difluoride

Ozone

Paraffin wax fume

Paraquat, respirable dust

2234-13-1

$111-65-9$

8012-95-1

20816-12-0

144-62-7

7783-41-7

10028-15-6

8002-74-2

4685-14-7

1910-42-5

2074-50-2

56-38-2

Parathion

Particulates not otherwise regulated

Total dust

Respirable fraction
5

100

(C) 5

10

(C) 0.2

100

25

25

1

310

(C) 9

29

(C) 2

250

90

90

30

500

0.1

2350

5

0.002

0.05

0.1

0.2

0.5

$x$

0.1

$x$

15 $x$

Skin

Designation

$x$

-
-
$X$
-
-

$x$

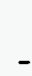

$x$

- 
TABLE Z-1-A, part 1. (contd)

Substance

Pentaborane
Pentachloronaphthalene
Pentachlorophenol
Pentaerythritol
Total dust
Respirable fraction
Pentane
2-Pentanone (Methyl propyl
ketone)
Perchloroethylene
(Tetrachloroethylene)
Perchloromethyl
mercaptan
Perchloryl fluoride
Perlite
Total dust
Respirable fraction
Petroleum distillates
(Naphtha)
Phenol
Phenothiazine
p-Phenylene diamine
Phenyl ether, vapor
Phenyl ether-biphenyl
mixture, vapor
Phenylethylene; see
Styrene
Phenyl glycidyl ether
(PGE)
Phenylhydrazine
Phenyl mercaptan
Phenylphosphine
Phorate
Phosdrin
(Mevinphos[R])
Phosgene (Carbonyl
chloride)
Phosphine
Phosphoric acid
Phosphorus (yellow)
Pothe

CAS No. [f]

$$
\begin{array}{r}
19624-22-7 \\
1321-64-8 \\
87-86-5 \\
115-77-5
\end{array}
$$

$109-66-0$

$107-87-9$

$127-18-4$

594-42-3

$7616-94-6$

108-95-2

92-84-2

106-50-3

$101-84-8$

$122-60-1$

$100-63-0$

$108-98-5$

638-21-1

298-02-2

7786-34-7

$75-44-5$

$7803-51-2$

$7664-38-2$

7723-14-0

\section{Transitional \\ Limits PEL*}

ppm[a] $\mathrm{mg} / \mathrm{m}[3] \quad$ Designation

$\begin{array}{rr}0.005 & 0.01 \\ - & 0.5 \\ - & 0.5\end{array}$

15
5
2950
700

See Table Z-2

\begin{tabular}{rr}
0.1 & 0.8 \\
3 & 13.5 \\
& \\
- & 15 \\
\hline
\end{tabular}

500

2000

$5 \quad 19$

$-$

$-$

1

0.1

1

7

10

5

60

22

$\bar{X}$

$-$

$-$

0.

0.1
0.3

$-$

$-$

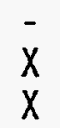

$$
-
$$$$
\begin{aligned}
& - \\
& - \\
& - \\
& - \\
& - \\
& - \\
& X \\
& \bar{x} \\
& -
\end{aligned}
$$ 
TABLE Z-1-A, part 1. (contd)

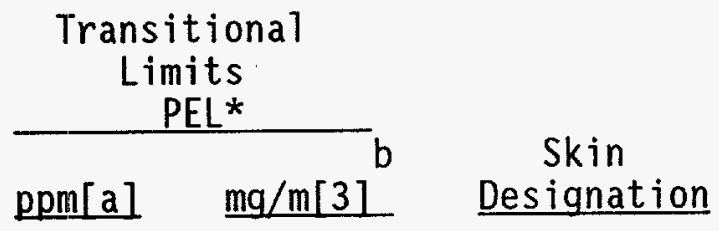

Phosphorus oxychloride

CAS No. [f]

$\mathrm{ppm}[\mathrm{a}] \quad \mathrm{mg} / \mathrm{m}[3]$

Designation

Phosphorus pentachloride

10025-87-3

Phosphorus pentasulfide

10026-13-8

$1314-80-3$

$7719-12-2$

85-44-9

Phthalic anhydride

$626-17-5$
$1918-02-1$

m-Phthalodinitrile

Total dust

Respirable fraction

Picric acid

Piperazine dihydrochloride

Pindone (2-Pivalyl1,3 -indandione)

Plaster of Paris

Total dust

Respirable fraction

$\mathrm{Pl}$ atinum (as Pt)

Metal

Soluble salts

Port 7 and cement

Total dust

Respirable fraction

Potassium hydroxide

Propane

Propargyl alcohol

beta-Propriol actone; see 1910.1013

Propionic acid

88-89-1

142-64-3

83-26-1

26499-65-0

$7440-06-4$

65997-15-1

Propoxur (Baygon)

n-Propy 1 acetate

$n$-Propyl alcohol

n-Propyl nitrate

Propylene dichloride

Propylene glycol

dinitrate

Propylene glycol monomethyl ether

Propylene imine

1310-58-3

74-98-6

$107-19-7$

$57-57-8$

0.1

15

0.1

$x$

79-09-4

114-26-1

$109-60-4$

71-23-8

627-13-4

78-87-5

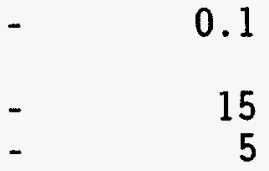

$\begin{array}{ll}- & - \\ - & 0.002\end{array}$

See Table Z-3

See Table Z-3

6423-43-4

107-98-2

$75-55-8$

1000

1800

$-$

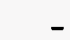

200

840

200

500

25

110

75

350

2

5

X 
TABLE Z-1-A, part 1. (contd)

Substance

Propylene oxide
Propyne; see Methyl
acetylene
Pyrethrum
Pyridine

Quinone

Resorcinol

Rhodium (as Rh), metal fume and insoluble compounds

Rhodium (as Rh), soluble compounds

Ronnel

Rosin core solder pyrolys is products, as formaldehyde

Rotenone

Rouge

Total dust

Respirable fraction

Selenium compounds

(as Se)

Selenium hexafluoride (as Se)

Silica, amorphous, precipitated and gel

Silica, amorphous, diatomaceous earth, containing less than $1 \%$ crystalline silica

Silica, crystalline cristobalite (as quartz), respirable dust

Silica, crystalline quartz (as quartz), respirable dust

\section{Transitional \\ Limits PEL*}

CAS No. [f]

$75-56-9$

8003-34-7

110-86-1

106-51-4

$108-46-3$

$7440-16-6$

7440-16-6

299-84-3

$83-79-4$

-.

$7782-49-2$

7783-79-1

112926-00-8

61790-53-2

0.1

0.4

$\mathrm{ppm}[\mathrm{a}] \mathrm{mg} / \mathrm{m}[3]$

100

240

$\overline{5}$

5
15

0.1

0.001

15

5

15

5

0.2

0.05

0.4

See Table Z-3

See Table Z-3

$14464-46-1$

See Table Z-3

14808-60-7
Skin

Designation

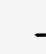


IABLE Z-1-A, part 1. (contd)

Substance

CAS No.[f] ppm[a] $\mathrm{mg} / \mathrm{m}[3]$

Skin

Transitional

Limits

PEL*

\begin{tabular}{l}
\hline Silica, crystalline \\
tripoli (as quartz), \\
respirable dust \\
Silica, crystalline \\
tridymite (as quartz), \\
respirable dust \\
Silica, fused, \\
respirable dust \\
Silicates (less than \\
l\% crystalline silica) \\
Mica (respirable dust) \\
Soapstone, total dust \\
Soapstone, respirable dust \\
Talc (containing asbestos) \\
use asbestos limit \\
Talc (containing no asbes \\
tos), respirable \\
dust \\
Tremolite \\
Silicon \\
Total dust \\
Respirable fraction \\
Silicon carbide \\
Total dust \\
Respirable fraction \\
Silicon tetrahydride \\
Silver, metal and \\
soluble compounds (as Ag) \\
Soapstone; see Silicates \\
Sodium azide \\
(as HN(3)) \\
(as NaN(3)) \\
Sodium bisulfite \\
Sodium fluoroacetate \\
Sodium hydroxide \\
Sodium metabisulfite \\
Starch dot \\
Total dust \\
Respirable fraction \\
\end{tabular}

1317-95-9

See Table Z-3

15468-32-3 See Table Z-3

60676-86-0 See Table Z-3

$12001-26-2$

See Table Z-3

See Table Z-3

See Table Z-3

See Table Z-3

$14807-96-6$

See Table Z-3

See Table Z-3

$7440-21-3$

409-21-2

$7803-62-5$

$7440-22-4$

26628-22-8

$7631-90-5$

$62-74-8$

$1310-73-2$

7681-57-4

9005-25-8

0.01

Designation 
TABLE Z-1-A, part 1. (contd)

\begin{tabular}{|c|c|c|c|c|}
\hline \multirow[b]{2}{*}{ Substance } & \multirow[b]{2}{*}{ CAS No.[f] } & \multicolumn{2}{|c|}{$\begin{array}{c}\text { Transitional } \\
\text { Limits } \\
\text { PEL* } \\
\end{array}$} & \multirow[b]{2}{*}{$\begin{array}{c}\text { Skin } \\
\text { Designation }\end{array}$} \\
\hline & & $\mathrm{ppm}[\mathrm{a}]$ & $\mathrm{mg} / \mathrm{m}[3]^{\mathrm{D}}$ & \\
\hline $\begin{array}{l}\text { Stibine } \\
\text { Stoddard solvent } \\
\text { Strychnine } \\
\text { Styrene } \\
\text { Subtilisins (Proteolytic }\end{array}$ & $\begin{array}{r}7803-52-3 \\
8052-41-3 \\
57-24-9 \\
100-42-5\end{array}$ & $\begin{array}{l}0.1 \\
500 \\
- \\
\end{array}$ & $\begin{array}{l}0.5 \\
2900 \\
0.15 \\
\text { Table Z-2 }\end{array}$ & $\begin{array}{l}- \\
- \\
-\end{array}$ \\
\hline $\begin{array}{l}\text { enzymes) } \\
\text { Sucrose }\end{array}$ & $\begin{array}{r}9014-01-1 \\
57-50-1\end{array}$ & - & - & - \\
\hline $\begin{array}{l}\text { Total dust } \\
\text { Respirable fraction }\end{array}$ & & - & $\begin{array}{r}15 \\
5\end{array}$ & - \\
\hline $\begin{array}{l}\text { Sulfur dioxide } \\
\text { Sulfur hexafluride }\end{array}$ & $\begin{array}{l}7446-09-5 \\
2551-62-4\end{array}$ & 5 & 13 & - \\
\hline Sulfuric acid & $\begin{array}{l}25 b 1-62-4 \\
7664-93-9\end{array}$ & 1000 & $\begin{array}{r}6000 \\
1\end{array}$ & $\begin{array}{l}- \\
-\end{array}$ \\
\hline Sulfur monochloride & $10025-67-9$ & 1 & 6 & - \\
\hline $\begin{array}{l}\text { Sulfur pentafluoride } \\
\text { Sulfur tetrafluoride }\end{array}$ & $\begin{array}{l}5714-22-7 \\
7783-60-0\end{array}$ & 0.025 & 0.25 & - \\
\hline $\begin{array}{l}\text { Sulfuryl fluoride } \\
\text { Sulprofos } \\
\text { Systox[R], see Demeton }\end{array}$ & $\begin{array}{r}2699-79-8 \\
35400-43-2\end{array}$ & 5 & 20 & - \\
\hline $\begin{array}{l}2,4,5-T \\
\text { Talc; see Silicates } \\
\text { Tantalum, metal }\end{array}$ & $93-76-5$ & - & 10 & - \\
\hline $\begin{array}{l}\text { and oxide dust } \\
\text { TEDP (Sulfotep) } \\
\text { Tellurium and }\end{array}$ & $\begin{array}{l}7440-25-7 \\
3689-24-5\end{array}$ & - & $\begin{array}{r}5 \\
0.2\end{array}$ & $\bar{x}$ \\
\hline $\begin{array}{l}\text { compounds (as Te) } \\
\text { Tellurium hexafluoride }\end{array}$ & $13494-80-9$ & - & 0.1 & - \\
\hline $\begin{array}{l}\text { (as Te) } \\
\text { Temephos }\end{array}$ & $\begin{array}{l}7783-80-4 \\
3383-96-8\end{array}$ & 0.02 & 0.2 & - \\
\hline $\begin{array}{l}\text { Total dust } \\
\text { Respirable fraction }\end{array}$ & & - & $\begin{array}{r}15 \\
5\end{array}$ & - \\
\hline $\begin{array}{l}\text { TEPP } \\
\text { Terphenyls } \\
1,1,1,2 \text {-Tetrachloro- }\end{array}$ & $\begin{array}{r}107-49-3 \\
26140-60-3\end{array}$ & (C) $\overline{1}$ & $\begin{array}{l}0.05 \\
\text { (C) } 9\end{array}$ & $x$ \\
\hline $\begin{array}{r}\text { 2,2-difluoroethane } \\
1,1,2,2 \text {-Tetrachloro- }\end{array}$ & $76-11-9$ & 500 & 4170 & - \\
\hline $\begin{array}{r}\text { 1,2-difluoroethane } \\
1,1,2,2 \text {-Tetrachloro- }\end{array}$ & $76-12-0$ & 500 & 4170 & - \\
\hline $\begin{array}{l}\text { ethane } \\
\text { Tetrachoroethylene; } \\
\text { see Perchloroethylene }\end{array}$ & $79-34-5$ & 5 & 35 & $x$ \\
\hline
\end{tabular}


TABLE Z-1-A, part 1. (contd)

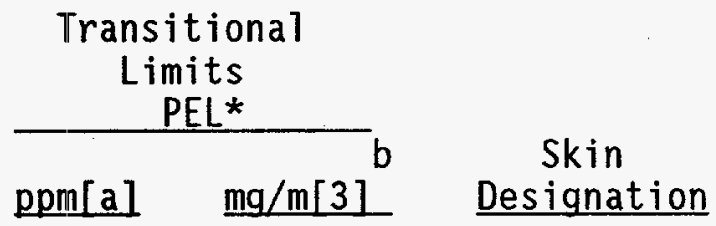

Tetrachloromethane; see Carbon tetrachloride

Tetrachloronaphthalene

Tetraethyl lead (as $\mathrm{Pb}$ )

Tetrahydrofuran

Tetramethyl Tead, (as $\mathrm{Pb}$ )

Tetramethyl succinonitrile

Tetranitromethane

Tetrasodium pyrophosphate

Tetryl $(2,4,6$-Trinitrophenyl-methyl-nitramine)

Thallium, soluble compounds (as T1)

4, 4' -Thiobis(6-tert, Butyl-m-cresol) Total dust Respirable fraction

Thioglycolic acid

Thionyl chloride

Thiram

Tin, inorganic compounds (except oxides) (as Sn)

Tin, organic compounds (as Sn)

Tin oxide (as Sn)

Titanium dioxide Total dust

$1335-88-2$

$3333-52-6$

$7722-88-5$

$7440-28-0$

7719-09-7

$7440-31-5$

7440-31-5

21651-19-4

$13463-67-7$

Toluene

Toluene-2,4-di i socyanate (TDI)

m-Toluidine

o-Toluidine

p-Toluidine

Toxaphene; see Chlorinated camphene

Tremolite; see Silicates

Tributyl phosphate

$126-73-8$
78-00-2

109-99-9

$75-74-1$

509-14-8

$479-45-8$

$96-69-5$

$68-11-1$

$137-26-8$

$108-88-3$

584-84-9

108-44-1

95-53-4

$106-49-0$

(C) 0.02

(C) 0.14

0.075

590

0.075

0.5

1

3

8

1.5

0.1

$X$

\section{See Table Z-2}


TABLE Z-1-A, part 1. (contd)

\section{Substance}

Trichloroacetic acid

1,2,4-Trich7orobenzene

1,1,1-Trich1oroethane; see Methyl chloroform

1,1,2-Trichloroethane

Trichtoroethylene

Trichloromethane; see

Ch7oroform

Trichloronaphthalene

1,2,3-Trich1oropropane

1,1,2-TrichToro-1,2,2trifluoroethane

Triethylamine

Trifluorobromomethane

Trimellitic anhydride

Trimethyl amine

Trimethyl benzene

Trimethyl phosphite

2, 4,6-Trinitrophenyl; see Picric acid

2, 4,6-Trinitropheny Imethyl nitramine; see Tetryl

2, 4,6-Trinitrotoluene (TNT)

Triorthocresy1

phosphate

Triphenyl amine

Triphenyl phosphate

Tungsten (as W) Insoluble compounds Soluble compounds

Turpentine

Uranium (as U)

Soluble compunds

Insoluble compounds
CAS No. [f]

76-03-9

120-82-1

79-00-5

79-01-6

$1321-65-9$

$96-18-4$

76-13-1

$121-44-8$

75-63-8

$552-30-7$

$75-50-3$

25551-13-7

$121-45-9$

Transitional

Limits

$\frac{\mathrm{PEL}^{*}}{b}$

$\mathrm{ppm}[\mathrm{a}] \mathrm{mg} / \mathrm{m}[3]$

Skin

Designation

10

45

See Table Z-2

5

300

50

7600

1000

100

1000

6100

$-$

118-96-7

78-30-8

$603-34-9$

115-86-6

7440-33-7

$8006-64-2$

7440-61-1 
TABLE Z-1-A, part 1. (contd)

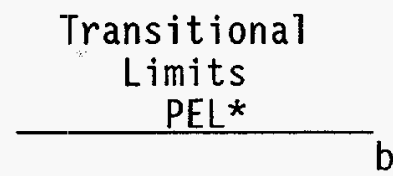

CAS No.[f]

$110-62-3$

$1314-62-1$

n-Valeraldehyde

Vanadium

Respirable dust

(as V(2)0(5))

Fume (as V(2)0(5))

Vegetable oil mist

Total dust

Respirable fraction

Vinyl acetate

Vinyl benzene;

see Styrene

Vinyl bromide

Vinyl chloride; see 1910.1017

Vinylcyanide; see Acrylonitrile

Vinyl cyclohexene

dioxide

Vinylidene chloride

(1,1-Dichloroethylene)

$V$ inyl toluene

VM \& P Naphtha

Warfarin

Welding fumes (total

particulate) $* * *$

Wood dust,

all soft and hard

woods, except Western

red cedar

Wood dust,

Western red cedar

Xylenes (o-, m-, pisomers)

m-Xylene alpha, alpha' -

diamine

$1330-20-7$

1477-55-0

Xylidine

$593-60-2$

75-01-4

$106-87-6$

75-35-4

25013-15-4

8032-32-4

$81-81-2$

$\begin{array}{ll}- & \text { (C) } 0.5 \\ -- & \text { (C) } 0.1\end{array}$

$\begin{array}{lr}- & 15 \\ - & 5\end{array}$

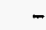

100

480

$\begin{array}{lr}- & 0.1 \\ - & -\end{array}$

100

435

5

25 ppm[a] $\mathrm{mg} / \mathrm{m}[3]$

Skin

Designation

$x$ 
IABLE 2-1-A, part 1. (contd)

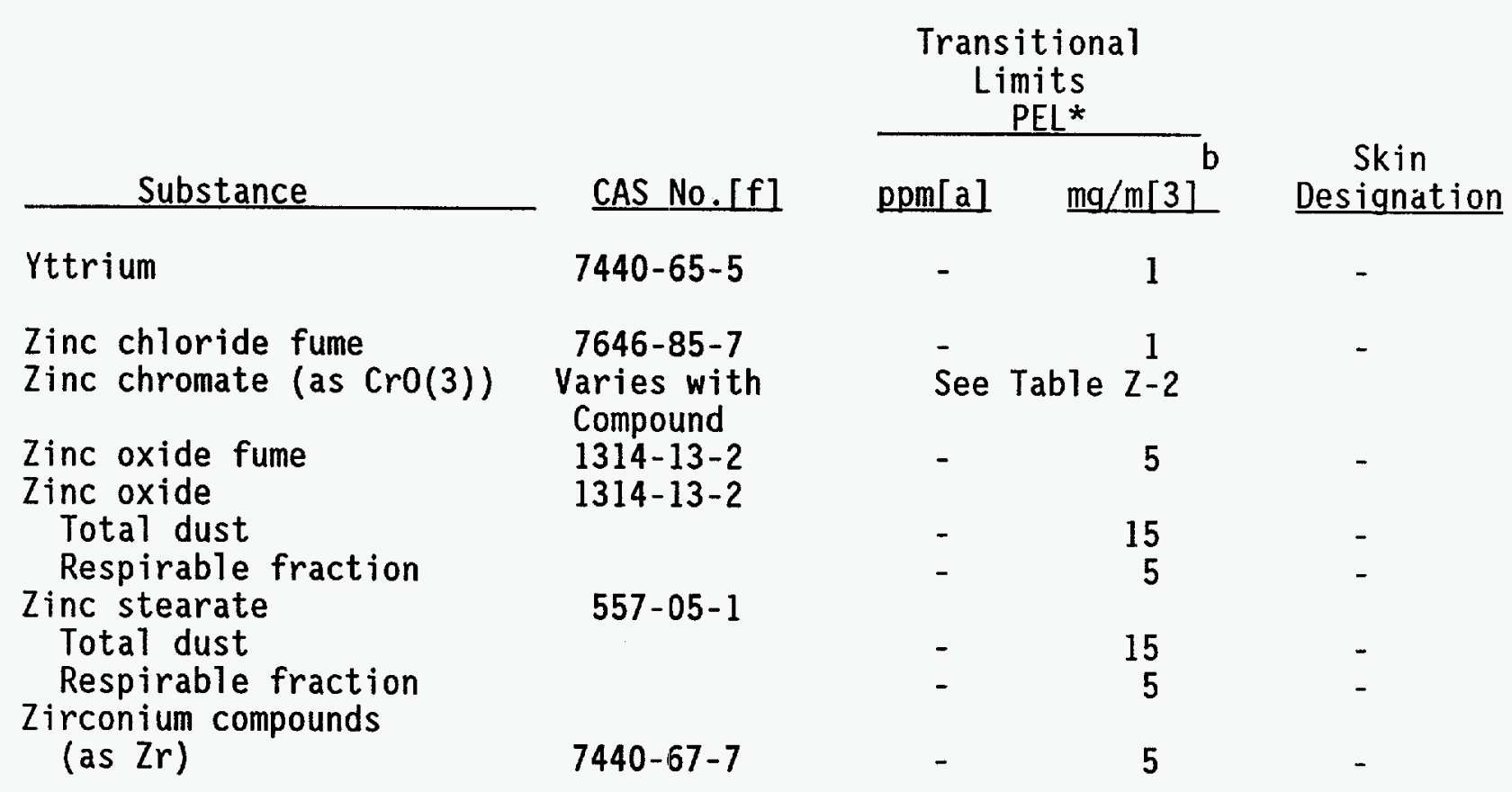


TABLE Z-1-A, part 2. Limits For Air Contaminants

Final Rule Limits**

Substance

Acetaldehyde

Acetic acid

Acetic anhydride

Acetone [h]

Acetonitrile

2-Acetyl aminofluorine;

see 1910.1014

Acetylene dichloride;

see 1,2-Dichloroethylene

Acetylene tetrabromide

Acetylsalicylic acid

(Aspirin)

Acrolein

Acrylamide

Acrylic acid

Acrylonitrile;

see 1910.1045

Allyl alcohol

Allyl chloride

Al lyl glycidyl ether

(AGE)

Allyl propyl disulfide

a) pha-Alumina

Total dust

Respirable fraction

Aluminum (as Al)

Meta]

Total dust

Respirable fraction

Pyro powders

Welding fumes***

Soluble salts

Alkyls

4-Aminodipheny1;

see 1910.1011

2-Aminoethanol;

see Ethanolamine

2-Aminopyridine

Amitrole

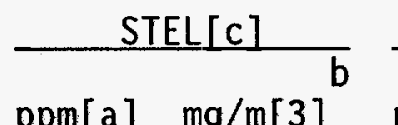

$\mathrm{ppm}[\mathrm{a}] \mathrm{mg} / \mathrm{m}[3]$

$\mathrm{ppm}[\mathrm{a}] \mathrm{mg} / \mathrm{m}[3]$

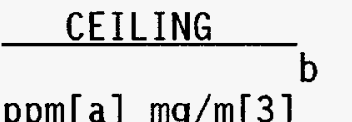

Skin

Desig-

nation

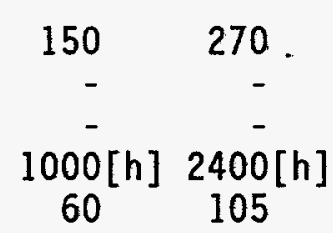

14

$\begin{array}{rr}100 & 180 \\ 10 & 25 \\ - & - \\ 750 & 1800 \\ 40 & 70\end{array}$

5

0.1

0.25

$-$

0.03

10

30

0.25

2

5

3

$5 \quad 22$

22

10

$\begin{array}{rr}- & - \\ 0.3 & 0.8 \\ - & - \\ - & -\end{array}$

10

5

15

5

5

2

0.5

2
0.2

$x$

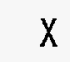

2

10

44

$\begin{array}{ll}3 & 18\end{array}$

$x$
$x$

Aldrin 
TABLE Z-1-A, part 2. (contd)

Final Rule Limits**

TWA

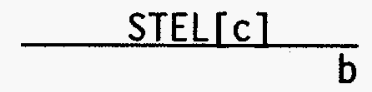

CEILING $b$

Skin

Substance

$\mathrm{ppm}[\mathrm{a}] \mathrm{mg} / \mathrm{m}[3]$

$\mathrm{ppm}[\mathrm{a}] \mathrm{mg} / \mathrm{m}[3]$

$\mathrm{ppm}[\mathrm{a}] \mathrm{mg} / \mathrm{m}[3]$

Desig-

nation

Ammonia

Ammonium chloride

$$
\text { fume }
$$

Ammonium sulfamate Total dust

Respirable fraction

$n$-Amyl acetate

sec-Amyl acetate

Aniline and homologs

Anisidine (o-, $p$-isomers)

Antimony and compounds (as Sb)

ANTU (Alpha Naphthy1thiourea)

Arsenic, organic compounds (as As)

Arsenic, inorganic compounds (as As); see 1910.1018

Arsine

Asbestos; see $1910.1001 \& 1910.1101$

Atrazine

Azinphos-methyl

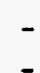

Barium, soluble compounds (as $\mathrm{Ba}$ )

Barium sulfate

Total dust

Respirable fraction

Benomy

Total dust

Respirable fraction

Benzene;

see 1910.1028

Benzidine;

see 1910.1010

p-Benzoquinone;

see Quinone

0.05
$35 \quad 27$

$\begin{array}{rrrrrrr}- & 10 & - & 20 & - & - & - \\ - & 10 & - & - & - & - & - \\ - & 5 & - & - & - & - & - \\ 100 & 525 & - & - & - & - & - \\ 125 & 650 & - & - & - & - & - \\ 2 & 8 & - & - & - & - & X \\ - & 0.5 & - & - & - & - & X\end{array}$

0.5

0.3

0.5

0.2

- 5

0.2

$\begin{array}{ll}- & 0.5\end{array}$

$\begin{array}{rr}- & 10 \\ - & 5\end{array}$

- $\quad 10$

See Table Z-2 for the limits applicable in the operations or sectors excluded in 1910.1028[d] 
TABLE Z-1-A, part 2. (contd)

Final Rule Limits**

TWA

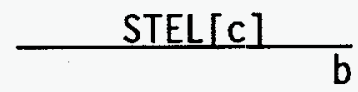

$\begin{array}{cl}\text { CEILING } & \text { Skin } \\ & \text { Desig- }\end{array}$

Substance

$\mathrm{ppm}[\mathrm{a}] \mathrm{mg} / \mathrm{m}[3]$

$\mathrm{ppm}[\mathrm{a}] \mathrm{mg} / \mathrm{m}[3]$ nation

Benzo(a)pyrene;

see Coal tar pitch

volatiles

Benzoyl peroxide

Benzyl chloride

$\begin{array}{ll}- & 5 \\ 1 & 5\end{array}$

Beryllium and beryllium See Table Z-2 compounds (as Be)

Biphenyl; see Diphenyl

Bismuth telluride, Undoped

Total dust

Respirable fraction

Bismuth telluride,

Se-doped

Borates, tetra, sodium salts

Anhydrous

Decahydrate

Pentahydrate

Boron oxide

Total dust

Respirable fraction

Boron tribromide

Boron trifluoride

Bromacil

Bromine

Bromine pentafluoride

Bromoform

Butadiene (1,3-

Butadiene)

Butane

Butanethiol; see Butyl mercaptan

2-Butanone (Methyl ethyl ketone)

2-Butoxyethanol

n-Buty 1 -acetate

sec-Butyl acetate

tert-Butyl acetate

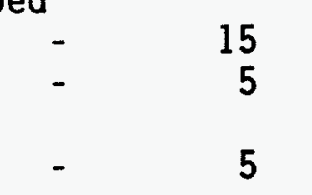

$\begin{array}{ll}- & 10 \\ - & 10 \\ - & 10\end{array}$

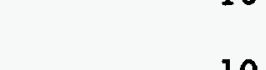

$\begin{array}{rr}- & 10 \\ - & - \\ - & - \\ - & - \\ 0.1 & 10 \\ 0.1 & 0.7 \\ 0.5 & 0.7 \\ 100 & 5\end{array}$

$1000 \quad 2200$

$800 \quad 1900$

$200 \quad 590$

$25 \quad 120$

$150 \quad 710$

$200 \quad 950$

$200 \quad 950$
300

885

200

950 
TABLE Z-1-A, part 2. (contd)

Final Rule Limits**

Substance

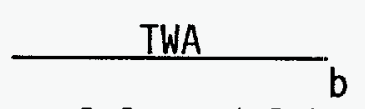

$\mathrm{ppm}[\mathrm{a}] \mathrm{mg} / \mathrm{m}[3]$

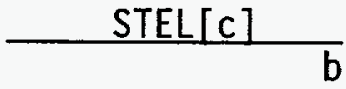

$\mathrm{ppm}[\mathrm{a}] \mathrm{mg} / \mathrm{m}[3]$

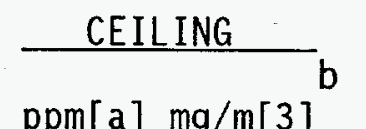

Desig-

nation

$\mathrm{ppm}[\mathrm{a}] \mathrm{mg} / \mathrm{m}[3]$

$\begin{array}{lr}\text { Butyl acrylate } & 10 \\ \text { n-Butyl alcohol } & \\ \text { sec-Butyl alcohol } & 100 \\ \text { tert-Butyl alcohol } & 100 \\ \text { Butylamine } & \\ \text { tert-Butyl chromate } \\ \text { (as Cro(3)) } \\ \text { n-Butyl glycidyl ether } \\ \text { (BGE) } \\ \text { n-Butyl lactate } \\ \text { Butyl mercaptan } \\ \text { o-sec-Butylphenol } \\ \text { p-tert-Butyltoluene } \\ \text { Cadmium fume (as Cd) } \\ \text { Cadmium dust (as Cd) } \\ \text { Calcium carbonate } \\ \text { Total dust } \\ \text { Respirable fraction } \\ \text { Calcium cyanamide } \\ \text { Calcium hydroxide[i] } \\ \text { Calcium oxide[j] } \\ \text { Calcium silicate } \\ \text { Total dust } \\ \text { Respirable fraction } \\ \text { Calcium sulfate } \\ \text { Total dust } \\ \text { Respirable fraction } \\ \text { Camphor, synthetic } \\ \text { Caprolactam } \\ \text { Dust } \\ \text { Vapor }\end{array}$

Captafol (Difolatan[R])

Captan

Carbaryl (Sevin[R])

$\begin{array}{rr}10 & 55 \\ - & - \\ 100 & 305 \\ 100 & 300\end{array}$

$\bar{x}$

135

$5 \quad 25$

$0.5 \quad 1.5$

$5 \quad 30$

$10 \quad 60$

0.1

0.2

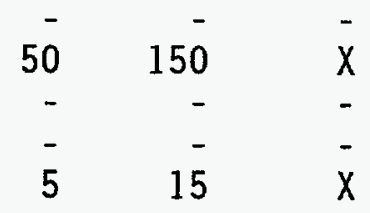

$0.1 \quad x$

Carbofuran (Furadan[R])

Carbon black

Carbon dioxide

Carbon disulfide

Carbon monoxide

Carbon tetrabromide

\begin{tabular}{|c|c|c|}
\hline 5 & 20 & 10 \\
\hline - & 0.1 & \\
\hline & 5 & \\
\hline & & \\
\hline & 0.1 & \\
\hline, 000 & 18,000 & 30,000 \\
\hline $\begin{array}{r}4 \\
35\end{array}$ & $\begin{array}{l}12 \\
40\end{array}$ & 12 \\
\hline 0.1 & 1.4 & 0. \\
\hline
\end{tabular}

15
5

0.5

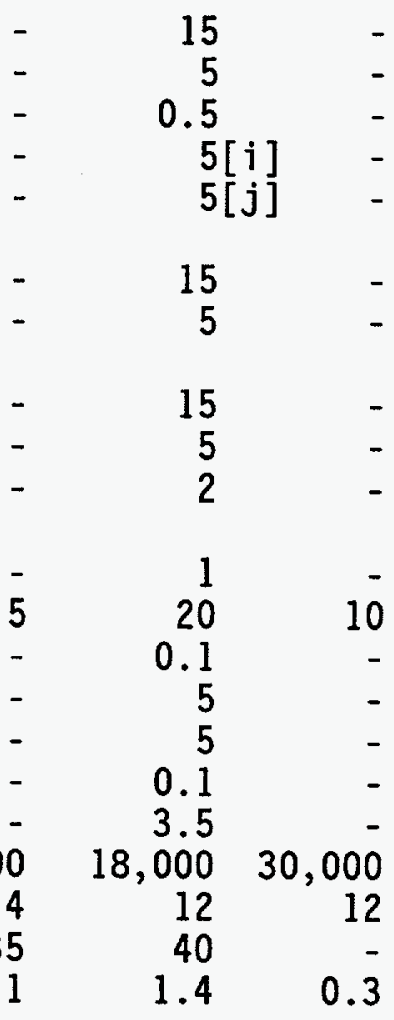

20

450

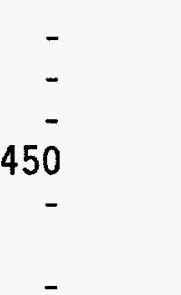

$x$

0.3

0.6

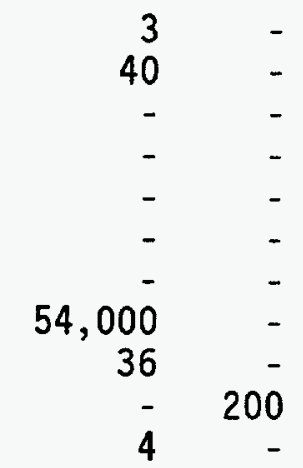

A. 38 
TABLE Z-1-A, part 2. (contd)

Final Rule Limits**

Substance

Carbon tetrachloride

Carbonyl fluoride

Catechol

(Pyrocatechol)

Cellulose

Total dust

Respirable fraction

Cesium hydroxide

Chlordane

Chlorinated camphene

Chlorinated diphenyl oxide

Chlorine

Chlorine dioxide

Chlorine trifluoride

Chloroacetaldehyde

a-Chloroacetophenone

(Phenacyl chloride)

Chioroacetyl chloride

$\mathrm{Ch}$ lorobenzene

o-Chlorobenzylidene

malononitrile

Chlorobromomethane

2-Chloro-1,3-butadiene; see b-Chioroprene

Chlorodifluoromethane 1000

Chlorodiphenyl (42\%

Chlorine) (PCB)

Chlorodipheny 1 (54\%

Chlorine) (PCB)

1-Chloro,2,3-epoxypropane;

see Epichlorohydrin

2-Chloroethanol;

see Ethylene chlorohydrin

Chloroethylene;

see Vinyl chloride

Chloroform

(Trichloromethane)

0.05

0.05

75

TWA

STEL $[c]$

CEILING

Skin

Desig-

$\mathrm{ppm}[\mathrm{a}] \mathrm{mq} / \mathrm{m}[3]$ ppm[a] $\mathrm{mq} / \mathrm{m}[3]$ nation

$0.5 \quad 1.5$

$0.1 \quad 0.3$

12.6

5

5

15

$5 \quad 20$

$-\quad 15$

$-\quad 5$

-
$-\quad 0.5$
$-\quad 0.5$

$-\quad 0.5$

0.5

0.5

.3

0.3

3
0.9

-

nation 
TABLE Z-1-A, part 2. (contd)

Final Rule Limits**

TWA

Substance

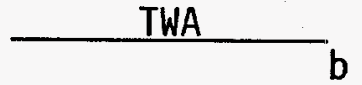

$\frac{\mathrm{STEL}[\mathrm{C}]}{\mathrm{b}}$

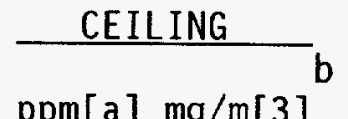

Skin

$\mathrm{ppm}[\mathrm{a}] \mathrm{mg} / \mathrm{m}[3]$

$\mathrm{ppm}[\mathrm{a}] \mathrm{mg} / \mathrm{m}[3] \mathrm{ppm}[\mathrm{a}] \mathrm{mg} / \mathrm{m}[3]$

Desig-

nation

Chloromethyl methyl ether; see 1910.1006

1-Chloro-1-nitropropane 2

Chloropentafluoro-

ethane

1000

Chloropicrin

0.1

beta-Chloroprene

0 -Chlorostyrene

10

50

0 -Chlorotoluene

2-Chloro-6-trichloro-

50

6320

0.7

35

285

250

methyl pyridine

Total dust

Respirable fraction

15

Chlorpyrifos

Chromic acid and chromates (as $\mathrm{CrO}(3)$ )

Chromium (II) compounds (as $\mathrm{Cr}$ )

Chromium (III) compounds (as $\mathrm{Cr}$ )

Chromium metal (as $\mathrm{Cr}$ )

Chrysene;

see Coal tar pitch

volatiles

Clopidol

Total dust

Respirable fraction

Coal dust (less than

$5 \% \mathrm{SiO}(2))$,

Respirable fraction

Coal dust (greater than

or equal to $5 \% \mathrm{SiO}(2)$ ),

Respirable quartz

fraction

Coal tar pitch vola-

tiles (benzene sol-

uble fraction), anthra-

cene, BaP, phenan-

threne, acridine,

chrysene, pyrene

0.2

5
0.2

0.5

0.5

1

75

428

$\bar{X}$

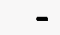$$
-
$$ 
TABLE Z-1-A, part 2. (contd)

Final Rule Limits**

TWA

Substance
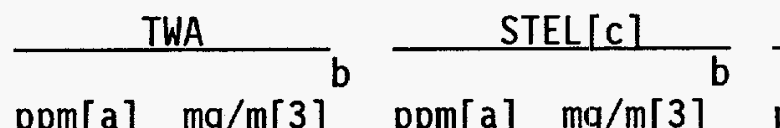

CEILING

Skin ppm[a] $\mathrm{mg} / \mathrm{m}[3]$

ppm[a] $\mathrm{mg} / \mathrm{m}[3]$

Desig-

Cobalt metal, dust, and fume (as Co)

Cobalt carbonyl (as Co)

Cobalt hydrocarbonyl (Co)

Coke oven emissions;

see 1910.1029

Copper

Fume (as $\mathrm{Cu}$ )

Dusts and mists (as $\mathrm{Cu}$ )

Cotton dust (raw),

0.05

0.1

0.1

This 8-hour TWA applies to respirable dust as measured by a vertical

elutriator cotton dust sampler or equivalent instrument. The time-weighted average applies to the cotton waste processing operations of waste recycling (sorting, blending, cleaning, and willowing) and garretting. See also

1910.1043 for cotton dust limits applicable to other sectors.

Crag herbicide (Sesone)

Total dust

Respirable fraction

Cresol, all isomers

Crotonaldehyde

Crufomate

Cumene

Cyanamide

Cyanides (as $\mathrm{CN}$ )

Cyanogen

Cyanogen chloride

Cyclohexane

Cyclohexanol

Cyclohexanone

0.1

cyclohexene

Cyclohexyl amine

Cyclonite

Cyclopentadiene

Cyclopentane

cyhexatin

$\begin{array}{rrrrrrr}- & 10 & - & - & - & - & - \\ - & 5 & - & - & - & - & - \\ 5 & 22 & - & - & - & - & X \\ 2 & 6 & - & - & - & - & - \\ - & 5 & - & - & - & - & - \\ 50 & 245 & - & - & - & - & X \\ - & 2 & - & - & - & - & - \\ - & 5 & - & - & - & - & - \\ 10 & 20 & - & - & - & - & - \\ - & - & - & - & 0.3 & 0.6 & - \\ 300 & 1050 & - & - & - & - & - \\ 50 & 200 & - & - & - & - & X \\ 25 & 100 & - & - & - & - & X \\ 300 & 1015 & - & - & - & - & - \\ 10 & 40 & - & - & - & - & - \\ - & 1.5 & - & - & - & - & - \\ 75 & 200 & - & - & - & - & - \\ 600 & 1720 & - & - & - & - & - \\ - & 5 & - & - & - & & \end{array}$




\section{TABLE Z-1-A, part 2. (contd)}

Final Rule Limits**

Substance
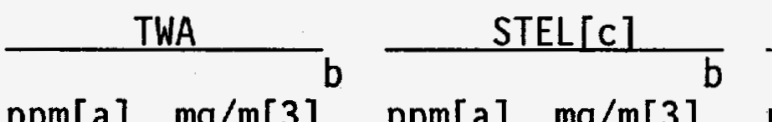

CEILING

Skin

$\mathrm{ppm}[\mathrm{a}] \mathrm{mg} / \mathrm{m}[3] \mathrm{ppm}[\mathrm{a}] \mathrm{mg} / \mathrm{m}[3] \mathrm{ppm}[\mathrm{a}] \mathrm{mg} / \mathrm{m}[3]$ nation

$2,4-D$

(Dichlorylphenoxyacetic acid)

Decaborane

10

Demeton (Systox[R])

0.05

0.3

Dichlorodiphenyltri-

0.1

chloroethane (DDT)

Dichlorvos (DDVP)

Diacetone alcohol

(4-Hydroxy-4-methyl2 -pentanone)

50

240

1,2-Diaminoethane;

see Ethylenediamine

Diazinon

Diazomethane

Diborane

0.2

0.1

1,2-Dibromo-

0.1

0.4

3-chloropropane;

see 1910.1044

2-N-Dibutyl aminoethanol

Dibutyl phosphate

Dibutyl phthalate

Dichloroacetylene

0 -Dichlorobenzene

p-Dichlorobenzene

3, 3'-Dichlorobenzidine;

see 1910.1007

Dichlorodifluoromethane

1,3-Dich7oro-5,5dimethyl hydanto in

1,1-Dichloroethane

1,2-Dichloroethylene

Dichloroethyl ether

Dichloromethane; see Methylene chloride

Dichloromonofluoromethane

10

0.1

0.15

0.9

$-$

$-$

1

1,1-Dich7oro-1-nitroethane

0.2

400

200

790

5

30

0.4

10

10

2

$-$

$-$

110

675

0.1

50

0.4

300

210


IABLE Z-1-A, part 2. (contd)

Final Rule Limits**

Substance
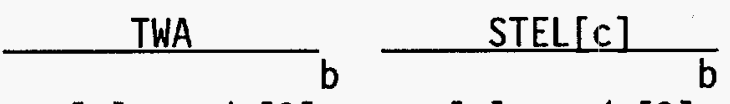

CEILING

Skin

Desigppm[a] $\mathrm{mg} / \mathrm{m}[3]$ ppm[a] $\mathrm{mg} / \mathrm{m}[3]$ ppm[a] $\mathrm{mg} / \mathrm{m}[3]$ nation

1,2-Dichloropropane;

$$
\text { see Propylene }
$$

dichloride

1,3-Dichl oropropene

2,2-Dichloropropionic acid

Dichlorotetrafluoroethane

Dicrotophos

Dicyclopentadiene

Dicyclopentadienyl iron Total dust

Respirable fraction

Dieldrin

Diethanol amine

Diethylamine

2-Diethy 1 aminoethanol

Diethylene triamine

Diethyl ether; see Ethyl ether

Diethyl ketone

Diethyl phthalate

Difluorodibromomethane

Diglycidyl ether (DGE)

Dihydroxybenzene; see Hydroquinone

Di isobutyl ketone

Di i sopropylamine

$\begin{array}{rrrrrrr}1 & 5 & - & - & - & - & X \\ 1 & 6 & - & - & - & - & - \\ 1000 & 7000 & - & - & - & - & - \\ - & 0.25 & - & - & - & - & X \\ 5 & 30 & - & - & - & - & - \\ - & 10 & - & - & - & - & - \\ - & 5 & - & - & - & - & - \\ - & 0.25 & - & - & - & - & X \\ 3 & 15 & - & - & - & - & - \\ 10 & 30 & 25 & 75 & - & - & - \\ 10 & 50 & - & - & - & - & x \\ 1 & 4 & - & - & - & - & -\end{array}$

4-Dimethy 7 aminoazobenzene; see 1910.1015

Dimethoxymethane; see Methylal

Dimethyl acetamide

Dimethyl amine

200

705

100

5

$0.1 \quad 0.5$

Dimethyl aminobenzene; see Xylidine

Dimethylaniline

(N-Dimethyl-aniline) 
TABLE Z-1-A, part 2. (contd)

Final Rule Limits**

TWA

Substance
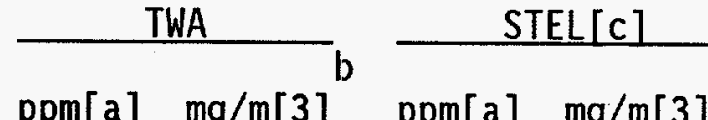

$\frac{\text { CEILING }}{b}$

Skin

Desig-

$\mathrm{ppm}[\mathrm{a}] \mathrm{mg} / \mathrm{m}[3] \quad \mathrm{ppm}[\mathrm{a}] \mathrm{mg} / \mathrm{m}[3] \quad \mathrm{ppm}[\mathrm{a}] \mathrm{mg} / \mathrm{m}[3]$ nation

Dimethylbenzene; see Xylene

Dimethyl-1,2-dibromo2,2-dichioroethyl phosphate

Dimethyl formamide

2,6-Dimethy1-4-heptanone; see Dijsobutyl ketone

1,1-Dimethylhydrazine

Dimethylphthalate

Dimethyl sulfate

Dinitolmide $(3,5$ -

Dinitro-o-toluamide)

Dinitrobenzene (a11 isomers)

Dinitro-o-cresol

Dinitrotoluene

Dioxane (Diethylene dioxide)

Dioxathion (Delnav)

Diphenyl (Biphenyl)

Diphenyl amine

Diphenylmethane diisocyanate; see Methylene

0.5

$\begin{array}{rr}- & 3 \\ 10 & 30\end{array}$

$\begin{array}{rr}- & 5 \\ 0.1 & 0.5\end{array}$

bisphenyl isocyanate

Dipropylene glycol

methyl ether

Dipropyl ketone

Diquat

Di-sec octyl phthalate

(Di-2-ethylhexy]-

phthalate)

Disulfiram

Disulfoton

2,6-Di-tert-buty1-p-

cresol

Diuron

Divinyl benzene

0.5

150

900

$X$
$X$

0.2

1.5

$\begin{array}{rr}25 & 90 \\ - & 0.2\end{array}$

0.2

1

10

50
$-\quad 0.5$

-

$\begin{array}{rr}- & 5 \\ - & 2 \\ - & 0.1 \\ - & 10 \\ - & 10 \\ 10 & 50\end{array}$


TABLE Z-1-A, part 2. (contd)

Final RuTe Limits**

TWA $b$

Substance

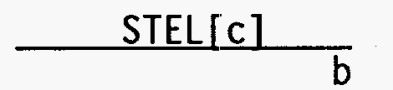

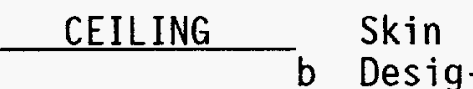

ppm[a] $\mathrm{mg} / \mathrm{m}[3] \quad \operatorname{ppm}[\mathrm{a}] \mathrm{mg} / \mathrm{m}[3] \quad \mathrm{ppm}[\mathrm{a}] \mathrm{mg} / \mathrm{m}[3]$ nation

Emery

Total dust

Respirable fraction

Endosulfan

Endrin

Epichlorohydrin

EPN

1,2-Epoxypropane; see

Propylene oxide

2,3-Epoxy-1-propanol; see Glycidol

Ethanethiol; see Ethyl mercaptan

$\begin{array}{lr}- & 10 \\ - & 5\end{array}$

- $\quad 0.1$

0.1

$\overline{2}$

8
0.5

2
$-\quad 0.5$

Ethanolamine

Ethion

2-Ethoxyethanol

2-Ethoxyethyl acetate

(Cellosolve acetate)

Ethyl acetate

Ethyl acrylate

Ethyl alcohol (Ethanol)

Ethyl amine

Ethyl amyl ketone

(5-Methy 1-3-heptanone)

Ethyl benzene

Ethyl bromide

Ethyl butyl ketone (3-Heptanone)

Ethyl chloride

Ethyl ether

Ethyl formate

Ethyl mercaptan

Ethyl silicate

Ethylene chlorohydrin

Ethylenediamine

Ethylene dibromide

Ethylene dichloride

Ethylene glycol

$\begin{array}{ll}3 & \\ - & 0.4\end{array}$

$200 \quad 740$

$100 \quad 540$

400

5

1000

10

25

100

200

50

1000

400

100

0.5

10

10

Ethylene glycol

dinitrate[k] 
TABLE Z-1-A, part 2. (contd)

Final Rule Limits**

Substance

TWA
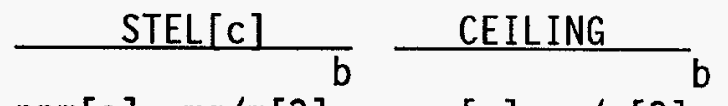

Skin

Desig-

ppm[a] $\mathrm{mg} / \mathrm{m}[3]$ ppm[a] $\mathrm{mg} / \mathrm{m}[3]$ ppm[a] $\mathrm{mg} / \mathrm{m}[3]$ nation

Ethylene glycol methyl

acetate; see Methyl

cellosolve acetate

Ethyleneimine;

see 1910.1012

Ethylene oxide;

see 1910.1047

Ethylidene chloride; see 1,1-Dichloroethane

Ethylidene norbornene

$\mathrm{N}$-Ethylmorphol ine

23

Fenamiphos

Fensulfothion

(Dasanit)

Fenthion

Ferbam

Total dust

Ferrovanadium dust

Fluorides (as F)

Fluorine

Fluorotrichloro-

0.1

0.1

methane (Trichloro-

fluoromethane)

Fonofos

Formaldehyde; see 1910.1048;

Formamide

Formic acid

Furfural

Furfuryl alcohol

Gasoline

Germanium tetrahydride

Glutaraldehyde

Glycerin (mist)

Total dust

Respirable fraction

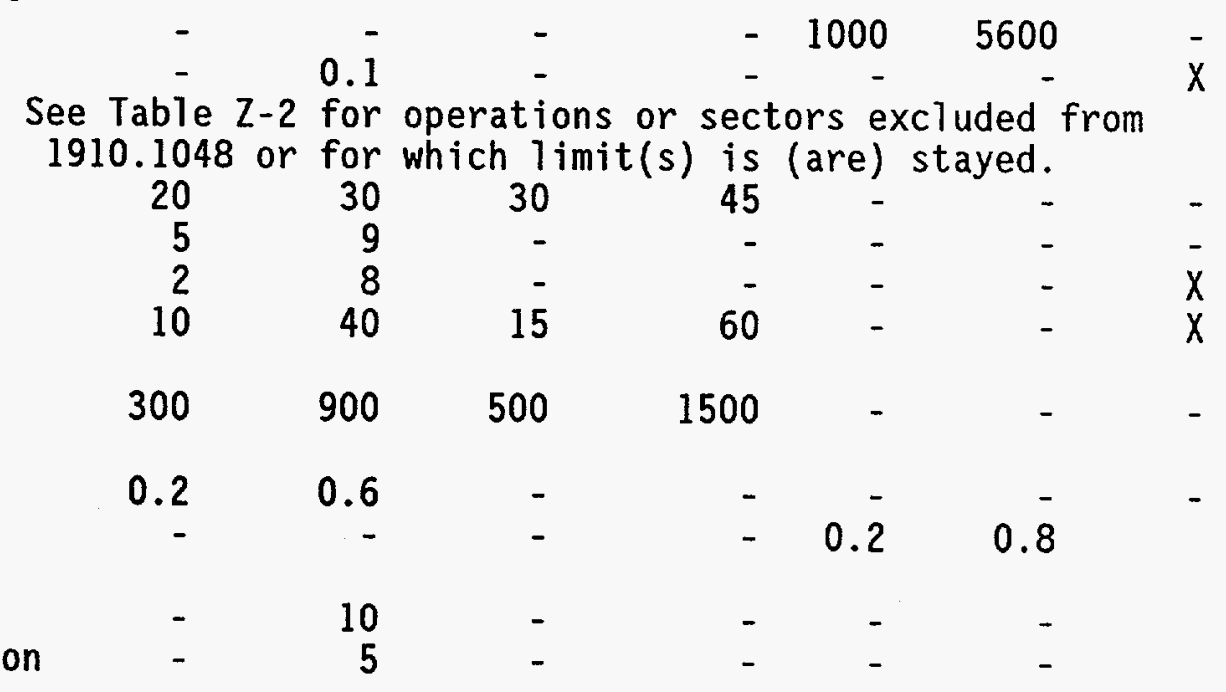


TABLE Z-1-A, part 2. (contd)

Final Rule Limits**

Substance
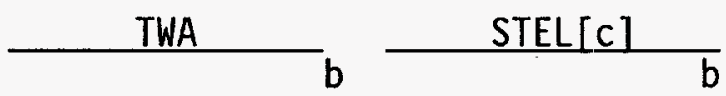

CEILING $b$

Skin

ppm[a] $\mathrm{mg} / \mathrm{m}[3]$

$\mathrm{ppm}[\mathrm{a}] \mathrm{mg} / \mathrm{m}[3]$

Desig-

Glycidol

25

75

Glycol monoethyl ether; see 2-Ethoxyethanol

Grain dust (oat, wheat, barley)

Graphite, natural

respirable dust

Graphite, synthetic

Total dust

Respirable fraction

Guthion $[R]$; see Azinphos methy 1

Gypsum

Total dust

Respirable fraction

Hafnium

Heptachlor

Heptane (n-Heptane)

Hexachlorobutadiene

Hexachlorocyclo-

$$
\text { pentadiene }
$$

Hexachloroethane

Hexachl oronaphthalene

Hexafluoroacetone

$\mathrm{n}$-Hexane

Hexane isomers

2-Hexanone (Methy1

n-butyl ketone)

Hexone (Methyl isobuty ketone)

sec-Hexyl acetate

Hexylene glycol

Hydrazine

Hydrogenated

terphenyls

Hydrogen bromide

Hydrogen chloride

Hydrogen cyanide

$\mathrm{ppm}[\mathrm{a}] \mathrm{mg} / \mathrm{m}[3]$ nation 
TABLE Z-1-A, part 2. (contd)

Final RuTe Limits**

TWA

Substance
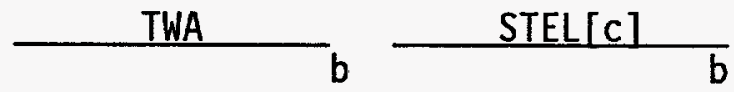

$\frac{\text { CEILING }}{\mathrm{ppm}[\mathrm{a}] \mathrm{mg} / \mathrm{m}[3]}$

Skin

$\mathrm{ppm}[\mathrm{a}] \mathrm{mq} / \mathrm{m}[3]$

$\mathrm{ppm}[\mathrm{a}] \mathrm{mg} / \mathrm{m}[3] \quad \mathrm{ppm}[\mathrm{a}] \mathrm{mg} / \mathrm{m}[3]$ nation

Desig-

Hydrogen fluoride

$$
\text { (as F) }
$$

Hydrogen peroxide

Hydrogen selenide

$$
\text { (as Se) }
$$

Hydrogen sulfide

Hydroquinone

2-Hydroxypropy 1

\begin{tabular}{|c|c|c|c|c|c|}
\hline $\begin{array}{l}3 \\
1\end{array}$ & 1.4 & $\begin{array}{l}6 \\
-\end{array}$ & - & - & - \\
\hline $\begin{array}{r}0.05 \\
10 \\
-\end{array}$ & $\begin{array}{r}0.2 \\
14 \\
2\end{array}$ & $\overline{15}$ & 21 & $\begin{array}{l}- \\
-\end{array}$ & 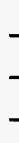 \\
\hline 0.5 & 3 & - & - & - & - \\
\hline 10 & 45 & - & - & - & - \\
\hline $\begin{array}{r}\overline{-} \\
0.6\end{array}$ & $\begin{array}{r}0.1 \\
- \\
10\end{array}$ & $\begin{array}{l}- \\
-\end{array}$ & - & $0 . \overline{1}$ & 1 \\
\hline - & 10 & - & - & - & - \\
\hline 0.1 & 0.8 & 0.2 & 1.6 & - & - \\
\hline $10 \overline{-}$ & $\begin{array}{r}1 \\
525\end{array}$ & - & - & - & - \\
\hline $\begin{array}{r}100 \\
150 \\
50 \\
50 \\
4\end{array}$ & $\begin{array}{r}360 \\
700 \\
150 \\
270 \\
23\end{array}$ & $\begin{array}{r}125 \\
- \\
- \\
- \\
-\end{array}$ & $\begin{array}{r}450 \\
- \\
- \\
- \\
-\end{array}$ & $\begin{array}{l}- \\
- \\
-\end{array}$ & $\begin{array}{l}- \\
- \\
-\end{array}$ \\
\hline $\begin{array}{r}0.005 \\
25 \\
250 \\
400 \\
5 \\
2 \\
500\end{array}$ & $\begin{array}{r}- \\
105 \\
950 \\
980 \\
12 \\
10 \\
2100\end{array}$ & $\begin{array}{r}0.02 \\
- \\
310 \\
500 \\
10 \\
- \\
-\end{array}$ & $\begin{array}{r}- \\
- \\
1185 \\
1225 \\
24 \\
- \\
-\end{array}$ & $\begin{array}{l}- \\
- \\
-\end{array}$ & \\
\hline 50 & 240 & 75 & 360 & - & - \\
\hline
\end{tabular}

acrylate

0.53

$10 \quad 45$

Indium and compounds (as In)

Iodine

Iodoform

Iron oxide dust and fume (as $\mathrm{Fe}$ )

Total particulate Iron pentacarbonyl (as Fe)

Iron salts (soluble) (as Fe)

Isoamyl acetate

Isoamyl alcohol (primary and secondary)

Isobutyl acetate

Isobutyl alcohol

Isooctyl alcohol

Isophorone

Isophorone diiso-

$$
\text { cyanate }
$$

2-Isopropoxyethanol

Isopropyl acetate

Isopropyl alcohol

Isopropyl amine

$\mathrm{N}$-Isopropylanil ine

Isopropyl ether

Isopropyl glycidyl

ether (IGE) 
TABLE Z-1-A, part 2. (contd)

Final Rule Limits**

Substance

TWA $b$

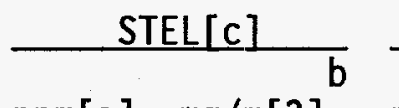

CEILING

Skin ppm[a] $\mathrm{mg} / \mathrm{m}[3]$ ppm[a] $\mathrm{mg} / \mathrm{m}[3]$ nation

Kaolin

Total dust

Respirable fraction

Ketene

$\mathrm{ppm}[\mathrm{a}] \mathrm{mg} / \mathrm{m}[3]$

Lead inorganic (as Pb) see 1910.1025

Limestone

Total dust

Respirable fraction

Lindane

Lithium hydride

L.P.G. (Liquefied

petroleum gas)
0.5
10
5
0.9
$1 . \overline{5}$

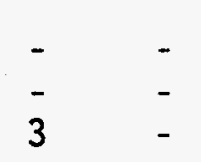

Magnesite

Total dust

Respirable fraction

Magnesium oxide fume Total dust

Malathion

Total dust

Maleic anhydride

Manganese compounds

(as Mn)

Manganese fume

(as Mn)

Manganese cyclopentadienyl tricarbonyl (as Mn)

Manganese tetroxide (as $\mathrm{Mn}$ )

Marble

Total dust

Respirable fraction

Mercury (aryl and inorganic) (as $\mathrm{Hg}$ )

$\begin{array}{rr}- & 15 \\ - & - \\ - & 0.5 \\ - & 0.025 \\ 1000 & 1800\end{array}$

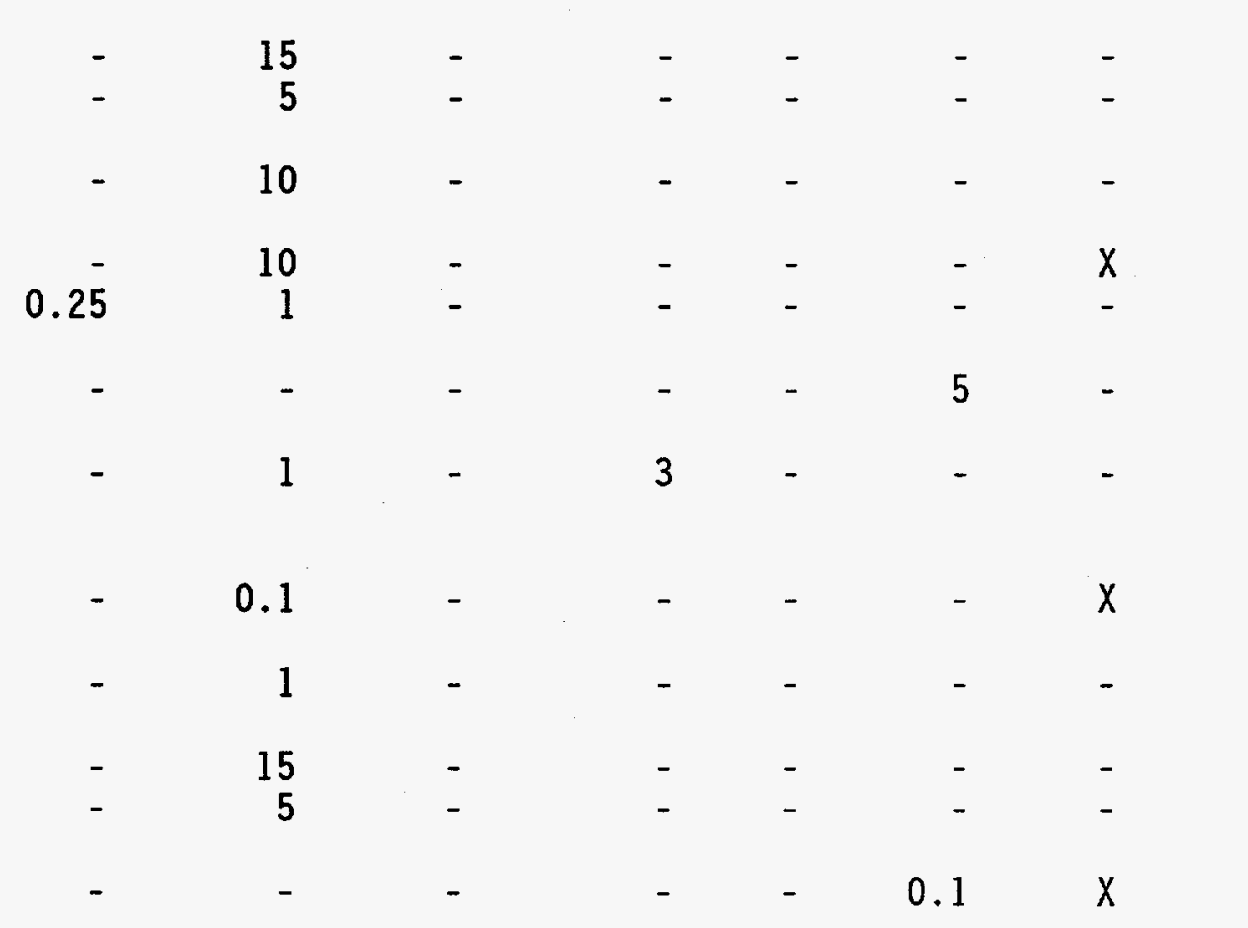


TABLE Z-1-A, part 2. (contd)

Final Rule Limits**

Substance

\begin{tabular}{l} 
Substance \\
\hline Mercury (organo) \\
alkyl compounds \\
(as Hg) \\
Mercury (vapor) \\
(as Hg) \\
Mesityl oxide \\
Methacrylic acid \\
Methanethiol; see Methyl \\
mercaptan \\
Methomyl (Lannate) \\
Methoxychlor \\
Total dust \\
2-Methoxyethanol; see \\
Methyl cellosolve \\
4-Methoxyphenol \\
Methyl acetate \\
Methyl acetylene \\
(Propyne) \\
Methyl acetylene- \\
propadiene mixture \\
(MAPP) \\
Methyl acrylate \\
Methylacrylonitrile \\
Methylal (Dimethoxy- \\
methane) \\
Methyl alcohol \\
Methylamine \\
Methyl amyl alcohol; \\
see Methyl isobutyl \\
carbinol \\
Methyl n-amyl ketone \\
Methyl bromide \\
Methyl butyl ketone; \\
see 2-Hexanone \\
Methyl cellosolve \\
(2-Methoxyethanol) \\
Methyl cellosolve acetate \\
(2-Methoxyethyl \\
acetate) \\
\end{tabular}

TWA

[a] $\mathrm{mg} / \mathrm{m}$ [3]

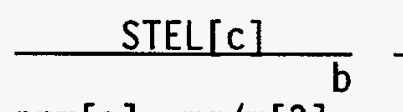

$\mathrm{ppm}[\mathrm{a}] \mathrm{mg} / \mathrm{m}[3] \mathrm{ppm}[\mathrm{a}] \mathrm{mg} / \mathrm{m}[3]$
Skin

Desig- 
TABLE Z-1-A, part 2. (contd)

Final RuTe Limits**

Substance

Methy] chloride

Methyl chloroform

(1,1,1-Trichloroethane)

Methyl 2-cyanoacrylate

Methylcyclohexane

Methylcyclohexanol

o-Methylcyclohexanone

Methylcyclopentadienyl

manganese tricarbonyl (as Mn)

Methyl demeton

$4,4^{\prime}$-Methylene bis

(2-chloroaniline)

(MBOCA)

Methylene bis(4-cyclo-

hexylisocyanate)

Methylene chloride

Methyl ethyl ketone

(MEK); see 2-Butanon

Methyl ethyl ketone

peroxide (MEKP)

Methyl formate

Methyl hydrazine (Monomethyl hydrazine)

Methyl iodide

Methyl isoamyl ketone

Methyl isobutyl carbinol

Methyl isobutyl ketone; see Hexone

Methyl isocyanate

Methyl isopropy? ketone

Methyl mercaptan

Methyl methacrylate

Methyl parathion

Methyl propyl ketone;

see 2-Pentanone

Methyl silicate

alpha-Methyl styrene
TWA

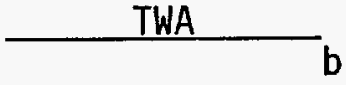

ppm[a] $\mathrm{mq} / \mathrm{m}[3]$

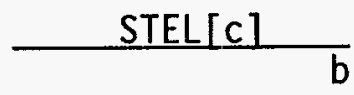

$\frac{\text { CEILING }}{b}$

Skin

Desig-

$\mathrm{ppm}[\mathrm{a}] \mathrm{mg} / \mathrm{m}[3] \mathrm{ppm}[\mathrm{a}] \mathrm{mg} / \mathrm{m}[3]$ nation

$\begin{array}{llll}50 & 105 & 100 & 210\end{array}$

$\begin{array}{rr}350 & 1900 \\ 2 & 8 \\ 400 & 1600 \\ 50 & 235 \\ 50 & 230\end{array}$

$\begin{array}{rr}450 & 2450 \\ 4 & 16 \\ - & - \\ - & - \\ 75 & 345\end{array}$

$\begin{array}{ll}- & 0.2 \\ - & 0.5\end{array}$

0.02

0.22

See Table Z-'

0.01

0.11
100

250

150

0.7

375

0.02

0.05

200

0.5

100

$$
705
$$

410

0.2

5

6
240

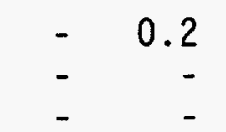

0.35

165

$x$

X

$x$

100

485 
TABLE Z-1-A, part 2. (contd)

Final Rule Limits**

TWA $b$

Substance

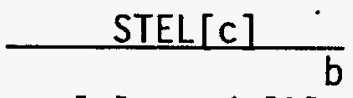

CEILING $b$

Skin

Desig-

ppm[a] $\mathrm{mg} / \mathrm{m}[3] \quad \mathrm{ppm}[\mathrm{a}] \mathrm{mg} / \mathrm{m}[3] \quad \mathrm{ppm}[\mathrm{a}] \mathrm{mg} / \mathrm{m}[3]$ nation

Methylene bisphenyl isocyanate (MDI)

Metribuzin

Mica; see Silicates

Molybdenum (as Mo)

Soluble compounds

Insoluble compounds

Total dust

Monocrotophos

(Azodrin[R])

Monomethyl aniline

Morpholine

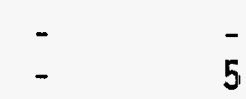

$-0.02$

0.2

Naphtha (Coal tar)

Naphthalene

alpha-Naphthyl amine;

see 1910.1004

beta-Naphthylamine;

see 1910.1009

Nickel carbony?

$$
\text { (as } \mathrm{Ni} \text { ) }
$$

Nickel, metal and

insoluble

compounds

(as $\mathrm{Ni}$ )

Nickel, soluble compounds (as $\mathrm{Ni}$ )

Nicotine

Nitric acid

Nitric oxide

p-Nitroaniline

Nitrobenzene

$\mathrm{p}$-Nitrochlorobenzene

4-Nitrodiphenyl; see 1910.1003

Nitroethane

Nitrogen dioxide

Nitrogen trifluoride

Nitroglycerin [1]

Nitromethane

$0.001 \quad 0.007$

0.5

20

30

105

100

10

400

50

15

75

$x$
$x$

$\begin{array}{rr}- & 0.1 \\ - & 0.5 \\ 2 & 5 \\ 25 & 30 \\ - & 3 \\ 1 & 5 \\ - & \end{array}$

0.1

0.5

5
30
3

3

1

$x$

$\begin{array}{rr}- & - \\ 10 & 29 \\ 100 & 250\end{array}$


IABLE Z-1-A, part 2. (contd)

Final Rule Limits**

TWA

Substance

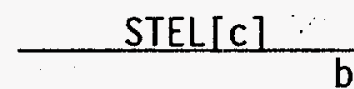

CEILING

Skin

Desig-

ppm[a] $\mathrm{mg} / \mathrm{m}[3]$

ppm[a] $\mathrm{mg} / \mathrm{m}[3]$

$\mathrm{ppm}[\mathrm{a}] \mathrm{mg} / \mathrm{m}[3]$

nation

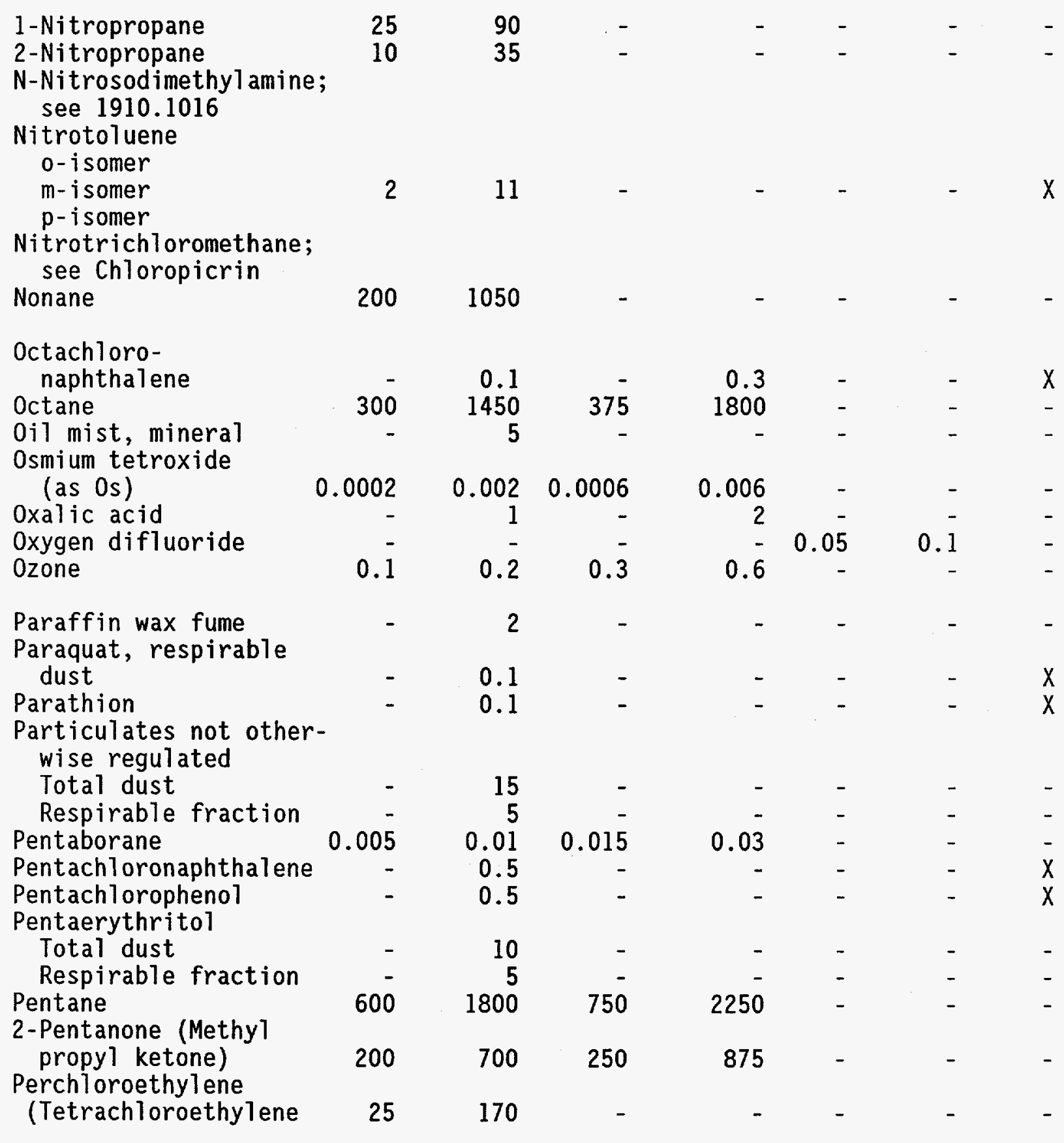

A. 53 
TABLE Z-1-A, part 2. (contd)

Final Rule Limits**

Substance

TWA

$b$

$\mathrm{ppm}[\mathrm{a}] \mathrm{mg} / \mathrm{m}[3]$

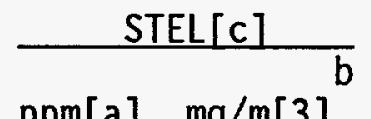

$\mathrm{ppm}[\mathrm{a}] \mathrm{mg} / \mathrm{m}[3]$
CEILING $b$

$\mathrm{ppm}[\mathrm{a}] \mathrm{mg} / \mathrm{m}[3]$
Skin

Desig-

nation

Perchloromethyl

mercaptan

Perchloryl fluoride

Perlite

Total dust

Respirable fraction

0.

.1

0.8
14

$\overline{6}$

28

Petroleum distillates (Naphtha)

Phenol

Phenothiazine

$\mathrm{p}$-Phenylene diamine

Phenyl ether, vapor

Pheny 1 ether-bipheny 1 mixture, vapor

Phenylethylene; see Styrene

Phenyl glycidyl ether (PGE)

Phenylhydrazine

Phenyl mercaptan

Phenyl phosphine

Phorate

Phosdrin

(Mevinphos [R])

Phosgene (Carbonyl chloride)

Phosphine

Phosphoric acid

Phosphorus (yellow)

Phosphorus oxychloride

Phosphorus penta-

chloride

Phosphorus penta-

sulfide
Phosphorus trichloride

Phthalic anhydride

m-Phthalodinitrile

Picloram

Total dust

Respirable fraction

Picric acid

$\begin{array}{rrrrrrrr}- & 15 & - & - & - & - & - \\ - & 5 & - & - & - & - & - \\ 400 & 1600 & - & - & - & - & - \\ 5 & 19 & - & - & - & - & X \\ - & 5 & - & - & - & - & x \\ - & 0.1 & - & - & - & - & X \\ 1 & 7 & - & - & - & - & - \\ 1 & 7 & - & - & - & - & -\end{array}$

$\begin{array}{rrrrrrr}1 & 6 & - & - & - & - & - \\ 5 & 20 & 10 & 45 & - & - & X \\ 0.5 & 2 & - & - & - & - & - \\ - & - & - & - & 0.05 & 0.25 & - \\ - & 0.05 & - & 0.2 & - & - & X\end{array}$

$\begin{array}{lllllll}0.01 & 0.1 & 0.03 & 0.3 & - & - & X\end{array}$

$0.1 \quad 0.4$

$0.3 \quad 0.4$

$-\quad 1$

$0.1 \quad 0.6$

1

$0 . \overline{2} \quad 1.5$

$0 . \overline{5}$

1

6

$-10$

$\begin{array}{rr}- & 10 \\ - & 5\end{array}$

0.1

A. 54 
TABLE Z-1-A, part 2. (contd)

Final Rule Limits**

TWA

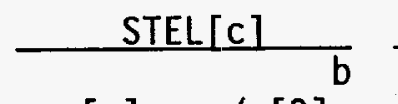

CEILING

Skin

Desig-

Substance

$\mathrm{ppm}[\mathrm{a}] \mathrm{mg} / \mathrm{m}[3]$

$\mathrm{ppm}[\mathrm{a}] \mathrm{mg} / \mathrm{m}[3]$

$\mathrm{ppm}[\mathrm{a}] \mathrm{mg} / \mathrm{m}[3]$

nation

Piperazine dihydrochloride

Pindone (2-Pivaly)1,3-indandione)

Plaster of Paris

Total dust

Respirable fraction

$\mathrm{Pl}$ atinum (as Pt)

Metal

Soluble salts

Portland cement

Total dust

Respirable fraction

Potassium hydroxide

Propane

Propargyl alcohol

beta-Propriolactone;

see 1910.1013

Propionic acid

Propoxur (Baygon)

n-Propyl acetate

$n$-Propyl alcohol

n-Propyl nitrate

Propylene dichloride

Propylene glycol

dinitrate

Propylene glycol monomethyl ether

Propylene imine

Propylene oxide

Propyne; see Methyl acetylene

Pyrethrum

Pyridine

Quinone

Resorcinol

Rhodium (as Rh),

metal fume and

insoluble compounds

$\begin{array}{rrrr}10 & 30 & - & - \\ - & 0.5 & - & - \\ 200 & 840 & 250 & 1050 \\ 200 & 500 & 250 & 625 \\ 25 & 105 & 40 & 170 \\ 75 & 350 & 110 & 510\end{array}$

0.05

0.3

100

2

360

150

540

20

50

5

5 15

$0.1 \quad 0.4$

10

45

20

90

0.1 
TABLE Z-1-A, part 2. (contd)

Final Rule Limits**

Substance

Rhodium (as Rh), soluble compounds

Ronne 1

Rosin core solder pyrolysis products, as formaldehyde

Rotenone

Rouge

Total dust

Respirable fraction

Selenium compounds

(as $\mathrm{Se}$ )

Selenium hexafluoride (as $\mathrm{Se}$ )

Silica, amorphous, precipitated and gel

Silica, amorphous,

diatomaceous earth, containing less than

$1 \%$ crystalline silica

Silica, crystalline

cristobalite (as quartz), respirable dust

Silica, crystalline quartz (as quartz), respirable dust

Silica, crystalline tripoli (as quartz), respirable dust

Silica, crystalline tridymite (as quartz), respirable dust

Silica, fused, respirable dust

Silicates (less than

$1 \%$ crystalline

silica)

Mica (respirable dust)
TWA

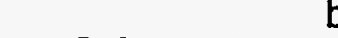

STEL $[\mathrm{c}$

ppm[a] $\mathrm{mq} / \mathrm{m}[3]$

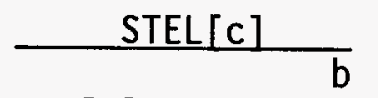

$\frac{\text { CEILING }}{\mathrm{ppm}[\mathrm{a}] \mathrm{mg} / \mathrm{m}[3]}$

Skin

Desig-

ppm[a] $\mathrm{mg} / \mathrm{m}[3] \mathrm{ppm}[\mathrm{a}] \mathrm{mq} / \mathrm{m}[3]$ nation
0.001

10

0.1

10

5

0.2

$0.05 \quad 0.4$

6

6

0.05

0.1

0.1

0.05

0.1

3 
TABLE Z-1-A, part 2. (contd)

Final Rule Limits**

TWA

Substance
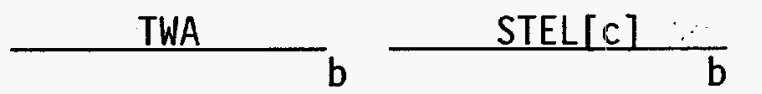

CEILING $b$

Skin

Desig-

ppm[a] $\mathrm{mg} / \mathrm{m}[3]$ ppm[a] $\mathrm{mg} / \mathrm{m}[3]$ ppm[a] $\mathrm{mg} / \mathrm{m}[3]$ nation

Soapstone, total

dust

Soapstone, respirable

dust

Talc (containing

asbestos): use asbestos

limit

Talc (containing no asbestos), asbestos), respirable dust

Tremolite

Silicon

Total dust

Respirable fraction

Silicon carbide

Total dust

Respirable fraction

Silicon tetrahydride

Silver, metal

and soluble compounds

(as $\mathrm{Ag}$ )

Soapstone; see

Silicates

Sodium azide

(as $H N(3)$ )

(as $\mathrm{NaN}(3)$ )

Sodium bisulfite

Sodium fluoroacetate

Sodium hydroxide

Sodium metabisulfite

Starch

Total dust

Respirable fraction

Stibine

Stoddard solvent

Strychnine

Styrene

6

3

See 29 CFR 1910.1001

Subtilisins

(Proteolytic enzymes)

See $29^{2}$ CFR $191 \overline{0} .1101$

$\begin{array}{rr}- & 10 \\ - & 5 \\ - & 10 \\ - & 5 \\ 5 & 7\end{array}$

0.01

$\begin{array}{rr}- & - \\ - & - \\ - & 5 \\ - & 0.05 \\ - & - \\ - & 5 \\ & \\ - & 15 \\ - & 5 \\ 0.1 & 0.5 \\ 100 & 525 \\ - & 0.15 \\ 50 & 215\end{array}$

100

425

0.15

0.1

0.3

$x$

$x$

$-$

X

$-\quad 0.00006$ 
TABLE Z-1-A, part 2. (contd)

Final Rule Limits**

$\begin{array}{cll}\text { STEL[C] } & \text { CEILING } & \text { Skin } \\ \underline{\mathrm{ppm}[\mathrm{a}] \mathrm{mg} / \mathrm{m}[3]} & \begin{array}{l}\text { Desig- } \\ \mathrm{ppm}[\mathrm{a}] \mathrm{mg} / \mathrm{m}[3]\end{array} & \text { nation }\end{array}$

Sucrose

TWA $b$

$\mathrm{ppm}[\mathrm{a}] \mathrm{mg} / \mathrm{m}[3]$

\section{ppm[a] $\mathrm{mg} / \mathrm{m}[3]$}

Total dust

Respirable fraction

Sulfur dioxide

Sulfur hexafluoride

Sulfuric acid

Sulfur monochloride

Sulfur pentafluoride

Sulfur tetrafluoride

Sulfuryl fluoride

Sulprofos

Systox $[R]$, see Demeton

$\begin{array}{rrr}- & 15 & - \\ - & 5 & - \\ 2 & 5 & 5 \\ 1000 & 6000 & - \\ - & 1 & - \\ - & - & - \\ - & - & - \\ - & - & - \\ 5 & 20 & 10 \\ - & 1 & -\end{array}$

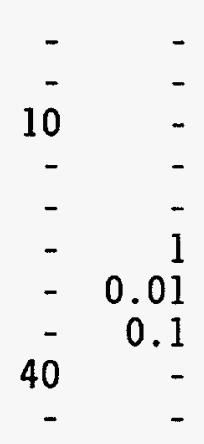

10

$2,4,5-T$

Talc; see Silicates

Tantalum, metal and oxide dust

TEDP (Sulfotep)

Tellurium and compounds (as Te)

Tellurium hexafluoride (as Te)

Temephos

Total dust

Respirable fraction

TEPP

Terphenyls

$1,1,1,2$-Tetrachioro2,2-d ifluoroethane

1,1,2,2-Tetrachloro1,2-difluoroethane

1,1,2,2-Tetrachloroethane

Tetrachoroethylene; see Perchloroethylene

Tetrachloromethane; see Carbon tetrachloride Tetrachloronaphthalene Tetraethy 1 lead (as $\mathrm{Pb}$ )

Tetrahydrofuran

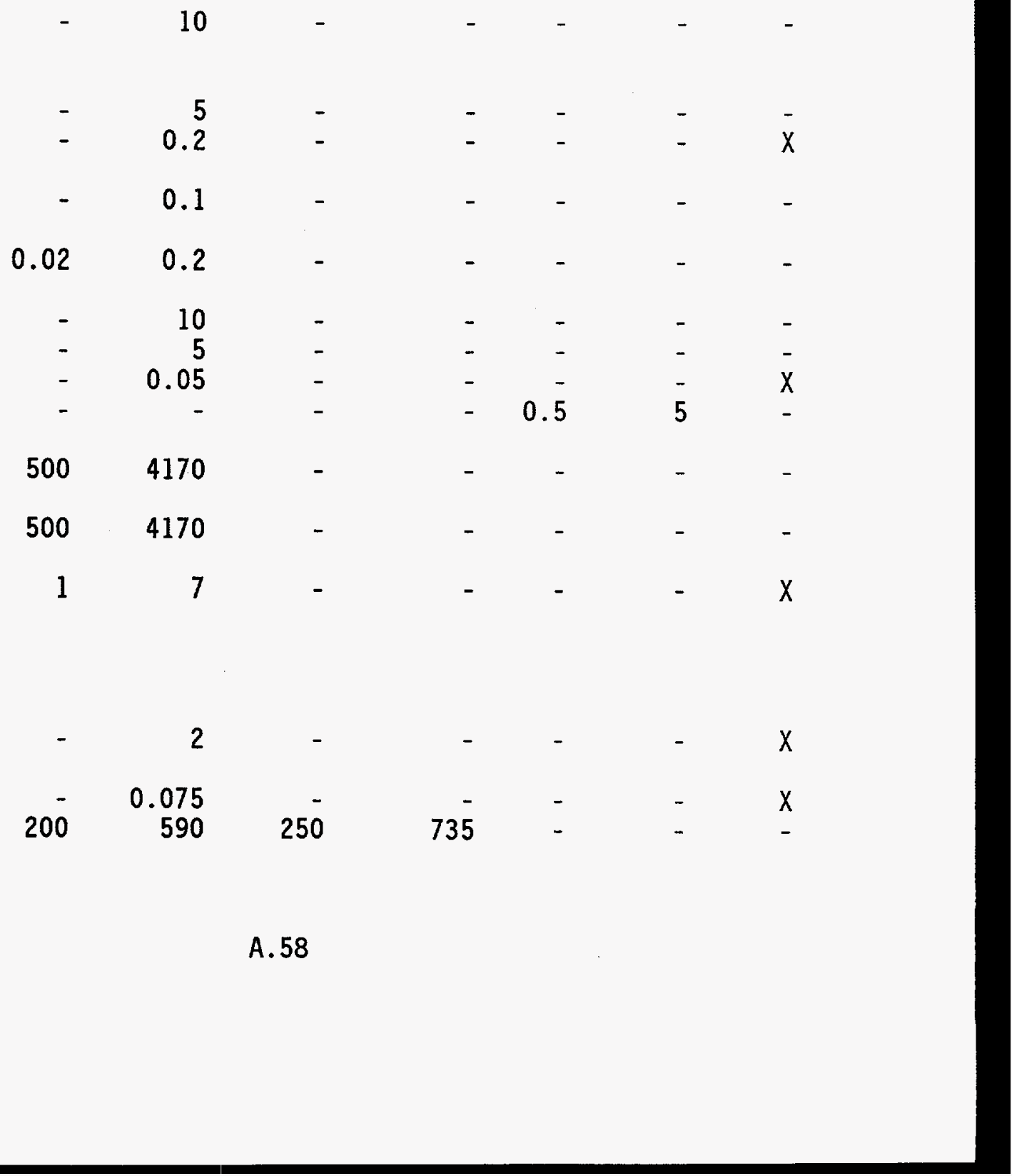


TABLE Z-1-A, part 2. (contd)

Final Rule Limits**

Substance

Tetramethyl lead, (as $\mathrm{Pb}$ )

Tetramethyl succinonitrile

Tetranitromethane

Tetrasodium pyrophosphate

Tetryl (2,4,6-Trinitropheny 1 -methylnitramine)

Thallium, soluble compounds (as $\mathrm{T} 1$ )

4, 4' -Thiobis(6-tert, Butyl-m-cresol)

Total dust

Respirable fraction

Thioglycolic acid

Thionyl chloride

Thiram

Tin, inorganic compounds (except oxides) (as Sn)

Tin, organic compounds (as $\mathrm{Sn}$ )

Tin oxide (as Sn)

Titanium dioxide Total dust

Toluene

Toluene-2,4-di i socyanate (TDI)

m-Toluidine

o-Toluidine

p-Toluidine

Toxaphene; see Chlorinated camphene

Tremolite; see Silicates

Tributyl phosphate

Trichloroacetic acid

1,2,4-Trichlorobenzene

1,1,1-Trichloroethane; see Methyl chloroform 1,1,2-Trich1oroethane

0.005

0.2

1
TWA

0.075

0.5

1

3

8

5

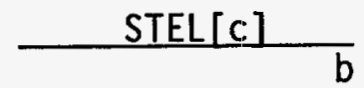

CEILING

Skin

Desig-

ppm[a] $\mathrm{mg} / \mathrm{m}[3] \mathrm{ppm}[\mathrm{a}] \mathrm{mg} / \mathrm{m}[3]$ nation

$x$

$x$ $x$

$x$

0.1

10

5

4

5

2

0.1

2

10

375

150

560

0.04

0.02

0.15

9
22

9

2.5

7

$-$

45 
TABLE Z-1-A, part 2. (contd)

Final Rule Limits**

\section{Substance}

Trichloroethylene

Trichloromethane; see Chloroform

Trichloronaphthalene

1,2,3-Trichloropropane

1,1,2-Trich1oro-1,2,2trifluoroethane

Triethylamine

Trifluorobromomethane

Trimellitic anhydride

Trimethy 7 amine

Trimethyl benzene

Trimethyl phosphite

2,4,6-Trinitrophenyl; see Picric acid

2,4,6-Trinitropheny 1 methy 1

nitramine; see Tetryl

2,4,6-Trinitrotoluene (TNT)

Triorthocresyl phosphate

Triphenyl amine

Triphenyl phosphate

Tungsten (as W) Insoluble compounds Soluble compounds

Turpentine

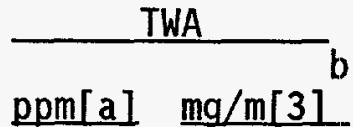

50

270

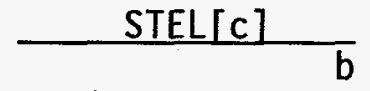

CEILING

Skin

$\mathrm{ppm}[\mathrm{a}] \mathrm{mg} / \mathrm{m}[3]$

$\mathrm{ppm}[\mathrm{a}] \mathrm{mg} / \mathrm{m}[3]$

Desig-

$200 \quad 1080$

nation

-

5

10

1000

0.005

10

25

2

60

7600

40

6100

0.04

24

125

10

\section{0 \\ 15}

9500

60

36

0.5

$\begin{array}{lr}- & 0.1 \\ - & 5 \\ - & 3\end{array}$

3

$\begin{array}{rr}- & 5 \\ - & 1 \\ 100 & 560\end{array}$

Uranium (as $U$ )

Soluble compunds

Insoluble compounds

$\begin{array}{ll}- & 0.05 \\ - & 0.2\end{array}$

0.05
0.2

$50 \quad 175$

$50 \quad 175$

n-Valeraldehyde

Vanadium

Respirable dust

$$
\text { (as V(2)0(5)) }
$$

Fume (as V(2)0(5))

Vegetable oil mist

Total dust

Respirable fraction

$-\quad 0.05$

- $\quad 0.05$

$\begin{array}{rr}- & 15 \\ - & 5\end{array}$ 
TABLE Z-1-A, part 2. (contd)

Final Rule Limits**

Substance

Vinyl acetate

Vinyl benzene;

see Styrene

Vinyl bromide

Vinyl chloride; see 1910.1017

Vinylcyanide; see Acrylonitrile

Vinyl cyclohexene dioxide

Vinylidene chloride (1,1-Dichloroethylene)

Vinyl toluene

VM \& P Naphtha

Warfarin

Welding fumes (total particulate) $)^{* * *}$

wood dust, a11 soft and hard woods, except Western red cedar

Wood dust,

Western red cedar

Xylenes (o-, m-, pi somers)

m-Xylene alpha, alpha' diamine

Xylidine

Yttrium

Zinc chloride fume

Zinc chromate (as Cro(3))

Zinc oxide fume
TWA

$b$

$\mathrm{ppm}[\mathrm{a}] \mathrm{mg} / \mathrm{m}[3]$

10

30

20

60

5

20

10

60

100

300

4
480
1350

1350

$\begin{array}{lr}- & 0.1 \\ - & 5\end{array}$ 
TABLE Z-1-A, part 2. (contd)

Final Rule Limits**

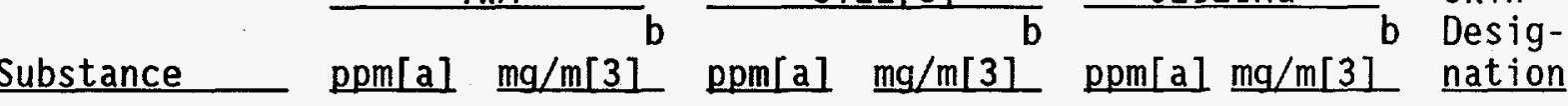

Zinc oxide

Total dust

Respirable fraction

ppm[a] $m g / m[3]$

Zinc stearate

Total dust

Respirable fraction

Zirconium compounds

(as $Z r$ )

$\begin{array}{rr}- & 10 \\ - & 5 \\ - & 10 \\ - & 5 \\ - & 5\end{array}$

$\begin{array}{ll}- & - \\ - & - \\ - & - \\ - & 10\end{array}$

* The transitional PELS are 8-hour TWAs unless otherwise noted; a (C) designation denotes a ceiling limit.

* Uniess otherwise noted, employers in General Industry (i.e., those covered by 29 CFR 1910) may use any combination of controls to achieve these limits until December 31, 1992 as set forth in 29 CFR 1910.1000(f).

*** As determined from breathing-zone air samples.

[a] Parts of vapor or gas per million parts of contaminated air by volume at $25^{\circ} \mathrm{C}$ and 760 torr.

[b] Approximate milligrams of substance per cubic meter of air.

[c] Duration is for 15 minutes, unless otherwise noted.

[d] The final benzene standard in 1910.1028 applies to all occupational exposures to benzene except some subsegments of industry where exposures are consistently under the action level (i.e., distribution and sale of fuels, sealed containers and pipelines, coke production, oil and gas drilling and production, natural gas processing, and the percentage exclusion for liquid mixtures); for the excepted subsequents, the benzene limits in Table Z-2 apply.

[e] Exposures under $10,000 \mathrm{ppm}$ to be cited de minimus.

[f] The CAS number is for information only. Enforcement is based on the substance name. For an entry covering more than one metal compound measured as the metal, the CAS number for the metal is given - not CAS numbers for the individual compounds.

[g] Compliance with the subtilisins PEL is assessed by sampling with a high volume sampler (600-800 liters per minute) for at least 60 minutes.

[h] The acetone STEL does not apply to the cellulose acetate fiber industry. It is in effect for all other sectors.

[i] The Final Rule Limit of $5 \mathrm{mg} / \mathrm{m}[3]$ is not in effect as a result of reconsideration. Calcium hydroxide is covered by the exposure limits for particulates not otherwise regulated of $5 \mathrm{mg} / \mathrm{m}[3]$ respirable dust and $15 \mathrm{mg} / \mathrm{m}[3]$ total dust. 


\section{TABLE Z-1-A, part 2. (contd)}

[j] The Final RuTe Limit TWA of $5 \mathrm{mg} / \mathrm{m}$ [3] is not in effect as a result of reconsideration. The calcium oxide Transitional Limit of $5 \mathrm{mg} / \mathrm{m}[3]$ remains in effect and employee exposures shall be kept below that level pursuant to the methods of compliance specified in 29 CFR 1910.1000(e).

[k] The Final RuTe Limit STEL of $0.1 \mathrm{mg} / \mathrm{m}$ [3] is not in effect as a result of reconsideration for the industrial sector of civilian manufacture and distribution of explosives and propellants for civilian use. The Final rule limits skin designation and the Transitional 1imits ceiling 1 imit of $1 \mathrm{mg} / \mathrm{m}[3]$ remain in effect for this sector until completion of the reconsideration.

[1] The Final Rule Limit STEL of $0.1 \mathrm{mg} / \mathrm{m}$ [3] is not in effect as a result of reconsideration for the industrial sector of civilian manufacture and distribution of explosives and propellants for civilian use. The Final rule limits skin designation and the Transitional limits ceiling limit of $2 \mathrm{mg} / \mathrm{m}[3]$ remains in effect for this sector until completion of the reconsideration.

NOTE: Pursuant to administrative stays effective September 1, 1989 and published in the FEDERAL REGISTER on September 5, 1989, and extended in part by notices published in the FEDERAL REGISTER on October 6, 1989, December 6, 1989, February 5, 1990, Apri1 6, 1990, May 9, 1990 and November 8, 1990, the September 1, 1989 start-up specified in 29 CFR 1910.1000(f)(2)(i) is stayed as follows:

1. Until decision on the merits of the Eleventh Circuit Court of Appeals in the case of Courtaulds Fibers Inc. V. U.S. Department of Labor, No. 89-7073 and consolidated cases, for the Ceiling for carbon monoxide for blast furnace operations, vessel blowing at basic oxygen furnaces and sinter plants in the steel industry (SIC 33). OSHA will publish in the Federal Register notice of the termination of the carbon monoxide stay.

2. For employees exposed between $2 \mathrm{mg} / \mathrm{m}[3]$ and $0.1 \mathrm{mg} / \mathrm{m}[3]$ as a STEL for nitroglycerin as of December 15, 1990 in the manufacture of nitroglycerin and nitroglycerin based explosives and propellants for military and space use: until July 1, 1991 for all of those employees per facility, until January 1,1992 for $1 / 2$ of those employees per facility, until March 1 , 1992 for $1 / 4$ of those employees per facility.

In addition the December 31, 1992 start-up date for feasible engineering controls specified in 29 CFR 1910.1000(f)(2)( $i$ i)(A) for employees exposed between $2 \mathrm{mg} / \mathrm{m}[3]$ and $0.1 \mathrm{mg} / \mathrm{m}[3]$ as a STEL for nitroglycerin without regard to respirator use on December 15, 1990 in the manufacture of nitroglycerin and nitroglycerin based explosives and propellants for military and space use is stayed until December 31,1994 for $3 / 4$ of those employees per facility, until December 31, 1996 for $1 / 2$ of those employees per facility and until December 31, 1998 for $1 / 4$ of those employees per facility. 
TABLE Z-2.

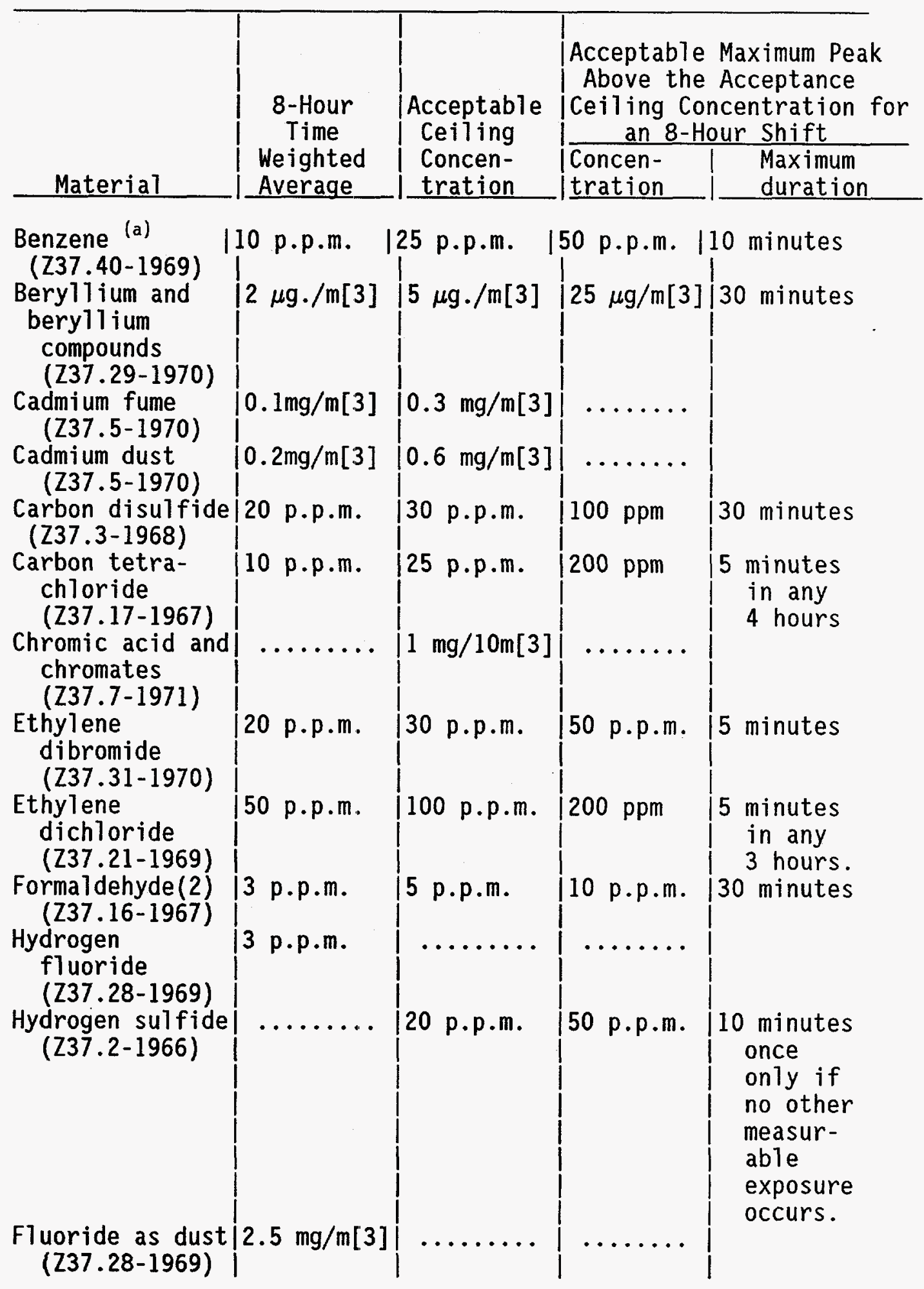

A. 64 
TABLE Z-2. (contd)

\begin{tabular}{|c|c|c|c|}
\hline & $\begin{array}{l}\text { 8-Hour } \\
\text { Time }\end{array}$ & $\begin{array}{c}\text { Acceptable } \\
\text { Ceiling }\end{array}$ & $\begin{array}{l}\text { Acceptable Maximum Peak } \\
\text { Above the Acceptance } \\
\text { Ceiling Concentration for } \\
\text { an 8-Hour Shift }\end{array}$ \\
\hline Material & $\begin{array}{l}\text { Weighted } \\
\text { Average }\end{array}$ & $\begin{array}{l}\text { Concen- } \\
\text { tration }\end{array}$ & $\begin{array}{l}\text { Concen- } \\
\text { tration }\end{array}$ \\
\hline
\end{tabular}

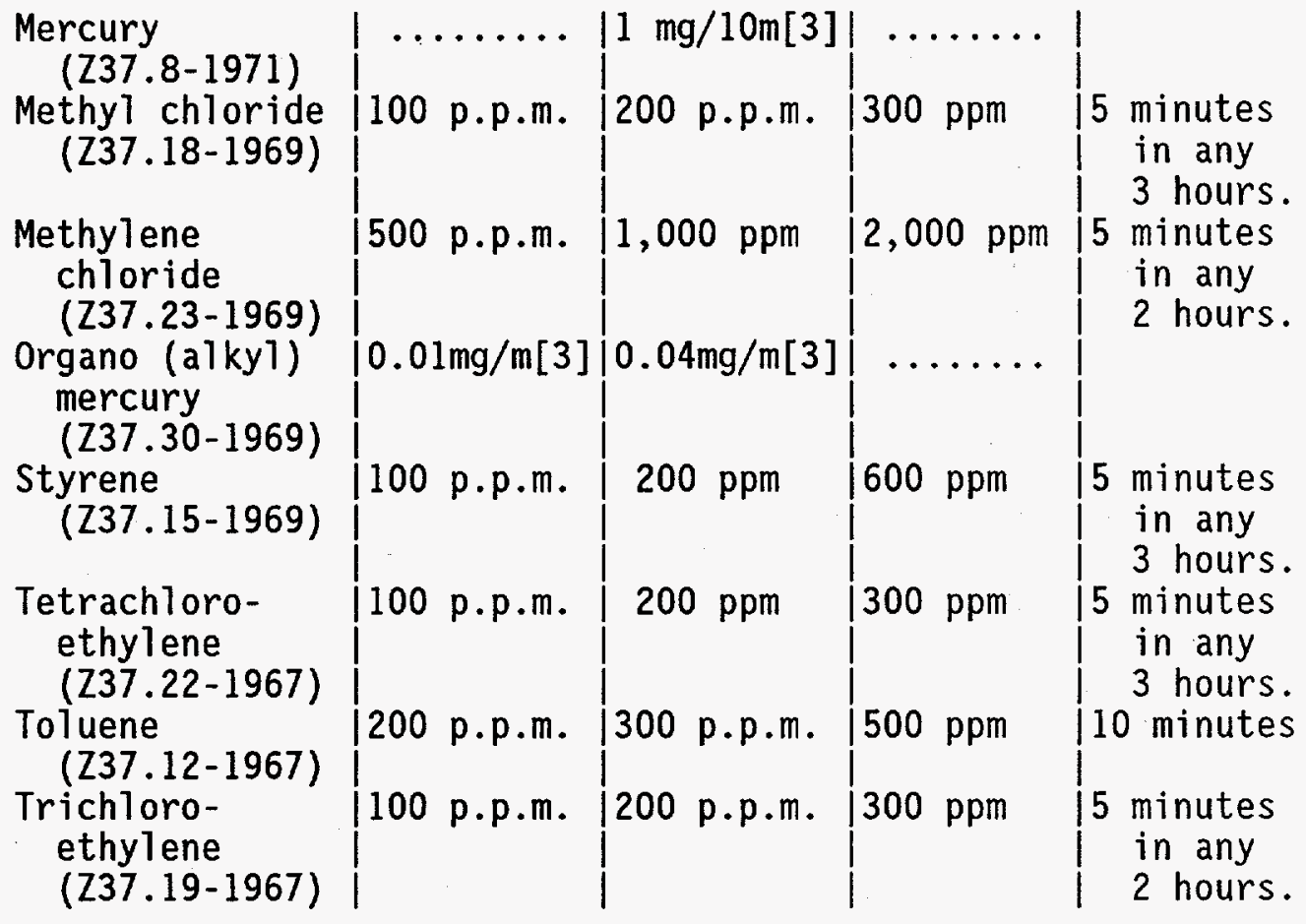

Note 1. This standard applies to the industry segments exempt from the 1 ppm 8 hour TWA and $5 \mathrm{ppm}$ STEL of the benzene standard at 1910.1028. This standard also applies to any industry for which 1910.1028 is stayed or otherwise not in effect.

Note 2. This standard applies to any industry for which 1910.1048 is stayed or otherwise not in effect. 
TABLE Z-3. - MINERAL DUSTS

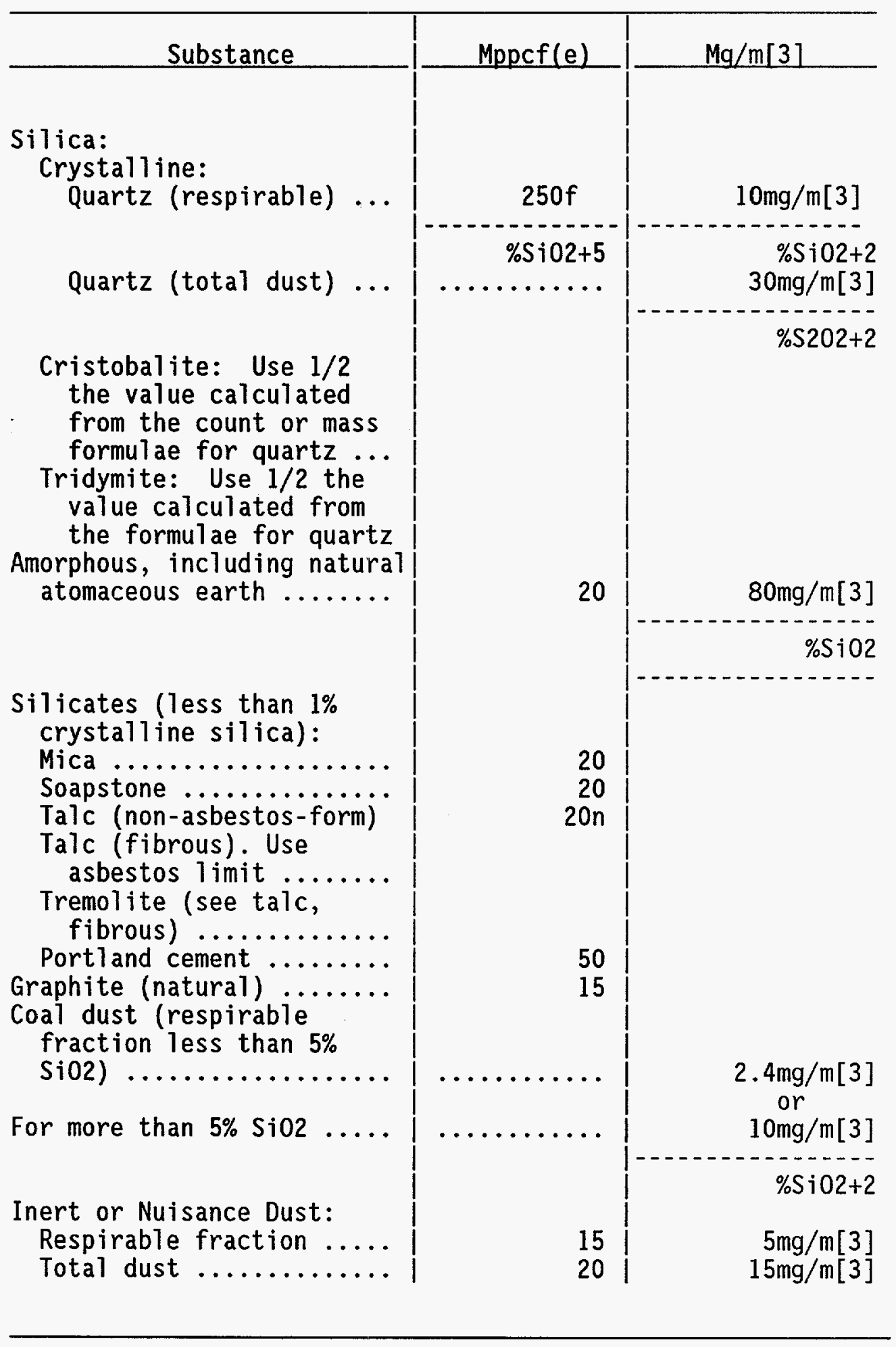


NOTE: Conversion factors -- mppcf $\times 35.3=$ million particles per cubic meter = particles per $c . c$.

\begin{tabular}{c|c}
$\begin{array}{c}\text { Aerodynamic diameter (unit } \\
\text { density sphere) }\end{array}$ & Percent passing selector \\
\hline 2 & 90 \\
2.5 & 75 \\
3.5 & 50 \\
5.0 & 25 \\
10 & 0 \\
\hline
\end{tabular}

The measurements under this note refer to the use of an AEC instrument. The respirable fraction of coal dust is determined with a MRE; the figure corresponding to that of $2.4 \mathrm{Mg} / \mathrm{m}[3]$ in the table for coal dust is $4.5 \mathrm{Mg} / \mathrm{m}[3]$. 


$$
\text { APPENDIX B }
$$

DERIVED AIR CONCENTRATIONS FOR CONTROLLING

RADIATION EXPOSURE TO WORKERS AT DOE FACILITIES

(from DOE 5480.11, Change 1, 7-20-89) 
APPENDIX B

\section{DERIVED AIR CONCENTRATIONS FOR CONTROLLING RADIATION EXPOSURE TO WORKERS AT DOE FACILITIES}

(from DOE 5480.11, Change 1, 7-20-89)

The derived air concentrations (DAC) for limiting radiation exposures through inhalation of radionuclides by workers are listed in Table 1 . The values are based on either a stochastic (committed effective dose equivalent) dose 1 imit of $5 \mathrm{rem}(0.05 \mathrm{~Sv}$ ) or a nonstochastic (organ) dose limit of $50 \mathrm{rem}$ (0.5 Sv) per year, whichever is more limiting. (Note: the 15 rem [0.15 Sv] dose limit for the lens of the eye does not appear as a critical organ dose 1imit.)

Table 1 contains five columns of information: (1) radionuclide; (2) inhaled air DAC for lung retention class $D$ (micro $\mathrm{Ci} / \mathrm{mL}$ ); (3) inhaled air DAC for lung retention class $W$ (micro $\mathrm{Ci} / \mathrm{mL}$ ); (4) inhaled air DAC for lung retention $\mathrm{class} Y$ (micro $\mathrm{Ci} / \mathrm{mL}$ ); and (5) an indication of whether or not the DAC for each class is controlled by the stochastic (effective dose equivalent) or nonstochastic (tissue) dose. The classes D, W, and $Y$ have been established by the International Commission on Radiological Protection (ICRP) to describe the clearance of inhaled radionuclides from the lung. This classification refers to the approximate length of retention in the pulmonary region. Thus, the range of half-times is less than 10 days for class $D$ (days), from 10 to 100 days for class $W$ (weeks), and greater than 100 days for class $Y$ (years). The DACs in Table 1 are listed by radionuclide, in order of increasing atomic mass, and are based on the assumption that the particle size distribution of the inhaled material is unknown. For this situation, the ICRP recommends that an assumed particle size distribution of $1 \mathrm{micro} \mathrm{m}$ be used. For situations where the particle size distribution is known to differ significantly from 1 micro $\mathrm{m}$, appropriate corrections (as described in the DOE report Internal

\section{B.1}


Dose Conversion Factors for Calculation of Dose to the Public) ${ }^{(a)}$ can be made to both the estimated dose to workers and the DACs.

Alternative absorption factors and lung retention classes for specific compounds are listed by element in Table 2 for cross-referencing with the inhalation DACs in Table 1. The data shown in Table 2 are listed by element in alphabetical order.

The following assumptions and procedures were used in calculating these DAC values for inhalation by workers:

1. The worker is assumed to inhale $2,400 \mathrm{~m}^{3}$ of air during a 2000-hour work year, as defined by the ICRP in its Publication No. 23. (b)

2. The internal dose factors used in calculating the DAC values were taken from the report Internal Dose Conversion Factors for Calculation of Dose to the Public. (a) These factors are based on the metabolic data and dosimetry models recommended by the ICRP in its Publication No. 30. (c)

The air immersion DAC values shown in Table 3 are based on a stochastic limit of 5 rem ( $0.05 \mathrm{~Sv}$ ) per year or a nonstochastic (organ) dose limit of 50 rem $(0.5 \mathrm{~Sv})$ per year. Table 3 contains three columns of information: 1) radionuclide; 2) half-life in units of seconds (s), minutes (min), hours $(h)$, days $(d)$, or years $(y)$; and 3 ) air immersion DAC (micro $\mathrm{Ci} / \mathrm{mL}$ ). The data in Table 3 are listed by radionuclide in order of increasing atomic mass. The air immersion DACs were calculated for a continuous, nonshielded exposure via immersion in a semi-infinite atmospheric cloud. The dose conversion factors used to calculate the DAC values for air immersion were taken from the DOE report External Dose-Rate Conversion Factors for Calculation of Dose to the

(a) U.S. Department of Energy (DOE). 1988. Internal Dose Conversion Factors for Calculation of Dose to the Public. Washington, D.C.

(b) International Commission on Radiological Protection (ICRP). 1975. ICRP Publication 23; Report of the Task Group on Reference Man. Pergamon Press, New York, New York.

(c) International Commission on Radiological Protection (ICRP). 1979-1982. ICRP Publication 30: Limits for Intakes of Radionuclides by Workers. Parts 1 to 3 and supplements 2(3/4) through 8(4), Pergamon Press, New York, New York. 
Public. (a) The DAC value for air immersion listed in Table 3 for a given radionuclide is determined either by a limit on annual effective dose equivalent, which provides a limit on stochastic radiation effects, or by a limit on annual dose equivalent to any organ, which provides a limit on nonstochastic radiation effects. For most of the radionuclides listed in Table 3 , the DAC value is determined by the limit on annual effective dose equivalent. Thus, the few cases where the DAC value is determined by the limit on annual dose equivalent to skin are indicated in the tables by an appropriate footnote. Again, the DACs listed in Table 3 account only for immersion in a semiinfinite cloud and do not account for inhalation or ingestion exposures. Three classes of radionuclides are included in the air immersion DACs given in Table 3, as described below.

1. Class 1. The first class of radionuclides includes selected noble gases and short-lived activation products that occur in gaseous form. For these radionuclides, inhalation doses are negligible compared to the external dose from immersion in an atmospheric cloud.

2. Class 2. The second class of radionuclides includes those for which a DAC value for inhalation has been calculated (using the ICRP inhalation dose equivalent factors), but for which the DAC value for external exposure to a contaminated atmospheric cloud is more restrictive (i.e., results in a lower DAC value). These radionuclides generally have halflives of a few hours or less, or are eliminated from the body following inhalation sufficiently rapidly to limit the inhalation dose.

3. Class 3. The third class of radionuclides includes selected isotopes with relatively short half-lives that were not considered in ICRP Publication 30. These radionuclides typically have half-lives that are less than 10 minutes, they do not occur as a decay product of a longerlived radionuclide, or they lack sufficient decay data to permit internal dose calculations. These radionuclides are also typified by a radioactive emission of highly intense, high-energy photons and rapid removal from the body following inhalation.

The DAC values are given for individual radionuclides. For known mixtures of radionuclides, the sum of the ratio of the observed concentration of a particular radionuclide and its corresponding DAC for all radionuclides in the mixture must not exceed 1.0 .

(a) U.S. Department of Energy (DOE). 1988. External Dose-Rate Conversion Factors for Calculation of Dose to the Public. Washington, D.C. 
Table 1

Derived Air Concentrations (DAC) for Controlling Radiation Exposures to Workers at DOE Facilities

Radionuclide

H-3 (Water)2I

H-3 (Elemental)2/

Be-7

$\mathrm{Be}-10$

C-11 (Org)2/

$\mathrm{C}-11(\mathrm{CO}) 21$

$\mathrm{C}-11(\mathrm{CO}) 2 /$

C-14 (Org)2/

$\mathrm{C}-14(\mathrm{CO}) 2$ /

F-18

m $\mathrm{Na}-22$

is $\mathrm{Na}-24$

$\mathrm{Mg}-28$

Al-26

Si -31

Si -32

$P-32$

$\mathrm{S}-35$

S-35 (Gas)

Cl -36

C1 -38

C1 -39

$K-40$

$K-42$

$\mathrm{K}-43$

$K-44$

$K-45$
Inhaled Air - Lung Retention Class

$0 \quad W \quad Y$

5. E-01

2. E-05

2. E-05

5.E-OI

9. E-06

8. E-06

6. E-08

6.E-09

2. E-04

5. E-O4

3.E-04

1.E-06

7. $E-04$

9.E-05

3. E-05

2. E-04

5. E-04

2. E-04

5.E-04

3. E-04

1.E-06

1.E-06

T. $E-04$
9. E-O5

7.E-04

9.E-05

4. E-05

3. E-05

3. E-07

2.E-06

7.E-07

5. E-07

3. E- 08

3. $E-08$

1.E-05

1.E-05

5. E-08

1.E-05
2.E-09

2. E-07

4.E-07

1.E-06

7.E-06

9. E-07

6. E-06

1.E-06

1. E-06
2. E-05

2. E-05

J.E-07

2. E-05
2. E-05

2.E-07

2.E-06

4. E-06

3. E-05

5. E-05
Stochastic

or Organ 1/

Radionuclide

Ca- 41

$\mathrm{Ca}-45$

$(D / W / Y)$

St/St/St

St/St/St

/St/St

$/ S t / S t$

St/St/St

St/St/St

St/St/St

St/St/St

$\mathrm{St} / \mathrm{St} / \mathrm{St}$

St/St/St

St/St/St

st//

St/St/

St/St/

St/St/St

St/St/St

St/St/

St/St/

St/St/

St/St/

St/St/

St/St/

St/ /

st/ $/$

St/ /

St/ $/$

Sc -43

Sc $-44 m$

Sc -44

$\mathrm{Sc}-46$

Sc -47

Sc -48

Sc-49

$T i-44$
$T i-45$

Ti-45

$V-47$
$V-48$

$v-49$

$\mathrm{Cr}-48$

$\mathrm{Cr}-49$

$\mathrm{Cr}-51$

$\mathrm{Mn}-51$

$M n-52 m$

$M n-52$

$\mathrm{Mn}-53$

$\mathrm{Mn}-54$

$M n-56$

$\mathrm{Fe}-52$

$\mathrm{Fe}-55$

$\mathrm{Fe}-59$

$\mathrm{Fe}-60$

Co-55

Co-56

Co-57

Co-58m

Co-58

Co-60m

Co-60

Co-61

Co-62m
Inhaled Air - Lung Retention Class

$(\mu \mathrm{Ci} / \mathrm{mL}) \quad(\mu \mathrm{Ci} / \mathrm{mL}) \quad(\mu \mathrm{Ci} / \mathrm{mL})$

Stochastic

or Organ 1/

2.E- -06
3. E- 07

4. E- -07

/E / $/$

/St/

\begin{tabular}{|c|c|}
\hline $\begin{array}{l}1 . E-05 \\
\text { 3.E-07 } \\
5 . E-06 \\
1 . E-07 \\
1 . E-06 \\
6 . E-07 \\
2 . E-05\end{array}$ & $\begin{array}{ll}/ & / S t \\
/ & / S t \\
/ & / S t \\
/ & / S t \\
/ & / S t \\
/ & / S t \\
/ & / S t\end{array}$ \\
\hline
\end{tabular}

5.E-09

1. E-05

1.E-08

1. E-05

4. E-05

4.E-05

4. E- 07

1. E- 05

5. E-06

3. E-05

2. E-05

2. E-05

4. E-05

5. $E-07$

5.E-06

4. $E-07$
$6 . E-06$

1.E-06

8.E- -07

1. $E-07$

3. E-09

3. E-07

7.E-06

3. E-06

4. $E-05$

1. E-05

3. E-06

E-05

8. E-06

2. E-05

4.E-O5

4.E-O

5. E-0

3. $E-07$

1.E-06

2. E-06

2. E- 07

8. E-09

1.E-06

1. $E-07$

1.E-06

4. E-05

5. E-07

2. $\mathrm{E}-\mathrm{O3}$

7. E-OB

3. E-05

7. E-05
1. E-06

$3 . E-07$
$3 . E-05$

3.E-07

1. $E-03$

1.E-08

2. -05

7.E-05
St/St/St

St/St/St

St/St/

St/St/

St/St/St

$\mathrm{St} / \mathrm{St} / \mathrm{St}$

St/St/St

St/St/

St/St/

St/St/

BS/St/

St/St/

St/St/

St/St/

St/St/

St/St/

St/St/

/St/St

/St/St

/St/St

/St/St

/St/St

/St/St

/St/St

/St/St

/St/St 
Inhaled Air - Lung Retention Class Stochastic

Radionuclide

Ni-56 (Inorg)

$\mathrm{Ni}-56$ (Vapor)

$\mathrm{Ni}-57$ (Inorg)

$\mathrm{Ni}-57$ (Vapor)

$\mathrm{Ni}-59$ (Inorg)

$\mathrm{Ni}-59$ (Vapor)

Ni-63 (Inorg)

$\mathrm{Ni}-63$ (Vapor)

N1-65 (Inorg)

$\mathrm{Ni}-65$ (Vapor)

$\mathrm{Ni}-66$ (Inorg)

$\mathrm{Ni}-66$ (Vapor)

Cu-60

$\mathrm{Cu}-61$

$\mathrm{Cu}-64$

Cu-67

$2 n-62$

(1) $Z n-63$

ir $2 n-65$

$2 n-69$

$\mathrm{Zn}-71 \mathrm{~m}$

Zn-72

Ga-65

$\mathrm{Ga}-66$

Ga-67

Ga-68

$\mathrm{Ga}-70$

$\mathrm{Ga}-72$

Ga-73

Ge-66

Ge-67

$\mathrm{Ge}-68$

Ge-69

$\mathrm{Ge}-71$

$\mathrm{Ge}-75$

Ge -77

$\mathrm{Ge}-78$

As -69
Stochastic
or Organ $1 /$

$\begin{array}{llll}(\mu \mathrm{Ci} / \mathrm{mL}) & (\mu \mathrm{Ci} / \mathrm{mL}) & (\mu \mathrm{Ci} / \mathrm{mL}) & (D / \mathrm{W} / \mathrm{Y})\end{array}$

$\begin{array}{ll}\text { 8.E-07 } & 5 . E-07 \\ & 5 . E-07 \\ \text { 2.E-06 } & 1 . E-06 \\ & 3 . E-06 \\ \text { 2.E-06 } & 3 . E-06 \\ & 8 . E-07 \\ \text { 7.E-07 } & 1 . E-06 \\ & 3 . E-07 \\ 1 . E-05 & 1 . E-05 \\ & 7 . E-06 \\ \text { 7.E-07 } & 3 . E-07 \\ & 1 . E-06 \\ & \\ \text { 4.E-05 } & 5 . E-05 \\ 1 . E-05 & 2 . E-05 \\ 1 . E-05 & 1 . E-05 \\ 3 . E-06 & 2 . E-06\end{array}$

7.E-O

5. E-07

7.E-05
$1 . E-06$
$6 . E-06$
$2 . E-05$
$7 . E-05$
$2 . E-06$
$6 . E-06$
$1 . E-05$
$4 . E-05$
$2 . E-06$
$6 . E-06$
$2 . E-04$
$3 . E-05$
$4 . E-06$
$9 . E-06$

8.E-05

1.E-06

4.E-06

.E-05

8.E-05
$1 . E-06$

6. E- -06

8. E-06

4.E-05

4. E-08

3.E-06

2. E-05

3. E-05

2. E-O6

9.E-O6

5.E-05
St/St/

$/ S t /$
St/St/

St/St/

St/St/

/St/

St/St/

/St/

/St/

St/St/

/St/

St/St/St

St/St/St

$\mathrm{St} / \mathrm{St} / \mathrm{St}$

St/St/St

$1 / 5 t$

$/ /$ /St

/ / $/ \mathrm{st}$

/ / St

$/ /$ st

Radionuclide

As -70

As -71

As -72

As -73

As -74

As -76

As-77

As -78

Se-70

Se $-73 \mathrm{~m}$

$\mathrm{Se}-73$

$\mathrm{Se}-75$

$\mathrm{Se}-79$

$\mathrm{Se}-81 \mathrm{~m}$

$\mathrm{Se}-81$

$\mathrm{Se}-83$

$\mathrm{Br}-74 \mathrm{~m}$

$\mathrm{Br}-74$

$\mathrm{Br}-75$

$\mathrm{Br}-76$

$\mathrm{Br}-77$

$\mathrm{Br}-80 \mathrm{~m}$

$\mathrm{Sr}-80$

$\mathrm{Br}-82$

$\mathrm{Br}-83$

St/St/

St/St/

St/St/

St/St/

St/St/

St/St/

St/St/

St/St/

St/St/

St/St/

St/St/

St/St/

St/St/

St/St/

/St/

$\mathrm{Rb}-79$

$\mathrm{Rb}-81 \mathrm{~m}$

$\mathrm{Rb}-81$

$\mathrm{Rb}-82 \mathrm{~m}$

$\mathrm{Rb}-83$

$\mathrm{Rb}-84$

$\mathrm{Rb}-86$

$\mathrm{Rb}-87$

$\mathrm{Rb}-88$

$\mathrm{Rb}-89$

$\mathrm{Sr}-80$

$\mathrm{Sr}-81$

$\mathrm{Sr}-83$

$\mathrm{Sr}-85 \mathrm{~m}$
Inhaled Air - Lung Retention Class $D$

$(\mu \mathrm{Ci} / \mathrm{mL})$

$\mathrm{W}$
$(\mu \mathrm{Ci} / \mathrm{mL})$

2.E-05

2.E-06

$6 . \mathrm{E}-07$

7.E-07

3. E- -07

6. E-07

2. E-06

9.E-06
1.E-05

6.E-05

6.E-06

3. E-07

3.E -07

3. E-05

9. E-05

5.E-05

1.E-05

3. E-05

2. E-05

2. E-06

1.E-05

7. E-06

8.E-05

2. E-06

3. E-05

2.E-05

5. E-05

2. E-05

6. $E-05$

3.E-O

3.E-O

2. E-07

3.E-05

1.E-04

5. E-05

2.E-05

3.E-05

2.E-05

2.E-06

8. E-06

6.E-06

9.E-05

2.E-06

3.E- 05

3. E-05

2.E-05

7.E-O6

4. E-07

3. E-07

3. E-OZ

6. E-07

3. E -05

6.E-05

5. E-06

3.E-05

3.E-06

3. E-04
Stochastic

or Organ 1/

(D/W/Y)

/St/

/St/

/St/

/St/

/St/

/St/

St/St/

St/St/

St/St/

St/St/

St/St/

$\mathrm{St} / \mathrm{St} /$

St/St/

St/St/

St/St/

St/St/

St/St/

St/St/

St/St/

St/St/

St/St/

St/St/

St/ /

st/ /

St/ /

St//

st/ /

st/ /

St/ /

st//

St/ /St

5. E-06

3. E-05

2. E-06

St/ /St

St/ /st

st//St 
Radionuclide

Sr-85

$\mathrm{Sr}-87 \mathrm{~m}$

$\mathrm{Sr}-89$

Sr-90

Sr-91

$\mathrm{S} r-92$

$Y-86 m$

$Y-86$

$Y-87$

$Y-88$

$Y-90 m$

$Y-90$

$\gamma-91 m$

$\gamma-91$

$\gamma-92$

$Y-93$

$Y-93$
$Y-95$

एా

$2 r-86$

$Z r-88$

$\mathrm{Zr}-89$

$\mathrm{Zr}-93$

$\mathrm{Zr}-95$

Zr-97

$\mathrm{Nb}-88$

$\mathrm{Nb}-89$ (66 $\mathrm{min})$

$\mathrm{Nb}-89$ (122 min)

$\mathrm{Nb}-90$

$\mathrm{Nb}-93 \mathrm{~m}$

$\mathrm{Nb}-94$

$\mathrm{Nb}-95 \mathrm{~m}$

$\mathrm{Nb}-95$

$\mathrm{Nb}-96$

$\mathrm{Nb}-97$

Nb-98

Mo-90

Mo-93m

Mo-93

Mo-99

Mo-101
Inhaled Air - Lung Retention Class

$$
\begin{gathered}
(\mu \mathrm{Ci} / \mathrm{mL}) \\
1 . \mathrm{E}-06 \\
5 . \mathrm{E}-05 \\
3 . \mathrm{E}-07 \\
\text { B.E-09 } \\
\text { 2.E-06 } \\
\text { 4.E-06 }
\end{gathered}
$$

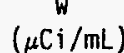

$\begin{array}{ll}\text { 2.E-05 } & 2 . E-05 \\ 1 . E-06 & 1 . E-06 \\ 1 . E-06 & 1 . E-06 \\ 1 . E-07 & 1 . E-07 \\ 5 . E-06 & 5 . E-06 \\ 3 . E-07 & 3 . E-07 \\ 1 . E-04 & 7 . E-05 \\ 7 . E-08 & 5 . E-08 \\ 3 . E-06 & 3 . E-06 \\ 1 . E-06 & 1 . E-06 \\ 3 . E-05 & 3 . E-05 \\ \text { 6.E-05 } & \text { 6.E-05 }\end{array}$

2.E-06

9. $E-08$

2.E-06

3.E-09

6. E-08

8.E-07

2.E-07 1.E-07

$\begin{array}{ll}\text { 1.E }-06 & 1 . E-06 \\ 1 . E-08 & 2 . E-08\end{array}$

2. $E-07 \quad 1, E-07$

6. E-07 5.E-07

1.E-04 9.E-05

2. $E-05$

8. E-06

1. $E-06$

5.E-07

8. $E-08$

1.E-06

5.E-07

1. $E-06$

3. E-05

2. E-05

3.E-06

7.E-06

2. $E-06$

1.E-06

6. E-05

1.E-06 1.E-06

(uci/mit)

Stochastic

or Organ 1/

(D / W/Y)

St/ /St

st/ /st

St/ /St

St/ /St

St/ /St

/St/St

/St/St

/St/St
$/ S t / S t$

/St/St

/St/St

/St/St

/St/St
/St/St

$/ \mathrm{St} / \mathrm{St}$

$/ S t / S t$

St/St/St

St/St/St

St/St/St

BS/St/St

$\mathrm{St} / \mathrm{St} / \mathrm{St}$

/St/St

$/ S t / S t$

/St/St

/St/St

/St/St

/St/St
/St/St

/St/st

/St/St

/St/St

/St/St

2. E-06

6. E-06

6.E-07

St/ /St

St/ /St

St/ /St

St//St

St/ / St
Radionuclide

Tc-93m

Tc-93

Tc-94m

Tc-94

Tc-96m

Tc-96

Tc-97m

Tc-97

Tc-98

Tc-99m

Tc-99

Tc-101

Tc-104

Ru-94

Ru-97

Ru-103

$\mathrm{Ru}-105$

Ru-106

Rh-99m

Rh-99

Rh-100

Rh-101m

Rh-101

Rh-102m

$\mathrm{Rh}-102$

Rh-103m

Rh-105

Rh-106m

Rh-107

$P d-100$

$P d-101$

Pd-103

$\mathrm{Pd}-107$

Pd-109

$\mathrm{Ag}-102$

Ag-103

Ag-104m

$\mathrm{Ag}-104$

$\mathrm{Ag}-105$
Inhaled Air - Lung Retention Class

D
$(\mu \mathrm{Ci} / \mathrm{mL})$

$7 . \mathrm{E}-05$
$3 . \mathrm{E}-05$
$2 . \mathrm{E}-05$
$8 . \mathrm{E}-06$
$1 . \mathrm{E}-04$
$1 . \mathrm{E}-06$
$3 . \mathrm{E}-06$
$2 . \mathrm{E}-05$
$7 . \mathrm{E}-07$
$6 . \mathrm{E}-05$
$2 . \mathrm{E}-06$
$1 . \mathrm{E}-04$
$3 . \mathrm{E}-05$

W

$\mathrm{Y}$
$(\mu \mathrm{Ci} / \mathrm{mL})$

Stochastic

or Organ 1/

$(D / W / Y)$

St/St/

St/St/

St/st/

$\mathrm{St} / \mathrm{St} /$

St/St/

St/St/

SW/St/

St/St/

St/St/

St/St/

SW/St/

St/St/

St/St/

2.E-05

B.E-06

7.E-OT

6.E-06

4. E-08

2. E-05

1.E-06

2. E-06

5.E-06

2.E-07

2.E-07

4. E-08

4.E-04

5. E-06

1. E-05

1. E-04

6. E-07

1.E-05

3.E-06

9. E-06

3. E-06

8.E-05

4. E-05

4.E-05

3. E-05

4.E-07

1.E-07

3.E-07

4.E-05

3. E- 05

5.E-06

4. E-07

6. E-06

2. E-008

2.E -05

.E-06

3.E-07

5.E-06

St/St/St

St/St/St

St/St/St

St/St/St

St/St/St

3.E-05

9. E-07

3.E-05

8.E-07

2.E-06

3. E-06

3.E-0.6

3. E-07

2. E-07

7.E-08

5.E-04

3. E-06

1.E-05

1.E-04

.E-08

5.E-08

E-08

2.E-06

1. E-05

1.E-04

5. E- 07

1.E-05

2.E-06

3. E-06

6.E-07

1.E-05

J.E-06

2. $E-07$

2. E-06

9. E-05

6. E-05

5. E-05

8. E-05

5.E-05

5. E-05

6. E-05

7. E-07

7.E-07

St/St/St

$\mathrm{St} / \mathrm{St} / \mathrm{St}$

St/St/St

St/St/St

St/St/St

St/St/St

St/St/St

St/St/St

St/St/St

St/St/St

St/St/St

$S t / S t / S t$

$S t / S t / S t$

St/St/St

$S t / S t / S t$

St/St/St

St/St/St

St/St/St

St/St/St 
Inhaled Air - Lung Retention Class

Radionuclide $\mathrm{Ag}-106 \mathrm{~m}$ $\mathrm{Ag}-106$ $\mathrm{Ag}-108 \mathrm{~m}$

$A g-110 \mathrm{~m}$

$\mathrm{Ag}-111$

$\mathrm{Ag}-112$

$\mathrm{Ag}-115$

Cd-104

Cd-107

Cd-109

Cd-113m

Cd-113

Cd-115m

Cd-115

$\mathrm{Cd}-117 \mathrm{~m}$

Cd-117

In-109

In-110 (69 min)

tor In-110 (5 h)

In-111

In-112

In-113m

In-114m

In-115m

$\ln -115$

In-116m

$\ln -117 m$

$\ln -117$

In-119m

$S n-110$

Sn-111

Sn-113

Sn-117m

$\mathrm{Sn}-119 \mathrm{~m}$

$\mathrm{S} n-121 \mathrm{~m}$

$\mathrm{Sn}-121$

$\mathrm{S} n-123 \mathrm{~m}$

Sn-123

Sn-125

Sn-126

Sn-127

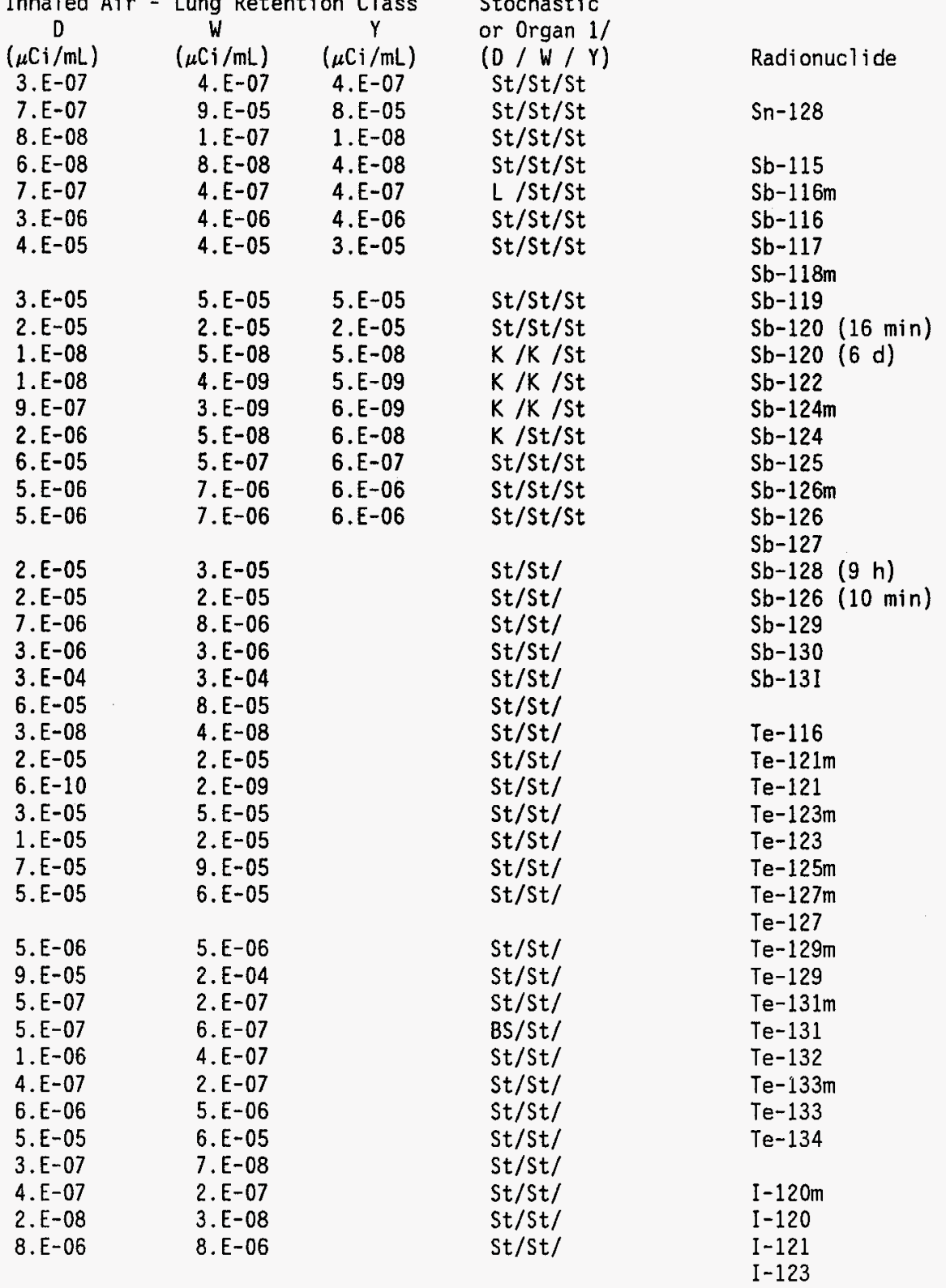

Inhaled Air - Lung Retention Class

$$
\begin{array}{ccc}
D & W & Y \\
(\mu \mathrm{Ci} / \mathrm{mL}) & (\mu \mathrm{Ci} / \mathrm{mL}) & (\mu \mathrm{Ci} / \mathrm{mL})
\end{array}
$$

Stochastic

1. E-05 1. E-05

(D $/ W / Y)$

1.E-04

3.E-05

1.E-04

$9 . E-05$

8.E-06

2. E-05

2.E-04

9. $\mathrm{E}-07$

1.E-06

3.E-04

4.E-07

1.E-06

8.E-05

4. E-07

9.E-07

2.E-06

2.E-04

4.E-06

3.E-05

1.E-05

9.E-06

8.E-08

2.E-06

$9 . E-08$

8.E-08

2. E-O

1. E-07

9.E-06

3. E-07

3. E-05

2. E-07

2.E-06

9. E-08

2. $E-06$

9.E-06

1.E-05

1. E-04

6. E-05

1. E-04

1. E-04

9. E-06

1.E-05

2.E-04

6. $E-07$
4. E-07

3. E-04

1. E-07

2. $E-07$

8.E-05

2. E-07

4. E-07

1.E-06

2.E-04

4. E-06

3. E-05

1. E-05

1. E-05

2. E-07

1. E-06

2. E-07

2. E-07.

3. E-07

1. $E-07$

7.E-06

1. E-07

3. E-05

2. E- 07

2.E-06

9. E-08

2. $E-06$

9. E-06

1. $E-05$

9. E-06

4. E-06

7.E-06

3.E-06
St/St/

St/St/

St/St/

St/St/

St/St/

St/St/

St/St/

St/St/

St/St/

St/St/

$\mathrm{St} / \mathrm{St} /$

St/St/

St/St/

St/St/

St/St/

St/St/

St/St/

St/St/

St/St/

$\mathrm{st} / \mathrm{St} /$

$T / T$ /

St/St/

BS/St/

St/St/

BS/St/

BS/05/

BS/St/

BS/St/

St/St/

St/St/

St/St/

$T / T /$

$\mathrm{T} / \mathrm{T} /$

$\mathrm{T} / \mathrm{T} /$

$T / T$

$\mathrm{T} / \mathrm{T} /$

$\mathrm{T} / \mathrm{T} /$

St/ /

T / /

т 11 
Inhaled Air - Lung Retention Class

Radionucl ide

I-124

$\mathrm{I}-125$

I -126

I-128

$1-130$

I -131

$\mathrm{I}-132 \mathrm{~m}$

$1-132$

I -133

I -134

Cs-125

Cs -127

Cs-129

Cs -130

Cs-131

Cs-132

$\infty$

Cs-134m

$\infty \quad \mathrm{Cs}-134$

Cs -135

Cs -136

Cs-137

Cs -138

Ba-126

$B a-128$

$\mathrm{Ba}-131 \mathrm{~m}$

Ba-131

$\mathrm{Ba}-133 \mathrm{~m}$

$\mathrm{Ba}-133$

$\mathrm{Ba}-139$

$\mathrm{Ba}-141$

Ba-142

La-131

La -132

La-135

La-137

La-138

$(\mu \mathrm{Ci} / \mathrm{mL}) \quad(\mu \mathrm{Ci} / \mathrm{mL}) \quad(\mu \mathrm{Ci} / \mathrm{mL})$

tochastic

or Organ 1/

3. E-08

3. E-08

1.E-08

5.E-05

4.E-09

3. E-07

2. E-08

4.E-06

3.E-06

$1 . \mathrm{E}-07$

2.E-05
$7 . E-07$

6. E-05

4. E-05

1.E-05

8. E-0.

2.E-06

6. $E-05$

8. E-05

5. E-07

3. $E-07$

7.E-08

2.E-05

6. E-06

7.E-07

6. E-04

3. E-06

4.E-06

3. $E-07$

5.E-06

1.E-05

6. E-07

3. E-05

6. E-05

5.E-05

4.E-06

4. $\mathrm{E}-05$

3.E-08

2. E-09

7.E-05

4.E-05

1. $E-07$
6. $E-09$

6.E-09

(1)
Inhaled Air - Lung Retention Class

Radionuclide

D
$(\mu \mathrm{Ci} / \mathrm{mL})$
$6 . E-07$
$4 . E-06$
$9 . E-06$
$4 . E-05$

$\begin{array}{ll}5 . E-07 & \mathrm{St} / \mathrm{St} / \\ 5 . \mathrm{E}-06 & \mathrm{St} / \mathrm{St} / \\ 1 . \mathrm{E}-05 & \mathrm{St} / \mathrm{St} / \\ 4 . \mathrm{E}-05 & \mathrm{St} / \mathrm{St} /\end{array}$

$\mathrm{Nd}-149$

Nd-151

Pm-141

Pm-143

Pm-144

Pm-145

Pm-146

Pm-147

$P m-148 m$

Pm-148

Pm-149

Pm-150
$\mathrm{W}$

$(\mu \mathrm{Ci} / \mathrm{mL})$

3. E-07

2. E-06

2.E-06

6. E-05

3. E-07

3. E-07

8.E-07

1.E-06

1. E-04

6. E-05

2. E-05

5. E-05

7.E-05

8. E-07

3. E-07

5. E-05

4. E-06

8. E-05

2. E-05

3. E-06

7.E-06

1.E-04

3.E-04

4. E-07

1. E-05

8. E-05

3. E-07
2. E-06

2. E-06

5.E-05

3.E-07

3.E-07

7.E-07

6. E-09

9. E-05

6. E-05

2. E-05

5.E-05

6.E-05

8. E-07

3. E-07

5. E-05

3. E-06

8. E-05

2. E-05

2. E-06

6. E-06

1.E-04

3 E-04

3. E-07

1.E-05

Stochastic

or Organ $1 /$

$(D / W / Y)$

8.E-05

3. E-07

5.E-08

7.E-08

2. $E-08$

6.E-08

1.E-07

2. E-07

8. E-07

8. $E-05$

7. E-05

3.E-07

5. E-08

8. E-08

2. $E-08$

6. E-08

1. E-07

2.E-07

8. E-07

7. E-06

$/ S t / S t$

/St/St

/St/St

/St/St

/St/St

/St/St

/St/St

$/ S t / S t$

$/ S t / S t$

/St/St

/St/St

/St/St

/St/St

/St/St

/St/St

/St/St

/St/St

/St/St

/St/St

/St/St

/St/St

/St/St

/St/St

/St/St

/St/St

/St/St

$/ S t / S t$

/St/St

BS/St

/BS/St

/St/St

/St/St

/St/St

/St/St 
Inhaled Air - Lung Retention Class Stochastic

Radionuclide

Pm-151

$\mathrm{Sm}-141 \mathrm{~m}$

$\mathrm{Sm}-141$

Sm-142

Sm-145

$\mathrm{Sm}-146$

Sm-147

Sm-151

Sm-153

Sm-155

Sm-156

Eu-145

Eu-146

Eu-147

Eu-148

Eu-149

Eu-150 (12 h)

Eu-150 (34 yr

क. Eu-152m

$E u-152$
$E u-154$

Eu-155

Eu-156

Eu-157

Eu-158

Gd-145

Gd-146

Gd-147

Gd-148

Gd-149

Gd-151

Gd-152

Gd-153

Gd-159

Tb-147

$\mathrm{Tb}-149$

Tb-150

$T b-151$

Tb-153

$\mathrm{Tb}-154$

\begin{tabular}{|c|c|c|c|c|}
\hline $\begin{array}{c}D \\
(\mu \mathrm{Ci} / \mathrm{mL})\end{array}$ & $\begin{array}{c}W \\
(\mu \mathrm{Ci} / \mathrm{mL})\end{array}$ & $\begin{array}{c}Y \\
(\mu \mathrm{Ci} / \mathrm{mL})\end{array}$ & $\begin{array}{l}\text { or Organ } 1 / \\
(D / W / Y)\end{array}$ & Radionuclide \\
\hline & $\begin{array}{l}2 . E-06 \\
4 . E-05 \\
7 . E-05 \\
1 . E-05 \\
2 . E-07 \\
1 . E-11 \\
2 . E-11 \\
4 . E-08 \\
1 . E-06 \\
9 . E-05 \\
4 . E-06 \\
8 . E-07 \\
5 . E-07 \\
7 . E-07 \\
2 . E-07 \\
1 . E-06 \\
3 . E-06 \\
8 . E-09 \\
3 . E-06 \\
1 . E-08 \\
8 . E-09 \\
4 . E-08 \\
2 . E-07 \\
2 . E-06 \\
2 . E-05\end{array}$ & 1.E-06 & $\begin{array}{l}\text { /St/St } \\
\text { /St/ } \\
\text { /St/ } \\
\text { /St/ } \\
\text { /St/ } \\
\text { /BS/ } \\
\text { /BS/ } \\
\text { /BS/ } \\
\text { /St/ } \\
\text { /St/ } \\
\text { /St/ } \\
\text { /St/ } \\
\text { /St/ } \\
\text { /St/ } \\
\text { /St/ } \\
\text { /St/ } \\
\text { /St/ } \\
\text { /St/ } \\
\text { /St/ } \\
\text { /St/ } \\
\text { /St/ } \\
\text { /BS/ } \\
\text { /St/ } \\
\text { /St/ } \\
\text { /St/ }\end{array}$ & $\begin{array}{l}\text { Tb-155 } \\
\text { Tb-156m }(24 \mathrm{~h}) \\
\text { Tb-156m }(5 \mathrm{~h}) \\
\text { Tb-156 } \\
\text { Tb-157 } \\
\text { Tb-158 } \\
\text { Tb-160 } \\
\text { Tb-161 } \\
\text { Dy-155 } \\
\text { Dy-157 } \\
\text { Dy-159 } \\
\text { Oy-165 } \\
\text { Dy-166 } \\
\\
\text { Ho-155 } \\
\text { Ho-157 } \\
\text { Ho-159 } \\
\text { Ho-161 } \\
\text { Ho-162m } \\
\text { Ho-162 } \\
\text { Ho-164m } \\
\text { Ho-164 } \\
\text { Ho-166m } \\
\text { Ho-166 } \\
\text { Ho-167 }\end{array}$ \\
\hline $\begin{array}{l}\text { 7.E-05 } \\
5 . E-08 \\
2 . E-06 \\
\text { 3.E-12 } \\
9 . E-07 \\
2 . E-07 \\
4 . E-12 \\
6 . E-08 \\
3 . E-06\end{array}$ & $\begin{array}{l}7 . E-05 \\
1 . E-07 \\
2 . E-06 \\
1 . E-11 \\
1 . E-06 \\
5 . E-07 \\
2 . E-11 \\
3 . E-07 \\
2 . E-06 \\
1 . E-05 \\
3 . E-07 \\
9 . E-06 \\
4 . E-06 \\
3 . E-06 \\
2 . E-06\end{array}$ & & $\begin{array}{l}\text { St/St/ } \\
\text { St/St/ } \\
\text { St/St/ } \\
\text { BS/BS/ } \\
\text { St/St/ } \\
\text { BS/St/ } \\
\mathrm{BS} / \mathrm{BS} / \\
\mathrm{BS} / \mathrm{St} / \\
\mathrm{St} / \mathrm{St} / \\
\\
\text { /St/ } \\
\text { /St/ } \\
\text { /St/ } \\
\text { /St/ } \\
\text { /St/ } \\
\text { /St/ }\end{array}$ & $\begin{array}{c}\mathrm{Er}-161 \\
\mathrm{Er}-165 \\
\mathrm{Er}-169 \\
\mathrm{Er}-171 \\
\mathrm{Er}-172 \\
\\
\mathrm{Tm}-162 \\
\mathrm{Tm}-166 \\
\mathrm{Tm}-167 \\
\mathrm{Tm}-170 \\
\mathrm{Tm}-171 \\
\mathrm{Tm}-172 \\
\mathrm{Tm}-173 \\
\mathrm{Tm}-175 \\
\\
\mathrm{Yb}-162 \\
\mathrm{Yb}-166\end{array}$ \\
\hline
\end{tabular}

Inhaled Air - Lung Retention Class

Stochastic

D $\quad W \quad Y$

or Organ $1 /$

$(\mu \mathrm{Ci} / \mathrm{mL})$

$(\mu \mathrm{Ci} / \mathrm{mL})$

$(\mu \mathrm{Ci} / \mathrm{mL})$

(D / W / Y)

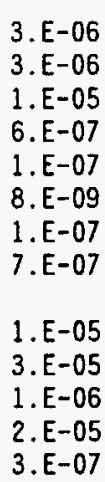

/St/

/St/

/St/

/BS/

/St/

/St/

/St/

/St/

/St/

/St/

/St/

/St/

/St/

/St/

/St/

/St/

/St/

/St/

/St/

St $i$

/St/

/St/

/St/

St/

/St/

/St/

/St/

/BS/

/St/

/St/
$/ \mathrm{St} /$

/St/

5. E-06

1.E-04

1.E-04 1.E-04

$/ S t / S t$

/St/St 
Inhaled Air - Lung Retention Class Stochastic

or Organ $1 /$

Radionuclide

$\mathrm{Yb}-167$

$\mathrm{Yb}-169$

$Y b-175$

Yb-177

$\mathrm{Yb}-178$

Lu-169

Lu-170

Lu-171

Lu-172

Lu-173

Lu-174m

Lu-174

Lu $-176 m$

Lu-176

Lu-177m

Lu-177

Lu-178m

(1)

$\dot{0}$

$\begin{array}{ll}H f-170 & 2 . E-06 \\ H f-172 & 4 . E-09 \\ H f-173 & 5 . E-06 \\ H f-175 & 4 . E-07 \\ H f-177 m & 2 . E-05 \\ H f-178 m & 6 . E-10 \\ H f-179 m & 1 . E-07 \\ H f-180 m & 9 . E-06 \\ H f-181 & 7 . E-08 \\ H f-182 m & 4 . E-05 \\ H f-182 & 3 . E-10 \\ H f-183 & 2 . E-05 \\ H f-184 & 3 . E-06\end{array}$

$\mathrm{Ta}-172$

$\mathrm{Ta}-173$

$T a-174$

Ta-175

$\mathrm{Ta}-176$

Ta-177

Ta-178

$\mathrm{Ta}-179$

Ta-180m

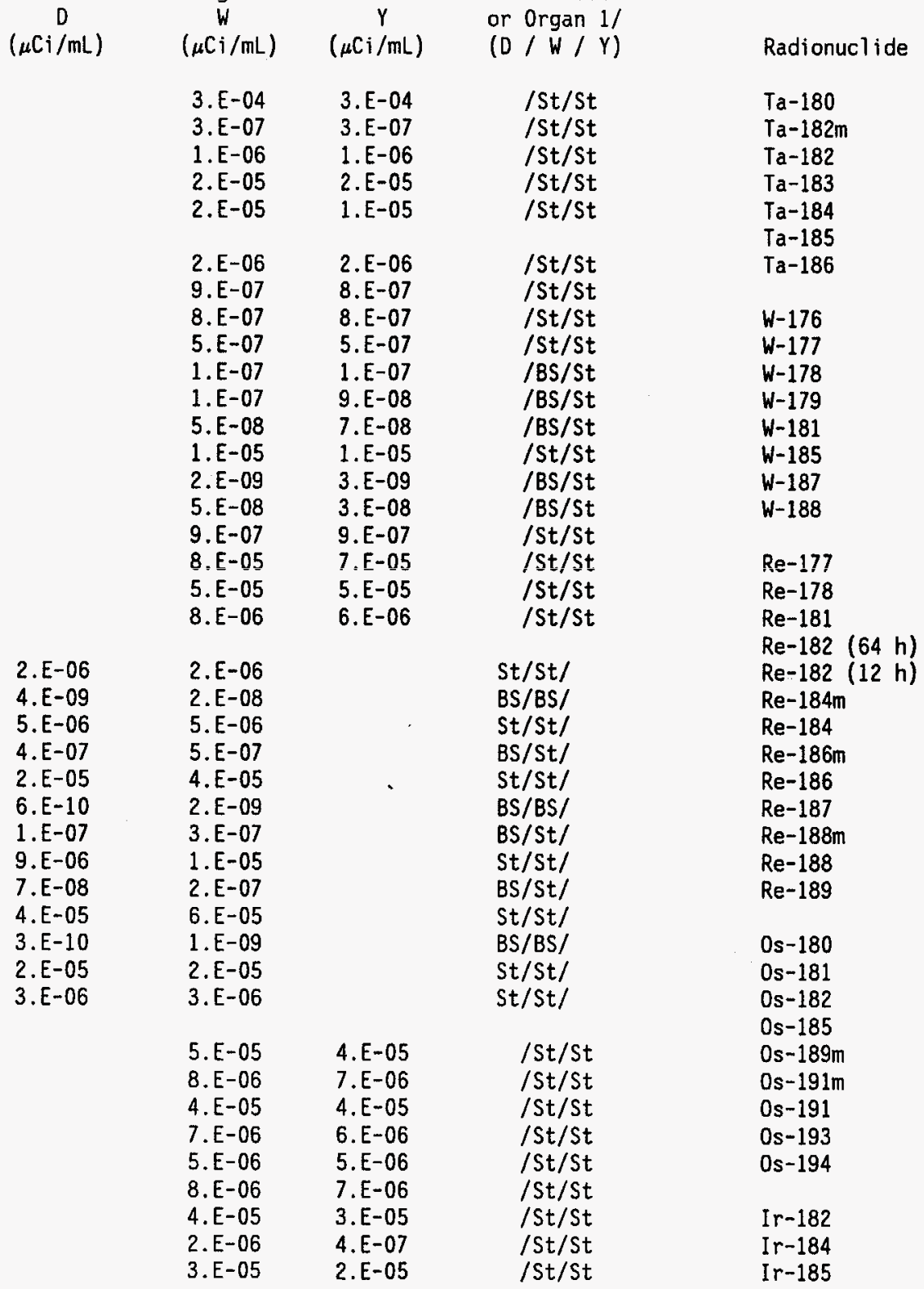

Inhaled Air - Lung Retention Class D

$\begin{array}{cc}\begin{array}{c}W \\ (\mu \mathrm{Ci} / \mathrm{mL})\end{array} & \begin{array}{c}Y \\ (\mu \mathrm{Ci} / \mathrm{mL})\end{array} \\ 2 . E-07 & 1 . \mathrm{E}-08 \\ 2 . \mathrm{E}-04 & 2 . \mathrm{E}-04 \\ 1 . \mathrm{E}-07 & 6 . \mathrm{E}-08 \\ 5 . \mathrm{E}-07 & 4 . \mathrm{E}-07 \\ 2 . \mathrm{E}-06 & 2 . \mathrm{E}-06 \\ 3 . \mathrm{E}-05 & 3 . \mathrm{E}-05 \\ 1 . \mathrm{E}-04 & 9 . \mathrm{E}-05\end{array}$

$2 . E-05$
$4 . E-05$
$8 . E-06$
$7 . E-04$
$1 . E-05$
$3 . E-06$
$4 . E-06$
$5 . E-07$
$1 . E-04$
$1 . E-04$
$4 . E-06$
$1 . E-06$
$5 . E-06$
$1 . E-06$
$2 . E-06$
$7 . E-07$
$1 . E-06$
$3 . E-04$
$6 . E-05$
$1 . E-06$
$2 . E-06$
2.E-04
$2 . E-05$
$2 . E-06$
$2 . E-07$
$1 . E-04$
$1 . E-06$
$9 . E-07$
$2 . E-06$
$2 . E-08$
$6 . E-05$
$1 . E-05$
$5 . E-06$

1.E-06

2.E-06

2.E-04

2. E-05

2. E-06

3. E-07

9.E-05

9. E-06

7. E-07

1. E-06

2. E-08

2. $E-04$

2. E- 05

2. E-06

3. E-07

7. $E-05$

7.E-06

6. E-07

1. E-06

3. E-OO

6. E-05

1. E-05

5. E-05

5. $E-05$
1. $E-05$
Stochastic or Organ 1/

$(D / W / Y)$

/St/St

/St/St

/St/St

/St/St

/St/St

/St/St

/St

St/ I

St/ /

st/ I

St/ /

St/ /

st/ /

St/St/

St/St/

St/St/

St/St/

St/St/

St/St/

St/St/

SW/St/

St/St/

SW/St/

$\mathrm{St} / \mathrm{St} /$

St/St/

St/St/

St/St/St

St/St/St

St/St/St

St/St/St

St/St/St

St/St/St

$\mathrm{St} / \mathrm{St} / \mathrm{St}$

St/St/St

St/St/St

$\mathrm{St} / \mathrm{St} / \mathrm{St}$

St/St/St

St/St/St 


\section{Radionuclide}

I $r-186$

I $r-187$

I $r-188$

I $r-189$

I $r-190 m$

I $r-190$

I $r-192 m$

I $r-192$

I $r-194 m$

Ir -194

I $r-195 \mathrm{~m}$

I $r-195$

Pt-186

Pt-188

Pt-189

Pt-191

Pt-193m

Pt-193

(ए) Pt-195m

i. Pt $-197 \mathrm{~m}$

Pt-199

Pt-200

Au-193

Au-194

Au-195

$\mathrm{Au}-198 \mathrm{~m}$

$\mathrm{Au}-198$

Au-199

$\mathrm{Au}-200 \mathrm{~m}$

$\mathrm{Au}-200$

$\mathrm{Au}-201$

$\mathrm{Hg}-193 \mathrm{~m}$ (Org)

$\mathrm{Hg}-193 \mathrm{~m}$ (Inorg)

$\mathrm{Hg}-193 \mathrm{~m}$ (Vapor)

$\mathrm{Hg}-193$ (Org)

$\mathrm{Hg}-193$ (Inorg)

Hg-193 (Vapor)

$\mathrm{Hg}-194$ (Org)

Hg-194 (Inorg)

$\mathrm{Hg}-194$ (Vapor)
Inhaled Air - Lung Retention Class

$\begin{array}{ccc}\begin{array}{c}D \\ (\mu \mathrm{Ci} / \mathrm{mL})\end{array} & \begin{array}{c}W \\ (\mu \mathrm{Ci} / \mathrm{mL})\end{array} & \begin{array}{c}Y \\ (\mu \mathrm{Ci} / \mathrm{mL})\end{array} \\ 3 . \mathrm{E}-06 & 3 . \mathrm{E}-06 & 2 . \mathrm{E}-06 \\ 1 . \mathrm{E}-05 & 1 . \mathrm{E}-05 & 1 . \mathrm{E}-05 \\ 2 . \mathrm{E}-06 & 2 . \mathrm{E}-06 & 1 . \mathrm{E}-06 \\ 2 . \mathrm{E}-06 & 2 . \mathrm{E}-06 & 2 . \mathrm{E}-06 \\ 8 . \mathrm{E}-05 & 9 . \mathrm{E}-05 & 8 . \mathrm{E}-05 \\ 4 . \mathrm{E}-07 & 4 . \mathrm{E}-07 & 4 . \mathrm{E}-07 \\ 4 . \mathrm{E}-08 & 9 . \mathrm{E}-08 & 6 . \mathrm{E}-09 \\ 1 . \mathrm{E}-07 & 2 . \mathrm{E}-07 & 9 . \mathrm{E}-08 \\ 4 . \mathrm{E}-08 & 7 . \mathrm{E}-08 & 4 . \mathrm{E}-08 \\ 1 . \mathrm{E}-06 & 8 . \mathrm{E}-07 & 8 . \mathrm{E}-07 \\ 1 . \mathrm{E}-05 & 1 . \mathrm{E}-05 & 9 . \mathrm{E}-06 \\ 2 . \mathrm{E}-05 & 2 . \mathrm{E}-05 & 2 . \mathrm{E}-05\end{array}$

2.E-05

7.E-07

1. E-05

3. E-06

2.E-06

1. E-05

2.E-06

2. E-05

4. E-O6

6.E-05

1.E-06

1. E-05

3. E-06

5. E-06

1. E-06

2.E-06

4. E-06

1. E-06

3. E-05

6. -06

6. $E-06$
4. E-06

3. E- 05

2.E-05

1. E-08

2. E-08

8.E-06

2. E-06

6.E- -07

5. E-07

7. E-07

2.E-06

1.E-06

3.E-05

1.E-O4

8. E-06

2. E-06

2. $\mathrm{E}-07$

$5, E-07$

7.E-07

2.E-06

1. E-06

3. E-05
Stochastic

or Organ 11

(D $/ W / Y$ )

St/St/St

St/St/St

St/St/St

St/St/St

St/St/St

St/St/St

St/St/St

$\mathrm{St} / \mathrm{St} / \mathrm{St}$

St/St/St

St/St/St

St/St/St

St/ /

St/ /

st/ /

St/ /

St/ /

St/ /

St/ /

St/ /

St/ I

st/ $/$

St/St/St

St/St/St

St/St/St

St/St/St

$\mathrm{St} / \mathrm{St} / \mathrm{St}$

St/St/St

$\mathrm{St} / \mathrm{St} / \mathrm{St}$

St/St/St

St/ /

St/St/

/St/

St/st/

/St/

/St/
st// /
st/st/

St/St/
Radionuclide

$\mathrm{Hg}-195 \mathrm{~m}$ (Org)

Hg-195m (Inorg)

$\mathrm{Hg}-195 \mathrm{~m}$ (Vapor)

$\mathrm{Hg}-195$ (Org)

$\mathrm{Hg}-195$ (Inorg)

Hg-195 (Vapor)

$\mathrm{Hg}-197 \mathrm{~m}$ (Org)

$\mathrm{Hg}-197 m$ (Inorg)

$\mathrm{Hg}-197 \mathrm{~m}$ (Vapor)

$\mathrm{Hg}-197$ (Org)

$\mathrm{Hg}-197$ (Inorg)

$\mathrm{Hg}-199 \mathrm{~m}$ (Org)

$\mathrm{Hg}-199 \mathrm{~m}$ (Inorg)

$\mathrm{Hg}-199 \mathrm{~m}$ (Vapor)

$\mathrm{Hg}-203$ (Org)

$\mathrm{Hg}-203$ (Inorg)

Hg-203 (Vapor)

Tा-194m

T1-194

T1-195

T1-197

T1 $-198 \mathrm{~m}$

Tा-198

T1-199

T1-200

T1-201

T1-202

$\mathrm{Pb}-195 \mathrm{~m}$

$\mathrm{Pb}-198$

$\mathrm{Pb}-199$

Pb-200

$\mathrm{Pb}-201$

$\mathrm{Pb}-202 \mathrm{~m}$

$\mathrm{Pb}-202$

$\mathrm{Pb}-203$

Pb-205

$\mathrm{Pb}-209$

$\mathrm{Pb}-210$

$\mathrm{Pb}-211$

$\mathrm{Pb}-212$
Inhaled Air - Lung Retention Class

$\begin{array}{ccc}D & W & Y \\ \mu \mathrm{Ci} / \mathrm{mL}) & (\mu \mathrm{Ci} / \mathrm{mL}) & (\mu \mathrm{Ci} / \mathrm{mL})\end{array}$

Stochastic

or Organ $1 /$

$(D / W / Y)$

3. E-06

2. $\mathrm{E}-06$

2. E-06

2. $E-06$

2. E- 05

1. E-05

1.E-05

4. E-06

1. E-05

3. E-06

2.E-06

2.E-06

6. E-06

5. E-06

4.E-06

7.E-05

3.E-05

6. E-05

7. E-05

3. E-05

3. E-07

5. E- -07

5. E-07

6. E-05

3. E-04

5. E-05

5. E-05

2. E-05

1.E-05

3. E-05

5. E-06

9. E-06

2. E-0S

9.

8.E-05

3. E -05

3. E-05

3. E-06

9. E-06

1. E-05

2.E-08

4. E-06

6. E-07

2. E-05

1.E-10

3. $E-07$
$1 . E-08$
St/ /

St/St/

St/

$\mathrm{St} / \mathrm{St} /$

/St/

st/ /

t/St/

/St.

st/ /

$\mathrm{St} / \mathrm{St} /$

/St/

St/St/

/St/

St/ I

St/St/

/St/

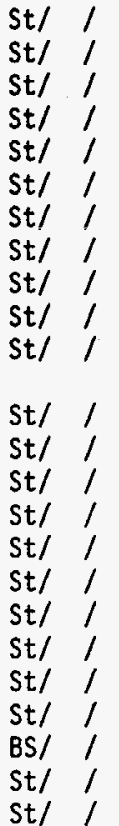


Inhaled Air - Lung Retention Class $D \quad W \quad Y$

Stochastic

Radionucl ide

3.E-07

3. E- 05

1. E-05

2. $E-05$

3.E-06

1.E-06

6.E-07

7.E-07

2. E-09

1.E-07

1. $E-07$

1. $E-07$

3.E-07

3.E-05

2. E-05

1.E- 05

3.E-10

1.E-06
3. E-08

8.E-09 $4 /$
3.E-08 $4 /$

2.E-07

3.E-07

$\mathrm{Fr}-222$

$\mathrm{Ra}-223$

$\mathrm{Ra}-224$

$\mathrm{Ra}-225$

$\mathrm{Ra}-226$

$\operatorname{Ra}-227$

$\mathrm{Ra}-228$

Ac- -224

Ac -225

Ac-226

Ac-227

Ac -228

Th-226

Th-226
Th-227
(D / W / Y )

\begin{tabular}{|c|c|}
\hline St/ $/$ & $\begin{array}{l}\text { Th-228 } \\
\text { Th-229 }\end{array}$ \\
\hline$S t / S t /$ & Th-230 \\
\hline$S t / S t /$ & Th-231 \\
\hline St/St/ & Th-232 \\
\hline $\mathrm{St} / \mathrm{St} /$ & Th-234 \\
\hline St/St/ & \\
\hline $\begin{array}{l}\mathrm{St} / \mathrm{St} / \\
\mathrm{St} / \mathrm{St} /\end{array}$ & $\begin{array}{l}\mathrm{Pa}-227 \\
\mathrm{~Pa}-228\end{array}$ \\
\hline $\mathrm{k} / \mathrm{st} /$ & $\mathrm{Pa}-233$ \\
\hline $\mathrm{K} / \mathrm{st} /$ & $\mathrm{Pa}-231$ \\
\hline St/St/ & $\mathrm{Pa}-232$ \\
\hline $\mathrm{St} / \mathrm{St} /$ & $\mathrm{Pa}-233$ \\
\hline St/St/ & $\mathrm{Pa}-234$ \\
\hline $\mathrm{St} / \mathrm{St} /$ & $U-230$ \\
\hline $\mathrm{st} / \mathrm{st} /$ & $U-231$ \\
\hline $\mathrm{St} / \mathrm{St} /$ & $U-232$ \\
\hline$E / S t /$ & $\mathrm{U}-233$ \\
\hline & $U-234$ \\
\hline St/St/ & $U-235$ \\
\hline $\mathrm{St} / \mathrm{St} /$ & $U-236$ \\
\hline & $U-237$ \\
\hline $4 /$ & $\mathrm{U}-238$ \\
\hline $4 /$ & $U-239$ \\
\hline & $U-240$ \\
\hline st/ / & \\
\hline st/ / & $\mathrm{Np}-232$ \\
\hline & $\mathrm{Np}-233$ \\
\hline /St/ & $\mathrm{Np}-234$ \\
\hline /St/ & $N p-235$ \\
\hline /St/ & $\mathrm{Np}-136 \quad(1 . E+05 \mathrm{yr})$ \\
\hline /St/ & $\mathrm{Np}-236(22 \mathrm{~h})$ \\
\hline /BS/ & $\mathrm{Np}-237$ \\
\hline /St/ & $N p-238$ \\
\hline & $N p-239$ \\
\hline BS/St/St & $\mathrm{Np}-240$ \\
\hline $\mathrm{BS} / \mathrm{St} / \mathrm{St}$ & \\
\hline BS/St/St & $\mathrm{Pu}-234$ \\
\hline$B S / B S / S t$ & $\mathrm{Pu}-235$ \\
\hline $\mathrm{BS} / \mathrm{BS} / \mathrm{St}$ & Pu-236 \\
\hline & $\mathrm{Pu}-237$ \\
\hline$/ S t / S t$ & $P u-238$ \\
\hline$/ S t / S t$ & $P u-239$ \\
\hline
\end{tabular}

Inhaled Air - Lung Retention Class

$$
\begin{gathered}
D \\
(\mu \mathrm{Ci} / \mathrm{mL})
\end{gathered}
$$$$
(\mu \mathrm{Ci} / \mathrm{mL})
$$$$
\begin{gathered}
\mathrm{Y} \\
(\mu \mathrm{Ci} / \mathrm{mL})
\end{gathered}
$$

2. $E-10$

3.E-06

9. $E-11$

5. E- 10

5. E-10

6. E-10

6.E-10

1.E-06

6.E-10
8. E-05

2.E-06$$
\text { 4. E-12 }
$$$$
\text { 4. } E-13
$$$$
\text { 3. E-12 }
$$$$
\text { 3.E-06 }
$$$$
\text { 5. E-13 }
$$$$
\text { 9.E-08 }
$$

5.E-08

5.E-09

2. E-09

7.E-13

9. E-09

3. $E-07$

3.E-06

1. $E-10$

2.E-06

2. $E-10$

3. E-10

3. E-10

3. $E-10$

3.E-10

$E-10$

7. E-05

1. E-06

7.E-12

1. $\mathrm{E}-12$

7.E-12

3.E-06

1. $E-12$

6.E-08

4.E-08

5. E-09

1.E-09

2.E-12

2. E-08

2. $E-07$

3. E-06

1. E-10

2.E-06

2. $E-11$

2.E-11

2.E-11

2. $E-11$

6. $E-07$

2. $E-11$

6.E-05

1.E-06

1.E-06 5/

1.E-03 5/

1.E-06 5/

5. E-07 5/

1.E-11 5/

2.E-08 $5 /$

2. $E-125 /$

4.E-08 $5 /$

1.E-06 5/

3.E-05 5/

9.E-08 5/ 8.E-08 5/

1. $E-035 / \quad 1 . E-035 /$

7.E-12 5/ 1.E-11 5/

1.E-06 5/ 1.E-06 5/

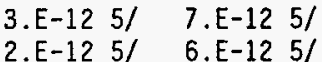

Stochastic

or Organ 1/

$(D / W / Y)$

/BS/St

/BS/BS

/BS/BS

/St/St

/BS/BS

/St/St

/St/St

/BS/St

$/ \mathrm{St} / \mathrm{St}$

/BS/BS

/BS/BS

/St/St

/St/St

BS/St/St

St/St/St

BS/St/St

BS/St/St

BS/St/St

BS/St/St

BS/St/St

St/St/St

BS/St/St

St/St/St

St/St/St

/BS/

/St/

/St/

/BS/

/BS/

/BS/

BS,

BS/

/St/
/St/

/St/St

/St/St

/BS/St

/St/St

/BS/BS

/BS/BS 
Inhaled Air - Lung Retention Class

Radionuclide

$\mathrm{Pu}-240$

Pu-241

$\mathrm{Pu}-242$

$\mathrm{Pu}-243$

Pu-244

Pu-245

Am-237

Am-238

Am-239

Am-240

$A m-241$

Am-242m

Am-242

Am-243

Am-244m

Am-244

$A m-245$

Am-246m

Am-246

ஸr

$\mathrm{Cm}-238$

$\mathrm{Cm}-240$

$\mathrm{Cm}-241$

$\mathrm{Cm}-242$

$\mathrm{Cm}-243$

$\mathrm{Cm}-246$

$\mathrm{Cm}-247$

$\mathrm{Cm}-248$

$\mathrm{Cm}-249$

Bk-245

Bk -246

Bk-247

Bk-249

Bk-250

Cf -244

Cf -246

Cf -248

Cf -249

Cf -250

.E- -05 5/

1.E-04 5/

1.E-06 5/

5.E-06 5/

1.E-06 5/

2.E-12 $5 /$

2.E-12 $5 /$

3.E-08 5/

2. E-12 5/

2.E-06 5/

7.E-08 5/

3.E-05 5/

7.E-05 5/

4.E-07 5/

2.E-10 5/

9.E-09 5/

1.E-10 5/

3.E-12 5/

4. E-12 $5 /$

2.E-12 5/

2. E-12 5/

2.E-12 $5 /$

6. $E-135 /$

$6 . \mathrm{E}-065 /$

5. E- -07

1.E-06

2. $E-12$

9. $E-10$

2. E- 07

2.E-07 5/ 2.E-07 5/

4.E-09 5/ 4.E-09 5/

4.E-11 5/ 5.E-11 5/

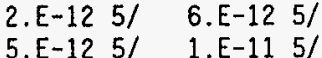

Stochastic

or Organ 1/

$(\mu \mathrm{Ci} / \mathrm{mL}) \quad(\mu \mathrm{Ci} / \mathrm{mL}) \quad(\mu \mathrm{Ci} / \mathrm{mL}) \quad(\mathrm{D} / \mathrm{W} / \mathrm{Y})$

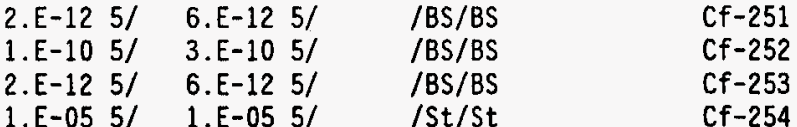

2.E-12 5/ 6.E-12 5/ /BS/BS

2.E-06 5/ 2.E-06 5/ /St/St
/St/

/BS/

/BS/

/BS/

/BS/

IBS/

/BS/

/BS/

/BS/

/St/

/St/

/BS/

IBS/

/St/St

$/ S t / S t$

/BS/St

/BS/BS

/BS/St
Radionuclide

Cf -251

Cf -252

Cf -253

Inhaled Air - Lung Retention Class

Stochastic

$D \quad W \quad$ or Organ 1/

Es -250

Es-251

Es-253

Es-254m

Es-254

$\mathrm{Fm}-252$

$\mathrm{Fm}-253$

$\mathrm{Fm}-254$

$\mathrm{Fm}-255$

$\mathrm{Fm}-257$

Md-257

$\mathrm{Md}-258$

$(\mu \mathrm{Ci} / \mathrm{mL}) \quad(\mu \mathrm{Ci} / \mathrm{mL})$

$(0 / W / Y)$

2.E-12 5/ 5.E-12 5/ /BS/BS

1.E-11 5/ 2.E-11 5/ /BS/St

8.E-10 5/ 7.E-10 5/ /St/St

9.E-12 5/ 7.E-12 5/ /St/St

3. E- -07

4. E-07

6. $E-10$

4. E-09

4. E-11

6. E-09

4. E-09

4. E- -08

9.E-09

1. $E-10$

4.E-08

1.E-10

/BS/

/BS/

/St/

/St/

BS/

/St/

/St/

/St/

/E /

/St/ 
1/ A determination of whether the DACs are controlled by stochastic (St) or nonstochastic (organ) dose, or if they both give the same result (E) for each lung retention class is given in this column. The key to the organ notation for nonstochastic dose is: BS Bone surface, $K=$ Kidney, $L=$ Liver, $\operatorname{Sin}=$ Stomach wall, and $T=$ Thyroid. A blank Indicates that no calculations are performed for the lung retention class shown.

2/ The ICRP identifies tritiated water and carbon as having immediate uptake and distribution; therefore no solubility classes are designated. For

purposes of this table, the DAC values are shown as being constant, independent of solubility class. For tritiated water, the inhalation DAC values allow for an additional 50\% absorption through the skin. as described in ICRP Publication No. 30: Limits for Intakes of Radionuclides by Workers. For elemental tritium, the DAC values are based solely on consideration of the dose-equivalent rate to the tissues of the lung from inhaled tritium gas contained within the lung, without absorption in the tissues.

3/ A dash indicates no values given for this data category.

4/ These values are appropriate for protection from radon combined with its short-lived daughters and are based on information given in ICRP Publication 32: Limits for Inhalation of Radon Daughters by Workers and w Federal Guidance Report No. 11: Limiting Values of Radionuclide Intake and Air Concentrations, and Dose Conversion Factors for Inhalation.

1 Submersion, and Ingestion (EPA 520/1-88-020). The values given are for $100 \%$ equilibrium concentration conditions of the radon daughters with the parent. To allow for an actual measured equilibrium concentration or a demonstrated equilibrium concentration, the values given in this table should be multiplied by the ratio (100\%/actual \%) or $(100 \% /$ demonstrated $\%)$. respectively. Alternatively, the DAC values for $\mathrm{Rn}-220$ and $\mathrm{Rn}-222$ may be replaced by $1 \mathrm{WL}^{*}$ and $1 / 3 \mathrm{WL}$, ${ }^{*}$ respectively, for appropriate $1 \mathrm{imiting}$ of daughter concentrations. Because of the dosimetric considerations for radon, no $f 1$ or lung clearance values are listed.

* A "Working Level" (WL) is any combination of short-lived radon daughters, in one liter of air without regard to the degree of equilibrium, that will result in the ultimate emission of $1.3 \mathrm{E}+05 \mathrm{MeV}$ of alpha energy.

5/ For the calculations, fl values were obtained from ICRP Publication 48: The Metabol ism of Plutoni um and Related Elements. It is assumed that the effective dose equivalents for inhalation are unchanged even though the $f 1$ values have changed. This is because the contribution to organ dose from inhalation is dependent mainly on transfer from lung to blood when $f 1$ values are sma11. Also, the gastrointestinal tract dose would be unchanged because the fraction of activity passing through the tract is $(1.0-f 1)$. 
Table 2

Alternative AbsorptIon Factors and Lung Retention Classes for Specific Compounds

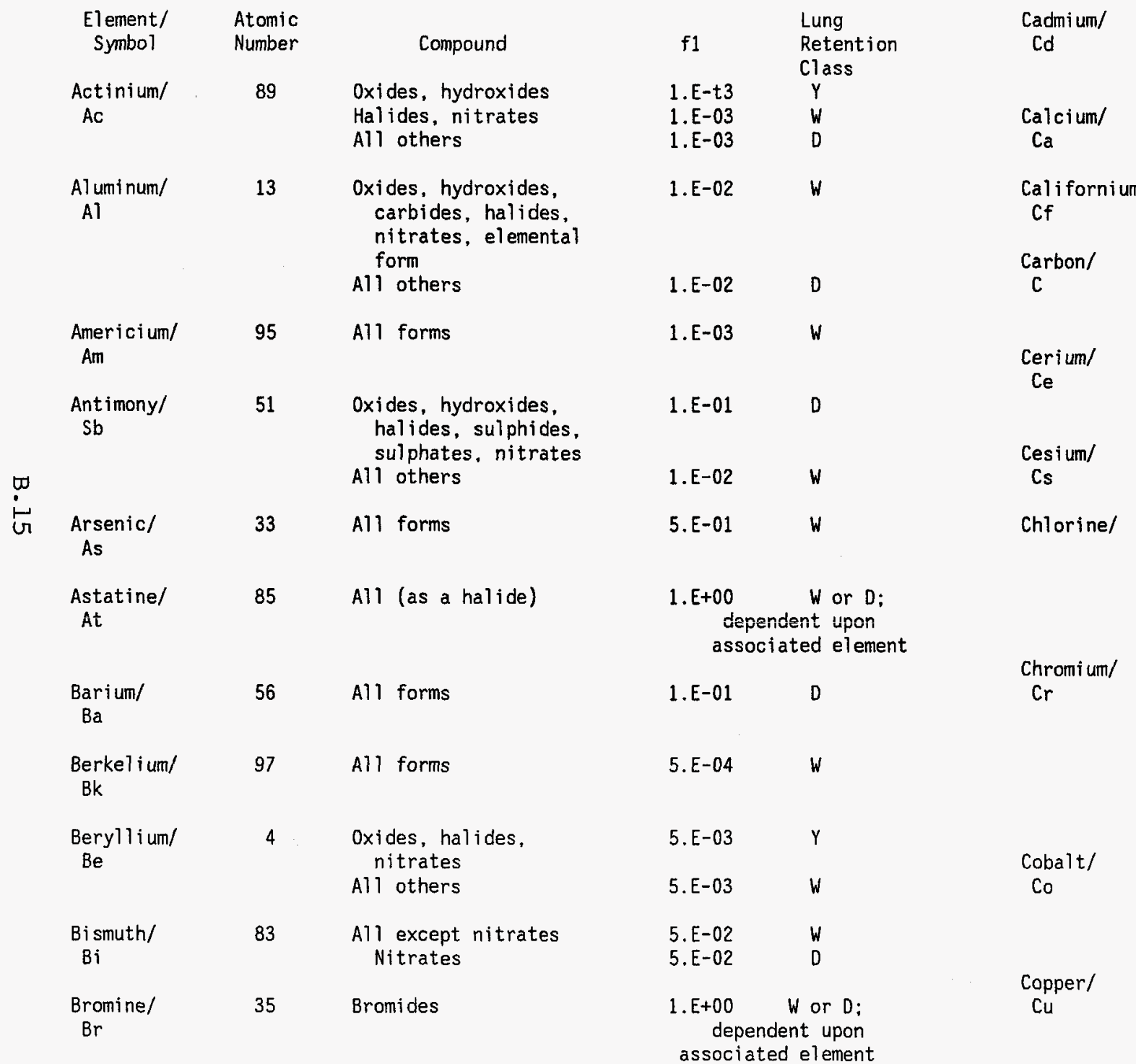

\begin{tabular}{|c|c|c|c|c|}
\hline $\begin{array}{l}\text { Element/ } \\
\text { Symbol }\end{array}$ & $\begin{array}{l}\text { Atomic } \\
\text { Number }\end{array}$ & Compound & f1 & $\begin{array}{l}\text { Lung } \\
\text { Retention } \\
\text { Class }\end{array}$ \\
\hline $\begin{array}{l}\text { Cadmi um/ } \\
\text { Cd }\end{array}$ & 48 & $\begin{array}{l}\text { Oxides, hydroxides } \\
\text { Sulphates, halides } \\
\text { All others }\end{array}$ & $\begin{array}{l}5 . E-02 \\
5 . E-02 \\
5 . E-02\end{array}$ & $\begin{array}{l}Y \\
W \\
D\end{array}$ \\
\hline $\begin{array}{l}\text { Calcium/ } \\
\mathrm{Ca}\end{array}$ & 20 & A11 forms & 3. E-01 & $W$ \\
\hline $\begin{array}{l}\text { Cali fornium/ } \\
\text { Cf }\end{array}$ & 98 & $\begin{array}{l}\text { Oxides, hydroxides } \\
\text { All others }\end{array}$ & $\begin{array}{l}\text { 1.E-03 } \\
1 . E-03\end{array}$ & $\begin{array}{l}Y \\
W\end{array}$ \\
\hline Carbon/ & 6 & $\begin{array}{l}\text { Oxides } 1 / \\
\text { Organic (11C) } \\
\text { Organic (14C) }\end{array}$ & $\begin{array}{l}1 . E+00 \\
1 . E+00\end{array}$ & $\begin{array}{l}D \\
W \\
W\end{array}$ \\
\hline $\begin{array}{l}\text { Cerium/ } \\
\text { Ce }\end{array}$ & 58 & $\begin{array}{l}\text { Oxides, hydroxides, } \\
\text { fluorides } \\
\text { All others }\end{array}$ & $\begin{array}{l}\text { 3.E-04 } \\
\text { 3.E-04 }\end{array}$ & $\begin{array}{l}Y \\
W\end{array}$ \\
\hline $\begin{array}{l}\text { Cesium/ } \\
\text { Cs }\end{array}$ & 55 & A11 forms & 1. $E+\infty 0$ & $D$ \\
\hline Chlorine/ & 17 & Chloride & $1 . E+\infty$ & $\begin{array}{l}W \text { or } D ; \\
\text { dependent } \\
\text { upon } \\
\text { associated } \\
\text { el ement }\end{array}$ \\
\hline $\begin{array}{l}\text { Chromi um/ } \\
\mathrm{Cr}\end{array}$ & 24 & $\begin{array}{l}\text { Oxides, hydroxides } \\
\text { Halides, nitrates } \\
\text { All others } \\
\text { Ingestion 2/ } \\
\text { Trivalent } \\
\text { Hexavalent }\end{array}$ & $\begin{array}{l}\text { 1. E-O1 } \\
\text { 1.E-O1 } \\
1 . E-01 \\
\text { 1.E-O2 } \\
1 . E-01\end{array}$ & $\begin{array}{l}Y \\
W \\
D\end{array}$ \\
\hline $\begin{array}{l}\text { Cobalt/ } \\
\text { Co }\end{array}$ & 27 & $\begin{array}{l}\text { Oxides, hydroxides, } \\
\text { halides, nitrates } \\
\text { All others } \\
\text { Ingestion only } 2 /\end{array}$ & $\begin{array}{l}\text { 5.E-02 } \\
\text { 5.E-02 } \\
\text { 3.E-01 }\end{array}$ & $\begin{array}{l}\text { Y } \\
W\end{array}$ \\
\hline $\begin{array}{l}\text { Copper/ } \\
\text { Cu }\end{array}$ & 29 & $\begin{array}{l}\text { Oxides, hydroxides } \\
\text { Sulphites, halides, } \\
\text { nitrates } \\
\text { All others }\end{array}$ & $\begin{array}{l}\text { 5.E-01 } \\
5 . E-01 \\
\text { 5.E-01 }\end{array}$ & $\begin{array}{l}Y \\
W\end{array}$ \\
\hline
\end{tabular}




\begin{tabular}{|c|c|c|c|c|c|c|c|c|c|}
\hline $\begin{array}{l}\text { Element/ } \\
\text { Symbol }\end{array}$ & $\begin{array}{l}\text { Atomic } \\
\text { Number }\end{array}$ & Compound & $f 1$ & $\begin{array}{l}\text { Lung } \\
\text { Retention } \\
\text { Class }\end{array}$ & $\begin{array}{l}\text { Element/ } \\
\text { Symbol }\end{array}$ & $\begin{array}{l}\text { Atomic } \\
\text { Number }\end{array}$ & Compound & f1 & $\begin{array}{l}\text { Lung } \\
\text { Retention } \\
\text { Class }\end{array}$ \\
\hline$\underset{\text { Cm }}{\text { Curium/ }}$ & 96 & All forms & 1.E-03 & W & $\begin{array}{l}\text { Gold } \\
\mathrm{Au}\end{array}$ & 79 & $\begin{array}{l}\text { Oxides, hydroxides } \\
\text { Halides, nitrates } \\
\text { All others }\end{array}$ & $\begin{array}{l}1 . E-01 \\
1 . E-01 \\
1 . E-01\end{array}$ & $\begin{array}{l}Y \\
W \\
D\end{array}$ \\
\hline $\begin{array}{l}\text { Ey } \\
\text { Es }\end{array}$ & 99 & All forms & 5.E-04 & w & $\begin{array}{l}\text { Hafnium/ } \\
\mathrm{Hf}\end{array}$ & 72 & $\begin{array}{l}\text { Oxides, hydroxides, } \\
\text { halides, carbides, } \\
\text { nitrates } \\
\text { All others }\end{array}$ & $\begin{array}{l}2 . E-03 \\
2 . E-03\end{array}$ & W \\
\hline$\underset{E r}{E r b i u m / ~}$ & 68 & A11 forms & 3.E-04 & w & $\begin{array}{l}\text { Holmiuin/ } \\
\text { Ho }\end{array}$ & 67 & All forms & 3.E-04 & $w$ \\
\hline $\begin{array}{l}\text { Europium/ } \\
\text { Eu }\end{array}$ & 63 & Al1 forms & 1.E-03 & w & Hydrogen/ & 1 & Water, elemental & 1. E+oo & \\
\hline $\begin{array}{l}\text { Fermi um/ } \\
\text { Fm }\end{array}$ & 100 & All forms & 5.E-04 & w & $\begin{array}{l}\text { Indium/ } \\
\text { In }\end{array}$ & 49 & $\begin{array}{l}\text { Oxides, hydroxides, } \\
\text { hal ides }\end{array}$ & 2.E-02 & w \\
\hline Fluorine' & 3 & Fluoride & $1 . E+00$ & $\begin{array}{l}Y, W, \text { or } \\
D: \\
\text { dependent }\end{array}$ & lodine/ & 53 & All forms & $\begin{array}{l}2 . E-02 \\
1 . E+00\end{array}$ & $\begin{array}{l}0 \\
0\end{array}$ \\
\hline & & & & $\begin{array}{l}\text { upon } \\
\text { associated } \\
\text { element }\end{array}$ & $\begin{array}{l}\text { Iridium/ } \\
\text { Ir }\end{array}$ & 77 & $\begin{array}{l}\text { Oxides, hydroxides } \\
\text { Halides, nitrates, } \\
\text { metallic form }\end{array}$ & $\begin{array}{l}\text { 1.E-02 } \\
1 . E-02\end{array}$ & $\begin{array}{l}Y \\
W\end{array}$ \\
\hline Francium/ & 87 & All forms & $1 . E+00$ & $D$ & & & All others & 1.E-02 & $D$ \\
\hline & & & & & $\begin{array}{l}\text { Iron/ } \\
\mathrm{Fe}\end{array}$ & 26 & $\begin{array}{l}\text { 0xides, hydroxides, } \\
\text { hal ides }\end{array}$ & 1.E-01 & w \\
\hline $\begin{array}{l}\text { Gadol inium/ } \\
\text { Gd }\end{array}$ & 64 & $\begin{array}{l}\text { Oxides, hydroxides, } \\
\text { fluorides }\end{array}$ & 3.E-04 & w & & & All others & 1.E-01 & $D$ \\
\hline Gallium/ & 31 & 0xides, hydroxides, & 3.E-04 & W & $\begin{array}{l}\text { Lanthanum/ } \\
\text { La }\end{array}$ & 57 & $\begin{array}{l}\text { Oxides, hydroxides } \\
\text { All others }\end{array}$ & $\begin{array}{l}\text { 1.E-03 } \\
1 . E-03\end{array}$ & $\begin{array}{l}W \\
D\end{array}$ \\
\hline Ga & & $\begin{array}{l}\text { carbides, halides, } \\
\text { nitrates, } \\
\text { All others }\end{array}$ & 1. E-03 & D & $\begin{array}{c}\text { Lead/ } \\
\mathrm{Pb}\end{array}$ & 82 & All forms & 2.E-01 & 0 \\
\hline $\begin{array}{l}\text { Germanium/ } \\
\text { Ge }\end{array}$ & 32 & $\begin{array}{l}\text { Oxides, sulphides, } \\
\text { halides } \\
\text { All others }\end{array}$ & $\begin{array}{l}\text { 1. } E+00 \\
1 . E+00\end{array}$ & $\begin{array}{l}W \\
D\end{array}$ & $\begin{array}{l}\text { Lutet i um/ } \\
\text { Lu }\end{array}$ & 71 & $\begin{array}{l}\text { Oxides, hydroxides, } \\
\text { fluorides } \\
\text { A11 others }\end{array}$ & $\begin{array}{l}3 . E-04 \\
3 . E-04\end{array}$ & $\begin{array}{l}\text { Y } \\
\text { w }\end{array}$ \\
\hline & & & & & $\begin{array}{l}\text { Magnesium/ } \\
\mathrm{Mg}\end{array}$ & 12 & $\begin{array}{l}\text { Oxides, hydroxides, } \\
\text { carbides, halides, } \\
\text { nitrates } \\
\text { All others }\end{array}$ & $\begin{array}{l}\text { 5.E-01 } \\
\text { 5.E-01 }\end{array}$ & W \\
\hline
\end{tabular}




\begin{tabular}{|c|c|c|c|c|c|c|c|c|c|}
\hline $\begin{array}{l}\text { Element/ } \\
\text { Symbol }\end{array}$ & $\begin{array}{l}\text { Atomic } \\
\text { Number }\end{array}$ & Compound & f1 & $\begin{array}{l}\text { Lung } \\
\text { Retention } \\
\text { Class }\end{array}$ & $\begin{array}{l}\text { Element/ } \\
\text { Symbol }\end{array}$ & $\begin{array}{l}\text { Atomic } \\
\text { Number }\end{array}$ & Compound & f1 & $\begin{array}{l}\text { Lung } \\
\text { Retention } \\
\text { Class }\end{array}$ \\
\hline $\begin{array}{l}\text { Manganese/ } \\
\text { Mn }\end{array}$ & 101 & $\begin{array}{l}\text { Oxides, hydroxides, } \\
\text { halides, nitrates } \\
\text { All others } \\
\text { All forms }\end{array}$ & $\begin{array}{l}\text { 1.E-01 } \\
\text { 1.E-01 } \\
\text { 5.E-04 }\end{array}$ & $\begin{array}{l}W \\
D\end{array}$ & $\begin{array}{l}\text { Phosphorus/ } \\
P\end{array}$ & 15 & Phosphates & 8.E-01 & $\begin{array}{l}\text { W or } D ; \\
\text { dependent } \\
\text { upon } \\
\text { associated } \\
\text { element }\end{array}$ \\
\hline $\begin{array}{l}\text { Mercury/ } \\
\mathrm{Hg}\end{array}$ & 80 & $\begin{array}{l}\text { Oxides, hydroxides, } \\
\text { halides, nitrates, } \\
\text { sulphites } \\
\text { Sulphates, elemental } \\
\text { form } \\
\text { Organic forms } \\
\text { Vapor } 1 /\end{array}$ & $\begin{array}{l}\text { 2.E-02 } \\
\text { 2.E-02 } \\
1 . E+00\end{array}$ & $\begin{array}{l}D \\
D \\
D\end{array}$ & $\begin{array}{l}\text { Platinum/ } \\
\text { Pt } \\
\text { Plutonium/ } \\
\text { Pu }\end{array}$ & 78 & $\begin{array}{l}\text { Oxides, hydroxides } \\
\text { Nitrates } \\
\text { All other } \\
\text { [Note: Use same } \\
\text { values for } \\
\text { ingestion] }\end{array}$ & $\begin{array}{l}1 . E-05 \\
1 . E-04 \\
1 . E-03\end{array}$ & $\begin{array}{l}Y \\
W \\
W\end{array}$ \\
\hline $\begin{array}{l}\text { Mol ybdenum/ } \\
\text { Mo }\end{array}$ & 42 & $\begin{array}{l}\text { Oxides, hydroxides, } \\
\text { Mos2 } \\
\text { All others }\end{array}$ & $\begin{array}{l}5 . E-02 \\
8 . E-01\end{array}$ & $\begin{array}{l}Y \\
D\end{array}$ & $\begin{array}{l}\text { Polonium/ } \\
\text { Po }\end{array}$ & 84 & $\begin{array}{l}\text { Oxides, hydroxides, } \\
\text { nitrates } \\
\text { A11 others }\end{array}$ & $\begin{array}{l}1 . E-01 \\
1 . E-01\end{array}$ & W \\
\hline & & $\begin{array}{l}\text { Ingestion 2/ } \\
\text { MoS2 } \\
\text { All Others }\end{array}$ & $\begin{array}{l}\text { 5.E-02 } \\
\text { 8.E-01 }\end{array}$ & & $\begin{array}{l}\text { Potassium/ } \\
\mathrm{K}\end{array}$ & 19 & All forms & 1. $E+\infty 0$ & D \\
\hline $\begin{array}{l}\text { Neodymi um/ } \\
\text { Nd }\end{array}$ & 60 & $\begin{array}{l}\text { Oxides, hydroxides, } \\
\text { carbides, } \\
\text { fluorides } \\
\text { All others }\end{array}$ & $\begin{array}{l}\text { 3.E-04 } \\
\text { 3.E-04 }\end{array}$ & $\mathbf{Y}$ & $\begin{array}{l}\text { Praseodymium/ } \\
\mathrm{Pr}\end{array}$ & 59 & $\begin{array}{l}\text { Oxides, hydroxides, } \\
\text { carbides. } \\
\text { fluorides } \\
\text { All others }\end{array}$ & $\begin{array}{l}3 . E-04 \\
3 . E-04\end{array}$ & $w$ \\
\hline $\begin{array}{l}\text { Neptuni um/ } \\
\text { Np }\end{array}$ & 93 & Al1 forms & 1.E-03 & $W$ & $\begin{array}{l}\text { Promethium/ } \\
\text { Pm }\end{array}$ & 61 & $\begin{array}{l}\text { Oxides, hydroxides } \\
\text { carbides, } \\
\text { fluorides }\end{array}$ & 3.E-04 & $Y$ \\
\hline $\begin{array}{l}\text { Nickel/ } \\
\mathrm{Ni}\end{array}$ & 28 & $\begin{array}{l}\text { Oxides, hydroxides } \\
\text { All others (vapor) 1/ }\end{array}$ & 5.E-02 & $\begin{array}{l}W \\
D\end{array}$ & & & All others & 3.E-04 & $W$ \\
\hline $\begin{array}{l}\text { Niobium/ } \\
\text { Nb }\end{array}$ & 41 & $\begin{array}{l}\text { Oxides, hydroxides } \\
\text { All others }\end{array}$ & $\begin{array}{l}\text { 1.E-02 } \\
1 . E-02\end{array}$ & $\begin{array}{l}Y \\
W\end{array}$ & $\begin{array}{l}\text { Protactinium/ } \\
\mathrm{Pa}\end{array}$ & 91 & $\begin{array}{l}\text { 0xides, hydroxides } \\
\text { All others }\end{array}$ & $\begin{array}{l}\text { 1.E-03 } \\
1 . E-03\end{array}$ & $\begin{array}{l}Y \\
W\end{array}$ \\
\hline $\begin{array}{l}\text { Osmium/ } \\
\text { Os }\end{array}$ & 76 & $\begin{array}{l}\text { Oxides, hydroxides } \\
\text { Hal ides, nitrates } \\
\text { All others }\end{array}$ & $\begin{array}{l}1 . E-02 \\
1 . E-02 \\
1 . E-02\end{array}$ & $\begin{array}{l}Y \\
W \\
D\end{array}$ & $\begin{array}{l}\text { Radium/ } \\
\operatorname{Ra}\end{array}$ & 88 & All forms & 2.E-01 & W \\
\hline $\begin{array}{l}\text { Palladium/ } \\
\text { Pd }\end{array}$ & 46 & $\begin{array}{l}\text { Oxides, hydroxides } \\
\text { Nitrates } \\
\text { All others }\end{array}$ & $\begin{array}{l}\text { 5.E-03 } \\
\text { 5.E-03 } \\
\text { 5.E-03 }\end{array}$ & $\begin{array}{l}Y \\
W \\
0\end{array}$ & & & & & \\
\hline
\end{tabular}




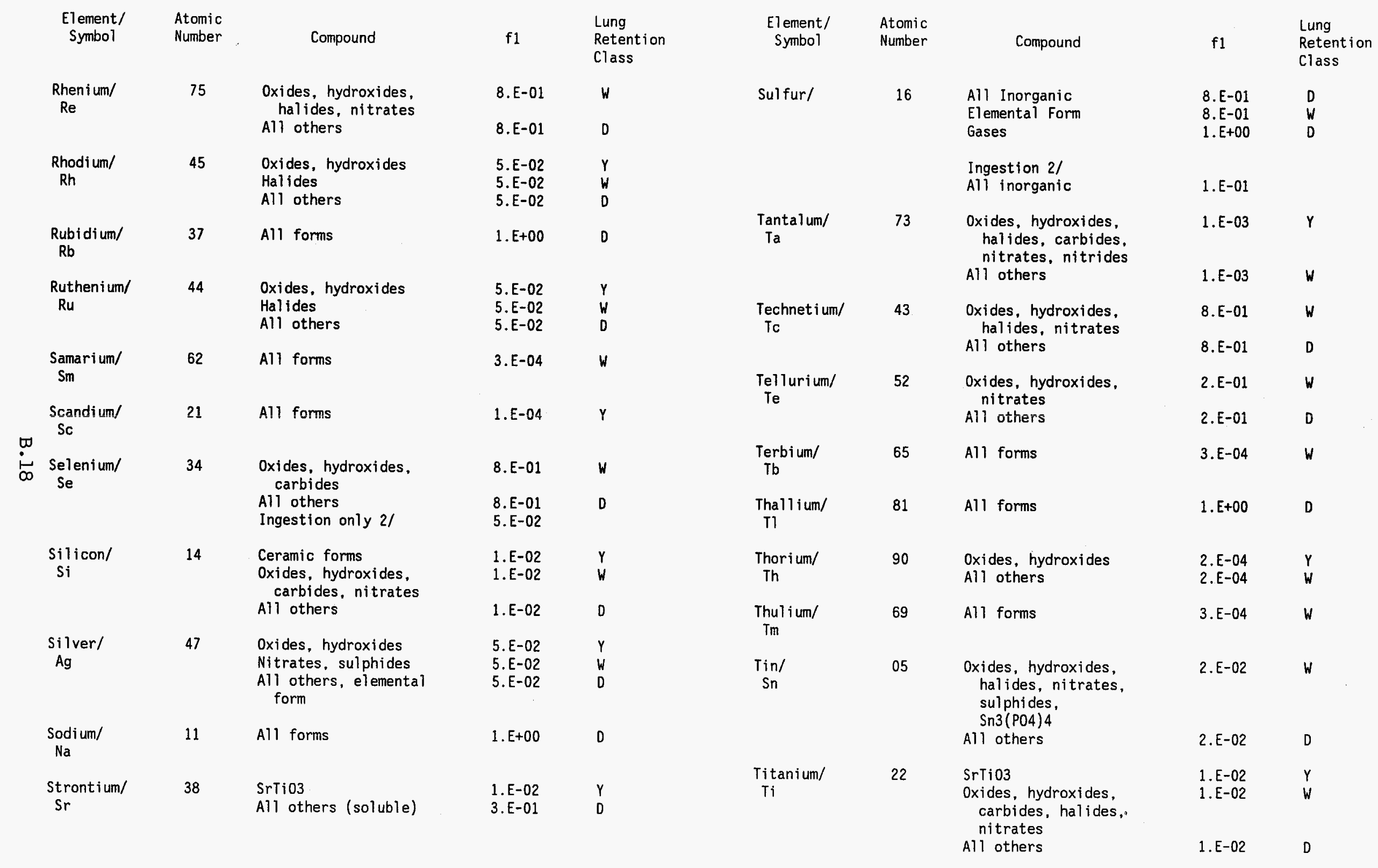




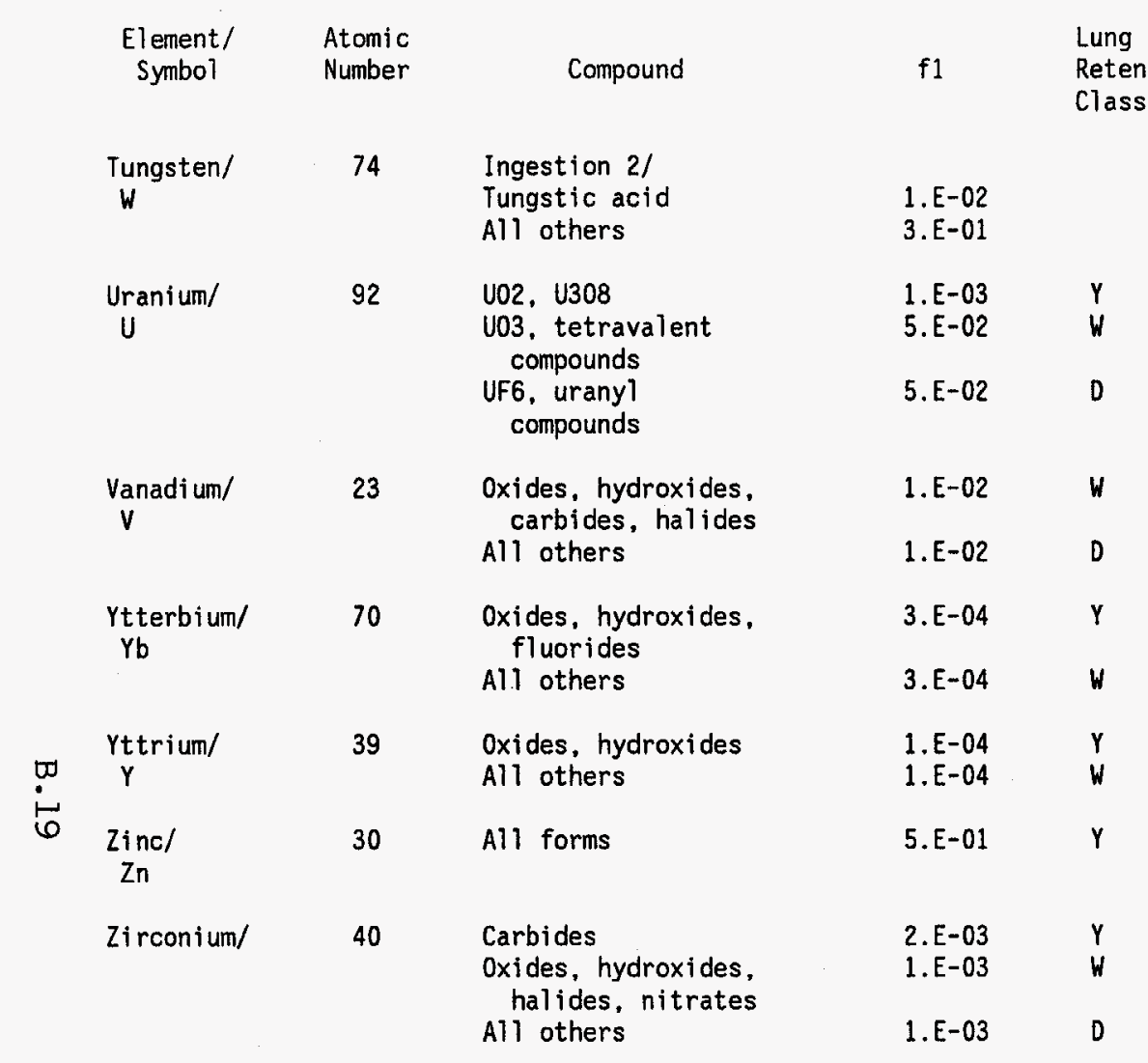

1/ A dash indicates no data for the value shown.

2) For ingestion, no lung retention classes are listed. 
Table 3

Derived Air Concentrations (DAC) for Workers from External Exposure During Immersion in a Contaminated Atmospheric Cloud

\begin{tabular}{|c|c|c|}
\hline Radionuclide & Half-Life & $\begin{array}{l}\text { Air Immersion } \\
\text { DAC }(\mathrm{uCi} / \mathrm{mL})\end{array}$ \\
\hline$c-11$ & $20.48 \mathrm{~min}$ & 4. E-06 \\
\hline $\begin{array}{l}N-13 \\
N-16\end{array}$ & $\begin{array}{l}9.97 \mathrm{~min} \\
7.13 \mathrm{~s}\end{array}$ & $\begin{array}{l}\text { 4.E-06 } \\
\text { 7.E-07 }\end{array}$ \\
\hline $0-15$ & $122.24 \mathrm{~s}$ & 4. E-06 \\
\hline$F-18 \quad 1 /$ & $109.74 \mathrm{~min}$ & 4.E-06 \\
\hline $\mathrm{Na}-241 /$ & $15.00 \mathrm{~h}$ & $9 . E-07$ \\
\hline$M g-272 /$ & $9.458 \min$ & 5. E-06 \\
\hline A $1-28 \quad 2 /$ & $2.240 \mathrm{~min}$ & 2.E-06 \\
\hline $\mathrm{Cl}-38 \mathrm{1} /$ & $37.21 \mathrm{~min}$ & 3.E-06 \\
\hline $\begin{array}{l}A r-37 \\
A r-39 \\
A r-41\end{array}$ & $\begin{array}{l}35.02 \mathrm{~d} \\
269 \mathrm{yr} \\
1.827 \mathrm{~h}\end{array}$ & $\begin{array}{l}\text { 3.E-00 } \\
2 . E-043 / \\
3 . E-06\end{array}$ \\
\hline$K-431 /$ & $22.6 \mathrm{~h}$ & 5.E-OG \\
\hline $\mathrm{Ca}-492 /$ & $8.719 \mathrm{~min}$ & 1.E-06 \\
\hline $\begin{array}{l}\text { Sc }-44 \text { 1/ } \\
\text { Sc }-46 m 2 /\end{array}$ & $\begin{array}{l}3.927 \mathrm{~h} \\
18.72 \mathrm{~s}\end{array}$ & $\begin{array}{l}\text { 2.E-06 } \\
5 . E-05\end{array}$ \\
\hline $\begin{array}{ll}\mathrm{Ti}-45 & 1 / \\
\mathrm{Ti}-51 & 2 /\end{array}$ & $\begin{array}{l}3.08 \mathrm{~h} \\
5.752 \mathrm{~min}\end{array}$ & $\begin{array}{l}5 . E-06 \\
1 . E-05\end{array}$ \\
\hline$V-52 \quad 2 /$ & $3.75 \mathrm{~min}$ & $3 . E-06$ \\
\hline $\mathrm{Cr}-49 \mathrm{I} /$ & $42.09 \mathrm{~min}$ & 5.E-06 \\
\hline 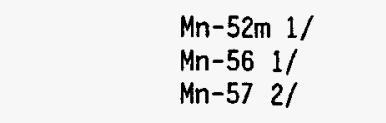 & $\begin{array}{l}21.4 \mathrm{~min} \\
2.5785 \mathrm{~h} \\
1.47 \mathrm{~min}\end{array}$ & $\begin{array}{l}\text { 2.E-06 } \\
2 . E-06 \\
6 . E-05\end{array}$ \\
\hline Co-60m I/ & $10.47 \mathrm{~min}$ & 1.E-03 \\
\hline $\begin{array}{l}\mathrm{Ni}-57 \mathrm{1}, 4 / \\
\mathrm{Ni}-651 /, 5 /\end{array}$ & $\begin{array}{l}36.08 \mathrm{~h} \\
2.520 \mathrm{~h}\end{array}$ & $\begin{array}{l}\text { 2.E-06 } \\
\text { 8.E-06 }\end{array}$ \\
\hline
\end{tabular}

Air Immersion DAC $(\mathrm{uCi} / \mathrm{mL})$

5.E-06

5. E- 06

2. $E-06$

5.E-06

1.E-06

4. E-06

1.E-05 6/

5. E-05

1. E-06

2. E-06

5.E-05

2. E-05

5.E-04

5.E-02

1.E-04 3/

3. E-05

5.E-06

2.E-06

2. E-06

3.E-06

8.E-06

2.E-06

7.E-06

2. $E-06$

2. $\mathrm{E}-0.06$

1.E-06

2.E-05

6.E-05

3. E-06

2.E-06

1.E-06

5.E-06 6/

$14.74 \mathrm{~h}$

$49.71 \mathrm{~min}$

$14.60 \mathrm{~h}$

$6.26 \mathrm{~min}$

9.E-06

1. E-07

9. $\mathrm{E}-\mathrm{O} 4$ 


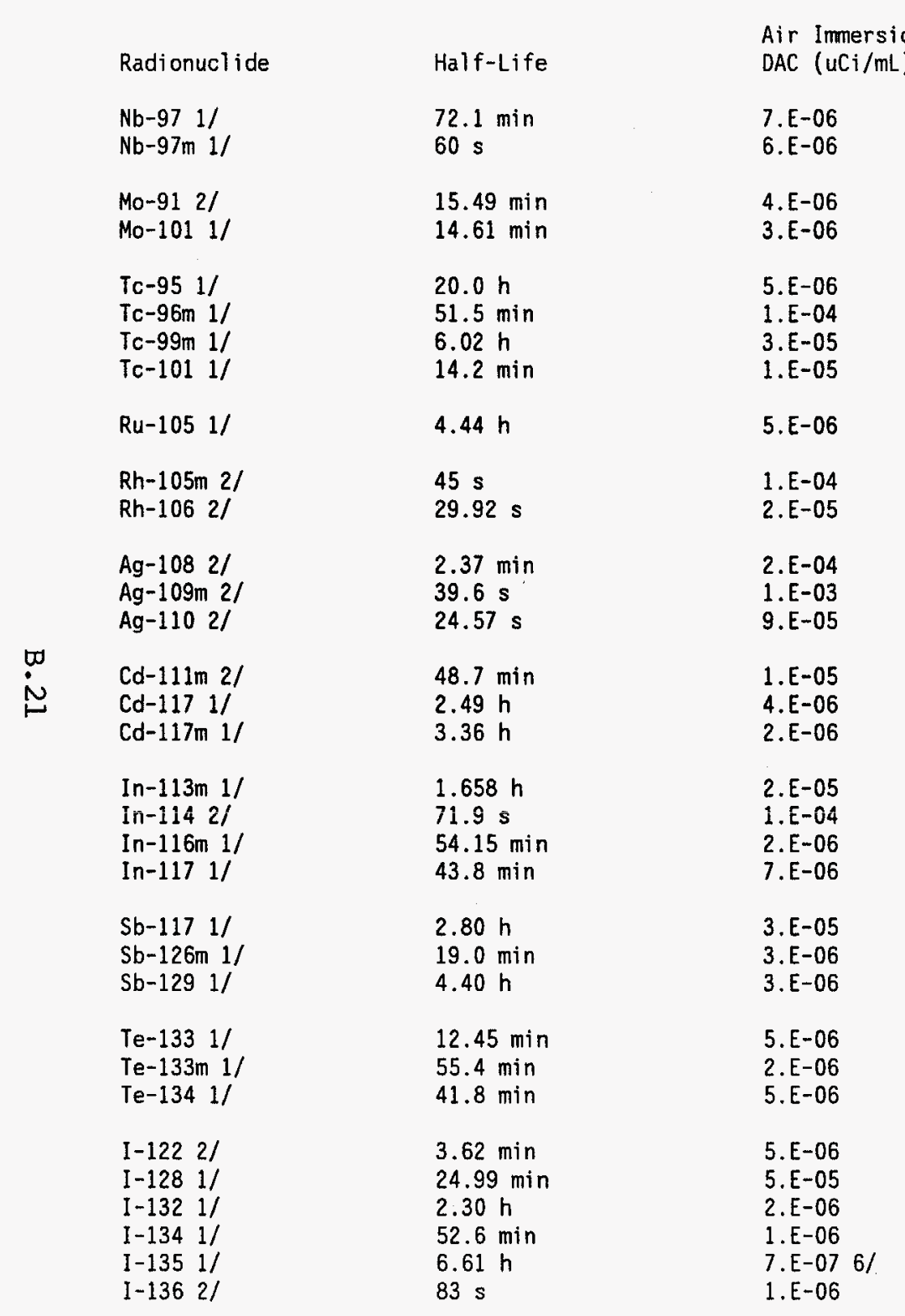

\begin{tabular}{|c|c|c|}
\hline Radionuclide & Half-Life & $\begin{array}{l}\text { Air Immersion } \\
\text { DAC }(\mu \mathrm{Ci} / \mathrm{mL})\end{array}$ \\
\hline$x_{e-122}$ & $20.1 \mathrm{~h}$ & 8.E-05 \\
\hline$X e-123$ & $2.14 \mathrm{~h}$ & 7. $E-06$ \\
\hline$x_{e}-125$ & $16.8 \mathrm{~h}$ & 2.E-05 \\
\hline$X e-127$ & $36.406 \mathrm{~d}$ & 1. E-05 \\
\hline$x e-129 m$ & $8.89 \mathrm{~d}$ & 2.E-04 \\
\hline$x e-131 m$ & $11.84 \mathrm{~d}$ & 5.E-04 \\
\hline$x e-133$ & $5.245 \mathrm{~d}$ & 1.E-04 \\
\hline$X e-133 m$ & $2.19 \mathrm{~d}$ & 1.E-04 \\
\hline$x e-135$ & $9.11 \mathrm{~h}$ & 2. E-05 \\
\hline$x e-135 m$ & $15.36 \mathrm{~min}$ & 1.E-05 \\
\hline$x e-137$ & $3.83 \mathrm{~min}$ & 2.E-05 \\
\hline$X e-138$ & $14.13 \mathrm{~min}$ & 4.E-06 \\
\hline $\begin{array}{lll}C s-126 & 2 /\end{array}$ & $1.64 \mathrm{~min}$ & 4.E-06 \\
\hline $\begin{array}{lll}C s-129 & 1 / \\
C s-138 & 1 /\end{array}$ & $32.06 \mathrm{~h}$ & 1.E-05 6/ \\
\hline $\begin{array}{lll}C s-138 & 1 / \\
C s-139 & 2 /\end{array}$ & $9.40 \mathrm{~min}$ & $\begin{array}{l}2 . E-06 \\
1 . E-05\end{array}$ \\
\hline $\mathrm{Ba}-137 \mathrm{~m} 2 /$ & $2.552 \mathrm{~min}$ & 7.E-06 \\
\hline $\mathrm{Ba}-141 \quad 1 /$ & $18.27 \mathrm{~min}$ & 5.E-06 \\
\hline$B a-142 \quad 1 /$ & $10.70 \mathrm{~min}$ & 5.E-06 \\
\hline La-142 I/ & $95.4 \mathrm{~min}$ & 1.E-06 \\
\hline $\operatorname{Pr}-144 \mathrm{~m} 2 /$ & $7.2 \min$ & 9.E-04 \\
\hline $\mathrm{Nd}-149 \mathrm{i} /$ & $1.73 \mathrm{~h}$ & 1.E-05 \\
\hline Gd-162 2/ & $9.7 \min$ & 1.E-05 \\
\hline$T d-162 \quad 2 /$ & $7.76 \mathrm{~min}$ & 4. E-06 \\
\hline Dy-157 $1 /$ & $8.06 \mathrm{~h}$ & 1.E-05 \\
\hline Re-182m 1/ & $12.7 \mathrm{~h}$ & 4. E-06 \\
\hline $0 \mathrm{~s}-190 \mathrm{~m} 2 /$ & $9.9 \min$ & 3. E-06 \\
\hline $\mathrm{Ir}-190 \mathrm{~m} 1 /$ & $3.2 \mathrm{~h}$ & 8.E-05 6/ \\
\hline $\mathrm{Au}-195 \mathrm{~m} 2 /$ & $30.6 \mathrm{~s}$ & 2.E-05 \\
\hline
\end{tabular}




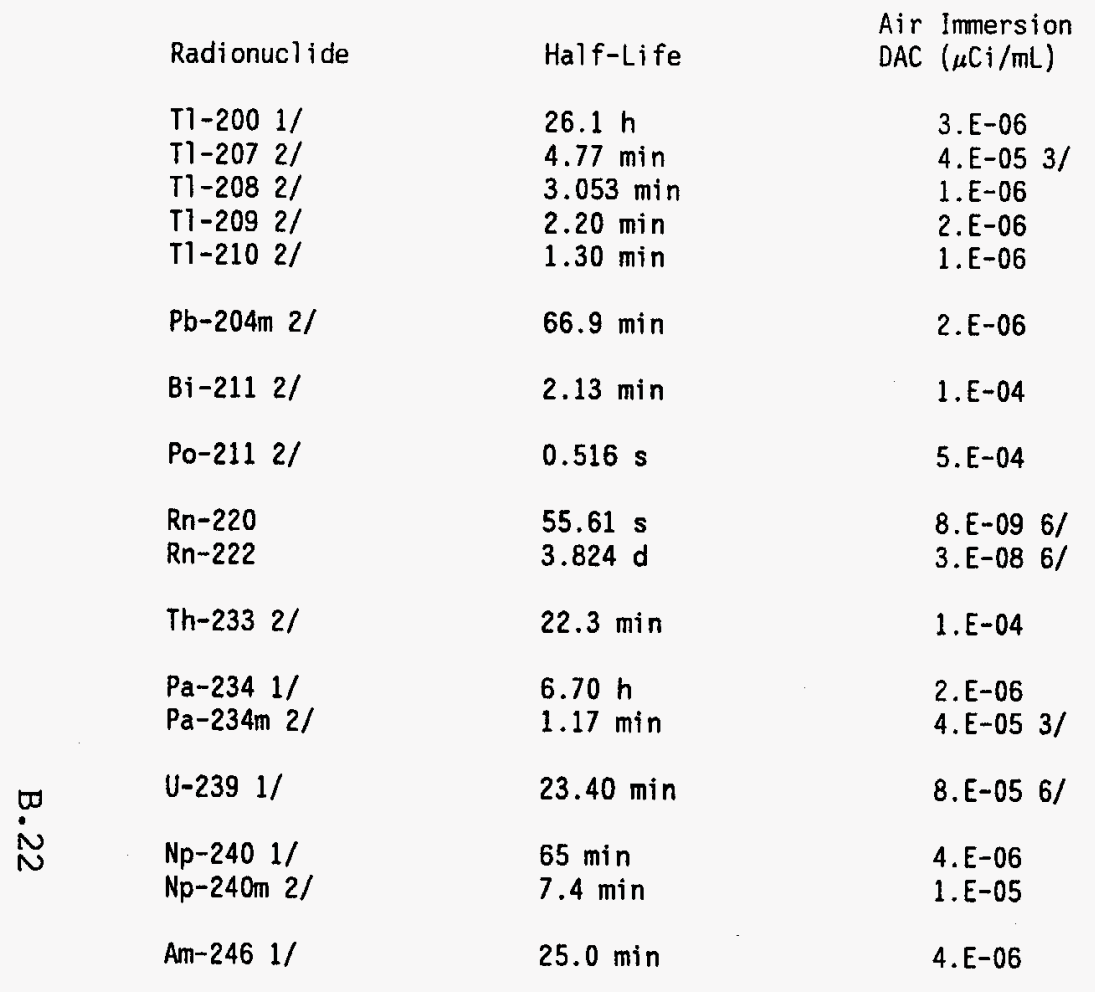

1/ Committed effective dose equivalent from inhalation is calculated in ICRP Publication 30, but the DAC value for external exposure to a contaminated atmospheric cloud is more restrictive than the DAC value for inhalation.

2) Committed effective dose equivalent from inhalation is not calculated in ICRP Publication 30 , but DAC value for external exposure to contaminated cloud should be more restrictive than DAC value for inhalation due to relatively short half-life of radionuclide.

3/ DAC value is determined by limit on annual dose equivalent to skin, rather than limit on annual effective dose equivalent.

4/ DAC value applies to radionuclide in vapor form only: DAC value for inhalation is more restrictive for radionuclide in inorganic form.

5/ DAC value applies to radionuclide in inorganic or vapor form.

6/ DAC value for exposure to contaminated atmospheric cloud is the same as DAC value for inhalation. See footnote 4/ to Table 1 on page 24 of Attachment 1. 
APPENDIX C

SURFACE RADIOACTIVITY GUIDES

(from DOE 5480.11, Change 1, 7-20-89) 


\section{SURFACE RADIOACTIVITY GUIDES}

\section{NUCLIDE $1 /$}

$U$-nat, U-235, U-238,

and associated

decay products

Transuranics

Ra-226, Ra-228,

Th-230, Th-228,

$\mathrm{Pa}-231, \mathrm{Ac}-227$,

I-125, I-129

Th-nat, Th-232,

$\mathrm{Sr}-90$, Ra-223,

Ra-224, U-232.

I-126, I-131,

I -133

Beta-gamma emitters

(nuclides with decay modes

other than alpha emission

$?$ or spontaneous fission) except

$\checkmark$ sr-90 and others noted above. 5/
TOTAL 2/3/

(FIXED PLUS REMOVABLE)

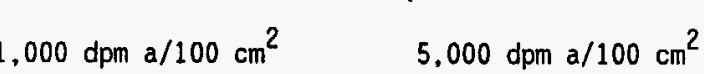

$20 \mathrm{dpm} / 100 \mathrm{~cm}^{2}$

$300 \mathrm{dpm} / 100 \mathrm{~cm}^{2}$

$1,000 \mathrm{dpm} / 100 \mathrm{~cm}^{2}$

$200 \mathrm{dpm} / 100 \mathrm{~cm}^{2}$

$1,000 \mathrm{dpm} / 100 \mathrm{~cm}^{2}$

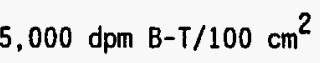

1/ Where surface contamination by both alpha- and beta-gamma-emitting nuclides exists, the limits established for alpha- and beta-gamma-emitting nuclides should apply independently.

2) As used in this table, dpm (disintegrations per minute) means the rate of emission by radioactive material as determined by correcting the counts per minute observed by an appropriate detector for background, efficiency, and geometric factors associated with the instrumentation.

3 The levels may be averaged over one square meter provided the maximum surface activity in any area of $100 \mathrm{~cm}^{2}$ is less than three times the guide values. For purposes of averaging, any square meter of surface shall be considered to be above the activity guide $G$ if: (1) from measurements of a representative number $n$ of sectigns it is determined that $1 / n$ n $S i>G$, where $S i$ is the dis/min-100 $\mathrm{cm}^{2}$ determined from measurement of section $i$; or (2) it is determined that the sum of the activity of all isolated spots or particles in any $100 \mathrm{~cm}^{2}$ area exceeds $3 G$.

4/ The amount of removable radioactive material per $100 \mathrm{~cm}^{2}$ of surface area should be determined by wiping that area with dry filter or soft absorbent paper, applying moderate pressure, and assessing the amount of radioactive material on the wipe with an appropriate instrument of known efficiency. (Note - The use of dry material may not be appropriate for tritium.) When removable contamination on objects of surface area less than $100 \mathrm{~cm}^{2}$ is determined, the activity per unit area should be based on the actual area and the entire surface should be wiped. Except for transuranics and Ra-226, Ra-228, Ac-227. Th-228, Th-230, and $\mathrm{Pa}-231$ alpha emitters, it is not necessary to use wiping techniques to measure removable contamination levels if direct scan surveys indicate that the total residual surface contamination levels are within the limits for removable contamination.

5/ This category of radionuclides includes mixed fission products, including the SR-90 which is present in them. It does not apply to SR-90 which has been separated from the other fission products or mixtures where the SR-90 has been enriched. 
APPENDIX D

DERIVED CONCENTRATION GUIDES FOR AIR AND WATER (from DOE 5400.5, Change 1, 6-5-90) 


\section{DERIVED CONCENTRATION GUIDES FOR AIR AND WATER}

(from DOE 5400.5, Change 1, 6-5-90)

1. PURPOSE. The Derived Concentration Guide (DCG) values listed in this chapter are provided as reference values for conducting radiological environmental protection programs at operational DOE facilities and sites. Derived Air Concentrations (DAC) guides for controlling occupational intake of radionuclides through inhalation are listed in DOE 5480.11 .

2. BASIS. The DCG values are presented for each of three exposure modes: 1) ingestion of water; 2) inhalation of air; and 3) immersion in a gaseous cloud. The DCG values for internal exposure shown in Figure III-1 are based on a committed effective dose equivalent of $100 \mathrm{mrem}$ for the radionuclide taken into the body by ingestion or inhalation during one year. To use the DCGs for comparison with the DOE drinking water systems criterion of $4 \mathrm{mrem} / \mathrm{yr}(0.04 \mathrm{mSv} / \mathrm{sr})$, use 4 percent of the DCG values for ingestion. Compliance with the 40 CFR Part 61, Subpart $H$, criterion of $10 \mathrm{mrem} / \mathrm{yr}(0.10 \mathrm{mSv} / \mathrm{yr})$ effective dose equivalent is demonstrated using the AIRDOS/RADRISK models prescribed by the EPA. Alternative gastrointestinal-tract (GI-tract) absorption factors (fI) and lung retention classes (noted as $D, W$, or $Y$ in the Task Group Lung Model used to produce the inhalation dose factors reported in ICRP Publication 30) are listed for specific compounds, by element, in Figure III-2 for cross-referencing with the internal DCGs in Figure III-1. The data in Figure III-2 are listed in alphabetical order, by element name. Removal half-times assigned to the compounds with lung retention classes $D, W$, and $Y$ are $0.5,50$, and 500 days, respectively. The air immersion DCG values shown in Figure III-3 are based on an effective dose equivalent of 100 mrem from exposure during one year. Figure III-1 contains six columns of information: Radionuclide/Chemical Form/Isomer 
Half-Life; fl Value (GI-tract absorption); Ingested Water DCG $(\mu \mathrm{Ci} / \mathrm{mL})$; Inhaled Air DCG for Lung Retention Class $D(\mu \mathrm{Ci} / \mathrm{mL})$; and Inhaled Air DCG for Lung Retention $\mathrm{Cl}$ ass $\mathrm{W}(\mu \mathrm{Ci} / \mathrm{mL})$; and Inhaled Air $D C G$ for Lung Retention $\mathrm{Cl}$ ass $Y(\mu \mathrm{Ci} / \mathrm{mL})$. Figure III-2 contains five columns of information: Element/Symbol; Atomic Number; compound; fl value; and Lung Retention Class. Figure III-3 contains three columns of information: Radionuclide; Half-life in units of seconds (s), minutes (min), hours $(h)$, days (d), or years $(y r)$; and Air Immersion DCG $(\mu \mathrm{C} ; ; m L)$.

a. Exposure Conditions for Ingestion of Water and Inhalation. Under conditions of continuous exposure, members of the public are assumed to ingest 730 liters of drinking water or to inhale 8,400 cubic meters of air (for exposure of 24 hours per day, 365 days per year), as given for the "reference man" in ICRP Publication 23. Only single modes of exposure were considered in the calculation of the DCGs - that is, they apply to either inhalation or ingestion, not to a combination of both. The dose factors used to calculate the DCG values for internal exposure were taken from the report "Internal Dose Conversion Factors for Calculation of Dose to the Public" (DOE/EH-0071). For ingestion, DCG values are tabulated for all values of $\mathrm{fl}$ for each radionuclide given in ICRP Publication 30, as modified for several transuranic elements by ICRP Publication 48. For inhalation, DCG values are given for all combinations of $f l$ and lung retention class ( $D$, in, or $Y$ ) given by the ICRP, as tabulated in Figure III-2. For radionuclides with multiple fl listings, where specific data for an airborne or liquid release are lacking, the $f l$ value that results in the most restrictive DCG for ingested water or inhaled air should be used.

b. Exposure Conditions for Air Immersion. The air immersion DCGs were calculated for a continuous, nonshielded exposure via immersion in a semi-infinite atmospheric cloud. The dose conversion factors used to calculate the DCG values for air immersion were taken from the report "External Dose-Rate Conversion Factors for Calculation 
of Dose to the Public" (DOE/EH-0070). The DCG value for air immersion listed in Figure III-3 for a given radionuclide is determined either by a limit on annual effective dose equivalent, which provides a limit on stochastic radiation effects, or by a limit on annual dose equivalent to any organ, which provides a limit on nonstochastic radiation effects. For most of the radionuclides listed in Figure III-3, the DCG value is determined by the limit on annual effective dose equivalent. Thus, the few cases where the DCG value is determined by the limit on annual dose equivalent to skin are indicated in the figure by an appropriate footnote. Again, the DCGs listed in Figure III-3 account only for immersion in a semiinfinite cloud and do not account for inhalation or ingestion exposures. Three classes of radionuclides are included in the air immersion DCGs given in Figure III-3, as described below.

(1) Class 1. The first class of radionuclides includes selected noble gases and short-lived activation products that occur in gaseous form. For these radionuclides, inhalation doses are negligible compared to the external dose from immersion in an atmospheric cloud.

(2) Class 2. The second class of radionuclides includes those for which a DCG value for inhalation has been calculated (using the ICRP inhalation dose equivalent factors), but for which the DCG value for external exposure to a contaminated atmospheric cloud is more restrictive (i.e., results in a lower DCG value). These radionuclides generally have half-lives of a few hours or less, or are eliminated from the body following inhalation sufficiently rapidly to limit the inhalation dose.

(3) Class 3. The third class of radionuclides includes selected isotopes with relatively short half-lives that were not considered in ICRP Publication 30. These radionuclides typically have half-lives that are less than 10 minutes, they do not 
occur as a decay product of a longer-lived radionuclide, or they lack sufficient decay data to permit internal dose calculations. These radionuclides are also typified by a radioactive emission of highly intense, high-energy photons and rapid removal from the body following inhalation.

c. Application to Mixtures of Radionuclides. The DCG values are given for individual radionuclides. For known mixtures of radionuclides, the sum of the ratios of the observed concentration of each radionuclide to its corresponding DCG must not exceed 1.0.

3. LIMITATIONS. The values given in Figures III-1 and 111-3 account for only three exposure pathways (ingested water or inhaled air or air immersion) and do not include other potentially significant pathways. When more complex environmental pathways are involved, a more complete pathway analysis is required for calculating public radiation doses resulting from the operation of DOE facilities. 
Figure III-1

Derived Concentration Guides (DCGs) for Members of the Public from Ingested Water and Inhalation Resulting in $100 \mathrm{mrem} / \mathrm{yr}$

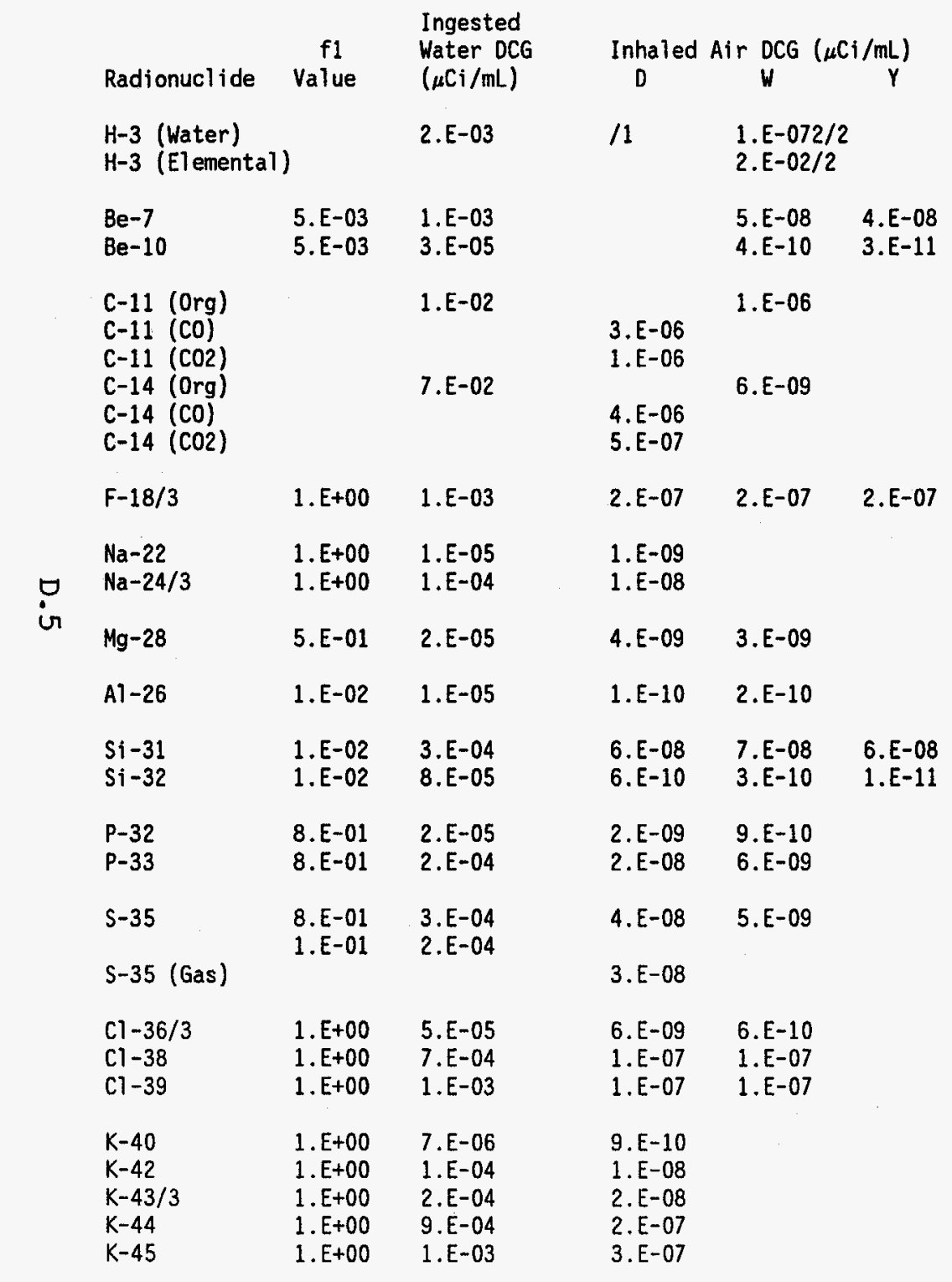

Ingested Water DCG $(\mu \mathrm{Ci} / \mathrm{mL})$ 1.E-04 5. E-05

2. $E-05$ $\mathrm{Ca}-45 \quad 3 . \mathrm{E}-01$

$\mathrm{Ca}-47$ 3.E-01

2. E-04

Sc -43

Sc $-44 m$ Sc $-44 / 3$

Sc -46

Sc -47

Sc -48

Sc -49

$\mathrm{Ti}-44$

$T i-45 / 3$

1.E-04

$1 . E-04$
$1 . F-04$

1.E-05

1.E-04 1.E-04

1.E-04 7.E-05

1.E-04 2.E-05

1.E-04 6.E-04

1.E-02

7.E-06

2. E-04

$V-47$

$V-48$
$V-49$

1.E-O2

1. E-02

9.E-04

2. E-05

3. E-03

Cr-48

$\mathrm{Cr}-49 / 3$

1.E-01

2. E-04

$1 . E-02$ 2.E-04

$\begin{array}{ll}1 . E-01 & 8 . E-04 \\ 1 . E-02 & 8 . E-04\end{array}$

$\mathrm{Cr}-51$ 1.E-01 1.E-03

1.E-02 1.E-03

$M n-51$

$M n-52 m / 3$

1. E-01

6. E-04

1.E-01 9.E-04

1.E-01 2.E-05

$M n-53$ 1.E-01 1.E-03

$\mathrm{Mn}-54$ 1.E-01 5.E-05

$\mathrm{Mn}-56 / 3$ 1.E-01 1.E-04

$\mathrm{Fe}-52 \quad 1 . \mathrm{E}-01$ 3.E-05

$\mathrm{Fe}-55$ 1.E-01 2.E-04

$\mathrm{Fe}-59$ 1.E-01 2.E-05

$\mathrm{Fe}-60 \quad 1 . \mathrm{E}-01 \quad 9 . \mathrm{E}-07$

Co-55

5. E-02

3. E-05

Co-56

5. 3-02

1.E-05
Inhaled Air DCG $(\mu \mathrm{Ci} / \mathrm{mL})$

$\begin{array}{lll}D & \text { W } \\ & 9 . \mathrm{E}-09 & Y\end{array}$

2. E-09

2.E-09

5.E-08

2.E-09

3. E-08

6. $E-10$

7.E-09

3.E-09

1.E-07

3.E-11 7.E-11 1.E-11

2. E-07 2.E-07

$\begin{array}{ll}\text { 3. E-09 } & \text { 2.E-09 } \\ \text { 8.E-08 } & 4 . E-08\end{array}$

3.E-08 2.E-08 2.E-08

2.E-07 2.E-07 2.E-07

1.E-07 6.E-08 5.E-08

1. E-07 1.E-07

2.E-07 2.E-07

3.E-09 2.E-09

3.E-08

2.E-09

4.E-08 5.E-08

7.E-09 6.E-09

5.E-09 1.E-08

8.E-10 1.E-09

2.E-11 4.E-11

7.E-09 6.E-09

7.E-10 5.E-10 


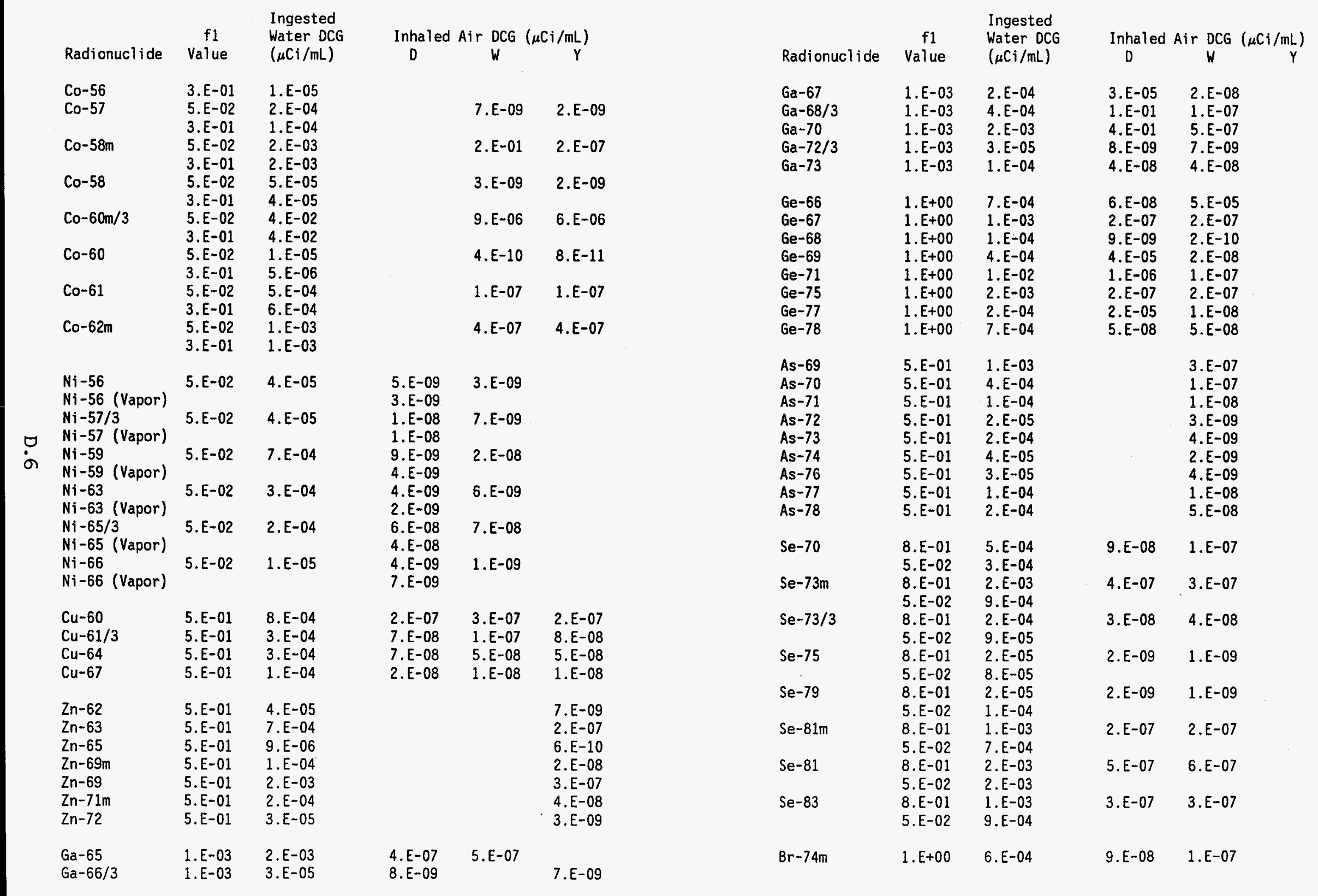




\begin{tabular}{|c|c|c|c|c|c|c|c|c|c|c|}
\hline Radionuclide & $\begin{array}{c}f 1 \\
\text { Value }\end{array}$ & $\begin{array}{l}\text { Ingested } \\
\text { Water DCG } \\
(\mu \mathrm{Ci} / \mathrm{mL})\end{array}$ & \multicolumn{2}{|c|}{ Inhaled Air DCG $(\mu \mathrm{Ci} / \mathrm{mL})$} & Radionuclide & $\begin{array}{c}f 1 \\
\text { Value }\end{array}$ & $\begin{array}{l}\text { Ingested } \\
\text { Water DCG } \\
(\mu \mathrm{Ci} / \mathrm{mL})\end{array}$ & \multicolumn{3}{|c|}{ Inhaled Air DCG $(\mu \mathrm{Ci} / \mathrm{mL})$} \\
\hline $\mathrm{Br}-74$ & 1. $E+\infty 0$ & 1.E-03 & 2.E-07 & 2.E-07 & $\gamma-86 / 3$ & 1.E-04 & $3, E-05$ & & 8.E-09 & 7.E-09 \\
\hline $\mathrm{Br}-75$ & $1 . E+\infty 0$ & 1. E-03 & 1.E-07 & $1 . \mathrm{E}-07$ & $y-87$ & 1.E-04 & 6.E-05 & & 8.E-09 & 7.E-09 \\
\hline $\mathrm{Br}-76$ & 1. $E+00$ & 1.E-04 & 1.E-08 & 1.E-08 & $Y-88$ & 1. E- -04 & 3.E-05 & & 6.E-10 & $6 . E-10$ \\
\hline $\mathrm{Br}-77 / 3$ & $1 . E+00$ & 4.E-04 & 6.E-08 & 5.E-08 & $Y-90 m / 3$ & 1.E-04 & 2.E-04 & & 3.E-08 & 3.E-08 \\
\hline $\mathrm{Br}-80 \mathrm{~m}$ & 1. $E+\infty$ & 6.E-04 & 4. $E-08$ & 3.E-08 & $Y-90$ & 1.E-04 & 1.E-05 & & 2.E-09 & $1 . E-09$ \\
\hline $\mathrm{Br}-80 / 3$ & $1 . E+00$ & 2. $E-03$ & 4.E-07 & 5. E-07 & $Y-91 \mathrm{~m} / 3$ & 1. E-04 & 4.E-03 & & $6 . E-07$ & $4 . E-07$ \\
\hline $\mathrm{Br}-82 / 3$ & $1 . E+00$ & 8.E-05 & 1.E-08 & 9.E-09 & $y-91$ & 1.E-04 & 2.E-05 & & 4.E-10 & 3.E-10 \\
\hline $\mathrm{Br}-83$ & 1. $E+00$ & 2.E-03 & 2.E-07 & 1.E-07 & $Y-92$ & 1.E-04 & 7.E-05 & & 2.E-08 & 2.E-08 \\
\hline $\mathrm{Br}-84 / 3$ & 1. $E+00$ & 9.E-04 & 1.E-07 & 2. E-07 & $Y-93$ & 1.E-04 & 3.E-05 & & 7.E-09 & 6.E-09 \\
\hline & & & & & $Y-94$ & 1.E-04 & 8.E-04 & & 2.E-07 & 2.E-07 \\
\hline $\begin{array}{l}\mathrm{Rb}-79 \\
\mathrm{Dh}-81 \mathrm{~m}\end{array}$ & 1. E+00 & 2.E-03 & 3.E-07 & & $Y-95$ & 1.E-04 & 1.E-03 & & 4.E-07 & 3.E-07 \\
\hline $\mathrm{Rb}-81 \mathrm{~m}$ & 1. $E+00$ & 1. E-03 & 8.E-07 & & & & & & & \\
\hline $\mathrm{Rb}-81 / 3$ & $1 . E+00$ & 1.E-03 & $1 . E-07$ & & $\mathrm{Zr}-86$ & 2.E-03 & 4.E-05 & 9.E-09 & 6.E-09 & 6.E-09 \\
\hline $\mathrm{Rb}-82 \mathrm{~m}$ & $1 . E+00$ & 3.E-04 & 4.E-08 & & $\mathrm{Zr}-88$ & 2. E-03 & 1.E-04 & 5. E-10 & 1. E-09 & 7.E-10 \\
\hline $\mathrm{Rb}-83$ & 1. $E+00$ & 2.E-05 & 2.E-09 & & $\mathrm{Zr}-89$ & 2. $\mathrm{E}-03$ & 4.E-05 & $8 . E-09$ & 6.E-09 & $6 . E-09$ \\
\hline$R b-84$ & 1. $E+00$ & 1.E-05 & $2 . E-09$ & & $\mathrm{Zr}-93$ & 2. E- 03 & 9.E-05 & 4.E-11 & 1.E-10 & 2.E-10 \\
\hline $\mathrm{Rb}-86$ & 1. $E+00$ & 1.E-05 & 2. $E-09$ & & $\mathrm{Zr}-95$ & 2. E- 03 & 4. E- 05 & $6 . E-10$ & 9.E-10 & 7.E-10 \\
\hline $\mathrm{Rb}-87$ & $1 . E+00$ & 3.E-05 & 4.E-09 & & $\mathrm{Zr}-97$ & 2. E-03 & 2.E-05 & 5. E-09 & 3.E-09 & 3.E-09 \\
\hline $\mathrm{Rb}-88 / 3$ & $1 . E+00$ & 8.E-04 & $1 . E-07$ & & & & & & & \\
\hline $\mathrm{Rb}-89 / 3$ & 1. $\mathrm{E}+00$ & 2.E-03 & 3.E-07 & & $\mathrm{Nb}-88$ & 1.E-02 & 2.E-03 & & 5.E-07 & 5.E-07 \\
\hline & & & & & $\mathrm{Nb}-89(66 \min )$ & 1.E-02 & 3.E-04 & & 1.E-07 & $9 . E-08$ \\
\hline $5 r-80$ & 3.E-01 & 3.E-02 & 5.E-06 & & $\mathrm{Nb}-89(122 \mathrm{~min})$ & 1.E-02 & 1.E-04 & & 4.E-08 & 4.E-OB \\
\hline & 1.E-02 & 3.E-02 & & 6.E-06 & $\mathrm{Nb}-90$ & 1.E-02 & 3.E-05 & & 6.E-09 & 6.E-09 \\
\hline$S r-81$ & 3.E-01 & 1.E-04 & 2.E-07 & $0.2-00$ & $\mathrm{Nb}-93 \mathrm{~m}$ & 1.E-02 & 3.E-04 & & 3.E-09 & $4 . E-10$ \\
\hline & 1. $\mathrm{E}-02$ & 6.E-04 & & 2. E-07 & $\mathrm{Nb}-94$ & 1.E-02 & 3.E-05 & & 5. $E-10$ & 4.E-11 \\
\hline $\mathrm{Sr}-83$ & 3.E-01 & 8.E-05 & 2.E-08 & & $\mathrm{Nb}-95 \mathrm{~m}$ & 1. E-02 & 7.E-05 & & 6.E-09 & 5.E-09 \\
\hline & 1.E-02 & 6.E-05 & & 8.E-09 & $\mathrm{Nb}-95$ & 1. E-02 & 6.E-05 & & 3. $E-09$ & 3.E-09 \\
\hline$S r-85 \mathrm{~m} / 3$ & 3.E-01 & 6. E-03 & 1.E-06 & & $\mathrm{Nb}-96$ & 1.E-02 & 3.E-05 & & 6. E-09 & 6.E-09 \\
\hline & 1.E-02 & 6.E-03 & & $2 . \varepsilon-06$ & $\mathrm{Nb}-97 / 3$ & 1.E-02 & $6 . E-04$ & & 2.E-07 & 2.E-07 \\
\hline $\mathrm{Sr}-85$ & $3 . \mathrm{E}-01$ & 7.E-05 & $6 . E-09$ & & $\mathrm{Nb}-98$ & 1.E-02 & 4.E-04 & & 1. $E-07$ & 1.E-07 \\
\hline & 1.E-02 & 1.E-04 & & 4. E-09 & & & & & & \\
\hline$S r-87 \mathrm{~m} / 3$ & 3.E-01 & 1.E-03 & 3.E-07 & & Mo-90 & 8.E-01 & 1.E-04 & 2.E-08 & & \\
\hline & 1.E-02 & 1.E-03 & & 4.E-07 & & 5. E-02 & 6.E-05 & & & 1.E-08 \\
\hline $\operatorname{sr}-89$ & 3.E-01 & 2.E-05 & 2.E-09 & & Mo-93m & 8.E-01 & 3.E-04 & 4.E-08 & & \\
\hline & 1.E-02 & 2. E-05 & & $3, E-10$ & & 5.E-02 & 1.E-04 & & & 3.E-08 \\
\hline $\mathrm{Sr}-90$ & 3.E-01 & 1.E-06 & 5.E-11 & & Mo-93 & 8.E-01 & 1.E-04 & 1.E-08 & & \\
\hline & 1.E-02 & 1.E-05 & & 9. E-12 & & 5.E-02 & 6.E-04 & & & 4.E-10 \\
\hline$s r-91$ & 3.E-01 & 6.E-05 & 1.E-08 & & Mo-99 & 8.E-01 & 5.E-05 & 6.E-09 & & \\
\hline & 1. E-02 & 5.E-05 & & 8.E-09 & & 5.E-02 & 3.E-05 & & & 3.E-09 \\
\hline $\mathrm{Sr}-92 / 3$ & 3.E-01 & 9.E-05 & 2.E-08 & & Mo-101/3 & 8.E-01 & 2. E-03 & 3.E-07 & & \\
\hline & 1.E-02 & 7.E-05 & & 2.E-08 & & 5.E-02 & 1.E-03 & & & 4.E-07 \\
\hline$\gamma-86 m$ & 1.E-04 & 6.E-04 & & 1. E-07 & Tc-93m & 8.E-01 & 2.E-03 & 4.E-07 & 7.E-07 & \\
\hline
\end{tabular}




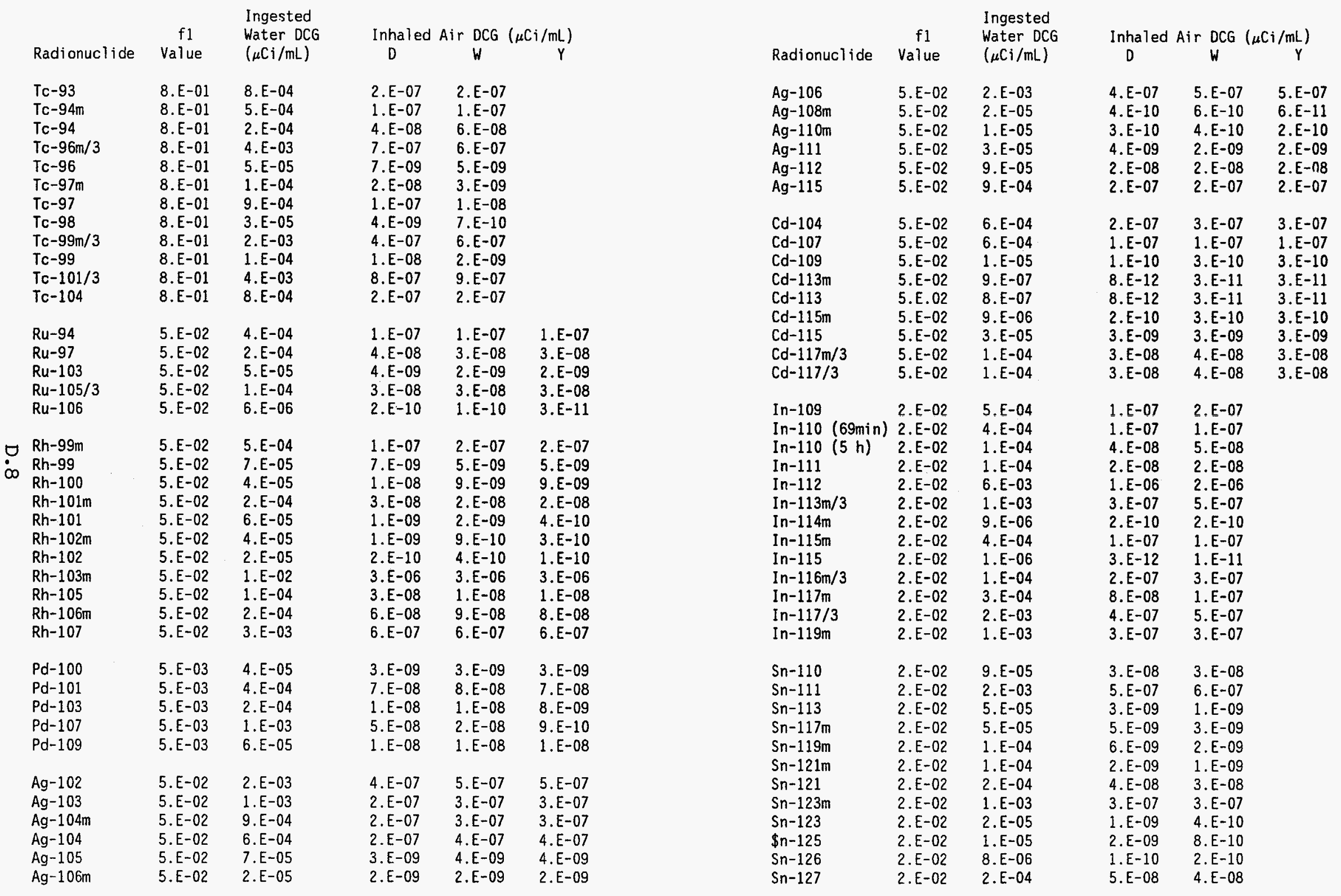




\begin{tabular}{|c|c|c|c|c|c|c|c|c|c|}
\hline Radionucl ide & $\begin{array}{c}\text { f1 } \\
\text { Value }\end{array}$ & $\begin{array}{l}\text { Ingested } \\
\text { Water DCG } \\
(\mu \mathrm{Ci} / \mathrm{mL})\end{array}$ & $\begin{array}{c}\text { Inhaled } \\
0\end{array}$ & $\underset{W}{\operatorname{DCG}}(\mu \mathrm{Ci} / \mathrm{mL})$ & Radionuclide & $\begin{array}{c}\text { f1 } \\
\text { Value }\end{array}$ & $\begin{array}{l}\text { Ingested } \\
\text { Water oCG } \\
(\mu \mathrm{Ci} / \mathrm{mL})\end{array}$ & $\underset{D}{\text { Inhaled }}$ & Air $\underset{W}{\text { DCG }}(\mu \mathrm{Ci} / \mathrm{mL})$ \\
\hline$S n-128$ & 2.E-02 & 3.E-04 & 6. E-08 & 9.E-08 & $\begin{array}{l}\text { Te-116 } \\
\text { Te-121m }\end{array}$ & $\begin{array}{l}2 . E-01 \\
2 . E-01\end{array}$ & $\begin{array}{l}\text { 2.E-04 } \\
\text { 2.E-05 }\end{array}$ & $\begin{array}{l}\text { 5.E-08 } \\
9 . \mathrm{E}-10\end{array}$ & $\begin{array}{l}\text { 7.E-08 } \\
\text { 1.E-09 }\end{array}$ \\
\hline \multirow{2}{*}{$S b-115$} & 1.E-01 & 2.E-03 & $6 . E-07$ & & Te-121 & 2.E-01 & 9.E-05 & 1.E-08 & 7.E-09 \\
\hline & 1.E-02 & 2.E-03 & & 7.E-07 & $T e-123 m$ & 2. $E-01$ & 3.E-05 & 1.E-09 & 1.E-09 \\
\hline \multirow{2}{*}{$S b-116 m$} & 1.E-01 & 6. E-04 & 2.E-07 & & Te-123 & 2.E-01 & 3.E-05 & 1.E-09 & 3.E-09 \\
\hline & 1.E-02 & 6.E-04 & & 3.E-07 & Te-125m & 2.E-01 & 4.E-05 & 2.E-09 & 2.E-09 \\
\hline \multirow[t]{2}{*}{$S b-116$} & 1. E-01 & 2. E-03 & 7.E-07 & & Te-127m & 2.E-01 & 2.E-05 & 1.E-09 & 6.E-10 \\
\hline & 1.E-02 & 2. E-03 & & 8.E-07 & $\mathrm{Te}-127$ & 2.E-01 & 2.E-04 & 5.E-08 & 4. E-08 \\
\hline \multirow[t]{2}{*}{$\mathrm{Sb}-117 / 3$} & 1.E-01 & 2.E-03 & 5.E-07 & & Te-129m & 2.E-01 & 1.E-05 & 1.E-09 & $6 . E-10$ \\
\hline & 1.E-02 & 2. $E-03$ & & 7.E-07 & $\mathrm{Te}-129$ & 2. E-01 & 7.E-04 & 2.E-07 & 2.E-07 \\
\hline \multirow[t]{2}{*}{$\mathrm{Sb}-118 \mathrm{~m}$} & 1. E-01 & 2. $E-04$ & 5.E-08 & & Te-131m & 2.E-01 & 9.E-06 & 3.E-09 & 2.E-09 \\
\hline & 1.E-02 & 1. E-04 & & 5.E-08 & Te-131 & 2.E-01 & 7.E-04 & 1.E-07 & 1.E-07 \\
\hline \multirow{2}{*}{$S b-119$} & 1. E-01 & 4. E-04 & 1. E-07 & & Te-132 & 2.E-01 & 2.E-05 & 2.E-09 & 2.E-09 \\
\hline & 1.E-02 & 4. E-04 & & 6.E-08 & $\mathrm{Te}-133 \mathrm{~m} / 3$ & 2.E-01 & 2.E-04 & 3.E-08 & 3.E-08 \\
\hline \multirow{2}{*}{$\mathrm{Sb}-120(16 \mathrm{~min})$} & 1. E-01 & 5. $E-03$ & 1.E-06 & & $T e-133 / 3$ & 2.E-01 & 9.E-04 & 1. $E-07$ & 1.E-07 \\
\hline & 1.E-02 & 5.E-03 & & 1.E-06 & $\mathrm{Te}-134 / 3$ & 2. E-01 & 7.E-04 & 1.E-07 & 1.E-07 \\
\hline \multirow{2}{*}{$S b-120(6 d)$} & 1.E-01 & 3.E-05 & 5.E-09 & & & & & & \\
\hline & 1.E-02 & 3.E-05 & & 3.E-09 & $\mathrm{I}-120 \mathrm{~m}$ & $1 . E+00$ & 4. $E-04$ & 5.E-08 & \\
\hline \multirow{2}{*}{$S b-122$} & 1.E-01 & 2.E-05 & 5.E-09 & & $\mathrm{I}-120$ & 1. $E+00$ & 2.E-04 & 3. $E-08$ & \\
\hline & 1.E-02 & 2.E-05 & & 3.E-09 & $\mathrm{I}-121$ & $1 . E+00$ & 8.E-04 & 1.E-07 & \\
\hline \multirow{2}{*}{$S b-124 m$} & 1.E-01 & 7. E-03 & 2.E-06 & & $1-123$ & $1 . E+00$ & 3. E-04 & 4. E-08 & \\
\hline & 1.E-02 & 7.E-03 & & 1.E-06 & $1-124$ & 1. $E+\infty 0$ & 4. $E-06$ & $6 . E-10$ & \\
\hline \multirow{2}{*}{$S b-124$} & 1.E-01 & 2. $\mathrm{E}-05$ & 2.E-09 & & I-125 & $1 . E+\infty 0$ & 4.E-06 & 5.E-10 & \\
\hline & 1.E-02 & 1. E-05 & & 6.E-10 & $\mathrm{I}-126$ & $1 . E+\infty 0$ & 2. E-06 & 3.E-10 & \\
\hline \multirow[t]{2}{*}{$\mathrm{Sb}-125$} & 1.E-01 & $6 . E-05$ & 6.E-09 & & $\mathrm{I}-128 / 3$ & 1. $E+00$ & 2. $\mathrm{E}-03$ & 3.E-07 & \\
\hline & 1. E-02 & 5. E-05 & & 1.E-09 & $1-129$ & $1 . E+\infty 0$ & $5 . E-07$ & 7.E-11 & \\
\hline \multirow[t]{2}{*}{$\mathrm{Sb}-126 \mathrm{~m} / 3$} & 1.E-01 & 2. $\mathrm{E}-03$ & 4.E-07 & & $I-130$ & 1. $E+\infty 0$ & 3. E-05 & 5.E-09 & \\
\hline & 1.E-02 & 2. E-03 & 4.L-6r & 5.E-07 & I-131 & 1. $E+\infty 0$ & 3.E-06 & 4. E-10 & \\
\hline \multirow[t]{2}{*}{$S b-126$} & 1. E-01 & 2.E-05 & 3.E-09 & & $\mathrm{I}-132 \mathrm{~m}$ & 1. $E+00$ & 3. E-04 & 5.E-08 & \\
\hline & 1.E-02 & 1.E-05 & & 1.E-09 & $1-132 / 3$ & 1. E+00 & 2. E-04 & 4.E-08 & \\
\hline \multirow[t]{2}{*}{$S b-127$} & 1.E-01 & 2. $\mathrm{E}-05$ & 5.E-09 & & I-133 & $1 . E+00$ & 1. E-05 & 2. E-09 & \\
\hline & 1.E-02 & 2.E-05 & & 2.E-09 & I-134/3 & 1. $E+00$ & 7.E-04 & 1.E-07 & \\
\hline \multirow[t]{2}{*}{$\mathrm{Sb}-128(9 \mathrm{~h})$} & 1.E-01 & 3.E-05 & $1 . \mathrm{E}-08$ & & I-135/3 & 1. $E+00$ & 7.E-05 & 1.E-08 & \\
\hline & 1.E-02 & 3.E-05 & & 8.E-09 & & & & & \\
\hline \multirow[t]{2}{*}{$\mathrm{Sb}-128(10 \mathrm{~min})$} & 1.E-01 & 3.E-03 & 9.E-07 & & Cs- 125 & 1. $E+00$ & 2.E-03 & 3.E-07 & \\
\hline & 1. $\mathrm{E}-02$ & 3. $\mathrm{E}-03$ & & 1.E-06 & Cs-127 & 1. $E+00$ & 2. $\mathrm{E}-03$ & 2.E-07 & \\
\hline \multirow[t]{2}{*}{$\mathrm{Sb}-129 / 3$} & 1.E-01 & 5.E-05 & 2.E-08 & & Cs-129/3 & 1. $E+00$ & 6.E-04 & 8.E-08 & \\
\hline & 1.E-02 & 8.E-05 & & 2.E-08 & $C_{s}-130$ & 1. $E+00$ & 3. E-03 & 4.E-07 & \\
\hline \multirow[t]{2}{*}{$S b-130$} & 1.E-01 & 5.E-04 & 1.E-07 & & $C s-131$ & 1. $E+00$ & 6.E-04 & 7.E-08 & \\
\hline & 1. $E-02$ & 5.E-04 & & 2.E-07 & Cs-132 & $1 . E+00$ & 7. $\mathrm{E}-05$ & 1.E-08 & \\
\hline \multirow{3}{*}{$S b-131$} & 1.E-01 & 5.E-04 & 9. E-08 & & $\mathrm{Cs}-134 \mathrm{~m}$ & 1. $E+00$ & 3.E-03 & 3.E-07 & \\
\hline & 1.E-02 & 5.E-04 & 0.600 & 1.E-07 & $C s-134$ & 1. $E+00$ & 2. E-06 & 2. $E-10$ & \\
\hline & & & & & Cs-135m & 1. $E+00$ & 3.E-03 & 5.E-07 & \\
\hline
\end{tabular}




\begin{tabular}{|c|c|c|c|c|c|}
\hline Radionuclide & $\begin{array}{c}f 1 \\
\text { Value }\end{array}$ & $\begin{array}{l}\text { Ingested } \\
\text { Water } \mathrm{OCG} \\
(\mu \mathrm{Ci} / \mathrm{mL})\end{array}$ & $\begin{array}{c}\text { Inhaled } \\
D\end{array}$ & Air $\underset{W}{D C G}$ & $\underset{Y}{(\mu \mathrm{Ci} / \mathrm{mL})}$ \\
\hline $\begin{array}{l}\mathrm{Cs}-135 \\
\mathrm{Cs}-136 \\
\mathrm{Cs}-137 \\
\mathrm{Cs}-138 / 3\end{array}$ & $\begin{array}{l}1 . E+00 \\
1 . E+00 \\
1 . E+00 \\
1 . E+00\end{array}$ & $\begin{array}{l}2 . E-05 \\
1 . E-05 \\
3 . E-06 \\
9 . E-04\end{array}$ & $\begin{array}{l}\text { 3.E-09 } \\
\text { 2.E-09 } \\
\text { 4.E-10 } \\
1 . E-07\end{array}$ & & \\
\hline $\begin{array}{l}\mathrm{Ba}-126 \\
\mathrm{Ba}-128 \\
\mathrm{Ba}-131 \mathrm{~m} \\
\mathrm{Ba}-131 \\
\mathrm{Ba}-133 \mathrm{~m} \\
\mathrm{Ba}-133 \\
\mathrm{Ba}-135 \mathrm{~m} \\
\mathrm{Ba}-139 \\
\mathrm{Ba}-140 \\
\mathrm{Ba}-141 / 3 \\
\mathrm{Ba}-142 / 3\end{array}$ & $\begin{array}{l}1 . E-01 \\
1 . E-01 \\
1 . E-01 \\
1 . E-01 \\
1 . E-01 \\
1 . E-01 \\
1 . E-01 \\
1 . E-01 \\
1 . E-01 \\
1 . E-01 \\
1 . E-01\end{array}$ & $\begin{array}{l}2 . E-04 \\
1 . E-05 \\
1 . E-02 \\
8 . E-05 \\
7 . E-05 \\
4 . E-05 \\
9 . E-05 \\
3 . E-04 \\
2 . E-05 \\
7 . E-04 \\
1 . E-03\end{array}$ & $\begin{array}{l}4 . E-08 \\
4 . E-09 \\
3 . E-06 \\
2 . E-08 \\
2 . E-08 \\
2 . E-09 \\
3 . E-05 \\
7 . E-08 \\
3 . E-09 \\
2 . E-07 \\
3 . E-07\end{array}$ & & \\
\hline $\begin{array}{l}\text { La-131 } \\
\text { La-132 } \\
\text { La-135 } \\
\text { La-137 } \\
\text { La-138 } \\
\text { La-140 } \\
\text { La-141 } \\
\text { La-142 } \\
\text { La-143 }\end{array}$ & $\begin{array}{l}1 . E-03 \\
1 . E-03 \\
1 . E-03 \\
1 . E-03 \\
1 . E-03 \\
1 . E-03 \\
1 . E-03 \\
1 . E-03 \\
1 . E-03\end{array}$ & $\begin{array}{l}1 . E-03 \\
9 . E-05 \\
1 . E-03 \\
3 . E-04 \\
2 . E-05 \\
2 . E-05 \\
1 . E-04 \\
2 . E-04 \\
1 . E-03\end{array}$ & $\begin{array}{l}3 . E-07 \\
2 . E-05 \\
3 . E-07 \\
2 . E-10 \\
8 . E-12 \\
3 . E-09 \\
2 . E-08 \\
5 . E-08 \\
2 . E-07\end{array}$ & $\begin{array}{l}4 . E-07 \\
3 . E-08 \\
2 . E-07 \\
6 . E-10 \\
3 . E-11 \\
3 . E-09 \\
3 . E-08 \\
8 . E-08 \\
2 . E-07\end{array}$ & \\
\hline $\begin{array}{l}\mathrm{Ce}-134 \\
\mathrm{Ce}-135 \\
\mathrm{Ce}-137 \mathrm{~m} \\
\mathrm{Ce}-137 \\
\mathrm{Ce}-139 \\
\mathrm{Ce}-141 \\
\mathrm{Ce}-143 \\
\mathrm{Ce}-144\end{array}$ & $\begin{array}{l}3 . E-04 \\
3 . E-04 \\
3 . E-04 \\
3 . E-04 \\
3 . E-04 \\
3 . E-04 \\
3 . E-04 \\
3 . E-04\end{array}$ & $\begin{array}{l}2 . E-05 \\
4 . E-05 \\
7 . E-05 \\
1 . E-03 \\
1 . E-04 \\
5.3-05 \\
3 . E-05 \\
7 . E-06\end{array}$ & & $\begin{array}{l}2 . E-09 \\
9 . E-09 \\
1 . E-08 \\
3 . E-07 \\
2 . E-09 \\
2 . E-09 \\
4 . E-09 \\
6 . E-11\end{array}$ & $\begin{array}{l}2 . E-09 \\
8 . E-09 \\
9 . E-09 \\
3 . E-07 \\
2 . E-09 \\
1 . E-09 \\
4 . E-09 \\
3 . E-11\end{array}$ \\
\hline $\begin{array}{l}\text { Pr }-136 \\
\text { Pr }-137 \\
\text { Pr-138m } \\
\text { Pr-139 } \\
\text { Pr-142m } \\
\text { Pr }-142 \\
\text { Pr-143 }\end{array}$ & $\begin{array}{l}3 . E-04 \\
3 . E-04 \\
3 . E-04 \\
3 . E-04 \\
3 . E-04 \\
3 . E-04 \\
3 . E-04\end{array}$ & $\begin{array}{l}2 . E-03 \\
1 . E-03 \\
3 . E-04 \\
1 . E-03 \\
2 . E-03 \\
3 . E-05 \\
3 . E-05\end{array}$ & & $\begin{array}{l}6 . E-07 \\
4 . E-07 \\
1 . E-07 \\
3 . E-07 \\
4 . E-07 \\
5 . E-09 \\
2 . E-09\end{array}$ & $\begin{array}{l}\text { 5.E-07 } \\
3 . E-07 \\
1 . E-07 \\
3 . E-07 \\
3 . E-07 \\
4 . E-09 \\
2 . E-09\end{array}$ \\
\hline
\end{tabular}

\begin{tabular}{|c|c|c|}
\hline Radionuclide & $\begin{array}{c}f 1 \\
\text { Value }\end{array}$ & $\begin{array}{l}\text { Ingeste } \\
\text { Water } D \\
(\mu \mathrm{C} i / \mathrm{mL}\end{array}$ \\
\hline $\begin{array}{l}\mathrm{Pr}-144 \\
\mathrm{Pr}-145 \\
\mathrm{Pr}-147\end{array}$ & $\begin{array}{l}\text { 3.E-O04 } \\
\text { 3.E.04 } \\
\text { 3.E-04 }\end{array}$ & $\begin{array}{l}\text { 1.E-03 } \\
9 . E-05 \\
2 . E-03\end{array}$ \\
\hline $\begin{array}{l}N d-136 \\
N d-138 \\
N d-139 m \\
N d-139 \\
N d-141 \\
N d-147 \\
N d-149 / 3 \\
N d-151\end{array}$ & $\begin{array}{l}3 . E-04 \\
3 . E-04 \\
3 . E-04 \\
3 . E-04 \\
3 . E-04 \\
3 . E-04 \\
3 . E-04 \\
3 . E-04\end{array}$ & $\begin{array}{l}4 . E-04 \\
5 . E-05 \\
1 . E-04 \\
2 . E-03 \\
4 . E-03 \\
4 . E-05 \\
3 . E-04 \\
2 . E-03\end{array}$ \\
\hline $\begin{array}{l}P_{m}-141 \\
P_{m-143} \\
P_{m-144} \\
P_{m}-145 \\
P_{m}-146 \\
P_{m}-147 \\
P_{m}-148 m \\
P_{m}-148 \\
P_{m}-149 \\
P_{m}-150 \\
P_{m-151}\end{array}$ & $\begin{array}{l}3 . E-04 \\
3 . E-04 \\
3 . E-04 \\
3 . E-04 \\
3 . E-04 \\
3 . E-04 \\
3 . E-04 \\
3 . E-04 \\
3 . E-04 \\
3 . E-04 \\
3 . E-04\end{array}$ & $\begin{array}{l}2 . E-03 \\
1 . E-04 \\
3 . E-05 \\
3 . E-04 \\
4 . E-05 \\
1 . E-04 \\
2 . E-05 \\
1 . E-05 \\
4 . E-05 \\
1 . E-04 \\
5 . E-05\end{array}$ \\
\hline $\begin{array}{l}S m-141 m \\
S m-141 \\
S m-142 \\
S m-145 \\
S m-146 \\
S m-147 \\
S m-151 \\
S m-153 \\
S m-155 \\
S m-156\end{array}$ & $\begin{array}{l}3 . E-04 \\
3 . E-04 \\
3 . E-04 \\
3 . E-04 \\
3 . E-04 \\
3 . E-04 \\
3 . E-04 \\
3 . E-04 \\
3 . E-04 \\
3 . E-04\end{array}$ & $\begin{array}{l}8 . E-04 \\
2 . E-03 \\
2 . E-04 \\
2 . E-04 \\
7 . E-07 \\
8 . E-07 \\
4 . E-04 \\
5 . E-05 \\
2 . E-03 \\
1 . E-04\end{array}$ \\
\hline $\begin{array}{l}\text { Eu-145 } \\
\text { Eu-146 } \\
\text { Eu-147 } \\
\text { Eu-148 } \\
\text { Eu-149 } \\
\text { Eu-150 (12 h) } \\
\text { Eu-150 (34 rr }\end{array}$ & $\begin{array}{l}\text { 1.E-03 } \\
1 . E-03 \\
1 . E-03 \\
1 . E-03 \\
1 . E-03 \\
1 . E-03 \\
1 . E-03\end{array}$ & $\begin{array}{l}\text { 4.E-05 } \\
3 . E-05 \\
\text { 8.E-05 } \\
3 . E-05 \\
3 . E-04 \\
9 . E-05 \\
\text { 2.E-05 }\end{array}$ \\
\hline
\end{tabular}

Inhaled Air DCG $(\mu \mathrm{Ci} / \mathrm{mL})$

\begin{tabular}{|c|c|}
\hline $\begin{array}{l}\text { S.E-07 } \\
\text { 2. E-08 } \\
\text { 5.E-07 }\end{array}$ & $\begin{array}{l}\text { 3.E-07 } \\
\text { 2.E-08 } \\
\text { 4.E-07 }\end{array}$ \\
\hline $\begin{array}{l}1 . E-07 \\
2 . E-08 \\
4 . E-08 \\
\text { 8.E-07 } \\
\text { 2.E-06 } \\
\text { 2.E-09 } \\
6 . E-08 \\
5 . E-07\end{array}$ & $\begin{array}{l}1 . E-07 \\
1 . E-08 \\
3 . E-08 \\
7 . E-07 \\
1 . E-06 \\
2 . E-09 \\
6 . E-08 \\
4 . E-07\end{array}$ \\
\hline $\begin{array}{l}\text { 4.E-07 } \\
1 . E-09 \\
\text { 3.E-10 } \\
\text { 5.E-10 } \\
\text { 1.E-10 } \\
\text { 5.E-10 } \\
\text { 7.E-10 } \\
1 . E-09 \\
\text { 4.E-08 } \\
\text { 4.E-08 } \\
\text { 8.E-09 }\end{array}$ & $\begin{array}{l}4 . E-07 \\
2 . E-09 \\
3 . E-10 \\
4 . E-10 \\
1 . E-10 \\
3 . E-10 \\
7 . E-10 \\
1 . E-09 \\
4 . E-09 \\
4 . E-08 \\
7 . E-09\end{array}$ \\
\hline
\end{tabular}

2. E-07

1.E-09

2. E-13

2.E-13

4. E-10

7. E-09

5. E-07

2. E-08

5.E.09

3. E-09

4. $E-09$

8. E-10

7.E-09

2. E-08

4. $E-11$ 


\begin{tabular}{|c|c|c|}
\hline Radionuclide & $\begin{array}{c}\text { fl } \\
\text { Value }\end{array}$ & $\begin{array}{l}\text { Ingested } \\
\text { Water DCG } \\
(\mu \mathrm{Ci} / \mathrm{mL})\end{array}$ \\
\hline $\begin{array}{l}E u-152 m \\
E u-152 \\
E u-154 \\
E u-155 \\
E u-156 \\
E u-157 \\
E u-158\end{array}$ & $\begin{array}{l}1 . E-03 \\
1 . E-03 \\
1 . E-03 \\
1 . E-03 \\
1 . E-03 \\
1 . E-03 \\
1 . E-03\end{array}$ & $\begin{array}{l}7 . E-05 \\
2 . E-05 \\
2 . E-05 \\
1 . E-04 \\
2 . E-05 \\
6 . E-05 \\
5 . E-04\end{array}$ \\
\hline $\begin{array}{l}G d-145 \\
G d-146 \\
G d-147 \\
G d-148 \\
G d-149 \\
G d-151 \\
G d-152 \\
G d-153 \\
G d-159\end{array}$ & $\begin{array}{l}3 . E-04 \\
3 . E-04 \\
3 . E-04 \\
3 . E-04 \\
3 . E-04 \\
3 . E-04 \\
3 . E-04 \\
3 . E-04 \\
3 . E-04\end{array}$ & $\begin{array}{l}1 . E-03 \\
4 . E-05 \\
5 . E-05 \\
7 . E-07 \\
8 . E-05 \\
2 . E-04 \\
9 . E-07 \\
1 . E-04 \\
7 . E-05\end{array}$ \\
\hline $\begin{array}{l}\text { Tb-147 } \\
\text { Tb-149 } \\
\text { Tb-150 } \\
\text { Tb-151 } \\
\text { Tb-153 } \\
\text { Tb-154 } \\
\text { Tb-155 } \\
\text { Tb-156m }(24 \mathrm{n}) \\
\text { Tb-156m }(5 \mathrm{~h}) \\
\text { Tb-156 } \\
\text { Tb-157 } \\
\text { Tb-158 } \\
\text { Tb-160 } \\
\text { Tb-161 }\end{array}$ & $\begin{array}{l}3 . E-04 \\
3 . E-04 \\
3 . E-04 \\
3 . E-04 \\
3 . E-04 \\
3 . E-04 \\
3 . E-04 \\
3 . E-04 \\
3 . E-04 \\
3 . E-04 \\
3 . E-04 \\
3 . E-04 \\
3 . E-04 \\
3 . E-04\end{array}$ & $\begin{array}{l}2 . E-04 \\
1 . E-04 \\
1 . E-04 \\
1 . E-04 \\
1 . E-04 \\
5 . E-05 \\
2 . E-04 \\
2 . E-04 \\
4 . E-04 \\
3 . E-05 \\
1 . E-03 \\
3 . E-05 \\
2 . E-05 \\
5 . E-05\end{array}$ \\
\hline $\begin{array}{l}\text { Dy-155 } \\
\text { Dy-157/3 } \\
\text { Dy-159 } \\
\text { Dy-165 } \\
\text { Dy-166 }\end{array}$ & $\begin{array}{l}3 . E-04 \\
3 . E-04 \\
3 . E-04 \\
3 . E-04 \\
3 . E-04\end{array}$ & $\begin{array}{l}2 . E-04 \\
5 . E-04 \\
3 . E-04 \\
4 . E-04 \\
2 . E-05\end{array}$ \\
\hline $\begin{array}{l}\text { Ho-155 } \\
\text { Ho-157 } \\
\text { Ho-159 } \\
\text { Ho-161 }\end{array}$ & $\begin{array}{l}3 . E-04 \\
3 . E-04 \\
3 . E-04 \\
3 . E-04\end{array}$ & $\begin{array}{l}\text { 1.E-03 } \\
\text { 7.E-03 } \\
6 . E-03 \\
3 . E-03\end{array}$ \\
\hline
\end{tabular}

$\underset{D}{\operatorname{Inhaled}} \underset{W}{\operatorname{Air}} \underset{\mathrm{DCG}}{\operatorname{Dr}} \underset{\mathrm{Y}}{(\mu \mathrm{Ci} / \mathrm{mL})}$

$\begin{array}{ll} & 2 . E-08 \\ & 5 . E-11 \\ & 5 . E-11 \\ & 3 . E-10 \\ & 1 . E-09 \\ & 1 . E-08 \\ & 1 . E-07 \\ & \\ 4 . E-07 & 4 . E-07 \\ 3 . E-10 & 7 . E-10 \\ 1 . E-08 & 8 . E-09 \\ 4 . E-14 & 1 . E-13 \\ 5 . E-09 & 6 . E-09 \\ 1 . E-09 & 3 . E-09 \\ 5 . E-14 & 2 . E-13 \\ 6 . E-10 & 1 . E-09 \\ 2 . E-08 & 1 . E-08 \\ & \\ & 7 . E-08 \\ & 2 . E-09 \\ & 5 . E-08 \\ & 2 . E-08 \\ & 2 . E-08 \\ 1 . E-08 \\ & 2 . E-08 \\ & 2 . E-08 \\ 6 . E-08 \\ 3 . E-09 \\ 1 . E-09 \\ 5 . E-11 \\ & 5 . E-10 \\ 4 . E-09 \\ & 6 . E-08 \\ & 2 . E-07 \\ 6 . E-09 \\ 1 . E-07 \\ 2 . E-09 \\ & 4 . E-07 \\ & 3 . E-06 \\ 2 . E-06 \\ 1 . E-06\end{array}$

Ingested

Water DCG

$(\mu \mathrm{Ci} / \mathrm{mL})$

Radionuclide

Value

2. E-03

2. E-02

3. E-03

6.E-03

2. $E-05$

2.E-05

4.E-04

4.E-04

2. E-03

1.E-04

1.E-04

4. E-05

Er-171

Er-172

Tm-162

Tm-166

$\mathrm{Tm}-167$

$\mathrm{Tm}-170$

Tm-171

Tm-172

Tm-173

$\mathrm{Tm}-175$

Yb-161

Yb-166

Yb-167

Yb-169

$\mathrm{Yb}-175$

Yb-177

Yb-178

Lu-169

Lu-170

Lu-171

Lu-172

Lu-173

Lu-174m

Lu-174

Lu-176m

Lu-176

Lu-177m

Lu-177

Lu-178m

2. E-03

1.E-04

7.E-05

3.E-05

4. E-04

2.E-05

1.E-04

3. E-03

2. E-03

4.E-05

8.E-03

5. E-05

9. E-05

4. E-04

4.E-04

3.E-04 7.E-05

3.E-04 3.E-05

3.E-04 5.E-05

3.E-04 3.E-05

3.E-04 1.E-04

3.E-04 8.E-05

3.E-04 1.E-04

3.E-04 2.E-04

3.E-04 2.E-05

3.E-04 2.E-05

3.E-04 7.E-05

3.E-04

2. E-03
Inhaled Air DCG $(\mu \mathrm{Ci} / \mathrm{mL})$

$D$ W

6. E- -07

6.E-06

7.E-07

1.E-06

2. E-11

4. E-09

1. E-07

2.E-07

4. E- -07

6. E-09

2.E-08

3. E-09

7.E-07

3.E-08

5. E-09

5. E-10

1.E-09

3. E-09

3.E-08

6. E-07

7.E-07 7.E-07

5.E-09 4.E-09

2.E-06 2.E-06

2.E-09 2.E-09

8.E-09 8.E-09

1.E-07 1.E-07

1.E-07 9.E-08

1.E-08 1.E-08

5.E-09 5.E-09

4. E-09 5.E-09

3.E-09 3.E-09

1.E-09 6.E-10

8. E-10 5.E-10

6. $E-10$ 4. $E-10$

6. E-08 5.E-08

3.E-11 2.E-11

3. $E-10 \quad 2 . E-10$

5.E-09 5.E-09

4. E-07 4.E-07 


\begin{tabular}{|c|c|c|c|c|c|}
\hline Radionuclide & $\begin{array}{c}\text { f1 } \\
\text { Value }\end{array}$ & $\begin{array}{l}\text { Ingested } \\
\text { Water } \mathrm{OCG} \\
(\mu \mathrm{Ci} / \mathrm{mL})\end{array}$ & $\begin{array}{c}\text { Inhaled } \\
D\end{array}$ & $\underset{W}{A i r} \underset{W}{D C G}$ & $\underset{\gamma}{(\mu \mathrm{Ci} / \mathrm{mL})}$ \\
\hline $\begin{array}{l}\text { Lu-178 } \\
\text { Lu-179 }\end{array}$ & $\begin{array}{l}\text { 3.E-04 } \\
3 . E-04\end{array}$ & $\begin{array}{l}\text { 1.E-03 } \\
\text { 2.E-04 }\end{array}$ & & $\begin{array}{l}3 . E-07 \\
4 . E-08\end{array}$ & $\begin{array}{l}\text { 3.E-07 } \\
\text { 4.E-08 }\end{array}$ \\
\hline $\begin{array}{l}H f-170 \\
H f-172 \\
H f-173 \\
H f-175 \\
H f-177 m \\
H f-178 m \\
H f-179 m \\
H f-180 m \\
H f-181 \\
H f-182 m \\
H f-182 \\
H f-183 \\
H f-184\end{array}$ & $\begin{array}{l}2 . E-03 \\
2 . E-03 \\
2 . E-03 \\
2 . E-03 \\
2 . E-03 \\
2 . E-03 \\
2 . E-03 \\
2 . E-03 \\
2 . E-03 \\
2 . E-03 \\
2 . E-03 \\
2 . E-03 \\
2 . E-03\end{array}$ & $\begin{array}{l}7 . E-05 \\
3 . E-05 \\
1 . E-04 \\
8 . E-05 \\
5 . E-04 \\
7 . E-06 \\
3 . E-05 \\
2 . E-04 \\
3 . E-05 \\
1 . E-03 \\
1 . E-05 \\
6 . E-04 \\
7 . E-05\end{array}$ & $\begin{array}{l}1 . E-08 \\
4 . E-11 \\
3 . E-08 \\
2 . E-09 \\
1 . E-07 \\
6 . E-12 \\
1 . E-09 \\
5 . E-08 \\
9 . E-10 \\
2 . E-07 \\
4 . E-12 \\
1 . E-07 \\
2 . E-08\end{array}$ & $\begin{array}{l}1 . E-08 \\
1 . E-10 \\
3 . E-08 \\
3 . E-09 \\
2 . E-07 \\
2 . E-11 \\
1 . E-09 \\
6 . E-08 \\
1 . E-09 \\
3 . E-07 \\
2 . E-11 \\
1 . E-07 \\
2 . E-08\end{array}$ & \\
\hline $\begin{array}{l}T a-172 \\
T a-173 \\
T a-174 \\
T a-175 \\
T a-176 \\
T a-177 \\
T a-178 \\
T a-179 \\
T a-180 m \\
T a-180 \\
T a-182 m \\
T a-182 \\
T a-183 \\
T a-184 \\
T a-185 \\
T a-186\end{array}$ & $\begin{array}{l}1 . E-03 \\
1 . E-03 \\
1 . E-03 \\
1 . E-03 \\
1 . E-03 \\
1 . E-03 \\
1 . E-03 \\
1 . E-03 \\
1 . E-03 \\
1 . E-03 \\
1 . E-03 \\
1 . E-03 \\
1 . E-03 \\
1 . E-03 \\
1 . E-03 \\
1 . E-03\end{array}$ & $\begin{array}{l}1 . E-03 \\
2 . E-04 \\
7 . E-04 \\
2 . E-04 \\
1 . E-04 \\
3 . E-04 \\
5 . E-04 \\
6 . E-04 \\
6 . E-04 \\
4 . E-05 \\
6 . E-03 \\
2 . E-05 \\
3 . E-05 \\
5 . E-05 \\
7 . E-04 \\
2 . E-03\end{array}$ & & $\begin{array}{l}3 . E-07 \\
5 . E-08 \\
2 . E-07 \\
4 . E-08 \\
3 . E-08 \\
5 . E-08 \\
2 . E-07 \\
1 . E-08 \\
2 . E-07 \\
1 . E-09 \\
1 . E-06 \\
7 . E-10 \\
3 . E-09 \\
1 . E-08 \\
2 . E-07 \\
\text { 5.E-07 }\end{array}$ & $\begin{array}{l}2 . E-07 \\
4 . E-08 \\
2 . E-07 \\
3 . E-08 \\
3 . E-08 \\
4 . E-08 \\
2 . E-07 \\
2 . E-09 \\
1 . E-07 \\
6 . E-11 \\
1 . E-06 \\
3 . E-10 \\
2 . E-09 \\
1 . E-08 \\
2 . E-07 \\
5 . E-07\end{array}$ \\
\hline$W-176$ & 1.E-02 & 3.E-04 & & & \\
\hline$W-177$ & $\begin{array}{l}3 . E-01 \\
1 . E-02 \\
3 . E-01\end{array}$ & $\begin{array}{l}4 . E-04 \\
6 . E-04 \\
7 . E-04\end{array}$ & 2.E-07 & & \\
\hline$W-178$ & $\begin{array}{l}\text { 1.E-02 } \\
\text { 3.E-01 }\end{array}$ & $\begin{array}{l}\text { 1.E-04 } \\
\text { 2.E-04 }\end{array}$ & 5.E-08 & & \\
\hline $\begin{array}{l}W-179 \\
W-181\end{array}$ & $\begin{array}{l}\text { 1. E-02 } \\
\text { 3.E-01 } \\
1 . E-02\end{array}$ & $\begin{array}{l}2 . E-02 \\
2 . E-02 \\
4 . E-04\end{array}$ & 4.E-06 & & \\
\hline
\end{tabular}

\begin{tabular}{|c|c|c|c|c|c|}
\hline Radionuclide & $\begin{array}{c}f 1 \\
\text { Value }\end{array}$ & $\begin{array}{l}\text { Ingested } \\
\text { Water DCG } \\
(\mu \mathrm{Ci} / \mathrm{mL})\end{array}$ & $\begin{array}{c}\text { Inhaled } \\
D\end{array}$ & $\underset{W}{A}$ & $i / \mathrm{mL})$ \\
\hline $\begin{array}{l}W-181 \\
W-185 \\
W-187 \\
W-188\end{array}$ & $\begin{array}{l}3 . E-01 \\
1 . E-02 \\
3 . E-01 \\
1 . E-02 \\
3 . E-01 \\
1 . E-02 \\
3 . E-01\end{array}$ & $\begin{array}{l}5 . E-04 \\
7 . E-05 \\
1 . E-04 \\
5 . E-02 \\
7 . E-05 \\
2 . E-05 \\
2 . E-05\end{array}$ & $\begin{array}{l}\text { 8.E-08 } \\
\text { 2.E-08 } \\
\text { 2.E-08 } \\
\text { 3.E-09 }\end{array}$ & & \\
\hline $\begin{array}{l}\operatorname{Re}-177 \\
\operatorname{Re}-178 \\
\operatorname{Re}-181 \\
\operatorname{Re}-182(64 \quad \mathrm{~h}) \\
\operatorname{Re}-182(12 \mathrm{~h}) / 3 \\
\operatorname{Re}-184 \mathrm{~m} \\
\operatorname{Re}-184 \\
\operatorname{Re}-186 \mathrm{~m} \\
\operatorname{Re}-186 \\
\operatorname{Re}-187 \\
\operatorname{Re}-188 \mathrm{~m} \\
\operatorname{Re}-188 \\
\operatorname{Re}-189\end{array}$ & $\begin{array}{l}8 . E-01 \\
8 . E-01 \\
8 . E-01 \\
8 . E-01 \\
8 . E-01 \\
8 . E-01 \\
8 . E-01 \\
8 . E-01 \\
8 . E-01 \\
8 . E-01 \\
8 . E-01 \\
8 . E-01 \\
8 . E-01\end{array}$ & $\begin{array}{l}3 . E-03 \\
3 . E-03 \\
1 . E-04 \\
4 . E-05 \\
2 . E-04 \\
6 . E-05 \\
6 . E-05 \\
4 . E-05 \\
5 . E-05 \\
2 . E-02 \\
2 . E-03 \\
5 . E-05 \\
9 . E-05\end{array}$ & $\begin{array}{l}6 . E-07 \\
6 . E-07 \\
2 . E-08 \\
6 . E-09 \\
3 . E-08 \\
7 . E-09 \\
8 . E-09 \\
5 . E-09 \\
7 . E-09 \\
2 . E-06 \\
3 . E-07 \\
7 . E-09 \\
1 . E-08\end{array}$ & $\begin{array}{l}\text { 8.E-07 } \\
7 . E-07 \\
2 . E-08 \\
\text { 5.E-09 } \\
4 . E-08 \\
1 . E-09 \\
3 . E-09 \\
4 . E-10 \\
4 . E-09 \\
2 . E-07 \\
3 . E-07 \\
6 . E-09 \\
1 . E-08\end{array}$ & \\
\hline $\begin{array}{l}0 s-180 \\
0 s-181 \\
0 s-182 \\
0 s-185 \\
0 s-189 m \\
0 s-191 m \\
0 s-191 \\
0 s-193 \\
0 s-194\end{array}$ & $\begin{array}{l}1 . E-02 \\
1 . E-02 \\
1 . E-02 \\
1 . E-02 \\
1 . E-02 \\
1 . E-02 \\
1 . E-02 \\
1 . E-02 \\
1 . E-02\end{array}$ & $\begin{array}{l}3 . E-03 \\
4 . E-04 \\
6 . E-05 \\
7 . E-05 \\
2 . E-03 \\
4 . f-04 \\
7 . E-05 \\
4 . E-05 \\
\text { 2.E-05 }\end{array}$ & $\begin{array}{l}9 . E-07 \\
1 . E-07 \\
1 . E-08 \\
1 . E-09 \\
2 . E-07 \\
7 . E-08 \\
5 . E-09 \\
1 . E-08 \\
1 . E-10\end{array}$ & $\begin{array}{l}\text { 1.E-06 } \\
1 . E-07 \\
1 . E-08 \\
\text { 2.E-09 } \\
\text { 5.E-07 } \\
\text { 5.E-08 } \\
\text { 4.E-09 } \\
\text { 7.E-09 } \\
1 . E-10\end{array}$ & $\begin{array}{l}1 . E-06 \\
1 . E-07 \\
9 . E-09 \\
2 . E-09 \\
4 . E-07 \\
4 . E-08 \\
3 . E-09 \\
6 . E-09 \\
2 . E-11\end{array}$ \\
\hline $\begin{array}{l}\text { I } r-182 \\
\text { I } r-184 \\
\text { I } r-185 \\
\text { I } r-186 \\
\text { I } r-187 \\
\text { I } r-188 \\
\text { I } r-189 \\
\text { I } r-190 \mathrm{~m} / 3 \\
\text { I } r-190 \\
\text { I } r-192 \mathrm{~m} \\
\text { I } r-192\end{array}$ & $\begin{array}{l}1 . E-02 \\
1 . E-02 \\
1 . E-02 \\
1 . E-02 \\
1 . E-02 \\
1 . E-02 \\
1 . E-02 \\
1 . E-02 \\
1 . E-02 \\
1 . E-02 \\
1 . E-02\end{array}$ & $\begin{array}{l}1 . E-03 \\
2 . E-04 \\
1 . E-04 \\
6 . E-05 \\
3 . E-04 \\
5 . E-05 \\
1 . E-04 \\
5 . E-03 \\
3 . E-05 \\
9 . E-05 \\
3 . E-05\end{array}$ & $\begin{array}{l}3 . E-07 \\
6 . E-08 \\
3 . E-08 \\
2 . E-08 \\
7 . R-08 \\
1 . E-08 \\
1 . E-08 \\
5 . E-07 \\
2 . E-09 \\
2 . E-10 \\
6 . E-10\end{array}$ & $\begin{array}{l}\text { 4.E-07 } \\
\text { 7.E-08 } \\
\text { 3.E-08 } \\
\text { 1.E-08 } \\
\text { 7.E-08 } \\
\text { 8.E-09 } \\
9 . E-09 \\
5 . E-07 \\
2 . E-09 \\
2 . E-10 \\
9 . E-10\end{array}$ & $\begin{array}{l}3 . E-07 \\
6 . E-08 \\
2 . E-08 \\
1 . E-08 \\
6 . E-08 \\
8 . E-09 \\
9 . E-09 \\
5 . E-07 \\
2 . E-09 \\
4 . E-11 \\
5 . E-10\end{array}$ \\
\hline
\end{tabular}




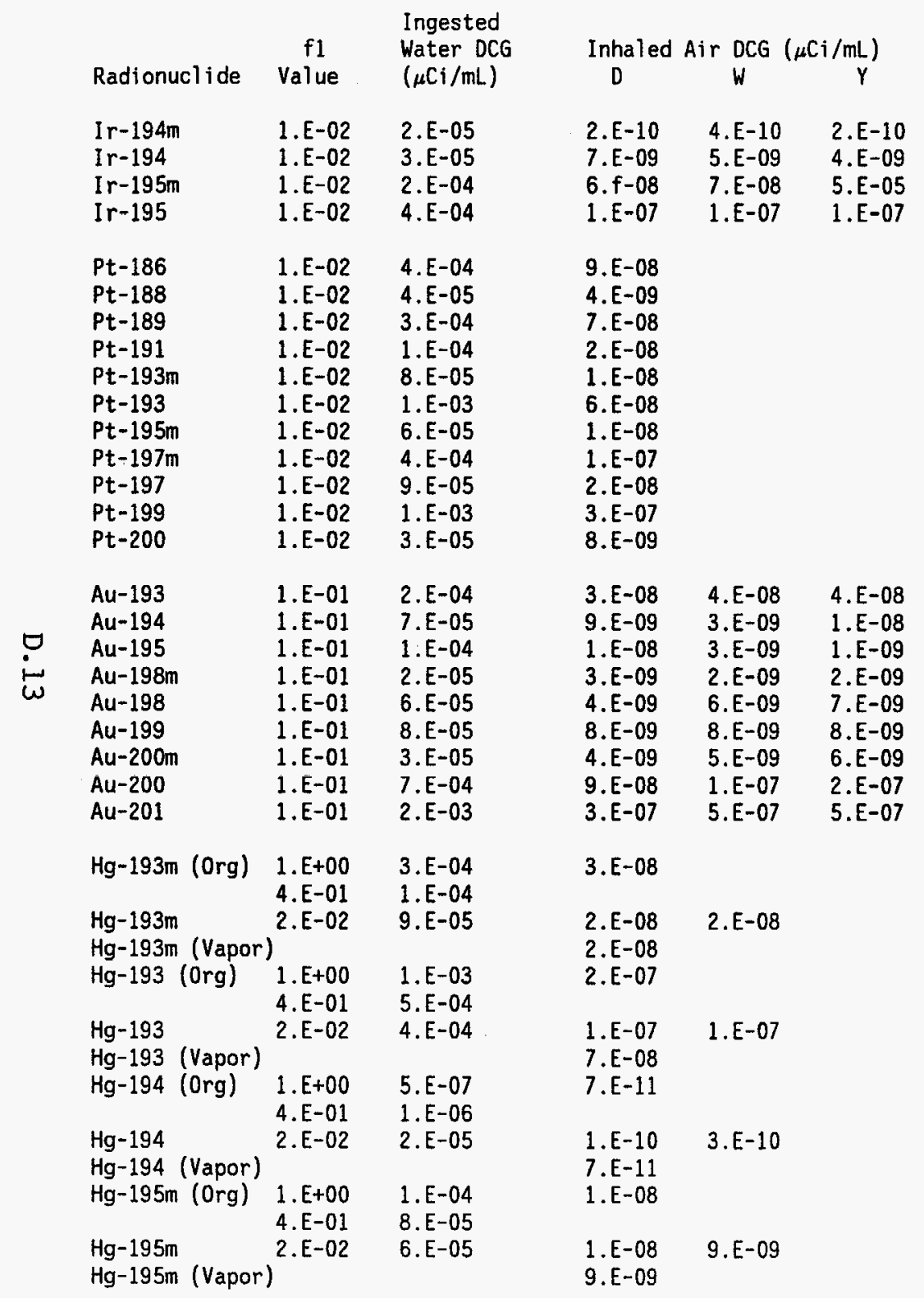

\begin{tabular}{|c|c|c|c|c|}
\hline Radionuclide & $\begin{array}{c}\text { fl } \\
\text { Value }\end{array}$ & $\begin{array}{l}\text { Ingested } \\
\text { Water } D C G \\
(\mu \mathrm{Ci} / \mathrm{mL})\end{array}$ & $\begin{array}{c}\text { Inhaled } \\
D\end{array}$ & $\underset{W}{\operatorname{Air}} \underset{W}{\operatorname{DCG}}(\mu \mathrm{Ci} / \mathrm{mL})$ \\
\hline $\mathrm{Hg}-195$ (0rg) & $\begin{array}{l}\text { 1. } E+00 \\
\text { 4. } E-01\end{array}$ & $\begin{array}{l}\text { 1.E-03 } \\
\text { 5.E-04 }\end{array}$ & 1.E-07 & \\
\hline $\begin{array}{l}\mathrm{Hg}-195 \\
\mathrm{Hg}-195 \text { (Vapor) }\end{array}$ & 2. $E-02$ & 4.E-04 & $\begin{array}{l}\text { 8.E-08 } \\
\text { 7.E-08 }\end{array}$ & 8.E-08 \\
\hline $\mathrm{Hg}-197 \mathrm{~m}(\mathrm{Org})$ & $\begin{array}{l}1 . E+00 \\
4 \cdot E-01\end{array}$ & $\begin{array}{l}\text { 2. E-04 } \\
1 . E-04\end{array}$ & 2. $E-08$ & \\
\hline $\begin{array}{l}\mathrm{Hg}-197 \mathrm{~m} \\
\mathrm{Hg}-197 \mathrm{~m} \text { (Vapor) }\end{array}$ & )$^{2 . E-02}$ & 8.E-05 & $\begin{array}{l}\text { 2. } E-08 \\
1 . f-08\end{array}$ & 1.E-08 \\
\hline $\mathrm{kg}-197$ (Org) & $\begin{array}{l}\text { 1. } E+00 \\
\text { 4. } E-01\end{array}$ & $\begin{array}{l}\text { 3. E-04 } \\
\text { 2.E-04 }\end{array}$ & 3.E-08 & \\
\hline $\begin{array}{l}\mathrm{Hg}-197 \\
\mathrm{Hg}-197 \text { (Vapor) }\end{array}$ & 2. E-02 & 1.E-04 & $\begin{array}{l}\text { 3.E-08 } \\
\text { 2.E-07 }\end{array}$ & 2.E-08 \\
\hline $\mathrm{Hg}-199 \mathrm{~m}(\mathrm{Org})$ & $\begin{array}{l}1 . E+00 \\
4 . E-01\end{array}$ & $\begin{array}{l}3 . E-03 \\
2 . E-03\end{array}$ & 4.E-07 & \\
\hline $\begin{array}{l}\mathrm{Hg}-199 \mathrm{~m} \\
\mathrm{Hg}-199 \mathrm{~m} \text { (Vapor) }\end{array}$ & $2 \cdot E-02$ & 2.E-03 & $\begin{array}{l}\text { 4.E-07 } \\
\text { 2.E-07 }\end{array}$ & 4.E-07 \\
\hline $\mathrm{Hg}-203(\mathrm{Org})$ & $\begin{array}{l}\text { 1. E+00 } \\
\text { 4. E-01 }\end{array}$ & $\begin{array}{l}\text { 1.E-05 } \\
\text { 2.E-05 }\end{array}$ & 2.E-09 & \\
\hline $\begin{array}{l}\mathrm{Hg}-203 \\
\mathrm{Hg}-203 \text { (Vapor) }\end{array}$ & 2. E-02 & 7.E-05 & $\begin{array}{l}\text { 3.E-09 } \\
\text { 2.E-09 }\end{array}$ & 3.E-09 \\
\hline $\begin{array}{l}\text { Tl-194m } \\
\text { Tl-194 } \\
\text { Tl-195 } \\
\text { Tl-197 } \\
\text { Tl-198m } \\
\text { Tl-198 } \\
\text { Tl-199 } \\
\text { Tl-200/3 } \\
\text { Tl-201 } \\
\text { Tl-202 } \\
\text { Tl-204 }\end{array}$ & $\begin{array}{l}1 . E+00 \\
1 . E+00 \\
1 . E+00 \\
1 . E+00 \\
1 . E+00 \\
1 . E+00 \\
1 . E+00 \\
1 . E+00 \\
1 . E+00 \\
1 . E+00 \\
1 . E+00\end{array}$ & $\begin{array}{l}2 . E-03 \\
7 . E-03 \\
2 . E-03 \\
2 . E-03 \\
8 . E-04 \\
5 . E-04 \\
2 . E-03 \\
2 . E-04 \\
5 . E-04 \\
9 . E-05 \\
4 . E-05\end{array}$ & $\begin{array}{l}4 . E-07 \\
1 . E-06 \\
3 . E-07 \\
3 . E-07 \\
1 . E-07 \\
7 . E-08 \\
2 . E-07 \\
3 . E-08 \\
5 . E-08 \\
1 . E-08 \\
5 . E-09\end{array}$ & \\
\hline $\begin{array}{l}\mathrm{Pb}-195 \mathrm{~m} \\
\mathrm{~Pb}-198 \\
\mathrm{~Pb}-199 \\
\mathrm{~Pb}-200 \\
\mathrm{~Pb}-201 \\
\mathrm{~Pb}-202 \mathrm{~m} \\
\mathrm{~Pb}-202 \\
\mathrm{~Pb}-203 \\
\mathrm{~Pb}-205 \\
\mathrm{~Pb}-209\end{array}$ & $\begin{array}{l}2 . E-01 \\
2 . E-01 \\
2 . E-01 \\
2 . E-01 \\
2 . E-01 \\
2 . E-01 \\
2 . E-01 \\
2 . E-01 \\
2 . E-01 \\
2 . E-01\end{array}$ & $\begin{array}{l}2 . E-03 \\
9 . E-04 \\
6 . E-04 \\
9 . E-05 \\
2 . E-04 \\
2 . E-04 \\
4 . E-06 \\
1 . E-04 \\
9 . E-05 \\
7 . E-04\end{array}$ & $\begin{array}{l}5 . E-07 \\
2 . E-07 \\
2 . E-07 \\
1 . E-08 \\
5 . E-08 \\
7 . E-08 \\
1 . E-10 \\
2 . E-08 \\
3 . E-09 \\
1 . E-07\end{array}$ & \\
\hline
\end{tabular}




\begin{tabular}{|c|c|c|c|c|c|c|c|c|c|c|c|}
\hline Radionucl ide & $\begin{array}{c}f 1 \\
\text { Value }\end{array}$ & $\begin{array}{l}\text { Ingested } \\
\text { Water DCG } \\
(\mu \mathrm{Ci} / \mathrm{mL})\end{array}$ & $\begin{array}{c}\text { Inhaled } \\
D\end{array}$ & $\underset{W}{\text { DCG }}($ & ${ }_{\mu \mathrm{Ci} / \mathrm{mL})}$ & Radionucl ide & $\begin{array}{c}\text { f1 } \\
\text { value }\end{array}$ & $\begin{array}{l}\text { Ingested } \\
\text { Water DCG } \\
(\mu \mathrm{Ci} / \mathrm{mL})\end{array}$ & $\begin{array}{c}\text { Inhaled } \\
D\end{array}$ & Air $\underset{W}{D C G}($ & Ci/mL) \\
\hline$P b-210$ & 2.E-01 & 3.E-08 & 9. E-13 & & & Th-226 & 2.E-04 & 1.E-04 & & 4. E-10 & $3 . E-10$ \\
\hline $\mathrm{Pb}-211$ & 2. $E-01$ & 3. E-04 & 1.E-09 & & & Th-227 & 2.E-04 & 4.E-06 & & 8.E-13 & 7.E-13 \\
\hline $\mathrm{Pb}-212$ & 2.E-01 & 3.E-06 & $8 \cdot E-11$ & & & Th-228 & 2.E-04 & 4.E-07 & & 5. E-14 & 4. $E-14$ \\
\hline \multirow{2}{*}{$\mathrm{Pb}-214$} & 2.E-01 & 2.E-04 & 2.E-09 & & & Th-229 & 2. E-04 & 4.E-08 & & 6. E-15 & 7.E-15 \\
\hline & & & & & & Th-230 & 2.E-04 & 3.E-07 & & 4.E-14 & $5 . E-14$ \\
\hline$B i-200$ & 5.E-02 & 8. E-04 & 2.E-07 & 2.E-07 & & Th-231 & 2.E-04 & 1.E-04 & & 1.E-08 & 2.E-08 \\
\hline $\mathrm{Bi}-201$ & 5.E-02 & 3.E-04 & 7.E-08 & 9. E-08 & & Th-232 & 2.E-04 & 5.E-08 & & 7.E-15 & 1.E-14 \\
\hline$B i-202$ & 5. $\mathrm{E}-02$ & 4. E-04 & 1.E-07 & 2. E- 07 & & Th-234 & 2.E-04 & 1.E-05 & & 5. E-10 & 4.E-10 \\
\hline$B i-203$ & 5.E-02 & 7.E-05 & 2.E-08 & 1. E-08 & & Th-Natural & 2.E-04 & 5.E-08 & & 7.E-15 & $1 . E-14$ \\
\hline$B i-205$ & 5.E-02 & 4. E- 05 & 6.E-09 & 3.E-09 & & & & & & & \\
\hline$B i-206$ & 5. E-02 & 2. E-05 & 3.E-09 & 2.E-09 & & $\mathrm{Pa}-227$ & 1.E-03 & 1.E-04 & & 3.E-10 & 2. E-10 \\
\hline$B i-207$ & 5.E-02 & 3.E-05 & 4.E-09 & 8.E-10 & & $\mathrm{Pa}-228$ & 1.E-03 & 3.E-05 & & 5.E-11 & 3.E-11 \\
\hline$B i-210 m$ & 5.E-02 & 2. E-06 & 1.E-11 & 2. $E-12$ & & $\mathrm{~Pa}-230$ & 1.E-03 & 2.E-05 & & 1.E-11 & $8 . E-12$ \\
\hline$B i-210$ & 5.E-02 & 2.E-05 & 9.E-10 & 6.E-11 & & $\mathrm{Pa}-231$ & 1.E-03 & 1.E-08 & & $9 . E-15$ & 1. $E-14$ \\
\hline$B i-211$ & 5. E-02 & 1. E-04 & 6.E-10 & 7.E-10 & & $\mathrm{Pa}-232$ & 1.E-03 & 4.E-05 & & 1. E-10 & 2.E-10 \\
\hline$B i-213$ & 5. E-02 & 2.E-04 & 7.E-10 & 8.E-10 & & $\mathrm{Pa}-233$ & 1.E-03 & 4.E-05 & & 2.E-09 & $1 . E-09$ \\
\hline $\mathrm{Bi}-214$ & 5.E-02 & 6.E-04 & 2.E-09 & 2.E-09 & & $\mathrm{Pa}-234 / 3$ & 1.E-03 & 7.E-05 & & 2.E-08 & 2.E-08 \\
\hline $\mathrm{Po}-203$ & 1. $\bar{E}-\overline{0} \mathbf{1}$ & T.E- $0 \overline{4}$ & 2.E-07 & 2. E-07 & & $U-230$ & 5.E-02 & 2.E-07 & 1.E-12 & 8.E-13 & \\
\hline Po-205 & 1.E-01 & 6.E-04 & 9. E-08 & 2.E-07 & & & 2.E-03 & 1.E-06 & & & $6 \cdot E-13$ \\
\hline Po-207 & 1.E-01 & 2.E-04 & 6.E-08 & 7.E-08 & & $U-231$ & 5.E-02 & 1.E-04 & 2.E-08 & 1.E-08 & \\
\hline \multirow[t]{2}{*}{ Po-210 } & 1.E-01 & 8.E-08 & 1.E-12 & 1.E-12 & & & 2. E- 03 & 1.E-04 & & & 1.E-08 \\
\hline & & & & & & $U-232$ & 5.E-02 & 1.E-07 & 1.E-12 & $9 . E-33$ & \\
\hline At -207 & 1. $E+00$ & 2.E-04 & 6.E-09 & 5. E-09 & & & 2.E-03 & 2.E-06 & & & 2.E-04 \\
\hline At-211 & 1. $E+00$ & 3.E-06 & 2.E-10 & 1. E-10 & & $U-233$ & 5. E-02 & 5.E-07 & 4.E-12 & 2. $E-12$ & \\
\hline \multirow{3}{*}{$\begin{array}{l}R n-210 \\
R n-211\end{array}$} & & 14 & 14 & 14 & 14 & $y-234$ & $\begin{array}{l}2 . E-03 \\
5 . E-02\end{array}$ & $\begin{array}{l}3 . E-00 \\
5 . E-07\end{array}$ & $4, E-12$ & $2 . E-12$ & Y.E-14 \\
\hline & & $\begin{array}{l}14 \\
15\end{array}$ & $\begin{array}{l}14 \\
15\end{array}$ & $\begin{array}{l}14 \\
15\end{array}$ & $\begin{array}{l}14 \\
15\end{array}$ & $0-<34$ & $\begin{array}{l}5 . E-0 L \\
2 . E-03\end{array}$ & $\begin{array}{l}5 . E-0 / \\
5 . E-06\end{array}$ & $4 . E-12$ & & 9.E-14 \\
\hline & & & & & & $U-235$ & 5.E-02 & $6 . E-07$ & $5 . \varepsilon-12$ & 2.E-12 & \\
\hline \multirow{2}{*}{$\begin{array}{l}\mathrm{rr}-222 \\
\mathrm{Fr}-223\end{array}$} & 1. $E+00$ & 6.E-05 & 1.E-09 & & & & 2.E-03 & 5.E-06 & & & 1. E-13 \\
\hline & 1. $E+00$ & 2. E- -05 & 2. E-09 & & & U-236 & $\begin{array}{l}\text { 5.E-02 } \\
\text { 2. E-03 }\end{array}$ & $\begin{array}{l}\text { 5.E-07 } \\
6 . E-06\end{array}$ & 5. E-12 & 2.E-12 & 1.E-13 \\
\hline $\begin{array}{l}\mathrm{Ra}-223 \\
\mathrm{Ra}-224\end{array}$ & $\begin{array}{l}\text { 2. E-01 } \\
\text { 2 E-OI }\end{array}$ & $\begin{array}{l}\text { 3. E-07 } \\
\text { 4. F-07 }\end{array}$ & & 2.E-12 & & U-237 & 5. E-02 & $\begin{array}{l}\text { 5.E-05 } \\
\text { 5. }-0.05\end{array}$ & $6 . E-09$ & 4.E-09 & \\
\hline $\begin{array}{l}R a-224 \\
R a-225\end{array}$ & $\begin{array}{l}\text { 2. } E-01 \\
\text { 2.E-01 }\end{array}$ & $\begin{array}{l}\text { 4. E-07 } \\
\text { 4. E-07 }\end{array}$ & & $\begin{array}{l}\text { 4.E-12 } \\
\text { 2.E-12 }\end{array}$ & & & $\begin{array}{l}\text { 2.E-03 } \\
\text { 5.F-0? }\end{array}$ & $\begin{array}{l}\text { 5.E-05 } \\
6 . F-07\end{array}$ & $5 F-12$ & $2 F-12$ & 4.E-09 \\
\hline $\mathrm{Ra}-225$ & 2.E-01 & $\begin{array}{l}4 . E-07 \\
1 . E-07\end{array}$ & & 2.E-12 & & $U-238$ & 5.E-02 & 6.E-07 & $5 . E-12$ & $2 . E-12$ & \\
\hline $\mathrm{Ra}-226$ & 2.E-01 & 1.E-07 & & 1.E-12 & & & 2. E-03 & $6 . E-06$ & & & 1.E-13 \\
\hline \multirow{3}{*}{$\begin{array}{l}\mathrm{Ra}-221 \\
\mathrm{Ra}-228\end{array}$} & 2.E-01 & $6 . E-04$ & & 4.E-08 & & $U-239 / 3$ & 5.E-02 & 2.E-03 & 4.E-07 & 4.E-07 & \\
\hline & 2.E-01 & 1.E-07 & & 3. $\mathrm{E}-12$ & & & 2. E- 03 & 2. E-03 & & & 4.E-07 \\
\hline & & & & & & $U-240$ & 5.E-02 & $3 . E-05$ & 9.E-09 & 7.E-09 & \\
\hline$A C-224$ & 1.E-03 & 5.E-05 & 9.E-11 & 1. E-10 & 1. E-10 & & 2. $\mathrm{E}-03$ & 3.E-05 & & & 6.E-09 \\
\hline Ac- -225 & 1.E-03 & 1. E-06 & 1. E-12 & 2. $E-12$ & 1. $E-12$ & U-Natural & 5.E-02 & $6 . E-07$ & $5 . E-12$ & 2.E-12 & \\
\hline$A C-226$ & 1.E-03 & 3.E-06 & 9.E-12 & $1 . E-11$ & $1 . E-11$ & & 2. E- -03 & 6. $\mathrm{E}-06$ & & & 1. E-13 \\
\hline$A C-227$ & 1.E-03 & 1.E-08 & 2.E-15 & 7.E-15 & 1. E-14 & & & & & & \\
\hline AC-228 & 1.E-03 & 6.E-05 & 4.E-11 & 1. $E-10$ & 1.E-10 & $\mathrm{Np}-232$ & 1. $E-03 / 6$ & 6. E- $-03 / 7$ & & 1.E-08/8 & \\
\hline
\end{tabular}




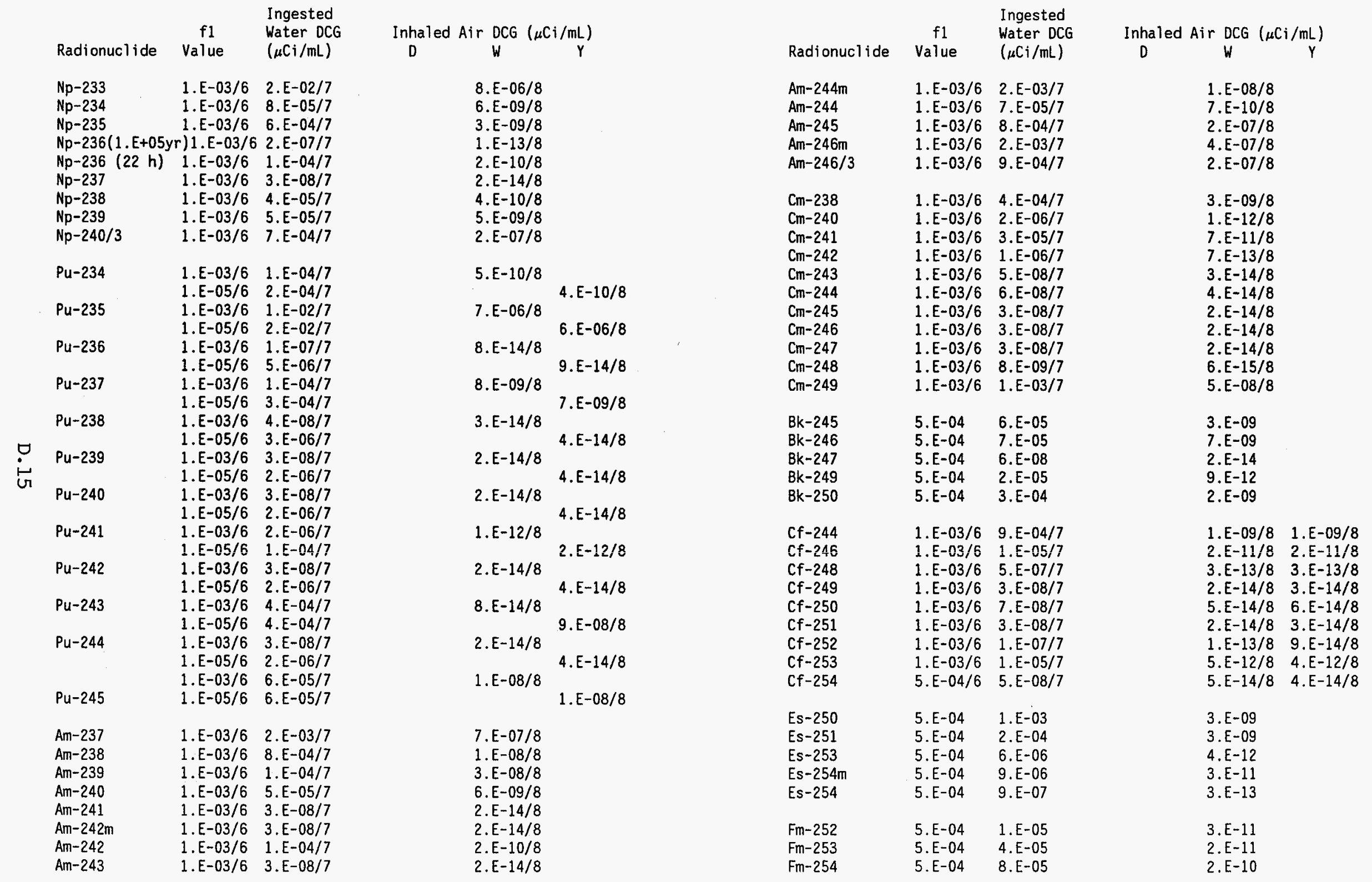




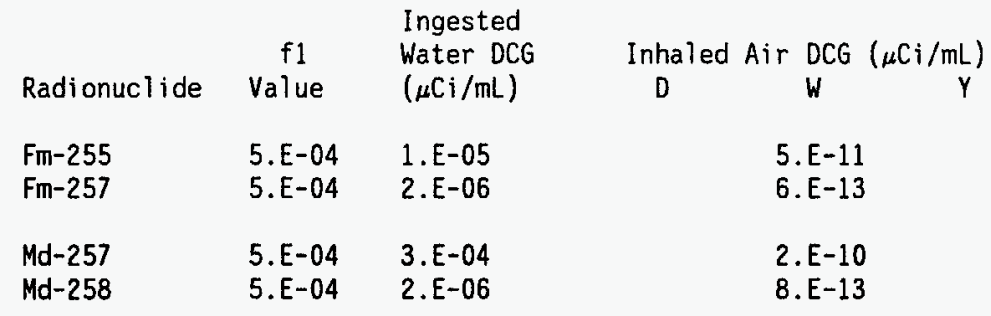

11 A dash indicates no values given for this data category.

12 The inhalation DCG values allow for an additional $50 \%$ absorption through the skin, as described in ICRP Publication No. 30: "Limits for Intakes of Radionuclides by Workers." for elemental tritium, the lung dose equivalent is used as the basis for the DCG value shown.

$/ 3$ For the radionuclide shown, the DCG for external exposure from immersion in a contaminated plume ( 1 isted in figure III-2) is more restrictive.

14 DCGs for Rn-220 are being assessed by DOE. Unti 1 the review has been completed and new values issued, the value of $3 . \mathrm{E}-09 \mathrm{micro} \mathrm{Ci} / \mathrm{mL}$ given in figure III-3 shall be used.

/5 DCGs for Rn-222 are being assessed by DOE. Until the review has been completed and new values issued, the value of $3 . \mathrm{E}-09 \mathrm{micro} \mathrm{Ci} / \mathrm{mL}$ given in Figure III-3 shall be used for Rn-222 releases from DOE facilities. in Figure III-3 shall be used for Rn-222 releases from DOE facilities.
In addition, the requirements of Chapter IV, Sections $4 b, 6 b$, and $6 d$, shall be used when they are applicable.

/6 ICRP Publication No. 48: "The Metabolism of Plutonium and Related Elements."

17 Based on the listed $f 1$ value. It is assumed that individual organ doses, except for the gastrointestinal tract, change in proportion to f1 for all organs, including the "Remainder." Gastrointestinal doses are unchanged because very little material is absorbed in the upper portions of the tract.

18 It is assumed that the effective dose equivalents are unchanged even though the fl values have changed. This is because the contribution to organ dose from inhalation is dependent mainily on transfer from lung to blood when $f 1$ values are small. Also the gastrointestinal tract dose would be unchanged because the fraction of activity passing through the tract is $(1.0-f 1)$ 
Figure III-2

Alternative Absorption factors and Lung Retention Classes for Specific Compounds

\begin{tabular}{|c|c|c|c|c|c|c|c|c|c|}
\hline $\begin{array}{l}\text { El ement/ } \\
\text { Symbol }\end{array}$ & $\begin{array}{l}\text { Atomic } \\
\text { Number }\end{array}$ & Compound & f1 & $\begin{array}{l}\text { Lung } \\
\text { Retention Class }\end{array}$ & $\begin{array}{l}\text { El ement/ } \\
\text { Symbol }\end{array}$ & $\begin{array}{l}\text { Atomic } \\
\text { Number }\end{array}$ & Compound & f1 & $\begin{array}{l}\text { Lung } \\
\text { Retention Class }\end{array}$ \\
\hline $\begin{array}{l}\text { Actini um/ } \\
\text { Ac }\end{array}$ & 89 & $\begin{array}{l}\text { Oxides, hydroxides } \\
\text { Halides, nitrates } \\
\text { All others }\end{array}$ & $\begin{array}{l}1 . E-03 \\
1 . E-03 \\
1 . E-03\end{array}$ & $\begin{array}{l}Y \\
W \\
D\end{array}$ & $\begin{array}{l}\text { Cadmi um/ } \\
\text { Cd }\end{array}$ & 48 & $\begin{array}{l}\text { Oxides, hydroxides } \\
\text { Sulphates, halides } \\
\text { All others }\end{array}$ & $\begin{array}{l}5 . E-02 \\
5 . E-02 \\
5 . E-02\end{array}$ & $\begin{array}{l}Y \\
W \\
D\end{array}$ \\
\hline \multirow[t]{2}{*}{$\begin{array}{l}\text { Al uminum/ } \\
\text { Al }\end{array}$} & \multirow[t]{2}{*}{13} & \multirow{2}{*}{$\begin{array}{l}\text { Oxides, hydroxides, } \\
\text { carbides, halides, } \\
\text { nitrates, elemental } \\
\text { form } \\
\text { A11 0thers }\end{array}$} & 1.E-02 & w & $\begin{array}{l}\text { Calcium/ } \\
\mathrm{Ca}\end{array}$ & 20 & All Forms & 3.E-01 & w \\
\hline & & & 1.E-02 & D & $\begin{array}{l}\text { Californium/ } \\
\text { Cf }\end{array}$ & 98 & $\begin{array}{l}\text { 0xides, hydroxides } \\
\text { All others }\end{array}$ & $\begin{array}{l}\text { 1.E-03 } \\
1 . E-03\end{array}$ & $\stackrel{Y}{Y}$ \\
\hline $\begin{array}{l}\text { Americium/ } \\
\text { Am }\end{array}$ & 95 & All forms & 1.E-03 & w & $\begin{array}{l}\text { Carbon/ } \\
\text { C }\end{array}$ & 6 & $\begin{array}{l}\text { Oxides } \\
\text { Organic (11C) } \\
\text { Organic (14C) }\end{array}$ & $\begin{array}{l}\text { /1 } \\
1 . E-02 \\
7 . E-05\end{array}$ & $\begin{array}{l}D \\
W \\
W\end{array}$ \\
\hline $\begin{array}{l}\text { Antimony/ } \\
\text { Sb }\end{array}$ & 51 & $\begin{array}{l}\text { Oxides, hydroxides, } \\
\text { hal ides, sulphides, } \\
\text { sulphates, nitrates } \\
\text { All others }\end{array}$ & 1.E-02 & w & $\begin{array}{l}\text { Cerium/ } \\
\text { Ce }\end{array}$ & 58 & $\begin{array}{l}\text { Oxides, hydroxides, } \\
\text { fluorides } \\
\text { All others }\end{array}$ & $\begin{array}{l}3 . E-04 \\
3 . E-04\end{array}$ & $\begin{array}{l}Y \\
W\end{array}$ \\
\hline $\begin{array}{l}\text { Arsenic/ } \\
\text { As }\end{array}$ & 33 & All Forms & 5. E-01 & $w$ & $\begin{array}{l}\text { Cesium/ } \\
\text { Cs }\end{array}$ & 55 & All Forms & $1 . E+00$ & $D$ \\
\hline $\begin{array}{l}\text { Astatine/ } \\
\text { At }\end{array}$ & 85 & All (as a halide) & 1. E+00 & $\begin{array}{l}\text { W or } D \text {; dependent } \\
\text { upon associated } \\
\text { element }\end{array}$ & $\begin{array}{l}\text { Chlorine/ } \\
\mathrm{Cl}\end{array}$ & 17 & Chloride & $1 . E+00$ & $\begin{array}{l}W \text { or } D ; \text { dependent } \\
\text { upon associ ated } \\
\text { element }\end{array}$ \\
\hline $\begin{array}{l}\text { Barium/ } \\
\text { Ba }\end{array}$ & 56 & All Forms & 1.E-01 & $D$ & $\begin{array}{l}\text { Chromium/ } \\
\mathrm{Cr}\end{array}$ & 24 & $\begin{array}{l}\text { Oxides, hydroxides } \\
\text { Halides, nitrates } \\
\text { AlI Others }\end{array}$ & $\begin{array}{l}1 . E-01 \\
1 . E-01 \\
1 . E-01\end{array}$ & $\begin{array}{l}Y \\
W \\
D\end{array}$ \\
\hline $\begin{array}{l}\text { Berkel i um/ } \\
\text { Bk }\end{array}$ & 97 & A11 Forms & 5.E-04 & w & & & $\begin{array}{l}\text { Ingestion } / 2 \\
\text { Trivalent } 1 \\
\text { Hexavalent }\end{array}$ & $\begin{array}{l}\text { 1.E-02 } \\
1 . E-01\end{array}$ & \\
\hline $\begin{array}{l}\text { Beryllium/ } \\
\text { Be }\end{array}$ & 4 & $\begin{array}{l}\text { Oxides, halides, } \\
\text { nitrates } \\
\text { All others }\end{array}$ & $\begin{array}{l}5 . E-03 \\
5 . E-03\end{array}$ & $\begin{array}{l}Y \\
w\end{array}$ & $\begin{array}{l}\text { Cobalt/ } \\
\text { Co }\end{array}$ & 27 & $\begin{array}{l}\text { 0xides, hydroxides, } \\
\text { halides, nitrates } \\
\text { All others }\end{array}$ & $\begin{array}{l}5 . E-02 \\
5 . E-01\end{array}$ & $\begin{array}{l}Y \\
W\end{array}$ \\
\hline $\begin{array}{l}\text { Bismuth/ } \\
\mathrm{Bi}\end{array}$ & 83 & $\begin{array}{l}\text { All except nitrates } \\
\text { Nitrates }\end{array}$ & $\begin{array}{l}5 . E-02 \\
5 . E-02\end{array}$ & $\begin{array}{l}W \\
D\end{array}$ & & & Ingestion only & 3.E-01 & \\
\hline $\begin{array}{l}\text { Bromine/ } \\
\text { Br }\end{array}$ & 35 & Bromides & 1. $E+00$ & $\begin{array}{l}\text { W or } D \text {; dependent } \\
\text { upon associated } \\
\text { element }\end{array}$ & & & & & \\
\hline
\end{tabular}




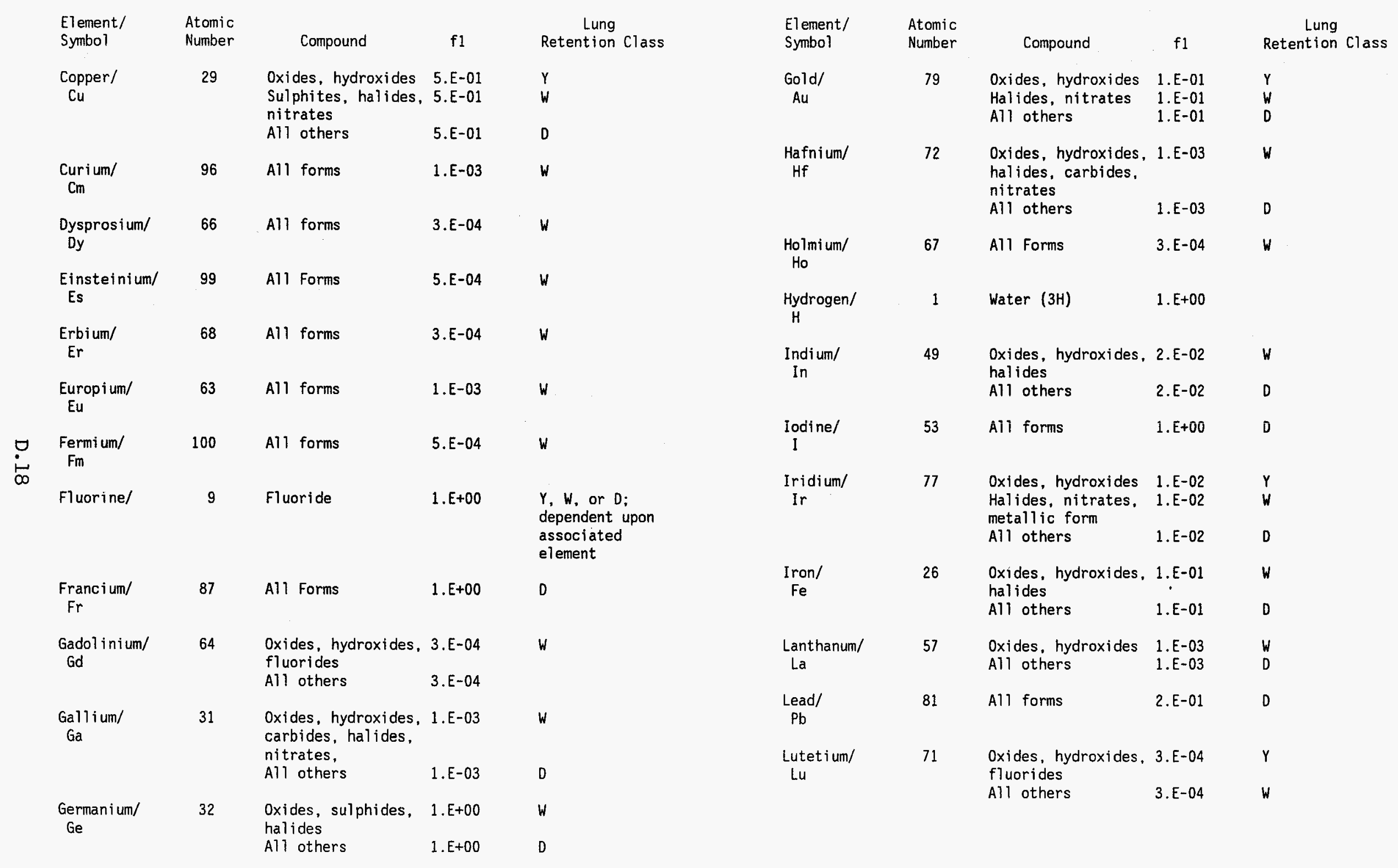




\begin{tabular}{|c|c|c|c|c|c|c|c|c|c|}
\hline $\begin{array}{l}\text { Element/ } \\
\text { Symbol }\end{array}$ & $\begin{array}{l}\text { Atomic } \\
\text { Number }\end{array}$ & Compound & f1 & $\begin{array}{c}\text { Lung } \\
\text { Retention Class }\end{array}$ & $\begin{array}{l}\text { Element/ } \\
\text { Symbol }\end{array}$ & $\begin{array}{l}\text { Atomic } \\
\text { Number }\end{array}$ & Compound & f1 & $\begin{array}{l}\text { Lung } \\
\text { Retention Class }\end{array}$ \\
\hline $\begin{array}{l}\text { Magnesium/ } \\
\text { Mg }\end{array}$ & 12 & $\begin{array}{l}\text { 0xides, hydroxides, } \\
\text { carbides, halides, } \\
\text { nitrates }\end{array}$ & 5.E-01 & $W$ & $\begin{array}{l}\text { Osmium/ } \\
\text { Os }\end{array}$ & 76 & $\begin{array}{l}\text { Oxides, hydroxides } \\
\text { Hal ides, nitrates } \\
\text { All others }\end{array}$ & $\begin{array}{l}1 . E-02 \\
1 . E-02 \\
1 . E-02\end{array}$ & $\begin{array}{l}Y \\
W \\
D\end{array}$ \\
\hline $\begin{array}{l}\text { Manganese/ } \\
\text { Mn }\end{array}$ & 25 & $\begin{array}{l}\text { 0xides, hydroxides, } \\
\text { halides, nitrates } \\
\text { All others }\end{array}$ & $\begin{array}{l}\text { 5.E-01 } \\
1 . E-01\end{array}$ & $D$ & $\begin{array}{l}\text { Palladium/ } \\
\text { Pd }\end{array}$ & 46 & $\begin{array}{l}\text { Oxidés, hydroxides } \\
\text { Nitrates } \\
\text { All others }\end{array}$ & $\begin{array}{l}5 . E-03 \\
5 . E-03 \\
5 . E-03\end{array}$ & $\begin{array}{l}Y \\
W \\
D\end{array}$ \\
\hline $\begin{array}{l}\text { Mendelevium/ } \\
\text { Md }\end{array}$ & 101 & All others & $\begin{array}{l}\text { 1.E-01 } \\
\text { 5.E-04 }\end{array}$ & D & $\begin{array}{l}\text { Phosphorus/ } \\
\text { p }\end{array}$ & 15 & Phosphates & 8.E-01 & $\begin{array}{l}W \text { or } D \text {; dependent } \\
\text { upon associated } \\
\text { element }\end{array}$ \\
\hline \multirow[t]{3}{*}{$\begin{array}{l}\text { Mercury/ } \\
\mathrm{Hg}\end{array}$} & \multirow[t]{3}{*}{80} & $\begin{array}{l}\text { 0xides, hydroxides, } \\
\text { halides, nitrates, } \\
\text { sulphites }\end{array}$ & 2.E-02 & $W$ & $\begin{array}{l}\text { Platinum/ } \\
\text { Pt }\end{array}$ & 78 & A11 forms & 1.E-02 & 0 \\
\hline & & $\begin{array}{l}\text { Sulphates, el emental } \\
\text { form }\end{array}$ & $12 . E-02$ & $D$ & \multirow[t]{3}{*}{$\begin{array}{l}\text { Plutonium/ } \\
\mathrm{Pu}\end{array}$} & \multirow[t]{3}{*}{94} & $\begin{array}{l}\text { Oxides, hydroxides } \\
\text { Nitrates }\end{array}$ & 1.E-05 & $\begin{array}{l}Y \\
W\end{array}$ \\
\hline & & $\begin{array}{l}\text { Organic Forms } \\
\text { Vapor }\end{array}$ & 1. $E+00$ & $\begin{array}{l}D \\
D\end{array}$ & & & $\begin{array}{l}\text { A11 others } \\
\text { [Note: Use same } \\
\text { values for }\end{array}$ & $\begin{array}{l}\text { 1.E- }-04 \\
1 . E-03\end{array}$ & W \\
\hline \multirow[t]{3}{*}{$\begin{array}{l}\text { Molybdenum/ } \\
\text { Mo }\end{array}$} & \multirow[t]{3}{*}{42} & $\begin{array}{l}\text { Oxides, hydroxides, } \\
\text { MoS2 }\end{array}$ & 5.E-02 & Y & & & ingestion] & & \\
\hline & & All others & 8.E-01 & $D$ & $\begin{array}{l}\text { Polonium/ } \\
\text { Po }\end{array}$ & 84 & $\begin{array}{l}\text { Oxides, hydroxides, } \\
\text { nitrates }\end{array}$ & 1.E-01 & W \\
\hline & & $\begin{array}{l}\text { Ingestion } / 2 \\
\text { MoS2 } \\
\text { Al1 others }\end{array}$ & $\begin{array}{l}\text { 5.E-02 } \\
\text { 8.E-01 }\end{array}$ & & $\begin{array}{l}\text { Potassium/ } \\
\mathfrak{K}\end{array}$ & 19 & $\begin{array}{l}\text { A11 others } \\
\text { A11 forms }\end{array}$ & $\begin{array}{l}\text { 1. E-01 } \\
\text { 1. E+00 }\end{array}$ & $D$ \\
\hline $\begin{array}{l}\text { Neodymi um/ } \\
\text { Nd }\end{array}$ & 60 & $\begin{array}{l}\text { Oxides, hydroxides, } \\
\text { carbides, } \\
\text { fluorides } \\
\text { All others }\end{array}$ & $\begin{array}{l}3 . E-04 \\
3 . E-04\end{array}$ & $Y$ & $\begin{array}{l}\text { Praesodymium/ } \\
\mathrm{Pr}\end{array}$ & 59 & $\begin{array}{l}\text { Oxides, hydroxides, } \\
\text { carbides, } \\
\text { fluorides } \\
\text { All others }\end{array}$ & $\begin{array}{l}3 . E-04 \\
3 . E-04\end{array}$ & $W$ \\
\hline $\begin{array}{l}\text { Neptunium/ } \\
\mathrm{Np}\end{array}$ & 93 & A11 forms & 1.E-03 & $W$ & \multirow[t]{2}{*}{$\begin{array}{l}\text { Promethlum/ } \\
\text { Pm }\end{array}$} & \multirow[t]{2}{*}{61} & $\begin{array}{l}\text { 0xides, hydroxides, } \\
\text { carbides, }\end{array}$ & 3.E-04 & $Y$ \\
\hline $\begin{array}{l}\mathrm{Nickel/} \\
\mathrm{Ni}\end{array}$ & 28 & $\begin{array}{l}\text { Oxides, hydroxides } \\
\text { A11 others (vapor) }\end{array}$ & 5.E-01 & $\begin{array}{l}W \\
D\end{array}$ & & & $\begin{array}{l}\text { fluorides } \\
\text { All others }\end{array}$ & 3.E-04 & W \\
\hline \multirow[t]{2}{*}{$\begin{array}{l}\mathrm{Ni} \text { obi um/ } \\
\mathrm{Nb}\end{array}$} & \multirow[t]{2}{*}{41} & $\begin{array}{l}\text { Oxides, hydroxides } \\
\text { A11 others }\end{array}$ & $\begin{array}{l}1 . E-02 \\
1 . E-02\end{array}$ & $\begin{array}{l}Y \\
W\end{array}$ & $\begin{array}{l}\text { Protactinium/ } \\
\mathrm{Pa}\end{array}$ & 91 & $\begin{array}{l}\text { Oxides, hydroxides } \\
\text { All others }\end{array}$ & $\begin{array}{l}\text { 1.E-03 } \\
1 . E-03\end{array}$ & W \\
\hline & & & & & $\begin{array}{l}\text { Radium/ } \\
\text { Ra }\end{array}$ & 88 & Al1 forms & 2.E-01 & $w$ \\
\hline
\end{tabular}




\begin{tabular}{|c|c|c|c|c|c|c|c|c|c|}
\hline $\begin{array}{l}\text { Element/ } \\
\text { Symbol }\end{array}$ & $\begin{array}{l}\text { Atomic } \\
\text { Number }\end{array}$ & Compound & fl & $\begin{array}{c}\text { Lung } \\
\text { Retention Class }\end{array}$ & $\begin{array}{l}\text { El ement/ } \\
\text { Symbol }\end{array}$ & $\begin{array}{l}\text { Atomic } \\
\text { Number }\end{array}$ & Compound & f1 & $\begin{array}{c}\text { Lung } \\
\text { Retention Class }\end{array}$ \\
\hline $\begin{array}{l}\text { Rheni um/ } \\
\text { Re }\end{array}$ & 75 & $\begin{array}{l}\text { Oxides, hydroxides, } \\
\text { halides, nitrates } \\
\text { All others }\end{array}$ & $\begin{array}{l}\text { 8.E-01 } \\
8 . E-01\end{array}$ & $\begin{array}{l}W \\
D\end{array}$ & $\begin{array}{l}\text { Sulfurf } \\
\text { S }\end{array}$ & 16 & Sulphates, sulphides & 1.E-01 & $\begin{array}{l}W \text { or } D \text {; dependent } \\
\text { upon associated } \\
\text { element }\end{array}$ \\
\hline $\begin{array}{l}\text { Rhodi um/ } \\
\text { Rh }\end{array}$ & 45 & $\begin{array}{l}\text { Oxides, hydroxides } \\
\text { Halides } \\
\text { All others }\end{array}$ & $\begin{array}{l}5 . E-02 \\
5 . E-02 \\
5 . E-02\end{array}$ & $\begin{array}{l}Y \\
W \\
D\end{array}$ & & & $\begin{array}{l}\text { All inorganic } \\
\text { Elemental form } \\
\text { Gases }\end{array}$ & $\begin{array}{l}\text { 8.E-01 } \\
1 . E-01 \\
1 . E+00\end{array}$ & $\begin{array}{l}W \\
D\end{array}$ \\
\hline $\begin{array}{l}\text { Rubidium/ } \\
\text { Rb }\end{array}$ & 37 & All forms & $1 . E+00$ & 0 & $\begin{array}{l}\text { Tantalum/ } \\
\text { Ta }\end{array}$ & 73 & $\begin{array}{l}\text { Oxides, hydroxides, } \\
\text { halides, carbides, } \\
\text { nitrates, nitrides } \\
\text { All others }\end{array}$ & $\begin{array}{l}\text { 1.E-03 } \\
\text { 1.E-03 }\end{array}$ & $Y$ \\
\hline $\begin{array}{l}\text { Rutheni um/ } \\
\text { Ru }\end{array}$ & 44 & $\begin{array}{l}\text { Oxides, hydroxides } \\
\text { Halides } \\
\text { All others }\end{array}$ & $\begin{array}{l}\text { 5.E-02 } \\
5 . E-02 \\
5 . E-02\end{array}$ & $\begin{array}{l}Y \\
W \\
D\end{array}$ & $\begin{array}{l}\text { Technetium/ } \\
\text { Tc }\end{array}$ & 43 & $\begin{array}{l}\text { Oxides, hydroxides, } \\
\text { halides, nitrates } \\
\text { All others }\end{array}$ & $\begin{array}{l}\text { 8.E-01 } \\
\text { 8.E-01 }\end{array}$ & $\begin{array}{l}W \\
D\end{array}$ \\
\hline $\begin{array}{l}\text { Samari um/ } \\
\text { Sm }\end{array}$ & 62 & All forms & 3.E-04 & W & $\begin{array}{l}\text { Tellurium/ } \\
\text { Te }\end{array}$ & 52 & $\begin{array}{l}\text { Oxides, hydroxides, } \\
\text { nitrates }\end{array}$ & 2.E-01 & $W$ \\
\hline $\begin{array}{l}\text { Scandium/ } \\
\text { Sc }\end{array}$ & 21 & Al! forms & 1.E-04 & $Y$ & & & All others & 2.E-01 & $\mathbf{D}$ \\
\hline $\begin{array}{l}\text { Selenium/ } \\
\text { Se }\end{array}$ & 34 & $\begin{array}{l}\text { 0xides, hydroxides, } \\
\text { carbides }\end{array}$ & 8.E-01 & W & $\begin{array}{l}\text { Terbium/ } \\
\text { Tb }\end{array}$ & 65 & All forms & 3.E-04 & $W$ \\
\hline & & $\begin{array}{l}\text { All others } \\
\text { Ingestion only }\end{array}$ & $\begin{array}{l}\text { 8.E-01 } \\
\text { 5.E-02 }\end{array}$ & $D$ & $\begin{array}{l}\text { Thalli ium/ } \\
\text { Tl }\end{array}$ & 81 & All forms & 1. $E+00$ & $D$ \\
\hline $\begin{array}{l}\text { Silicon/ } \\
\text { Si }\end{array}$ & 14 & $\begin{array}{l}\text { Ceramic forms } \\
\text { Oxides, hydroxides, } \\
\text { carbides, nitrates }\end{array}$ & $\begin{array}{l}1 . E-02 \\
1 . E-02\end{array}$ & $\begin{array}{l}Y \\
W\end{array}$ & $\begin{array}{l}\text { Thorium/ } \\
\text { Th }\end{array}$ & 90 & $\begin{array}{l}\text { Oxides, hydroxides } \\
\text { All others }\end{array}$ & $\begin{array}{l}\text { 2.E-04 } \\
\text { 2.E-04 }\end{array}$ & $\begin{array}{l}Y \\
W\end{array}$ \\
\hline & & A11 others & $1 . E-02$ & D & $\begin{array}{l}\text { Thul ium/ } \\
\text { Tm }\end{array}$ & 69 & All forms & 3.E-04 & $W$ \\
\hline $\begin{array}{l}\text { Silver/ } \\
\mathrm{Ag}\end{array}$ & 47 & $\begin{array}{l}\text { Oxides, hydroxides } \\
\text { Nitrates, sulphides } \\
\text { A11 others, } \\
\text { elemental form }\end{array}$ & $\begin{array}{l}5 . E-02 \\
5 . E-02 \\
5 . E-02\end{array}$ & $\begin{array}{l}Y \\
W \\
D\end{array}$ & $\begin{array}{c}\text { Tin/ } \\
\text { Sn }\end{array}$ & 50 & $\begin{array}{l}\text { 0xides, hydroxides, } \\
\text { halides, nitrates, } \\
\text { sulphides, } \\
\text { Sn3(PO4) } 4\end{array}$ & 2.E-02 & $W$ \\
\hline $\begin{array}{l}\text { Sodium/ } \\
\mathrm{Na}\end{array}$ & 11 & All Forms & $1 . E+\infty 0$ & D & & & All others & $2 . E-02$ & $D$ \\
\hline $\begin{array}{l}\text { Strontium/ } \\
\text { Sr }\end{array}$ & 38 & $\begin{array}{l}\text { SrTi03 } \\
\text { All others (soluble) }\end{array}$ & $\begin{array}{l}1 . E-02 \\
3 . E-01\end{array}$ & $\begin{array}{l}Y \\
D\end{array}$ & $\begin{array}{l}\text { Titanium/ } \\
\mathrm{Ti}\end{array}$ & 22 & $\begin{array}{l}\text { SrTi03 } \\
\text { Oxides, hydroxides, } \\
\text { carbides, halides, } \\
\text { nitrates } \\
\text { All others }\end{array}$ & $\begin{array}{l}\text { 1. E-02 } \\
\text { 1. E-02 } \\
\text { 1.E-02 }\end{array}$ & $\begin{array}{l}Y \\
W \\
D\end{array}$ \\
\hline
\end{tabular}




\begin{tabular}{|c|c|c|c|c|}
\hline $\begin{array}{l}\text { Element/ } \\
\text { Symbor }\end{array}$ & $\begin{array}{l}\text { Atomic } \\
\text { Number }\end{array}$ & Compound & f1 & $\begin{array}{c}\text { Lung } \\
\text { Retention Class }\end{array}$ \\
\hline Tungsten/ & 74 & $\begin{array}{l}\text { Ingestion/2 } \\
\text { Tungstic acid } \\
\text { All others }\end{array}$ & $\begin{array}{l}\text { 1.E-02 } \\
\text { 3.E-01 }\end{array}$ & \\
\hline \multirow[t]{2}{*}{$\begin{array}{l}\text { Uranium/ } \\
U\end{array}$} & 92 & $\begin{array}{l}\text { U02, U308 } \\
\text { U03, tetravalent } \\
\text { compounds }\end{array}$ & $\begin{array}{l}\text { 2.E-03 } \\
\text { 5.E-02 }\end{array}$ & $\begin{array}{l}Y \\
W\end{array}$ \\
\hline & & $\begin{array}{l}\text { UF6, uranyl } \\
\text { compounds }\end{array}$ & 5.E-02 & D \\
\hline \multirow[t]{2}{*}{$\underset{V}{\text { Vanadium/ }}$} & 23 & $\begin{array}{l}\text { Oxides, hydroxides, } \\
\text { carbides, halides }\end{array}$ & 1.E-02 & w \\
\hline & & All others & 1.E-02 & $D$ \\
\hline \multirow[t]{2}{*}{$\begin{array}{l}\text { Ytterbium/ } \\
\text { Yb }\end{array}$} & 70 & $\begin{array}{l}\text { Oxides, hydroxides, } \\
\text { fluorides }\end{array}$ & 3.E-04 & $\gamma$ \\
\hline & & All others & 3.E-04 & w \\
\hline$\underset{Y}{\text { Yttrium/ }}$ & 39 & $\begin{array}{l}\text { Oxides, hydroxides } \\
\text { All others }\end{array}$ & $\begin{array}{l}\text { 1.E-04 } \\
1 . E-04\end{array}$ & $\begin{array}{l}Y \\
W\end{array}$ \\
\hline $\operatorname{Zincl}_{\mathrm{Zn}}$ & 30 & All forms & 5.E-01 & $\gamma$ \\
\hline \multirow[t]{2}{*}{$\begin{array}{l}\text { Zirconium/ } \\
\text { Zr }\end{array}$} & 40 & $\begin{array}{l}\text { Carbides } \\
\text { Oxides, hydroxides, } \\
\text { halides, nitrates }\end{array}$ & $\begin{array}{l}\text { 2.E-03 } \\
2 . E-03\end{array}$ & $\begin{array}{l}Y \\
W\end{array}$ \\
\hline & & All others & 2.E-03 & D \\
\hline
\end{tabular}


Figure III-3

Derived Concentration Guides (DCGs) For Members of the Public from External Exposure During Immersion in a Contaminated Atmospheric Cloud

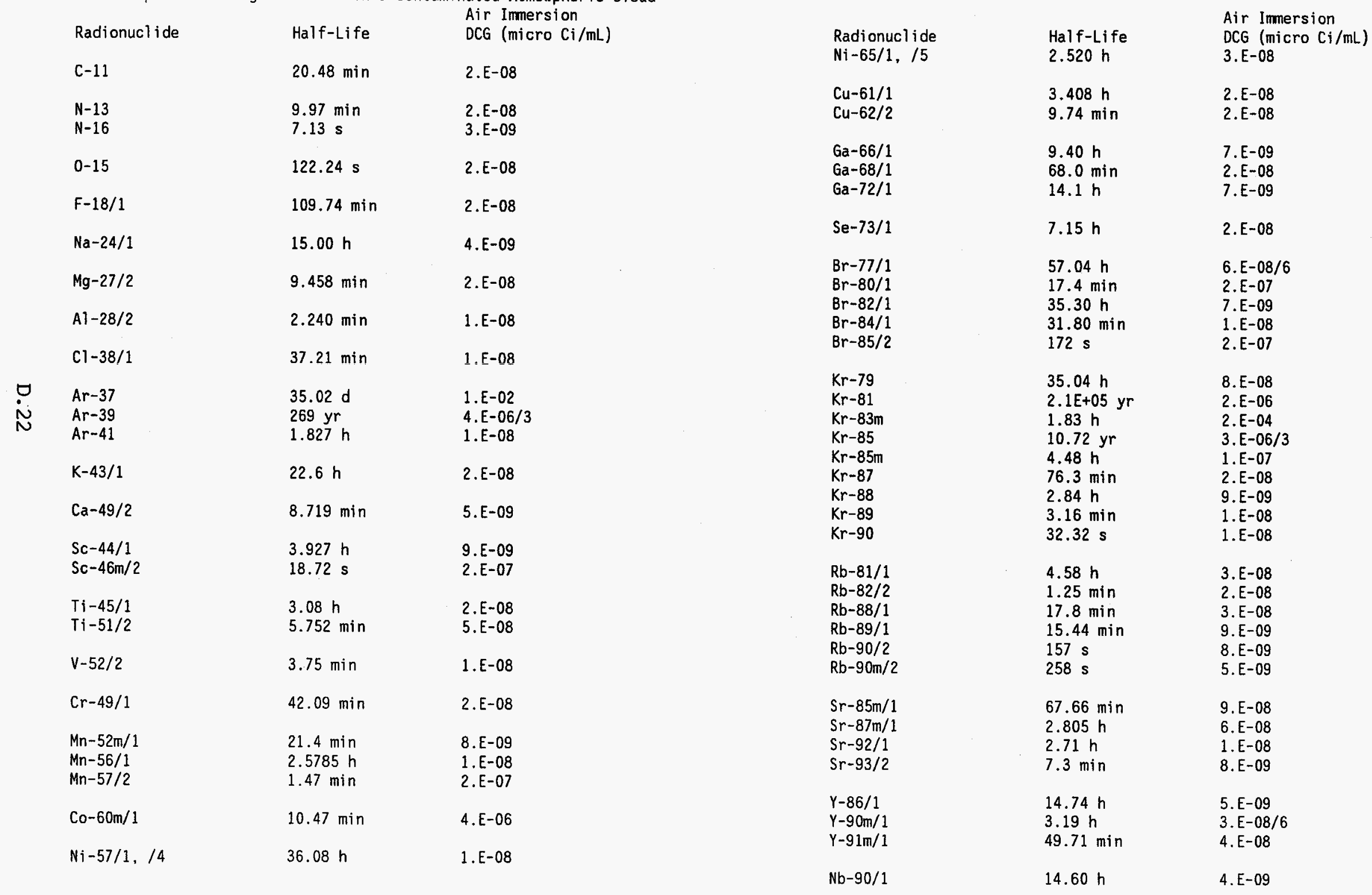




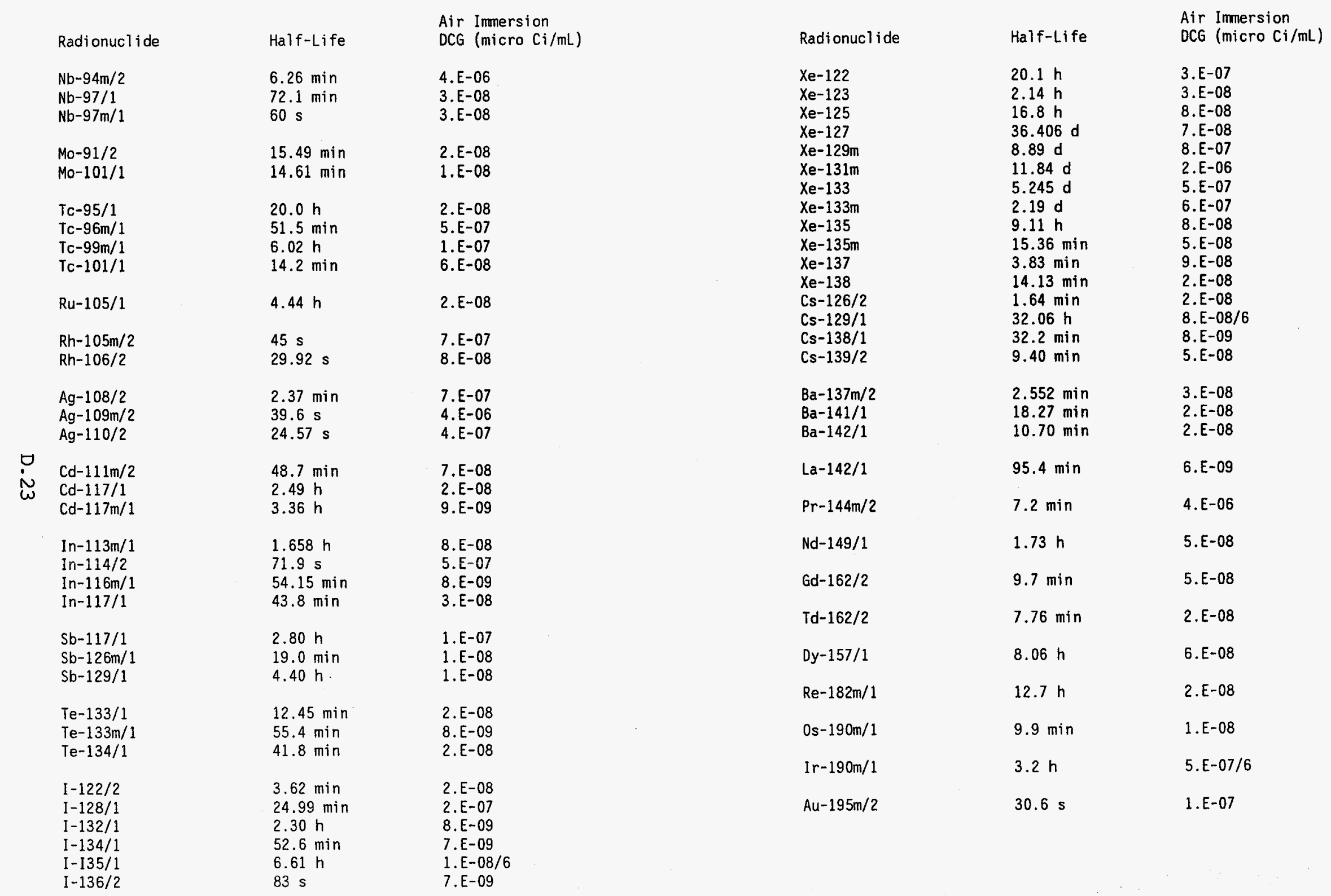




$\begin{array}{lll}\text { Radionuclide } & \text { Half-Life } & \begin{array}{l}\text { Air Imersion } \\ \text { OCG (micro Ci/mL) }\end{array} \\ \mathrm{Tl}-200 / 1 & 26.1 \mathrm{~h} & 1 . \mathrm{E}-08 \\ \mathrm{Tl}-207 / 2 & 4.77 \mathrm{~min} & 1 . \mathrm{E}-06 / 3 \\ \mathrm{Tl}-208 / 2 & 3.053 \mathrm{~min} & 5 . \mathrm{E}-09 \\ \mathrm{Tl}-209 / 2 & 2.20 \mathrm{~min} & 9 . \mathrm{E}-09 \\ \mathrm{Tl}-210 / 2 & 1.30 \mathrm{~min} & 7 . \mathrm{E}-09 \\ \mathrm{~Pb}-204 \mathrm{~m} / 2 & 66.9 \mathrm{~min} & 9 . \mathrm{E}-09 \\ \mathrm{Bi}-211 / 2 & 2.13 \mathrm{~min} & 4 . \mathrm{E}-07 \\ \mathrm{Po}-211 / 2 & 0.516 \mathrm{~s} & 2 . \mathrm{E}-06 \\ \mathrm{Rn}-220 / 7 & 55.61 \mathrm{~s} & 3 . \mathrm{E}-09 \\ \mathrm{Rn}-222 / 7 & 3.82 \mathrm{~d} & 3 . \mathrm{E}-09 \\ \mathrm{Th}-233 / 2 & 22.3 \mathrm{~min} & 5 . \mathrm{E}-07 \\ \mathrm{~Pa}-234 / 1 & 6.70 \mathrm{~h} & 1 . \mathrm{E}-08 \\ \mathrm{~Pa}-234 \mathrm{~m} / 2 & 1.17 \mathrm{~min} & 8 . \mathrm{E}-07 / 3 \\ \mathrm{U}-239 / 1 & 23.40 \mathrm{~min} & 4 . \mathrm{E}-07 / 6 \\ \mathrm{~N} & 65 \mathrm{~min} & 2 . \mathrm{E}-08 \\ \mathrm{~Np}-240 / 1 & 7.4 \mathrm{~min} & 6 . \mathrm{E}-08 \\ \mathrm{~Np}-240 \mathrm{~m} / 2 & 25.0 \mathrm{~min} & 2 . \mathrm{E}-08\end{array}$

11 Committed effective dose equivalent from inhalation is calculated in ICRP Publication 30 , but the DCG value for external exposure to a contaminated atmospheric cloud is more restrictive than the DCG value for inhalation.

12 Committed effective dose equivalent from inhalation is not calculated in ICRP Publication 30, but DCG value for external exposure to contaminated cloud should be more restrictive than DCG value for inhalation due to relatively short half-life of radionuclide.

13 DCG value is determined by 1 imit on annual dose equivalent to skin, rather than limit on annual effective dose equivalent.

14 DCG value applies to radionuclide in vapor form only; DCG value for inhalation is more restrictive for radionuclide in inorganic form.

15 DCG value applies to radionuclide in inorganic or vapor form.

16 OCG value for exposure to contaminated atmospheric cloud is the same as OCG value for inhalation.

17 The value shown for radon gas is a result of unit conversion from $3 \mathrm{pCi} / \mathrm{L}$ to 3 micro $\mathrm{Ci} / \mathrm{mL}$. 